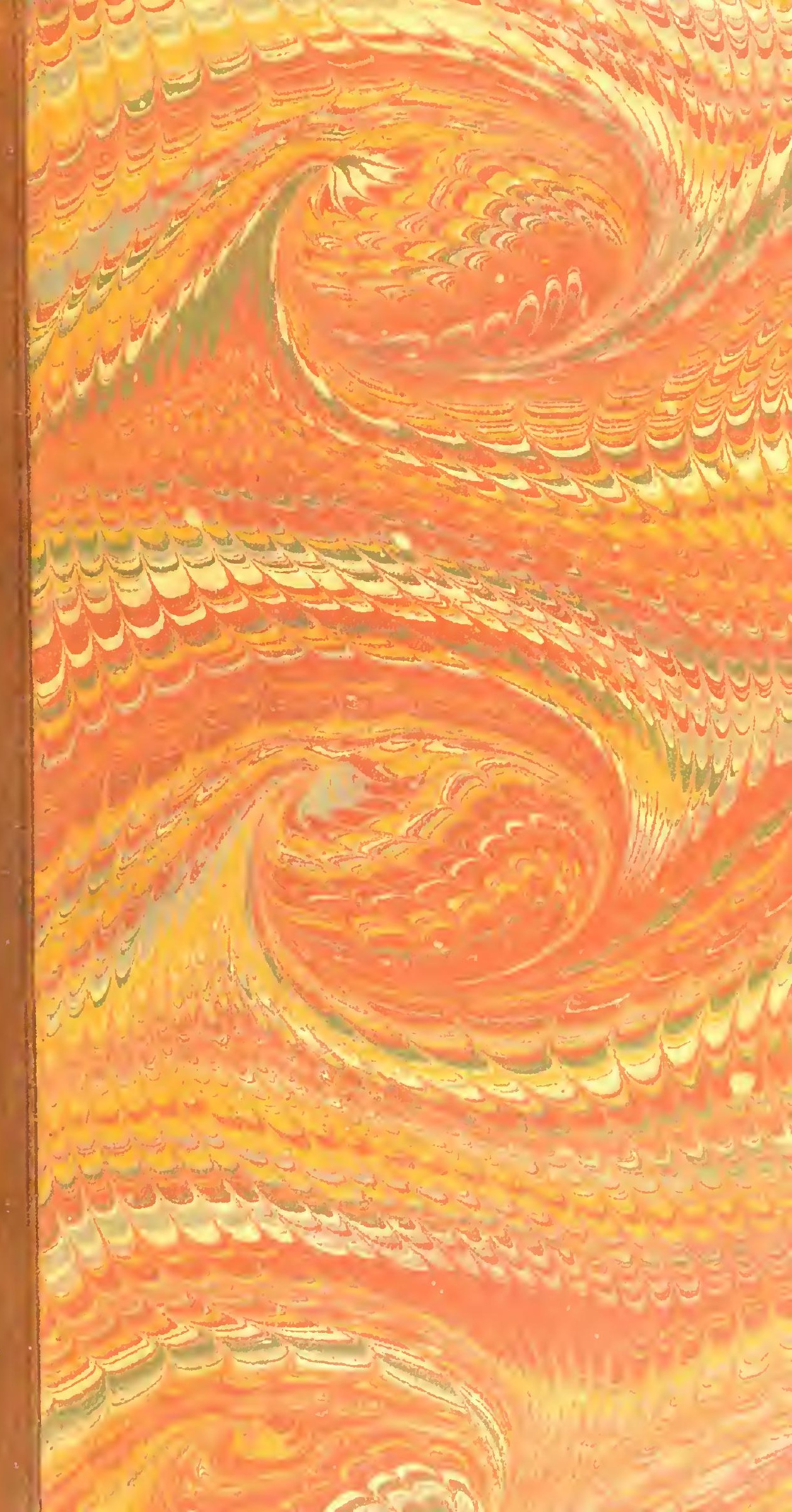




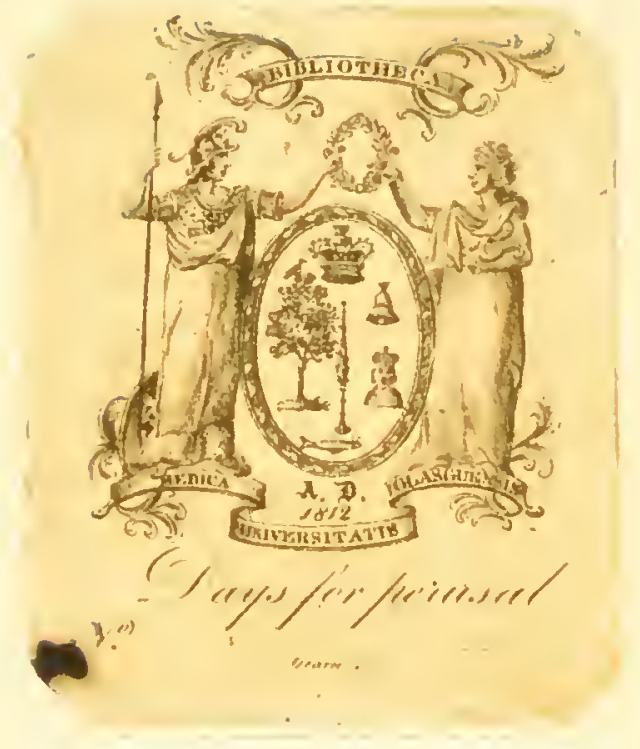

(5) ingatw

alnibrsity Eibrarn

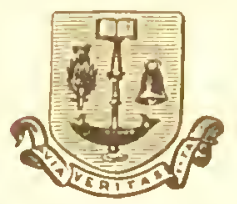

$N N-4.3$ 
Digitized by the Internet Archive in 2016 





\section{RECHERCHES}

ANATOMIQUES ET PHYSIOLOGIQUES

SUR

\section{LA STRUCTURE INTIME}

DES ANIMAUX ET DES VÉGETAUX,

IT SUR LEUR MOTILITÉ,

P A R

11. H. DUTHOCHET,

DOCTEOR EN MEDECINE, CORRESPONDAXT DE L'IXSTITUT DF FRAXCE DANS

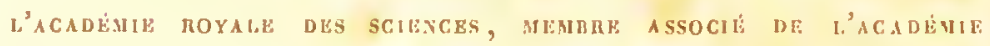
ROXALE DI MEDECINF, DES SOCIETES PHILOMATIQUE, LTYNLENNE кT

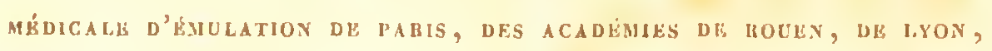
DR Toulovsk, etc.,

AVEC DEUX PLANCIIES.

A PARIs,

C II EZ J. B. BAICI, I ERE, I I B R I R I,

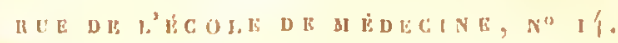
1824 . 



\section{REGHERCHES}

ANATOMIQUES ET PHYSIOLOGIQUES

\section{SUR LA STRUCTURE INTIME}

\section{DES ANIMAUX ET DES VÉGE'TAUX,}

ET SUR LEUR MOTILITÉ.

\section{INTRODUCTION.}

Tous les êtres vivants sont susceptibles de subir certaines modifieations vitales, par l'influence de eertains agents qui leur sont extérieurs. Les physiologistes on donné le nom de sensibilité à la faculté, i la propriété vitale, en vertu de laquelle a lieu cettc influence des causes extérieures sur l'être vivant. Ce que nous appelons sentir ne se peut guère délinir; ehaeun sait ee que e'est par sa propre expéricnee. Ce sont nos sensatious qui nous donnent la conseience de l'existence, qui font que nous avons un moi. Toutes les fois que nous observerons, dans un être vivant, des preuves bien certaines qu'il possèle la conseicrice de l'existence, nous pourrons aflimer, 
par cela même, qu'il possède la sensibilité; nous serons autorisés à lui refuser cette faculté lorsqu'au contraire il nous sera bien démontré qu'il ne possède point la conscience de son existence individuelle. Les végétaux sont daus ce dernier cas: personne, je pense, ne sera tentć de leur accorder un moi; et par conséquent des sensations; cependant. ils manifestent souvent, par les mouvements qu'ils exécutent à l'occission de l'influence de certaines causes extérieures, qu'il se passe chez eux un phénomène analogu' i celui que l'on appelle sensation chez les animaux. Les physiologistes de l'école de Bichat considèrent ce phénomène comme appartenant à la scnsibilité que cet auteur nomme organique; sensibilité d'une nature particulière, qui n'est point une source de sensations, et qui existe de même dans les organes intćricur's des aninaux. Chacun connaît la distinction que $\mathrm{Bi}-$ chat a établic de deux vies, l'une animale, l'autre organique, chez les animaux. Selon ce physiologiste, ces deux vies possèdent chacune une sensibilité particulière: la sensibilité animale est la seule qui soit une source de sensations; la sensibilité organique n'en procure aucune. $\mathrm{Or}^{\mathrm{x}}$, si l'on prétend que, dans l'exercice de la sensibilité organique, la sensation est bornće à la parie sur laquelle agit la cause qui la met en jeu, on est conduit par cela même à admeure dans cette partie des sensations individuclles et un moi particulier. Le corps d'un animal devient de cette manière un assemblage d'êtres qui ont tous leurs sensations, leurs appétences, leur's aversions parli- 
culières. Cette tínéorie entraîne nécessairement l'idée d'un moi particulier, d'une volonté particulière dans chaque organe. Ceute hypothèse est évidemment inadnissible. On ne peut véritablement point dire que les organes qai ne procurent jamais de sensations aient de la sensibilité; cependant les organes intérieurs des animaux exécutent des inouvements sous l'influence de certaines canses qui leur sont extéricures; ils ont done ane proprióté vitale analogne à la sensibilité. Cés conséquences contradictoires prouvent que c'est à tort que l'on sc sert en physiologie du mot sensibilité. Que l'on supprime ce mot, lequel ne réveilie que des idées purement morales, et qu'on le remplace par une cxpression qui représente la nature matérielle du phénoricne en question, et tontes les difficultés disparaîtront à cet égard. Nous pouvons trouver cette expression nouvelle dans l'étude de la manière dont nos sensations sont produitcs. Les aggents extérieurs, lorsqu'ils nons fontéprouver des sensations, produisent une modification d'ime nature quelconque dans les sens sur lesquels ils agissent; il y a par conséquent production d'un monvement particulier; l'organe est remué. Nous ignorons quelte est la nature de ce mouventent, mais son existence n'en est pas moins incontestable. Cic nouvement est transmis, par le canal des nurls, au cerveau, siége unique du moi, et par conséquent des sensations. de donne ì ee phénomène de mouvement, produit dins les sens par les agents du dehors et transmis par les nerfi, le nom de nervimotion, a i la proprictiv vitak 
en vertu de laquelle il a lieu, le nom de mervimotilité '; je donne aux agents extérieurs qui sont susceplibles de produire la nervimotion, le nom d'agrents nervimoteurs. La nervimotion est un phénoniène purement physique; il précède constamnent le plénomène noral de la sensation, nrais il n'en est pas toujours suivi: ainsi nos organes intérieurs possèdent la nervimotilité, ils éprouvent li nervimotion; mais il n'en résulte point de sensation, cumme cela a lieu pour nos organes extérienrs; ceci tient à des secrets particuliers de la vie. Celte distinclion étant une fois bien établie entre les phénomènes moraux el les phénomènes physiques, la science de la vie devient plus simple et plus facile; elle peut même devenir unc science exacte. Il était impossible d'appliquer des mesures à la sensibilité et à la sensation, tandis que la nervimotilité et la nervimation sont susceptibles de mesures, comne tous les pliénoniènes physiques. Je le répète, ce n'est qu'en bamnissant de la physiologrie toutes les expressions qui n'éveillent que des idées morales, qu'on se inetura sur la voie de lui faire laire de nouveaux progrès. La nature de la sensibilité, comme celle de la sensation, est totalentent inaccessible à notre investigation. Notre taculté de sentir est

M. Flourens, dans ses Rechorches sur les fonctions dusystcme nerveux, uomme irritabilité la propriété vilale que je désignc ici suus le nom de ncrvimotilitc. Je regrelte de ne pouroir adopter ave lai celte expression, qui, détournéc ainsi du sens qui lui a été donné par tous les plysiologistes, ne pourrait que produire la plus grande ennfusion dans les idées. 
culle à l'aide de laquelle nous connaissons, il nous est par conséquent impossible de la connaîlre ellenême. Il est done eontrairc à la saine raison, à la boune philosophie, de placer dans une scienee d'observation, telle que la physiologie, celui de tous les phénomènes de la nature qui est le plus néeessairement soustrait ì nos recherches; l'étude de la sensibilité et de la sensation appartient exclusivement ì la psychologrie.

La vie, eonsidérée dans l'ordı’e physique, n'est autre chose qu'un mouvement: la mort est la eessation de ee mouvement. Les êtres vivants nous offrent diverses laeultés de mouvement; à leur tête est la nervimotilité, faeulté d'éprouver certaines nodifiealions, certains changements dans leur être, par l'influenee de eertains agents du dehors, ou des agents nervimoleurs. Ce premier mouventent, qui est invisible, est la source des mouvenients visibles qu'exéeutent les parties vivantes. Ĺa faeulté d'exéeuter ees mouvencnts qui dćplacent les parties pent recevoir le nom de locomolilicé : Alle offie denx mouvements opposés, la conlraclion el la largescence. Tontes ces faeultés de mouvements se rattaehent ì mue scule - laculé générale, que je désigne sous le nom de molilélé vilalc ': c’est lit vie elle-même.

Ia motiliti vitale nous offre, chez tous les êtres

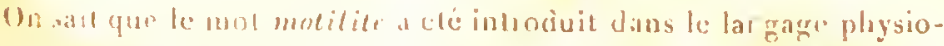

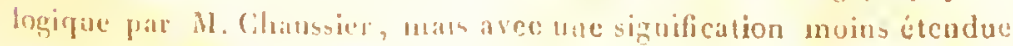
que celle que je lui donne ici. 
vivants, les mệmes phénomènes principaux. Partout il y at nervimotilité, et par conséquent nervimotion sous l'influence des-agents nervimoteurs; pariout aussi il y a locomotilité ou faculté de ehanger la position des parties. Les végétaux offrent, comme les animaux, ces deux facultés de mouvement; mais elles sont, chez eux, bien moins énergiụnes, bien moins développées: Il esi fort peu de.végétaux dont les parties soient susceptibles d'exécuter ces mouvemenis brusques, rapides qui, tels que ceux que l'on observe chez la sensitive, frappent d'étonnement pai leur ressemblance avec les mouvements des animaux; mais tous les végétaux ont la faculić de donner une direction spéciale à leurs diverses parties, et cette facnlté se ratlache aux lois générales de la motilité vitale, ainsi quic cela sera démontré dans le cours de cet ouvrage. L'étude des lois qui présidert à la moıilité vilale esı, clıcz les animaux, d'une difficulıé peutétre insurmontable, à raison de l'extrêne consplication-des causes, tant intérieures qu'extérieures, qui peuvent influer sur l'état de cetre motilité. Liétude, ¿̀ cet égard, se simplifie beaucoup chez les végétaux, et c'est probablement à eux seuls que l'on devra lat solution des principaux problèmes de la science de la vie. Les secrets de cette science sont disséminés dans tout le règne organique; aucun être en particulier et même ancune classe d'êtres ne fournit les moyens faciles d'apercevoir tous ces secrets. Le physiologiste doil donc interroger tous les êtres vivants sans exception: chacun d'eux lui dira son mol; 
INTRODUCTION.

chacun d'eux soulèvera à ses yeux une portion particulière du voile dont la nature couvre ses nrystères; et c'est de l'universalité de ces recheŕches que sortira la connaissance complète des phénomènes de la vic. 


\section{SECTION Ire.}

()BSERVATIONS SUR L'ANATOMIE DES VÉgétaUX, ET SPÉCIALENENT SUR C'ANATONIE DE LA SENSITIVE ( mimosa pudica. L.).

L'anatomie végétale, étudiée avee le plus grand soin par les obscrvateurs les plus exereés, est eertainement arrivée au dernier degré de perfeetion auquel il soit possible de la conduire par les moyens mis en usage pour eettc étude. Que pourrait-on, en effet, attendre de nouveau de l'observation mieroscopique des organes des végétaux, après les reeherches de Leuwenhoeek, de Grew, de Malpighi, d'Hedwig; après les travaux réeents de nessieurs Mirbel, Link, Tréviranus, Sprengel, ete.? On doit penser qu'après de pareils observateurs il y a bien peu de chose : faire, à moins que l'on ne trouve de nouveaux moyens d'investigation. Bien persuadéde cctte véritć, ja ai eherché, par des essais nombreux, à rendre plus facile qu'elle ne l'a étć jusqu'à ce jour l'étude de l'anatomie végétale, et j'y suis parvenu au moyen d'un proeédé bien simple. Le plus grand obstacle que la nature ait mis à l'élude des organes intérieurs des végétanx n'est pas leur extrême petitesse; c'est la difficulté d'isoler ces petits oryanes les uns des autres pour les étudier sćparément. Lcur forte adhérence mutuelle rend cet 
isolement presque impossible; de plus, ces organes sont opaques pour la plupart, ce qui augmente la difficulté de leur observation, qu'on ne peut faire qu'avec le secours du microscope. J'ai essayé divers moyens pour renıédier à ee double ineonvénient, et j'en ai trouvé un qui a parfaitement rempli le but que je me proposais. Je place un fragment du végrétal que je veux étudier dans une petite frole remplie d'acide nitrique, et je plonge cette fiole dans l'eau bouillante. Par eette opération, les parties qui composent le tissu végétal perdent leur agrégation et deviennent transparentes, ce qui facilite singulièrement leur étude. En même temps les trachées et les autres vaisseaux se remplissent d'un fluide aériforme, ce qui leur donne au microscope un aspect tout particulier, et foumit un nouveau moyen pour les observer. On sent qu'il ne faut pas que cette opération soit poussée trop loin, car le tissu végétal seraic tout-à-fait désorganisé: c'est à l'observateur à limiter le temps que le végétal doit rester dans l'acide nitrique, et cela selon la délicatesse phus ou moins grande de son tissu. Moins l'ébullition est prolongréc, mieux cela vaut: cn sénéral, il ne faut pas attendre que le tissu végétal soit devenu tout-ì-fait transparent, et qu'il se divise spontanément. Avant cette éporque de dissolution, il est déjà devenu lacile à dóchirer dans l'eatu avec des pinces, et ses éléments organiques dissociés sont devenus ıres faciles à étudier. Pour laire cette obscrvation, je place dans l'ean, contenue dans un cristal

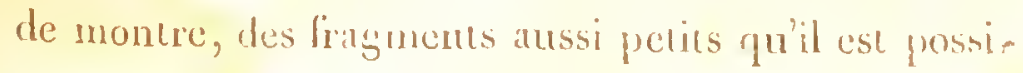


ble de se les procurer par la division mécanique, et je les soumets au microseope.

C'est le désir de connaître l'anatomie particulière de la sensilive (mimosa pudica L.) qui m'a engagé lans ces recherches, que j'ai ćtendues ensuite à beaucoup d'autres végétaux. Ce sera donc l'anatomie de cette plante qui me servira de texte. J'y rattacherai des considérations sur l'organisation des autres végétaux, lorsque cela me paraîtra nécessaire pour éclaicir des points obscurs, et résoudre certaines questions.

Je commiencerai l'étude anatomique de la sensitive par l'examen de la moclle. Elle est, comme celle de tous les végétaux, entièrement composée de tissu cellulaire. Les cellules qui lia composent offrent une forme hexagonale assez régulière dans quelques endroits, et, dans d'autres, leur lorme est tout-à-fait irrégulière; en général, elles sont disposées en séries longitudinales. Girew a comparé le tissu cellulaire à l'écume d'une liqueur en feimentaion, et M. Mirbel adopte cette comparaison, qui s'accorde partivitement avec la manière dont il considère le tissu cellulaire. En effet, il admet que les cellules ont une parni commune là où elles se touchent, en sorte qu'elles seraient pratiquées dans un tissu nembraneux continu; unais l'observation infirme cette assertion. Eu eflet, lor'syu'on soumet à l'ébullition dans l'acide nitrique la noelle de la sensitive ou celle de tom antre végétal, on voil tontes les celluies se séparer les unes des antres, el se présenter conme autant de vésicules con- 
plètes qui conservent lenr forme, laquelle leur avait été donnée par la compression que les cellules voisincs exerçaient sur elles: ainsi, partout où deux cellules se touchent, la paroi qui les sépare offre une double membrane. On voit d'après cela que la comparaison du tissu cellulaire à l'écume manque tout-àfait de justesse.

Dans la moelle de la sensitive, chaque cellule porte plusieurs corpuscules arrondis, opaques dans leurs bords, et trans parents dans leur milieu. (Fig. 1.) Ces petits corps à demi opaques, et percés, en apparence, dans. leur milieu, ont été observés dans le tissu cellulaire de beaucoup de végétaux par M. Mirbel : il les considere comme des pores environnés d'un bourrelet opaque et saillant. L'observation de la moelle de la sensitive ne me permettait guère d'admeture cette assertion; en effet, le tissu cellulaire dont elle est composée est incolore et d'une transparence parfaite, tandis que le trou prétendu qui est au centre des petits corps dont il est ici question transmet à l'ceil une lumière verdâtre. Il me parut que ces petits corns n'étaicnt autre chose que des petites cellules golobuleuses, remplies d'une matière verdâtre transparente, lesquelles, en leur qualité de corps sphériques transparents, rassenblaíent les rayons lumineux rlans un loyer central, ci devaient, par conséquent, paraître opayues dans leur ponrtour. Cliacun sait que tel est l'ellet de la réliaction des rayons luninem par les corps transparents sphériques on lenticularies. Ce sompen s'est changri 
en certitide par l'observation de l'effet que produit l'acide nitrique sur ces corpuscules transparents: en effet, lor'squ'on fait chanffer la moelle de la sensitive dans cet acide, les cellules acyuièrent une grande uransparence, et les corpuscules dont il est ici question deviennent complètenicnt opaques; leur centre ne transmet plus aucun rayon de lumière. Cette ohservation prouve d'une nanière incontestable que les petits corps qui sont situés sur les parois des cellules ne sont pas des pores environnés d'un bourrelet opaque, comme le pense MI. Mirbel, nıais que ce sont véritablement des petites cellules globuleuses, remplies d'un fluide qui cst concrété et rendu opaque par l'acide nitrique. On sait que les fluides qui ont été concrétés par les acides sont ordimairement dissous et fluidifiés de nouveau par les alkalis. Il était inportant de savoir si ce phénomène chinnique se manificsterait par rapport aux corpuscules concrétés de la moelle de la sensitive. J'ai donc placésur une lane de verre quelques fraggnents de cette moelle dont les corpuscules étaient devenus opaques par l'action de l'acide nitrique; je les ai couverts d'une grosse goulte de solution aqueuse de potasse carstique ', et j’ai présenté la lame de verre avec précaution à la flamne d'une lampe ì esprit de viu, afin que la chaleur favorisât la dissolution. Au bout de quelques minutes, ayant cximminc

- Cost de la potasse causlique a la chanx, ou hydrate de pulisane, yue j'ai lait usage. 
ce tissu cellnlaire au microscope, j’ai tronvé tous les corpuscules transparents dans leur milicu, avec une teinte verdâtre, conme cela avait lieu dans l'état naturel : ainsi il est évident que l'alkali avait dissous et rendu transparent le fluide que l'acide avait concrétć et rendu opaque. Cette double expérieuce, qui sera répétée souvent dans la suite de cet ouvrage, ne permet done plus de douter que les corpuscules arrondis dont il est ici (puestion ne soient, comme je l'ai dit plus haut, de petites cellules globulcuses remplies d'un flide concrescible par les acides et soluble dans les alkalis. Il n'y a point de végétal dont le tissu cellulaire ue soit nuni avec pius ou moins d'abondance de ces petites cellules globulcuses, qui sont situćes dans l'épaissenr des parois des grandes cellules; nous verrons plus bas qu'on les trouve aussi à la surface de certains tubes végétaux. Quelle est la nature, quels sont les usiges de ces corpuscules globuleux vésiculaires?' c'est ce qu'il est impossible de déterminer par l'étude des seuls véscétaux. Ce n'est que l'étude comparée de la structure intime des animaux qui peut ici uous foumio des lımic̀res. Les recherches microscopiques de plusieurs observatcurs, recherches qui seront exposćes plus bas, ont appris que tous les organes des animaux sont composés de corpuscules gुlobuleux ağglonıérés. Il est évident que ces corpuscules sont les analogues de ceux que nous venons d'ohserver dans le tissu organique des végétaux, chez lesquels ils sont infuniment moins nombreux qu'ils ne le sont chez les animanx. 
Cette observation nous montre une certaine analogie de structure organique entre les végétaux et les animaux, mais elle ne nous éclaire point sur les fonctions de ces petits organes ylobuleux. Comme ils eomposent tous les organes des animaux, cela prouve que ee n'est point de leur forme qu'il fnut tirer des inductions pour déterminer leurs fonctions; mais, chez les animaux, la nature chimique de ces corpuseules globuleux n'est point partout la même. Ainsi, ceux qui composent lcs museles sont solubles dans les aeides, tandis que cenx qui composent le système nerveux somt insolubles dans ces mêmes acides, mais seulement solubles dans les alkalis. Or, telle est aussi la nature chimique des corpuseules globu'eux que l'on observe dans les végétaux, ainsi que nous venons de l'exposer. Ceei peut donc autoriser à penser que ces corpuscules globuleux sont des organes nerveux, ou plutôt que ce son les éléments" épars d'un système nerveux diffus, ou qui n'est point réuni en nuasses, comme il l'est ehez les animaux. Celte considération, appuyée sur l'analoģic de la nature chimique des corpuscules globuleux, est encore fortifiée par l'observation de la strueture intime du système nerveux de certains animaux: ainsi, chez les mollusques gastéropodes, la substance médullaire du cerveau est composéc de cellules globuleuses agghlomérées, sur les parois desquelles il existe une grrande quantité de corpuscules globuleux on ovoïdes, comme on le voit dans la figure 20. Ces corpusculcs, de couleur blanehe, sont évidem- 
ment de très petites eellules remplies de substanee médullaire nerveuse; elles sont situées sur les parois des grandes cellules qui contiennent une substanee deni-transparente. La similitude de ectte organisation avec eelle dn tissu eellulaire médullaire des véçétaux est évidente : nous voyons ici de même de petites ecllules globuleuses, remplies de substanee conereseible par les aeides, et situées sur les parois des grandes eellules. Cette analogie très remarquabie de strueture qui existe entre le tissu cellulaire médullaire des végétaux et la substance du cervean des mollusques est done une analogie de plus, qui sert it étayer l'opinion que nous venons d'émeture sur la nature et sur les fonctions des eorpuscules véggétaux que nous eonsidérerons eomme des molécules nerveuses éparses sur les parois des ecllules; et, en effet, les phénomènes singuliers que présentent les végétaux irritables ne permettent guìre de donter qu'il n'y ait chez eux quelque chose d'analogue anx fonctions que remplit le systène nervenx chez les animaux. Ces phénomènes teudent, par conséquent, à prouver qu'il y a ehez les plantes, sinon un système nervenx, au moins quelques éléments de ce système. On sent qu'il serait impossible de trourer un plus grand nonibre de prenves iirées de l'analogic entre les animaux et les végétaux, pour étahlir , chez ees demier's, l'existence des éléments du sysic̀me nervenx. L'immense distance qui sépare ces deux classes d'êtres ne laisse subsister entre elles alicune de ces analogies comprimbiós de la forme générale or 
de la position des masses qui nous servent, dans l'anatomie comparée des animaux, à déterminer la nature des organes. Déjà ces analogics ont disparn graduellement chez les zoophytes; il ne reste, che: les végétaux, lorsquion veut les comparer aux animanx, que les analogies emprnntées de la formı, de la position, et de la nature chimique des particules qui composent le tissu organique. Lor's donc que nous avons saisi ces analogies des particules, nous avons saisi tout ce qu'il y a de comparable dans la structure organique des végétaux et des animaux. Fondé sur les observations qui viennent d'être exposćes, je n'hésiterai donc point à considérer les corpuscules globuleux de nature concrescible qui sont situćs dans les parois des cellules des régćtaux, comme des corpuscules nerveux; je les désignerai dorénavant sous ce nom, que l'on devra considérer commc une expression abrégéc qui signifie une cellule globulense microscopique, remplie de substance nerveuse.

Les cellules de la moelle ne contiennent que de l'air dans les liges de la sensitive un peu âgées; mais lorsque ces tiges sont naissantes, comme elles le sont aux extrémités des rameaux, les cellules de la moelle contiennent un fluide diaphane concrescible par la chaleur et par les acides, et soluble cependant dans ces dernicrs. Pour voir cela, il faut eouper une tranche de moclle extrênement mince, ec la niettre dans un peu d'eau. Cette tranche transparente, obscrvéc au microscope, ne fail apercevoir que des eellnles dia- 
phanes dans les parois desquelles on distingue une grande quantité de corpusculès nerveux; mais si l'on trempe ceue tranche dans l'acide nitrique froid pendant une ou deux minutes, on voit que plusieurs de ces cellules deviennent opaques, tandis que les autres conservent leur transparence, comme on le voit dans la fignnre 2. C'est spécialement auprès de l'ćtui médullaire que ces cellniles opaques sont nombreuses. Cette observation prouve que ces cellules contiennent, dans l'état naturel, un fluide diaphane qui est concrété par l'action à froìd le l'acide nitrique. Si l'on fait chauffer dans cet acide la petite tranche dont il vient d'être question, toutes les cellules qui étaient devenues opaques reprennent leur transparence; il y a dissolution complète de la substance concrétce qu'elles contenaient.

L'étui médullaire de la sensitive est composé d'une assez grande quantité de trachécs qui, dans l'état naturel, ne se dérouient point; elles sont extrêmement. petites. Lorsqu'on fait bouillir la tige de ceue plante dans l'acide nitrique, les trachées se remplissent d'air; on les voil alor's très facilement, d'antant plus que te lissn végrétal environnant a acquis de la transparence. La propriété de l'acide nitrique à chand étant de tétruire l'agrégation des organes qui composent les vérgétaux, il en résulte que par ce moyen les trabhées s'isolent tout-ì-fait du tissu végétal environnant, (1 qu'elles deviennen plus laciles à déronler yrielles ne: l'éarient auparavant. J'esperais, par ce moyen, nhenir le déroulement des barahres de la sensiitve; ar- 
pendant, quoique je lenr eusse fait subir unc ébullition de dix minutes, elles refusèrent de se dérouler: oll pourrait penser, d'après cela, que ce ne sont point des trachces. On sait que M. Mirbel a admis chez les vérgétaux des fausses trachées, c'est-à-dirc des tubes qui, par leur aspect, resscmblent extérienrement :tux traehées, mais qui en diffèrent parcequ'ils ne se déroulent point eonme clles : mais tcls ne sont point les vaisseanx de la sensitive dont il vient d'être question; car, en les faisant bouillir pendant long-temps dans l'aeide nitrique, ils finissent par devenir susceptibles de se dérouler. Ainsi, l'impossibilité qu'il y avait de dérouler ces trachées dans l'ćtat naturel provenait de l'adhérenec nutuclle de leurs spires qui étail plus fortc que la ténacité du fil spiral, en sorte que celui-ci se rompait plutôt que de quitucr l'adhérence qui l'unissait aux spircs roisincs. Une longue ébullition dans l'acide nitrique détruit ectue adhérence, et alors les apparcntes fausses trachées se trouvent être des traehées véritables. M. Link, dans ses Recherches sur l'anatomie des plantes', a fait mention de ees trachées qui ne se déroulent point; il les nomme vaisseaux en spirale soudée. Au reste, j'ai observé que les spires des traehćes sont. unies entre elles par une membrane transparentc qui se déehire lorsyu'on déroule le fil spiral; cela sc voit avec facilité lorsque, par l'ćbullition dans l'acide nitrique, on a eomplètement isolé des traehćcs qui

- Innales du Muscum d'tistoire nalurctic, lome in. 
se trouvent remplies d'irr, ef dont les spires sont un peu éloignées les unes des autres. L'un des végétaux yui se prête le mieux à ce genre d'observations est le solanum tuberosum. Les pétioles des leuilles de cette plante contiennent des trachées très grrosses et plongées dans un tissu très délicat, ce qui rend lenr observation très facile, surtont à l'aide de l'aeide niuriqunc. On peut, sur ce végétal, voir avec flacilité la membrane qui unit entre elles les spires des trachées. MI. Mirbel a fait mention de cette membrane dans son Traité d'anntomie et de plyssiologie végétale; car il dit positivement que la trachée, en se déroulant, présente quelquefois deux filets réunis par une membrane internédiaire. Il dit un pen plus bas: On peut conjecturer'as'ec quelque apparence de raison que, dans beaucoup de cas, les trachées ne se déroulent. que par'cequ'on déchiv'e les membranes qui unissent les spir'es entr'e elles. Mais, quittant bientôt cette manière de voir', qui ne s'aceordait pas avee sa tlıéorie, M. Mrirbel considère les traehées eomme formées d'une lame roulće en spirale, l,ordée souvent de petits bourrelets calleux ${ }^{2}$; à l'appui de celle opinion, il donne la lignure très grossie d'unc portion de trachce, figure dont je reproduis ici l'analogue (fig. 5, a). Dans l'explication que 11. Hirbel dome de cette tigure, il considère la trachóc conne ayaut des fentes uransversales horiées en dessus el en dessous par le

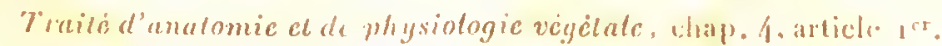

Eténents al: physiotogic rigetule et de botuneque, page ja. 
bourrelet on cordon saillant que l'on voit icide cliaque côté de la lame qu'a formée la trachée en se déroulant. D'abord je dois faire observer que la lorme de trachée représentée iei est assez rare; je ne l'ai observée que dans quelques uraehées du surean (sambucus nigrra). Ici la trachéc est composéc de deux fils spiraux juxtaposés et formant une lame opaque par leur réunion; cette lame est roulce en spirale dont les spires sont ćeartées les unes des autres, et leurs intervalles sont remplis par une membrane transparente $c$. Lorsquion essaie de dérouler cette trachée, le déroulement s'opère par la séparation des deux fils qui forment la lame opaque, en sorte que la membrane transparente qui remplissait les intervalles des spires se trouve rester intacte el bordée de ehaqne eôté par un fil opaque, qui est la moitié de la lame opaque qui eomposirt la spire de la trachéc avant son déronlement. J'ai représenté la eontinuation de cette trachée non déroulée en $b$. Celte figure fera voiv, mieux que l'explieation que j'en pourrais donner, l'erreur oil est tombé M. Mirbel, en prenant pour une lane spirale de trachée ee qui n'est dans le lail que la nembrane intermédiaire anx spires, bordée de chaque côté par un des deux fils spiraux qui forment cette lame par leur réunion. L'adhérenee mutuelle de ees deux fils étant moins lorte que ne l'est la résistanee de la membrane internédiaire aux spires, il en résulte que le déroulentent de la urachée s'opère seulement par la séparation de ces denx fils qui, dans l'état naturel, ne sont point séparés par nne lente conme l'admet 
M. Mirbel. An reste, on sait que les trachées, qui sonveme n'ont qu'un seul fil spiral, en possèdent 'puelquefois deux, trois el quatre, ainsi que je l'ai observé moi-même; M. Link en a compté jusqu'i: sept: Ces fils spiranx, yui se snivent parallèlement, forment, par leur assemblage, une lame en spirale phus on moins large; ct la réunion de ces fils, opérée par une unembrane interméliaire quelyuefois aperecvable, ne laisse point subsister de fentes entre cux. Ainsi les trachées n'ont poim de lentes trmsversales en spirale, comme le pense M. Mirbel, qui trouve dans ees fentes et dans les bourrelets prétendus qui les bordent, une transitiou heureuse pour passer des trachées aux fausses trachées, dans lesquelles il a crul recommintre des fentes transper'sales bordées de borurrelets, fintes qui, selon lui, ne diflërent que par lem forme alongée, des pores, égalentem borresés d'un boumelet. Nous avous prouvé plus ha:ll que ces prétendus pores n'existem point dans le tissu cellulaire; nous verrons tom à l'heure yu'ils n'existent poim non plus sur les lubes frue il. Hirtbel appelle: poreux. Nous venous de voir yue les larachées n'ont point de fentes transversales en spirale; nous verrons dans mu iustaut que les fiuster luachíes ne som point non p!us lendues traunversalenent.

Les trachées sont, en général, les tuber dont

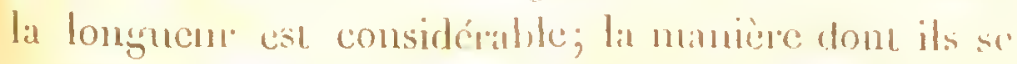

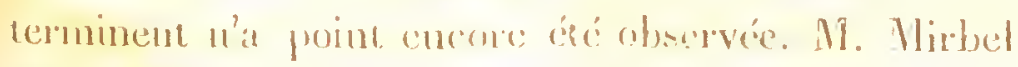
prétend que ces mbes se mótmorplosent vers leme

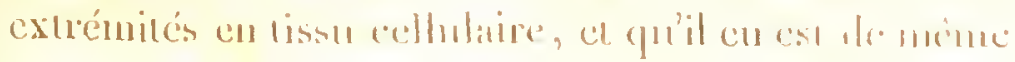


des autres tubes végétaux. Cette assertion est encorc infirmée par l'observation. J'ai vu dans les pétioles des feuilles du noyer (juglans regia), et dans l'éui médullaire dn surean ( sambncus nigra), que les trachées se terminent en devenant des spirales coniques dont la pointe devient très aiguë, comme on le voit dans la figure 4 ; jai vu que cctte terminaison des trachées étai la même en haut et en bas, c'està-dirc à la basc et au sommet de ces tubes spiraux.

Les trachées. sont très souvent munics extérieurement de corpuscules nerveux plus ou moins nombreux. On peut faire cette observation avec lacilité dans les tiges du solanum tuberosum et du cucurbita pepo, en dissociant leurs parties constituantes par le moyen de l'ébullition dans l'acide nitrique, qui rend opaques les corpuscules nerveux, lesqucls, dans l'état naturel, ne sont point apercevables, à cause de leur transparencc. On voit, dans ces deux végétaux, les trachées accompagnées souvent de deux rangées de corpuscules nerveux qui restent adhércnts à leurs spires lorsqu'on les déroule, conme on le voil dans la figure 5. Ces corpuscules concrétés par l'acide nitrique, étant mis dans la solution acqucusc de potasse caustique, y deviennent fluides ct transparents : ainsi il n'y a pas de doute qu'ils ne soient tout-à-fait semblables à ceux yui sont situés dans les parois du tissu cellulaire. Quelquefois les trachées sont couvertes de rangées transversales de corpuscules nerveux, comme on le voit dans la figurc 6, qui représente une trachéc du clematis vitalba. Une portion 
de cette trachée se trouve dépourvine de corpuscules nerveux, el cela ne provient évidemmient que de ce que ces corpuscules out été enlevés par la manière dont s'est opérée la déchirure du tissu végétal, ear ils n'adhèrent que faiblement aux trachées sur lesquelles ils sont applirqués; ils ne font point partie cssenticlle de leur organisation. Il n'en est pas de même des corpuscules que l'on observe ì ha surface des tubes que M. Mirbel a nommés tubes poreux ( figg. 7 ), pareequ'il prend les corpusenles nerveux qui les couvrent pour des pores environnés d'un hourtelet opaque et saillant. I c ubbe que je représente est emprunté all surean ( sambucus nigra). Ces corpuscules sont ici contenus dans les prarois mêues du whe qui tes porte; ils ne peuvent jannais en être séparés. J'ai démontré plus haut que M. Mirbel était tombé dans l'erreur en prenant les corpuscules nerveux du tissu cellulaire pour des pores; les mêues preuvés me serviront ici pour démontrer la vérinable nature des prétendus pores de ses tubes porerux. Mans m grant nosubre d'observations et d'expériesses que j’al laites sur les vaisseaux corpusculifêres de beaucoup de végétanx, j’ai conjours vu que les coppuscules quits offiaient se comportaicm exatemem comme cens du issu celhulaire, lor'squ'ou les somucutio à l'action de l'acidentripue on de la potasse camstiple. Le premier les rend oparques el paraít les coneréter ; la seconde les rend hansparents et les dissoun. Ainsi il uo

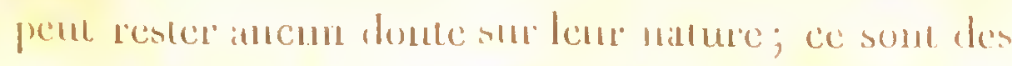

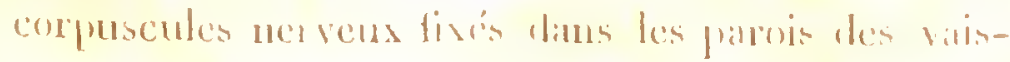


seaux, conme ils sont situés dans les parois des cellules. Il n'y a donc point de vaisseaux poreux, suivant l'acception que M. Mirbel donne à cette expression. Déjà M. Link avait émis l'opinion que les points obscur's que l'on remarque dans le tissu cellulaire et à la surface des vaisseaux ne sont pas des pores entourés d'un bourrelet saillant, mais que ce sont des petits grains transparents an milien"; il pense qu'il èn est de même des liğnes transversales obscures et interrompues qu'on observe dans les vaisseaux, auxquels cet observateur donne, avee M. Mirbel, Je nom de fausses trachées. On sait que ce dernier naturaliste considère ces lignes transversales interrompues comme des fentes bordées d'un bourrelet. Si l'on veut observer ces vaisseaux avee faeilité, il faut soumeure à l'éloullition dans l'acide nitrique un morcean de bois de vigne (vitis vinifer $a$ ), et cela pendant un espace de temps suffisant pour que l'agrégation de ses parties constituantes soil presque complètement délruite; alors on observe avec la plus grande facilité tous les organes qui entrent dans sa composition. Lorsque l'on coupe transversalement le bois de la vigne, on déeouvre, à l'œil nu, les ouvertures d'une grande quantité de gros tumes: ce sont des fausses trachées de M. Mirbel. Ces tubes, que l'ébullition dans l'acide niurique remplit d'air, sont articulés et chaeun des articles dont ils sont eomposés est environ trois à quatre fois plus 
lonğ qu'il n'est large. Les cavitćs de ces articles ne communiquent point entre elles; cela se voit facilement, parcecque l'air qui les remplit forme autant de bulles alongées et sćparćes lcs uncs des autres qu'il y a d'articlcs; cela prouve bien évidemment qu'il y a une cloison intéricure à chaque articulation. Je donne ( fig. 8 ) la figure de l'un de ces articles; on voit qu'il est couvert de lignes transversales interrompues. Ces lignes, que leur opacibétiaj paraître noirces, r'essemblent assez bien à des spros de trachées qui seraicnt interrompues de distance en distance : je ne sais si ce sont ces lignes ou bien leurs intervalles demi-transparents que M. Mirbel considère conme des fentes transversales. Pour savoir à quoi m'en tcnir sur la nature de ces lignes opaques, j’ai eu recours au moyen dort j'ai déjà fait mention; j’ai fait chauffcs: dans une forte solution acqueuse de potasse canstique le tissu de la vigne déjà préparé, comme il a été dit cidessus, par le moyen de l'acide nitrique. Ce second réactif a complètement fait disparaitre les lignes opaques dont il vient d'être question; et les articles des gros vaisscaux, sur lesquels on les observail auparavant, n'ont plıs prćsenté qu'un aspect et unc demitransparence uniformes. Nous avons vu phus liaul que tel ćtait constamment l'effet prodıit sur les corpuscules nerveux par la potasse caustique; elle les rend transparents, et les fail ainsi disparầtre quand ils ne possèdent aucune coloration. La potasse caustique ne produit point le même effet sur les fils spiraux des rrachées: nualgré l'action prolongécéc de cet alkali, il. 
conservent constamment leur opacité; ainsi, il n'y a aucune analogie entre ces fils spiraux et les lignes opaques dont il vient d'êtrc fait mention; ces dernières sont évide mment des corpuscules nerveux alonggés et linéaires. Peut-être ces lignes sont-elles formées par des séries de corpuscules grlobuleux placés à la file et qui se touchent; nous verrons bientôt un exemple qui pourra fortifier ce soupcon. Le clematis vitalba contient, conmritg vigne, une grande quantité dc ces gros tubes ariycalés, dont les orifices sont visibles à l'œil nu; leurs articles sont très courts, et ils sont couvcrts de corpuscules nervenx qui représentent des lignes transvcrsales extrêmement conrtes, comme on le voit dans la figure 9. C'est en vain que je cherche ici ce qui a pu induire M. Mirbel en erreur, en lui faisant voir, dans les tubes qu'il appelle des fausses trachées, des fentes transversales bordées d'un bourrelet. On pourrait croire que cc naturaliste a vu cela sur d'autres vésétaux que ceux que j’ai observés. A cela je répondrai que M. Mirbel a louné spécialcment la figure du gros vaisseau de la vignnc ${ }^{x}$ dont je viens d'exposer la structure, et yu'i! y dessine les fentes ouvcrtes à jour qui consituent ses fausses trachées. Il est donc certain que M. Mirłel s'est laissé induirc en erreur par quelque illusion (l'oplique; et, dans le fait, il n'est pas étommant yu'ay ant pris des corpuscules nerveux semblables à des points

' Elémculs de physiotogie vigitale el de folanigue. planclse 12, jigure 10. 
pour des pores, il ait pris des eorpuscules nerveux linéaircs pour des lentes. Ainsi il n'y a point de fausses trachées, dans le sens que M. Mirbel attache à eette expression; il $y$ a des trachées qui ne se déroulent point, parceque leurs spires sont fortement soudées; il y a des tubes eouverts de eorpuscules nerveux linéaires dont la direetion est transversale : voila les deux sortes de vaisseaux que M. Mirbel a pris ponr des fausses trachées. Ces organes n'existent pas plus que les tubes poreux, pas plus que le tissu cellulaire poreux, dans le sens que M. Mirbel attache à, ees dénominations. J'en dirai autani des tubes que ee naturaliste appellc mixtes, et qui, véritables trachées dans nue portion de leur longueur, seraient, dans les portions suivantes, successivenent fausses tractiécs et tubes poreux, en sorte que le même uube ofrrirait une organisation différente dans les diverses portions de son étendue. La source de eette erreur est facile à déeouvrir. Les traehées sunt quelquefois couvertes de eorpusenles nerveux qui masquent leurs spires en partie, eonme nous venons de le voir ( fisure 6); M. Mirbel, eousidérant ces corpuscules comme des pores, et voyant les lignes transversales de la traehée interrompries par les eorpuseules nerveux qui les masquent, a ćtć conduit par là à penser que la traehée qu'il observait avait quitté sa structure en spiriale, pour devenir un tube muni de pores et de petites lentes transversales. P'our moi, j'ai toujours vu les trachées conserver l'organisation qui les caractérise daus toutc leur ćcndue; rependant le 
moyen d'analyse que j'emploie m'a sonvent pernis de suivre ces tuhes dans une portion considérable de leur longueur. Mes ohservations à cet égard ont été tellement inulipliées et tellement précises, que je ne crains point d'affirmer que jamais un même tuhe végétal ne présente successivement l'organisation en spirale des trachées et la structure particulière aux tuhes corpusculilères que M. Mirhel désigne sous les noms de tubes por'eux et de fausses trachées. Ainsi, il n'existe point de tubes mixtes, swroins qu'on ne veuille appliquer ce nom aux tubes dont la surface présente simultanément des lignes transversales obscures et des points ohscurs, c'est-à-dire des corpuscules nerveux linéaires dirigrés transversalement, et des corpuscules nerveux globuleux. On trouve cette réunion, par exemple, sur les gros tubes dont on voit les orifices à l'veil nu dans le hois du chêne (quercus robur ). La figure 10 représente l'un de ces tubes, que l'on pourrait appeler mixtes, si la forme des corpuscules nerveux qui les couvrent leur donnait un caractère particulier d'organisation, ce que je ne pense pas. En effer, quand on considère la forne et la position des gros tuhes corpusculifères, on ne peut se dispenser de recomnaitre que tous ces tuhes sont idenliques, bien qu'ils different souvent par la forme et par la position des corpuscules nerveux qui sont sitnés dans l'épaisseur de leurs parois. S'il fallait reconnaître autant de sortes de tuhes qu'il y a de formes particulières dans les corpuscules nerveux yui les couvrent, on multiplicrati d'sne nuanière indéfinie les distinc- 
tious et les dénominations; car il est probable qu’il y a beancoup de diversité à ect égard. La sensitive, à elle seule, nous offie denx variétés toutes nouvelles dans la configuration des corpuseules nerveux de ees gros tubes; en effet, dans l'étui médullaire de cette plante, à côté des trachées, on trouve des tubes dont le diamètre est cnviron le donble de celui de ces dernières, et dont les parois offrent des corpusenles nerveux disposés en losanges irrégulières, comme on le voit dans la firgure 1 1. Lorsquion observe ces tubes cncore adhérents aux organes qui les environnent, on les prendrait volontices pour un faiscean de trachées à moitié déronlées; tel est, en effet, l'aspect que prósentent, au premier coup d'veil, les lignes en losanges qui parcourent ces tubes dans le sens longitulinal. J'avoue que j’ai moi-mêne douté si ecte apparenee n'était point produite par des I rachées fort petices, collées sur le tube dont il est ici question; mais ayant plusicurs fois obtenu ce tube parfaitement isolé, j’ai pu l'eximiner dans tous les sens, el me convainere que les liggnes en losanges que présente sa surtice sont bien récllement des eorpuscules nerveux contenus dans l'épaisscur de ses parois. Dans les pétioles des feuilles rle la sensitive, on rouve des tubes dont les corpuseules nerveux offrent unc antre configuration; ils présentent des lignnes longrudinales disposées symétriquement, conme on le voit dans la figure 12.

Quelles sont tes fonctions de ces ubes corpusenliteres! quelles sont des fonctions des trachées qui tem. sont associées daus l'éui módullaire? V'oiti des ques 
tions auxquelles il est impossible de répondre d'une manière satisfaisante dans l'état aetuel de nos eonmaissances. Nous ne pouvons offrir iei sur eet objet que des conjectures plus ou moins probables. Je pense que les gros tubes eorpusculifères sont les eanaux par lesquels la sève opère son ascension dans le végétal. Ces tubes n'uceupent pas seulement l'étui médullaire, ils existent dans tout le système central du végétal, et se remarquent spécialement chez les végétaux ligneux dans les intervalles des eouches annuelles du bois; ils sont très nombreux dans le bois de la vigne, et il m'a paru que e'était par leurs orifiees que sortait la sève qui coule si abondanıment au printemps des rameaux tronqués de ee végétal. Une foree eonsidérable préside à ee mouvement d'aseension de la sève, ainsi que l'a expérimenté Hales; eette foree n'est pas le seul résultat de la eapilłarité, puisque l'ascension de la sève n'a plus lieu dans les branehes mortes qui tiennent encore au vésétal vivant, branches dont la eapillarité est cependant toujours la nûne.

Les fonctions des trachées ont été l'objet de bien des discussions. Les premiers naturalistes qui les déeonvirient, séduits par leur analogie avee les trachées des insectes, n'hésitèrent pas à les considérer eomme des organes respiratoires; d'autres observateurs atfirmèrent que ees tubes ne eontiennent jamais d'air, mais bien de la sève; mes observations ni'ont prouvé la vérité de eette dernière opinion. Les trachées conduisent bien certainement m liquide diaphane, et 
jamais on ne trouve une seule bulle d'air dans leur intérienr. Le moyen d'analyse que jemploie, l'ébullition dans l'acide nitrique, remplit les trachées, comme tous les autres tmbes, l'un fluide aćriforne; clles offrent alors un aspect tout particulicr et très différent de celui qu'elles présentent dans l'état naturel. Ainsi il est bien certain que, dans ce dernier état, elles ne contiemnent jamais d'air. () aelles sont donc leurs lonctions? Adnettra-t-on, avec M. Mirbel, qu'elles servent, conme les ubes corpusculifères, ì conduire la sève dans son ascension? mais il répugne à eroire que la nature ait auribué des fonctions senblables à des unbes aussi différents dans leur organisation, surtout lorsquion voit ces tubes placés les uns à côté des autres dans l'étui nrédullaire; car on conecviail peut-être qu'une position wès dillérente d'un mêne organe entraînàt une modification dans son organisation. Ce qu'il y a de certain, c'est que les fonctions des trachées ont un rapport nécessaire et immédiat avee les fonctions des feuilles; on ne les trouve que dans les feuilles et dans l'étui médullaire, parties qui, dans les jeunes tiges, on une correspondance intine et immédiatc. Les fonctions des fenilles ne som pas encore bien connues; il est certain cependant que la lunière exerce spécialement sur les feuilles une action vivifiante, soil par elle-même, soit en déterminant certaines combinaisons chimiques daus les fluides que contiennent lents vaisscanx. Ceeci est nn

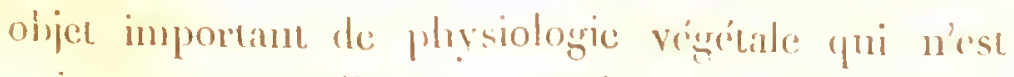
point encore stlfismmutnt éclairé. malgré les re- 
cherehes d'Ingrenhouz el de Sennebier, malgré les travaux encore plus étendus de M. Théodore de Saussure. Quoi qu'il en soit, il me paraît probable que les trachées sont destinées à transmettre dans le eorps du végétal un liquide modifié dans les feuilles par les agents du dehors, et propre à propager l'action vivifiante dont nous avons parlé plus haut; ainsi elles seraient eomparables, jusqu'à un eertain point, aux trachées des inseetes qui transportent dans toutes les parties de l'animal l'air atmosphérique qui doit y produire une influence vivifiante. Considérées sous ec point de vue, les trachées des végétaux seraient des organes respiratoires qui conduiraient un liquide vivifiant.

Après avoir étudié les organes qui composent l'étui médnllaire, nous arrivons naturellement à l'exanien de la eouehe ligneuse qui le reeouvre. En effer, la sensitive, plante frutiqueuse, possède des fibres ligneneses tout-à-fait semblables à celles qui composent le bois des arbores. Ce mot fibre ligniense, employé par quelques naturalistes, doit être banni de la science comme n'offrant aucune idée exacte; il indique seulement que les parties dont le bois est composé sont susceptibles de se diviser en filets très fins; cette division, eomme on le sait, s'opère dans le sens de la longueur de la tigge. Rien n'est plus difficile, dans l'état naturel, que l'observation microscopique dn tissn qui compose le bois proprement dit, ou la partic ligneuse du systemme central; celte difficulte: disparât cntièrement par le mog̣cu que jemploie. 
En firsant chanfier un petit fragnent d'un bois quelconque dans l'acide nitrique, ses partics constimantes ne tardent pas à perdre leur agrégation, elles sc séparent au moindre effort, ct alors leus ósscrvation au microscope ne présente plus ancune difficultć. On voit de cette manière que le bois est en majeure partic composé de tubes renflés dans lenr milien, et rui so terminent en pointe aiguë par lenrs deux extrémitćs, conme on le voii dans la figgrne 15. Je désignerai ecs tubes finsilormes par le non de clostres' $^{1}$; ils sont appliqunés les uns à côté des autres. Les clostres voisins se touchent par leur partic renflée, et laissent entre leurs pointes des intervalles qui sont remplis par les pointes des elostres qui les suivent en-dessus et en-dessons.' Chez la scnsitive, plusieurs de ces clostres sont divisês dan. leur nilieu par nue cloison transversale (fig. $15 a)$, d'autres offrent deux ou trois cloisons, bb. La membrane qui forme ces tubes est très solide; clle est d'un aspect nacré. J'ai vu qu'ils étaient ercux jusque dans leurs pointes, par les bulles d'air que l'action de l'acide nitrique produit souvent dans lenr intéricur. leurs parois ne contiennent aucun corpuseule nervcux. Ces organes finsiformes appartiennent spécialement aux végétaux ligneux; cependant on les rencontre aussi dans les partics des véçétaux herbacés, qui présentent ıne certainc solidité; les végćtanx dont le tissu est mou de délicat en sont tont-à-fait

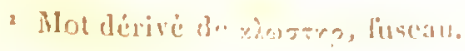


dépourvis. Ainsi il parait que les clostres sunt les organes auxquels les végétaux doivent spécialement la solidité de leur tissu. Cependant je noterai, comme un fait remarquable, que la tige du clematis vitalba, quoique ligneuse, ne contieni point de elostres; elle est, en majeure partie, composée de petits tubes articulés qu'on peut eonsidérer eomme du tissu eellulaire alongé et articulé. Les elostres ne présentent pas toujours exaetement la forme de fusealu que nous venons de leur reeonnaître. Quelquefois ils représentent des tubes parallèles qui se teminent brusquement en pointe aiguë; c'est sous eetle forme que se présentent, par exemple, les clostres du pinuts picea (fig. 14). La forme des clostres a étć figurée d'une manière assez exacte par M. Link; il désigne l'assemblage de ees organes, sous le nom de tissu d'aubier. M. Mirbel a également aperçu, quoique d'une nranière peu distincte, celle organisation ; il regarde le bois comme formé de tissu cellulaire alongé. Nul doute en effet que les clostres ne soient engendrés par un développement particulier des celJules, mais on conviendra que leur forme les éloigne trop du tissu cellulaire pour leur en conseiver le riom. ís clostres sont les réservoirs d'un suc qui est susceptible de se concréter, et qui, presque toujours, aequiert en vieillissant une couleur plus ou moins foncée et une plus grande dureté. C'est ainsi que s'opère le changrement de l'aubier en bois de caurr. En effet, ee n'est point par cux-mèmes que les clostres sont durs et colorés, e'est par la substance 
concrétce qu'ils contiennent. Si l'on fait chauffer du bois d'ádjène dans l'acide nitrique, cet acide dissont la substance noire que contiennent les clostres, qui peu à peu aequièrent ainsi de la transparence, tandis que l'acide nitrique se colore fortement en noir. Ce fait prouve bien évidemment que la couleur du bois de coum est due au suc coloré et endurci, que contiennent les clostres. Ceux-ci sont, par leur nature, d'un Jlane naeré; c'est dans leur intéricur qu'est contenue la substance colorante des bois employés dans la teinture. On pourrait penser que la durcté plus ou moins grande du bois proviendrait de la ténuité plus ou moins considérable des clostres; mais il u'en est rien. En effet, $j$ ai vu que les clostres qui forment le bois ont des dimensions semblables. dans le buis (buxus sempervirens) et dans le peuplier (populıs fastigiata), c'est-à-dire dans les deux bois indigènes dont la.dureté et la pesanteu: spécifique offrent les plus grandes différences. Ce fait achève de prouver que la dureté ci la pesanteur spcéeifique du bois dépendent exclusivement de la sulsstance endurcie que contiennent les clostres; il parait que ees organes son $t$ vides dansle penplier; aussi ce bois est-il tendre, extrêmement léger, et d'une couleur blanehe, qui est la couleur naturelle des clostres. C'est par la mème raison qu'il n'offie point la distinction de l'aubier et du bois de coum'; les clostres. partout également vides, sont partout également blancs, puisçu'ils ne doivent lenr coloration qu'à la substance qu'ils contiennent chrz les bois colorés. 
Au reste, la coloration et la dureté qu'acquiert cetıc substance en vieillissant, et d'où résulte là trannsformation de l'aubier en bois de cœur', est un phénomène chimique dont l'essence n'est point connue.

Les clostres, dans l'aubier de formation récente, me paraissent être les réservoirs de la sève élaborée qui sert spécialement à fournir les matériaux de l'accroissement en diamètre du végétal, et qui, transmise de clostre en clostre par un mouvement descendant, va fournir aux racines les matériaux de leur accroissement. Je pense que cette sève élaborée, transmise à travers le tissu perméable du végétal, se mêle à la sève ascendante pour fournir aux bourgeons les matériaux de leur accroissement, et qu'elle va fournis aux vaisseaux propres les matériaux de ‘a sécrétion qu'ils opèrent. On sait que c'est au moyen d'une diffusion semblable d'un suc élaboré que s'opèrent -et la nutrition ct les sécrétions chez les insectes. Lorsque cette sève élahorée est tout entière employée à l'accroissement du végétal, l'accroissement de ce dernier cst rapide, et ses clostres restent vides; alors le bois est blanc, tendre et léger: lorsque, au contraire, la plus grande partie de celte sève élaborce demeure dans les clostres, et n'cst point emploỵce it l'accroisscment, ce dernier est plus ou moins lent, et le bois demeure lourd, dur el coloré.

Les clostres, quoique contenant un liquide diflérent de la sève ascendante, ne doivent cependant point être confondus avec les vaisseaux propres, lescuucls sont des organes sécrétemrs. Ces dernicrs soul des 
unbes dont le dianietre est toujours plus grand yue celui des clostres; ils sont, comme enx, tonjours privés de corpuscules nerveux, mais les substances quilis contiennent sont bien différentes, et paraissent être purement excrémentitielles. 'Telle est, par cxemple, la résinc pure que conticnnent les vaisscaux propres des arbres résineux. Cette substance n'est bien certainement pas destinée à l'accroissement et ì la nutrítion du viggétal; mais ne serait-clic point le résidu de la substance alimentaire, qui aurait éte absorbée, et avec laquelle elle était mêlée dans le principe? Les sucs laitenx, que l'on comprend généralement dans la classe des sucs propres, me paraissent devoir être considérés comme des liqquides, au moins en partie excrémentitiels. Cette partie de la physiologie vérétale demande, comme on le voit, de nouvelles recherches, et je ne mi'y arrèterai pas davantage; je nue contenterai de laire obscrver ici incidemnent que les sucs résineux, qui sont abondants dans l'écorce de la plupart des coniferes, ne sont point contenus dans des lacunes ou dans des cavités produites par le déchirement du tissu cellulaire, comme te pense M. Mirbel. Ces sucs résineux sont contenus dans des vaisseaux irrégulièrement renflés et tortucux. On les isole complètement par le moyen de l'acide nitrique. Ce fail et quelques autres me font penser que la théorie de M. Mirbel sur les lacmes a besion de recevoir des modilications.

Les faisceaux des clostres som melés, clec\% la seusilive, avec un lissu cellulaire quit se divise mécani- 
quement en filets longitudinaux, composćs de séries de cellules, comne eela se voit dans la figure 15 , $a b, c d$. Ici je crois devoir rappeler que, dans mes Recherches sur' l'accroissement et la reproduction des végélaux ', j'ai désigné sons le nom de fibres ees assemblages de cellules qui se prêtent avec facilité à la division longitudinale en filets, parceque les cellules qui les composent adhèrent plus les unes aux autres dans le sens de la longueur de la tige que dans le sens transversal, ce qui n'a point lieu pour le tissu cellulaire irrégulier. Mais, reconnaissant que ce mot fibre a été appliqué à plusienrs sortes d'organes linéaires très différents entre eux, et que par consćquent il est difficile d'y attacher une idée exacte, j'ai résolu de désigner ces assemblages rectilignes de '"ellules articulées les unes avec les autres par le simple $110 \mathrm{~m}$ de tissu cellulaire articulé. Pour peu qu'on multiplie un peu ses observations sur la structure intérieure des végétaux, on ne tarde pis à trouver des cellules articulées qui, par leur alongensent dans le sens longitudinal, tendent ì devenir des tubes. C'est ce que Link ${ }^{2}$ a désignné sous le nom de tissucellulaire alongé. On trouve, enfin, de véritables tubes articulés les uns avec les antres dans le sens longitudinal. Ces observations prouvent que, du tissu cellulaire articulé aux tubes articulés, il y a une transition évidente, ct que ces organes ne diffèrent que par

Himoires du Muscum d'hisloirc nalurelle, tome 7 .

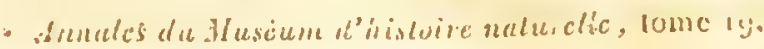


les proportions respectives de leurs parties. Aprés cette petite digression, je revicns an tissu cellulaire articulé, qui m'y a conduit. Ce tissu cellulaire est assez généralement semblable à celui de la moelle; il est, comme ce dernier, tout courert de corpuscules nerveux placés d'une manière fort irrégulière. Quelquefois cependant j'ai observé des portions de ce tissu cellulaire articulé qui offraient dans le milien de chacunc des cellules un seul corps linéaire phacé longitudinalement, comme on le voit en $b$ ( fiçure 15); c'est un corpuscule nerveux qui, vu avec une forte lentille, paraît formé par une série de quatre ou cirry corpuscules globulcux placés à la file, conme on le voit en $a$. Ce lait justifie le soupçon que j'ai émis précédemment touchant la nature des corpuscules nerveux linćaires, que j'ai considérés comme probablement formés de très petits corpuscules placés à la file. Le tissu cellulaire articulé dont il est ici quuestion est l'organe générateur des rayons médullaires dans les végétaux ligneux et frutiquenx. Les végétaux totalement lierbacés ne possèdent point ces rayons, qui existent dans la tige frutiqueuse de la sensitive. Dans les jeunes tiges de cette plante, ce tissu cellulaire mêlé aux elostres est articulé dans le sens longitudinal ( $c d$, fig. 15$)$; ce n'est que dans ee sens qu'il se divise mécaniquement en filets. Dans les grosses branches ou dans le trone, le sens de cette articulation est clanngé, et ce même lissu se trouve articulé dans le sens do, c'est-ì-dire dans le sens transversil, pour former les rayous médullaires. Ainsi, dians, 
les tiges naissantes ou dans les jeunes branches des végrétaux ligneux dicotylés, le tissu cellulaire articulé et ecrpusculifère qui est mêlé aux faiseeaux des elostres, et qui est évidemment une énanation latérale de la moelle, est articulé dians le sens lorgitudinal, eomne cela a lieu dans les petites plantes herbacées dieotylées. Lorsque ces liges ou ces bran.. ches prennent de l'accroissement en diamètre, ee tissu cellulaire cesse de présenter une articulation longitudinale; il en prend une transversale, et c'est ainsi que se forment les rayons médullaires qui sont exclusivement formés de tissu cellulaire articulé.

Le système cortical de la sensitive est composé de elostres beaucoup plus alongés que eenx qui existent dons le système central, leur diamètre est également plus grand. An resie, en parlant de la longrueur de ces organes, je n'entends faire mention que de leur apparenee au mieroscope ; ear, dans le hit, ils sont toujours d'une cxtrêne petitesse. J'ai mesuré les clostres de la sensitive, et j’ai trouvé que. les plus alongés, dans le système eortieal, ont ì peine un millimc̀tre et demi de longneur sur $\frac{r}{55}$ de millimètre de largeur; les clostres du système central n'ont guère que la moitié de ces deux dimensions . Lcs clostres du système eortieal sont, eomme ees

Se me scrs du microseope solairr: pour mesurer les objels d'une cxtrène petifesse. Je eompare l'iragge ou l'ombre produite à une dislance delermines par l'objet que je reux mesures, avec l'umbre que moduit, á la mềue distance, un pelit norccau de fil mélallique dont le dismelre isact m'est counu. 
derniers, privés de corpuscules ncrveux; leurs faisccaux sont plongés dans un tissu cellulaire corpusculifère tout-ìtfait semblable à celui de la moelle. On y trouve de même, et en assez grande quantité, des cellules remplies de ec fluide conercscible par l'acide nitrique froid, et soluble dans le même acide chaud; cellules dont j'ai fait mention plus laut en étudiant la moelle. Ccue identité parfaite de structure et de composition chimique cntre la moelle et le parenchyme cortical est une preuve à ajoutcr à celles que j’ai cxposćes dlans un précédent ouvrages ", pour démontrer que ces deux tissus organiques ne diffèrent en aucunc façon et ont des fonctions scmblables; c'est donc avec raison que, dans eet ouvrage, j'ai domné ì la moclle le nom de médulle centrale, et au parenchynue de l'écorce le nom de médulle corlicale.

Les feuilles de la sensitive sont portées sur un long pétiole, à la base ducquel existe une portion renflée $a b, c d($ figg. 18) que je désignerai par le nom bour'relet. Des renflements scmblables, mais plus petits, existent à l'insertion des pinnules sur le sommét du pétiole, et à l'insertion des folioles sur les pinnules; c'est en enx que réside le principe des nouvements (qu'crécutent les féuilles de la sensitive, conme nous le verrons plus bas. Le hourrelet qui est situé à la base du puétiole est le seul qui présente une grosscur sufisante pour qu'il soit possible d'cn obscrver la struchure intériente: en le fendan Innogindim? lement, el e: l'examinant à la loupe, on voit qune ce

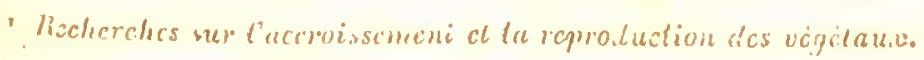


bourrelet est prineipalement formé par un développement considérable du parenchyıne cortical; le centre est oecupé par les tubes qui établissent la communication vasculaire de la feuille avec la tige: si l'on veut voir avec faeilité l'organisation intérieure du parenchyme cortical qui constitue essentiellement ce renfiement, il fant, avec un rạsoir, enlever d'abord l'épiderme sur l'un de ses côtés; ensuite on enlève une tranche de parenchyme, aussi minee qu'il est possible de l'obtenir, et on la soumet au microscope, plongée dans un peu d'eau. On voil de celie manière que le parenchyme du bourrelet est composé d'une grande quantité de cellules globuleuses et diaphanes dont les parois sont couvertes de corpusenles nerveux. Si on supprime l'eau dans laquelle est plongée la petite tranche, et qu'on mette en place un peu d'acide nitrique, on voit, en peu d'instants, les cellules diaphanes devenir d'abord jaunâtres, et ensuite complètement opaques. On reconnaît alors que ce sont des cellules tout-à-lait semblables à celles que nous avons déjà observées dans la moelle et dans le parenchyme cortical, excepté que celles-ci sont de formo globuletsse. Ces cellules, qui ne sont point en contact immédiat, sont alignées dans le sens longitudinal, comme on le voit dans la figure 16. J'ai représenté, dans eette figure, quelques unes de ces cellules alignées, et les autres dans un ordre confus, parceque c'est ordinairement ainsi qu'clles se présentent à l'observation, l'instrument tranchant avec lequel on enlève la lame mince du bourrelet, ne rencontrant que 
par hasard la direction alignée des cellules. La figure 17 représente ces cellules globuleuses plus grrossies; on voit qu'il existe entre elles des intervalles qui sont occupés par un tissu cellulaire très délicat, et rempli d'unc irmmense quantité de corpuscules nerveux semblables à des points opaques. Si l'on fait chauffer l'acide nitrique où se trouve la petite tranche de bourrelet mentionné plus haut, en présentant avec précaution le cristal de montre qui le contient au-dessus d'une lampe à esprit de vin, on ne tarde pas à voir disparaître complètement toutes les cellules grlobuleuses. La substance qu'elles conticnnent est entièrcment dissonte par l'acide; il ne reste plus alors que les cellules et le tissu extrêmement délicat qui les environne. J'ai vu qu'il suffisait d'une chaleur de 40 à 50 degrés R. pour que l'acide nitrique opérât la dissolution de la substauce contenue dans ces ecllules globuleuses. J'ai essayé sur ces organes l'action de la solution aqueuse de potasse caustique. Je n'ai observé à froid aucun ehangenıent dans leur transparence, wais it chaud j'ai vu que tout le parenchyme prenait une teinte verte uniforme; on n'apercevait plus les cellules globuleuses, ce qui me fit peuser que lit substance qu'elles contenaient avait été dissoutc. Cependant, ayant soumis à la même épreıve une lame de parenchyme du bourrelet dont les cellules globuleuses avaient été rendues opąues par l'acide nitrique lroid, je vis ces cellules globuleuses devenir encore phus opaques, el acyucrir une coulcur noire: ceci prouve que la potasse canstique car'bonisc ces cellules, lorsque son 
aetion succède à cellc de l'acide vitrique, car elle nc froduit point du tout cet effet lorsqu'elle agit sur ces cellıles dans lcur ćtat naturel. Ce scrait à tort que l'on croirait pouvoir conclure de cette ex péricnee que la potasse caustiquc ne dissout point la substanee que conticnnent les cellules globuleuses; $\mathrm{cn}$ effet, la solubilivé de ccuc substance dans la solution alkaline est bien pronvée par les cxpéricnces suivantes. Si l'on fait bonillir dans l'eau un bourrelet de sensitive, les celluies globuleuses qu'il contient deviennent toutes opaques, cc qui provient de la conerétion de la substance contenue dans ces cellules; alors si l'on verse sur cettc substanee concrétée un peu de solution aqueusc de potasse caustique, cclte smbstance concrétée se dissout et disparaît avee une extrême rapiditć. Je me suis un peu étendu sur les propriétés de la substanee contenue dims les cellnles globuleuses du bourrelet, parceque ce dernier organe est la partie la plus intéressante à étudier dans la sensitive, commc étant, clicz cctte plante, l'organe immédiat du mouvenient.

Les lonrrelets situés à linscrtion des pinnules sur le sommet du pétiole on la même organisation que le bourrelet situé à la base de ce dernier, sculement leur's cellulcs globulcuses sont plus petites.

Le pétiole de la feuille de sensitive offre it sa partie extérieure unc grrande quantité de clostres fort alongés; ils forment, pour ainsi dire, l'ćcoree du pétiole; dans son intérieur, on trouve du tissu eellulaire articulé cl corpusculifè̀re, cl de ğros tubes corpusen- 
liferes, dont nous avons deji fait mention p! tus hant (fig. 12). Au centre dn pétiole, cxistent des traclées à spires qui ne se déroulent point dans l'état naturel, mais que l'on parvient à dírnuler au moyen d'une lonģue ébullition dans l'acide nitrique.

les folioles de la sensitive contiennent une inmense quantité de corpusenles nervenx; pour les voir, il fant plonger une feuille de cente phante dans l'acide nitrique, à la température de l'ean bonillinte, pendant une minute seulement, et la transportci de suitc dans l'eau purce. Par cette opération, les folioles deviennent fort transparentes, et laissent apercovoir, an microscope, lcurs innombrables corpuscules nerveux, qui sont devenus opaques. Ils sontd'une cxirême petitessc; lenrs groupes sont spécialement placés antour des nervares, ou plutôt des vaisseaux qui parcourent la foliole. Lees raneaux les plus fins de ces vaisscaux, chargés de ces corpuscnles globuleux, ressemblent tout - à - fait ì un végétal charegré de firuits.

La racine de la sensitive offre, dans son système central, des clnstres mêlés avec de gros tubes tontà-fait semblahles par leur forme, leur grensscur et leur position anx tubes corpusculifères de la tige; mais on n'aperçoit point de corpusenles nerveux dans leurs parois; cela tient probablement à la petitesse ot à la grande transparence de ces corpursculcs.

Le tissu cellulaire articulé est disposé en rayons médullaires conccurrignes dans les prosses racines, ct en filcts longitialinaux dans les ratiredles I I's cor- 
puscules nerveux qu'il contient sont fort transparents' et l'acide nitrique ne les rend point opaques, ce qui fait qu'ils sont bien moins visibles que ceux du système central de la tige. On sait qu'il n'y a, dans les racines, ni noelle, ni étui mćdullaire, ni trachées. Ce fait est général. Cependant MMI. Link et Tréviranus prétenrlent avoir trouvé des trachées dans les racines: n'en ayant jamais trouvé dans des recherches assez nombrcuses quc j'ai laites, je suis porté à penser que ces deux naturalistes ont observé des tiges souterraines, en croyant observer des racines véritables. Il est en effet facile de les confondre; j'ai indiqué les moyens de les distinguer dans mes Recherches sur l'accroissement et la reproduction des végétaux'. Ces tiges souterraines possèdent en effet des trachées, de même que les tiges aériennes, ainsi que je l'ai observé.

Le systèmc cortical de la racine de sensitive ne diffère point essentiellement du système cortical de la tigre, sous le point de vue de sa composition anatomique; seulement je n'ai point vu que les cellules de son parenchyme contiussent un fluide concrescible par les acides.

Iorsqu'on coupe une jcune tige de sensitive, on le bourrelet du pétiole de l'une de ses feuilles, on en voit sortir sur-le-champ unc goutte d'un liquide dinphane qui, vu au microscopc, paraît composé d'une immense quaniité de globules transparents. J'ai re-

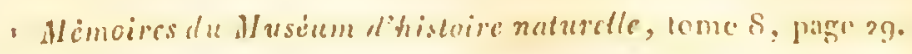


cueilli mne certaine quantité de ce fluide sur une lame de verre; el ayant nis dedans une goutle d'acide nitrique très alfitibli, il s'y est lómé sur-le-champ un coagulum membraneux qui, vu au microscope, s'est trouvé entièrement composé de globules opaques agrghlonérés: ces gylobules sont ceux que l'on apercevail à peine auparavant, à canse de lcur transparence. Ayant mis une goutte de solution aqueuse de potasse caustique sur ce coagnlum membraneux, les grobules dont il était composé ont été entièrement dissous. La propriété que possède ce fluide d'êtru concrété et rendn opaque par l'acide nitrique, met ì nểine de déterminer quels sont les vaisseaux daus lesquels il est contenu. Une lame mince et transparente, conpée longritudinalement dans le milieu d'une jeune tigge, étant plongée daus l'acide nitrique froid, et examinée ensuite an microscope, on voil que les seuls organs qui soien rendus opaques par cette općration sont quelques unes des cellules des deux médulles centrale et corticale, cellules que nous avons vies contenir un fluide concrescible; tous les autres organes conservent leur transparence. Ainsi il n'y a point de doute que le fluide concrescible dont il est ici question ne soit celui qui sort de celles de ces cellules qui ont élé ouvertes parla section, ou par la lacération du tissu végútal.

Les divers organes creux que nous avons ohservés dans le tissu végétal, c'est-ì-dire les cellules, les trachécs, les tubes membraneux et les closercs, n'ont entre cux que des rapports de contiguite; it nexiste 
jamais de communiention directe entre lcurs cavités. Ainsi les fluides qu'ils eomtiennent ne peuvent ĉtre transmis des uns aux autres que par les pores de leurs parois. L'existence des pores n'est done point douteuse, mais on s'en ferait une idée bien fausse, si on les eonsidérait comme des trous laits exprès pour livier passage aux fluides; ee ne sont, dans le fait, que des espaces intermolćculaires. Les solides organiques sont généralement composćs de noléeules intégrantes globuleuses, ainsi que nous le verrons plus bas, en étudiant la structure organique des animaux. Or, on eonȩoit que ees moléeules ģlobnleuses doivent laisser entre elles des espaces vides qui n'existent point entre les moléeules polyèdres des minéraux; molécules dont les faecttes s'appliquent exactement les unes sur les autres. De là vient la grrande perméabilité pour les fluides aquenx que présenient en grénéral tous les tissus organiques, quoiqu'on n’aperęoive aueun trou, ou aneun pore proprement dit dans leurs membranes, même dans eclles que nons savons être les plus perméables. L'épiderme humain, par exemple, dont la perméabilité est si grande, ne laisse cependant aperecroir aueun pore avec les plus forts mieroseopes. Ainsi la doctrine émise par M. Mirbel, touchant l'existence des pores visibles dans les parois des tubes et d̀u tissu eellulaire des végrétaux, serait douteuse, par le seul fail de la grandeur et de la forme de ees pores prétendus, quand bien même eette doetrine ne serait pas infirmée directement par l'observation. 
Les fluides, pour passer d'un organe ereux dans un autre, ont besoin de traverser les dcux parois contiguës de ces organes; car l'obscrvation démontre que tons ces organes ont chacun une mcmbrane propre, el qu'ainsi ils n'ont jamais de paroi comunune là oǹ ils sont contigus. En effet, nons avons va que, par le moyen de l'ébullition dans l'acide nitrique, on isole les uncs des autres tontes les celhulcs de la moclle, lesquelles, ainsi isolées, sc trouvent former cliacune une vésicule complète; il en cst de même du tissu cellulaire articulé, chacun des articles dont il se compose se détache en formant une vésicule sans ancmue ouverture. Ainsi les cellules sont des vésicules simplentent asgglomérées, et sans aucunc continuité entre clles; leur forme originelle est la forme globuleuse: e'est par l'érgalité de li compression qu'elles éprouvent dans tous les sens, qu'clles prennent souvent unc forme polyc̀dre symétriquc. Les cellules isolées et extrêmement pelites conservent eette forme gobuicuse que nons avons obscrvée dans les corpuscules nerveux. J'ai encore observé cette forme globuleusc des cellules dans la substance dure qui lorme le noyau ou l'endocarpe de l'abricon; cette substance étant soumise à l'ébullition dans l'acide nitrique, perd eomplitement sa dureté, scs éléments organiques se dissocient avec facilité, et on voil qu'elle est entièrentent composée de peties celIules vésiculaires el ghloluleuses, qui sont agrghnéjées, comme on le roil dans la figure 19. Cus cellules contenaient une substince consrète et fort durc 
dont l'acide nitrique a opéré la dissolution. C'est ici spécialement que l'on voil avec évidence que les cellules sont tout-ì-fait indépendantes les unes des autres, et que leur forme originelle est la torne ghobuleuse. Les clostres, qui ne sont que des eellules uubuleuses soumises à un mode particulier de développement, n'ont de même jamais de parois eommunes dans les endroits où ils sont contigus; il en est de mềne de tous les tubes végétaux : on les obtient toujours, par le moyen que j'ai indiqué, parfuitement nuss et complètement isolés de tous les orgảnes qui les environnaient, et auxquels ils étaient simplement contigus. Les tubes qui sont réunis en faisceaux n'ont point non plus de paroi commune là où ils se touchent; car j'ai toujours vu ces tukes se séparer les uns des autres, en formant ehacun un tube complet. Ce n'est point sans regret que je me trouve eneore ici dans la néeessité de combattre les assertions d'un naturaliste eélèbre que je semble avoir entrepris de eontredire en tout, tant il y a de disparité entre ses observations et les miennes. Selon M. Mirbel, les cellules auraient une paroi eommune là où elles se tonchent; il en serait de même des tubes rassemblés en laisceaux : les tubes isolés seraient latéralement continus avee le tissu eellulaire qui les environne. Sur ees assertions, que l'observation infirme, M. Mirbel fonde une théorie de l'organisation végétale dont on voit de suite le peu de solidité. Sclon ee naturaliste, toutes les cellule's et tous les tubes seraient le résuliat des diverses ma- 
nières d'être d'un seul el même tissu nembraneux eontinu dans toute l'étendue dı végétal, et dont l'épiderne ferait la limite. Considéré de eette manière, el pour me scrvir d'une eomparaison grossière, mais assez juste, le tissu végétal, renupli de eavités de différentesformes, ressemblerait, en quelque sorte, it $1 . m$ pain dont la substance, eontinue dans toutes ses parties, offre une immense qiantité de cavités cellulaires; mais l'obscrvation, eomnc je viens de le dire, n'est point d'aecord avec cette théoric: ellc prouve que ehaque tube et chaque eellule est un organe eirconscrit qui posséde des parois yui lui sont cxclusivement propres, et qui se détache d'une manière nette des autres organes qui l'environnent, ee qui peut fairc penser que ces or granes contigus étaient simplement agglutinés. On peut. supposer, il est vrai, que l'acide nitrique ne séparerait ecs organes les uns des aultes qu'en détruisant nu tissu internédiaire qui ćtablissait leur continuité, mais ecei est me prure hypolhèse. Nous verrons a la fin de eet ouvrage des observations sul la eomposition organique des animaux qui viendront ¿ l'appui de la théorie nouvelle que l'on pourraiı déduire de mes observations, et qui tendraient à faire eonsidérer le lissu organique eoume formé par l. réunion d'une immensc quantité de vésicules cellulcuses ou tubulenses dont les parnis sont en contaet, et qui tiemnent les mes aux autres par mo. simple force d'adhrision ou d'agghlutination. 


\section{SEGTION II.}

OESERVATIONS SUR LES MOUVEMENTS DE LA SENSITIVE (mimosa pudica).

Depuis long-temps les mouvements de la sensitive attirent les regards des curicux, et sont devenus l'objet de l'étude des savants. On a fait sur cette plante beaneoup d'observations et d'expériences, sans parvenir a connaitre la cause des mouvements singyuliers qu'on lui voit exécuter. On connaît les travaux de Duhamel et Dufay sur cet objet '. Les expériences de ces deux savants sont nombreuses et intéressantes; cependant elles laissent beaneoup ì désirer. On ignore encore quel est, chez la sensitive, le tissu organique auquel appartient la faculté que l'on nomme l'irritabilité végétale; faculté que les physiologristes n'ont point encore distingruće de la sensibilité chez les végétaux : ponr parler le langage que jài adopté, je dirai que l'on ignore si la nervimotilité et la locomotilité unt une existence à part cliez la sensitive. On ignore si des organes, si des tissus particuliers sont allectés à l'exerciee de ehacune de ces deux facultés de mouvenent; on ignore, enfin, quelle est la nature de ce mouvement organique el intéricur auquel est due la loconotion végéc-

- Wén:oires de t'acodimie myale des scicuses, 1,56 . 
tale. Comment serait-on parvenu à la solution de oes problèmes sans la connaissance de l'anatomic de ta plante qui les présente? C'est eette anatomie, que nons avons préseutée dans la section précédente, 'qui va guider nos recherehes. Elle nous a appris que la sensitive possède un appareil nervenx très développé, spécialenent dans les feuilles et dans les bourrelés qui sont situés dans leur's articulations. Cet appareil nervenx, siége de la nervimotilité de la plante est-il anssi le siége de la locomotilité? L'expérience va nous apprendre ce que nous devolns penser à ect égard.

Les monvements de déplacentent qu'offrent les pariies des végétaux ne s'exécntent point exactement comme les mouventents de déplacement des membres des animanx articulés. Cliez ces derniers, il y a des articnlations mojiles, et les organes du monvement, les muscles, sont plus on moins éloignés de ees articulations. Clez les végétanx, il u'y a jamais d'articulations molsiles; Icnr locomotion s'opère toujours au moyen de l'inflexion de parties douces d'une souplesse et d'une mollesse remar|puahles; ici les organes dı mouvement sont dans le lieu mêne où la flexion s'opiere; le tissu organifue éprouvedans cet endroit un monvement iutérieur qui détermine la flexion on le redressement de la partic. C'est de cente manière que se menvent égalenent les membes de certains animanx inarticulés, tels que les mollusques céphalopodes, les hydres, cte. On ne donte point phe diez ces derniers le monvenent ne soit dia a l'action mus- 
culaire; mais en est-il de même chez les végétaux? L'anatomie ne nous a rien fait voir ehez la sensitive que nous puissions comparer à des nuuscles. Étudions donc la manière dont s'opèrent les mouvements de déplacement dans les feuilles de cette plante. On sait qu'au moindre attouehement, à la moindre secousse, ces feuilles se ploient avec rapidité; cette plieature s'opère de la manière suivante. Les folioles se ploient par paires en se joignant par leurs faces supérieures; par ce mouvement, elles se rapproehent de leur axe commun qui est la pinnule; les pinnules se ploient en se rapprochant égalenient de leur axe eommun, qui est le pétiole, au sommet duquel elles sont implantées par paires; le pétiole se ploie en s'éloignant de la tige sur laquelle il est implanté. Ce mouvement d'éloignement du pétiole est si étendu que ee dernier s'incline vers la tcre en se rapprochant de la partie de la tige qui est située au-dessous de son insertion; ainsi le mouvement du pétiole s'opère en sens inverse de celui des pinnules et des folioles. Cos deux dernières se rapprochent de la partie supérieure de l'axe duquel elles émanent; le pétiole, au contraire, s'éloigne de la partie de la tige qui lui est supérieure, et se rapproche de la partie de eette même tige qui lui est inférieure. Tous ces nouvements s'opèrent au moyen dé la flexion de eertains bourrelets alongés qui sont situés à la base de ees parties mobiles. Le bour'clet dı pétiole présente seul une grosseur suffisante pour qu'il soit possible de le soumettre à l'expérience. Ce bourreles, lorsque la leuille est redres- 
sée, est disposé comme on le poit en ab (figure 18); on voit en ed la forme qu'il prend lorsque le pétiole est fléchi et la feuille inclinée vers la terre. Droit dans le prenicr cas, ce bourrelet forme dans le second une combe dont la convexité est diriggée vers le cicl : cette courbure n'est point un état d'affaissement, car elle résiste à l'effort que l'on lait pour la redresser; c’est véritablement le résnltat d'une action organique des parties qui composent intérienrement le bourrelet. Nous avons vu plus haut que ce dernicr est principalement composé de cellules globuleuses qui contiennent un fluide concrescible et qui sont enviromnées par un tissu cellulaire wès délicat, dans lequel il existe une immense quantité de corpuscules nerveux; ce tissu est un développement particulics du parenchyme cortical; le centre du bourrelet cst occupé par un petit faisceau de tubes. Il fallait d'a bord savoir (quelle est celle de ces deux parties qui est l'organe du mouvement; pour y parvenir, j’ai fait l'expérience suivante. J'ai enlevé tout le parenchyme cortrical du bourrelet, en le grattant doucenent avec un canif bien acéré, en sorte que le laisceau central de tubes est resté à nu. Cette opriration n'a point fait monrir la fenille, dont seulentent les folioles ont été pendant plusieurs jours saus se déployer. Le résultat de cette expérience a été que le pétiole a conplètement perdn la ficulté de se mouvoir"; ee qui prouve que les tubes contenus dans le bourrelet ne sont pas les orgaues de son monvement, qui m'a parı ainsi revoir résiler uniquement lans le parenchume 
cortical. Les deux mouvements opposés, de flexion et de redressement, que présente le bourrelet me paraissant devoir être en rapport avec les fonctions de la portion supérieure $a$ et de la portion inféricure $b$ (figure 18 ), j'enlevai, par une section longitudinale, tout le parenchyme cortical sur le cóté supérieur $a$, de plusieurs bourrelets; je fis la même opération au côté inférieur $b$ de plusieurs autres bourrelets. Les fenilles continuèrent à vivre et à présenter leurs plıénomènes habituels, excepté seulement en ce qui concerne le mouvement du pétiole. Ce mouvement fut tout-à-fait anéanti dans les pétioles dont le bourrelet avait été dépouillé de son parenchyme cortical à son côté inférieur $b$; le pétiole resta constanment fléchi russ la terre et ne fit aucun effort pour se relever. Ge fait me prouva que le redressement du pétiole If est point opéré par le côté supérieur du bourrelet; car ce côté supérieur étant resté intact n'aurait pas manqué d'opérer le redressement du pétiole. Au eontraire, tous les pétioles dont le hourrelet avait été dépouillé du parenchyme cortical par son côté supérieur $a$ ne tardèrent point à se redresser, et même ils se redressèrent beaucoup plus qu'ils ne le faisaient avant l'opération; et je remarquai quiils ne se fléchirent plus vers la terre pendant la nuit, comme ils le faisaient auparavant: les secousses que je leur imprimais n'avaient plus le pouvoir de déterminer cette flexion. J'employai, dans la vue de provoquer cette dernière, les moyens que je savais être les plus énergiques; telle est, par exemple, l'ustion lúrère des 
folioles. Ce moyen produisil une légère hésilation de flexion sur quelques uns de mes pétioles el laissa les aulres conplètement immobiles. Je m'aperçus que ( cette différence de résultat tenail à ce que, dans les premiẹs, le côté supérieur du bourrelet n'avait pas r été enlcvé exactement jusqu'à la noitiéde ce dernier : ij'acherai cet enlèvement, et alors les pétioles demeui rèrent immobiles dans leur état de redressement. Le : fail du redressement du pétiole après l'ablation diu - parenchyme cortical au côté supérieur du bourrelet, me prouva de nouveau que ce redressement n'est point dû à l'action de ce cóté supérieur'; il ne proura en mêne temps qu'il est dù à l'action dù côté inférieur. Ainsi c'est l'action organique de la moitié supérieurc $a$ du bourrelet, considéré comme fendu longitudinalement, qui opère seule la flexion du pétiole; et c'est l'action organique de la moitié inférieure $b$ qui opère seule son redressement. Dans la dernière expérience, le redressement fut plus considérable qu'il ne l'est dans l'état naturel, parceque l'action redressante du còté inférieur n'était plus contre-balancée par l'action fléchissante du côté supéricur. Quelques jours après cette dernière expérience les feuilles qui y étaient soumises fléchirent leurs pétioles vers la terre, randis que les autres feuilles de la plante conservaient lou état de redressement. Ce fuit étail en contradiction avec mes expériences précélentes; j’en recherchai la cause, el soupenonnaul que la plante n'avait pas assez d'eau, je l'arrosai; bientơt apres les péfioles abaltus se redressèremt. Ce 
fait me prouva que la flexion de ccs pétioles n'était point due, dans ce cas, à une action vitale, mais qu'elle était seulement le résultat de l'affaissement des cellules par le manque d'eau. L'afflux de la sève dans les cellules occasiona le redressement du pétiole, qui ne se fléchit plus, moyennant que j'eus soin d'arroser suffisamment la plante. Ce dernier fait me prouva que l'action organique par-laquelle le cóté inférieur $b$ redresse le pétiole ne peut avoir lieu qu'à l'aide d'une sève abondante, et cela ine donna lieu de penser qu'il en était de même de l'action organique par laquelle le côté supérieur $a$ opérait la flexion du pétiole. Je voulus toutefois m'en assurer par l'expérience. Pour cela, je retranchai le côté inféricur $b$ aux pétioles des trois dernières feuilles d'une lige fort alongée, puis ayant courbé cette tige de sorte que son sommet était dirigé vers la terre, je la fixai dans cette position; de cette manière, le côté supérieur $a$, qui restait seul à chaque pétiole, regardait la terre; il était devenu inférieur. Le premier jour le poids de la feuille l'entraina un peu vers la terre, mais dès le second jour la feuille et son pétiole se portèrent vers le ciel par lc moyen de la courbure du côté $a$, qui, dans cettc expérience, était devenu inférieur. Celte position ne varia point pendant la nu\&, et les irritants extérieurs appliqués aux folioles ne la firent point varier non plus. Cet état de redressement constant, ou plutôt de flexion ascendante, dura pendant quinze jours. Je n'avais point arrosć la plante pendant cet espace de temps, et je l'avais tcuue à l'ombre pour éviter 
que ses feuilles, trop peu fournies de sève, ne fussent lesséeliées par les rayons du soleil. Je vis alors les métioles s'incliner vers la terre par la cessation de la courbure du cóté a du bourrelet; les autres feuilles le la plante avaient perdu la plus grande partie de cur motilité : lorsqu'on les frappail vivement avec le toigt, les folioles se ployaient imparfaitentent et le pćiole demenrait immobile. Alors jarrosai la plante, et juelques heures après je vis les pétioles inclinés se sorter de nouveau ver's le ciel, par le rétablissement le la courbure du cóté $a$, dont la convexité regarlait la terre, par l'effet du renversement de la ligre. Cette expérienee me prouva deux choses, $1^{0}$ que ha conrbure du côté supérieur $a$ est le résultat d'ıne action organique; $2^{0}$ que cette action organique perr de son énergie lorsque l'abondance de la sève est diminuéc, ct qu'elle récupère cette énergyie par le retour d'une sève abondante. Il résulte en outre de ces expériences que les deux côtés $a$ et $b$ du bourrelet présentent le même phénomène, mais en sens inverse: le côté $a$, par son aecion orgranique prédominante, fléchic le péliole vers la terre, et le eôté $b$, par son action organique à son tom prédominante, relève le pétiole vers le ciel. Dans l'expérience précédente, nous avons vu que le manque d'une sève suffisammant abondante avait oceasioné l'extrême diminution de la motilité des feuilles de la sensitive; cette observation achève de prouver le róle important que joue la sève abourlante dans lin production des mouvements de celte plitite. 
J'ai dit, dans la section précédente, que pour voir l'organisation intéricure du bourrelet je le divisais cn tranches minces. Cettc opération m'a fait apercevoir un nouvcau phénomène. En plongeant ces tranches dans l'cau, on ne tarde point à les voir se ployer en ccrcle. Si ces tranches sont cnlevécs sur le cótć supćricur $a$, leur concavité occupe toujours la partic qui regardait le ecatre ou l'axe du bourrelet; il en est de même si les tranches sont culcrées sur le côtć inférieur $b$; cn sorte que le bourrelct se trouve ainsi composć de deux ressorts antagonistes, et qui tendent à se courber en scns inverses: Ic ressort inféricur $b$ redressc le pétiole, ct le ressort supćricur $a$ le fléchit. L'action de ecs ressorts ne se manifestc d'une manière bien sensible dans les tranches cnlevées aux bourrelets que lorsqu'on plonge ces tranches dans l'eau, qui jouc certainenient. un rổle important dans le développement de cette lorce élastiquc. En effet, nous avons vu que, dans l'état naturel, c'cst la présenec d'une sève abondante qui donne l'énergic à ces ressorts. Ceci pourrait laire penser que leur force élastique dépendrait d'une sorte de turgesence des cellules gonflées par l'abondance dụ liquide; mais comment concevoir une turgescence qui courberait et qui redresserail alternativement le même organe? It fostrait done admettre que le liquide se portcrait avec excès, tantôt vers le côtcé supéricur du bourrelet, tantôt vers son côté inféricur. Il faudrait çrgalement admettre aue dans les tranches minces dn bourrelel, lesquelles se courbent en cercle, le liquide remplirait 
vec excès les cellules du côté convexe, et se porterait vec moins d'abondance dans les cellules du côté oncave. Ces explications, purement hypothétiques, eraient nulles ponr la science. Nous n'apercevons ici n'un seul fail, c'est l'existence d'unc force élastique ni dininue on qui même cesse d'exister par l'absence 'une quantité suffisante d'enu, ct qui, snivant certaines irconstances, tantôt courbe lc bourrelet vers lia terre, matôt le redresse vers le ciel. Le résultat de cette forece lastique est une courbure du lissu organique dans un ens déterminé; je donnerai à ce phénomène le nom imctenation. Les còtés supéricur et i.férieur du onrrelei tendent ì s'incurver dans des sens inverses; etie incurvation se manifeste dans toutes les tranches, fuclque minces qu'elles soient, dans lespurlles ces zôtés penvent être mécilnicquentent divisés; elle :esse tout-ii-coup d'avoir lieu par l'iumersion des ranches contubes dans un fhide quil ancéantit la vie, el qu'un acide ou une solution alkaline. Le contact le ces substances fait ì l'instant cesser la condunre: es tranches deviennent droites, et ne sont plus susceptibles de se condior de nonvenu; änsi ecte incurvalion élastique est un phénomène vital.

Les deux ressolts vitanx dont l'antagonisme opère alternativenent le reabesscunent et ia flexion du pétiole sont en rapport, sonss le point de vue de leur action, avec des causes occisionelles différentes. L’incurvation du ressort supérienr a est déterminée

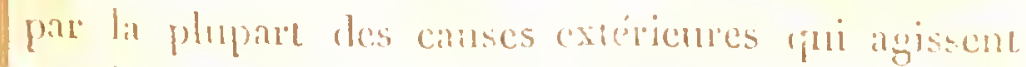
sur la plinte cutiere, on sulemem sur l'une de ses 
parties; telles sont principalement les secousses, l'aetion subite du froid ou d'me trop forte ehalcur, l'action des substanecs eaustiques, cte. Alors le ressolt inféricur $b$ épronve une incurvation qui s'effeetue dans un scns opposć à celui dans lequcl son incurvation naturelle tend à s'opérer ; c'est le résultat d'une augmentation momentanéc et d'une prédominance dans la forec du ressort supćricur $a$. L'incurvation naturellc du ressort inféricur $b$ sc manifestc à son tour d'unc manière prédominante, par lc seul effet de l'absence des causes oecasionelles qui avaient déterminé l'ineurvation du ressort supéricur, c'està-dire par le repos. L'influeiree de la lumière détermine égalcment eelte prédominanec du ressort inférieur; aussi la perd-il et le ressort supérieur devient-il prédominant par le seul fait de l'absence de ect agent; c'est pour cela que les fenilles sc ploient lc soir.

Jusqu'ici nous avons considéré le bourrelet de la sensitive comme eomposé sculement de dcux ressorts antaugonistes, l'ur supérieur $a$, qui flćchit le pétiole, et l'autre inférienr $b$ qui le redresse. Ces mouvements sont en effet, dans l'état naturel, les seuls qu'excrec le bourrelet, mais on peut lui en faire exćeuter d'autres : ainsi, si l'on pleic une ligge de manière à déranger la direction naturelle des leuilles vers la lumic̀re, on voit celte direction se rétablir bientôt, et ccla s'opère sonvent par l'inflexion latérale dủ bourrelet; il y a donc aussi des ressorts latćraux. Effectivement, si l'on culeve des tranehes minces sur les partics la- 
dérales du bourrelet, ces tranches plongées dans l'can se courbent en cercle, de la núme nanière que cela arrive aux tranches enlevécsmax côtés supérienr et inférieur; en un mol, quelle que soit la partie du bourrelet sur laquelle on enlève une tranche, celle-ci jouit tonjours de la propriécé d'affecter, lorsqu'on lit plonggedans l'eau, une courbedont la concavitéregarde l'axe du bourrelet. Ainsi, le bourrelet du pétiole est organisé pour se mouvoir dans tous les sens; cependant il ne se meil ordimaircment que dans deux sens seulement, celui de la flexion, qui est une abduction du pétiole, et celni dn redressement, yni est nne adduction de ce méne pétiole. Or il est fort reniarquable que dans le même moment, et sous l'influence d'une même cause, les folioles et les pinnules se meuvent dans des sens opposés à celni du pétiole. En effer, lorsqu'on provoque les monvements d'unc: fenille de sensitive, les lolioles et les pinnules se menvent dans le sens de l'adduction, elles se rapprochent. de la partic supérieure de l'axe commun qui les porte; le pétiole, au contraire, se meut dans le sens de l'abduction, il s'éloirgne de la partic supéricure de la ligse sur laquelle il est implanté, et ce monvement d'ab;duction est tellement étendu, que le pétiole se rapproche de la partic de la ligge qui lui est iuférieure. Ces organes étant abandonnés à eux-mêmes, ne tardeni point à se monvoir spontanenuent dans des sens opposés à celui de lenr mouvement provogné, c'est-ii-dire les folioles et les pinnules dans le sens de l'abducion, et le péliole dams le sous de l'adduction. 
Nous venons de voir que c'est dins une incurvation vitale, et qui s'exeree dans des sens alternativement opposés, sous l'influenee de eertaines eauses extéricures, que consiste l'irrilabilité de la sensitive. Si actuellement nous jetons les yeux sur les autres plantes chez lesquelles on observe eette irritabilité, nous voyons partout le même phénomène, c'est-àdire une ineurvation vitale du tissu organique. l'hedisarum girans nous montre dans les pétioles de ses feuilles, sans eesse oscillantes, une incurvation oscillatoire, c'est-à-dire qui s'exeree dans des sens alternativement. opposés. Les étamines du cactus opuntia cu du berberis valgaris offrent de même, lorsqu'on les touehe, un simple phénomène d'ineurvation dans un sens déterminé et snivi de redressement quelque temps après que la cause oecasionelle de lineurvation a eessé d'aggir: il en est de même des feuilles de la dionée (dionea muscipula). Dans toutes ces eirconstanees l'ineurvation ne s'effeetue que dans un sens; il n'y a qu'une seule courbmre qui alterne avec $u n$ état de redressement ou avec une eourbure dans un sens opposé; mais il est quelques cas oì eette incurvation oseillatoire s'effectue dans plusieurs sens différents, tel est, par exemple, le phénoniène que présente une plante du genre ypomcea, observée aux Antilles par M. Turpin, plante eneore inédite, qu'il désigne sous le nom d'ypomcea sensitiva. Le tissu membraneux de la corolle eampanulée de cette plante est soutenu par des filets ou par desnervires qui, au moindre attouchement, se plissent ou s'in- 
'curvent simucusement, de manière à cnưâner le tissı membrancux de la corolle, laquelle, de cetie nianière, se ferme complètement; clle ne tarde point à s'ouvrir de nouveau lorsque la causc qui avait déterminé sa plicature a cessć d'aggir. Ce phénomène, dont l'observation appartient ì M. Turpin, n'est point, air reste, essenticllement différent de celui que présente la corolle des compolvulus dont le genre yponter est très voisin. C'est, cn cffet, par le nıême mécanisme que la corolle de ces plantes se ferme le soir et s'ouvre le matin; c'est encorc par le mênre mécanisme que s'ouvre el se ferme la corolle des nyctaginées. Il n'y a de particulier dans l'ypomace sensitiva que la propriété que possède sa corolle de se former sous l'influencedes aģents mécaniques. Ces diverses observations prouvent que l'incurvation oscillatoire des végéctaux est tantôt simple on ì courbure unique, cltantôt simuense on à coübures muliplićcs.

Outre l'incurration oscillatoire, il y a chez les végétanx une incmrration fixe, c'est-à-dire une incurvation qui n'alterne point avec un état de rerlresscment. Ce second phenomène est beauconp plus conmmu que le premier, dont il ne diflère pas essenliellement. L'ovaire de la balsanine en offre un exemple très remarquable. A l'épopue de la maiurité, les valyes de cet ovaire se sćparent les unes des autres, et se roulent cn spirale. A vant leur séparation elles se pressaient mutucllement par l(ur forec élastique, on par leur tendance it lineurvation. Les vrilles et les tiges grimpantes qui se roulent on spirale antour de 
leurs appuis offrent de même un phénomène d'incurvation fixe. Ainsi on peut établir en thèse générale que la locomotilité végétale consiste dans une tendance à l'incurvation fixe ou oscillatoire. Je ne chercherai point ici à déterniner la cause de ce phénomène de la vie vérgétale. Ce serait d'ailleurs en vain que l'on essaierail de le faire avec les seules notions que nous ayons acquises jusqu'ici. On ne peut expliquer les phénomènes de la nature que par un rapprochement de faits; or, le fait de l'incurvation vitale est encore pour nous un phénomène isolé. Ce ne sera que dans l'étude des animaux que nous trouverons de nouveaux faits du même grenre, à l'aide desquels nous pourrons tenter l'explication de ce phénomène. Je me contenterai donc de prouver ici que l'incurvation végétale est un résultat de l'action nervense mise en jeu par les agents du dehors.

Les chocs ou les secousses sont les moyens les plus généralement employés par les curieux pour provoquer les mouvements de la sensitive. Lorsqu'une feuille se ploie sous l'influence d'un choc, on peut penser avec raison que cette influence s'est fait sentir. directement et sans intermédiaire aux bourrelets qui exécutent le mouvement; on en peut dire autant, lorsqu'une secousse imprimée à la plante entière détermine la plicature de toutes les feuilles. Ainsi ces expériences laissent douter s'il existe un mouvement nerveux antérieur an mouvement de flexion des bourrelcts; elles ne permettent pas de distingsuce la nersimotilité de la locomotilité. 11 n'cn cal pans ainsi lors- 
'qu'on sollicite les nouvements de la sensitive par des agrents dont l'influenec ne s'exerce que sur une par: ic détermincee, qui est plus ou moins éloignćc des bourrelets ou des organcs locomolcur's. Les mouvements qu'exćcutent alors ces organes prouvent bien évidemment que lenr action est la suite d'un mouvement nerveux, et que par conséquent la ncrviniotilité et la locomotilité existent d'une manière distincte chez la sensilive. Ainsi, lor'squ'on brúle une seule foliole avec les rayons du soleil rasscmblés par une lentille, ou avec une flamme légère, on voit à linstant cette foliole se ployer avec son opposcé; les folioles voisines se ploient ensuite, et le mouvement se comnunique ainsi de proche en proche et de haut en bas jusqu'ì la hase de la pinnule qui porte ces folioles: les autres pinnules se ploient, et ensuite on voit le nouvement se communiquer de même de proche en proche et de bas en haut aux folioles qu'clles supportent. Pendant que cela s'exécute, et après un certain intervalle de ternps, on voil le pétiole se flćchir. Ce n'est pas kout, les autres fenilles qui garnissent la tige ari-dessus et au-dessous de la feuille qui a ćté brûtée se mettent aussi cn mouvenient les unes après les autres, et l'on voit la plicature de leurs pimnules et de leurs folioles succéder à la flexion de leur pétiole. Il est impossibic de ne pas reconnaître qu'il existe ici un mouvenent invisible qui se transmet de proche en proche. It existe done un pliénomène vital antéricur à la locomotion, et postérieur à l'influence de la cause excérienre. Ce phénomène est. la nersi- 
motion; monvement vital invisible par lui-mêne, appréciable sculement par ses effets; mouvement dont on pent suivre el calculer la marche; mouvement, enfin, qui détermine la locomotion végétale, lorsqu'il parvient aux parties qui, en verlu de leur: organisation, possèdent cette liculté de mouvement. La nervimotion paraît être un mouvensent vital passif, c'est-⿳亠丷⿵-dire communiqué par les agents nervimoteurs; ce premier mouvement vital est la cause immédiate du mouvement vital sceondaire ou de la locomotion qui opère le déplacenıen des parties. Ce mouvement vital secondaire, dépendant immédiatement d'une cause intérieure et vilale, est par celte raison nommé spontané.

La faculté locomotrice n'apparticnt qu'aux bourrelets des feuilles chez la sensitive; coutes les autres parties de celte plante sont étrangères à celte faculté vitale; il n'en est pas de même de la nervimotilité; cette dernière existe dans toutes les parties de la plante. Aussi avons-mous vu que toutes possèdenı des organes nerveux en quanticé plus ou moins considérable. Ainsi, si l'on dirige un verre ardent sur les fleurs de la sensilive, il ne se manifeste à l'extéricur aucun mouvement dans ces fleurs ni dans leur long pédoncule rommun; cependant la nervimotion y est prodrite, car quelques instants après on voil les leuilles de la cige se ployer les nnes après les autres. Le mîne phémoniene a licu lorsqu'on agril sur les fleurs non encore développées et en bonton. Une clialeur un pen vive appliquée par le mine moyen à l'é- 
coreede la tige produit les mêmes nouvemonts dansles feuilles de ecte tige. Lorsqu' une fenille est complèreincut ployée, et quil n'est phus possible de provoquer chez elle aneun mouvement appréciable, clle ne laissc Ipas eependant d'être encore suseeptible de nerviI motion, ear l'ustion de ses folioles provoque la plicat ture des autres feuilles de lir tige à laquelle elle appartient. Ces faits prouvent que la nervimotilité appart lient à tontes les parties de la plante, et yu'eile est très - distinete de la locomotilité. Ici une question fort importante se présente: nous voyons que la nervinıtion prodnite dans une partie quelconque de la plante se transmet de proche en proche aux autres paries. Ce mouvement invisibic se transmet-il par tous les organes intérieurs du végrétal, ou bien y a-t-il des organes spécialement affeetés à cette transmission? Pour arriver a la solution de cette question, j’ai fait des expérienees assez nombrenses, el la plupart fort délieates: je vais les exposer. J'enlevai un annean d'éeorce sur une tigge; les feuilles, comme on le pense bien, se ployèrent toutes par l'elfet de leur agyitation pendant celte općration; mais elles ne tardèrent pas à reprendre leur position de déploiement. Alors je brûlailégèremeñ quelques folioles de la feuille situéc au-dessus de la dé cortication annuhire. Cette fenille se ploya, et quelques instants après les anues leuilles situées ant-dessous de l'endroit décortiqué se ployèrent tour à tour. Je répútai cette cxpérienee, en brullant les folioles de la fenille siluée an-dessons de l'endroit décortiqué. Les feuilles situés au-dessus de cet curlroit ne tardèent 
point à se ployer. Ces expérienees me prouvèrent que la nervimotion se transımet ésalement bien en montant et en descendant, malgré l'enlèvement de l'écoree.

Après avoir enlevé un anneau d'écoree, j'ouvris latéralement le canal médullaire, et j'erlevai toute la moelle; après eette préparation et le repos néeessaire, je brùlai quelques folioles de la feuille située au-dessus du lieu de l'opération. Les feuilles subjacentes ne tardèrent pas à se ployer. Cette expérienee me prouva que la nervimotion se transmet malgré l'enlèvement simultané de l'écorce et de la moelle. Les parties de la plante situées au-dessus et au-dessous du lieu de l'opération ne eommuniquaient plus iei que par la partic ligneuse du système central.

Je voulus savoir si la moelle seule était susceptible de transmettre la nervimotion. A eet effet, je choisis l'un des derniers artieles d'une tige dont la moelle était eneore verte et pleine de sève; j'enlevii tout le tissu végétal jusquàa la moelle sur trois de ses côtés avec un instrument bien tranchant; ensuite je fortifiai la tige, affaiblie par cette opération, an moyen d'une petite attelle de bois que j'attachai avec du fil au-dessus et au-dessous du lieu de l'opération. Cela fait, j'enlevai le tissu végétal jusqu'àla moelle surle côté de la tige qui était resté intact. Je m'assurai que la moelle était parfaitement à nu dans tout son pourtour en l'examinant à la loupe. J'enveloppai la plaie avec du eoton imxbibé d'eau, afm d'empêeher que la moelle ne se desséchât, et j'attendis que les feuilles situées au-dessous du lieu de l'opération se fiussent déployces, 
a car la feuille située au-dessus ne se déploya point. Je Hrùlai légèrement eette dernière, saehant, par mes rexpćrienees préeédentes, que la fcuille dans l'état de plieature cst tout aussi suseeptible de nervimotion que dans l'état de déploiemont. Les feuilles, situées an-dessous du lieu de l'opération, n'éprouvc̀rent aneun mowvement, quelque forte que fùt l'ustion de la feuille supérieure. Cettc expérienec me prouva que la moelle ne transmet point du tout la nervimotion.

Il me restait à savoir si l'ćeoree était susecptible de transmettre ce mouvenent. Je préparai done une tige de manicre que sa partie supćrienre ne eommuniquait avec sa partic inférieurc que par un lambeau d'écoree, qui n'était guère que le tiers de l'écorcc entièrc. Cettc opération fut faite avee assez dc légèreté pour que les feuilles de la partie inféricure de la tigcc soumise à l'expérienee ne se ployassent point, en sortc qu'il me fut possible de laire cette expérienec immédiatcment après l'općration. Ayant done brûlé les feuilles de la partie supérieurc de la tige, eelles dc la partic inférieure ne sc ployc̀rent point, ee qui nuc prouva que l'éeorec ne transmet point la nervimotion. Dans un essai tenté antérienrement, j'avais obtenu un résnltat opposé, lcquel n'avait fait penser que l'ćcoree était sisseptible de transmettre la nervimotion. Mais, ayant répété plusieurs fois ccite expérienee avec beauconp de soin, je nuc suis pleiuement convaincu que l'écorce ne jonissait point du tonu de coue faculté, el que si quelquefois clle paraissait transmettre la nervimntion, cela provenait 
de ee qu'en détachant l'éeoree, j’avais entrainé avec elle quelques filets lighneux du système eentral. C'était par ces filets que la nervimotion se transmettait darıs ees expérienees trompeuses.

Il était important de savoir si le tissu eellulaire rempli de corpuscules nerveux, qui forme la majeure partie des bourrelets, était suseeptible de transmettre la nervimotion. Pour faire eette expérienee, il s'agissait de laisser une portion de ee tissu cellnlaire subsister seule, en enlevant eomplètement le faiseeau de tuhes qui oecupe le eentre du bourrelet. Cette opération est extrêmernent délieate : je vins eependant à bout de l'exéeuter, et j'eus une feuille qui ne eommuniquait plus avee la tige que par le moyen d'une portion du tissu cellulaire de son bourrelet pétiolaire. Je brûlai les folioles de eette feuille; mais les autres feuilles de la tigge restèrent parfaitement immobiles, ce qui me prouva que le tissu eellulaire rempli d'organes nerveux, qui constitue essentiellement le bourrelet, ne transmet point du tout la nervimotion. Je fiș une contre-épreuve : jenlevai tout le tissu eellulaire du bourrelet, et je ne laissai subsister que le très petit faisecau de tubes qui en oceupe le centre, en sorte que la feuille ne communiquait plus avec la tire que par ee petit faiseeau. Je brûlai ses folioles, et bientôt après les autres feuilles de la tige se ployèrent.

Il résulte de ces expérienees, que la moelle, l'écoree, et le tissu eellulaire rempli de eorpuscules herveux, qui constitue le bourrelet, sont également incapabjles 
de transmente la nervimotion, el que ee mouvement iinvisible cst exclusivement transmis par la portion lligneuse du sysième central. L'anatomic que nous avons présentée plus haut, de toutes les parties de Ila sensitive nous met à mếme de rechercher les coauses de ceuc différence qui cxiste cntre les ficultés des diverses parties de la plante. La moelle est cntièrement conposćc de tissu cellulaire qui contient des corpuscules nerveux. Conme elle ne uransmet point la nervimotion, cela prouve, $1^{\circ}$ que ce nouwenent u'est point transmis par le tissu cellulaire, $2^{\circ}$ qu'il ne se propage point non plus par le moyen des corpuscules nerveux que contient ce lissu ecllulaire. Ceue inaplitude des corpuscules nerveux à transmettre la nervimotion est encore démontrée d'une manière plus évilente par le tissu cellulaire , corpusculifere du bourrelet. Ici les corpuseules nerveux sont extrêuncment nombrenx; cependant ce 1 lissu cellulaire corpusculilère ne transmet poinc la nervintotion. Nous sommes done forećs de reconnaitre que les corpuscules nervenx, qui somt, dans ma manière de voir, les agents de la prissance nerveuse, ne sont cependant point les organes de la tramsmission de celle puissance.

Il nous reste à comparer l'organisation du système cortical qui ne transmet point la nervimotion avec l'organisation de la partic du systène cemural qui lrumsmet ce nouvencut. L'écorce est exclusivement composéc de clostres el de lissu cellulaire articulé corpusculiferere. La partic ligneuse du systeme ecn- 
tral contient des trachées, des tubes corpusculifères, des clostres et du tissu cellulaire artieulé eorpusculifère. L'inaptitude des clostres et du tissu cellulaire articulé corpuseulifère à transmettre la nervimotion dans le système cortieal doil nous porter à refuser celte fonetion à ees mênes organes dans' le système central. Il ne nous reste donć, dans ce dernier système, que les trachées et les tubes corpuseulitères, auxquels, par voie d'exelusion, nous puissions at tribuer la faculté de transmettre la nervimotion. Mais l'expérience prouve que cette transmission s'opère sans le coneours des trachées. En effet, j'ai vu qu'en laissant subsister le plus petit filet de la partie extérieure du système central comme seul moyen de communication entre les' deux parties d'une tige, cela suflisait pour transmettre la nervimotion de l'nne à l'autre. Or les traehées oeeupent exelusivement l'étui médullaire : elles sont done, dans eette expérience, étrangères à la transmission de la nervimotion. Il ne reste done, en définitive, que les tubes corpuseulifères auxquels nous puissions attribuer cette transmission. Ces tubes, mêlés aux clostres, se trouvent en effet dans toute l'épaisseur de la couclie ligneuse. Iei l'on peut se demander si c'est par le moyen de la sève qu'ils conduisent, ou par le moyen des eorpuscules nerveux qui sont pheés dans leurs parois, que ces tubes transmettent la nervimotion. Nous avons eonstaté plus haut linaptitude des corpuseules nerveux pour opérer ectle transmission, il reste donc démòntré qu'elie s'opière 
par l'intermédiaire de la sève. Cette conclusion est mise hor's de doute par les observations suivantes. Il lest eertain que les parties qui conduisent la sève sont Hes seules qui conduisent également la nervimotion. Lorsque deux portions de igge ne communiquent plus enure elles que par le moyen de la moclle ou par le moyen de la seule écorce, la portion supérieure ne tarde point à se flétrir et à nourir, parceque la moelle et l'éeoree ne transmettent point la sève d'une portion à l'autre. Elles ne transmettent point non plus lla nervinotion. Lorsqu'une feuille de sensitive ne communique plus avec la tige que par le noyen du tissu eellulaire lu bourrelet de son pétiole, clle se fane promptement, pareeyue ee tissu cellulitire ne transmet point la sève; il ne transunet point non plus la nervimotion. Lorsqu'au eonlraire une feuille ne communique plus arec la tige que par le moyen du petit faisceau de tubes qui occupe le centre du bourrelet du pétiole, ee petit faiseeau de tubes eontinue à nourrir la leuille; en lui transmettant la sève, il transmet également la nervinotion. 'Toutes les portions du systène central qui conticnnent des tubes propres à transmeure la sève, sont également propres à transnettre da nervinotion. En un not, nous voyons toujonrs la mansmission de la sève liće d'une nanière exclusive et insciparable i la transunission de la nervimolion; il n'y a done pas de doute que la transmission de la puissance nervense, ehe\% la sensilive, ne sopore par l'internédiane du liquide séveux. Les corpuseules nerveux sont ćtran- 
grers à eetue transmission, bien qu'ils soient les organes producteurs de eette puissance, au moyen de l'influence des agents nervimoteurs.

La nervimotilité n'appartient pas exelusivement aux diverses parties de la tige de la sensitive, on l'observe aussi dans les raeines de eette plante; l'expérience qui prouve eelte assertion appartient à l'illustre Desfontaines, et je l'ai répétée. Si l'on arrose les racines de la sensitive avee de l'acide sulfurique, on ne tarde point à voir les feuilles de la tige se ployer les un€s après les autres; celles qui sont les plus voisines de la racine se ploient les premières; les feuilles qui occupent les extrémités des rameaux se ploient les dernières. Il y a évidemment, dans ee phénomène, une transmission de la nervimotion qui provoque la plieature des fenilles à mesure qu'elle parvient jusqu'à elles, el qui tire son origine de l'action exereée sur les racines par l'acide qui les baigne. Je n'avais versé de l'acide que dans un seul endroit sur les racines de ma sensitive. Lorsque je vis toutes les feuilles ployées, j'enlevai, en les cernant avee un conteau, toutes les racines offensées, ainsi que la terre impréynée d'acide: la plante, quelques heures après, redressa ses pétioles, mais elle ne déploya ses folioles que le lendemain; cette opération ne la fit point mourir.

La transmission de la puissance nerveuse ou la nervimotion s'opère avee assez de lenteur chez la sensilive. Il s'écoule en effet un temps assez considérable cntre le moment où l'on brile légèrement une fo- 
nioic avec un verre ardent, et eelui on la nervimoion produite pa: cette action parvient aux autres iolioles, aux pinnules, au bourrelet du pétiole, et enfin aux autres feuilles de la tige. II ne parut donc flu'il n'ćtait point impossible de inesurer le temps qui "écoulail entre ces diverses actions, et de comparer" des espaces parcourus par la nervimotion avec les ıcnips cmployés à parcourir ces espaces. Il était intportant de savoir si les variations de la température unlluaient sur la vitesse de la transnission de ce monvement intérienr. J'ai fiat dans cette vue un grant inombre d'expérienecs; voici la méthode que jempployais : je brûlais légèrement les folioles terminales de l'une des pimnules d'une feuille, soil avec un verre ardent, soit avec une flamme légère. A l'instant les folioles comrençaient à se ployer par paires les unes après les antres. Je tenais près de non coreille une montre dont le balancier effectuait ses oscillations, composées chacune de deux battements, dans une deni-seconde; je complais le nombre de ses oscillations, à partir du moment de l'nstion jusiqu ì céhi où les pinnules opéraient leur flexion; je mesurais de la même manière le temrps qui s'écoulait jusqu'au monent de la flexion du pétiole; j'appliquais ensuite la même mesure an temps yui s'écoulait jus'qu'an moment de la flexion suceessive des pétioles des antres fienilles de la tige. Cete première partie de l'observation ćant faite, je nuesurais la longuene de lis pinnule, celle du pétiole, el celle des articles

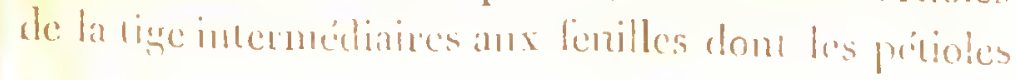


s'étaient fléehis. De cette manière il n'était facile de eomparer les espaces pareourus par la nervimotion avec les temps employés pour les pareourir. J'ai fait eette expérience la température de l'atmosphère étant à 10 , à 13 , à 15 , à 18 , à 20 et à 25 degrés de ehalenr au thermomètre de Réaumur. Voiei les résulıats généraux que j'ai obtenus : la progression de la nervimotion est toujours beauconp plus rapide dans les pinnules et dans les pétioles qu'elle ne l'est dans les articles de la tige. La vitesse ordinaire de ee mouvement dans les pétioles est de huit ì quinze millimètres par seeonde, tandis que dans les arlicles de la tige ce même mouvement, n'exeède pas deux à trois millimèires par seconde, et souvent est encore plus lent. La température de l'atmosphère ne m'a paru exereer aueune influence sur la vitesse : le ee mouvenent; ear j'ai obtenu des résultats peu différents les uns des autres aux divers degrés de température dont je viens de faire mention. Les variations que j'ai obtenues dans ees résultats ont été purement aeeidentelles, et sans aueun rapport fixe avee les variations de la tentpérature extérieure; seulement j’ai observé que, lorsque la température était à + dix degrés, la nervimotion provoquée par l'ustion se transmettait à une distance moindre que eclle à laquelle elle parvenait lorsque la température était plus élevée.

Nous venons de voir que la nervimotion a constamnient une vitesse plus cousidćrable dans les pétioles que dans la tige, lorsque $\bullet$ inouvement provoqué dans les folioles traverse le pétiole en desecn- 
Hant pour gagner le corps de la tige. J'ai observé que ic même phénomène a lieu lorsque la nervimotion orovoquéc dans la tigoc par l'nstion de son écorce arrive aux pétioles et les traverse en remontant pour zagner les pinnules et les folioles. Voici comment jo aisais cette expérience: après avoir brûlé vivcment l'écorce de la tige avee un verre ardent, je ne tardais pas à voir les feuilles les plus voisines fléchir leur tpétiole. Bientôt après, les pinnules et les !olioles de ces funilles se ployaient à lemr tour; je mesurais le temps qui s'écoulait entre le moment de la flexion du pétiole et le mo:nent de la flexion des pinnules; puis je comparais le temps écoulé avee la longuenr du pétiole. J'ai trouvé, de cette manière, que la nervimotion avait, en remontant dans le pétiole, la même i vitesse que nous avons observé qu'elle avait cn descendant dans ee mêne pétiole, c’est-à-dire que ce I mouvęment parcourait loujours de huit ì quinze milllimètres par seconde, tandis que dans le corps de la uligg ce même monvement ne parcou:l que deux à trois millimètres dans le même temps. L'étude comparalive que nous avons faite plus liant de la structure anatomique de ces partics ne nons apprend point du tont la canse d'une différence anssi considérable. If me parait done probable que cente différence tient spécialement à la différence du diamètre des partics; la nervinotion est plus rapide dans les pétioles, lesquels ont peu de dianètre, fu'elle ne l'est dans lit tige, dont le diancitre est phus considérable. Ce monvenent nervens messmblerait par conséfuent, sons 
ce point de vue, au mouvement des fluides qui, nus avec une vitesse détcrminće dans un canal étroit, perdent de cette vitesse en proportion de l'élargissement du canal qui les transmet, et la reprennent de nouveau lorsque le canal se rétrécit. Cette explication du phénomène dont il s'agit devient encore plus plausible par l'observation que nous avons déjà faite, que c'est par l'intermédiaire du liquide séveux que la nervimotion se transmct.

La nervimotion provoquéc par l'ustion d'une feuille se propage quelquefois jusqu'aux branches voisines de celle qui porte cette feuille, en sorte qu'on voit quelquefois se ployer des feuilles très éloignées de celle sur laquelle on fait l'expérience. Il m'a scmblé que l'intensité de l'ustion influait sur l'étendue de la propagation de la nervimotion; ce mouvement ne s'étcndait qu'à peu de distanec lorsque l'ustion était extrêmement légère. On scnt qu'il est difficile dę déıerminer d'une manière certaine le degré d'intensité de l'ustion que l'on opère; ecpendant je pouvais jagger approximativement de son intensité comparative lorsque jemployais le verre ardent; car je modérais à volonté la chalcur produite en parcil cas, en plaçanı le verre de nıanière à ce que la feuille soumisc à son action fìt situce plus ou moins en-deçà ou au-delà de son foyer. De cette manière on peut provoquer dans la feuille une nervimotion qui ne s'étend pas plus loin que la base de son pétiole.

La communication en ligne droile, an moyen des tubes sćveux, influe beancoup sur la promptitude 
ce la propagation de la nervimotion. On sent que cela doil être ainsi, puisque e'est le fluide séveux qui ransmet ce mouvement. Aussi ai-je obscrvé que, orsyu'on brule une feuille de sensitive, il arrive onvent que la nerrimotion parvient à la feuille qui st située du même côté deux articles plus has, avant le se manifester dans la feuille située dans l'article oisin, mais du côté opposé de la tiğc; car on sait que es feuilles de la sensitive sont alternes.

ILes leuilles de la sensitive perdent complètement rear motilité, lorsque la température de l'atnosphère e trouve à sept degrés environ au-dessus de gुlace, u thermomètre de Réaumur; on peut alors les bruer sans qu'il en résuite chez elles aucun phénomène le mouvement appréciable.

La lumière solaire exeree sur l'énergie de la moilité de la sensitive une influenec extrêmement renarquable, et qui pourtani n'a point eneore été obervée. Cependant plusieurs naturalistes, et notamnent MM. Duhamel, Dufay et Decandolle, ons herché à ćtudier les phénomènes que préscnte ecte slante, lorsqu'elle est plongré dans nne profonde bseuritć. Ces naturalistes ont toujours choisi des aves pour faire cette expérience; mais, la température le ces lieux souterrains me paraissant peu favorable u libre et plein exerciee des laculés vilates de la sensitive, je résolus d'employer, pour: somstraire cette plante à l'influence de la lumière, 111 procédé qui laissât subsister sur clle l'influence nécessaire l'une cempérame plus élevée. A cer effer, je placai un 
pied de sensilive, planté dans un pot sous un réeipient tait avee du earton fort épais. Toutes les préeautions possibles avaient été prises dans la fabrieation de ce réeipient pour qu'aucun rayon de lumière ne pénétrît dans son intérieur. J'aceumulais de la seiure de bois autour de son orifiee, afin d'intercepter tout-à-fait la faible lumière qui aurait pu pénétrer par cette voie. Cet appareil fut établi dans un appartement qui, situé sous la tuile et exposé au midi, éprouvait pendant le jour une forte chaleur, qu'il conservait avec peu de diminution pendant la nuit. C'était pendant les chaleurs de l'été; le thermomètre se tint coustamment, dans cet appartement, à une élévation de + 20 à 25 degrés pendant mon observation. La sensitive, ainsi plongée dans une profonde obseurité sans être soustraite à l'influenee de la ehaleur, commenea par ployer toutes ses feuilles. Vers le milieu du premier jour, clle les déploya à demi, et les ferma eomplètement le soir. Le lendemain au matin, je trouvai toutes les feuilles complètement déployées, ct déjà leur motilité était sensiblement diminuée; elles ne se fermèrent plus d'une manière complète, et le roisième jour; je les trouvai à moitié déployées, et leurs folioles avaient perdu leur motilité; le pétiole seul avait eneore la faeulté de se fléehir. Je voulus voir si, dans eette diminution eonsidérable de la motilité, la nervimotion aurait éprouvé de l'altération dans la rapidité de sa progression. Je brûlai légèrement l'une des folioles d'une, feuille; la nervimotion se transmit, eorume à l'ordinaire, à la 
base du pétiole et de là aux pétioles de deux autres feuilles de la tige. Dans cette progression, la nervimotion parcourut dix millimètres par seconde dans ta pimnule de la feuille et dans son pétiole; elle parconrut deux nillimètres par seconde dans la tige. La même expérience, faite sur un autre pied de sensitive qui était dans le même appartement, et qui jouissait de toute sa motilité, me donna des résultats à peu près ppareils. Ainsi il me fut prouvé que la diminution He la motilité n'en apporte aucune dans la rapidité Hc la progression de la nervimotion. Seulement je remarrquai que ce mouvement se propagea moins Loin ehez la sensitive dont la motilité était diminuée. Je la remis sous le récipient pour continuer mon observation. Le quatiième jour, les pétioles des feuilles se ployaient encore, mais faiblement lorsqu'on Hes frappait vivement; les folioles étaient immobiles: le cinquième jour, toute espèce de motilité appréciable avait disparu. L'ustion elle-même ne provoquait pplus aucun mouvement dans les feuilles qui étaient à moitié ouvertes, et dont les pétioles étaient redressés. J'exposai alors cette sensitive à la lumière du soleil; les folioles tardèrent peu à se déployer complètement, et, au bout de denx heures, elles commencèrent ì se mouvoir légèrement lorsqu'on les frappait. CepenIdant le pétiole continuait ì demeurer imnobile. Après 'deux heures el demie d'insolation, les pótioles commencèrent à manifester de la motilité; elle augunenta peu à peu, et, dans le courant de la journée suivante,

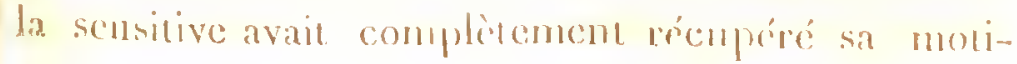


lité. Il résulte de cette expérience qu'il suffit de priver la sensitive de l'influence de la lumière pour lui faire perdre les conditions de sa motilité, et que c'est dans l'influence de cet agent qu'elle puise de nouveau ces conditions, lorsqu'elle les a perdues. J'ai voulu voir quelle était l'influence qu'exerçait la température extérieure sur ce phénomene. J'ai donc répété cette expérience de la même manière sur d'autres pieds de sensitive, car celui sur lequel cette expérience avait été faite avait un peu souffert; plusieurs de ses feuilles étaient tombées. Je plaçai donc une de ses plantes sous mon récipient; la chaleur de l'appartentent étail alors de +22 degrés Réaunır, et elle monta jusqu’à 24 deggrés pendant la durée de l'expérience. Au bout de quatre jours et demi d'obscurité, la sensitive avait complètement perdu sa motilité. Je fis alors, sur le phénomène du retournement des feuilles, une expérience qui sera, rapportée dans l'une des sections suivantes. Dans cette seconde expérience, l'abolition de la motilité fut un peu plus rapide que dans la première; cela me parut devoir dépendre du degré de la température extérieure, qui avait été constamment de +22 à 24 degrés, tandis que dans la première expérience cette même température avait été assez constamment de +20 à $2 \tilde{J}$ degrés; elle ne s'était élevée qu'un seul jour à 25 degrés. Pour m'assurer davantage du değré de l'influence qu'exerçaiı la température extérieure sur la production de ce phénomène, je fis de nouveau cette même expérience par une température qui varia de +14 à 20 degrés. Il 
ifallut dix jours d'obscurité à la sensitive pour lui faire perdıe complètement sa motilité. Il me parut bien śvident, par cette troisième expérience, qu'une température modérée retardait l'extinction de la motilité alicz la sensitive, plongée dans l'obscurité; les expériences précédentes m'avaient appris que cette extinction était bien plus rapide lorsque la température ćtait ćlcvée. J'avais vu précédemment que l'exposition aux rayons directs du soleil rendait assez promptement lles conditions de la motilité à la sensitive qui les avait perdues. Je voulus voir, dans cette circonstance, si lc même effet serait produit par la lumière diffuse du jour. J'exposai donc la sensitive tirée de dessous le récipient, en plein air, dertière un bâtiment qui la garantissait des rayons directs du soleil. Le premier jour, la sensitive ne nranifesta aucune motilité, mais lorsque la nuit arriva, quelques unes de ses feuilles, celles qui avaient le plus récemment atteint leur complet développentent, se ployèrent, et présentèrent ainsi le phénonène du sommeil qui avait cessé d'avoir lieu sous le récipicnt. Le lendemain, les folioles se déployèrent, mais elles ne manifestaient aucune motilité sons l'inftuence des plus fortes secousses. Les vicilles leuilles avaient presciue toutes perdu leurs folioles; celles qui restivient con-mencèrent à présenter le phénomène du sonmeil le second jour. Le troisiène jour, les folioles' commencèrent ì se monvoir sous l'influence des choes; le pélinles chaicnt encore immobiles. İe quatrième jour, les pétioles commenciencint ì se monvoir assez ló- 
gèremeni, et, le cinquième jour, la sensitive avait récupéré sa motilité. Ainsi il fallut cinq jours d'exposition à la lumière diffuse du jour pour rendre à la sensitive les conditions de sa motilité : nous avons vu qu'il suffisait de quelques heures d'exposition à la lumière dirccte du soleil pour produire le même effet. Je recommençai cette expérience une quatrième fois par une température qui varia de +13 à 17 degrés. Il falluz onze jours d'obscurité pour opérer l'extinction complète de la motilité de la sensitive. Cette fois je ne pus observer le retour de la motilité, parceque la sensitive rendue à la lumière perdit toutes ses feuilles. Je répétai une cinquième fois l'expérience dont il est ici question par une température qui varia de +10 à 15 degrés dans l'appartement où était le récipient sous lequel était placéc la sensitive. Cette plante, plongée dans une obscurité complète, conserva sa motilité sans aucune altération bien sensible pendant dix jours. Le douzième jour, les folioles cessèrent de se mouvoir lorsqu'on les frappait; les pétioles seuls possédaient encore leur motilité. Le quinzième jour, toute motilité appréciable avait disparu. La sensitive avait souffert par cette longue obscurité; plusieurs de ses feuilles avaient jauni et leurs folioles tombaient à la moindre seeousse. Cependant un assez grand nombre de ces feuilles avaient conservé lcur couleur verte cı me paraissaient suseeptibles de réeupérer leur motilité. Je voulus voir si cet effet pouvait être produit par l'exposition de la plante à la lumière diffuse 
Hn jour, telle qu'elle parvient dans une chambre ar les fenctures au moyen de la réllexion des nuages ul des objets du dehors. Ayant done tirć na sensitive We dessous son récipient, je la plaçai dans un lieu !e l'appartement qui était bien éclairé, mais qui ne cecevait point la lunière directe du soleil; dès le soir la premice jour quelques unes des fenilles les moins iguées conmencèrent à présenter le phénomène dt: commsil, qui avait cessé d'avoir lieu sous le récipient. ILe lendemain, les folioles se déployèrent à la lumière, mais restèrent immobiles sous l'inflnence des phis fortes secousses. Les feuilles plus âgées ne comnencèrent ì présenter le phénomène du sommeil que le quatrième jour. Alors les folioles des jeunes leuilles se mouvaient fort légèrement lorsqu'on les cloquai vivement avec le doigr ; les pétioles étaicnt immobiles. Le cinquième jour, la plante continua de présenter I les mêrnes plićnomènes d'une motilitć langruissante. I Le sixième jour, je plaçai la sensitive aux rayous d'un solecil ardent; au bout de quatre beures, les jemes I leuilles avaient complèicment récupéré leur motilité, et les vieilles fentilles l'avaient récupéréc en partic. Ces dernières avaicm jusqu'alors relusé de se momoir sous l'influence des chocs. Liexposition de lit plinte au soleil pendinu la duréc du sejptiène jour acheva de hui rendre complètcment sil molilité. Il résulte de ces expériences que la privation de la lumière occasione chez la sensitive l'abolition des conditions. de lit motilite, et que l'exposition de cette plante it la lumiere lui rend ace conditions perdues. Ceme 
perte des conditions de la motilité dans l'obscurité est fort rapide quand la température est très ćlevée, elle est beaucoup plus lentelorsque cette température offre un ccrlain degré d'abaissement. En effet, nous avons vu qu'il n'a fallu que quatre à cinq jours d'absence de la lumière, par une température de +20 à 25 degrés , pour abolir complètement la motilité d'une sensilive, tandis que, par une température de +15 à 20 degrés il a fallu dix jours d'obscurité pour produire cette abolition ; et qu'il a fallu quinze jours d'obscurité pour produire ec même effet, lorsque la température était de +10 à 15 degrés. La rapidité du retour des conditions de la motilité chez la sensitive qui les a perdues dans l'obscurité est en raison de l'intensité de la lumière à laquelle elle est soumisc. Nous avons vu en effet qu'il ne faut que quelques heures d'cxposition à la lumière directe du soleil pour réparer ces conditions perdues, tandis que pour produirc le même effet il faut plusieurs jours d'exposition à la lumière diffuse du jour. Il résulte de ces expériences que la lumière, et spécialement la lumière solaire, est l'agent extérieur dans l'influenee duquel les végétaux puisent le renouvellement des conditions de leur motilité. J'ignore en quoi consiste cette influence réparatrice, mais le fait de cette réparation est certain, comme l'est eelui de l'abolition de ces conditions dans l'obscurité. Dans les expériences qui viennent d'être exposées, j'ai observé que les folioles ont perdu leur motilité avant les pétioles, et l'ont récupéréc avant eux. Jai observé de même que les 
jeunes feuilles ont récupéré ícur motilité avant les vicilles feuilles, et que, chez les unes comme chez les autres, les piemiers indices de la motilité réparée se sont manilestés par les sculs phénomicnes du sommeil et du réveil. Ces phénomènes de motilité vitale ont été pendant quelque temps les seuls qu'ait présentés la scnsitive dont la motilité n'était pas cncore cntièrement x'écupérée. Il résulte de là qu'en privant une sensitive d'une portion des conditions de sa moilité, on la réduit au mode d'existence des véçétaux vulgaires, c'est-à-dire qu'clle ne meut point ses feuilles sous l'influence des aggents nervimoteurs mécaniques, bicn qu'clle les meuve cncore pour présenter les phénomènes du sommeil et du réveil. Il est enfin un état d'épuisentent des conditions de la motilité qui, sans occasioner chez la sensitive la mort de la feuille, fait qu'elle demeure quelque temps dans un état d'immobilité parfaite, et qu'clle est ineapable de sommeil et de réveil appréciables, comme le sont tant d'autres végétaux. Cela prouve que toutes les différences qui existent à cet égrard cntre les plantes dérivent seulement de ec qu'elles possèrdent en quautité différente les conditions de la motilité, conditions dont la nature est cncore inconnue. Ces conditions sont réparées chéz les véçétaux par la lumière solaire; par conséquent l'influcnce qu'exeree la lumière sur les véģétaux est comparable ì celle qu'exerce l'oxiggénation respiratoire sur les animaux. Onl sait que chez ces dernices l'énerouie de la inotilité est généalenent en raisun de la puanticéde la res- 
piration, c'est-à-dire cn raison de la quantité de l'oxigène absorbć; toute motilité cesse rapidement lorsque l'oxigćnation du sang n'a plus lieu. Le genre de l'influence qu'exerce l'oxigénation des fluides sur' l'éncrgie de la motilité animale est inconnu; lc fait seul de cette influence est bien constaté. Il en est de même de l'influence qu'exerce la lumière solairc sur l'ćnergie de la motilité végćtale; Ic genre de cette influence est inconnu, mais le fail de cette influence est constaté. Donc l'inssolation est pour les végétaux ce que l'oxigénation est pour lcs animaux. Cic sont deux sortcs de visification, si je puis m'expriner - ainsi. Il résulte de ce rapprochement que l'étiolement des végétaux cst un état analogue à celui de l'asphyxie des animaux; dans l'un comme dans l'autre il $\mathrm{y}$ a diminution ou abolition des conditions de la motilité, par cause de l'absence de l'agent cxtéricur qui sert à les entrctcnir. Ce rapprochement inattendu est encorc fortifié par la considération suivante. On sait combien l'asphyxic est rapide chez les animaux à sang chaııd; on sait combicn elle est lente chez les animaux à sang froid; on sail enfin, par les expériences dc M. Edwards, quc chcz ces dernicrs l'asphyxie peut être à volonté accélérée ou rcetardée, cn augmentant ou en diminuant la température extérieure dans certaines limites. $\mathrm{Or}_{\mathrm{r}}$, chez la sensitive, nous observons le mêmc phénomìne. Nous voyons son asphyxie arriver promptement quand il fait chaud, c. tardivement quand la température est phus basse. 'Tout concourt done à prouver qu'une même lonc;- 
on réparatrice de la molilité est exereée de deus nanières différentes par les animaux et par les végéux. Les premiers exereent eetle fonction réparatrice u moyen de l'oxigénation, et les seeonds au moyen e l'insolation. Il est à remarquer que ee sont lì les eux eauses les plus universelles de la production de it clisleur.

La eonclusion définitive que nous tirerons de ces sxpériences est que la motilité de la sensitive décend de trois conditions principales, $1^{\circ}$ de l'exissnee d'une température plus élevée que le septiène cegré au-dessus de zéro, au thermomètre de Réanaur; $2^{\circ}$ de l'influence de la lumière; $5^{\circ}$ de la préenee d'une sève suffisamment abondante. L'absenee l'une seale de ees conditions suffil pour anéantir comslètenıent la motilité de ecue plante. 


\section{SECTION III.}

DES DIRECTIONS SPECIALES QU'AFFECTENT LES DIVERSIS PARTIES DES VÉgÉTAUX',

Les phénomènes les plus généraux de la nature, ceux qu'elle présente sans cesse à nos yeux, sontén ģénéral ceux que la plupart des hommes remarquent le moins. Celui qui n'a point appris à méditer sur les phénomènes naturels, a peine à se persuader, par exemple, qu'il existe un mystère profond dans l'ascension des tiges des végétaux, et dans la progression descendante de leurs racines. Ce phénomène, cependant, est un des plus curieux parmi ceux que nous offre la vie végrétale. Le mouvement descendant des racines parâtra facile à expliquer pour la plupart des esprits: elles tendent, dira-t-on, comme tous les autres corps, vers le centrẹ de la terre, en vertu des lois connues de la pesanteur; mais comment expliquera-t-on l'ascension verticale des tiges, qui est en opposition manifeste avec ees lois? C'est ici qu'ont échoué ceux qui ont tenté d'expliquer ce phéno-

- Ce mémoire avait été présenté (à l'Académic royale des sciences) - pour le prix de physiologic, et l'Académic a dú regretter que ce prix - fut restrcint des cettc anncie il la plyysiologic animale. Anatyse des travau, de l'stcalimic royalc des scicnes pondant l'annéc $18_{21}$, par M. le biron Cinvier. 
ène. Dodart', le premier, à ce qu'il paraît, qui $\downarrow$ recueilli quelques observations sur cet objet, éétend expliquer le retournement de la radicule et : la plumule dans les graines semées à contre sens, ar l'hypothèse suivante: il admet que la racine est omposéc de parties qui se contractent par l'effet de sumidité, et que les parties de la tigge, au contraire, ; contractent par l'effet de la sécheresse. Il doit en śsulter, sclon lui, que, dans la graine semće à cone sens, la radicule tournée vers le ciel se contracte s'incline ver's la terre, siége de l'humidité; tandis true la plumule, au contraire, se contracte et se tourne u cóté du ciel, ou plutôt de l'atmosphère, nialicu lus sec ou moins humide que ne l'est la terre. On onnaît les expériences de Dulamel, è les tentatives qu'il a faites pour contraindre des graines à pousser eur radicule en haut, et leur plumule en bas, en les nfermant dans des tuhes qui ne permettaicnt pas le etournement de ces parties; ne pouvant obër ì leurs endances naturelles, la radicule et la plumule se :ontournèrent en spirale. Ces expériences prouvent que les tendances opposées de la radienle et de la plumule ne peuvent être interverties, mais elles nous aissent dans une ignorance complète de la cause ì aquelle sont dues ces tendances. Nous ignorons de même la cause du retournencut des levilles. Bonnet ${ }^{2}$ a cru pouvoir appliguer a l'explication de ce

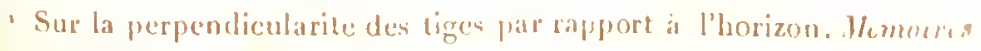
de t'Académic des scicnees. 1700.

" Rerherches sur l'usage des feuille. 
phénomène l'hypothèse imaginée par Dodart pour expliquer le retournement de la radicule et de la plumule dans les graines scmées à contre sens. Selon ce naturaliste, la face inférieure des feuilles est, comme la radicule, composée de fibres qui se contractent à l'humidité, tandis que leur face supérieure est, comme la plumule, composée de fibres qui se contractent à la sécheresse. Cherchant à donner des preuves à ces assertions, Bonnet imagina de lảbriquer des feuilles artificielles, dont la face supérieure était en parchemin, qui se contracte par l'effet de la sécheresse, et dont la face inférieure était en toile, dont les fils se raccourcissent par l'effet de l'humidité. Il soumit ces feuilles à la chaleur et à l'humidité, et crut voir qu'elles se comportaient à peu près comme de véritables feuilles. Ce que prouve le mieux cette étrange expérience, c'est le danger qu'il y a d'observer la nature avec des systèmes faits à l'avance, et dans l'intention de leur trouver des preuves.

Convaincus del'insuffisance des hypothèses proposées pour expliquer les directions spéciales qu'affectent lesdiverses parties des végétaux, les physiologgistes se bornent aujourd'hui à dire que ces directions spéciales sont des phénomènes vitaux. Mais cette assertion, dont au reste tout concourt à prouver la vérité; cette assertion, dis-je, ne nous apprend rien sur la canse de ces phénomènes. Il en est du phénomène de la direction opposée des tiges et des racines comme de la plupart des phénomènes que la nature offre à notre observation: rarement, ils sont les effets d'une cause 
unique; la plupart du temps plusieurs causes concourent à les produire. La tâche de l'observatemr consiste ì démêler des causes diverses, et à assigner la part que prend cliaeuned'elles dansla production du phénomène.

En voyant les tiges se diriger constamment vers le ciel, et les racines se diriger toujours vers la terre, on peut penser qu'il existe un certain rapport entre I la cause de la gravitation et celle de la vie végétale; lla direction également constante des tiges vers la lumière peut aussi porter à penser que cet agent est ponr lles végétanx une cause de direction spéciale. Les tiges ipour se développer ont besoin d'être placées dans le sein de l'atmosphère; les racines au contraire ont lbesoin de se trouver dans le scin de la terre: existerait-il une tendance entre l'atmosphère et la tigge, entre la terre humide et la racine, tendanee de laquelle résulterait l'ascension de la tige, et le monvement descendant de la racine? C'està l'observation à éclaircir nos doutes sur ces différents objets.

J'ai rempli de terre une boite dont le fond était percé de plusieurs trous; j'ai placé des graines de haricot (phaseolus vulgaris) dans ces trous, et j’ai suspendn la boîte en plein air à une élévation de six mètres. De cette manière les graines, placées dans les trous pratiqués à la faee inférieure de la boite, recevaient de lbas en haut l'influence de l'atinosplière et de la lumière : la terre humide se tronvait placće ant-dessus d'elles. Si la cause de la direction de la plumnle et de la radieule existait dans une tendance de ces parties pour la terre humide el pour l'anmosphère, an 
devait voir la radicule monter dans la terre placée audessus d'elle, et la tige au contraire descendre vers l'atmosphère placée au-dessous; c'est .ce qui n'eut point lieu. Les radicules des graines descendirent dans l'atmosphère, où elles se desséchèrent bientôt; les plumules, au contraire, se dirigrèrent en haut dans l'intérieur de la terre. Je plaçai verticalement en hau la pointe de la radicule de quelques unes de ces graines germées, en les enfonçant dans les trous dont il vient d'être question; ces radicules, au lieu de se diriger ver's la masse de terre humide placée au-dessus d'elles, se courbèrent en bas. Je voulus voir si nne srande masse de terre, placée an-dessus des graines, exercerait plus d'influence sur la direction de leurs radicules. Je fixai donc des graines de haricot au plancher d'une excavation qui était recouverte d'environ six mètres de terre, et je les y maintins dans de la terre humide par des moyens appropriés. Les résultats de cette seconde expérience ne furent point différents de ceux de la première.

Ces expériences prouvent que ce n'est point ver's la terre humide que se dirige la radicule, et que ce n'est point vers l'atmosphère que se dirige la plumule. Ces deux parties se dirigrent toujours l'une vers le centre de la terre, l'autre dans une direction opposée. Quoiqu'il paraisse résulter des expéricnces précédentes que la radicule des embryons séminaux ne possède aucune tendance spéciale vers les corps humides, on pourrait cependant penser que, dans les expériences dont il s'agit, la tendance de la radicule 
vers le centre de la terre étant plus forte que la tendanee supposéc de cette même radicule vers les corps lummides, eetle derniere tendance n'aurait pas pu se manifester. J'ai vu évanouir ee soupeson par l'expérienee suivante : j'ai suspendu dans un bocal une petite soucoupe que j’ai remplie d'eau, et dans laquelle j’ai placé une éponģe taillée et placée de manière ì présenter une face plane verticale; ensuite, au moyen d'un fil de fer fixé au couvercle du bocal, j’ai suspendu dans l'intérieur de ee dernier une feve nouvellement germée, ayant soin de placer la radi- eule aussi près qu'il était possible de la face vertieale de l'éponge sans la toucher. De cette manière le eorps humide ćlait placé latéralement par rapport à la radicule, et eomme il n'y avait point d'eau an fond du boeal, et que la face vertieale de l'éponge dépassait un peu le hord de la soucoupe qui la eontenait, il en résultai que la radieule, si elle avait une tendanee tvers l'hunidité, devait se eomrber latéralement pour se diriger vers l'éponge qui l'avoisinait; car il n'y avait point d'eau ni de corps hunide de tout autre côté. Au reste, l'air de l'intérieur du boeal sc trouvint saturé d'eau, et la radieule étant extrêmenent rapprochée de l'épongre mouillée, ecla non seulement smpéchail cetre zadieule de se flétrir, mais fournissait i son absorption une quancité d'cau sullisante pour suffire à son développement el inĉrne à la production le nouvelles raeines latérates. Cette expérience rlonna les résultats suivants: Ia rarlicule ne nunilesta aucune tendance vers l'éponge indsibréc d'eau; les racines lat- 
térales qu'elle produisit du côté de l'éponge pénétrèrent dans les cellules de cette dernière; mais les autres racines latérales qui prireut naissance dans les autres points de la surlace de la radicule ne manifestèrent aucune tendance vers l'éponge, quoique plusicurs de ces racines latérales prissent leur origine très près de ce corps monillé. Il résulie de ces diver'ses expériences que les racines n'ont aucune tendance vers les corps humides, et que, par consćquent, cette cause n'est point une de celles qui déterninent ha direction des racines vers la terre. It est probable que les tiges n'ont pas plus de tendance spéciale ver's l'air atmosphérique, que les racines n'en ont vers l'cau, mais on ne peut gucuère s'en assurer par l'expérience.

Tous les végétaux ne sont pas destinés par la nature à plonger leurs racines dans la terre; les végétanx parasites enfoncent leurs racines dans la suljstance d'antres végétaux : les radicules de leur's cmbryons se dirigent-elles aussi vers le centre de la terre? L'observation de la germination de la graine du gui résout cette question par la négative. On sait depuis long-temps que la gaine du gui germe dans toutes les directions. Le premier développentent de l'embryon de cette graine consiste dans une élongaltion caulinaire de sa tige, qui puise la matière de cet accroissement dans la substance des cotylédons, auxquels elle aboutit par l'une de ses extrénités, et qui est terminée à son autre extrémité par un petit renflement d'un vert moins foncé qui est la radicule. Lorsque la graine est fixéc sur une branche d'arbre 
au moyen de sa glu naturelle, on voit la tigge de l'embryon se courber pour diriger la radicule dins un sens perpendiculaire à lá surlace de la branche; car cetce radicule elle-mêne, qui ne eonsiste qu'en un petit corps hémisphérique, ne subut ordinairement aucune inflexion. Lorsque la radicule touche la surface de la branclıe, clle s'épanouil dessus on me sorte de disque, résultat de l'aplatissenent du tubereule hémisphérique qui la constituait. C'est de la partic de ce disque qui est collée sur la branche que sortent les racines qui vont puiser leur nourriture dans la substance de la branche qui porte cetce plante parasite. Quelle que soit la place qu'oceupe la graine dn gui sur la branehe d'un artse, l'entbryon dirige constanment sa radieule ver's le centre de ectte branche; en sorte que cette radieule est, suivant la position de la graine, tantôt deseendante, tantôt ascendante, 'tantồt diriggée horizontalement, ete. Existet-il dans cette circonstance une tendance de la radicule vers les parties vivantes du végétal dans lequel elle doit s'implanter? Pour éclaireir ce donte, jai fixs des graines de grui sur du bois mort, sur des pierres, snr des eorps métalliques, sur du verre, etc., toujonrs jai vu la radienle prendre nue direction perpendiculaire au plan sur lequel la garaine était collée. Je fixai un zrand nombre de graines de gui snr la surface d'un gros boulet de ler; toutes les radientes se dirigerent vers le centre dn bonlet. Ces faits pronvent que ce n'est point vers nu milien prople à sit murrifion que l'embryou du gan dirier sa radicule, mais 
que celle-ei obéit à l'attraction des eorps sur lesquels la graine est fixée, quelle que soit leur naturc. Ainsi, les radicules des végétaux terrestres obéisscnt it l'attraetion de la terie, tandis que la radieule du gui par'asite obéit à l'attraction particulière des corps. Les tigcs des végétaux terrestres se dirigent dans le scns opposé ì cclui de l'auraetion du globe, et s'ćlèvent. ainsi au-dessus du sol, auquel clles deviennent perpendiculaires; la tige du gui affecte toujours une dircetion perpendiculairc à eelie de la branche sur laquelle elle est implantéc; cn sorte qu'clle cst descendante lorsque l'implantation a licu à la facc inféricurc de la branche, ascendante lorsque cette inplantation est taite à la face supérieure, eic.; cllc sc dirigc constamment dans un sens opposé à celui de l'attraction de la branche. Ainsi, l'cmbryon du gui se comporte, par rapport à la branche qui le nourril, comme les cmbryons terrestres se comportent par rapport à la terre. Ces deux phénomènes, différents au premier eoup d'œil, sc trouvent, au moyen de eette analysc, être du même genrc. Les moisissures nous offrent cneore un excmple remarquable de la perpendieularité des tiges par rapport aux corps sur lesquicls elles sont fixćes, et de l'absenec de ectte même perpendicularitć par rapport à la terre. Spallanzani a noté unc partic de ce phénomène dans ses observations sur l'origine des moisissures, mais il ne l'a point aperçu dans son entier; il n'a point vu que les moisissures affectent eonstamment unc direction perpendieulaire à celle de la surface sur laquelle ellcs 
sont implantées. J'ai observé ce liait chez les muisissures aquatiques conme ehcz les moisissures aériennes. Les poils des végétaux se comportunt à cel égard eomme les moisissures, c'est-it-dire qu'ils sont toujours perpendiculaires à leur surface d'implantation. Il paraît que l'extrême ténuité de ces produclions véģétales les soumet spéeialement à l’influence de l'atraetion particulicre des eorps sur lesquels elles sont implantées, et les soustrait à l'influenec de l'attraetion dü grobe terrestre. C'est ainsi que nous voyous les corps réduits en poussicre fine adlécer aux corps les plus polis, et manilester par là qu'ils obéissent à l'atuaction particulière de ces corps, de prélérence à l'atuaction du golobe terrestre. Lat tendanee des racines et des tiges, les unes dans le sens de la pesanteur, les autres dans le seris diantétralement opposé, ne se remarque d'mne manière spécials que dams les candex ascendauts et descendants, éest-à-dire dans l'axe du végétal considéré daus son cutice. I es productions latérales de cet axe prennent tonjours une direction plus ou novins differente. On sail que les branches qui maissent alux parties latérales de la vige principale, ainsi que les racines qui sout produites latéralement par la racine pivotante, n'aflectent point ordinairenent unc direction parfaitement verticale. Plusienrs canses influent sur la direction quelpquefois parfaitcuncent horizontale qu'elles prennent : nous tâcherons d'exposer ces causces diverses; l'une d'entre elles est indubitiblement la tent-

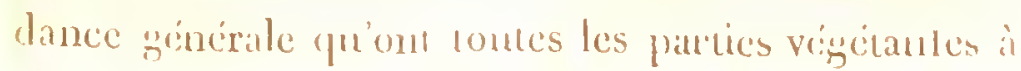


affecter une direction perpendiculaire à leur surface partieulière d'implantarion. La branehe latérale et la raeine latérale se comportent comme le grui par rapport à la branehe sur laqnelle il est implanté; la tige prineipale et la raeine pivotante sont des surfaces partienlières d'implantation auxquelles les branches et les raeines latérales tendent à devenir perpendieulaires : mais comme eette tendance est combinée avec les tendanees générales qui portent les tigres en haut et les racines en bas, il en résulte ordinairement une direetion moyenne, en sorte que les branches et les raeines font, avec l'axe vertieal du végétal, un angle plus ou moins ouvert. En faisant germer et développer des graines dans de l'eau ou dans de 'a moussc: humide, on est à même de voir que les racines latérales n'ont qu'une faible tendance vers le eentre de la terre. On voit de ces racines latérales, longues d'un ou de deux centimètres, qui sont dirigées dans une horizontalité parfaite; j'en ai même vu quelques unes qui étaient tout-à-fait ascendantes : ce n'est que lorsqu'elles ont acquis une certaine longneur qu'elles commencent à se diriger en bas; elles sont en eela bien différentes de la radieule pivotante, qui, dès qu'elle conmence à se manifester, tend vers le centre de la terre avec une énergie el une eonstanee qu'il est impossible de vainere. On peut faire, sur les branches, des observations semblables. J'ai vu des branches de chêne nées à la surface inférienre de grosses branehes horizontales se diriger verticalement en bas jusqu'à ce qu'elles eussent aequis environ la longuenr 
d'm décimètre; alors sentement elles commencèrent il releyer leur extrénité végétante vers le ciet. Dans heincoup d'anthes, les branches latérales végèeent dans une horizontalité phus on moins partaite; cette horizontalité qui, dans la branche naissante, parait due it la, tendance que possèle cette branche a se disposer perpendiculairement à sa surliace d'implantation, qui est ici la surfice de la tige verticale, cente horizontalité, dis-je, est duc à d'antres canses lorsque lir branche a acquis une certaine longuens. Son poids l'entraine vers la terre, et les branches supérienres qui s'étendent anrdessus d'elle, de même dans le sens horizontal, l'cmpêchent de se dresser vers le ciel. Ces denx causcs tendent à unaintenir sou horizontalité, yui est encore entretenne par l'action de la lunière, que les extrénités végétantes des hianches horizontales ne reçoivent que latéralement.

L'influence que les tiges, considérées conme surfaces dimptantation, exercent sur la perpendicnlarite des branches anxupucles elles donnent naissance prarait ne s'écendre qu'ì unc très petite distance; de parait mêtue quelquefois proportionuclle it la masse de ces tiones: je dis quelquefois, car il s'en faut beauconp que cente règle puisse être doméc comme générale. Cependant il est un lait qui tend à prouver qu'clle n'est pas sans fondenent. Nous avons vu plus laart que la graine du gुui tend constamment à implanter sa radicule perpendicularement is la strelince de la branche, on phus zénéralenene du corps sur lepuel ello est fixée; or, jait observe que sa la- 
dicule ne se dirige point vers ce corps lorsqu'il est trop délić, ou lorsqu'elle en est trop éloignćc. Une distance de cing à six millimètres suffic pour anćantir toute tendance de la radicule du gui vers les corps qui l'avoisinent. Il suffit cncore, pour anćantir cette tendance, de fixer la graine du gui sur des corps filiformes qui aient moins d'un millimètre de diamètre; dans ces deux circonstances, la radicule ne se dirige point vers le corps qui porte ou qui ayoisine la graine, clle prend une direction particulière, ainsi que je l'exposerai plus bas. Nous venons de voir, par l'exemple des moisissures et des poils des végétaux, que l'extrême ténuité de ces productions végétales les soumet spécialement à l'auraction particulic̀re des corps, comme cela a lieu pour les corps inorganiques. Ces faits prouvent que l'influence des surfaces d'implantation pour déterminer la direction perpendiculaire des prodactions végétales est en rapport avec l'étenduc de ces surfaces; ils prouvent en même temps que cette influence est en rapport avec la distance qui existe entre ces surfaces et les productions végétales qui leur deviennent perpendiculaires.

I.es faits qui viennent d'être exposés prouvent que la cáase inconnue de l'attraction générale agit sụ les végétaux comne cause de dircetion spéciale, mais ils prouvent en même temps qu'il s'en laut beaucoup que celte cause agrisse sur les végétaux conme elle agit sur les corps inertes. Chèz ces derniers, clle produit constanment la tendance vers le centre de gravité; chez les êtres vivants végétaux, elle ne produit 
cette tcndance que pour les racines; clle détcrmine une tendance opposćc dans les tigges. Ce phénomène, en apparence paradoxal, peut faire soupçonner que la cause de la gravitation n'est point la causc immédiate de la direction des tiges et des racines, mais qu'elle en cst sculement la causc éloighnée ou occasionelle; pour ćclaircir ce doute, j’ai fait l'expéricnec suivante. J'ai pris unc graine de gui, que j’avais fait préalablement germer suspendue à un fil délicé, d'où il était résulté que la tigge de l'embryon s'était développéc sans que la radicule hémisphérique qui la terninait cùt manilesté aucnne tendance ì se fixer. J'ai collé cette graine gyerméc à l'une des extrémités d'une aiguille de cuivre construitc comme unc aiguille de boussole et suspendue de même sur un pivol; une petitc boulc de cire placéc à l'autre extrémité de l'aiguruille formait contre-poids. Les choses étant ainsi disposécs, j’ai approché latéralement de la radicule une petite planche de bois que j’ai placéc à un millimètre environ de distance de la radicule. J'ai cnsuite couvert cet apparcil d'un récipicnt de verre, afin qu'ancune cause cxtérienre ne pît faire mouvoir l'aignille sur son pivot. Au bout de cing jour's j'ai vu la tige de l'embryon se fléchir et diriger la radicule vers la petite planche qui l'avoisinait, ct cela sans que l'aiguille cût changoé de position, quoiqu'clle fìt extrêmement moḅile sur sou pivot. Deux jours après, la radiculc était dirigée perpendiculairement vers ta planche, avec laquelle elle s'était mise cu contact; al cependant l'aiguille, qui 
portait la graine, n'avait point varié daus sa direetion. Celte expérience est fort délicate, et demande, pour réussir, des préeautions particnlières. Il faut que l'appareil soit nuis à l'ombre, car si le réeipient était ćchanffé par les rayons du soleil, il communiquerait à l'air qu'il contient un mouvement qui se ferait senlir à l'aiguille; il faut que cente expérience soit faite par un tenıps chaud, ear la gyérmination de la graine du gui ne s'opère qu'avec une extrême lentenr lorsque le thermomètre de Réannunr n'est pas au moins ì quinze rlegrés au-dessus de zéro. Comne il est facile de tronver des graines de gui mùres de l'année précédente jusque vers le milieu de l'été, j’ai pu faire l'expérience dont il s'agit pendant les jours les pliss chands de cette saison. Malgré ces précautions, mon expérience a quelquefois été dérangée par une autre cause. La ghu qui enveloppe la graine est fort hygrométrique; l'eau qu'elle ahsorbe de l'atmosphère ou qu'elle lui live angmente ou diminue son poids, en sorte que, suspeudne à l'une des pointes d'mue aignuille mobile, elle fait éprouver à cette dernière des mouvements de haseule qui penvent um peu déranger sa direction; anssi m'a-t-il fallu répéter plusieurs lois l'expérience pour la voir réussir ì souhair.

Cetue expérience pronve que la direction de la radieule dn grui vers les eorps qui l'avoisinent u'est point le résnltat inmmédiat de l'attraction exercée sur elle par ces corps, mais qu'elle est le résultat d'un monvement spontané exécutcé par l'embryon, à l'occasion de l'altraction exercée sur sal radicule, attrac- 
tion qui n'est ainsi que la cause médiate on occasionelle du phénomène. H est facilc, en effet, de comprendic que l'inflexion de la tige de l'embryon du grui ne peut être due à l'action irnmédiate excrcée sur la radicule par l'attraction de la petite planche de bois, car 1 une torce extérieure capable d'općrer cette inflexion eût opévé avee bien plus de facilité un changement dans la direction de l'aiguille à l'une des pointes de laquelle la graine était fixée. Il n'y a donc point de donle que ce monvement ne soit spontané, c'est-idire qu’il ne soit dû à une cause intérieure ct vilale mise en jeu par l'influcnce d'un aggent extérienr. Cette spontanéité de la direction de la radicule du gुnir sous l'influence de l'atuaction prouve d'une manière incontestablc que celle allracion n'a agi que sur la nervimotilité du véģćtal, et point du tont sur sa matière pondérable. Il en est indubitablement de même pour les végétaux terrestres. La cause inconnue de l'attraction n'est que la cause occasionelle du mouvement descendant des racines et de l'ascension des tigres; elle n'en est point la cause immédiate; elle asgit, dans cette circonstance, comme agent nervimotemr. Nous verrons plus bas de nouvelles preuves de la généralité de ce lait important en pliysiologie, savoir, que les mouvcments visibles des végctanx sont tous des monvements spontanés, exócutós à l'occasion de l'influence d'un aggent extérieur, et non des monvements imprimés par cet aggent.

La lunière est pour les végéranx muc caunse de direction speciale non moins énergigne que eclle dout 
nous venons d'observer l'influence. On sait qu'une plante renferméc dans un appartement qui ne reçoit la lumière que par une seule ouverture dirige eonstamment vers cette ouverture sa tige, qui cesse d'alfecter une position perpendiculaire à l'horizon. Nul doute que ecte tendance des tiges vers la lumière n'ait également lieu en pleiri air. La lumière affluant de toutes parts, à peu près en égale quantité par la réflexion des nuages ct de l'atmosphère, doil déterminer l'ascension des tiges vers le cicl; elle est en cela l'auxiliaire de 'la cause de là gravitation. On pourrait même penser que la tendance ver's la lumière serait la cause unique de l'ascension des tiges et de leur position verticale, si l'expérience ne prouvait le contraire. J'ai eouché sur le sol, dans un endroit sec et parfaitement obscur, des tiges d'allium cepa ct d'allium porrum, arrachées avec leurs bulbes; on sait que ces plantes, quoique déracinées, continuent long-temps à vivre : ces tiges se courbìrent dans runc portion de leur longnenr, et lemr partie supćrieure se dirigea vers le ciel. Je n'obtins ce résultal qu'an bout de dix jours, tandis qu'il ne me lallut que trois jour's pour l'obtenir en répétant la même expérience en plein air. L'absence de la lumière, dans la première expérienee, ne permé d'aturibuer le redressenent de la tige qu'ì la causc de la gravitation, seule cause connuc qui agisse dans le sens perpendiculaire à l'horizon ; ecpendant on pourrait peut-être penser que l'humidité agrirait ici pour rendre convexe le côtć de lia ligge en contiact avec le 
sol, et déterminer ainsi la flexion de la tige vers le haut. J'ai déjà dit que le lien où se faisait cette expérience ćtait fort see, ainsi il n'était pas probable qune le redresscment de la tige füt dù à la eause que je viens d'indiquer; cependant, pour dissiper tous les Ĺdoutcs à ect écrard, j'ai répété l'expérienee en eouehant une lige d'allium porrum dans une auge qui eontenail assez d'eau pour eouvrir entièrement eette tige retenue au fond. Iei l'influence de l'humidité devenait nulle, par eela mêne qu'elle s'excręait simulınément nur toutes les parties de la tige: celle-ei ne laissa pas ide se dresser vers le ciel. Je voulus voir si la spathe remplie de fleurs qui terminait eette tige avail quelque influence sur son redressentent: je l'enlevai; et la tige à laquelle j'avais fait cette amputation ne laissa pas de se redresser. Je variai l'expérience : ayant couché la tige et l'ayant eourbée en arc, je la fixai solidement au sol en deux points de son étendue. L'arc eouehé sur le sol se redressa el tourna sa eonvexité vers le cicl. Cette expérienee me réussit éģadement bien en plein air et dans l'obscurité; sculement il fallut, dans ce dernicr cas, un temps beancoup plus long. Ces expérienecs prouvent que le redressement des tiges ver's le cicl est dî simultanément à l'influenee de la cause de la guravitation ex à l'influcnce de la lumière. Ce n'est point seulcment par lenr partic supérieure que les liges iendent vers le cicl ou vers la lumière. Bumnet a prouvé cette vérité par des expcrienees que j’ai répétres, et qui m’ont donné des résultats semblables à cenx qu'il a obtenus. 
J'ai enfoncé le sommet d'une tige, encore jeune, dc mercurialis annua dans l'ouverture d'une fiole remplie d'ean, et placée verticalement; Fuis, fléchissant la partie inférieure de cette tige vers la terre, je l'ai maintenue dans cette flexion avec une ligatnre tixée au col de la fiole. La portion de tige ainsi fléchie était dépourvue de feuilles; exposée à linflnence de la lumière, cette plante ne tarda pas à dresser vers le ciel sa portion libre, qui était la partie iuférieure de la tige. Ainsi ce n'est point seulemenı par lèur sommet que les tiges tendent vers le ciel; nous verrons bientôt que cette tendance se manifeste dans toutes leurs parties mobiles lorsqu'elles sont colorées.

Les tiges se dirigent quelquetois vers la terre, dans laquelle elles tendent à s'enfoncer comme des racines. Ce phénomène mérite une attention tonte particulière, tant ponr lui-même que par rapport aux circonstances qui l'accompagnent et qui le déterninent. Beaucoup de végétaux, outre leurs tiges aériennes, possèdent des tiges souterraines, ainsi que je l'ai fait voir dans mes Recherches sur l'accroissement et la reproduction des végétaux'. Ces tiges souterraines rampent horizontalement dans l'intérieur de la terre, sans manifester aucune tendance ver's le ciel; elles sont blanches comne les raciues dont elles affectent la direction et dont elles habitent le séjour. Quelquefois cependant eiles sont de couleur de rose, comme cela s'observe, par exemple, chez le sparganium 
erectum; mais alors c'est l'épidernue qui se trouve coloré et non le pareneliyme subjacent. Lorsque lis pointe de ees tiges souterraines approche de la surf face du sol, elle verdit, et dès lors elle tend vers le cic!. Pourquoi cette tendanee, qui était mulle dans la tigge blanche ou plutôt décolorée, se manifeste-t-clle dans cette même tigge lorsqu'elle vient à veıdir? Y aurait-il donc un rapport secret entre la eoloration des parties des végétaux et les tendances diverses yu'elles aftectent? L'observation va nous éelairer sur ee mystère.

En général, les tiges se diriggent vers la lmuière, ce qui eoincide avee leur coloration, presque coujours en vert; les racines n'affectent ordinairement aucuns: direction vers la Immière, ce qui coüncide avec lemr défaut de coloration. La couleur des racines IL'est autre, en effè, que celle du tissu végétal décoloré; leur blancheur ne saurait être comparée au blane nat que présentent les pétales de plusieurs végétanx, et yai est dù à la présence d'ume natière colorante blanche. lLa lnmière, principale mais non pas seule cansc de la coloration des tiges et de leurs organes, ne posscide aucun pouvoir pour colorer les racincs, anissi yuion pent s'en assurer en faisant développer les racines d'une plante dans l'ean contenue dans nu bocal de verre; malgré l'influence de la lunière elles restent constamment insolores; ecei ne tieut point à leur inumersion dans l'eau, car les feuilles des vérgélaux aquatiques sont colorées nialgré leur submersion. En giinéral, les racines ne possèdent aucune tendance ver's la lunière, mais cette tendince se manileste Jorsque 
le bourgeon terminal d'une racine acquiert une teinte légèrement verdâtre, commc ccla arrive quelquefois. J'avais fait germer des graines de mirabilis jalappa dans de la mousse humide, et je remarquai que les rádicules, déjà de la longueır du doigt, étaient termi nées par un bourgeon de couleur légèrement verdâtre. Je voulus voir si ces racines dirigeraient leur pointe vers la lumière. A cet effét je les plaçai dans un bacal de verre rempli d'eau et dont le couvercle de bois ćtait percé de trous pour recevoir les raeines et fixer les graines. J'enveloppai le boeal avec une étoffe noirc; en laissant sculement une fente verticale de peu de largeur, par laquelle la lumière parvenait dans l'intérieur du bocal. Je dirigeai cette fente vers la lumière du soleil; quelques heures après, je vis que toutes mes racines en expérience avaient courbé leur pointe en croehet, pour la diriger vers la fentc qui leur transmettait Ja lumière. Je fis la mème expérience avec d'autres racines dont le bourgeon terminal n'était point vcrdâtre, elles demeurèrent immobiles. D'après cette expérience, il est évident que la coloration est une des conditions qui déterminent la tendance de parties des végétaux vers la lumière, et par conséquent vers le ciel. Cela est si vrai que, lorsqu'elles sont décolorées, les tiges naissantes se dirigent ver's la terre. J'ai observé ce fait curieux chez plusieurs plantes aquatiques, et notamment chez le sagittaria sagittifolia. Des tiges naissent des bourgeons situćs dans les aisselles des feuilles toutes radicales de cette plante, qui, conme on sait, crôt au fond 
des eaux. Ces bouryeons ont leur pointe dirigée vers lle ciel, comme cela a lieu chez tous les végétaux. Les jjemnes tiges qui naissent de ces bourgeons sont entièreiment déeolorées comme des racines: aussi, au lieu de se diriger vers le eiel, comme le font les tigres colorées, telles se courbent et dirigent leur pointe verticalement wers le centre de laterre; se comportant dans ce retourinement comme laradicule d'une graineseméc à contre sens. Pour parvenir à prendre cette position, la jeune tige peree de vive force toute l'épaisseur du pétiole engainant de la feuille dans l'aisselle de laquelle elle pris naissance, surmontant ainsi l'obstacle mécanique qui s'oppose à sa tendance vers la terre. Cette tige souterraine, munie de feuilles décolorées comme elle, se plonge dans la vase, où bientôt sa progression devient horizontale; ce n'est que lorsqu'elle a acquis une certaine longueur que son bourgeon terminal commence à acquérir une couleur verte; dès ors elle devient ascendante et sort de la vase, elle levient tige aérienne. Les racines offrent quelquelois un phénomène analogue quoique inverse. On sait que Jlusicurs végétaux produisent des racines sur difféentes parties de leur tige : lorsque ees raeines aéiennes sont ineolores, elles se dirigent toujours vers e centre de la terre; mais lorsqu'elles ont une couceur verte elles recourbent leur pointe et la dirigent vers le ciel. J'ai observé ce dernier phénomène chez e pothos crassinervia et ehez le cactus phyllanluc.s. Ainsi, ce n'est point en leur qualité de tiges fue les tiges se dirigent ver's le eiel, c'est parcequ'elles 
ont un parenehyme coloré; et ce n'est point en leur qualité de raeines que les racines descenden: vers la terre, c'est pareeque leur parenchyme est ineolore. Au reste, en indiquant la présence ou l'absence de la eoloration $d u$ parenchyme superficiel eomme la cause de la différenee de la direction des tiges et des raeines, je ne fais qu'indiquer une condition générale de l'organisation végétale qui accompagıe constamnent eette différenee de direetion. Nous reviendrons plus bas sur eette coïncidenee de phénomènes. Les racines des végétaux terrestres, ainsi que nous venons de le voir, se dirigent vers la lumière lorsque leur parenchyme est coloré; elles n'affeetent aucune tendanee ni vers la lumière, ni dans le sens opposé, lorsque leur parenehyme est incolore. La radicule de l'embryon du gui offre à cet égard un phénomène tout partieulier. Cette radieule, qui est d'un verd bien moins foneé que eelui de la tige de l'cnibryon, au lieu de se diriger vers la lumière eomme ecla semblerait devoir être, en sa qualité de partie verte, se dirige au contraire eonstamment en sens inverse, comme si elle était repoussée par la lumic̀re. Pour être témoin de ee phénomène, il faut, dans l'intérieur d'un appartement, et vis-à-vis d'une l'enêtre, tendre un fil sur lequel on collera des gráines de grui, au moyen de leur glu naturelle. Ces graines, si le temps est chaud, ne tarderont point à germer, et l'on verra toutes les radicules se diriger vers le fond de l'appartement. Cette tendanee à fuir la lumière est ici la seule à laquelle obéit la radicule de l'embryon du gui, 
parceque le fil dćlié sur lequel la graine est fixée n'exerce pas sur ectle radicule une altraction assez puissante pour la détermincr à se diriger vers lui. Plus on approche de la fenêtre le fil qui porte les graines, blus la tendance de la radicule à fuir la lumièrc de̛ient énergique. J'ai collé plusieurs de ces graines ur les carreaux de vitre cn dedans de l'appartemcnt; outes les radicules se sont dirigées vers le fond de et appartement, obéissant ainsi à leur tendance à uir la lumière, de préfércnee à la ıcndanee qui, dans onte autre position, les cût portées vers la sị̣rface u carreau sur lequel elles étaicnt fixées. J'avais cn aême temps eollé un pareil nombrede ces graines en ehors, sur la faee opposée du même carreau de vitre; zutes les radieules se diriģèrcnt vers la surface de ce arreau, obéissant ainsi aux deux tendances qui les solcitaient dans le mênıe sens, c'est-à-dire à la tendance fuir la lumière et à la tendance à obéir à l'attraction du vrps sur lequel elles étaient fixées. J'ai retourné quelues unes de ces graines, et je les ai placées en sens verse de celui qu'elles a vaient pris naturdlenıcnt : les aines, de l'intéricur dont javais dirigé les radicules ers le carreau de vitre, ne tardèrent joint à ramencr « Inêmes radieules vers l'intéricur de l'apparteınent; 's graines de l'extćrieur dont javais dirigré les radihes vers les objets du dehors, ramenèrent cn mêne mps ces mêmes radiculcs vers la surface du carrean : vitre. La lumière directe ne possède pas seulc l: onvoir de déterminer le monvement rétrograde do radicule de gui; la lumière réflćchie par les objors 
tcrestrcs produit le mêmc effet : je m’ẹn suis assuré par l'expériencc suivante : j’ai pris un tube de bois fermé à l'un de ses bouts par une lame de verre, et recouvert à l'autre bout par un couverele de bois fermant cxactement; jai collé plusieurs yraines de gui sur la face intérieurc de la lame de verre, et j’ai suspendu le tube vcrtiealcment sous l'abri du toit d'une lenêtre en mansarde, et de manièrc à ee que l'cxtrémité de ee tube qui était fermée par la lame de verre fût en bas : ainsi l'intérieur du tube n'était éclairé que par la lunière que rćfléchissaient les objets terrestres. Les radicules des graines de gui mises en expérience se dirigèrent toutcs vertiealement vers le eiel, fuyant ainsi la lumière qui leur arrivait de bas en haut. Il ćtait intéressant de savoir si cette tendance singulière de la radicule du gui était le résultat d'une répulsion exereéc sur clle par la lumière. Je pris une graine dc gui que j'avais fait préalablement germer sur un fil et vis-à-vis de la lumière. Cette graine portait deux embryons dont les radicules étaient fléchies du même côté. Je fixai cettc graine à l'une des cxtrémités de l'aiguille de cuivre qui m'avait déjà servi dans une expérience rapportée plus haut, aiguille qui se suspend sur un pivot à la manière des aiguilles de boussole; je eouvris d'un récipient de verre cet appareil que je plaçai auprès d'une fenêtre que n'éclairaient point les rayons dircets du soleil, et j'eus soin de diriger les deux radicules vers la lumière. Au bout de quelques jours, ees deux radiculcs changèrent de direction, et se dirigèrent vers le fond de l'appar- 
ttement, sans laire éprouver aucun changement à la alirection de l'aiguille. Gette expérience me pronva ique la radicule du grui fuit la lumière par un nonveiment spontané, et non par l'effet d'une répulsion qui scrait exercée sur elle; car une force extérieure qui serait capable de fléchir la tige de l'embryon du gui -serait bien plus que suffisante pour opérer un changement de direction dans l'aiguille extrêmıment mothile qui portait cet embryon. Il résulıe de ces expériences et de celles qui ont été rapportées plus haut que la radicule de l'embryon du sui affecte deux tendances spontanées à l'occasion de l'influence de deux agents nervimoteurs différents. Le premier de ces agents, qui est l'attraction particulière des corps, est la cause occasionelle de la tendance spontanée de cette radicule vers ces mêmes corps; le second de ces agents, qui est la lumière, est la cansc occasionelle de la tendance spontanée que inanifeste cctte radicule à fuir cette lumière elle-mếnı.

Pour compléter mes observations sur la graine du gui, il me restait à observer la tendance quaffecterait la radicule dans l'obscurité, la graine étant I fixée sur un fil, et par conséquent soustraite à !'inIfluence de l'attraction particulière des corps. Les expériences que j'ai faites à cet égard ne n'ont rien appris de hien positil; jai vu, dans cette circonstance, la radicule affecter tontes sorles de rirections; cependant j’ai observé que très rarement la radicule s'est dirigéc vers la terre; un peu plus souvent sa direction a ćté horizontale, on inclince diversenent ì 
l'horizon; dans le plus grand nombre des cas, la radicule a été ascendante. Le seul fait bien certain qui résulte de ces observations, c'est que la radicule du gui ne possède aucune tendance vers le centre de la terre, comme cela a lieu chez la radicule des végétaux terrestres. On peut tirer de là cette conclusion vraimenı paradoxale, que la radicule du gui, qui obéit à l'attraction particulière des corps, n'obéit point du tout à l'attraction du globe terrestre; attraction qui n'est cependant que la somme des auractions particulières exercées par les corps dont le globe est composé.

Dans les observations que je viens de rapporter sur la graine du gui, je n'ai point parlé de la direction de la plumule, parceque ce n'est qu'un an après la germination qu'elle se développe; il ne se manifeste d'abord du caudex ascendant de l'embryon du gui que la portion de la tige qui est comprise entre l'insertion des cotylédons et l'origine de la radicule. La plumule, située entre les cotylédons, reste pendant la première année à l'état rudimentaire, et ne prend ainsi aucune direction particulière pendant la germination; les cotylédons eux-mêmes, fixés sur les corps an moyen de la glu qui les environne, n'ont aucune liberté pour prendre une direction quelconque; ce n'est que dans le printemps de la seconde année que les cotylédons desséchés se détachent de la tige qui commence à développer ses premières feuilles.

Les végétaux offrent un autre phénomène de direction spéciale qui a beaucoup occupé Jes observateurs de la nature: je veux parler de la direction 
constante de la laee supérieure des feuilles vers le ciel, et de leur faec inférieure vers la terre. Lorsqu'on renverse une feuille, et qu'on maintient la lace inférienre dirigée vers le eiel, il s'opère, soit dans le eorps de la feuille, soil dans son pétiole, une torsion au moyen de laquelle la face inférieure est ramence vers la terre, et la face supérieure vers le ciel. Bonnet, a fait beaueoup de reeherehes sur ec phénomène qu'il a eru pouvoir expliquer par l'influenee qu'exereerait, sur la face inférieure des feuilles, l'humidité qui s'élève de la terre; mais eette tendance de la faee inférieure des feuilles vers l'humidite ne peut être admise, puisque le retournement de ees organes a lien dans l'eau comme dans l'air. Cette cxpérienee est due à. Bonnet lui-même, et il est bien singulier qu'il n'ait pas vu qu'elle renversait sa théoric. Au reste, e'est faute d'avoir observé le phénomène de la direction des feuilles dans toute sa généralité que Bonnet a affirmé que la face des feuilles appelée supérieure se dirige eonstamment vers le ciel, et la faec opposcé vers la terre; il existe à eet égard des exeeptions fort remarquables: il y a presque tonjours une différenee sensible d'organisation entre la face sujé́rieure et la faee inférieure des feuilles, la face supérieure est presque toujours plus colorée que ne l'est la face inférieure, qui est ordinairement d'un vert banehâtre. Cette différenee de la eoloration des denx liees de la feuille coïncide constamment avee la dif- 
férenee de la direction de ces faces; la face la plus eolorée se dirige toujours vers la lumière, ou plus gुenéralement vers le eiel; la face qui a le moins de coloration, e'est-à-dire dont la coloration est moins vive, se dirige toujours vers la terre : aussi lorsque la face supérieure est moins eolorée que la face inférieure, la feuille présente une position inverse de eclle qui s'observe chez presque tous les végétaux; sa position est renversée, c'est-à-dire que sa face supérieur'e est dirigée vers la terre, et que, par conséquent, sa face inférieure est dirigée vers le eiel. C'esı ce que j’ai observé ehez plusieur's graminées: beaueoup de plantes de eette famille ont leurs feuilles renversées, la faee supérieure de ees feuilles est d'un vert glauque; la faee inférieure de ees mêmes feuilles est au contraire d'un vert éclatant: aussi est-ee cette dernière qui se dirige eonstamment vers le eicl, au moyen d'une torsion qui s'opère dans le eorps même de la feuille. Ce phénomène est surtout facile à observer chez les graminées eéréales; ces plantes, avant l'apparition de l'épi, offrent une multitude de feuilles qui, élancées dans l'atmosphère, ramènent leur pointe vers la terre, et sont ainsi disposées en arceaux : or c'est toujours la face inférieure de la feuille qui, dans ees areeaux, est dirigée vers le ciel; la faee supérieure regarde la terre. Avec un peu d'attention, on voit la même disposition dans la feuille de plusieurs des humbles graminées que nous foulons tous les jours aux pieds. J'ai tronvé peu de graminées qui fussent. étrangères à cette disposition. ( ) 11 ne l'observe 
joint, par exemple, ehez le zea mays; elle n'existe Ipoint non plus ehez le triticum repens ni chez l'agrostis rubra: aussi, ehez ees végétaux, n'observet-or point la prédominanee de la eoloration de la faee inféricure de la feuille, eomme eela s'observe ehez la plupart des autres granincéc. J'ai remarqué que les substanees qui masquent extérieurement la eoloration des feuilles ne nuisent en rien à la direetion qu'clles affeetent en raison de eette coloration; ainsi la feuille du seigle dirige eonstamment sa faee inféricure vers le ciel, quoique ectle face soit eouverte d'une poussière glanque qui masque sa eouleur verte, et qui fail que eette faee inférieure parait moins eolorée quue la faee supérieure. Cette apparenee disparaît en cssuyant la feuille; alors on voil que sa faee inférieure, dirigée vers le eicl, cst effeetivement plus eolorée que ne l'esı sa face supéricure dirigée vers la terre. Les feuilles dont les deux faees sont également eolorécs ne dirigent aucune de ees fices vers la lumière, mais leur pointe s'élève ordinairement droit vers le ciel; telles sont les feuilles des typhinées et les feuilles subulćcs des alliaeées. L'aseension vertieale de ees feuilles résulte de la même eause que celle qui produit l'ascension vertieale des tiges dépourvucs de leuilles, et qui sont également eolnrées dans tout leur pourtour, telles que les tiges des plantes qui appartiennent aux genres allium, scirpus, juncus, ete. Les feuilles du grui, également colorées sur leurs deux faces, les dirigrent de nềme indifféremment vers la lnmière, è j’ai remarqué que la 
pointe de ces feuilles tend aussi vers le ciel, de mêne que les extrémités des tiges de cette plante lorsqu'elles ont acquis une certaine longueur. Il résulte de ces observations que les directions spéciales qu'affectent les faces opposées des feuilles sont constamment en rapport avec la différence de la coloration de ces faces. C'est toujours la face dont la couleur est la plus éclatante qui se dirige vers le ciel, la face la moins colorée se dirige toujours vers la terre; ainsi ce n'est point en leur qualité de face supérieure ou de face inférieure de la feuille, que ces faces affectent des directions spéciules, c'est en leur qualité de faces différemment colorées.

Les pétales des fleurs sont soumis, sous le point de vue de la direction de leurs faces, à des lois semblables à celles qui président à la direction des feuilles; c'est toujours leur face la plus colorée qui se dirige vers la lumière, et c'est en général, comme chez les feuilles, la face supérieure qui présente celte prédominance de coloration qui, quoique souvent peu scnsible, est cependant toujours réelle. On la remarque même dans les pétales de couleur blanche: que I'on observe, par exemple, un pétale de lis blanc (lilium album), on verra que sa face supérieure est d'un blanc mat et fort éclatant, tandis que sa face inlérieure offre une teinte beaucoup plus pâle; la conleur blanche des fleurs, cormme toutes les autres couleurs que l'on observe dans ces organes, est due à une matière colorante particulière qui est déposée dans le parenchyme subjaccnt à l'épiderme; il cn est 
de même de la couleur verte des feuilles. Ainsi la blancheur des pétales de certaines fleurs n'est point due à la même cause que la blancheur des racines ainsi que des tiges étiolées. Dans les pétales blancs, il y a existence d'une matière colorante blanche; dans les racines ainsi que dans les tiges étiolées, il y a absence de toute matière colorinte, ce qui laisse apereevoir la coulenr propre áu tissu végétal, couleur qui approche du blanc.

Les pétales tendent à se retourner comme les feuilles, lorsqu'on dirige leur face supérieure vers la terre, en maintenant renversée la fleur à laquelle ils appartiemnent. J'ai fait cette observation sur les pétales du lilium album; mais leur retournement, qui ne s'opère qu'au inoyen de leur torsion, n'est jamais aussi complet que l'est celui des feuilles que leur pétiole rend fort mobiles; on observe avec plus de facilité la tendance de la face supérieure de la fleur tout entière, vers la lumière, ce fait est si connu que je ne crois pas devoir m'y arrêter. Il est cependant des fleurs dont l'ouverture est constamment dirigée vers la terre, cela,sans nuldoute, provient souvent de leur pesanteur et de la faiblesserle leur pédoncule; rnais je pense que cela provient aussi quelquefois d'une tendance naturelle de la face inférieure de la fleur vers le ciel, comme étant plus colorée que la face supériemre. Dans les fleurs du digitalis purpur'ea, du symplytum officinale, du firtillaria imperialis, par exemple, la face supérieure est moins colorée que la lace inférieure, qui deit, par cela même, tendre de prélérence vers la 
lumière, et par conséquent vers le ciel: de là vierit que ces fleurs ont toujours leur orifice dirigé vers la terre; c'est par une action spontanée qu'elles se dirigent ainsi. Nous trouverons la preuve de eette assertion dans la seetion suivante. Dans les fleurs papilionaeées, il est presque général de voir le pavillon diriger sa faee supérieure vers la lumière, ee qui coïneide avee la plus forte coloration de cette face; les ailes, au contraire, appliquées ordinairement l'une eontre l'autre par leur faee supérieure, qui est peu colorée, présentent latéralement à l'influenee de la lumière leur face inféricure, dont la coloration est beaucoup plus forte. Dans le genre phaseolus, on remarque même que les ailes se tordent sur elles-mêmes pour diriger vers le eiel cette même face inférieure; le eontraire a lieu dans la fleur du melilotus officinalis; ehezelle, e'est laface supérieure des ailes qui se dirige en haut, au moyen de la torsion de ces mêrnes ailes, et cela coïneide eneore avee la plus forte coloration de la faee dirigée vers le eiel; ainsi les pétales se eomportent exaetement eomme les feuilles, sous le point de vue des direetions spéeiales qu'ils affectent : chez les uns eomme ehez les autres, la prédoninance de la coloration de l'une quelconque des deux faees est la condition organique qui détermine la direetion de cette faee vers la lumière et vers le ciel.

Les ovaires, après la chute de la fleur, affeetent souvent une dir eetion spéeiale et différente de eelle que présentait la fleur; chez lo digitalis purpurea, par exemple, après la chute de la fleur qui était diri- 
-géc vers la terre, l'ovaire se redresse el dirige sa pointe vers le ciel; ce fait coïneide avec la eouleut verte de l'ovaire; il se dirigye ver's le eiel comme le ferait mne tigge, et par la même raison. Un phénomène absolument inverse s'observe ehez les convolvulus ivolubilis et arensis: la fleur est dirigrée vers le ciel; à peine est-elle tombée, que l'ovaire tend à se diriger ivers la terre au moyen de la torsion da pédoneule : à coup sîr cettc torsion du pédoneule, lequel est fort trobuste, n'est point due à la pesanteur de l'ovaire qui, iimmédiatement après la chute de la fleur, èst eneore llort petit, et par eonséquent très léger; il faut donc, ebereher ailleurs la cause de eette direction 'spéeiale. I Lovaire qui est nu est blane, ou plutôt décolorć comme une racine, il tend eomme elle et par lat mềne raison vers la terre; le nềme phénomène n'a point 'lieu chez le convolvulus sepium, dont l'ovaire, après la chute de la fleur, reste enveloppé par deux larges bractées, qui, en leur qualitć de partics vertes, tendent vers le eiel et maintiennent l'ovaire dans eette direction.

Ainsi, les phénomènes de direction spéciale que nous observons dans les diverses parties des végćtaux eoincident constamment avec la nature de la coloralion de ees parties: nous ne pouvons donc nous dispenser de reeonnaitre que la différence de eoloration est la eondition organique à laquelle est attachée la différence de eette direction. Les tigres se dirigent. vers le ciel et vers la Jumière, pareequ'elles possèdent un parenchyme coloré; les racines se diriggent vers la terre, parecque leur parenchyne est incolore: 
les feuilles et les pétales diriggent l'une quelconque de leurs faces vers le eiel et vers la lumière, parceque dans cette faee le parenehyme subjaeent à l'épiderme est plus fortement eoloré que ne l'est eelui de la faee opposée, qui se dirige vers la terre. Ainsi la eoloration des tiges opposées à la déeoloration des raeines est un phénomène du même genre que la forte eoloration de la feuille sur l'une de ses faces, mise en opposition avee la moindre eoloration de l'autre faee.

A près avoir étudié les direetions spéeiales qu'affeetent les faees opposées des feuilles, il nous reste à déeider eette question: Ces directions spéciales sontelles mécaniquement imprimées à la fenille par des agents extérieurs, ou bien sont-elles les résultats d'actions spontanées, exécutées à l'occasion de l'influence de ces agents? Pour déeider eette question, j'ai fait les expérienees suivantes: j’ai pris des feuilles de diver's végétaux, el, apprès avoir retranehé leur pétiole, je l'ai remplaeé par un cheven fixé dans le eorps de la feuille au moyen d'un petit eroehet; à l'autre extrémité dı eheveu était attaehé un petit moreeau de plomb. J'ai ensuite plongé eet appareil dans un boeal plein d'eau, après avoir pris le soin de laisser ce boeal long-temps en repos, afin que l'eau qu'il eontenait n'eût aneun mouventent propre. La pesanteur du plomb préeipitait la feuille au fond du bocal; mais, eomme, en vertu de sa pesanteur spéeifique moindre que eelle de l'eau, la leuille tendait vers la surfaec de ce liquide, il en résultait qu'elle se plaẹait dans une position verticale, 
layant sa pointe dirigée vers le eiel, et j'avais soin de II a plaeer de telle façon qu'elle eût sa laee inlérieure diriggée vers la lumière. On sait, par les expériences de Bonnet, que les feuilles plongées dans l'ean se rettournent de la même manière que dans l'air : si done lle retournement de la feuille était dì à une attraetion cexercée par la lumière sur la faee supérieure de eet corgane, ee retournement devait s'opérer, dans l'exipérience en question, au moyen de la torsion du cheveu qui remplaęait le pétiole, et eela même avec plus de faeilité que dans l'ordre naturel, puisque ee ceheveu opposait moins de résistanee à la torsion que ı́en opposait le pétiole lui-même, qui eependant se tord en pareille cireonstanee. Le résultat de eette expérienee a été que la feuille est restée parfaitement innmobile, et n'a manifesté aueune tendanee au retourinement. Cependant, lorsque j'ai mis en expćrienee des feuilles alongées et fort jeunes, telles que des ffenilles de pêeher (amygdalus persica) ou des folioles de noyer (juglans regia), j’ai vu la partie su[péricure de la feuille se tódre sur elle-même et raimener sa faee supérieure vers la lumière, sans que le ieheveu éprouvât la moindre torsion, ee dont je jugeais à la direetion du eroehet au moyen duquel la Ifeuille était attaehée au eheveu. Ces expérienees eommeneent à prouver que la lumière n'exeree ancune attraetion sur la faee des feuilles qui se dirige ordinairement vérs elle, et que le retournement de ces organes est le résultat d'un mouvement spontané. Cette vérité est mise hor's de doute par l'expérience 
suivante: j’ai pris un fragnnent de lige de polygonum convolvulus, ehargée de deux feuilles situées du même côté et dirigées dans le même sens. J'ai fixé avee un petit erochet un cheveu à la partie supéricure de ce fragment de tige; un morceau de plomb, fixć à l'autre extrémité du cheveu a préeipité dans l'cau d'un bocal ee fragment de tige dans une situation renversée, en sorte que les deux feuilles qu'il portait avaient leur faec supéricure dirigée obliquement vers la terre et à l'opposite de la lumière. La plante se tenait suspendue au milicu de l'eau du bocal, sans toueher les parois de ee dernier, qui était plaeé auprès d'une fenêtre. Lcs deux feuilles ne tardèrent pas à se rctourner an moyen de la torsion de lenrs pétiolcs; le fragment de tige qui les portait ne changea point de position, et le cheveu qui lc retenait au milieu de l'eau n'éprouva pas la moindre torsion. Ce eheveu délié offrait à la torsion une résistance infiniment moindre quc eelle qui lui était opposée par les deux pétioles des feuilles; si done ees deux derniers ont été tordus par l'effet du retournement des feuilles, sans que le eheveu ait participé le moins lu monde à cette torsion, cela prouve d'une manière irréfragable que ee n'est point une attraction, ou une autre eause mécanique extérieure qui déterminc le retournement des feuilles, mais que ee retournement est le résultat d'un mouvement spontané, exćeuté à l'oceasion de l'influcnce d'un agent extérieur sur la f'euille.

La lumière n'est point le seul agent dont l'influence soit susceptible de determiner le retournement des 
ifenilles. J'ai observé, avec Bonnet, que ces organes se atetournent dans une obscurité complète, et tendent ainsi, sans le secours de la lumic̀re, à diriger l'une de l. leurs faces vers le ciel et l'autre vers la terre. Cette cobservation prouve que la cause de la pesanteur jone, Hans la production de ce phénomène, un rôle semHblable à celui de la lumière; la faee la moins colorée dde la feuille tend, eomme les racines, vers la terre, ou dans le sens de la pesanteur; la lace la plús colorrée tend, comme les tiges, vers le eiel, on dans le ssens opposé à celui de la pesanteur. On pourrait fpeut-être penser qu'il n'y aurait qu'ane seule des faces de la feuille qui affecterail une tendance déterrminée, et que l'autre face serait passive dans cetre cireonstance; il esĩ, je crois, impossible d'éclaircir chez les feuilles ce doute qui se trouve levé par l'obsservation de la tendance que manificste la radicule du sgui à fuir la lumière. Cette radieule est moins coHorée en vert que la tige à laqnelle elle fait suite, et c'est cette nioindre coloration qui est la cause de sa ttendance évidente à luir la lumière. On ne peut se refuser ici à admettre les inductions de l'analogie, et ià reconnaître que la face la plus colorée des feuilles Itend vers la lumière, et que la face la moins colorée ttend à la fuir; par la même raison on peut affirmer que les deux faces de la feuille ont une tendanee iniverse, par rapport à la cause inconnue de la pesanteur. Ainsi il est bien établi par l'observation que là difféa rence de la coloration est la condition organirgue qui accompagne constamment lin différence de la direction 
des parties végétales; il est également démontré que c'est toujours par des mouvements spontanés que les végétaux dirigent d'une manière spéciale leurs diverses parties, et. que, par conséquent, les agents extérieurs qui déterminent ces directions spéciales n'agissent sur le végétal qu'en qualité d'agents nervimoteurs. C'est la nervimotion, produite par ces agents, qui produit à son tour les mouvements spontanés dont il est ici question. Aussi, quand la nervimotilité de la plante est abolie, ses feuilles renversées ne se retournent plus. Nous avons vu, dans la section précédente, qu'on peut abolir la motilité de la sensitive, en la plaçant dans une obscurité complète pendant un temps plus ou moins long. Or j'ai expérimenté que, lorsque celte plante est réduite par ce procédé à ne plus mouvoir ses feuilles sous l'influence des secousses, elle n'est plus capable non plus de les mouvoir pour les retourner, lorsqu'on les place dans un état de renversement. Les feuilles de la sensitive étant renversées se retournent assez promptement, même dans la plus profonde obscurité. Or, ayant renversé plusieurs feuilles d'une sensitive qui était depuis quatre jours et demi dans une obscurité complète, par une température de +22 à 24 degrés, et dont les feuilles n'olfraient plus aucune motilité sous l'influence des agents nervimoteurs mécaniques, ces feuilles conservèrent leur position renversée, sans faire aucune tentative pour la quitter pendant trois jours que je les laissai en expérience. Ceci achève de prouver que le retournement des feuilles dépend entièrèment d'une 
ction intérieure et vitale, et que les agents extérieur's ui déterminent ce phénomène ne sont, dans cette arconstance, que des agrents nervimoteurs. Or, somme la différenee de la eoloration des parties des śgétaux apporte une différenee dans la direction u'clles affectent, il en rćsulte qu'il y a deux modes ifférents de la nervimotion, qui sont en rapport avec u différence en plus ou en moins de la eoloration des aarties végrétales.

On doit à Bomnet plusicurs observations qui tenreaient à faire penser que les végétaux cherchent à iir les abris desquels ils sont voisins. Ainsi les Hantes qui croissent près d'une muraille inclinent unr tige pour s'en éloiguer; les feuilles que l'on cou re d'une planche s'éloi wnent spontanément de cet abri. ai répété et varié les expériences que Bonnet a faites cet égard; je ne me suis pas contenté de les faire sur us plantes exposées à l'influence de la lumière, je les répétées sur des véçétaux plonģés dans la plus cofonde obscuritć. J'ai vu cque lorsqu'on couvre d'une etite planche la face supérieure d'unc fenille d'un igétal situé en plein air, eette feuille tend à se ustraire à cet abri par des moyens qui ne sont point ujours les mêmes, mais qui sont toujours ceux ui doivent arriver le plus facilement et le plus romptement à eette fin; ainsi c'est tantôt au moyen : Ia flexion latérale du pétiolc que la feuille est rerée de dessous l'abri, tantót c'est au moyen de la exion de ce même pétiole ver's la tigge. Lorsque la lanche est rop large pour que la fenille puisse être 
retirée de dessous, le pétiole se fléchit vers la terre, et la feuille se présente ainsi à l'influence de la lumière, quilui arrive latéralement par-dessous la planche. J'avais couvert d'une petite planche la foliole terminale d'une feuille de haricot (phaseolus vulgaris), leuille qui, comme on sait, possède trois folioles; cette foliole ne pouvait point se retirer de dessous la planche par l'inflexion de son pétiole parliculier, à cause du peu de longueur de ce dernier; ce fut le pétiole commun qui, par son inflexion, retira la foliole de dessous l'abri qui la recouvrait. En voyant cette diversité de moyens employés pour parvenir à une mème fin, on serait presque tenté de croire qu'il existe là une intelligence secrète qui choisit les moyens les plus convenables pour accomplir unc action déterminée.

Les feuilles plongées dans une profonde obscurité, et recouvertes par un abri, ne manifestent aucune tendance à s'y soustraire. C'est ce dont je me suis assuré par des observations multipliées et faitcs avec beaucoup de soin; seulement j'ai observé quelquefois que les feuilles recouvertes d'une petite planche s'en éloignaient en s'abaissant; mais, comme ce mouvement peut être causé par la pesanteur de la feuille, on n'en peut rien conclure pour l'existence chez cette derniëre d'uue tendance spéciale à fuir l'abri qui la recouvre. Il faut donc admettre que le mouvcment par lequel les feuilles exposées en plein air se retirent de dessous les abris qui les recouvrent est uniquement dù à la tendance naturelle que ces or- 
, ranes ont à diriger l'une de leurs faces vers la lumière; c'est un phénomène analogue à celui de leur etournement. On peut conclure de là que si les yges sinclinent en avant lorsqu'elles croisscnt dans soisinage d'un mur, cela ne provient point d'une cendance particulière qu'elles auraicnt à fuir cet abri, mais que eela est occasioné par la tendance de la igge vers la lunière qui lui arrive principalement en tvant, et à l'influencc de laquelle cllc est presque toalement soustraite cn arrière, c'cst-à-dire du côté Ha mur.

It existe chez les végétaux un autrc phénomènc de direction spéciale dont la cause s'cst jusqu'ici diobée ì toutes les reeherches des naturalistes; je rcux parter du phénonicne auqucl Linné a donnć e nom de sommeil des plantes. On sait qu'aux ap ,roches dc la nuit les feuilles et les fleurs de beaucoup de végétaux affectent des directions et des yositions différentes de celles qu'ellcs offraient penHant le jour. Bonnct, qui a beaucoup obscrvé ce phénomènc, eroit qu'il dépend de l'humidité qui s'élève de soir de la terre. Cette hypothèse est repousséc par l'observation; car j'ai vu qu'une fcuille de sensitive. plongćc dans l'cau ne laisse pas de présenter pendant ta nuit le phénomène du sommeil ou de la plicature the ses folioles, quise dćploient au retour de la lunière In jour. M. Decandolle, qui a lait de bclles expériences sur les deux états de sommeil et de veille des plantes, a reconnu que ces phénomènes dépendent exclusivement de l'absence ou de la présence de la 
lumièrc; mais cela ne nous apprend point la cause du phćnomènc lui-même, et ne nous instruit point sur sa nature.

La lumic̀re exerce deux influences bien distinctes sur les vćgétaux; clle est à la fois agent rćparatcur dc la nervimotilité vćgćtale, ct agcnt nervimotcur, c'est-à-dirc qu'elle répare et consommc tout à la fois les conditions vitales de la nervimotilité. J'ai fait voir, dans la $2^{\mathrm{e}}$ section, que la lumic̀re répare chez la sensitive la nervimotilitć que l'absence de cette lumière avait laissé éteindre ou épuiser. Cette rćparation de la nervimotilité par la lumière a lieu en vertu d'une proprićté de cet agent qui n'est pas connue. Nous venors de voir que la lumière agil comme cause de nervimotion pour déterminer certaines directions des parties végétales. 11 résulte de cette complication d'actions de la part dc la lumière sur lcs végétaux que ceux-ci doivent avoir un état diurne en rapport avcc la double influence rćparatrice et nervimotrice de la lumière, et un état nocturne en rapport avec l'absence de cette double influence. L'observation nous apprend que dans l'état diurne les feuilles de plusieurs végétaux offrent deux directions spéciales diffćrentcs; tantôt elles présentent directement l'une de leurs faces à la lumière, tantôt elles dirigent leur pointe vers elle; c'est ce que l'on re-narque, par excmple, chez la sensitive (mimosa pudica), chez le robinia pseudo acacia, etc. Le matin les feuilles dc ces végétaux présentent leur facc supéricure à la lumière, mais dans le milieu du jour, sur- 
tout si la lumière du soleil ést fort intense, les folioles Whirigent len pointe vers la lumière ou vers le ciel. (Cies deux directions différentes, qui se croisent à angle idroit, composent par leur assemblage l'état diurne des feuilles. Ces deux directions ne s'observent pas chez tous les végétaux, mais il est un phénomène. assez commun qui s'y rattache : cc phénomène, qui a été noté par Bonnet, est celui de la forme concave ique prement les feuilles un peu larges lorsqu'elles sont soumises à l'influence d'une forte lumière. Celte concavité de la feuillc est produite par la tendance de ses bords ou des extrémités de ses nombreuses nervures vers la lumière; ce phénomène est évidemment du même genre quc celui que l'on observe dans les feuilles qui, comise celles de la sensitive, dirigent vers une forte lumière la pointe de leur nervure unique. Ce phénomène provient de cc que lcs extrémités des nervures des feuilles se comportent comme si elles étaient des extrémités de tigres, et qu'elles tendent, en cette qualité, à se diriger vers la lumière. Ainsi, pendant le jour, les feuilles de certains végétaux obéissent successivement à deux tendances qui se croisent à angle droit; la preniièrc de ces tendances dirige leur face supérieure vers la lumière, la seconde dirigge leur pointe vers ce même agent. Il est à remarquer que la premiere est le plus coustamment prédominante, et qu'il laut une grande intensité de lumière pour faire prédominer la seconde, encore ce dernier effet ne s'observe-i-il que chez quelques végétaux. Dans leur état nocurne, les feuilles n'nflent 
qu'une seule direetion spéciale, et eette direction, eonsidérée, chez les divers végétaux, est assez vitriable, quoique eonstante et unique chez ehacun d'eux. On sait qu'alors les folioles de la sensitive sont ployées le long de leur pinnule ou de leur axe commun, que les folioles du robinia pseudo acacia on leur pointe dirigée vers la terre; que les folioles des casses tordent leurs pétioles pour se joindre par paires par leurs faees, supérieures en mêne temps qu'elles dirigent leur pointe en bas, etc. Ces phénomènes ont leur cause dans un état particulier de la nervimotilitó dı végétal; cette cause se trouve spéeialement dans la diminution des conditions de la nervịmotilité, conditions qui, sans eesse épuisées par le milieu environnant, ne sont plus réparées en suffisante quantité, à cause de l'absence de l'agent réparateur, qui est la lumière. En un mot, le sommeil des feuilles est la position partieulière qui doit résulter d'ure diminution eonsidérable et rapide des eonditions de leur nervimotilité : aussi toute cause qui produira eette diminution produira une position des feuilles semblable à celle du sommeil. C'est ce que l'on observe chez la sensitive; une secousse imprimée à ses feuilles, en épuisant momentanément une portion des conditions de leur nervimotilité, leur fait prendre la même position qu'elles affectent pendant le sommeil; leur plicature est véritablement alors un soinmeil diurne. Il n'y a point de différence entre ce sommeil diurne provoqué par un agent nervimoteur violent, lequel consomme ct diminue rapidement les 
conditions de la nervimotilité, et le sommeil noctturne, qui est provoqué par la diminution de ces ımênes conditions de la nervintotilité, par le lait de ll'absence de l'agent réparateur de ees conditions vitrales sans cesse consommées par le milieu ambiant. Les fleurs, comne on le sait, présentent, de même que les feuilles, ces deux états de sommeil et de veille, qui reconnaissent certainement pour cause un état particulier d'épnisement ou d'accumulation des conditions de la nervimotilité. La lumière agissant à la fois comme cause réparatrice de la nervinıtilité vérgétale, et eomme cause nervimotrice, ou comme cause d'épuiscment de cette même nervimotiiité, elle doit, eonsidérće dans un degré déterminé d'intensité, tantôt réparer plus qu'elle n'épuise, tantôt épuiser plus qu'elle ne répare, et eela suivan l'organisation partieulière des végćtaux. Ainsi, il n'est point étonnant que l'on rencontre des parties végétales qui offrent la plicature du sommeil pendant le jour, et qui se déploient à la faible lueur du crépuscule; tclle est, par exemple, la fleur de la belle de nuit ( mirabilis jalappa). La plicature de eette fleur est provoqućc par une forte lumière qui agit sur elle plus comme cause d'épuiscment que eomme cause de réparation, tandis que le même degrré de lunière produit un effet inverse sur la plupart des autres fleurs. 


\section{SECTION IV.}

DE I'INFLUENCE DU MOUVEMENT DE ROTATION SUR LES DIRECTIONS SPÉCIALES QU'AFFECTENT LES DIVERSES PARTIES DES VÉGÉTAUX.

Les expériences rapportées dans la section précédente nous ont prouvé que les direetions spéeiales qu'affectent les diverses parties des végétaux sont dues ì des aetions vitales et spontanées dont la cause immédiate se trouve dans l'influence qu'exereent sur la nervimotilité végétale deux agents extérieurs, la lumière. et la eause inconnue de lia pesanteur. Si nous pouvions imiter les procédés de la nature, si nous pouvions $\mathrm{cm}-$ ployer des agents nervimoteurs différents de ceux qu'elle met en usage pour déterminer ces direetions spéciales et spontanées des végétaux, cela nous mettrait à même de déterininer qucl est le mode d'aetion de ces agents sur la nervimotilité végétale. Deux naturalistes, MM. Hunter et Knight; ont déjà tenté ee genre d'expériences; ils ont voulu voir ee qui arriverait à des graines qui, soumises à un mouvement de rotation continuel, présenteraient ainsi leur radicule et leur plumule, chaeune suecessivement au ciel et à la terre. Hunter mit uue fève au centre d'un baril plein de terre et qui était aniné d'un monvement continucl de rotation sur son axe horizontal: laradicule sedirigea diuns le sens del'axe 
de la rotation du baril. M. Knight 'fixa des graines de harieots à la eirconférence d'une roue de onze pouees de dianière, laquelle, mue continuellennent par l'eau dans un plan vertieal, firisait ecnt einquante révolutions par minute. Il résulta de eette expérience que chaque graine dirigea sa radieule et sa plumule dans le sens des rayons de la roue; les radieules tendirent vers la eireonférence et les plumules vers le eentre. M. Kinighı répéta la même expérienee avec une roue de semblable diamètre et qui était mue dans un plan horizontal; elle faisait deux eent einquante révolutions par minute. 'Toutes les radieules se dirig̀èrent eneore vers la eireonférenee et les plumules vers le eentre, mais avec une inclinaison de 10 değrés des radienles vers la terre et des plumules yers le eiel. En réduisant à quatre-vingts révolutions par minutela vitesse de rotation de eette roue horizontale, l'inelinaison des radicules vers la terre, et des plumules vers le eiel, devint de $45 \mathrm{de}-$ grés. Ces expérienees sont extrêmement incéressantes, en ee qu'elles démontrent qu'il existe des muyens d'oecasioner artificiellement chez les plantes des directions différentes de eelles qu'elles prennent naturellement. Je résolus de répéter ees expériences et de les varier; mais comme je ne pouvais disposer d'un appareil mu par l'eau sans interruption, je pris le parti de faire eonstruire un mouvement d'horlogerie assez semblable à un tournebroehe. Il est mu par un poids de deux cent soixante-dix livres, que l'on remonte de douze 
heures en douze heures; son mouvement est régblé par un régulateur ou volant, dont la rotation s'opère dans le sens horizontal : les roues vertieales, qui sont au nombre de cinc, prolongent leur's axes de ehaque côtć au-delà des montants qui les supportent; ces prolongcments des axes sont carrés, en sorte qu'il est facile d'y adlaptcr une roue de bois, à la cireonfërence ou au centre de laquelle je place lcs graines don $\mathrm{je}$ veux observer la germination. Je place ees graines dans des ballons de verre munis de deux ouvertures diamétralement opposées, et que jc ferme avee des houchons après y avoir introduit la quantité d'eau nécessaire pour la végétation des embryons des graines. Celles-ci sont enfilées par leurs enveloppes, oú leurs eotylédons, au mayen de deux fils de euivie extrêmıement déliés, dont les cxtrémités sont fixées de part ct d'autre aux bouehons qui ferment les deux ouvertures des ballons de verrc. Ceux-ci sont ensuite fixés d'une manièrc solide à la roue avee laquelle ils doivent se mouvoir; de eette manière, les graines transportent avec elles dans leur mouvement eirculaire l'cau néeessaire à leur gernination; lesballons de verre au milieu desquels elles sont fixéesd'une manière invariable, ont l'avantage de les soustraire à l'influenee de toute action mécanique de la part du milieu dans lequel le nouvement s'opère. Le fil de cuivre Iont je me sers pour fixer les graines dans l'intérieur des ballons de verre est le plus fin que l'on emploie pour envelopper en spirale des cordes d'instruments. J'ai pris des graines de pois (pisım sativum') et 
des grames rle vesee ( vicia sativa) qui commencaient à germer; je les ai placées, suivant le procédé décrit plns haut, dans des ballons de verre yne j'ai fixés à la circonférence d'une roue d'un mèlre de diamètre. qui fiusait quarante révolutions par minutc. Le résultat de cette expérience fut que toutes las radicules se dirigèrent vers la circonférence, et quo toutes les plumules se dirigèrent vers lecentre de larotation; les radicules, qui s'étiaent trouvé origginairement tournées ver's le centre, se retournèrent vers la circonférence; les plumules se courbèrent de même pour sediriger vers le centre. Celteexpérience, répétéc plusieurs fois, mia donné constamment le mênıe résultat, qui cst également celui qui a été obtenu par M. Kinight.

A l'exemple de M. Knighth, j’ai voulu éprouver l'effet que produirait sur les graines en grermination une rotation rapide, opérée dans un plan horizontal; pour cela, j’ai remplacé le régulateur ou volant de mon mouventent d'horlogerie par une règle de bois, à chacune des extrémités de laquelle j’ai autaché solidement uu petit ballon de verre contenant des graines de vesce, fixées dans son intérieur, comnı je l'ali dit plus haut, an moyen de deux fils de cuivre; cette règle formait un diamètre de 58 centimètres de longueur, elle faisait cent vingt révolutions par minnte. Les radicules et les plumules se dirigèrent dans $m$ sens parfaitement horizontal, les premières vers lat circonférence, et les secondes vers le centre. Ici les graines n'avaient point cessé d'être sonmises à la cause qui, dans l'état natmrel, préside à la direction per- 
pendiculaire de la plumule el de la radicule; mais cette cause naturelle avait été surpassée en énergie par la cause artificielie employće dans celte circonstance, c'est-à-dire par la force centrilinge qui résultait de la rotation rapide. M. Knight n'avait pas obteinu un résultat anssi complet de son expérience sur les graines de haricols soumises au mouvement de rotation horizontale, puisqu'elles avaient conservé un peu de leur tendance verticale; cependanı la force centrifugre à laquelle elles étaient soumises était plus considérable qu'elle ne l'étail dans mon cxpérience, puisque sa roue, qui avait 11 pouces anglais (ou 28 centimètres) de diamètre, faisait deux cent cinquante révolutions par minute. Cette différence dans le résultat dépend entièrement de la nature des graines soumises à l'expérience. J'ai éprouvé que l'cmbryon de la graine de vesce esı beaucoup plus facile à influencer pour sa direction que ne le sont les cmbryons beaucoup plus gros des graines de haricots ou de pois; aussi est-ce presque toujours avec des graines de vesce que j'ai fait mes expériences. J'ai placé un certain nomìre de ces graines dans un ballon de verre, dont elles occupaient le diamètre intérieur, fixées, comme à l'ordinaire, dans cette place au moyen do deux fils de cuivre qui enfilaient leurs enveloppes. J'ai attaché ce ballon de verre sur une petite planche que j'ai adaptéc au pivot du volant horizontal de mon mouvement d'horlogerie, en remplacement de ce volant; cet appareil faisait deux cent cinqunnte révolutions par minite; le centre de la rotation répondait 
an milieu de eette série longitudinale ct horizontale tle graines; une de ces dernières était sitnéc anssi :xaetement que possible an centre même; eependant ta radicule de eelle-ci se trouva décrire un cercle exurênement petit, ear je ne pense pas qu'il cû, dans l'origine, plus d'un à deux millimètres de rayon. (Cette radieule se diriggea vers la eirconférenee, dans un sens parfaitement horizontal; la plumule s'éleva vertiealement vers le eiel; les radieules des autres toraines, qui étaient plus éloignées du centre, se diriừèrent à plus forte raison dans une horizontalité parffaite vers la cireonférenee; leurs plumules se dirigèrrent toutes vers le centre, mais avee différents degrés d'inelinaison par rapport à l'horizon: eelles qui étaient à plus de deux centimiètres du eentre dirigèremt leur's plunules vers ee dernier avee une horizontalité parfaite; cclles qui.étaient situées plus près du eentre s'en approchèrent en se dirigeant obliquement vers le reiel; enfin, toutes les plumules ayant continné de s'acceroître, se réunirent en laiseeau au eentre, où elles [prirent toutes une direction verticale vers le eiel. Je rrépétai ectte expérien ee avectes ģraines germées, dont je dirigeai la radicule vers la terre; au bout de quelques heures de rotation, les radieules abandonnèrent ceette direction naturelle, et, se courbant vers la eireonIférenee, se plaeèrent daus une situation horizontale.

La rotation horizontale la plus lente quill unat été possible d'obtenir avec mon mouvenent d'horlogeric a a été de cinquante-quatre révolutions par minute. Les -graines de vesec soumises à cette rotation ont ineliné 
leur radicule vers la terre, dans me position oblique, eloignée d'environ 45 degrés de la ligne verticale, et dirigrée vers la circonférence; les plumules ont affecté le même degré d'inclinaison vers le centre, en montant obliquement vers le ciel. Ces expériences démontrent deux faits généraux, savoir, $1^{\circ}$ que la radicule, dans l'action spontanće au moyen de laquelle elle se dirige, obéit au mouvement ou à la tendance qui l'influence; en effet, soumise au mouvement de rotation, la radicule se dirige dans le sens de la tendance centrifuge qui naît du mouvement circulaire, c'est-à-dire qu'elle prend la direction du rayon, en s'avançant vers la circonlérence; $2^{\circ}$ que la plumule, dans l'action spontanée au moyen de laquelle elle sc dirige, réagit contre le monvement ou la tendance qui l'influence; en effet, soumise au mouvement de rotation, la piumule se dirige dans le sens diamétralement opposéà celui de la tendance ccntrifuge qui nait du mouvement circulaire, c'est-à-dire qu'elle prend la direction du rayon en s'avançant vers le centre. Après avoir répété et vérifié les expériences de M. Knight, j'ai voulu essayer de reproduire l'expérience de Hunter, qui a vu qu'en faisant lourner unc graine sur elle-mêmıe, la radicule se dirigeait daus le sens de l'axe de la rotation; cette observation fort incomplète méritait d'être suivie. J'ai placé un ballon de verre, contenant des graines de vesce, au centre d'une roue qui faisait quarante révolutions par minute; j'avais fait en sorte que la série longitudinale des graines, que maintenaient les deux fils de cuivre, 
cut située aussi exaetement que possible sur le proongement de l'axe de rotation, lequel ćtait diriggé it eurès du noid-est au sul-oncst. Les rindienles et us plumules se dirigèrent également selon l'axe de cotation, mais daus des sens diamétralentent opposés; ias radieules s'avancèrent ver's le sud-ouest et les olumules vers le nord-est. Le niême effet cut lieu ivec lous les degrés de vilesse de rotation qu'il ne iul possible d'employer, ce qui me proinva que ee thénomène ne dépendait point du tout du degré de ctte vitesse. Je pensai que eettr direetion spćciale de plumule et de la radieule pouvait provenir du sens dans lequel la rotation s'opérait; je répétai done mon uxpérience en faisant tourner la roue dans le sens opposé à celui dans lequel sa rotation s'opérait préeélemment; nuas le résultat ne varia point: les radiules se dipjgèrent eonstamment vers le surl-ouest, et eas plumules avee le nord-est. Je ne savais à quelle :ause attribuer cette direetion spéeiale de la radienle tt de la plumule, lorsquil ne vint dans l'idéc de m'assurer de l'horizontalité de l'axe de ma roue; je ui appliquai un niveau, et je vis qu'il inclinait vers ce sud-ouest d'une quantité que je trouvai être l'un degrré et demi. Cette inelinaison, quoique léère, me parut devoir ćre la cause de la direetion péeiale des eaudex séminaux; pour m’en assurer, je wenchai légèrement mon mouvement d'horlogerie, en uelinant les axes des roues vers le nord-est, et dans cette position je reeommençai mon expérience. Alors es directious précédenies de la plumule et de la ra- 
dicule furent interverties: les radicules se dirigèrent vers le nord-est, et les plumules vers le sud-ouest. Ainsi, il me fur démontré que la radicule se dirige vers le côté déclive de l'axe dont elle suit la pente en descendant, et que la plumule, au contraire, se dirige vers le côté ascendant de l'aze dont elle suit la pente en remontant. Il est évident que, dans cette circonstance, la plumule et la radicule subissent l'influence de la cause qui les sollicite dans l'état naturel; mais ne pouvant, à cause de la rotation continuelle, inonter et descendre verticalement, elles montent et descendent par uno ligne inclinée. Après m'être éclairci sur ce point, j'ai voulu voir ce qui arriverait en plaçant l'axe dans. une horizontalité parfaite, et j'ai vu qu'alors la plumule et la radicule se sont dirigyécs commc les deux rayons d'un même diamètre d'un cercle vertical dont la graine occupait le centre. Ayant répété plusieurs fois de suite la même expérience, je vis que les caudex séninaux se dirigeaient constamment dans le sens d'un diamètre toujours le mê:ne, et que, par conséquent, la plumule tendait constamment vers un point déterminé de la circonférence de la roue au centre de laquelle la graine était fixée, et que la radicule tendait constamment vcrs le point diamétralement opposé, et toujours le méme de cette circonférence. J'ai cherché, sans succès, pendant fort long-temps, la cause de cctte tendance spéciale, et je l'xi enfin trouvće en observant des graines en germination soumises à un mouventent très lent de rotation. J'avais fixé deux ballons de verre, con- 
fienant eomme à l'ordinaire des graines de vesee prêtes ii germer, à la eirconférenee d'une roue de deux décsimètres de rayon qui faisait trente révolutions par lueure; un autre ballon de verre semblable était placé nu eentre de eette mênte roue, dont l'axe de rotation ¿́tait parfaitcment horizontal. Les radicules, dans ces irois Jallons de verre, prirent une même direetion, 'est-ì-dire qu'elles se diriggèrent suivant des lignes coutes parallèles entre elles; les plumules prirent géuéralement une direetion diamétralement opposée à celle des radieules. De cette nıanière, les graines siunées au centre de la roue avaient leurs radicnles dirigées selon l'un des rayons de eette roue, tandis que es graines situées à la eireonférence avaient leurs ralicules dirigées parallèlement à ee même rạyon et du même côté. Les réflexions que je fis sur ee phénonène me conduisirent à penser qu'il y avait de l'iné¿alité dans le mouvement de la roue, c'est-à-tlire qu'il avait un des points de eerte roue qui marehait vite oendant une demi-révolution, et qui marchait plus entement pendant l'autre demi-révolution. Comme thaque révolution s'exéeutait dans l'espaee de deux ninutes, il me fut faeile de mesurer et de comparer intre elles les diverses parties de eetıe révolution, au moyen d'un pendule qui marquait les demi-seeondes. He trouvai de cette manière que ee que javais soupconné avait lieu effeetivement; la rotation de la rone i'était point unilorme. Celui des points de sa eirconérence pour lequel celte inégralité de monvement útait la plus marquée parcomait l'une de ses denx 
demi-révolutions, observée en partant d'un point déterminé, en soixante-six sceondes, et l'autre demi-ré. volution en cinquante-quatre secondes; en sorte que les temps dans lesquels s'opéraient ees deux demi-révolutions étaient entre cux comme onze est à neul. Or, les caudex séminaux étaient tous perpendienlaires à celui des diamètres de la roue qui, en raison de l'inégalité de la rotation, restait le plus long-temps exposé à l'influence de la pesanteur par l'un de ses eôtés ou flanc.s pendent une demi-révolution, et le moins long-temps exposé à cette mêne influence par le flanc opposé pendant l'autre deni- révolution. Les radieules étaient perpendiculaires au côté ou flanc le plus long-temps tourné vers la terre, et les plumules se dirigeaient perpendiculairement sur le côté ou flanc opposé, lequel était le plus long-temps tourné vers le ciel; ainsi, dans cette eirconstanee, les caudex séminaux se dirigeaient sous l'influence de la peșanteur à laquelle ils étaient ineomplètement soustraits à cause de l'inégalité du mouvement de rotation. Cette inégalité du mouvement provenait de la eonstruetion défectueuse de mon mouvement d'horlogerie, qui avait été confectionné par un serrurier fabricant de tournebroches.Quelques tentatives que j'aie faites, il m'a été impossible de eorrigrer ee défaut et d'obtenir un mouvement de rotation parfaitement égal; en revanche, il m'a été faeile de rendre la rotation de mes roues plus inégale qu'elle ne l'était, en les chargeant aux denx extrémités d'un même dianictrc de ballons de verre d'inégale pesanteur, de manière 
cependant à ce yue le mouvement de rotation ne fiit pas arrêté par une trop forte inćgralité de poidłs entre czes ballons. J'ai pleinement confiruné de cette mamière les résultats de l'expérience précédente. Lor'sque lic ballon le plus pesant parcourait sa demi-révolution descendant, son excès de poids s'ajoutait à la force mnotrice el accélérait le nouvement : lorsqu'au conuraire ce même ballon parcourait sa demi-révolution n remontant, son excès de poids diminuait la force motrice el retardait le mouvement. Il résultait de là ique le diamc̀tre sur lequel ćtaient placés ces deux ballons présentail ses deux flancs à la terre pendant Hes espaces de tcmps inéganx : lorsque, par exemple, le ballon le plus pesant ćtait au point le plus déclive de sa révolution, il commençait à parcourir lentement sa demi-révolution ascendante, et le diamètre sur lequel il était placé présentait pendant long-temps à la terre l'uu de ses flanes, et cela sous tous les degrrés successif's d'inclinaison jusqu'à ce que le ballon pesant 'eût gagné le point le plus élevé de la révolution. A partir de ce moment, le ballon pesant parcourait rapidenent sa demi-révolution descendante, et le diamètre sur lequel il était placé présentait, pendant tpeu de temps, à la terre son autre flanc sous tous lles degarés d'inclinaison. Il résuliait de là que ces deux tflanes opposés du diamètre dont il est ici question - étaient dirigyés vers la tcrre pendant des temps inégaux, et que, par conséquent, la pesantcur devait igrir sur les entoryons sćminam avec une force proportionnolle à cette différence de temps. La direction 
des caudex séminaux devail, dans cette circonstance, étre la ligne moyenne entre toutes les inclinaisons sous lesquelles le flanc du diamètre sa présentait à la terre, c'est-à-dire que les caudex séminaux devaient être perpendiculaires au dimètre dont il s'agit : c'est aussi ce que l'expérience m'a prouvé. Ainsi, en observant l'appareil lorsque le ballon pesant parcourait sa demi-révolution ascendante, et au moment où le diamètre sur lequel il était situé était horizontal, on voyait toutes les radicules dirigées verticalement vers le centre de la terre, et toutes les plumules dirigées verticalement vers le ciel. Il n'y avait ainsi qu'une seule et même direction pour toutes les graines contenues dans les ballons dont la roue pouvait être chargée, soit à son centre, soit à sa circonférence. Ainsi me fut dévoilée la cause de la direction, selon les deux rayons d'un même diamètre, d'un cercle vertical qu'affectaient les deux caudex séminaux de mes graines lorsqu'elles tournaient sur elles-mêmes; l'axe étant parfaitement horizontal. Il m'était impossible d'apercevoir celte cause lorsque j'employais une rotation plus rapide, qui ne permettait pas de mesurer la durée des demi-révolutions, ni même de soupçonner leur inégalité; aussi la recherche de ce phénomène m'at-telle entraîné dans des erreurs que je m'empresse ici de désavouer. J'avais cru apercevoir dans le principe que les secousses étaient la cause de la direction spéciale dont je viens d'exposer la cause véritable; je soumis en couséquence des graines en germination et tournant sur elles-mêmes sur un axe ho- 
rrizontal, ì des secousses régulières, opérées dans un nenens toujours le mêne, an moyen d'un mécanisme particulier. Je vis que les radicules et les plumules thes graines contenues dans cel appareil affectaicnt des Hirections constantes en rapport apparent avec la direction des secousses, et je n'hésitai point à admettre frue le mouventent imprimé par secousses exerçait mue influence déterminéc sur la direction des caudex séninaux soustraits à l'influence de la pesanteur par Heur rotation. Ce ne fut que long-temps après que je 'm'aperçus de mon erreur : la direction spéciale qu'alliectaien les caudex séminaux, dans cette expérience, Iprovenait uniquement d'une inégalité dans le nouvement de rotation; inégalité qui était produite par le mécanisme au moyen duquel je produisais les secousses, et qu, étant toujour's la même, produisait des effets toujours semblables. L'expériencc a ses déceptions conme l'imagiuation a ses illusions, et il est 'quelquefois bien difficile de s'y soustraire.

On voil, par les expériences qui viennent d'être irapportées, que lorsque la rotation est lente, les emIbryons séminaux qui l'éprouvent cessent de dirigrer leur radicule vers la circonférence et lenr plumule vers le centre. Il me paraissait important ide trouver quel est le degré de vitesse de rotation oì ccte direction spéciale cesse d'aroir lieu. Les expériences que j'ai fai'es sur cet objet ne m'ont rien appris de bien posilil; d'ahord parceque je n'ai pu essayer toutes les vitesses de mouvíment; en second lieu, à cause rle la construction défectucusio 
de mon mouvement d'horlogerie. Le mouvement le plus lent que j'aic pu obtenir avec ma roue la plus élevéc a été de quinze révolutions par minute; les graines soumises à cette rotation avec un décimètre de rayon ont dirigé leurs radicules vers la circonférence et leur plumule vers le centre. Les graines parcouraient ici neuf mètres quatre décimètres par minute. Le mouvemeni le plus rapide de la rouc immédiatement subjacente étail de quatre révolutions par minute. J'ai soumis des graines à cette rotation, avec un rayon de cinq décimètres : ici les graines parcouraicnt douze mètres quatre décimètres par minute, par conséquent leur mouvement était plus rapide que dans l'expérience précédente; cependant la radicule ne se porta poinı vers la circonférence ni la plumule vers le centre; ces deux caudex se dirigèrent parallèlement à l'axe de rotation, -lequel ćtait incliné légèrement. La radicule se porta vers le côté déclive de l'axe et la plumule vers le côté ascendant; ce résultat, comine on le voit, est semblable à celui que j'avais obtenu en faisant tourner des graines sur clles-mêmes. Je recommençai l'expérience en plaçant l'axe dans une situation horizontale; alors les caudex séminaux affectèrent la direction particulière qui cst produite par l'inégalité de la rotation; c'est-àdire que toutes les radicules et toutes les plumules se dirigèrent perpendiculairement au même diamètre dans un plan vertical. Il me fut impossible de corriger cette inégalité de nouvement, dans la rone dont il est ici question; en sorte que je ne sais pas d'une 
manière bien positive quel est le degré de vitesse de imouvement rotatoire sous l'influence dinquel la pluimule cesse de se porter vers le centre et la radicule wers la circonférence; toutefois ces expériences pourraient porter à penser que la direction de la radicule vers Ha circonférence, et celle de la plumule vers le centre, seraient produites plutôt par le nombre des révolutions dans un temps donné, que par l'étendue du chemin parcouru par la graine dans le mêne temps; inous venons de voir en cffet que des graines qui Iparcourent environ douze mètrcs par minute, en tfaisant quatre révolutions dans le même temps, ne dirigent point leur radicule vers la circonférence et leur plumule vers le centre, tandis que l'on observe cette double direction chez les graines qui ne parcourent qu'environ neuf mètres par minute, en faisant quinze révolutions dans le même temps. Mais ici il $\mathrm{y}$ a une observation importante à fairc; la roue qui ne faisait que quatre révolutions par minute, ćprouvait des saccades multipliées qui résultaient de l'engrenage des dents avec les pignons; ainsi son unouvement de rotation n'était point uniforme, c'était plutôt un transport circulaire opéré à des reprises multipliées. On conçoit que, dans cette circonstance, il ne devait point y avoir de force centrifuge; elle ne peut exister d'une manière sensible que dans un mouvement rotatoire continu; le même inconvénient n'existait pas lorsque j’employais la roue la plus élevée de mon mouvement d'horlogrerie, i laquelle je pouvais foire executer depmis quinze juspu’’i quarante révo- 
lutions par minute, avec un rayon que je pouvais porter jusqu'à cinq décimètres; je sıpprimais son engrenage avec le volant. Les ballons de verre, situés sur leur roue verticale à long rayon : servaient alors de régulateurs pour le mouvement de rotation, qui était continu el complètement exempt de saccades. On concoil que, dans cette circonstance, rien ne s'opposail au développement de la force centrifuge, et ceci explique d'où vient la différence qui a été signalée plus haut.

Lorsque le mouvement de rotation est lent, et que par conséquent la force centrifuge est insuffisante pour opérer la direction des caudex séminaux, cenxci subissent l'influence de la pesanteır, tantôt en se dirigeant parallèlement à l'axe, lorsque cet axe est incliné à l'horizon, tantôt en prenant la direction particulière qui résulte de l'inégalité de la rotation. Lorsque le mouvement rotatoire s'effectue avec une vitesse modérée, l'axe étant un peu incliné, et qu'en même temps la rotation est inégale, les caudex séminaux affectent des directions variées : tantôt on voit, par exemple, toutes les radicules affecter une direction semblable, qui est la direction moyenne résultant des trois forces qui les sollicitent, tantôt on voit ces radicules subir chacune en leur particulier l'influence exclusive de l'une quelconque de ces trois forces, sans qu'il soil possible de sávoir d'oì provient cette irrégularité dans ces effets, sous l'influence d'un niême assemblagge de causes. Les plumules sont, à cét égard, encore plus irrégnlières que les rarlicules; il est rare que, dans cette circonstance, la plumulc 
tprenne la direction diamétralenient opposée à colle ide la radicule; souvent elle semble errer au hasard, ssonvent même elle se dirige dans le même sens que Ila radicule. Ccla s'observe spécialement lorsque, Ila rotation étant fort lente, et l'axe étant horizon ıal, les caudex sémirsaux subissent seulement l'inIlluence d'uné faible inégalité dans le mouvemeut irotatoire.

Les denx caudex séminaux sont absolument indéyendants l'un de l'autre pour leur direction; on peut supprimer l'un quelconque de ces deux caudex sans ique le caudex opposé cesse pour cela d'affecter la direction qui lui est propre; cette direction spéciale In'appartient qu'à l'axe du végétal, lequel axe est : représenté par l'assemblarge rectiligne de la tigelle tet de la radicule; j'ai vu, en effet, que les racines I produites latéralement par la radicule pivotante n'éprouvent point, ou presque point, l'influence des causes qui déterminent la direction de cette dernière; aussi, ne se dirigent-elles point comme elle vers la circonférence lorsqu'elles sont sommises à une rotation rapide. La direction de ces racines latérales offre généralement une tendance à la perpendicularité sur la racine pivotante; celte observation est concordante avec celles que j'ai rapportées dans la scction précédente; observations qui pronvent que les productions végétales tendent généralement à affecter une position perpendiculaire à celle de leur surlace d'implantation; cela nous apprend pourquoi les racines latérales de beancoup de végútanx, au lien de s'en- 
fonccr verticalenent dans la terre, rampent horizontalemicn à peu de distance de sa surface.

Lc procédé au moycn duquel j’ai fail mes cxpéricnces ne m'a pas permis de répéter unc cxpérience très curicuse de M. Knight. Cet observateur ayant tixé des grraines de haricot à la circonférence d'une roue de 11 pouccs de diamètrc que l'eau faisait mouvoir, observa lc développement des tiges qui, en s'alongeant, gagnèrent le centre de la rotation : il avait cu soin de les attacher aux rayons de la roue; sans ccttc précaution, ces tiges, grêles et flexibles, auraient été, ou brisées, ou déviées de leur direction par l'effet de leur pesanteur. Lorsque, par leur accroissement progressif, ces tiges eurcnt un peu dépassé le ccntre de la rotation, elles se recourbèrent ct ramenèrent lcurs sommets vers cc mêmc ccntre, unique but de lcur tendancc constante. Si je n'ai pu répéter cette expérience, cn revanche il m’a été possible d'en faire plusieurs autrcs que M. Knight ne pouvait pas entreprendre avec son apparcil. J'ai voulu voir si lcs feuilles étaicnt susceptibles d'affccter une direction spéciale sous l'influencc d'un mouvement de rotation rapide. Cette expéricncc était lacile à faire avec mon appareil; il ne s'agissail que de renfermer des tiges munies de feuilles dans des ballons de verre, de les fixer solidement dans leur intćrieur, et de soumcttre ces ballons à un mouvcnıent de rotation rapide. Je plaçai donc dans un ballon de verre une tige de convolvulus arvensis, munie de quatre feuilles; $j^{\prime} a-$ vais choisi pour cet cffet les feuilles les plus petites 
fyu'il m'avait été possible de tronver, afin de pouvoir cemployer des ballons de verre d'une nédioere dimension, et, par conséquent, afin d'obtenir une rotation rrapide, à laquelle il m'ent été impossible de soumettre ides ballons volumineux, à cause de leur pesanteur. ILa tigge grê̂le et flexible du convolvulus étail attaehée avee un fil à une tige de fer de peu de grosseur, que jintroduisis ensuite dans le ballon de verre, et dont je fixai les denx extrémités anx ouvertures opposées de cce ballon, dans lequel je mis seulement une ou deux ¿cuillerées d'eau. Un second ballon de verre fut préparé He la même manière, eı je plaçai ees deux ballons aux deux extrémités d'un même diamètre, sur une rone qui avait einq décimètres de rayon, et qui faisait quarante révolutions par minute. Les tigres des plantes étaient perpendienlaires au plan de la roue, cen sorte que pendant la rotation elles étaient toujours dans une situation horizontale; ainsi elles ne toucehaient point à l'ean, qui oceupait tonjours la partie la Iplus déclive des ballons de verre; les feuilles n'y tonchaient point non plus, cependant elles ne tardèrent fpoint à être mouillées par l'ean vaporisée dans l'intétrieur des ballons qui étaient hermétiquement bouchés, tet eela suffit pour entretenir leur vie et leur fraîcheur. ILes feuilles placées au hasard affeetaient des direetions variées par rapport au plan de rotation. Au bout de dix-huit heures, toutes les f'euilles soumises à l'expétrience avaient dirigé leur face supérieure vers le eentre de la rotation, et par conséquent lenr lace inlérrieure se tronva dirigée vers la circonférenee. Ce 
retournement s'était opéré au moyen de la torsion ou de l'inflexion des pétioles. Je répétai cette expérience avec les feuilles à long pétiole du fraisier (fragaria vesca) et de la violette (viola odorata); je choisis pour cela les plus petites feuilles qu'il me fut possible de trouver, et n'en laissant que deux sur chaque pied, auquel j'avais conservé la racine, j'attachai cette dernière avec un fil à la tigge de fer, que je plaçai ensuite dans l'intérieur de mes deux ballons de verre, disposés comme dans l'expérience précédente. Au bout de vingt-quatre heures de rotation par un temps très chaur, toutes les feuilles avaient dirigé leur face supérieure vers le centre, et par conséquent leur face inférieure vers la circonférence. J'observai ici un phénomène de. plus que dans l'expérience précédente, c'est que les feuilles s'étaient rapprochées du centre au moyen de l'inflexion et de la tendance du sommet de leur pétiole vers ce point. Ce phénomène, entièrement vital, est tout-à-fait contraire aux lois ordinaires du mouviement; car, en soumettant au môme mouvement de rotation un corps aussi pesant que le limbe de la feuille suspendu à un fil, ce corps se porterait vers la circonférence, en vertu de la force centrifuge. Il résulte de ces expériences que les deux faces opposées des feuilles possèdent des conditions vitales opposées dans leur nature, comme cela a lieu pour la plumule et la radicule des embryons séminaux. La face supérieure des feuilles possède les conditions vitales de la plumule, et se dirige comme elle vers le centre: la lace inféricure des feuilles possède 
Ues eonditions vitales de la radicule, et se dirige comme celle vers la circontérenee. Ainsi, la face inférieure des fifeuilles obéit, eonme la radicule, au mouvement ou à Ha tendanee qui l'influenee; leur face supérieurc, an :ontraire, réagit, eomme la plumule, contré ce mouvement ou contre cette tendanee. Cela explique pourquoi des feuilles dirigent ordinairement leur faee supérieure ivers la lumière, c'est-à-dire dans le sens diamétraleament opposé à eelui du mouvement de cet agent, et tpourquoi leur face intérienre fuil la lumière, c'esth̀-dire se dirige dans le sens nićme du mouvement the cet agent; il y a obéissance au mouvement dans Ha laee inféricure, el réaction contre le mouvement dans la face supérieure. Si les teuilles se retournent aussi dans la plus profonde obscurité, cela provient évidemment de ee que la feuille est égralcment en rapport avec la cause inconnue rle la gravittation, dont la tendance de haut en bas détermine ıune obéissance de la part de la face inférieure, et unne réaction de la part de la faee supéricure. Ainsi, con peut établir comme un fait grénéral que e’est le mouvement, ou la tendanee au mouvement dans un sens déterminé, qui provoque la direction opposée des tiges et des raeines et la direction opposée des deux faees des feuilles. C'est la gravitation, ic'est la tendance en ligne droite vers le eentre ade la terre qui provoque l'ascension des tiges et le I mouvenıent deseendant des racines; e'est le mouvement en ligne droite de la lumière qui provoque la a direction des tiges et de la face supérienre des feuilles 
ot des fleurs vers le lieu duquel cette lumière arrive, et qui porte en même temps la face inférieure des feuilles et des fleurs, de même que la radicule du gui à s'éloigner du lien duquel la lumière émane.

J'ai fait voir, dans la section précédente, que les pétales des fleurs se comportent de la même manière que les feuilies; dans les directions spéciales qu'ils affectent, c'est toujours leur face la plus colorcée qui se dirige vers la lumière. J'ai dit que lorsque les fleurs avaient habituellement lenr face supérieure, ou plutôt leur partie intérieure dirigée vers la terre, cela provenait le plus souvent, moins de la faiblesse du pédoncule qui se ployait sous le poids de la fleur, que d'une tendance spéciale de la lace intérieure de la fleur vers la terre. Pour m'assurer de la validité de ceţe opinion, j'ai soumis à une rotation rapide des tiges de bourrache chargées de fleurs et renfermées dans des ballons de verre. On sait que les fleurs de cette plante ont toujours leur lace intérieure dirigée vers la terre; or l'extrême légèreté de ces fleurs ne permeltait guère de croire que cet effet pût être dî̀ à leur poids, sous lequel le pédoncule se fléchirait. Dans l'expérience dont il s'agit, il y avait trente-six révolutions par minute et trente-deux centimètres de rayon. Au bout de seize heures de rotation toutes les fleurs avaient dirigé leur face intérieure vers la circonférence, el cela au moyen de la torsion ou de l'inflexion des pédoncules; cette expérience me prouva que la direction de la face intérieure des flemrs de bourraclie ver's la terre est le résultat d'une ten- 
alance spéciale pareille a celle de la radieule ex à celle de la face inférieure des feuilles. Cependan eette lace intérienre de la corolle de la bourrache ne parait pas inférienre en coloration à la laee opposée; jattribne donc la direction vers la terre qu'clle présentc constamment à l'existencc dans celte corolle d'un nectaire incolore (phycostême de M. T'urpin ). Cot organe, ineolore comme une raeine, affecte, par cola mème, ane direetion semblable, et produit la direction de La face intéricure de la corolle vers la terre dans ll'ćtat naturel, et vers la eireonférence dans l'expćrience préeédente.

J'ai démon tré plus lıaut, par des expériences décisives, que ee sont des mouvements spontanćs qui opèrent la dircetion spéeiale des caudex séminaux, ainsi que le retournement des feuilles; j'ai fai voir mue ces mouvements spontanćs sont exćentés à l'oesasion de l'influence de eertains agrents extćrieurs sur a nervimotilité des végétaux. Or nous voyons que sous l'influence d'un même agent, nervimoteur la olumule et la radicule sc dirigent dins des sens diamétralenrent opposés; ees denx parties, opposées par cur position, ont donc une nervimotilité différente, sinon daus sa nature, du moins dans quelques unes de ves eonditions, prisque l'une tend à produire J'obéisance cu l'autre la réaction, par rapport à un agent nervinoteur qui ne varie point. Il y a nue nervimoilité, prineipe d'obéissance, et une nervinntilite, rrincipe de réaction; cen denx modes opposcis de ta

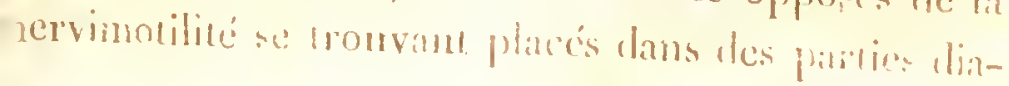


unétralement opposées, nous ne pouvons nous dispenser de reconnaître là un phénomène tout-à-läit analogue à ce que l'on nomme la polarisation en physiquue. La nervimotilité, ou plutôt son agent inconnu, cflire véritablement deux pôles chez les végétaux; les raeines sont le siége du pôle obéissant, les tiges sont le siégre du pôle réagissant. Ces mêmes pôles sont plaeés sur les deux faces opposées des feuilles: le póle réagissant est placé sur la faee qui se dirige ordinairement vers la lumière et vers le eiel; le pôle obéissant est plaeé sur la faee qui se dirige vers la terre. Nous avons vu, dans la seetion préeédente, que la différenee de direction de ees parties, qui sont iei eonsidérées eomme des pôles différents, eoïncide eonstamment avec une différenee en plus et en moins de la coloration de ees parties. Le pôle obéissant est toujours inlérieur en eoloration au pôle réagyissant; il y a par conséquent ehez l'un exeès de eertaines eonditions qui sont en moins ehez l'autre; je suis porté à penser que ees différenees visibles en plus et en moins coïneident avec des différenees semblables, c'est-à-dire en plus et en moins, dans les eonditions de la nervimotilité. 


\section{SECTION V.}

MSLRVATIONS SUR LA STRUCTURE INTIME DES SYSTEMES NERVELX IT MUSCUIAIRE, ET SUR IE MFCANISMF DE I. CONTRACTJON CHEZ LES ANIMAUX'.

L'étude de la physiologie végétale est presque géméralement négligée par ceux qui s'occupent de la icienee des animaux; il est rare de même yue les hotanistes cultivent la physiologic animalc. La science ¿ónérale de la vie ne peut que perdre à cet isolement He deux sciences qui n'en font véritablement quiune, at yui doivent mutucllement se fournir des lumières 't se prêter des sccours; carr il est des problèmes de a physiologie anima'c dont on ne peut trouver la sountion que dans l'ćtude des végétaux, et réciproquement il est des nyystères de l'organisation végétale qui ne peuvemt être dévoilés que par l'étude comparative He l'organisation animale. Nous avons déjà cu licu de unous convaincre de cette vérité cn étudiant l'anatomịe He la sensitive: sans le secours de l'anatomie microscopique animale, la nature des organes que nous uvons considérés comme nerveux dans celle plante

2 Les résultats généraux des observalions contrnues dans cetle section ont eté communiqués à ha Suciét philomatique, dans sis súnce du 6 diocrublure 18,5 
nous en̂t été totalement inconnue. Actuellement nous allons porter nos recherches sur le phénomène de l'irritabilité animale, et nous serons puissamment aidés daus cette investigation par les notions que nons avons précédemment acquises sur l'urritabilité vérgétale; mais avant de nous livrer à cette étude il est nécessaire de comnaître la struccure intime des systèmes nerveux et musculaire.

Le système nerveux des animaux, observé dans ses éléments microscopiques, est essentiellement composé de corpuscules globuleux aggrglomérés; cette organisation est connue depuis long-temps par les recherches de Leuwenhoeck, par celles de Prochaska, e de Fontana; par les observations de sir Everard Home, de Bauer, des frères Wensel, et en dernier lieu par les observations de M. Milne Edwards. Ces corpuscules globuleux paraissent être des ce!lules d'unc cxcessive petitesse, lesquels contiennent une substance médullaire ou nerveuse, substance qui est concrescible par l'action de la chaleur et par celle des acides. Cette opinion a été émise par'sir Everard Home $^{x}$, qui la empruntée à MM. Joseph et Charles Wensel "; on ne pourra se dispenser de l'adopter quand on aura jeté les yenx sur la structure microscopique du cerveau des mollusques gastéropodes.

Le cerveau des mollusques gastéropodes, comme on le sait, est composé de deux hémisphères, si toutefois on peut donner ce nom aux deux partics

- Philosophical Transactions, 1818.

- De pcnitiore structura cerctri hominis ct brutorum. 
ont se compose ce eorps symétrique. De ces dend émisphères partent denx cordons ncrveux yui enrassent l'cesophage, et se réunissent pour lormer un gangrlion. Le cerveau est enveloppé par une mcnmane fibrense dont on pent le dépouiller avec la pointe 'nne aignille et des pinees très fines; on obtient de ctue manière le petit noyan pulpeux qui occupe le centre de chaeun des hémisphères; culte opération, ttant fort délieate, ne pent guère être faile que sur ss grosses espèces; aussi est-ce exclusivement sur inelix pomatia, el sur le limax rufies que j'ai tail es observations. Les deux petits noyanx pulpeux qui composent essentiellenent le cerveau de ces mollusues doivent être placés dans l'ean pour les cxanier au microscope; ear on ne peut faire d'observalons délicates sur les tissus organiques qu'en les bservant dans ec fluide; e'est ainsi que j’al fait la Ilupart de mes observalions microscopiques; je dois in outre prévenir les observateurs (jui seraient eueux de les répéter, quils ne doivent point se servir u mieroseope composć, mais du microscope simple, ui senl pent procmrer une vision très netle et très istincte. Celle supérioritédu microscope simple, sur meilleur microscope composé, est conmue depris mb-lemps, mais je ne la croyais pas anssi considéwble quielle l'est réellenent; des lentilles de trois groses a une ligne do loyer suflisent pour fine la lupart des observations qui voul étre cxposées, et expluelles je mompresse de revenir aprós petile: igression. 
Le petit noyau pulpeux qui lorme chaeun des hémisphères du eerveau, chez le limax rufus et ehez l'helix pomatia, est composé de cellules globuleuses, aggnlomérées, sur les parois desquelles on voil unte grande quantité de eorpuseules globuleux ou ovoïdes, comme on le voit dans la figure 20. Ces eorpuseules grlobuleux sont très évidemment de petites celluies remplies d'une substanee médullaire ou nerveuse, demi-transparente, et eependant très sensiblement de eouleur blanehe. Les cellules globuleuses sur les parois desquelles ees corpuseules sont placés contiennent de même une substanee médullaire nerveuse, laquelle, autant qu'on en peut juger au microscope, est d'une couleur un peu grisâtre el demi-transparente: ainsi ces deux substanees nerveuses sont analogues aux deux substances grise et blanehe que contient le eerveau des animaux vertébrés; il n'y a de partieulier iei que la manière dont ees deux substances sont disposées l'une relativement à l'autre; la substance grise est eontenue dans de grosses eellules globuleuses, la substance blanche est eontenue dans de très petites cellules également ǵlobuleuses, et plaeées sur les parois des yrosses cellules, auxquelles elles n'adhèrent que faiblement: elles s'en détachent assez facilcment. Cette observation nous prouve que les corpuseules nerveux dont se compose le eerveau, et en général le système nerveux des animaux, sont véritablement des eellules remplies par la substanee nerveuse proprement dite. Ces cellules sont adhérentes les unes anx autes, sans aucun medium ap)- 
Iparent, aunsi que l'one pensé MM. Wensel pont des corpuscules vésicnlaires dont est composé le cervean des animanx vertébrés.

Les nerl's di l'helix pomatia et grisea offient extérienrement une tunique cellulense assez épaisse et Hemi-transparente; les cellules agglomérées qui composent ecte tunique sont globuleuses et contiennemt am fluide diaphane el incolore; les parois de ces celHules conticnnent des corpuseules égalenent diaphames, conume on le voil dans la figure 21, $a$. Cette organisation est, quant à la forme, tout-à-fait semblable a eelle que nous venons d'observ r dans le cerveau (ligg. 20); ruais elle en diffère essentiellement par lapparence et par la nature de la substance qui cst contemuedans les cellules. Au centre du canal que forme cencenveloppe celluleuse est te nerf propreunent dit, dont le tissu est représenté en b ( tigg. 21 ). ICe tissu est composé d'une immense yrantité de colIpuscules ncrveux d'unc excessive petitesse, adhérents ii rleux sortes de fibres, les unes longitudinales, et ' yui sont les plus grosses, les autres, d'une prodiggiense "cinuité, qui sont distribuées irrégulièrement dans les intervalles des précédentes. J'ai observé que le nerf $b$ pénètre seuí daus l'intérieur des oryanes anxquels il se distribue; l'enveloppe cellulense $a$ se contimne avee une enveloppe analogne, qui revêt lons les oršilles.

Chez tit grenouille, les nerf's sont composés de corpuscules nerveux, transparcuts, arihérents ì des fibres longitudiuales, égaleurent transparentes. ['our 
faire celte observation, il faut, avec lia pointe d'unc aigruille, diviser $u n$ nerf cn filets aussi déliés qu'il est possible de le faire: de cette manière on sépare les fibres nerveuses les mnes des autres. La figurc 22 représente unc seule de ces fibres considérablement grossie. Ces fibres paraissent être des ıubes renuplis I'un fluide diaphane; les corpuscules nerveux sont collés sur leur surface; la plapart du temps on ne voit d'une manière très distincte que les corpuseules qui sont situés sur les bords de la fibre, parcequ'ils forment une légrère saillie qui aide à les distinguer; les corpuseules qui sont situés sur le milieu de la fibre s'apercoivent plus diffieilentent, parceque lenr transparence les eonfond avee la fibre, qui est transparente elle-même. Fontana' avait déjà annoncé que les nerfs sont composés d'un grand nombre de eylindres transparcnts; M. Milne Edwards pense que ces eylindres longritudinaux sont formés par la réunion d'un eertain nombre de fibres éléntentaires, qui elles-mêmes sont composées de glohules plaeés ì la file. Iei les illusions du nicroscope permettent diffeilentent de distinguer la vérité; ccpendant il m'a paru évident que ees eylindres longitudinaux ne sont point composés de fibres élémentaires, formées clles mêmes dc globules alignnés, ainsi que le pense M. Milne Edwards, mais que ce sont des cylindres d'une substance diaphane dont la surfinee est hérissée de corpuseules globuleux, lesquels tantôt sont en 
contact et placés ì la file, tantôt sont séparés les uns Hes autres. Comme ils couvrent toute la surfice du ylindre, on est porté, dans l'observation microscotpique, it croire qu'ils le composent intérieurenent. Minsi les nerf's de la grenouille me paraissent compo:sés de filets transparents, cnvironnés de corpuscules nerveux : celtc organisation cst surtout évidente dans les nerfs de l'helix pomatia (fig. 21), ainsi que mous venons de le voir. Ici les fibres sont très disrinctes des corprseules globuleux qui les environment. Cétc manière de voir est d'ailleur's singulièrement. confirmée par linduction analogique, qui nous monttre, chez lcs végétaux, les corpuscules ylobnleux garnissant la surlace des cylindres tubuleux; nous allons voir d'ailleurs, chez les animaux, un autre exemple bien évident de cette disposition: je n'hésite idonc point à considérer les nerl's comme conıposćs de deux ćléments organiques; savoir, des cylindres idiaphanes ce des corpuscules glohule ux qui les enviromnent de toutes parts.

Le cerveau de la grenouille est emtierement composé par unc agglomération de corpuscules ncrvenx semblables à ceux qui existcnt dans les nerl's; quelques fibres diaphanes assez rares sont mêlées parmi ces corpuscules aggglomérés : la figgnre 25 représente ce lissu intine du cerveau de la grenouille. Ainsi la substance dir cervean de ce septile ne diffère de celle de ses nerf's que par une diflérente proportion dos mêmes éléments organigines: les corpuscules nerveux abondent dan le cerrean. les fibres nen reuses y sont 
rares; c'est le contraire dans les nerf's, qui oifrent des fibres nombreuses et très développées, tandis que les corpuscules nerveux y sont plus rares qu'ils ne le sont dans le cerveau.

Les inductions physiologiques que l'on pert tirer des observations préeédentes sont extrêmement inıportantes; en effet, nous voyons d'un eôté le cerveau, organe éminemment destiné à la production de la puissanee nerveuse, être éminemment composé de corpuscules nerveux; nous voyons d'un autre côté que les nerfs, qui sont éminemment destinés à la transinission de la prissanee nerveuse, ou de la nervimotion, sont éminenıment composés de fibres nervenses; eela nous donne droit de conclure que les corpuscules nerveux sont les organes producteurs de la puissance nerveuse, et que les fibres nerveuses sont destinées à la transmission de la nervimotion. Nous avons vu que, chez les végélaux, la nervimotion est rransmise par l'intermédiaire du liquide séveux; cela peut faire penser que les fibres nerreuses des animaux sont des tubes remplis d'un liquide parliculier, et que c'est par l'intermćdiaire de ee liquide que s'opère la transnission de la nervimotion.

Les polypes, comme on sait, n'ont point de nerfs; ils sont composés d'unc substance en apparence homogrène; cependant, eomme ils manifestent, par leurs mouvennents, 'pu'ils éprouvent l'influence des agents dur dehors, on doit penser qu'ils possèdent des organes nervenx. Effectiveneir, dius la pulpe transpatrente et en apparence lomogene yui les compose to 
microscope fait aperecvoir une grande quantite de :oglanulations qui ressenublent tout-à-fait aux corpuscules nerveux des autres animanx, et encore plus à ceux des végrétaux. Cette ressenublance peul autoriser ià les reconnâtre pour des organes nerveux épars dars tout le tissu organique: on se fera unc idće de cette organisation en jetant les yeux sur la figure 24, qui l'eprésente un tronçon de l'un des bras d'une hydre. ' Cics corpuscules nerveux sont bien moins nombreux, et sont proportionnellement plus gros chez les polypes à bouquets (vorticella convallaria); ils oceupent exclusivenent la partic centrale des rameaux, comme on le voil dans la figure 29.

Les muscles, chez les animaux vertéhrés, chez les crustacés et chez les insectes, sont composés de fibres ou de corps eylindriques filiformes auxquels on donne, par excellence, le nom de fibres musculaires. Ces fibres, comne chacun le sait, ont la propriété de se contracter, ou de se raccourcir dans le sens de leur longueur, en sc ridant transversalement, et en devenant plus g̀rosses qu'elles ne l'élaicnt dans leur état de relâchement. L'extrême petitesse de la fibre musculaire rend très difficile l'observation de sa structurc intime. Lcuwenhocek I a cherché ì obscrver cette structure chez divers yuartrupèdes, chez les poissons et chez quelques crustaces. Le seul résultat de ses recherehes est que la fibre musculaire est composée d'une srrande quantité d'autres fibres 
plus petites, lesquelles some réunics en faiscean par une niembrane enveloppante eommunc. Dans les premières observations yu'il publia sur cette natic̀re il affrma que les fibres musculaires étaient composées de globulcs; mais quclques années après, il revint sur cclte asscrition, et déelara que c'était nne erreur. Cependant Hook affirma avoir obscrvé ces grlobules dans les fibres musculaires des écrevisses $\mathrm{et}$ des erabes; il eonsidérait chaque fibrc comme comprosée de filaments semblables à des fils chargés de perles. Leuwenhoeck, auquel il fit part de cette observation, la rćpéta ct continua d'affirmer quc ces globules n'ćtaicnt aute ehose que les plis transversaux des fibres, et que cette apparence de petitcs boules ćtait causée par la ehute variće de la lumière sur ces plis plus ou moins élcvés ? Dans ccue circonstance Lcuwcnhocck, maļré son grand talcnt pour les observations microseopiques, a méconnu unc vérité qu'il avait d'abord cntrevue; en effet, lcs observations rapportćes par sir Everard Home ${ }^{2}$ ne laissent point de doute à cet égard. Ces observations, qui sont dues à M. Baucr, et quiont été faites sur les fibres museulaires de l'estomac humain, et sur eclles du mouton, du lapin et du saumon, prouvent que ces fibres sont composées de grobules plaeés à la suite les uns des autres, et qui sontde la griosscur des globules sanguins. Cettc dé-

- Tettre à Illook, insérce dans la Collcction philosophique de. ce Acrnics.

"Philosophical'Transaclions, 1818. 
couverte a été confirmée parles reeherches de MNI. L'reivost et Dumas ', qui alliment avoir vil lil mêne chose chez les mamnifères, les oiscaux et les poissons; mais malheureusement ils ne donnent auem ıdétail ì eet égard. La mêne structure a ćté vue depuis Ipar M. Milne Edwards. Ici je m'arrête un insiant ponr fprésenter une réflexion. Le not fibre est peut-être um de cenx dont on a le plus abusé en anatomie, aussi ne représente-t-il aucune idée cxaete; on donne, ien général, ce nom ì tons les eorps organiques Ilinéaires et très déliés. D’après eețe définition, on voit que le mot fibre n'est, pour ainsi dire, qu'une texpression provisoire dont on se sert en attendant 'que l'on eonnaisse exactement la nature véritable de I l'organe linéaire que l'on désigne sous ce nom. Ce que l'on appelle proprement les fibres musculaires sont des eorps eylindriques filiformes qui, par leur réunion en nombre immense, forment les museles dont ils sont les parties intégrantes, mais ces fibres ne sont point des eorps simples, elles ont une organisation intéricure qu'il est essentiel de dévoiler; c'est ce qu'ont tenté de faire les derniers observateurs que je viens de eiter, et le résultat de leurs recherches a été que les fibres musculaires étaient composées de globules placés à la file. Ainsi voilà l'expression, fibre musculaire, employéc par les anatomistes pour désigner des ohjets essenticllement difleirents, ear it cst évident yne ce ri'est pas 'le la libre 
musculaire intégrante que ces observateurs ont vonlu parler, mais des organes filiformes qui s'observent dans le tissu intime de cette fibre, ou des fibres musculaires constiluantes, si je puis employer cette expression; je crois donc que, pour rétablir l'ordre et la clarté dans cette discussion, il est nécessaire de réserver exclusivement le nom de fibre musculain'e aux organes filiformes qui composent immédiatement les muscles, et de donner le nom de fibrilles musculaires aux organes filiformes plus petits que l'on observe dans le tissu intime des fibres musculaires, et dont on ne distingue point l'organisation; enfin, je propose de désigner les assemblages rectiligrnes de corpuscules glnbuleux que l'on observe dans le tissu intime des organes musculaires, par cette expression, corpuscules musculaires articulés.

Ce n'est point clec les animanx vertébrés qu’il est. facile d'apercevoir la structure intime de la fibre musculaire, mais on la découvre assez facilement chez plusieurs animanx des classes inférieures, par exemple, chez l'écrevisse (astacus fuviatilis. Fab.). Pour faire cette observation, il faut prendre des fibres musculaires dans la queue de l'animal, et les diviser en. parties extrêmement fines avec la pointe d'une aiguille; de cette manière, on net à découvert le tissu intérieur de ces fibres, et le microscope fait voir que ce tissu est composé de fibrilles transparentes disposées longitudinalement, et dans les intervalles desquelles il existe une graude quantité de globuies uransparents ; ces globules sont tellcment semblables 
opar leur forme et par leur position anx corpuscules merveux que couvrent les fibres nerveuses, que j'aurais pu être porté à leur donner le même nom, si des cobservations qui seront rapportées plus has ne m'awaient éclairé sur la véritable nature de ces globules, ıque je désignerai sous le nom de corpuscules muscculaires. Ces corpuscules, remplis d'un fluide diaIplane, sont intercalés aux fibrilles et appliqués sur Ileur surface, à laquelle cependant ils ne paraissent adhérer quc faiblement, car on aperçoit des fibrilles "qui en sont entièrement dépourvues; ce sont ces (fibrilles et ces corpusenles musculaires qui constiunent par leur assenblage te tissu de la fibre muscuIlaire; je désignerai ce tissu sons le nom de tissu musculaire fibrillo-corpusculcire.

Les muscles sont composés de fibres chez les aniı maux vertébrés, chez les erustacés el lcs insectes; ımais il n'en est pas de nême chez les moilusques, idu moins à en juger par les gastéropodes: chez eux, Iles muscles ne sont point composés de fibres musculaires, dans le sens que nous venons de donner à ectte expression; ils sont composés inmédiatement de tissu musculaire fibrillo-corpusculaire. Cetue - organisation cst très lacile à voir chez l'helix pomatia; il faut prendre pour cela un des museles fort allongés qui servent à rentrer l'animal dans sa coquille; on le. place dans l'eau, el avec la pointe d'une aiguille on le divise en filets délićs que l'on soumet au microscope; on voit que lc corps du muscle est immédiatement composé de fibrilles transparentes qui portent des 
corpuscules musculaires, adhérents ì leur surface. La figure 25 représente cette organisation. On voit très distinctement que les corpuscules musculaires ne ne font point corps avec les fibrilles; ils s'en détachent même assez facilement. Cette observation, très facile à faire, ne peut laisser aucun doute dans l'esprit de l'observateur, relativement à la position des corpuscules grlobuleux, à la surface des fibrilles qui, elles-mêmes, ne se présentent que comme des corps diaphanes homogènes; ce fait est complètement en harmonic avec ce que j’ai exposé plus hiut touchant la structure de la fibre nerveuse, que j'ai considérée comme un corps cylindrique, diaphane et homogène, dont la surface est couverte de cerpuscules globulenx; j'ai fait remarquer alor's que cette organisation s'observe jusque dans le règne véģétal.

Le cour des animaux est, en général, l'organe musculaire dont l'organisation intime offre le moins de difficultés pour l'observation microscopique. Aussi est-ce sur le tissu de cel organe que j'ai spécialement fait mcs observations; c'est surtout chez les animaux des classes inférieures que la structure intime du cour est facile à voir. En généréral, on n'y rencontre point de fibres musculaires proprement dites, mais seulement des fibrilles et des corpuscules musculaires. Le cour de l'écrevisse, par exemple, est entièrement composé de tissu musculaire fibrillocorpusculaire; mais ici les corpuscules, musculaires abontent et les fibrilles sont ¡ssez rares; de plus, les 
corpuscules musculaires, que nons n'avons encore ujbservés que dans un ordie confus et sans aucun l:'apport immédiat entre cux, affectent ici la dispositiion en sćries longitudinales; ils forment ce que je nomme des corpuscules musculaires articulés. (C'est celte disposition des corpuseules en séries rectiliitignes qui a été observéc par M. Bauer, par MM. Prewost et Dumas, et par M. Milne Edwards, chez H'autres animaux. J'indique ici le cœur de l'écrevisse czomme l'un des organes musculaires où cetle orgámisation est le plus facile à apercevoir.

Le cour de la grenouille n'est point non plus composé de fibres musculaires proprement dites, comme le sont les autres muscles de ce reptile; il est immédiatement composé de tissı museulaire fibrillocorpusculaire. La figure 27 représente ce tissu intime thlu cour de la grenouille; on voit qu'il est entièreıment composé de fibrilles tortueuses extrêmenıent Héliées et transparentes, dans les intervalles desquelles ill existe une grande quantité de corpuscules museulaires transparents. Les fibrilles disparaissent tontì-fait dans le cœur des mollusques gastéropodes; en effet, chez l'helix pomatia et chez le limax infius, lle coeur est' entièrentent composé de corpuseules ımusculaires aygglomérés; ces corpuseules forment uantôt des séries longritudinales, tantôt des agréggats membraneux dans lesquels on ne distingue aneune idisposition rle ces corpuscules en ligne droite; lentr ordre d'agrégation est 1011-i-liait confus. Cette observation prouve yue l'existence des tibrilles, et urême 
celle des organes linéaires, en générál, n'est pas indispensable pour le mouvement musculaire, puisque nous voyolls ici ce mouvement exécuté par un tissu composé de corpuseules musculaires agrégés dans un ordre confus. Je donne à ce tissu musculaire, dans lequel il n'existe point de fibrilles, le nom de tissn. musculaire corpusculaire. Il y a grande apparence que les fibrilles, dont on ne peut apercevoir la structure intime, sont composées de ce tissu musculaire corpusculaire, soit articulé, soit confus, mais d'une tellc petitesse qu'il échappe à nos yeux armés des meilleurs microscopes.

Après avoir étudié la structure intine du tissu musculaire, j'ai fait plusieurs tentatives pour découvrir le mécanisme du mouvement qui lui est propre, c'est-à-dire du mouvement de contraction. Les insectes m'ont paru devoir se prêter avec plus de facilité que d'autres animaux à ce genre d'observations, parceque plusieurs d'entre eux ont leurs fibres nıusculaires complètement isolées les unes des autres; ıel est, par exemple, le cerf-volant, lucanus cervus. Les fibres musculaires de cet insecte sont fort grosses, et ne sont point liées les unes aux autres par du tissu cellulaire. C'est à tort, pour le dire en passant, que l'on a prétendu que cette organisation était généralc chez les insectes; chez la plupart d'entre eux, en effet, j'ai vu les fibres musculaires liées entre elles par du tissu cellulaire, comme cela a lieu chez les animaux des autres classes.

Pour observer au microscope la contraction des 
ibres musculaires chez le cerf-volant, j'enlevais avec in rasoir une partie du corselet mr un de ees inwectes vivant. De cette manière, je mettais à découwert les muscles du thorax, et je pouvais observer "action de celles de leurs fibres qui n'avaient point perdu leurs points d'attache par cette opération. Je hai vu, dans eette observation, que ce qui est connu Hepuis long-temps; savoir, que les fibres muscuaires, en se contractant, rentrent, pour ainsi dire, en elles-nêmes; elles deviennent plus grosses qu'elles te l'étaient dans l'étạt de relâchement, et elles se couzrent en même temps de plis transversaux plus ou moins irréguliers. La figure 26 représente une de ces iibres musculaires du cerf-volant, la portion a est Lans l'état de contraction, la portion $b$ dans l'état de ielâchement. On voit que les plis sont extrênement zapprochés et multipliés sur la portion contractée, lont la grosseur est beaucoup plus considérable que ne l'est celle de a portion relàchée. J'ai répété ces bservations sur les museles du thorax de plusicurs autres insectes, et j'ai vu partont que les fibres mus-ulaires se comportaient de la même manière. Ainsi 4 me fut démontré que le mottvement de contraction lie la fibre dépend d'un mécanisme intérieur qu'il a'est point possible d'apercevoir dans ces organes, a zause de leur défaut de transparence. Jẹ résolus donc Ide diriger mes recherches sur les organes musculaires qui n'ont point de fibres, mais dont le tisșn, considéré dans son entiex, est composé, comme le somt intériemrement ies tibres musculaires proprement 
dites; nous venons de voir que telle était l'organisation du cœur chez les batraciens et chez les mollusques gastéroporles. Cher les premiers, le cœur est composé de tissu musculaire fibrillo-corpusculaire; chez les seconds, cet organe est entièrement composé de tissı musculaire corpusculaire; mais ici il existe pour l'observation une très grande difficulté. On ne peut observer au microscope le tissu du cœur de ces animaux que dans l'état de mort: en effet, cet organe étant toujours dépourvu de transparence, et d'ailleurs étant trop épais, ne peut être observé au microscope pendant la vie ; il faut, pour observer son tissu intime, qui est le siége du mouvement de contraction, il faut, dis-je, le lacinier en parcelles d'une extrême petitesse, qui cessent d'être vivantes par le seul fait de cette opération; ainsi, il faut renoncer à observer au microscope la contraction du tissu intime des organes musculaires pendant la vie, mais il existe des moyens par lesquels on peut solliciter cette contraction dans les plus petites parcelles du tissu musculaire détaché de l'animal, et qui par conséquent n'est plus sous l'influence vitale de ce dernier. On sait, par exemple, que les acides provoquent énergiquement le mouvement de contraction tant pendant la vie qu'après la mort; on connaît leur action styptique: nul doute que la contraction qu'ils produisent en pareils cas sur le corps vivant ne soit une action vitale. Il reste à déterminer si le mouvement qu'ils produisent dans les organes musculaires privés de la vie est aussi une contraction, et si le 
mécanisure de cente contraction est semblable à celui de la coutraetion musculaire vitale. Pour résoudre ce problème, j'ai fail l'expérienee suivante : j'ai pris un umnscle longr de l'helix pomatia, je l'ai fixé solideument parl'une de ses extrémités, avec épingle, snr une llame de cire, puis l'ayant un peu alongé, pour déutruire la contraction, je l'ai fixé, ainsi distendu, sux lla lane de cire, au moyen d'une seconde épingle placéc à son autre extrémité, et enfoncée dans la cire d'une manière peu solide, afin qu'elle pî́t s'arracher au moindre effort. J'ai couvert le nuscle ainsi disposé d'une petite nappe d'eau, à laquelle j'ai ajouté ensuite une petite goutte d'acide nitrique. Un instant après cette addition le musele s'est contracté, et a arrachél'épingle qui fixait peu solidement l'une de ses - extrémités.Cette expérience me prouva que l'action des acides détermine, après la morı, dans le tissu musculaire, une contraction qui, par elle-même, ne diffère point de la contraction qui a lieu pendant la vie, mais qui en diffère seulement par sa cause déterminante. Cette similitude de l'action musculaire sons l'influence intéricure d'une cause vitale, et sous l'influence extérieure des acides ne fut encore démontréc par l'expérience suivante : ayant unis à nu les ınuscles de la cuisse d'une grenouille vivante, jen arrachai quelyues fibres avec des pinces très fines. En examinant au microscope ces fibres, placées dans de l'eau, je les vis se courber et se pelotonner les unes sur les autres, comme auraient fait des vermisseaux. Ce mouvement des fibres étail quelquelois assez 
rapide, d'autres fois il était d'une grande lenteur. Si dans ee dernier cas j'ajoutais une goutte d'acide à l'eau, je voyais à l'instant les fibres se ployer avec vivacité; ainsi, il est évident que l'influence des acides détermine dans les fibres musculaires des mouvements entièrement semblables à ceux qu'elles exécutent spontanément sous l'influence vitale. Je regarderai donc désormais cette proposition eomme démontrée, et je reviens à la dernière expérienee qui vient d'être exposée. Les fibres museulaires, séparées des muséeles auxquels elles appartiennent, et plongées dans l'eau, tendent à se ployer ou à s'incurver. Ce mouvement est le résultat d'une propriété vitale particulière de la fibre; car il n'y a eertainement là ni sensation déterminante de ee mouvement, ni volonté pour l'exéeuter. Si donc la fibre s'ineurve spontanément, cela prouve qu'il existe dans le tissu qui la eonstitue une disposition qui fait, ou que ce tissu se contracte du côté qui devient eoncave, ou que ee même tissu sedilate du côté eonvexe; peut-être ces deux états opposés du tissu de la fibre existent-ils à la fois; toujours en résulte-t-il qu'il existe dans la fubre une tendance au mouvement d'un seul côté, tendance de laquelle résulte son incurvation. Pendant que j'observais des fibres isolées qui venaient de s'ineurver, il me vint dans l'idée d'ajouter une goutte de solution aqueuse de potasse caustique à l'eau idans laquelle ces fibres flottaient; à linstant de eette addition, je vis les fibres qui étaient alors immobiles dans leur état d'ineurvation se déployer rapidement et demeurer 


\section{ET NUTHLT: DIS ANIMUX.}

$10 ั$.

smsuite intuobiles dans cet état de redressentent. J'apontai alors une goutce d'acide à l'ean; à l'instant les ifibres s’incurvèrent de nouvenu. J'ai répété un grand nombre de fois ces expériences, qui m'ont constamment donné les ınêmes résultats. Ainsi, les acides déterminent l'incurvation des fibres nutsculaires, ct Hes alkalis déterminent leur redressement ou la cessation de l'incurvation. Quelquefois les fibres exécuthent spontanéntent, et sous la seule influence de la ivie qui les anime, ces mouvements alternatil's d'inicurvation et de redressement. J'ai observé ces phénomènes, non seulement dans les fibres musculaires de lit grenouille, mais aussi dans celles de plusieurs iusectes. Ainsi je ne doute point que l'incurvation de La fibre musculaire ne coopère pendant la vic au raccourcissement des nuscles, et qu'elle ne soit ainsi ll'auxiliaire de la contraction de cette même fibre.

Nous allons actucllement nous livrer à l'étude de ce idernier mouvement que nous allons observer d'athord dans le tissu du cour de la gremouille. Cet or:gane, comme nous venons de le voir, est composé de ilibrilles et de corpuscules musculaires. Quel est te rôle que jouent ces deux sortes d'oryanes dans le phénomène de la contraction On regardera sams doute comme fort probable que les fibrilles se contractem conme les fibres, e'est-i-dire qu'elles rentrent en elles-nêmes en acquérant de lá grosseur aux dépens ' de leur longueur. Mais cette contraction des fibrilles, quoique probable, n'est point prouvée; elles sont si petites yu'on ne peut point, apereceroir leurs plis trans. 
versaux, si elles en possèdènt, comme on aperçoit ceux de la fibre musculaire. Ce qu'il y a de certain, c'est qu'on ne les voit point se raccourcir dans le sens de leur'longueur sous l'influence des acides; on les voil seulement s'incurver'; je m'en suis assuré par l'expérience suivante : ayant mis des fragnments laciniés du cour de grrenonille dans un petit cristal de montre rempli d'eau, je les ai soumis au microscope: quelques fibrilles flottantes dans l'eau débordaient le pourtour de ces fragments laciniés, comme on le voit en $u$ (figure 27). Je pris alors une petite goutte d'acide avec la pointe d'un cure-dent, et je la mis légèrement dans l'eau du cristal de montre; je mis à l'instant l'œil au microscope, et bientôt après je vis très distinctement les fibrilles flottantes se courber rapidement eri demi-cercle; les fibrilles de lintéricur du fragment musculaire s'incurvèrent de la même manière, et il en résulta un racourcissement accompagné de gonflement dans la petite portion de tissu nıusculaire que j'observais. Je ne pus voir quel était, dans cette circonstance, lc jeu des corpuscules musculaires intercalés en grand nombre aux fibrilles : toujours résulte-t-il de cette observation que c'est en s'incurvaut que les fibrilles opèrent la contraction du tissu musculaire fibrillo-corpusculaire, c'est-à-dire le raccourcissement accompagné de gonflement qui constitue cette contraction. Le cour des mollusques grastéropodes étant entièrement composé de corpuscules musculaires, cet organe pouvaiı seul me dévoiler le mécanisme de la contraction dans le lissu unuscu- 


\section{R'T MOTILITÉ DES ANIMAUX.}

aire eorpusculaire; je m'enpressai donc de le soumettre à l'expérience. Je pris le eœur d'une limace llimax rufus), et l'ayant lacinié dans l'eau en pareelles fort petites avec la pointe d'une aiguille, je blaçai quelques unes de ees parcelles dans un petir uristal de montre rempli d'eau, et je les soumis au microscope : ayant ajouté une goutte d'aeide nitrique I l'eau, je vis bientôt les fragments de eœur que j'ohwervais se contracter; mais il ne me fut point d'abord sossible de distinguer le méeanisme de cette conraction; je voyais seulement ce tissu se resserrer sur ıui-même, et par 'à devenir plus épais. Je découvrais Hans ce tissu, entièrement composé de corpuseules globuleux d'une extrême petitesse, des lignes parallèles, comme on le voit dans la figure 28 . Je jugeai que ces lignes parallèles obscures n'étaient point des ibrilles comme on aurait pu le croire, mais que c'écaient des plis formés par la membrane qui résultait He l'agglomération des eorpuseules musculaires; en effet, lors de la contraetion, je voyais ces lignes conserver leur longueur prenière, et le monvement de contraction resserrer le tissu que j'observais dans le sens $b c$. Ce soupçon fut confirmé par l'expérienee suivante : ayant soumis au microscope un autre firagment de eœur de limace qui offrait la même disposiition, j'ajoutai une petite goutte de solution aqueuse He potasse caustique à l'eau du cristal de montre dans. Hequel était ce fragment. Bientôt je vis les lignnes parallèles disparaître au voisinage des bords du liasment; il s'opéra un véritable déplisscment, an noyery 
duquel le fragment musculaire membraniforne prit une étendue plus grrande que celle qu'il possédait auparavant, et cessa de présenter des lignes parallèles, comme on le voit en $a$ (figure 28 ). Ce fait me confirma ce que j'avais précédemment observé, touchant la propriété qu'ont les alkalis de déployer les organes nıusculaires ineurvés; car le plissement observé dans cette circonstance est une véritable incurvation dont les courbures sont dirigées dans des sens alternativenıent inverses. Les choses étant dans eet état, j’ajoutai une goutte d'acide nitrique à l'eau du eristal ; un instant après, je vis la membrane déplissée $a$ se resserrer sur elle-même, et se plisser de nouveau de la même manière qu'elle l'était auparavant, offrant par conséquent des lignes parallèles obscures qui n'étaient autre chose que des plis. Je recommencai cette expérienee sur un autre fragment de cœur de limace, en employant pour déplisser son tissu une goutte d'ammoniaque ajoutée à l'eau dans lacjuelle flottait ce li'agment; jobtins le même effet qu'avec la solution de potasse caustique: l'incurvation sinueuse de ce tissu fut détruite par cet alkali, et je la rétablis ensuite au moyen d'une petite goutte d'aeide sulfurique ajoutée de même à l'eau. Il me fut prouvé de eette manière que, dans le tissu musculaire eorpusculaire, la eontraction consiste dans une incurvation sinueuse de ce tissu, lequel lorme de cette nianière des plis extrìmement fins.

Ces expériences achevèrent en outre de ne piouver que les alkalis ont la propriété de faire cesser 
l'incurvation du tissu musculaire, comme les acides out la propriété de provoqner cette incurvation.

Pour opérer d'une nınière heureuse le déplissement du tissu musculaire du cœur de la limace, il înut que la dose de solution alkaline ne soit point trop Corte, car elle opérerait la dissolution complète de ce xissu, quui disparâtrait tout-à-fait; mais il est une dose H'alkali qui, trop forte pour opérer le simple dépliswement, est trop faible pour opérer la dissolution comjlète et la disparution des corpuscules musculaires. H1lors, selon la dose de l'alkali, il y a, en sus du déplissement, tantôt écartement léger des corpuscules musculaires, tantôt dissociation complète de ces corpunscules; mais, je le répète, sans dissolution. CepenUant cette dernière ne tarderait pas à s'opérer, dans cette circonstance, si l'on tardait un peu à faire l'expérience qui va suivre. Lorsque l'alkali, après avoir péré le déplissement du tissu musculaire, eut en putre un peu écarté les uns des autres les corpuscules qui constituaient ce tissu, j'ajoutai une goutte d'atide nitrique à l'eau; un instant après, je vis ce tissu nusculaire corpusculaire se resserrer sur lni-même, mais sans offrir aucun plissement; ce resserrement u cette contraction consistait ainsi dans un simple approchement des corpuscules muscnlaires, qui auJaravant étaient lâchement unis; mais non tissociés. Jans une autre expérience du même genre, j'augmentai un pen la dose de l'alkali; alor's il y eut dissosiation des corpuscules musculaires, lesquels, quitant leur adhésion mutuclte, sc répandirent comme 
In fluide sur le fond du cristal, toutefois en conservant leur forme globuleuse; je me hâtai d'ajouter une goutte d'acide nitrique à l'eau, et dans l'instant je vis cette couche fluide, composée de corpuscules dissociés, se coaguler; les corpuscules se précipitèrent les uns sur les autres et s'agglomérèrent de la même manière que ccla a lieu dans la coagulation du sang, où l'on voit aussi des corpuscules globuleux dissociés se réunir et s'agglomérer.

Ces observations sont fécondes en résultats : clles prouvent, $1^{0}$ que le resserrement du tissu musculaire qui constitue la contraction dépend d'une doublc cause, c'est-à-dire d'un rapprochement corpusculaire et du plissement ou de l'incurvation sinueuse de ce tissu ; $2^{0}$ que la contraction et la coagulation som des degrés différents d'un seul et mênı phénonène: $3^{\circ}$ que les a!kalis on la propriété de faire cesser la contraction musculaire; on sait depuis long-temps que les acides ont la propriété de provoquer cette même contraction; et il est à remarquer que les acides produisent également la contraction des solides et la coagulation des fluides, et que les alkalis, au contraire, détruisent ce double effet. Nous allons étudier successivement ces résultats généraux, qui vont nous conduire à quelques autres résultats secondaires.

L'observation nous a démontré que la contraction des fibrilles et celle du tissu musculaire corpusculaire consistent dans un plissement ou dans une incurvation sinueusc; or, comme les fibres musculaires proprement dites sont composées de fibrilles et de 
rorpusenles musculaires, il en résulte que lenr eonraction résulte du plissement extrênement fin ou de ineurvation sinueuse du tissu qui les eompose intéiieurentent. Nous avons vu que le cour des nollustues gastéropodes ne contenait point de fibres, mais ru'il était entièrement composé de eorpuscules nusstulaires aggglomérés de manière à former nn tissu membraneux; ainsi il demeure prouvé que la conraetion ne s'opère pas exelısivement avee des organes néaires appelés fibres, mais qu'elle s'opère aussi wee des organes membraneux formés par la réunion "une grande quantité de eorpruseules musculaires ggolomérés. Le gonflement que présente le tissu musulaire eontraeté provient de son incurvation sinueuse, qui produit le plissement extrêmement fin des parties ntimes de ce tissu. On eonęoit en effet qu'un fil ou Iu'une membrane qui possèdent des plis qui les raecourcissent, doivemt, par cela même, offrir, dans leur nasse ainsi plissée; nne augmentation dans le dianètre transversai de cette masse. Les plis transversaux que l'on observe à la surface des fibres musculaires contractées sont le résultat de l'ineurvation sinueuse hes fibrilles superfieielles de la fibre; les fibrilles intérieures de eette même fibre possèdent indubitablement a même ineurvation sinueuse, à plis extrêmemen $t$ fins, aquelle opère leur raecoureissement, et pareonséquent celui de la fibre qu'elles forment par leur assemblage. Il résulte des observations qui ont été préeérlemment exposées, qu’il y a leux sortes de contraecions; l'une qui résulte du rapprochernent des cor- 
puscules musculaires, l'autre qui résulte de l'incurvation du tissu que ces corpuscules forment par leur agglomération. La premièrc de ces deux contractions est, par sa naturc mêmc, extrĉmement bornée; elle ne pourrait pas produirc le raccourcissement considćrable que l'on observe dans les organes musculaires, raccourcissement qui réduit quelquefois le muscle au cinquième de la longueur qu'il offre dans son etat de relâchement, ainsi que je l'ai vu dans les muscles qui servcut à opérer la rentréc de l'œil pédonculé des escargots. Il fallait, pour opérer un raccourcissement aussi considérable, un autrc mécanisme que cclui qui résulte du rapprochemcnt sịnple et uniforme dcs corpuscules musculaires; c'est cc que la nature a fait en employant l'incurvation sinueuse, incurvation qui est produite par un rapprochement inégal des corpuscules dans les diffćrcntes parties du tissu. Ce rapprochement existc spćcialcment et pcut-être cxclusivement au côté concave; en effet, le scul fait de la courburc prouve qu'il y a rapprochement des parties constituantes du tissu, spćcialement dansl'endroit où existe la concavité dc cette courbure. Ainsi, l'incurvation dépend de ce que la cause qui produit l'attraction corpusculaire, ou le resserremcnt, n'agit que sur un seul côté du tissu organique ; n'y aurait-il point là une sorte de polarisation transversale, cn vertu de laquelle les denx côtés opposćs dc la partie incurvće scraient modifiés en sens inverse l'un de l'autre? Mais ccci est une purc hypothc̀se, ct jc ne m'y arrête pas. Quoi qu'il en soit, le résultat positif quel'on 
ipeut tirer des observations qui viennent d'être exposées, est qu'il existe dans le tissu organique une foree ide resserrement out de rapproehement corpusculaire, Iforee qui peut être mise en jeu par divers agents. C'est Il'enploi de eette foree suivant un mécanisme parItieulier qui produit l'ineurvation du tissu organique, iincurvation qui produit à son tour des mouvements ¿d'une étendue à laquelle ne pourrait point arriver le mapproehement corpuseulaire tont seul. Ainsi ce que Il'on appeile la contraetion n'est point un phénomène :simple; c'est un phénomène eomplexe, eomposé du rapprochement corpusculaire et de l'incurvation, qui Irésulte elle-même de l'emploi de ee rapproehement corpusculaire, suivant un méeanisme partieulier. (Cette ineurvation sinuense est, ehez les animaux fproprement dits, un phénomène eaehé dans l'inténieur des organes et soustrait la plupart du temps à ınos yeux armés des meilleurs mieroseopes; mais, chez quelques zoophytes, ce phénomène devient tout-à-fait extérieur, et peut être aperçu presque ssans aucune diffieulté. Ainsi, chez les vorticelles ou ipolypes à houquets (vorticella convallaria), on iobserve des eontractions extrêmement rapides qui sse répètent à ehaque instant; ee sont les ramıeaux qui Iportent les polypes qui se contractent ainsi, et qui : se relâehent alternativement. On ignore le but de ee imouvement spasmodique continuel; il est fort eurieux à observer au mieroseope: on voil ces rameaux, dont lla ténuité est très considérable, prendre avee rapidité une ineurvation sinuense, comme on le voit en 
$a$ (figure 29); cette incurvation cesse un instant après, et lc ramcan relâché reprend sa rectitude, conıme on le voit en $b$; puis il recommence à se contracter, $\mathrm{ct}$ ainsi de suite. Ces polypes nous offrent ainsi à déeouvert, et en dchors, le mécanisme de la contraction, qu'il faut chercher dans l'intćricur des organes des autres animaux. Les bras des hydres offrent de mêne une incurvatiou sinueuse, dont les courbures offrent des directions très variées; c'est par le moyen dc ces courbures multipliécs que ces bras, en se pelotonnant, pour ainsi dire, portent ver's la bouche de l'animal la proic qu'ils ont saisie. Dans cctte incurvation sinueuse, lcs bras de l'hydre ne deviennent point plus gros qu'ils ne l'ćtaient auparavant. Cette incurvation, dans laquelle consiste essentiellement le mouvement musculaire, est évidemment un phénomène tout-à-fait semblable à celui de l'incurvation qu'offrent diverses parties des vćgétanx; les recherchcs qui ont ćté exposées plus haut nous ont appris, en effet, que le mécanisme du mouvement cxécuté par les bourrelets de la sensitive consistc dans une incurvation élastique, dont la puissancc nerveuse mise en jcu par les agents du dehors est causc occasionelle, et à laquelle succède, après un certain temps, un redressemcnt qui n'est autre chose que la cessation de cctte incurvation; il en est de même de toutes les parties des végétaux qui exécutcnt des mouvements visibles; car nous avons vu plus hant que tous ces mouvements, saus exception, c'est-à-dire non senlement ceux des végrétaux yue l'on appelle 
irritables par excellence, mais aussi les mouvements par lesquels les végétaux prennent des positions de sonmeil ou de réveil, ceux par lesquels les vrilles s'attachent ì leurs appuis, ctc., sont tous les résultats d'une incurvation. Chez les végétaux, ce phénomène se montre au dehors et dans toute sa simplicité ; chez enx l'ineurvation est le plus souvent simple, c'est-ì-dire ì courbure unique; tandis que généralement, chez les animaux, ce mêne phénomène est, Ipour ainsi dire, masqué; son mécanisme est caché dans l'intéricur des organes, et de plus, chez eux, l'incurvation est presque tonjours sinueusc; car je n'ai observé l'incurvation simple, ou à courbure unique, que dans la fihre musculaire considérée dans son enticr. Nous avons vil, en effet, que cette fibre jouit à la fois de la faculté de se contracter et de celle de s'incurver en demi-ccrcle; il résulte de cc rapprochement de faits que l'irritabilité animale et l'ir', ritabilité végétale sont deux phénomènes essenticlHement identiques; elles dépendent l'unc comme ll'autre de l'incurvabilité du tissn organique, on de Ila faculté vitale que possède ce tissn de se courber ret de se maintenir dans cet état de courburc d'une Imanièrc élastiqne. Les notions que nous venons d'acquérir sur la cause de cette incurvation chez les aniimaux nous mettent à même de rechercher la canse ide l'incurvation végétale; recherche que nous avons tété contraints d'abandonner plus haut, faute de points de comparaison. Nons venons de voir que le lissu musculaire est essentiellenent conuposé de corpus- 
cules vésiculaires agroglomérés, tantôt de manière ì lormer des organes linéaires, tantôl d'une manièrc confuse, et que ces corpuscules ont cela de particulier, qu'ils sont solubles dans les acides: ce qui les distingue essentiellement des corpuscules nerveux, qui sont insolubles dans ces agents ehimiques. Or, dans l'examen que nous avons fait plus haut du tissu organique des bourrelets de la sensitive (figr. 16), nous avons vu que ce tissu offre une grande quantité de eellules globuleuses alignées, et renıplies d'un fluide eoncrescible par l'acide nitrique froid, et soluble dans le mème aeidechaud; ces cellules globuleuses sont done de véritables corpuscules musculaires, plus gros que eeux des muscles des animaux, mais essentiellement semblables à ees derniers par leur alignement, et surtout par leurs propriétés chimiques; en cffet, les eorpuscules museulaires des animaux sont rendus opaques par les acides avant d'être dissous par eux, comme eela a lieu pour les cellules globuleuses des bourrelets de la sensitive. Or, eomme le phénomène de l'incurvation est essentiellement le inême chez les végétaux et chez les animaux, il en résulte que eette ineurvation dérive également chez les uns et ehez les autres d'un rapprochement corpusculaire qui n'a lieu que d'un seul cóté. Les corpuscules musculaires, ou les eellules globuleuses des bourrelets de la sensitive, ne sont point en effet en contaet immédiat, ainsi que nous l'avons vu; ils peuvent par eonséquent éprouver un rapprochement suffisant pour produire l'incurvation que l'on observe dans le tissu de ces bourrelets, et 
s'eloigner de nouveau lors du redressement de ces organes; il résulte de là que, sans posséder de véritables muscles, la sensitive possède réellement le tissı musculaire élémentaire, c'est-à-dire des corpusicules mnscnlaires organes de. l'incurvation; c'est ainsi que, sans posséder de véritables nerf's, cette même plante possède les éléments du système nervenx, ic'es-à-dire des corpuscules nerveux, qui du reste se rencontrent également chez tous les autres végétaux.

L'incurvation vitale, celle qui a lieu sous l'inflnence de la puissance nervéuse, est ordinairement un phénomène de peu de durée; la parti: incurvée reitourne plus ou moins promptement à l'état de redressement, qui, chez les animaux, constitue l'état de relâchement; les alternatives d'incurvation et de redressement ont lieu à des intervalles de temps assez i considérables chez les végétaux. Une feuille de sensitive, qui s'est ployée subitement par l'efiet d'une :secousse légère, se redresse lentemient au bout de quelques minutes: cette incurvation, toujours suivie du redressemient, se renouvelle autant de fois ' qu'on la sollicite. Ces alternatives d'incurvation et de iredressement ont lieu sous l'influence d'une cause déIterminanie intérieure chez l'hedysarum girans, dont. lles feuilles sont animées d'un mouvement oscillatoire perpétuel. Ces oscillations dépendent égralement d'une cause déterminante intérieure, et sont bien plus | fréquentes chez les oscillaires, êtres qui sont sitnés tont-í-lait sur la limite qui sépare le règne végétal dur règne animal, et dont les filaments offreut perpé- 
tuellement des phénomènes alternatif's d'incurvation et de redressement. Si des végétaux nous passons aux polypes, nous voyons, chezles vorticelles ( fiw 29) des alternatives, très firéquemment répétées, d'incurvation sinueuse et de redressement, sous l'influence de la volonté. Les muscles des animaux, proprement dits, nous offrent un phénomène toutà-fait semblable. Tout le monde sait que la contraction de ces organes, sous l'influence de la puissance nerveuse, est un phénomène de peu de durée, et qu'il est nécessaire qu'ils se relâchent lorsque leur contraction dure depuis un certain temps, après quoi ils sont susceptibles de se contracter de nouveau. D'ailleurs cette contraction, qui nous paraît fixe et permanente pendant certain temps, ne l'est point réellement: on sait que la contraction des muscles, sous l'empire de la volonté, n'est point un état d'immobilité, mais qu'elle est le résulıat d'une multitude d'oscillations ou de contractions partielles suivies de relâchement qui se succèdent à des intervalles de temps très courts; aussi, nos membres ne peuventils affecter une position qui exige une action musculaire soutenue, sans offrir un léger tremblement, qui est presque imperceptible chez les individus vigoureux, qui devient très sensible chez les personnes faibles, et notamment chez les vieillards. C'est ce tremblement, c'est ce frémissement oscillatoire des organes musculaires que l'on entend en se bouchant l'oreille avec la main; cette palpitation des organes musculaires est lacile a voir sur des muscles de gre- 
nouille mis à nu, et que l'on sampondre légèrement de sel commun finement pulvérisé; on voit de cette manicre que la contraction des muscles soumis à la volonté n'a que la durée de l'éclair, comme elle en a la rapidité. Si donc nos muscles se contractent d'une manière qui nous paraît fixe, cela provient de la petitesse de leurs oscillations ou de leurs alternatives de contraction et de relâchement. Ces oscillations sont beauconp plus lentes dans les fibres musculaires des mollusques, des annélides et même des inseetes, ainsi que je m'en suis assuré par diverses observations.

Il n'entre point dans le plan que je me suis tracé, d'offrir ici un traité complet snr la contraction considérée chez les animaux. Je me bornerai donc à présenter iei quelques considérations générales sur cet objet. Plusieurs des tissus de l'économie animale ont la propriété de se contracter; mais ee n'est que dans les organes muscurlaires que celte propriété existc à un degré éminent; c'est ce qui lait qu'elle peut être mise en jeu chez enx par des causes tont-à-fait insuffisantes pour en déterminer l'exerciced'une manière sensible dans les autres tissns. Airsi, la puissince nerveuse el l'électricilé galvanique provoquent vivernent la contraction des muscles, et n'ont point d'influence apercevable sur la contraction des autres parties; ces phénomènes ont fait penser qu'il existait plusieurs sortes de contractilité. Ainsi Bichat, reconnaît, outre une contractilité animale et unc contractilité organique sensible, une contractilité 
organique insensible et une contractilité de tissu qui est indépendante de la vie; il porte enfin ses regards sur le phénomène du racornissement, sur ce phénomène de mouvement que présentent plusieurs tissus animaux lorsqu'ils sont soumis à l'action du feu; et, par un rapprochement extrêmement philosophique, il considère tous ces phénomènes de mouvement comme dépendants également de la texture et de l'arrangement des molécules des tissus organiques, qui ont ainsi en enx la faculté de se raccourcir, faculté qui peut être mise en jeu par des causes très diverses, soit pendant la vie, soit après la mort '. Ce dernier aperçu d'un 'observateur profond, dont la plupart des idées sont marquées au coin du génie, est de la plus grande vérité; ainsi, en nous reportant aux causes et au mécanisme de la contraction, nous voyons, $x^{0}$ sous l'influence de la puissance nerveuse émanée des centres nerveux ou déterminée par des agrents extérieurs, les fibres musculaires éprouver une contraction qui alterne plus ou moins rapidement avec leur relâchement; c'est l'incurvation sinueuse oscillatoire et à mouvement très étendu; elle est propre aux seuls muscles. Cette incurvation rapide et de peu dée durée peut être sollicitée dans les muscles de l'aninual fraîchement tué par l'électricité galvanique, laquelle ressuscite la puissance nerveuse, ou peut-être la remplace. $2^{\circ}$ Sous l'influence de certaines causes intérieures ou extérieures, plusieurs tissus de l'économie

1 Ánatomié géndrale, considérations générales, $\$ 5$. 
animale épronvent une contraction firible, qui atterne, mais d'une manière lente, avee un élat de rolâchement: celte contraction est l'effet de ce que Bichat nomme la contractilité organique insensible. Quniqu'on n'ait point observé directement le méeanisme de cette contraction, on ne peut guère douter qu'elle ne consiste dans une incurvation sinueuse; cette incurvation, à ınouvenıcnt peu étendu, est lentement oscillatoire. $3^{\circ}$ Lors de la cessation de la vie, les fibres musculaires se contractent avec assez de force; c'est leur contraction qui produil, dans celte circonstance, la roideur des nembres, ainsi que l'a démontré Nysteu ${ }^{2}$ Cét auteur, considérant que cette contraction cesse spontanément quelques jours après la mort, lorsque la putréfaction commence à se manifester, a pensí qu'elle était le résultat d'un reste de vie organique quii ne s'éteignail que plusieurs jours après la mort. Eı cela, je pense qu'il est tombé dans l'erreur: la contraction des muscles après la mort est un phénomène du même genre que la coagulaion du sang, qui arrive, en même temps; ces deux phénomènes attestent également l'absence de la vie. Si les muscles cessent d'être contractés lorsque la putréfaction commence, cela provient évidemnent du dégagement, dans ces organes, de l'ammoniaque, qui, cn sá qualité d'alkali, hait cesser l'ineurvation du tissu musculaire; celte incurvation est sinuense, fixe, c'est-à-dire qu'elle n’alterne point spontanément avec un état de 
redressement ou de relâchement. La contraction des muscles occasionée par l'absence de la cause immédiate de la vie est un fait qui mérite toute l'attention des physiologistes; car il tend à prouver que la contraction de ces or ganes a lieu dans toutes les circonstances, comine dans celle-ci, par la soustraction d'un principe ou d'un élément inconnu, qui abonde au contraire dans le muscle relâché. $4^{\circ}$ Sous l'influence de l'cxtension mécanique, la fibre aninale, complètement morte, reprend, lorsqu'clle est abandonnéc à cllc-même, son état antécédent de raccourcissement: c'est l'effet de ce que Bichat appelle la contractilité de tissu. Cet effet résulte de l'élasticité des fibrilles, qui tendent à persister dans l'état d'incurvation qu'elles ont pris; elles agissent alors comne de véritables r'essor'ts : c'est encore une incurvation sinucuse fixc. $5^{\circ}$ Sous l'influence de l'action du feu, le tissu fibreux animal, complètement mort et mêrme desséché, s'agite presque conme le ferait un animal vivant: c'est un résultat des incurvations partielles et multipliées qui sont produites dans ce tissu, soil par le développement de gaz, soit par la dilatation ou par l'évaporation des fluides. Ainsi, partoul où nous observons des mouvements dans les tissus organiques, soil pendant la vie, soit après la mort, nous les voyons dépendre également d'incurvations élastiques, dout les causes occasionclles sent différentes, mais qui dépendent toutes de la texture organique de ces tissus, tous essenticllement cómposés de corpuscules ou de cellules vésiculaires agglomérées: tclle est, en effet, la 
composition intime de tous les organes des animaux, sans aucune exception. Leuwenhuek avail déjà annoncć une partie de celte vérité, qui a élé confirmée par les recherches récentes de M. Milnc Edwar:ls ', qui a examiné avec beaucoup de soin la structure microscopique des principaux tissus organiques des animaux: il n'a vu partout que des globules aggolomérés. J'ại vérifié l'exactitude de ces observations : partout, en effet, on ne trouve, dans les oryanes des animaix, que des corpuscules globuleux, tantôt rćunis en sćries longitudinales et linéaires, tantôt aggglomérés d'une manière confuse. C'cst sous ce dernier aspect que ces corpuscules globuleux sc présentent dans tous les oryanes sécréteurs, tels que le loie, les reins, les glandes salivaires, les testicules, etc.; la rate et les ovaires ne présentent pas une autre structure intime. Cettc similitude fondamentale du tissu de tous les organcs parenchymateux est telle, chez la grenouille, qu'il est presque impossible de distinguer les uns des autres, au microscope, les tissus du cerveau, du foie, des reins, de la rate, etc. : partout on n'aperçoit que des corpuscules globuleux aggrlomérés d'une manière confuse, et constituant ainsi le parenchyme de l'organe par leur assemblage. Chez les animaux vertébrés , les corpuscules globuleux sont tellentent petits qu'il est impossible de savoir si ce sunt des corps solides ou des corps vésiculaires; mais cela s'aperçoit

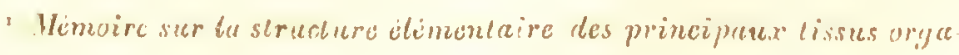
niquess. 
avee beiueoup de facilité che\% les mollusques. En effet, en examinant an mieroscope le tissu du foie, des testienles ou des grlandes salivaires des hélix et des limaees, on voit que ces organes sécréteurs sont eomposés, comme ceux des animaux vertébrés, de pelits eorps globuleux agclomérés d'une manière confuse; mais ici ees petits corps gylobuleux ne sont point d'une exeessive petitesse, ils sont même assez gros, si toutefois on peut se servir de cette expression en parlant d'objets microscopiques, et l'on voit de la manière la plus évidente que ce sont des eorps vésieulaires ou de véritables cellules, dans les parois desquelles on aperçoil même d'autres eorpuseules excessivement petits. On pourrait peut-être douter que ces cellules globuleuses soient les analogues des eorpuscules globuleux que l'on observe dans les organes sécréteurs des animaux vertébrés, mais l'exanıen le plus superficiel dissipera tous les doutes à cet égrard, en faisant voir que les eellules globuleuses des organes sécréteurs des inollusques, et les eorpuscules globuleux des organes sécréteurs des aniniaux vertébrés, eomposent de même immédiatement le parenchyme de ces organes; leurs nuasses entourem de même les vaisseaux sanguins et les canaux excréteurs Cette observation prouve que les corpuscules globulenx dont l'assemblage eompose les organes parenchymateux des animaux vertébrés sont des cellules d'une cxeessive petitesse, et dans les parois desquelles on distinguerait des corpuscules plus pelits, si le microscope pouvait faire pénétrer notre vue dans ces pro- 
fondeurs de l'infiniment petit. Nous avons déjà vu plus hau que, chez les molhusques gastéropodes, lit masse du cervenu est composée de vésicules globuleuses, contenant la substance nerveuse; nous avons également fait remarquer que cette observation confirmait l'opinion de MM. Wenzel, qui considèrent sous lc mênıe point de vue les eorpuscules globuleux du cerveau des animaux vertćbrés. On peut tirer de là celte conchusion générale, que les eorpuscules gुlobuleux qui composent par leur assemblage tous les tissus organiques des animaux sont véritablement des cellules globuleuses d'une excessivc pctitesse, lesquelles paraissent n'être réunies que par une simple force d'adhésion; ainsi, tous les tissus, tous les organes des animaux, ne sont véritablcment qu'un tissu eellulaire diversement modifié. Cette uniformité de structure intime prouve quc les organes ne diffèrent véitiablement entre eux que par la nature des substances que eontiennent les ccllules vésieulaires dont ils sont entièrentent eomposés : c'est dans ces cellules que s'opère la sécrétion du fluide propre à chaquc organe, fluide qui est probablement transmis par transsudation dans les canaux sécréteurs. Dans le cerveau, ces eellules agglomérécs opèrent la sécrétion de la substance nervcuse proprement dite, substance qui reste stationnaire dans lc tissu cellulaire qui l'a sécrćtée. Ainsi la cellule esı l'organe sćcréteur par exccllerice : elle sécrète, dans son intérieur, une substance qui tantôt est destinée à être portće aı dehors par le moy en des camaux excréteurs, et qui tantôt est 
destinée à rester dans l'intérieur de la cellule qui l'i sécrétée, et à faire aussi partie de l'éeonomie vivante, où elle joue un rôle qui lui est propre : telle est spéeialement la substance nerveuse proprentent dite qui remplit les cellules microscopiques du cerveau et des nerfs; substanee qui, dans le eorps vivant, jouit de propriétés si étonnantes et si inconnues dans leur nature. On ne peut guère douter que les organes parenchymateux, tels que la rate; qui n'ont point de eanal exeréteur, n'opèrent égralenent dans leurs cellules la sécrétion d'une substance qui est destinée, soit à y demeurer stationnaire, soit ì passer par transsudation dans les vaisseaux sanguins. Il faut bien que la cellule ait des qualités particulières dans chayue organe, puisqu'elle y séerète des substanees aussi dilférentes; et, à eet égard, on ne pent. s'empêcher d'admirer la prodigieuse diversité des produits de l'organisation, diversité qui est bien plus grande encore dans le règne végétal qu'elle ne l'est dans le règne animal. Quelle variété dans les qualités physiques et chimiques des substances séerétées par !es' cellules qui composent le parencliyme des firuits ou eelui des liges, des racines, des feuilles et des fleurs, dans tous les végćtaux répandus sur la surface du gylobe! On ne peut coneevoir qu'une si étonnante diversité de procluits soit l'ouvrage d'un seul organe, de la cellule. Cet organe étonnant, par la eomparaison que l'on peut faire de son extrêne simplicité avec l'extrême diversité de sa nature intime, est véritablement la pièee fondamentale de l'organisation; tout, en effel, 
dérive évidemment de la cellule cians le tissu organique des végétaux, et l'observation vient de nous prouver qu'il en esı de même chez les animaux.

Nous sommes arrivés plus laut, par le moyen de l'observation, à ce résultat, que la coagulation des liquides est un phénomène analogue à celui de la contraction des solides: ce fait est d'une grande importance en physiologie, car il prouve que certaines propriétés organiques appartiennent également aux solides et aux fluides; ces derniers, en effet, ne sont point semblables aux liquides inorganiques. Les fluides du corps vivant sont or'ganisés, e'est-à-dire que leur composition intime est tout-à-fait semblable à celle des solides; ils sont, conıme eux, entièrement eonıposés de corpuscules globuleux; mais, dans les solides, ees corpuseules sont adhérents les uns aux autres, tandis que, dans les fluides, ils sont libres et dissoeiés. Tout le monde connaît les globules dont le sang est composé; ees globules ont été observés avec soin par plusieurs naturalistes célèbres dont je vais exposer iei très sueeinctement les découvertes, en y ajoutant les résultats de mes propres recherches. Les globules sanguins ont été découverts, comme on le sait, par Leuwenhoek, et depuis ils ont été étudiés par un grand nombre d'observateurs, à la tête desquels on trouve Haller, Spallanzani et Hewson. Dans ees derniers temps, ils ont été de nouveau étudiés par sir Éverard Home, par M. Bauer, el tont récemment par MM. Prévost et Dumas. Le nom de globules, par lequel les premiers observa- 
teurs ont désigné ces corpuscules flottants dans le sang, prouve qu'ils les considéraient cuinme de petites sphères; certains observateurs, voyant que leur milieu était transparent, ınndis que leurs bords étaient opaques, crurent pouvoir en conclure que ces petits sphéroödes étaienı percés d'un trou dans leur milieu; mais cette assertion mérite peu d'attention, car il est de la plus grande évidence que cette apparence ne provient que de ce que ces globules transparents réfractent la lumière de manière à la rassembler dans -un foycr central, en sorte que leurs bords paraissent opaques et lcur milieu diaphane. Nous avons déjà fait cette observation plus haut, relativement aux corpuscules globuleux et transparents que M. Mirbel a pris pour des pores dans le tissu des végétanx. Jusqu'à Hewson, on s'accordait généralement à considérer les globules sanguins comme des sphéroïdes ou des cllipsoïdes; cet observateur prétendit que ielle n'était point leur forme, mais qu'ils avaient cellc d'un disque renflé dans son milieu '. M. Baner, reprenant ces observations, crut devoir leur restituer la forme sphérö̈de qui leur avait été atrribuée par la plupart des observateurs ${ }^{2}$. En dernier lieu, MM. Prévost et Dumas, revenant à l'opinion de Hcwson, ont considéré ces corpuscules comme ayant la fornne discoïde $^{3}$. Ce que l'on peut soupçonner, au milien de cette divergence d'opinions, c'cst quc les observateurs

' Transuclions philo:ophijucs, tome 63.

- Idcm, isis.

Examen du suriy, ctc. 
، yui les ont émiscs ont eu tous ćgalement raison. Si, , en effet, tous les corpuscules sanguins étaient discoïdes, comment cctte formc aurait-ellc échappé à tant d'excellcnts obscrvateurs? 'D'un autrc côlé, il , est inconiestable que telle est quclquefois lenr torme, ainsi quc jc l'ai observé moi-mênc; mais, il faut cn convcnir, cette forme se présente assez rarement, $\mathrm{ct}$, dans le plus grand nombre des cas, on pent même dire presque toujours, les crrpuscules sanguins se préscntent sous la forme sphériquc ou clliptique. Peut-être dira-t-on qu'ils ne sc préscntcnt sous cette forme quc parceque leur disque offre alors l'une de ses faces à l'œil de l'observateur; mais alors il faudrait adnettre qu'il y aurait un nombre immense de chances pour qu'ils affectassent cette position, tandis que le nombre des chances pour qu'ils sc' présentassent de champ seraicnt assez rares. On ponrrait penser que la direction de la pesanteur influcrait sur la position à plat de ces corpuscules prétendus discoïdes, et comme on observe ordinairement au inicroscope avcc un rayon visucl vertical, il en résultcrait, en efict, que ces corpuscules présenteraient le plus souvent une de leurs faces à l'œil de l'observateur. Quoiquc ccttc position à plat soit peu probable pendant la circulation, cependant j'ai voulu n'assurer si unc position différcute de l'animal changerait l'aspect sous lequel sc présentent ces corpuscules. J'ai donc dirigné lc rayon visuel de mon microscope dans le seus horizontal, ct dans cette pusition, j'ai observé la circulation daus la quene d'un têtard: Ic vaisscau que 
jobservais était dirigé dans le sens vertical. Si la pesanteur eût influé sur la position horizontale des corpuscules discoïdes, il en fî̀t résulté, dans mon observation, que ces disques se seraient tous présentés de champ. Or, j'ai continué à voir ces corpuscules ovö̈les; je n'ai même pu, dans cette observation, en apercevoir un seul qui fît discoïde. Tout concourt donc à prouver que cette dernière forme est rare, qu'elle est purement accidentelle, et que la forme normale des corpuscules sanguins est celle d'un sphéroïde ou d'un ellipsoïde; d'ailleurs, ce fait est prouvé par les changements de forme que les corpuscules sanguins sont susceptibles de prendre. Fontana et Spallanzani on t vu, le premier dans la grenouille, et le second dans la salamandre, les globules sanguins se former en un ellipsoïde très alongé quand ils étaient engagés dans un vaisseau d'un diamètre phus petit que le leur, se courber en forme de croissant dans les courbures anguleuses des vaisseaux, et reprendre enfin leur forme ordinaire quand ils étaient parvenus dans un vaisseau suflisanment large. Ces changements de forme ne peuvent dériver que d'un sphéroïde : on sent qu'un disque ne pourrait pas les présenter.

Une membrane d'une extrême délicatesse environne les corpuscules sangnins. Cette membrane vésiculaire est seule dépositaire de la matière rouge qui colore ces corpuscules; son extrême délicatesse lait qu'elle s'altèro très promptement après la mol't et que elle se détache du corpuscule, ainsi que l'ont observé MM. Bauer et 
Home; après cette séparation de l'cnveloppe colorée, Ile corpuseule sanguin parait hlane, et conserve sat I forme. On pourrait penser qu'il ne consiste plus alor's que dans un noyau de matière solide, mais la faculté ' qu'ont les corpuseules sanguins de changer de lorme, ainsi que nous venons de le voir, pronve que ce noyan , est composć d'une substance très molle ou mênie li'quide; par eonséquent, la eonservation de sa forme après la disparition de la membrane colorée qui l'enveloppait extérieurement semble prouver que le corIpuseule sanguin possède une seeonde membrane plus solide que la membrane eolorée ì laquelle elle est subjacente, et dans l'intéricur de laquelle est contenue lla matière molle ou liquide qui constitue le noyau du corpuseule: toujours résultc-t-il de l'existence de la imembrane vésiculaire colorée que les eorpuseules sanguins doivent être considérés comme des corps vésiculaires. L'existence de cette membrane, prouvéc I par les observations de Bauer et de Home, a été pleineıment confirméc par celles de MIM. Prévost et Dumas.

Un jeûne prolongé diminue considérablentent le inombre des corpuscules sanguins: je les ai vus disI paraître totalement chez un têtard de crapaud aceoulcheur que j'avais conservé une annće entière sans lui donner de nourriture. Leuwenhock avait anıuncé que les globules sanguins avaient un monvement de roItation sur cux-mêmes; mais les observations de $\mathrm{Hal}$ ler "et de Spallanzani" prouvèrent que ce mouve-

' Mémoire sui te mouventent du sang.

- Dc' fonomeni della circolluzione. 
ment n'existait point. Ces deux derniers observateurs ont tonjours vu les globules sanguins, plongés dans le fluide diaphane qui les environne, se tenir eonstamment éloignés les uns des autres : jamais, tant que ln vie subsiste, ees globules ne sont en eontaet inımédiat. Spallanzani a vu un grand nombre de fois que lorsque deux de ees globules se présentaient ensemble à l'orifiee d'un vaisseau qui ne pouvait admettre que l'un d'eux, l'autre, repoussé à l'instant, rétrogradait sans avoir touehé le globule qui le préeédait dans le passage; Haller ' a vu de mênıe que ees globnles se repoussaient réciproquement quand le mouvement progressif du sang tendait à les rapproeher. Ainsi, il a observé que l'un de ees globules étant plaeé lans une espèec de eul-de-sac, il repoussait, sans les avoir touchés, les globules qui venaient vers lui. Cet isolement constant des gylobules sanguins nu milieu du fluide séreux dans lequel ils nagent, et eette répulsion qu'ils exereent les uns sur les autres, quand une eause extérieure les rapproehe, ne eessent d'exister que lorsque la vie eommence à s'eteindre : e'est alors que Haller a vu ees globnles s'agglomérer en pérdunt leur forme sphérique; mais ils reprenaient eette forme en se séparant de nouveau, si la eireulation languissante venait à se ranimer. J'ai répété toutes ees observations de Haller et de Spallanzani, et je me suis assuré de leur exaetitude. J'ai beaueoup observé la eireulation du sang dans les parties transparentes des

- Douribme licmoire sur le mourement du sangr. 
jeunes salamandres et des jeunes têtards, et j'ai toujours vu les corpuscules sanguins être éloignés les uns des autres lant que la vie conserve une certaine énergie; mais aux approches de la z:oort, lorsque le sang avance dans les vaisseaux pendant la systole du cceur, el rétrograde dans ces mêmes vaisseaux pendant la diastole de cet organe; alors, dis-je, les corpuscules sanguins cessent de se tenir éloignés les uns des autres; ils s'agglomèrent et forment ainsi de petits caillots qui remplissent certaines parties des vaisseaux, tandis que les autres ne contiennent que du sérum. Il n'y a donc point de doute qu'il n'existe pendant la vic une force répulsive qui tient les corpuscules sanguins isolés les uns des autres, et qui disparaît lors de la mort; alors ces corpuscules, abandonnés à une force d'attraction qui les précipite les uns sur les autres, s'ağgolomèrent, et c'est leur réunion qui forme ce qu'on appelle le caillot. On sait, par des expériences positives, que ce n'est point le refroidissement qui est la canse de cette congulation, de laquelle il résulte tantôt des corps membraniformes ou couenneux, tantôt des corps fillilormes semblables à des fibres, ce qui a fail donner le nom de fibrine à la substance composante de ces corps, et cela avec d'autant plus de raison (que cette substance est tout-à-fait semblable à la substance musculaire, sous le rapport de ses proprićtés chimiques: aussi a-t-on appelé le sanğ de la chair coulante, eı cela n'est point une métaphore, c'est une vérité cxacte. La substance nusculaire, en effet, est essenticllement. 
composée de corpuscules globuleux comme le sang; mais, dans ce liquide, ces corpuscules flottent isolés, tandis que, dans lc muscle, ils sont aggromérćs, et forment ainsi un solide organique. Après la mort, le sang se coagule par lc rapprochement de ces corpuscules; dans le même temps, le tissu musculaire se contracte par le rapprochement et le resserrement de ses plis sinueux. Il y a, dans ces deux circonstances, égrale absencc d'une causc d'écartement corpusculaire ou de répulsion dans les parties intimes. Nous avons vu plus haut, par des cxpériences positives, la transition tout-à-fait insensible qui cxiste entre le phénomène de la contraction ct celui dc la coagulation; nous avons vu, en effet, que lc tissu musculairc corpusculaire, simplement déplissé par un faible alkali, était susccptible de se plisser de nouveau ou de s'incurver par l'accession d'un acidc, tandis quc ce même tissu, dont les corpuscules étaient dissociés par un alkali un peu plus fort, formait alors un liquide organique simplcment susceptiblc de se resserrer sur lui-même par le fait de sa coagulation; il n'est donc point douteux que les dcux phénomènes de l'incurvation et de la coagulation nc soient très voisins, $\mathrm{ct}$ ne tiennent au même principe; il rcstc à déterminer quel est le lien qui réunit ces deux phénomènes.

Les corpuscules sanguins, pendant la vie, ne sont jamais en contact immédiat; après la mort, ou lorsque le sang est tiré hors des vaisseaux, ccs corpuscules s'agglomèrent, et il en résulte une cspèce de solide organique: c'est le phénomènc de la coagulation; il 
dépend évidemment de l'attraction que les corpuscules sanguins exercent les uns sur les autres. J'ai voulu voir si cette espèce de solide organique étail susceptible de se contracter eomme le tissu musculaire. J'ai mis une groutte de sang de grenouille dans l'eau que contenait un cristal de moutre ; celte goulte de san: s'est coagulée en formant une membrane diaphane qui tapissait le fond du cristal; on pouvait enlever la membrane et l'agiter daurs l'eau sans que ces corpuscules quittassent leur adhérence mutuelle. Ayant ajouté à l'eau une goutte d'acide nitrique, je vis, au microscope, la membrane se resserrer sur elle-mêne par le sapprochement plus considérable des corpuscules dont elle était composée; ainsi le solide formé par la eoagulation du sang est susceptible de présenter seulement le mode primordial de la contraction, c'est-à-dire le resscrrement par rapproelıement général des corpuscules; il ne présente jamais le mode seeondaire de la contraction, c'est-à-dire l'incurvation sinueuse qui résulte du rapprochement eorpusculaire opéré d'un seul côté; ce mode secondaire de la contraction paraît dépendre essentiellement de la puissance nerveuse, laquelle est ćtrangère au solide formé par eoagulation.

Les propriétés vitales des liquides organiques sont encore peu eonnues: d'après ce que nous avons vu plus haut, il parait que la répulsion eorpusculaire, on plutôt que la faculté que possèdent les corpusculẹs des liquides de se tenir éloignnés les uns des autres, est la principale propriété vitale des fluides, puisque l"i- 
solement de ces corpuscules cesse généralement avec la vie. La contractilité est nécessairement étrangère anx fluides : elle ne peut appartenir qu'aux solides. P'our ce qui est de la nervimotilité, nous ignorons si elle appartient exclusivement à ces derniers. Nous avons vu que, chez les végétaux, la puissance nerveuse est transmise par l'intermédiaire d'un liquide organique, il n'est pas bicn certain qu'il n'en soit pas de mêmc chez les animaux, et même il paraît fort probable que la production de la puissance nerveuse est la propriété physique que possède pendant la vie le liquide contenu dans les cellules vésiculaires dont le cerveau est entièrement composé; cellules qui ne diffêrent peut-être pas, sous ce point dc vue, des cellules dont se composent les organes électriques de certains poissons.

Ce que nous venons de voir touchant la similitude de la composition organique des solides et des fluides du corps vivant pourrait faire penser que les globnles vésiculaires contenus dans le sang s'ajouteraient au tissu des organes et s'y fixeraient pour les accroitre et les réparer, en sorte que la nutrition consisterait dans une véritable intercalation des cellules toutes faites et d'une extrême petitesse. Cette opinion, tout étrange qu'elle puisse paraître, est cependant très londée, car l'observation parle en sa faveur. J'ai vu plusieurs fois les globules sanguins, sortis du torrent circulatoire, s'arrêter et se fixer dans le tissu or'ganique: j'ai été témoin de ce phénomène, que j'étais loin de soupconner, en observant le mouvement du sang an 
microscope dans la queue fort transparente des jeunes têtards du crapaud accoucheur. Des artères formant des courbures nombreuses se répandent dans la partie transparente de la queue de ces têtards; ces artères sont immédiatoment continues avec les veines, en sorte qu'il n'existe ici aucune distinction, aucune ligne de démarcation entre les deux circulations axtérielle et veineuse: le sang, dont on aperçoit parfaitement les globules, qui sont assez gros, offre un torrent dont le mouvement n'éprouve aucune inter'uption depuis son départ du cœur jusqun'à son retour à cet organe. Entre les courbures que forment les vaisseaux, il existe un tissu fort transparent dans lequel on distingue beaucoup de granulations de la grosseur des globules sanguins; or, en observant le mouvement du sang, j’ai vu plusieurs fois un globule scul s'échapper latéralement du vaisscau sanguin et se mouvoir dans le tissn transparent dont je viens de parler, avec une lenteur qui contrastait fortentent avec la rapidité du torrent circulatoire dont ce globule était échappé; bientôt après le globule eessait de se mouvoir, et il demeurait fixé dans le tissu transparent; or, en le comparant aux grauulations que eontenait ce même tissn, il était facile de voir qu’il n'en différait en rien; en sorte qu'il n'étail pas doutenx que ces granulations demi-transparentes ne fussent aussi des globules sanguins précédemment fixés. Par quelle voie ces globules sortent-ils du torrent circulatoire?' C'est ce qu'il n'est pas facile de déterminer. l'cui-être les vaisseaux ont-ils rles nuvertures latérales par lesquedles 
le sang pent verser ses éléments dans le tissu dcs organes; peut-être le mouvement de ces gglobules n'était-il ralenti d'abord, et ensuite arrêté que pareegu'ils étaient engagés dans des vaisseaux trop petits relativement à leur grosseur: On expliquera celte fixation des globules sanguins commc l'on voudra, mais le fait de eetle fixalion demeurcra toujours dćmontré; je l'ai observé un trop grand nombre de fois pour eroire que ce soit un phénomène aeeidentel. Cette fixation des globulcs est indubitablement un phénomène dans l'ordre de la nature vivante : eela explique le rôle que jouent les globulcs sanguins dans la nutrition; ee sont des eellules vagahondes qui finisscnt par se fixer et par se joindre au tissu des organes; aussi les cellules vćsiculaircs et microscopiques qui composent cssentiellement lc tissu de tous les organes, sans aucune exception, ne sont-elles généralement que de la grosseur des globules sanguins ehez les animaux vertébrés : Leuwenhoek le dit positivement par rapport au tissu du foie du mouton et de la vaehe '. Mes observations m'ont prouvé la même chose par rapport aux autres organes. Chez les mollusquucs, ces cellules microscopiques sont ineomparablement plus grosses que les globulcs contenus dans le sang de ecs animaux, ee qui peut provenir de ce qu'elles se sont développées après leur fixation. Au reste, le phénomène de cettc fixation explique pourquoi les globnles ont disparu tout-à-fait dans le sang du têtard que

- Transaclions philosophiqucs, i6-i. 
j'avais soumis à un jeûne très prolongé : cette disparition prouve en même temps que ces globules tirent leur origine des aliments : aussi Leuwenhoek les a-t-il trouvés en abondance dans le chyle. Cela peut faire penser que ces globules vésiculaices sont introduits tout formés dans l'économie. Les substances alimentaires, qui sont toutes des matières organiques, sont essentiellement composées de ces globules, et la digestion ne consiste probablement que dans leur dissociation, opérée par le menstrue stomacal. Ces observations paraitront sans doute très favorables au fameux système des molécules organiques de Buffon; système que je suis fort éloigné d'admettrc dans son entier, mais dont la base essentielle me paraît être appuyée sur les faits que je viens d'exposer. Ici je dois rappeler ce que j'ai exposé plus haut touchant la texture organique des végétaux: nous avons vu que ces êtres étaient cntièrement composés ou de cellules ou d'organes qui dérivent évidemment de la cellule; nous avons vu que ces organes creux étaient simplement contigus et adhérents les uns aux auires par ume force de cohésion, mais qu'ils ne formaient point, par leur assemblagge, un tissu réellement continu; en sorte que, dès lors, l'être organique nous a paru formé d'un nombre infini de pièces microscopiques qui n'ont entre elles que des rapports de voisinage. Les observations (que nous venons de faire sur les animanx tendent évidemment à confirmer ce premier aperç; il est encore confirmó par les observations si curiéuses de M. Bory re Saint-Vincent sur ces arthrodices, qui 
sont composées de pièces de rapport qui se réunissent successivement les unes aux autres, en sorte que ces êtres singuliers nous montrent en dehors le phénomène de l'agrégation corpusculaire, que les autres êtres vivants cachent dans l'intérieur de leurs tissus organiques. 


\section{APPENDIX.}

L'espèce d'avidité avec laquelle la nature est aujjourd'hui interrogée de toutes parts met les naturallistes dans la nécessité de publicr très promptement Heurs déeouvertes, s'ils ne veulent pas courir le risque ide se voir privés, par des observateurs plus diligents, ide l'homneur qui y est attaché. Mais cette précipitation expose à publier des travaux ineomplets et quelquefois fautifs; elle ne permet pas de rassembler et ide eoordonner une masse de faits. C'est eette dernière ' eonsidération qui m'a engagé à retarder de plusieurs mois la publication des observations qui m’ont dévoilé le méeanisme de la eontraetion musculaire. Pendant ce temps, deux observateurs très distingués, MM. Prévost et Dumas, se livraient à des recherehes sur le même sujet, et arrivaient, par une autre voie, au résultat auquel je suis parvenu. Le travail de ces deux observateurs, eommuniqué à la Soeiété philomatique et à l'Aeadémie des seiences, en juillet et août 1825, a paru, en extrait, dans le cahier de septembre du Bulletin des sciences de la Société philomatique, eahier yui ne m'est parvenu que dans le milieu du mois de novembre. Alors mon travail était complètemeut rérligé, et j'ai cru devoir le publier sans 
y rien changer, me réservant seulement d'y ajouter cet Appendix, dans lequel je vais exposer la découverte de MM. Prévost et Dumas, et la théorie qu'ils en déduisent. Le travail de ces deux savants a été imprimé en entier dans le numéro d'octobre du Journal de physiologie expérimentale de M. Magendie.

MM. Prévost et Dumas ayant placé sous le microscope un muscle de grenouille suffisamment mince pour conserver sa transparence, et y ayant excité des contractions, au moyen d'un courant galvanique, ont vu les fibres se fléchir en zig-zag d'une manière instantanée, et cette flexion a déterminé le raccourcissement de l'organe; ils on f fait, en même temps, cette importante observation, que les dernières ranifications des nerf's coupent à angle droit la direction des fibres musculaires, et que c'est toujours dans le lieu de leur intersection qu'existent les sommets des courbures qu'affectent les fibres musculaires en se courbant sinueusement. Ainsi, MM. Prévost et Dumas ont vu, comme moi, que la contraction des organes musculaires consiste dans une courbure sinueuse de leurs parties constituantes, el ta date de la publication de cette découverte leur en assure incontestablement la propriété, bien que j'eusse fait la mềme découverte de mon côté, au moyen d'expériences différentes. Toutefois, ceux qui liront mon travail et celui de MM. Prévost et Dumas avec attention verront qu'ils contiennent des faits différents, quoique du même genre. Je vais essayer d'établir ici la distinction de ce qui m'appartient et de ce qui constitue 
Ila pair de MM. Prévost et Dumas, dans la découverte ¿du mécanisme de la eontraetion museulaire.

MM. Prévost et Dumas ont observé la flexion siinueuse de la fibre musculaire, flexion tout-ì-fait semthlable à celle des tiges des vortieclles, et que j'ai treprésentée dans la tigrure 29, en $a$. Pour moi, je In'avais obscrvé que l'ineurvation semi-cireulaire de icette fibre, arrachée à l'animal vivant, et plongée dans Il'eau; j'avais cru pouvoir conclure de eette observattion que l'ineurvation semi-eireulaire de la fibre cooIpérait au raeeoureissement du muscle, et qu'elle était Il'auxiliaire de la contraetion de eette même fibre. Par ree mot de contraction, j'ai entendu exprimer l'acition par laquelle la fibre musculaire se raceoureit en devenant plus grosse, sans perdre de sa reetitude. Or j'ai prouvé que eette eontraetion de la fibre trouve sal cause dans le plissement extrênıement fin, ou dans l'inenrvation sinueuse des fibrilles et du tissu eorpusculaire qui eomposent intérieurement la fibre musculaire. C'est ici que mes observations ont été plus loin que eelles de MM. Prévost et Dumas. Ces observateurs ne regardent eomme une contraction que la courbure sinueuse de la fibre musculaire eonsidérée dans sa masse; ils ont bien observé que eette fibre se raceourcissait aussi sans aueune flexion, mais ils ont considéré ee raeeoureissement comme le résultat de eette propriété que Haller nomme l'élasticité de la fibre, et que Bichat désigne sous le nom de contractilité de tissu. Du reste, ils ne eherehent point à se rendre raison du méeanisme au moyen duquel cette 
élasticité est mise en jeu : ils admetient, dans la fíbre museulaire, un état de repos, qui est eelui qu'elle prend quand aucune eause ne tend plus à l'alonger. Ce n'est, selon ces observateurs, que lorsque la fibre a atteint, dans son raecoureissement élastique, eet état de r'epos, qu'elle devient suseepiible de se courber sinueusement pour se raecoureir de nouveau, et c'est à ee dernier phénomène seul qu'ils donnent le nom de contraction. Ici tout est exaetement vrai dans l'exposition des faits : il n'y a d'erreur que dans la théorie. MM. Prévost et Dumas, l1'ayant pas poussé assez loin leurs recherehes, n'out point vu que le raccourcissement de la fibre, sans aucunie flexion, est dû à l'ineurvation sinueuse à plis extrêmement fins du tissu intérieur de eette fibre, qui s'alonge par. le déplissement de ee tissu, et qui se raccourcit, en conservant sa rectitude, par le plissement ou par l'incurvation sinueuse élastique de ce même tissu intime. Lorsque ce plissement intérieur est parvenu au summum, la fibre ne peut plus se raecourcir de eette manière, elle se trouve dans l'état de repos, suivant l'expression fort inexacte de MM. Prévost et Dumas. C'est alors que commence le développement d'un second phénomène, celui de l'incurvation sinueuse de la fibre elle-même, qui se raccourcit en perdant sa reetitude, et cela par un mécanisme entièrement seniblable à celui qui avait opéré son raccoureissement avee conservation de rectitude. Toute Ta différenee. qu'il y a, c'est que, dans le premier cas, le phénomène que présente la fibre est extérieur, et que, dans 
He dernier cas, il est intérieur. Or, l'observation du Ipremier de ces phénomiènes appartient à MM. Préi vost et Dumas; l'observation du second m'appartient rexclusivement. C'est de l'ensemble de ces observaıtious que résulte l'explication complète du mécanisme ide la contraction musculaire. Au reste, c'est faute ¿d'avoir envisagé sous son véritable poini de vue le Iphénomène de la contraction de la fibre qui conserve :sa rectitude, que MM. Prévost et Dumas ont été conduits à le considérer comme le résuliat d'une simple iélasticité étrangère, en quuelque sorte, à la vie. Cette incurvation du tissu intime de la fibre est tout aussi vitale que son incurvation de masse; elle est fort différente, quant it la cause occasionelle, de la contracttilité de tissu ou de la propriété que possède la fibre complètement morte de se raccourcir quand on l'abandonne à elle-même après l'avoir distendue. Ce (dernier phénomène, comme je l'ai exposé plus haut, idépend de l'élasticité avec laquelle les parties intimes de la fibre tendent à conserver un certain état d'in(curvation qu'elles ont pris par le fait même de l'absence (de la cause immédiate de la vie, absence qui parait avoir coccasioné celle d'une cause d'écartement corpusculaire. Ainsi, la contractilité de lissu après la mort est l le résultat d'un état élastique fixe et permanent, tandis que la contraction vitale de la fibre, sans perte de rectitude de cette nềme fibre, est le résultat d'un état élastique susceprible d'éprouver des variations dans son intensité, et même de cesser d'exister, jusqu'à un certain point, par le fait du relâchement. MM. P'é- 
vost et Dumas ont observé que c'est au moyen de ce raccourcissement sans perte de rectitude de la fibre que s'opère la contraction des organes musculaires membraneux, tels que ceux qui existent dans les parois du canal alimentaire, et ils en ont conclu que la contraction de ces organes différe entièrement de celle des muscies de la locomotion. On a lieu de s'étonner qu'une assertion aussi hasardće ait pu ĉtre émise par des observateurs qui, habitués à envisager la nature sous plus d'une face, ont dû voir qu'elle rćunit constamment la simplicité et l'uniformité des causes prcmières, à la variété et à la fécondité des résultats. Ainsi, le seul raisonnement à priori devrait faire admettre qu'il n'existe point de différence essentielle cntre la contraction des muscles de la locomotion et celle des organies musculaires soustraits à l'empire de la volonté; et effectivcment l'observation apprend que, dans ces deux cas, la contraction dépend de niême de l'incurvation du tissu musculaire; dans l'un et l'autre cas, il existe un état élastique dont la cause est vitale: telle est l'idée que l'on doil se faire de l'incurvation sinueusc du tissu intime de la fibre musculaire, et de l'incurvation sinueuse de cclte fibre elle-même. En effet, l'obscrvation de l'incurvation végétale nous a démontré d'une manière bien ćvidente quc cet état est dû au développenınt d'une force ćlastique; nous avons établi l'analoyie de cettc incurvation végćtale avec l’incurvation animale; et en étudiant les phénomènes que présente cette dernière, nous avons vu qu'elle trouve sa cause dans un certain 
rapprochement corpusculaire. Ainsi, il nous a ćté dćmontré que l'incurvation végétale ct animale clépend idu développement d'une force élastique, qui cllemême trouve sa cause dans certains phénomènes moHéculaires ou corpusculaircs; les muscles, par consséquent, agissent comme des ressorts, mais ces ressorts cont une nature et un mécanisme tout particulier dont iil est facile de se fairc une idéc. Il y a deux choses ì considérer dans un ressort, sa position, et la lórce élastique avec laquelle il tend à conserver cette position, ou à y revenir quand il en est ćloigné. Un ouivrier qui vcut faire un ressort d'acier conmence par Hui donner la position, c'est-à-dire l'ćtat de rectitude ou de courbure particulic̀re qu'il veut que ce ressort possède dans l'état de repos; ensuite il lui communique, au moyen de la trempc, la force élastique qui llui donnc la tendance à persister dans cette position et à y revenir lor'squ'il en est éloigné. Or les fibres muscullaires sont des solides qui, sous l'influence de certaines ccauses intéricures ou cxtérieures, prennent, soit dans lileur masse, soit dans leurs parfies intimes, une position de courbure accompagnéc d'une force élastique qui tend à faire persistcr cette position. Ainsi la contraction musculaire est un véritable phénomène d'élasti' cité; mais c'est unc élasticité qui naît ct qui disparait successivement avec la position de courbure qui l'accompagnait; or', comme l'élasticité cst, en dernic̀re analyse, un phénomène d'action noléculaire, il en résulte que la contraction se trouve de même, en dernic̀re analyse, dépendre d'un cerlain mole 
d'action des molécules ou des corpuscules qui composent les solides organiques. Ceue thécrie est toutà-fail en opposition avec celle qui a été émise par MM. Prévost et Dumas: ces deux savants ayant observé que les dernières ramifications des nerf's coupent à auglc droit la direction des fibres musculaires, ont pensé que le courant galvanique excité au travers des filets nerveux déterminail le rapprochement de ces filets, qui s'attireraient réciproquement, et qui entraîneraient ainsi avec eux les faisceanx musculaires auxquels ils sont fix̣és, ce qui déterminerait le plissement des fibrcs. D'aprèes cette hypothèse, les nerl's seuls seraient les organes du mouvement de contraction, et les fibres musculaires seraient des organes inertes, destinés seulement par la "nature à assujettir les filets nerveux les uns aux autres. On sent tout ce qui s'opposerait à l'adruission d'une pareille hypothèse, quand bien même il ne serait pas prouvé qu'elle doit être rejetée. Mais si l'hypothèse disparait, les faits sur lesquels ellc paraissait pouvoir être établic subsistent, et cette découverte suffit pour la gloire de ses auteurs, auxquels la science doil déjà beaucoup, et qui l'cnrichissent tous les jours par d'importants travaux.

Je profiterai de l'occasion qui m'a été offerte d'ajouter cet appendix à mon ouvrage, pour discuter l'opinion d'un savant fort célèbre sur l'irritabilité végétale. J'avais d'abord résolu de n'en point parler', pensant que les faits que j'avais établis sur l'observation suffisaient pour combattre une théorie purcment 1:1- 
tionnelle, sans qu'il füt besoin d'entrer dans unc disicnssion à cet égard : cependant j'ai senti qu'il était méeessaire de ehanger ma prentière nuanière de voir ssur cel objet; car, bier que les faits soient tout dans Ha seience, cependant l'autorité des noms ne laisse Ipas d'avoir anssi qnelque influenee. Je discuterai donc rici brièvement l'opinion de M. de Lanarek sur l'irriunabilité. Ce naturaliste célèbre, dáns son Introduction ià l'histoire des animaux sans vertèbres, prétend ićtablir une démareation tranehée entre les mouvements des animaux el ceux des végétanx. Toici comunent il s'exprime (cliap. 5): "Les végétaux sont " des eorps vivants non irritables, et dont les ca"ractères sont, $1^{0}$ d'être incapables de contracter " subitement et itérativement, dans tons les temps, ") aneune de leurs parties solides, ni d'exéeuter, par ") ees parties, des mouvements subits ou instantanés, "répétés de suite autant de fois qu'une eause stimu") lante les provoquerait. "Partant de ce prineipe, il prétend qu'aueun des mouventents des végétaux m'est dî̀ à l'irritabilité; que ce ne sont que des nouvements de détente, ou des affaissements de parties, prorluits par l'évaporation de certains fluides subtils 'qui cessent de gronfler les eellules. Il affrrme qu'aucune des parties de la sensitive ne se contracte lor's' fri'on la touche, mais que les mouvements qu'on lui voil exéeuter ne sont que des mouventents articulaires opérés par détente, sans qu'aucume des dimensions des parties de cette plante soit iltérée, ce qui, selon lui, ćtablit une différence tranchéc entre ces 
mouvements et eeux qui résultent de l'irritabilité animale, dans laquelle il y a bien évidemment ehangement dans les dimensions de la partie eontraetće. Poursuivant, d'après les mêmes principes, le contraste qu'il établiı entre l'irritabilité anima!e et les nouvements des végétaux, M. de Lamarck donne comme une différence spécifique entre ees deux ordres de phénomènes, que ehez les animaux l'irritabilité reste la même dans les parties qui en sont doućes tant que l'animal est vivant, eı que leur contraetion peut se répéter de suite autant de fois que la cause exeitante viendra la provoquer, tandis que ehez les végétaux prétendus irritables la répétition de l'attou-

- chement on de la secousse ne peut plus produire aucun mouvement lorsque la plication articulaire est complètement effectuée.

D’après cet exposé, les différences tranchées que M. de Lamarck prétend établir entre l'irritabilité animale et l'irritabilité végétale se réduisent aux points suivants: $1^{\circ}$ les mouvements des végrétaux ne sont que des plications artieulaires; il n'y a point ehez cux de véritable enntraction ou de raccoureissement de parties; $2^{\circ}$ ces mouvements ne peuvent être produits itérativement, c'est-à-dire déterminés plusieurs fois de suile.

Il ne me faudra que quelques mots pour conıbattre ces diverses assertions. D'abord, c'est une crreur que de regarder les monvements de la sensitive comme des plications árticulaires. On a donné le nom d'ar'ticulation, dans les feuilles, à l'endroit où 
elles se séparent naturellement de la tige lorsqu'elles ont atteint le terme de leur vie: or, ce n'est point dans eet endroit que s'opère le mouvennent des feuilles de la sensilive, c'est dans une portion renfléc du pétiole, portion voisine de l'artieulation, et à laquelle j’ai donné le nom de bour'relet. C'est par l'ineurvation élastique de ce bourrelet que s'opère le mouvement du pétiole de la feuille; ee mouvenıent n'est. done point articulaire, comme le pense M. de Lamarek: on en doit dire autant des mouvenents des pinnules et des folioles de la sensitive; ces mouvements ne sont point non plus articulaiv'es, ils n'existent que dans les bour'relets, pareeque ees organes possèdent seuls la structure intime nécessairc pour l'exécution de ce mouvement.

M. de Lamarek prétend qu'il n'y a point de véritable eontraetion ou de raeeoureissement de parties chez les végétaux: l'observation infirme eneore cette assertion. Nous avons vu que, eliez l'ypomace sensitiva, les nervures de la eorolle présentent un raeeoureissement de parties ou une contraetion qui ne diffère en rien de eclle des filbres museulaires, car elle eonsiste de même dans une incurration sinuteuse. Le fait de la eontraction de la eorolle ehez l'ypomaca sensittva n'était point connu du publie, il est vrai, puisque je suis le premier qui l'ait publié, avee l'agrément de M. Turpin, qui a observé ce phénomène; mais tout le monde connaissait le phénomène essenticllement semblable que présente la corolle dés cortvolvulus et celle de la belle-de-nuil (mircatilis ja- 
lappa), qui se ploient au moyen d'une incurvation sinueuse pour présenter les alternatives de sommeil et de réveil. Mais il manquait à M. de Lamarck, pour établir l'analogie de ce mouvement avec l'irritabilité animale, de connaître le mécanisme de cette dernière, qui consiste de même dans une incurvation sinucuse.

Enfin, M. de Lamarck objecte que les mouvements des végétaux ne peuvent être produits itérativement. Cette objection tombera d'elle-même, au moyen d'une réflexion bien simple : l'incurvation ne peut être produite une seconde fois que lorsqu'elle a cessé d'exister, c'est-à-äire lorsqu'clle a été remplacée par le redressement ou par le relâchement, sclon l'expression ordinaire. Or, chez les végétaux, le redressement ou le relâchenent n'arrive que longtemps après l'acte de l'incurvation, en sorte que la partie reste long-t.mps incurvée, tandis que chez les animaux le redressenient ou le relàchement de la fibre arrive immédiatement après l'acte de son incurvation sinueuse ou de sa contraction; en sorte qu'il n'y a presque aucun intervalle entre ces deux phénomènes. De là vient que, chez les animaux, la contraction ou l'incurvation sinuense peut être produite itérativement un grand nombre de fois de suite dans un très court intervalle de temps, tandis que cliez les végétaux l'incurvation ne peut être produite itérativement qu'à des intervalles de temps assez longs : il faut attendre que le redressement ait succédé à l'incurvation. N'est-il pas évident que, dans celte circonstance, la longueur du temps qui s'écoule entre les deux actes 
de l'incurvation et du redressement n'apporte aucune différenee esscntielle entre les phénomènes de l'irritabilité animale et de l'irritabilité végétale? Dans l'une et dans l'autre, les mouvements sont produits itérativement, mais à des intervalles de temps différents.

Pour ce qui est de l'hypothèse émise par M. de Lamarck, que les mouvements des végétaux sont dus à des affaissements de cellules produits par l'évaporation des fluides, il ne me faudra, pour montrer son peu de fondement, que rappeler l'expérience suivante, que j’ai plusieurs fois répétée. La sensitive, entièrement plongée dans l'eau, meut ses feuilles sous l'influence des seeousses, eomme elle le fait dans l'air; elle y présente de même les phénomènes du sommeil. et du réveil. Or, il est évident que dans eette eirconstance il ne peut $y$ avoir rii ćvaporation ni affaissement de eellules.

Dans le cours de eet ouvrage j'ai opposé avee franchise mes opinions à celles de plusieurs savants eélèbres; et je l'ai fait sans crainte de les blesser, persuadé que tout philosophe observateur de la nature ne doit rechereher que la vérité, et qu'il ne pent. manquer de la voir avec plaisir, même lorsqu'elle heurte ses idées les plus favorites. 


\section{TAB LE \\ DES MATIERES.}

l'ages.

INTRODUCTION. . . . . . . . . . I

Section Ire. Observations sur l'anatomic des végétaux, et spécialement sur l'anatomie de la scnsitive. ........ 8

Section II. Observations sur les mouvements de la sensitive. . . . . . . 52

Section III. Des direclions spéciales qu'affectent les diverses pariies des végétaux.

Section IV. De l'influence du mouvement de rotation sur les directions spéciales qu'affectent les diverses parties des vé-

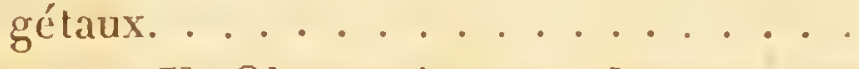

Section V. Observations sur la structure. intime des systèmes nerveux et musculaire, et sur le mécanisme de la contraction chez les animaux. . . . . . 163

APPENDIX. . . . . . . . . 2 I9

Tableau synoptique des diver'ses modifcations de l'incurvation organique dans les deux règnes animal et végétal. . . . 233 


\section{TABLEAU SYNOPTIQUE}

\section{DES DIVERSES MODIFICATIONS DE L'INGURVATION ORGANIQUE.}

\section{DANS LES DEUX RL̀GNES ANIUAL ET VÉGÉTAL。}

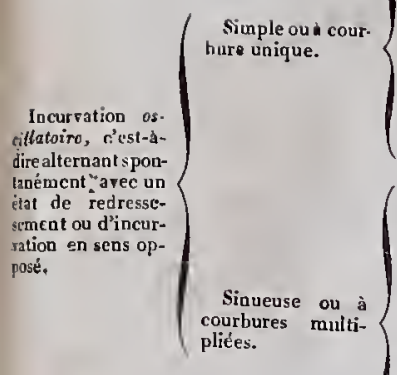

Iscurvation et redressementalternatifs des bourrclets de la sen sitive, des étamines du cactus opuntia et du borbcris,vulgaris, des feuilles du dionea muscipula; ascillation des folioles do l'todysarum gyrans; incurralions en sens iuverses, desquelles résultent les positions alternatives de somincil desquelles résulplantes; mourement des oscillaires. . . . . . . . . . . .

Incurvation de la fibre musculaire a rrachée à l'animal virant et

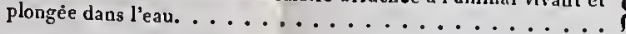

Plissement et déplissement des nervures de la corolle de l'y- ? pomaa sensitiva, des bras des hydres, et des tiges des rorticclles.

Plissement et déplissement du tissu intime de la fibre nusculaire, qui se raccourcit en devenant plus grosse et cn conservant

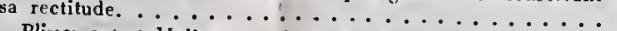
Plissernent et déplissement de la fibre musculaire clle-même, qui se raccourcit en perdant sa rectitude. . . . . . . . . .

Plissementet déplissement des tissus qui ne sont point muscu-

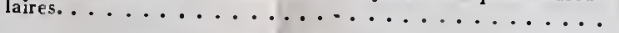

Simple ou à cour- $\{$ Incurration des valves de l'ovaire de la balsamine ; incurvation bure unique. $\quad\left\{\begin{array}{l}\text { des diverses parties des végétaux, pour affecter des oircctions spé- } \\ \text { cirate }\end{array}\right.$ ciales et fixc................................

Incurvation des vrilles des végéraux : elles s'effectuent sous l'in- ) fluence de la vie, et persistent après la mort et le déssícbement

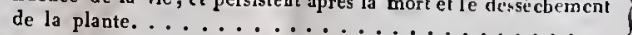

Sinucuse ou a courbures multipliées.

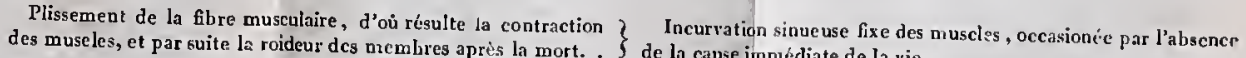

Plisscment de la fibre musculaire morte lorsqu'on l'abandonne ) Incurvation sinucuse fixe, suite de la précédente : rlle cst nom à elle-même après l'avoir distendue en l'alongeant....... fe tissu.
Inearvatiou simple oscillatoire de's vigélaux.

Incurvation simple uscillatoire des fibres musculaires.

Incurvation sinuéuse oscillatoire des végútuux ct des zoopbytes.

Incurvation sinueuse oscillatoire des muscles d'être rapide, lorte et trìs śtendue s's la contac et la contractilite animale de contractilité organique sensible de Bichat; c'est l'irritabilités

Incurvatipn sinueuse oxcillatoixs des organes non musculairs : son caractere est d'être lénte. faihle et trés peu étenduc : c'est la contractilité organique insensible de Bichat.

Incurvation simple fixe des vègétaux.

Incurvation sinueuse fixe des ivégétaux. 
.
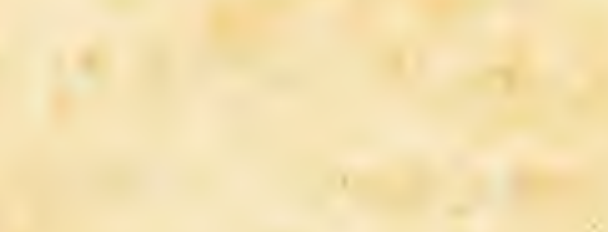

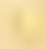

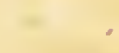

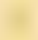
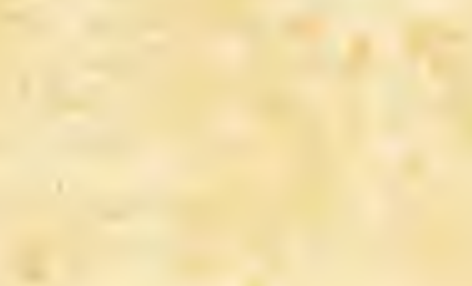

$\sqrt{2}+x^{2}$

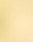

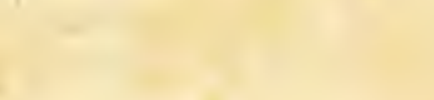

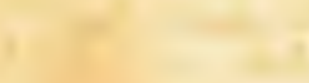

1

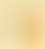

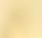

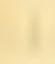
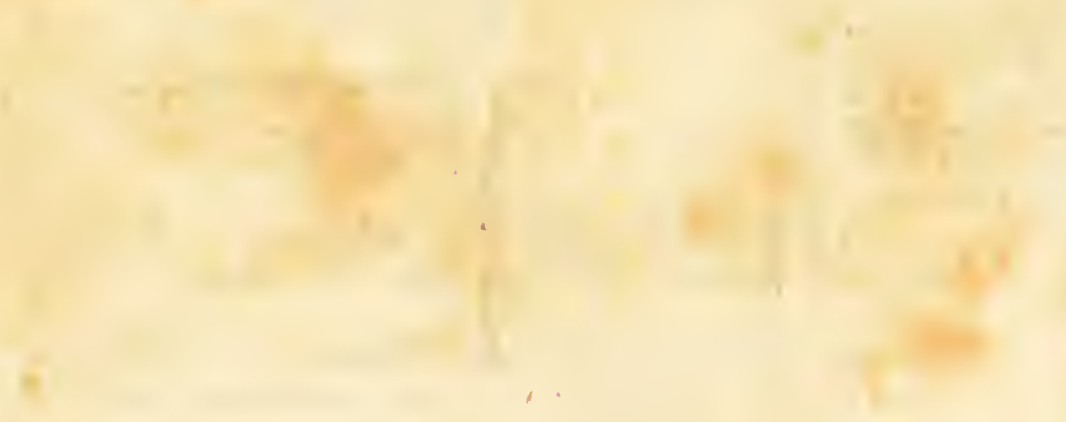


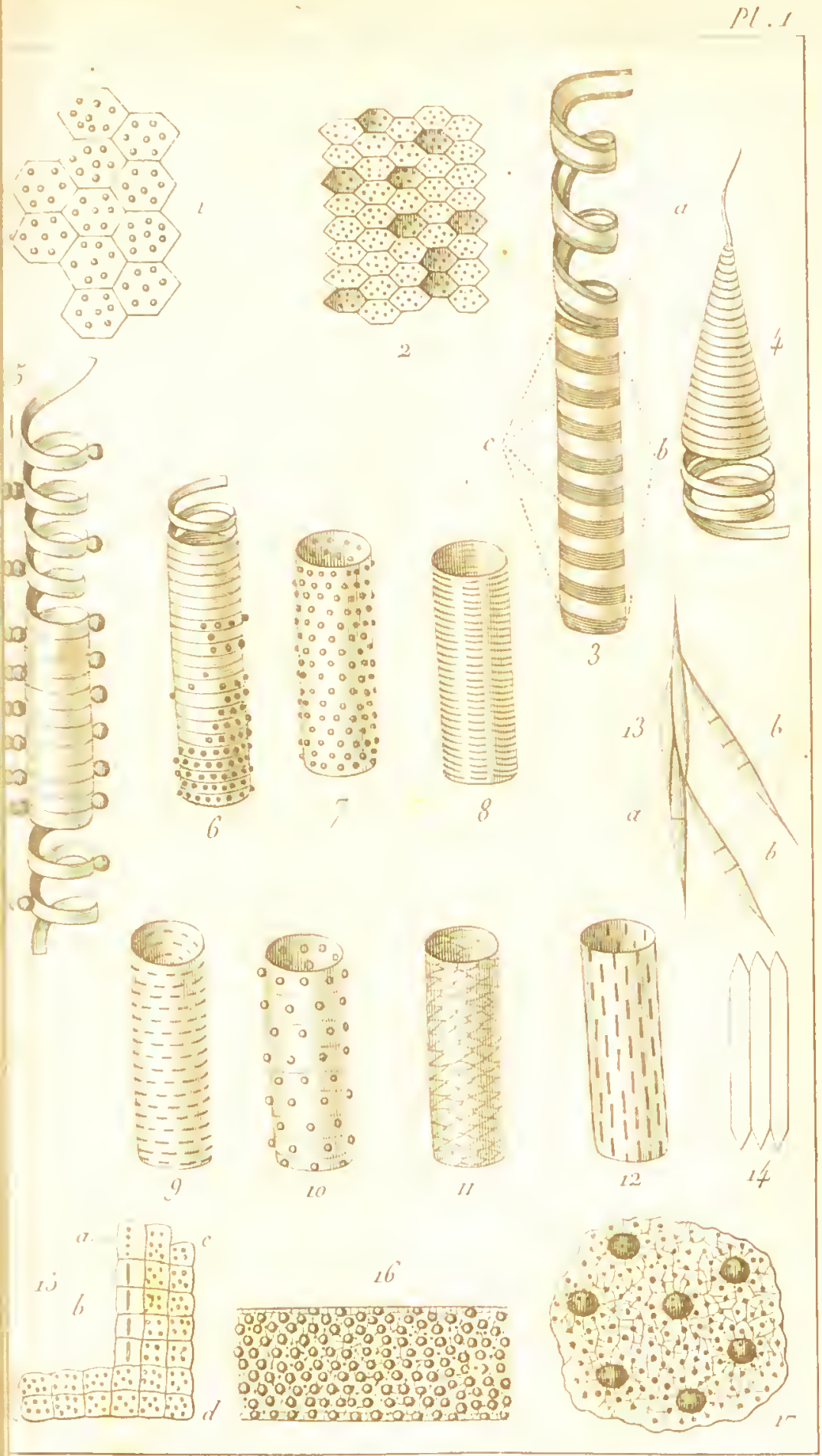

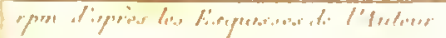

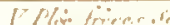





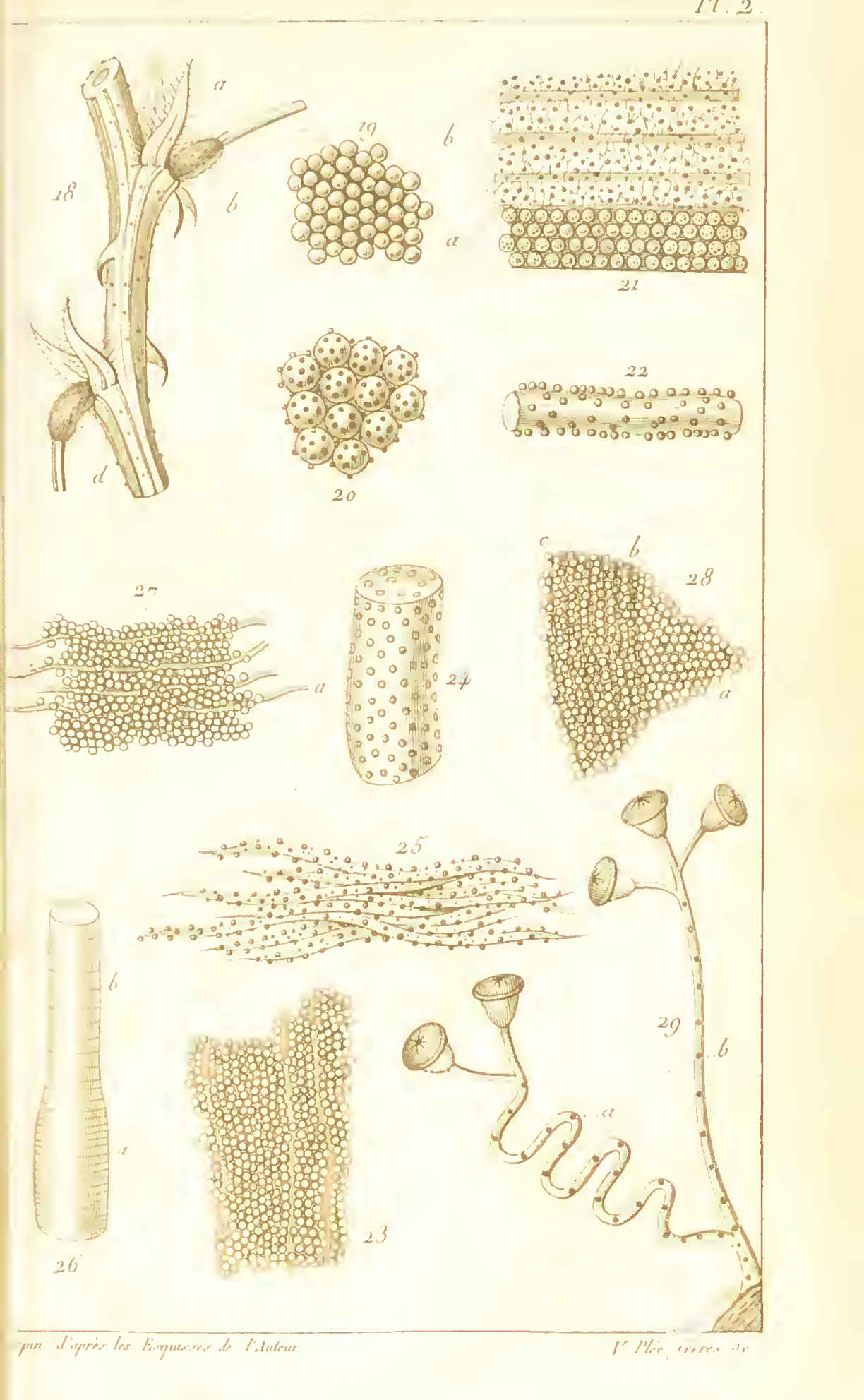




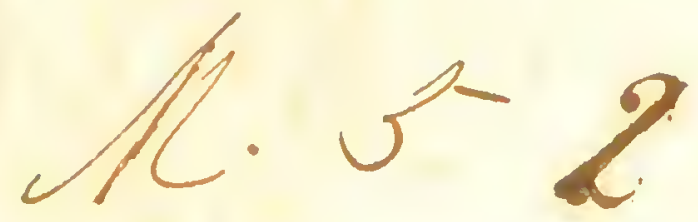

LAGENT IMMÉDIAT

DU

\section{MOUVEMENT VITAL}

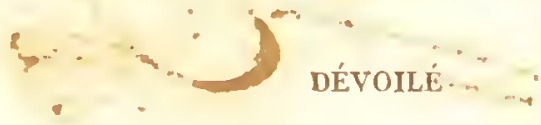

INS SA NATURE ET DANS SON MODE D'ACTION,

\section{CHEZ LES VÉGÉTAUX}

ET CHEZ LES A N MAUX. 


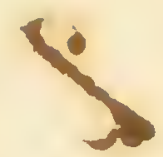

CET OUVRAGL SE IRUUVE AUSSI AU DLLOTT DE MA I.LBIALIL, Palais. Royal, galteries de bois, $10 \times 326 j$ el 266 .

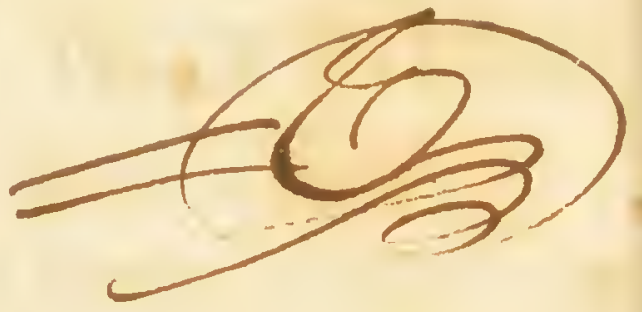

IMPRIMERIE JE J. G. DENTU, KUE DU COLUMBLFR, NO 2 I. 


\section{L'AGENT IMMÉDIAT}

LI

\section{IOUVEMENT VITAL.}

DÉVOILE $\because$

MXS SA MAURE ET DANS SOY HODE D'ACTION,

\section{CHEZ LES VÉGÉTAUX}

\section{ET CHEZ LES ANIVAUX.}

\section{PAR N. H. DUTROCHET,}

rorrespondant de l'hnstitut dans l'Acadénie royale des sciences, membre associr de l' lcalémie royale de médecine, ete.

Vil ara differle est quin quareudo investignari posit.

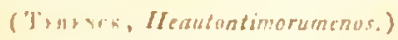

\section{A PARIS,}

IE' DENTU, IMPRIMEUR-LBBRALE,

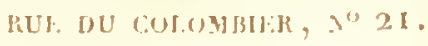

MIDCCGXXVI. 


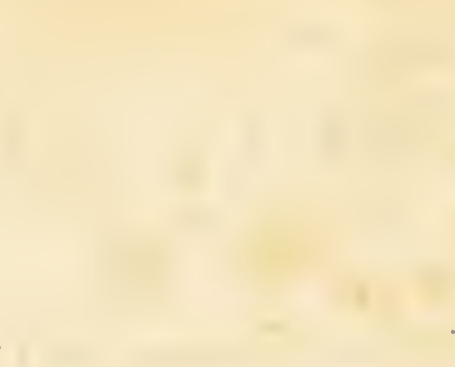




\section{AVERTISSEMENT.}

IL'ouvrage que je publie mettra dans ut son jour celte rérilé, qu'il n'existe int deux physiologies, l'une animale Il'autre végétale, entre lesquelles il soit ussible d'établir une ligne de démarcaon. La science de la vie est une, et l' on ? pent que perdre de précieux secours n isolant les unes des antres les divers parties qui la composent; car c'est ir le rapprochement des fails que la iience devient féconde. Ainsi, nous alms voir la physiologie végétale faire zarcher la physiologie animale, et réciroquement la physiologic animale prêir un puissant appui a la physiologie Égélale.

L'objet spécial et primitif de ce travail ull'élude du mouvement de la sève dans :s végétaux, phénomène dont l'explicaton, jusqu'à ce jour, a vainement élé 
cherchée, parce quion a voulu la trouver dans les faits déjà connus de la physique ou de la physiologie. L'étude attentive que jai faite de ce phénomène. m'a dévoilé pleinement sa cause mystérieuse. Cette découverte m'a conduit beaucoup plus loin que je ne devais le présumer. En effet, en trouvant le mécanisme et la cause du mourement de la sève, j’ai trouvé le mécanisme secret du mouvement vital, el cetle découverte m'a fait remonter à la connaissance de l'agent mystérieux auquel est immédiatement dû ce mourement. En annonçant celle découverte, je dois me hâter de. dire qu'elle ne fournira point d'armes au matérialisme. Les sciences, faites pour le bonheur et pour la perfection de l'homme, ne peuvent être hostiles envers la morale, cette autre source de bonheur et de perfection.

De tous les êtres vivans qui couvrent la surface du globe, l'homme est le seul qui possède l'intelligence, ce flambeau divin au moyen duquel il parvient à connaître une partie des mystères de la 
nture, et à remonles à la connaissance son auteur. Siége immatériel de celle Itclligence, l'âme est inaccessible à nule invesligalion des sens; elle est par. ynséquent inaccessible aux attaques qui raient fondées sur des observations; ur les observations ne peurent s'exerque sur des ohjets matériels. Liame ste nécessairement en dehors des renerches dont elle est l' agent; clle róside ans un sanchaire impónćtrable : c'est e là qui clie régit la machine organique ui hi est soumise. Or, tonte la série des itermédiaires par lesquels elle exerce " puissance, est du domaine de l'obrrvation physique. L'agent immédiat du couvement vital est pour nous le plus reulé de ces intermédinires; mais som xistence démontréc ne prouve point du but qu'il n'y ail rien au-delà : aussi l' exuression d'argent immédial. dont je me ors, laisse-t-elle toule la latilude possille pour: l'átablissement d'un agent méfiat de la vie, placé plus haul dans léhelle des faits. Ne serait-il pas absurble, in oflet de prélendere quil n'existe plus 
rien là où notre vue ne peut plus s'étendre, et que les bornes de notre horizon sont les bornes du monde? Ainsi la religion et la morale ne doivent point s'etfrayer des tentatives qui sont faites pour remonter à la source des phénomènes de la vie, puisque ces recherches ne peuvent jamais avoir pour résultat de prouver le matérialisme; ces recherches, d'ailleurs, sont commandées par le plus haut intérêt de l'humanité, celui de la conservation de la vie, conservation it laquelle on ne peut travailler avec efficacité qu'au moyen de la connaissance approfondie des ressorts qui font mouroir l'admirable machine organique. 


\title{
L'AGENT IMMÉDIAT
}

\section{DE LA VIE}

DÉVOILÉ

DANS SA NATURE ET DANS SON MODE D'ACTION,

\author{
CIIEZ IES VÉGÉTAUX
}

\section{ET CHEZ LES ANIMAUX.}

\section{SECTION I ${ }^{\mathrm{re}}$.}

Recherches sur la marche de la sève dans les plantes, ct sur la cause de sa progression.

\section{INTRODUCTION.}

Lorsque des faits dont la cause esi incomnue se Iprésentent à l'observation, on tâche de leur donmer une explication, en leur assignant poụr carıses certains phénomènes dont la marche bien conmuc semble concorder avec celle de la cause cncore ignorée des faits que l'on veut expliquer. On est naturellement porté à admetue que ce que l'on observe se rattache à ce que l'on commaît déjà. Mais les esprits 
philosophiques se mettent cu garde eontre ectuc tendanee que nous avons à eireonserire la nature dans le eerele étroit de ee que nous savons; persuadés qu'il ne suffit pas qu'une explication soit probable pour qu'elle soit viaie, ils savent rester dans le doute, el dire j'ignore, ee mot qui répugne tant à l'orğneil des esprits vulgaires. Combien de fois, ell effet, n'at-on pas vu les explieations les plus probables renversées, sans recour, par l'observation de eertains faits dont on n'avait pas auparavant la plus léçère idée, et qui venaient agrandir inopinément le ehamp de la seience? Cos réflexions maissent natmellement de la eonsidération du sujet que j'entreprends de traiter iei. On a eherehé à expliquer le mouvement de la sève en lui assignant pour eause la eapillaricé du tissu végétal, eause insuffisanıe évidemment à elle seule pour la produetion du phénomène, et à laquelle on a eru pouvoir adjoindre la eontraction supposée des parois des organes dans lesquels la sève se trouve eoutenue. La réunion de ees deux eauses donne sans doute une explieation assez plausible du mouvement de la sève; mais eette explieation n'a point le caraetère d'évidenee qui seul a le ponvoir de dissiper tous les dontes, et d'entraîner la eonvietion. Ineertains sur la eausc du mǫuvement de la sève, nous le sommes anssi sur les rontes que snil ce lluide. On sait que la sève monte des raeines dans la tige et dans ses appendices; on ne sail pas d'me manière bien posilive quels sont, daus le végcial, les organes de cette tra!ısmission. Cependant, les expériences de Duhamel, de 
nrrabat el de Bonnet ont fourni quelques données rcécicuses sur celle question. On admet généralenent que la sève élaboréc descend de la tigge vers les ucines; et certains faits prouvent que e'est par l'éwree que sopère ce motwement descendant : rien "a prouvé cependant que celtc fonction de transnettre la sève descendante soit le partage exchusif de iécorce. La scicnec réclame, à cet ćgard, des obserations plus étcndıes et plns précises que celles qui mt été faites jusqu'à cc jour. Ainsi, deux problêmes e présentent ici à résoudre : ${ }^{\circ}$ quelles sont les rontes que suit la sève? $2^{\circ}$ quelles sont les causes de la orogression de ce fluide? Nous allons aborder successivement ces denx questions, qui nous jettcront jécessairement dans le domaine de la physiologie unimale. 


\section{CHAPITRE PREMIER.}

Des roules de la sìre.

LE premier pas à faire dans l'examen des routes que suit la sève serait de déterminer si, comme on l'admet grénéralement, ce fluide possède deux mouvemens, l'un ascendant, l'autre descendant: le monvement ascendant de la sève n'est pas susceptible d'ètre.mis en doute; quant au mouvement descendant de ce fluide, les preuves sur lesquelles il doil être établi ressortiront de l'étude de certains faits qui ne peuvent ètre exposés qu'après l'examen préalable des routes que suit la sève dans son ascension.

Lorsque, dans nos climats, les premières chaleurs du printemps se font sentir, les végétaux se remplissent d'une sève abondante qu'ils pompent dans le sol, et ce mouvement ascendant de la sève est le prélude de leur développement végétatif. La vignne est certainement le végrétal qui offre le plus de facilité pour l'étude de ce mouvement ascendant de la sève. Tout le monde connaît l'abondance avec laquelle ce fluide s'écoule des plaies faites à cet arbuste au printemps. La surface de la section tranisversale d'unc branche de vigne offre une multitude de petits trous visibles à l'œil nu. Ce sont les ouvertures des tubes auxquels M. Mirbel donne le nom de fausses tra- 
thées; tubes qui, selon moi, sont une variété de ceux que j’ai désignés sous le nom général de tubes cormusculifères (1). M. Decandolle (2) nomme ces mêmes organes tubes lymphatiques; el j’adopte vocontiers cette expression, aujourd'hui qu'il m'est démontré que ces tubes servent effectivement, conme (e pensait M. Decandolle, à la transmission de la sève iscendante, qui est une lymphe incolore et insipide, 'est-ì-dire de l'eau presque pure.

Lorsque les premières chalenrs du printemps commencent à provoquer l'ascension de la sève, si l'on coupe transversalement, et en bisean, une branche de vigne, on voit ce fluide suinter lentement de la surface de la section. Si l'on essuie la sève à mesnre (qu'elle suinte, on voit, en armant son oeil d'une lloupe, les gonttelettes de cette sève sortir des onvertures des tubes lymphatiques; il n'en sort point du tissu compact qui les sépare. Celte observation est impossible ì faire lorsque la sève sort avec abondance; car à peine la surface de la section est-elle essuyée, qu'elle est reconverte par la sève, qui sort avec xapidité. A celte époque cependant, il est encore un. moyen de savoir quels sont les vaisseanx qui livrent passage à ce fluide. On prend une branche de vigne dont les deux extrémités sont coupées en biseau, afin de pouvoir observer plus facilement les orifices des

(1) Recherches amatomiques et physiologiques sur la slincture intime des animanx et des végútaux.

(2) Flore francaise, 3" ćctition. 
tubes lymphatiques; ees tubes sont alors entièrement remplis par la sève. Or, si l'on ploie légèrement ectte branehe par son milicu, ectte flexion comprimera la sève contenue dans les unbes situés à la partie eoncave de l'endroit fléehi, et la forcera à sortir par la surface des deux sections transversales qui terminent la branche. Lioil armé d'une loupe, on voit très-distinctement la sève sortir par les seules onvertures des iubes lymphatiques, et y rentrer rapidement lorsqu'on fait eesser la flexion qui opérail la eompression de ee fluidle, et le forçait de sortir. Il est à remarquer que, dans celtc eirconstance, la sève ne sort, anx sections terininales, que du côlé où se trouve la coileavité de la flexion, c'est-à-dirc dn seul eôté où il y a eomịression du fluide, et ccla quoiqu'il y ait plusieurs nœuds placés entre les extrémités et l’endroit ilćehi. Ceci proive $I^{\circ}$ que les tubes qui eontiennent la sève offrent nue cavité eontimue dans toute l'étenduc de la branehe, et que ecs tubes u'ont point de valvules qui favoriscraient lc mouventent ascendant de la sc̀ve en s'opposant à soll niouvement rétrogyrade; $2^{\circ}$ que e'est spécialement en ligue droite que s'opère la transunission de la sève dans ces tubes. Celle dernière assertion est mise hols de donte par l'expérience suivante.

A l'époque du printenps, oì la vigne pleure, jai choisi une tige de cet arbuste ấgéc d'un au, et des plis grosses. J'en ai retranché la partie supérienre par une section transversale, à l"instant la sève ascendante a coulé en abondance par eette blessure. Alors jai pratiqué à la tige une entaille, jusqu'au voisinage 
3a moelle, à mn pied environ au-dessous de l"exiémité tronquée : la sève a coulé par cette nouvelle ldessure, et a cessé de couler par la partie de la seccon transversale supérieure qui correspondait en rroite ligne à l'entaille. $\Lambda$ un pouce environ au-dessus de celue dernic̀re, j’ai pratiqué une nouvelle enviille semblable, qui a également donné issue à la sve, et qui a de même fail cesser l'émission de ce unide à la partie de la section supérieure qui lui corsspondait directement. Les deux entailles occupaient «s denx tiers de la circonférence de la tigge, el la sève ie sortait plus, ì la section supérienre, que par le icrs déla surface de cette section, correspondant, en gne droite, à la partic de la tige qui était restée inracte au-dessous. Je pratiquai une troisième cntaille, un pouce ant-dessous de la dernière, et de manière couper les unbes de la partie de la tigge qui était estéc intacte. De cette manière, toute communicaion directe se trouvait interronpue entre les denx rarties de la tigne supérieure el inférieure, aux trois entailles. An moment oì la dernière entaille fut faite, a sève cessa tout à fail de se montrer à la section ransvergale supérieure. Cette expéricnce prouve, l'une manière "certaine, que les tubes lymphatiques fui conduisent la sève ascendante ne comnuuiquent point latéralement les uns avec les autres, et quitils sont étendus en ligne droite dans la tigge, sans se dévier jamais du côté qu'ils occupent primitiventent. Cette dernière expérience scmble cn contradiction avec un fait bien connu, qui est celui-ci, que des en- 
tailles faites au tronc d'un arbre, de manière à couper tonte communication directe entre la partie supérieure et la partie inférieure, n'empêchent point la transmission de la sève des racines aux rameaux; mais celte contradiction n'est qu'apparente. La sève offre, outre son mouvement direct, un mouvement de diffusion générale, ainsi que nous le verrons plus bas. Les entailles faites au tronc d'un arbre interceptent senlement le premier de ces mouvemens, et laissent subsister le second, qui, dans certains cas, peut suffire pour nourrir ]'arbre.

Cies observations ne laissent point subsister de doutes sur la route que suil la sève dans son mouvement ascendant. Déjà les expériences de plusieurs physiciens, e s spécialement celles de Sarrabat ( I ) eide Bonnel (2), avaient prouvé que la sève ne monte ni par la moelle ni par l'écorce, el que son ascension s'opère exclusivement par les fibres ligneuses, expression vague, dont l'emploi ne peut paraître étonnant à une époque où l'anatomie des plantes étail encore imparfaite. Aujourd'hui, l'on sait que la substance ligneuse des végétaux contient plusieurs organes très-différens par leur structure, et par conséquent par leurs usages. Les tubes lymphatiques ou corpusculifères forment, avec les organes fusiformes, auxquels j’ai donné le nom de

(1) Dissertation sur la circulation de la sève, sous Je faux nom de Labaisse, 1733 .

(2) Recherches sur l'usage des feuilles, cinquième Mémoire. 
"iostres (I), les prineipales parties de la substanee gneuse; et ee sont ces organes différens que l'on a wuvent confondus ensemble sons le nom général de Hres ligneuses. J'ai répété les expérienees de Sarraut et de Bonnet, en faisant pomper des liqueurs eorécs à des végétaux, et je me suis convaineu que cest exclusivement dans les tubes lymphatiques et ans les traehées que ces liqueurs colorées s'introdui'snt : elles n'entrent point du tout dans les elostres. ues résultats de cette expérienee sont très-faciles à jir, spéeialement dans la vigne, dont les tubes lymthatiques, fort nombreux, se distinguent facilement ces faiseeaux de elostres, au milien desquels ils sont ttués. Il ne peut done rester aueun doute sur la desnation des tubes lymphatiques ì eonduire la sève seendante: ees tubes ne s'obstruent jamais, mème ans les couehes les plus aneiennes du duramen (2), iù ils ne cessent point de servir de canaux à la sève jmphatique ascendante. Ce fait est prouvé par l'exérience de Coulon (3) : cet expérimentateur perẹa

(1) Recherches anatomiques et physivlogiques sur la structure atime des animaux et des v'égétaux.

(2) Je propose de donner, cemme enl latin, ce nom de durazen à ce que l'on nomme viulgairenutule bois de coeur. Jusqua a e jour les botanistes ont désigué cette partie sous le simple nom c bois, la distinguant ainsi de l'anbier, qui, de cette manière, e serait pas du bois. Or, cela cst mavifestement contraire aus tés gévéralement reçues : l'aubier est du jeune bois cncore à 'état de moliesse et de blancherur; le duranuen est du vicux bois levenut dur et roloré.

(3) Journal de pligsique, 1. fis, 1' 3y's. 
le tronc d'un peuplier jusqu'au centre avec une tarière, et il vil que la sève ascendante sortait en abondance de la partie centrale de l'arbre. Une expérience d'un autre grenre nıa égaleınent prouvé cette vérité. Pendant l'hiver, je fis pratiquer une entaille circulaire au pied d'un gros chêne : cette entaille profonde avait emporté la totalité de l'aubier et une partie du duramen. Or, au printemps, l'arbre ne laissa pas de végéter comme à son ordinaire; et il continua de le faire pendant toute la belle saison, sans paraître souffrir de la profonde blessure qui avait été faite à son tronc. Cette expérience ne laisse aucun doute sur l'aptilude des tubes lymphatiques du duramen à transnettre la sève ascendante. Les tubes lymphatiques de l'aubier transmettent celte sève avec la même facilité, car il n'y a que de l'aubier dans les très-jeunes arbres; et il n'y a que cela non plus dans les troncs de quelques vieux arbres creux dont le duramen est entièrement pourri, ainsi que je l'ai observé quelquefois. Enfin, on peut ne laisser subsister qu'une portion de l'aubier d'un arbre, comme seul moyen de communication entre la tige et les racines, sans nuire d'une manière sensible à la transmission de la sève ascendante, ainsi que je m’en suis assuré souvent par l'expérience. Tout cela prouve d'une manière certaine que la sève ascendante se transmet avec une égrale facilité par les tubes lymphaiques de toutes les couches ligneuses, et que leur âge et leur dureté ne les privent en aucune manière de cette fonction. Il semblerait même résulter de l'expérience de Coulon, 
qui vient d'ĉtre citée, quc ce serait spécialenıent par Ilc centre de l'arbre quc la sèvc asccndante scrait transmise; mais cctuc asscrtion, appuyéc sur un fait particulier, nc doit pas ćtre généralisée. En cffct, j’ai observé quc dans la vigne c'est d'abord par la couche la plus extérieurc de l'aubicr quc s'effectue l'ascension ide la sèvc, au commencement du printemps. En coupant transversalement une branche de vigne, à cette époque, on voit la sève sortir à la circonférence, tandis que le centre reste sec.

La sève asccndante ne sc transmet point par le système cortical : ce résultat de l'expérience est d'accord avec l'obscrvation anatomiqne, qui prouve qu'il n'cxistc dans lc systc̀mc cortical aucun tube corpusculifère ou lymphatique. Cette considération vient encore à l'appui dc l'assertion que j’ai émise sur la fonction que j'attribue à ces tubes d'ĉtre les seuls conducteurs de la sève lymphatiquc ascendantc, car les trachées sont tout à fait étrangc̀res à cette fonction.

Les trachées, comme on le sait, ne se rencontrent à l'intérieur des tiges des végétaux dicotylés que dans l'étui médullaire seulcment: ellcs sont complètcment étrangères aux couches successivcs d'aubier qui se forment chaque annéc. Ccttc spćcialité dc position annonce indubitablcment une spécialité de destination dans les trachées. J'ai émis dans un antrc ouvragc (r) mon opinion sur lcs fonctions qui leur sont départies,

(1) Recherhes anatomiques et physiologiques sur la structure intime des animaux et des végétanx, cl sur leur motilité, p. 32. 
et je nc crois point supcrflu d'y revenir sommaircnıent ici.Les trachées contiennent bicn certaincment un liquide-diaphanc, ct non de l'air commc l'ont dit quelques physiologristes. Situées dans l'étui médullaire à côté des tubes lyıphatiques, on ponrrait croirc qu'elles scrvent comme cux à conduirc la sèvc ascendantc; mais on sera portć à cn doutcr, cn voyant qu’il n'existe jamais de trachées dans les racincs qui abondent commc les tigres cn tubes lymphatiques, conducteurs exclusif's de la sc̀ve asccndantc. Cepcudant, lorsqu'on met unc tige coupćc tremper par sa partie infériemrc dans un liquide coloré, ec dernicr sintroduil à la fois dans les tubes lymphatipues el dans les trachées. Plusicurs olsscrvateurs ont vu cc fait dont j’ai vćrifié l'exactitude, et qui cepondant ne doit point chre admis sans restriction. Lascension des liquides colorés dans les tubes lynuphatiques, porte ordisairement ecs liquides jusqu’an sommet des tigges, mais il n'cn est pas de micme pour les trachices. Les liquides colorés introduits dans lcur intérieur nc s’élèvent ordinairenent quc jusqu'an nivean du liquide dans lequel la tigge cst plonģée; à peinc, quelquefois, dépassent-ils de quclques liggnes le nivean de ce liquide. Cela pronve bicn évidemment que l'introduction des liquides colorés dans les trachćcs, n’est que le résuluat d'unc imbibition accidcntellc; cela prouvc cn mênc temps quc ces or granes nc sont point aptes, conıme le sont les tubes lymphatiques, à opérer l'ascension des liquides. Les trachées ont donc bicu certainenent diantes fouctions, et, commc le prouve 
l'observation anatomique, ces fonetions ont un rapport imnédiat avec les fonctions des feuilles dans lesquelles elles existent en gráande quantité. Or, les féuilles sont en quelque sorte les poumons des plantes. On sail que les animaus reçoivent de l'oxigène quiles cnvironne, et qui se fixe sur leur sanģ, une influenec vivifiante indispensable pour la conservation du nourcment vital : il ćtait naturel de penser que les végrétaux recevaient également d'un agent extéricur une influence vivifiantc. J'ai prowvé (I) que ectle influence viviflante était donnéc aux végcétanx par l'action de la lumière, en sorte qu'une même fonction qu'on pourrait appeler vivification, est excrećc par les animaux au nroyen de l'oxigénation, et par les végcétanx, au moyen de l'insolation. Or, il est permis de penser que, le liquide qui a reçı dans les fenilles l'influence vivifiante, la transporte avec lui par le canal des trachées jusque dans 'l'intéricur de la tigsc; car les trachées s'étendent des feuilles à l'étui médullaire de la jeune branche qui les porte. Ainsi les trachées des végrétaux auraient des fonctions analogues à celles des trachées des insectes : celles-ci conduisent l'air atmosphérique, qui cst un gaz vivifant; celles-là conduiraient un liquide vivifiant.

Les feuilles, les fleurs, les fruits des végétaux sont les parties auxquelles aboutit défnitivement la sève ascendante. I_à, une grande partic de ec fluide est

(1) Fecherches anatomiques ot phy-siologiques sur la structure intime des animarx et des végétaux, el sur leur motilité, p. S1. 
chassér par la transpiration, el livrée à l'atmosphère, qui la dissout; une autre partie, sournise à l'ćlaboration organique, se convertit en sève nourricière : cc sont surtout les fenilles qui sont chargées de ccttc importante fonction; aussi y a-t-il cessation de nutrition dans un végétal dont on enlève sans cesse les fcuilles; dès lors la tigge de cc végétal cesse de croître; ses fruits, devenus stationnaires dans lcur accroissement, ne tardent pas à sc faner ct à tomber. Les arbres que la nature appelle à porter le plus haut leur tige dans l'atmosphère, restent à l'état des plus faibles et des plus humbles arbrisseanx, lorsque leurs feuilles sont continuellement broutées par lc bétail. Tons ces faits prouvent incontestablement que les feuilles sont les organes élaboratcurs de la sève nourricière; elles joignent celtc fonction à celle d'être, cn quelque sorte, les poumons de la plante, ainsi que nous l'avons vu plus hant. Or, la sève élaboréc étant formée dans les feuilles, doit nécessairement prendre, à partir de là, un mouvement descendant : ce mouvement descendant de la sève élaboréc, est prouvé par une expérience très-connue. Si l'on enlève un anneau d'écorce à une branche d'arbre, la partie inférieure de la déeortication annulaire cesse de s'accroître en diamc̀tre, tandis que, dans la partic supérieure, cet accroissement reçoit un surcroît d'activité produit par la concentration, par la stase foreée du suc nourricier, lcquel manifeste la tendance qu'il a à descendre par le bourrelet qu'il produir à la partie supćrieure de la décortication annulaire. L'augmentation de nutrition 
qui survient en mène temps dans les liruits que porte la branehe à la partie inférieure de laquelle a été pratiquée cette décortieation annulaire, achève de prolıver que la sève élaborée y est aecumulée. L'enlèvement de l'éeorce a suffi pour opérer eette aecumulation, pour empêeher la sève élaborée d'obéir à la tendance qui la porte à descendre: on en a eonelu que c'est par l'éeorce que s'opère exelusivement la descente dé la sève nourrieière. Il faut l'avoner, eette eonclusion n’est point une déduetion rigoureuse du fait observé. En effel, la décortication occasionne la nort du tissu lignneux superficiel. Ne serait-il point possible que ce tissu ligneux de nouvelle formation, contribuât avec l'éeoree à la deseente de la sève? Cette opinion est celle d'un savant dont les ingénieuses expériences ont beaueoup contribué aux progrès réeens de la physiologie végétale. M. Knight, dans un Mémoire publié en 1806, dans les Transactions philosophiques (1), rapporte quelques expériences, desquelles il eonclut que la sève deseend par l'aubier lorsque cela est nécessaire pour la conservation de l" plante. Ainsi il regarde eette deseente de la sève par l'aubier eomme une marehe en quelque sorie accidentelle, paree que, selon lui, les vaisseanx de l'aubier servent ordinairement à l'aseension de la sève. Mais l'aubier n’est pas une substanee composéc d'une seule espèce d'organes végétaux; il eontient des tubes lymphatiques, bien eertainement destinés

(1) On the inverted action of ater allarmous vessels of trees 
à l'ascension de la sève, et des clostres qui bien certainement contiennent de la sève élaborée, et sont très-probablement les organes de la transmission descendante de cette sève. Ainsi, il n’est point nécessaire d'admettre, avec M. Knight, que l'action des vaisseaux de l'aubier soit quelquefois intervertie; et si l'expérience prouve qu'effectivement la sève élaborée descend dans l'aubier, il faudra reconmaître que c'est par une action naturelle et constante de l'une de ses deux parties constituantes, c'est - à - dire par les clostres, puisqu“il est prouvé que les tubes lymphatiques servent à l'ascension áe la sève. Voici les expériençes sur lesquelles M. Knight londe son opinion. Il avait remarqué que les tubercules ne sont point engendrés par un développement des racines, mais bien par un développement de certaines tiges sonterraines qu'il nomme coureüus (I) (rumners) : il supprima soigneusement ces coureurs sur un pied de solcnum tuberosum appartenant à une variété hâtive: de cette manière, il empêcha la formation des tubercules. La sève élaborée se trouvant alors en surabondance dans la plante, développa en grosseur plusieurs parties de la tige, et y forma des espèces de tubercules aériens : en même temps il se développa sur la plante des fleurs auxquelles succédèrent des fruits,

(1) Je croyais ávoir fitit cette remarque le premier, dans mẹ Recherches sur. l'accroissement et la reproduction des végétaux; je ne connaissais point encore, lorsque je les publiai, le hiémoire de M. Kunght dont il est ici question. 
"Le qui n’arrivait point ordinairennent à cetle varićté Hitive, dont toute la sève nourricière était employéc tuu développenuent précoce des tubercules. Cette obserration prouva à MI. Knight que c’est la sève élaborée Hescendante qui nourrit les ubbercules du solanum "uberosum. Mors il voulut savoir si ces tubercules -grossiraient malgré la décortication annulaire de la lïrgyc auprès du sol : il pratiqua celle opération, ct Ill vil que les tubercules situés au-dessous de celle Hécoltication s'acciurent un peu en ğlosscur, mais Ibicn moins qu’ils ne l’cussent fail si la tige fùt restée thans son intégrité. MI. Knight en conclut que c'est cordinairement par les válisseaux corticaux que la sève ¿élaboréc descend veis les tubercules, et que dans ll'expérience dont il est ici questiou, la voic ordinairc cde la desccnte de la sève ćtant supprimée par la décortication annulaire, celie sève, pour descendre vers lles tubercules, se fiaya une roule au travers des vaisseaux de l'aubicr, dont l'action se trouva ainsi intersvertie. Une autre expérience acheva de prouver à . MI. Knight que c'est effectivement la sc̀ve élaborée idescendante qui nourrit les tubercules : il coupa pres'qu'entièrement plusieurs tiges de solanum tuberosum près de la surface de la terre, et ne laissa subsister - de communication cntre la partic aérienne el la partic soutcrraine de ce végélal, qu’au moyen d'une petite portion d'aubicr revêtue de son écorce. Les tiges, ainsi préparées, produisirent toutes des tubcrcules aériens par le gonflement de leur's articulations, expression qui désigne d’une manière peu précisc les partics 
dont le développerment tuberenleux a été déterminé par la stase de la sève nourrieière, laquelle ne pouvant se transmettre en grande quantité à la partie souterraine du végétal, oeeasionna dans la partie aérienne un exeès de nutrition de eertaines parties.

Avant de eonnaître les expérienees de M. Knight, qui viennent d'être exposées, j'avais fait ces mêmes expérienees, et javais obtenu à peu près les mêmes résultats. Il est tout simple qu'on se reneontre dans le ehemin de la vérité. La déeouverte de ces faits appartient à M. Knight; et si je rapporte les expérienees du même genre que j’ai faites, e'est paree qu'elles offrent peut-être plus de détails que eelles de l'exeellent observateur qui m'a préeédé dans eette earrière.

Dans la vue d'étudier les effets de la déeortieation annulaire ehez les végétaux herbaeés, je pratiquai eette opération à plusieurs tiges de solanum tuberosum, près de la surfaee du sol. J'avais eu soin de ne laisser à ehaque tige qu'un seul tubereule dont j’avais soigneusement noté le diamètre, afin de voir s'il prendrait de l'aeeroissement : eette expérienee eut ull résultat auquel je ne m'attendais pas. $A_{11}$ bont d'un mois environ, je vis avee surprise que plusieurs de mes tiges de solanum tuberosum avaient produit des tubercules aériens, qui étaient situés dans les aisselles des feuilles, et étaient évidemment engrendrés par un mode partieulier de développenent du bourgeon axillaire de la feuille : e'étaient véritablement des tiges renflées; aussi ehaeun de ees tubereules était-il tcrminć par un petit bouquet de feuilles appartenant à l'extrémité de ees 
igres renflécs. Les tubercules aćriens dont il est ici question ćtaient généralement fort petils; les plus gros ggalaient la grosseur d'une noix; les plus petits n'éaaicnt pas plus gros qu'un pois : ces tubcrcules, qui ue tenaient à la tige que par un pédicule grềle, comme des fruits y tiennent par lcur pédonculc, étaicut de couleur rosc ou violacće; leur parenchime contenait He la fécule, comme celni des tubercules soutcrains. Toutes nues tigges de solanum tuberosum ne préscncaient pas ce phénomènc de production de tubercules mériens; cependant, tontcs étaient éğalcment dćcoritiquées circulairentent à lcur partie inféricure. Jc les soumis à un cxamen comparatif, pour savoir à quoi tenait cette différence; je m'aperçus que, dans les triges qui avaient produit des tubercules aéricns, la décortication anmulaire avait occasionné la mort de la plus grande partie du tissu ligneux du système central, en sorte que la tige ne communiquait plus avec lles racines que par un petil faisceau lignenx qui avait. conservé de la vie. An contraire, dans les tiges qui m'avaicnt point produit de tubercules aćricus, le tissı iligneux subjacent à la récortication annulairc avait ten cnticr conservé sa vic el sa fraîchcur, à l'exception? 'sculement d'unc très-légère couche superficielle de ce 'lissn lignenx, qui s'était desséchéc. An reste, dans toutcs ces tiges de solanum tubcrosum mises en expérience, les tubercules sonterrains avaicnt pris de l'accroissement; mais cet accroissement était bien plus considérable aux tiges qui n'avaicnt point produit des tubercules aéricns. Il me parut que je devais attribuer 
la production de ces derniers à ce que la sève élaboréc descendante éprouvait un obstacle à sa transmission vers les racines, par le fait de la très-faible communication qui existait entre la tige et les racines, au moyen d'un seul petit faiscean de tissu ligneux du système central. S’il n'y eut pas de production de tubercules aériens sur les tiges qui communiquaient largement avec les racines par la conservation intégrale du tissu ligneux subjacent à la décortication annulaire, cela me parut provenir de ce que la sève nourricière descendante n'était pas suffisamment retenue dans la tige, et qu'elle était transmise facilement aux racines par la communication établie au moyen du tissu ligneux. La validité de ce soupçon me fut confirmée par l'observation des tubercules souterrains, qui étaient considérablement développés aux tiges qui n’avaient point produit de tubercules aériens, tandis qu'ils n'avaient presque point pris d'accroissement aux tiges sur lesquelles ces tubercules aériens s'étaient développés. Ces phénomènes dépendaient évidemment de ce que la sève élaborée fournie par la partie aérienne de la tige, avait été transnise en grande quantité aux tubercules souterrains, et palt conséquent n'avait point été retenue et accumulée dans les tigges qui, à cause de cela, n'avaient point produit de tubercules aériens. La production de ces derniers dépendait, au contraire, de la stase forcée de la sève élaboréc dans les tiges, qui, par cette cause, n'avaient développé que fort peu les tubercules souterrains, auxquels elles ne pouvaient faire parvenir 
[u'ume très-petite quantité de sève élaborée. Il résulait de ces expériences, que le tissu ligneux du sysème central était propre, conme le système corrial, ì transmettre la sève descendante : ce r’ésultat, sour être incontestable, avait besoin d'être appuyé ur des observations nombreuses. Je répétai donc, sendant trois années de suite, les expériences que je iiens de rapporter. Il est bon de faire observer ici [ue, pour voir réussir ces diverses expériences, il iaut être favorisé par un temps constamment humide; ar lorsque les tigges n'ont qu'une très-faible commuication avec les racines, elles ne peuvent recevoir le ces dernières une quantité de sève lymphatique uffisante pour remplacer celle que la transpiration mlève à la plante par un temps sec. Lorsque le temps stuviens, les feuilles de la tige absorbent ellesmêmes du dehors la quantité de sève lymphatique nécessaire pour entretenir la vie el la fraîcheur de la olance.

Les résultats des expériences que je vicus de rapporter m'avaient donné lieu de penser quc la producxion des tubercules aériens était due à ce que la tige ne communiquait plus avec les racines qu'au moyen d'un petit faisceau de fibres ligneuses. Pour m'en assurer, je préparai un grand nombre de tiges de solctmum tuberosum, de manière à faire artiliciellement ce que le hasard avoit produit; après avoir opéré la ldécortication annulaire des tigges de cette plante auprès du sol, je ne laissai subsister qu'nne très-petite portion du lissu ligneux du systòme: central, et j’eus 
soin de soutenir la tige en l'attachant à un étai. Dans d'autres tigges semblablement décortiquées, je laissai le sytème central dans toute son intégrité. $\mathrm{Or}$, j’observai eonstamment que, dans toutes les tigges oì celte intégritédu système eentral avait été eonservée, il n’y eut point de production de tubercules aériens, tandis qu'il y en ent eonstamment sur les tiges où je n'avais laissé subsister qu'une petite portion de tissu ligneux à l'endroit de la décortication annulaire. On pourrait croire peut-être que la moelle serait le moyen de communication par lequel la sève descendante pourrait se transmettre aux raeines; mais je me suis convaincu du eontraire par l'observation d'une tige dont la moelle était. entièrement détruite au niveau de la décortication annulaire, et dont le tissu lignenx conserva dans eet endroit son intégrité presqu'entière. Or, cette tige ne produisit point de tubereules aériens, ee qui pronve que, malgré la destruetion de la moelle, la sève élaborée deseendante était facilement transmise anx raeines. 11 n'y a donc aucun doute que ce ne fùt par le tissu ligneux du système central que s'opérait eetle transmission. Pour varier ce genre d'expériences, je préparai plusieurs tiges de solanum tuberosum, de manière à ce que chaque tigge ne communiquât avee les racines que par un très-petit faisceau de tissu ligneux revêtu de sa petite portion d'éeorce. Or, quelque petite qu'ait été la communication ainsi établie entre les racines de la tige, jamais il n'y a eu sur cette dennière de production de tubercules aériens. La différenee qui se trouve iei entre les expériences 
M. Knight et les miennes, provient peut - être de que nons avons opéré sur des variétés différentes an solanum tuberosum. Quoi qu'il en soit, il résulte e ce fait que le tissu ligneux revêtu de soiı écorce, ist pour la sève descendante la voie de transmission plus libre et la plus facile. Ces expériences prouent en outre, d'une nanière décisive, que le tissu goneux du système central peut opérer à lui seul la cansmission de la sève élaborée descendante, eı cela ar une action qui lui est naturelle, et non par une stion intervertie, comme l'a pensé M. Knight. Cette ansmission de la sève élaborée descendante s'opère vec plus de facilité, lorsque le systènte central est zvêtu par le système cortical ou par l'écorce : cellei est, par conséquent, la voie de transmission la plus acile pour la sève descendante; mais elle ne jouit as exclusivement de ce privilégre. J'avais déjà énic nelques idées sur cette théorie, dans nes Recherches ur l'accroissement et la reproduction des végéalux (I), et j’y avais été porté par la considération de la structure des bourrelets qui se forment dans les arbres a la partie supérieure de la décortication annulaire. J'avais observé qne ces bourrelets reproducceurs étaient composés non senlement d'écorce, ınais aussi d'aubier, el j'avais cru pouvoir en conclure que da descente de la sève s'opérait par le tissu de récente production, et encore à l'état de mollesse du liber et de l'aubier tout à la fois. Les expériences que je viens

(1) Mómoires du Muséum d'histoive naturells, 1. 7, p. i17. 
de rapporter, prouvent que ce premier aperçu élait fondé; la sève élaborée descend effectivementà la fois. par le système cortical et par le système central, dans les plantes herbacées, ce qui prouve qu'elle doit descendre également par ces deux systèmes à la fois chez les végćtaux liggneux. Chez ces derniers, cette descente a spécialement lieu par les tissus fort jeunes, et pour ainsi dire herbacés, du nouveau liber et du nouvel aubier. Cependant il est probable que l'aubier dans son entier, ainsi que les couches d'écorce un pen plus àgées que le liber, contribuent aussi un peı à transmettre la sève descendante. Quant à la vicille écorce et au duramen, il paraît qu'ils sont l'un et l'autre étrangers à cette transmission. En effet, la métanıorphose successive des couches d'aubier en duramen, semble prouver que le plus vieil aubier reçoit encore de la sève élaborée; car ce changement de l'aubier en duramen, est un phénomène chimique dépendant de la nutrition; phénomène lent à s'effectuer, et qui ne s'opère qu'après plusieurs années dans les arbres. J'ai fail voir, dans mes Recherches sur la structure intime des animaux et des végétaux (1), que ce changement s'opère par le durcissement et par la coloration d'une substance contenue dans les clostres, substance dont la production est due à la sève élaborée, et qui n'est dans le fait que cette sève ellemême devenue concrète par la vaporisation de son eau surabondante. Je suis donc porté à considérer ces

(1) P. 3 \%. 
clostres qui abondent dans le bois et dans l'écorce, comme les organes par lesquels se transmet la sève ćélaborée. Cette transmission s'opère, comne nous le werrons plus bas, par une action particulière des clositres, qui sont les uns à l'égard des autres dans un état ide succion : c'est au travers des parois de ces organes (que doit s'opérer cette transmission, car ils n'ont auccune communication directe entre eux. Au reste, le Imouvement de la sève élaborée n'est pas tonjours desccendant, comme je l'ai établi jusqu'ici : en effet, il ddevient ascendant dans les pédoncules des fruits, puisque c'est à lui qu'est spécialement dû leur dévelloppement. Il devient également ascendant pour opérer l'élongation des tiges et le développenent des bonrgeons; car si la sève lymphatique ascendante était transmise aux bourgeons ell développement telle 9qu'elle est puisée dans le sol par les racines, elle serrait presqu'entièrement impropre à Jeur nutrition, ppuisqu'elle ne serait que de l'eau presque pure. Nous devons encore à M. Knight les expériences qui prouivenı que la sève lymphatique en montant dissout une ccertaine quantité de sève élaborée qui est conservée cdans le bois pendant l'hiver: or, j’ai prouvé que c'est (dans les clostres que se fait ce dépôt de la sève élabor rée et sa conservation; c'est donc de ces organes que sort la sève élaborée qui se mêle à la sève lymphatique : ascendante : ce mélange est prouvé par les expériences suivantes de M. Knight. II recueillit au printemps la - sève ascendante du sycomore prise à différentes haui teurs au - dessus du terrain, et il mesura comparati- 
vement sa pesanteur spécifique. Il mouva que la sève recueillie an niveau de la surface de la terre était insipide, el avait une pesanteur spécifique de I,004; recueillie à sept pieds de hautcur, sa pesantcur spécifique étå̉l de 1,008 ; et à douze pieds de hauteur, cette même pesanteur étail de 1,0 I 2, et la sève avait aequis une saveur douce (1). M. Knight fit les mêmes expériences, et avec les mêmes résultats, sur le bouleau (betula alba). Ces faits prouvent bien évidemment que la sève lymphatique ascendante dissout, à mesure qu'elle monte, une certaine quantité des substances extraclives nutritives que contient le tissu du végétal, et e'est à cette addition yu'elle doì de devenir cllemême une sève nourricière pour les bourģcons dont elle opère le développement.

La sivve lymphatique n'est pas toujours ascendante; on sait, en effet, que les feuilles pompent une grrande quantité d'eau, quand l'atmosphère esı humide: elles font alors, sous ce point de vue, l'office de racines; elles introduisent dans le végétal de la sève lymphatique; et celle-ci, introduite par la partie supérieure de la plante, doit nécessaircment affecter une marche descendante. Voici deux observations qui prouvent directement cette marche descendante de la sève lymphatique: Un éboulement de terrain avait mis à nu, dans une grande étendue, une racine verticale d'un arbre sur laquelle il s'était développé un rejeton de

(1) Philosophicul transactions 1825 , concerning the state in which the true sap of trees is deposited cluring winter. 
itige, à deux mètres environ de l'endroit où cette raccine aboutissait au tronc. L'extrémité de cette racine cétait restée implantée dans le sol, et continuait ainsi ù fournir de la sève à l'arbre. Je coupai, pendant l'hiver, celle racinc au-dessous de l'origine du rejeton, cen sorte que celui-ci fût privé de la sève lymphatique aascendante que cctte racine lui eùt fourni, et qu'il nıe pouvait plus en recevoir que du tronc, c'est-à-dire Ipar un mouvcment descendant. Or, au retonr du prinltemps, le rejeton végéta, et ne discontinua point, dans lla suite, de s'accroître: ce fait mc prouva que la sève Hymphatique avait affecté dans la racine un mouvement descendant. Ayant fait la même expérience sur tdes racines qui nc portaient point de rejetons de tige, celles se desséchèrent toutes: ce second fait me prouva que la descente de la sève dans la racine était déterminée uniquement par l'action vitale du rejeton de iige qu'elle portait à son extrémité. L'effet de cette action attractive, transmis de proche en proche, s'étendait jusqu'au tronc de l'arbrc, ct déterminait la sève ascendante à prendre daus la racine verticale un mouvement descendant. Cepcndant, la sève lymphaique ascendante pénètre en rétrogridant, et par son propre mouvement, dans les racines isolées de la terre et privées de rejetons de tige, ainsi que me l’a prouvé une autre expérience. J'ai ôté la terre qui couvrait une racine de vignnc, que j’ai déconverte ainsi jusqu’à son origine du tronc, et j’ai tronqué cette racine à son extrćmité : cette opération fut faite au printemps, à l'époque où la vigne versc abondamment de la sève 
par ses rameaus tronqués; je vis la sève couler goutte à gontute, pendant un jour, par l'extrémité de cette racine tronquéc, comme clle eût coulé par l'extrémité d'une branche coupéc. Ainsi, nul doute que, dans cette circonstance, la sève lymphatique n’éprouvât un mouvement descendant. Je reviendrai plus tard sur celle expéricnce.

Lia formation de la sève élaborée est le résultat d'une fonction qui appartient nécessairentent à tous les végétaux, car tous on besoin de se nourrir; et pour y parvenir, ils doivent préparer leur substance alimentaire. Cette production de la sève élaborée pent être considéréc comme le résultat d'une sorte de sécrétion. Duhamel a donné à cette sève élaborćc lc nom de suc propre. Or, il me paraît que, sous ce nom, on confond plusieurs fluides essentiellement dillérens. En eflet, il existe chez certains végétaux un fluide, également le produit d'une sécrétion, qui est souvent laiteux, qui d'autres fois est gommeux on résincux: ce fluide paraît essenticllement différent, du moins dans certains cas, de la sève élaboréc nourricière. Ainsi, chez le rhus typhinum, par exemple, il y a dans l'écorce plusieurs rangées de tubes qui conticnnent un fluide laiteux. Le système ccntral de cet arbre ne contient aucun fluide semblable, et cependant les elostres de ee système contiennent de la sève élaborée, comme il y en a dans les clostres du système cortical : cette sève élaboréc est donc différente du suc laiteux que renferment les tubes contenus dans l'écorce. D’un autre côté, il est difficile de 
nne pas considérer comme nue sève nourricière le Ifluide laiteux abondant qui remplit les deux systèmes cortical et central dans le fignuier (ficus carica). Il me paraît donc que les qualités extérienres des Ifluides ne sont pas des indices sulfisans pour déterminer leur nature et leur usage, par rapport au végétal; et il me paraît pronvé quon doit distingner la sève élaborée pour la nomriture du végétal, de ces sucs sécrétés qui paraissent êtı'e des résidus excrémentitiels de la substance nutritive : tels sont, parmi ces derniers, les fluides résineux ou gommo-résineux que contiennent les vaisseaux propres de certains vé¿étaux.

Le tissu cellılaire alvéolaire qui constitue la moelle, ou la medulle centrale, n'est point une voie de transmission pour la sève ascendante. J'ai expérimenté, en effet, qu'en laissant la moelle comme seul moyen de communication entre la partie inférieure et la partie supérieure d'une branche, cette partie supériemre se flétrissait, et se desséchait très-promptentent. J'ai fait cette expérience sur des branches fort jeunes d'églantier (rosa canina), dont la moelle était encore à l'état de fraîchenr; car sur des branches âgées, donı la moelle est à pen près desséchée, celle expérience n'eût pas été concluante. On sait d'aillenrs, par les expériences de Sarrabat et de Bonnet, que-les liquides colorés ne sont point aspirés par la moelle des branches coupées que l'on met tremper dans ces liquides par leur partie inférieure. Cependant, il est évident que le rissu cellulaire de la moelle des jeunes 
branches, et même des grosses tiges de certaines plantes herbacées, est rempli de sève. Nous allons rechercher quelle est la nature de ce liquide médullaire, dont la marche doit être fort lente, et qui ne doit s'introduire dans la moelle qu'en pénétrant au travers des parois des cellules, qui n'ont aucune communication directe les unes avec les autres; car j'ai démontré la non existence de ces pores, ou plutôt de ces trous par lesquels, selon M. Mirbel, les cellules auraient communiqué directement les unes avec les autres (I). M. Dupetit-Thouars regarde la moelle comme le réservoir de la substance nutritive qui doit servir au développement des bourgeons. Cette idée me paraît juste à certains égards, et plusieurs faits semblent la confirmer. La moelle, en effet, n'est à l'état de vie que dans les très-jeunes branches qui développent actuellement des bourgeons, ou qui en ont à développer. Elle est toujours désséchée et frappée de mort dans les vieilles branches qui n'ont point de bourgeons, et ses cellules y sont vides, tandis qu'elles sont toujours pleines de sucs dans les jeunes branches. Que l'on examine, en hiver, une branche de vigne poussée dans l'année précédente, et garnie par conséquent de bourgeons, on trouvera la moelle devenue roussâtre, et frappée de mort partout, excepté dans chaque noud, vis-à-vis de l'endroit où est situé le bourgeon qui paraît devoir se nourrir, au printemps, des sucs élaborés conservés dans cette

(1) Recherches sur la s'ructure intime des végétaux, ctc. 
ROUTES HE LA SEVE.

partie de la moelle avec laquelle il est cu communi¿cation. Des expériences positives miont pronvé d'aillleurs que la moelle est le réceptacle des substances lles plus élaborées, et les plus éuinemment propres à lla untrition. J'ai rapporté plus haut que je suis parwenu à faire produire des tubercules aériens aux tiges du solamum tuberasum, en retenant daus ces tiges lla sève élaborée, quii, dans l'état naturel, tend à descendre vers les racines, ponr servir au développement ides unbercules souterrains. Or, i] m'a été facile de voir que ces tubercules aériens étaient formés spé(cialement par un développenent considérable de la ımoelle des jeunes branches, on plutôt des bourgeons. IChez enx le tissu ligneux avait presque entièrement disparu. La moelle, ainsi développée, contenait de la fécule, comme il y en a dans les tubercules souterurains. Celte hypertrophic de la moelle ne s'était pas bornée à former des tubercules aériens, elle avait gonflé presque toutes les jeunes branches dans le voisinage de leur insertion à la tige principale. J'ai voulı voir eu quoi cette moelle, nourrie avec excès, différait, au microscope, de la moelle du même véyétal dans l'étal normal. Les cellules qui composent la moelle du solanum tuberosum offrent, coume e'est l'ordinaire chez tous les végétaux, une certaine quantité de ces corpuscules globulenx que j’ai considérés comme des organes nerveux ( I ). Or, j’ai vu que, dans la moelle qui avait un excès de nutrition, ces corpus-

(1) Recherches sur la structure intine des wégétanx, clc. 
cules étaicnt tellement nombreux, qu'ils ne laissaient aucnu intervalle entre eux dans les parois des cellules qui les portaient. C'est donc en partie par l'augmentation du nombre de ces corpuscules globuleux que se marque l'excès de nutrition de la moelle, dont les cellules augmentent aussi considérablement en nombre. Chacun de ces corpuscules est un grain de fécule dans les tubercules. Or, j’ai démontré, dans l'ouvrage que je viens de citer, que ces corpuscules sont de petites cellules globuleuses remplies d'une substance particulière; il en résulte que l'état de fécule est une manière d'être de cette substance que je considère comme nerveuse, et que chaque grain de fécule est contenn dans une petite enveloppe ınembraneuse. Cette dernière vérité, à laquelle j’étais arrivé par l'observation anatomique, a été dernièrement démontrée par M. Raspail, qui, par l'analyse de la fécule, a fait voir qu'elle est composée d'une substance extérieure insoluble dans l'eau, et qui sert d'enveloppe à la substance soluble qu'elle renferme. On ne pent douter que ces corpuscules globuleux contenus dans les parois des cellules, n'aiemt un usage quelconque dans l'économie végétale. Dira-t-on que c'est de la matière nutritive mise en réserve pour les besoins de la plante? Cette idée, qui se rattache à l'opinion de M. Dupetit-Thouars sur les usages de la moelle, me parâit vraie; et cependant, à mon avis, la saine philosophie de la science s'oppose à ce qu'elle soit admise sans restriction. Aucune espèce d'être, dans la nature, n'a la destination expresse de servir à 
11 nourriture d'une autre espèce : cette destination i'est qu'éventuelle. De même, dans les êtres vivans, tucune espèce d'organe n'a la destination expresse et ixclusive de nourrir les autres organes aux dépens lie sa propre substance; cette destination n'est de même qu'éventuelle; chaque organe a des fonctions ıqui lui sont propres, et qui sont plus ou moins né'eessaires à la vie génćrale de l’individu : c'est là la aaison de son existence. Un or'gane, après avoir r'embli ses fonctions pendant un certain temps, peut perHre son utilité; il peut devenir impropre à remplir :es mêmes fonctions : c'est alors que la matière qui zntre dans sa composition peut acquérir un nouvel usage purement éventuel, celui de servir à la nutritiion des autres organes. Le règne animal nous offre tan fait qui rendra cette vérité très-palpable. Les têtaards des batraciens ont une queue musculaire, au moyen de laquelle ils nagent comme les poissons. 10r, à l’époque de la métamorphose, la moelle épimière se retire de cette queue, selon M. Serres, et la Iprive ainsi de la vitalité énergique dont elle jouissait. IDès lors cette partie, devenue inutile sous le point ide vue de ses fonctions antécédentes, n'a plus que Il'utilité éventuelle de nourrir, anx dépens de sa subsItance, sans cesse absorbée, les antres parties de l'ani$1 \mathrm{mal}$, et spécialement les membres postérieurs, qui se ' développent rapidement à celte époque, sans que l'animal ait besoin de prendre de la nourriture dı dehors; il a dans sa queue musculaire une substance nutritive toute élaborée, et qui n'a besoin que de chan- 
yer de place. On en peut dire autant des autres matériaux organiques, qui, comme la graisse, sont absorbés pour servir à la nutrition, spécialement chez les animaux qui jeûnent pendant l'hiver. Ces substances organiques jouent un rôle particulier dans l'économie, ou sont les résultats nécessaires de l'action des organes; c'est d'une manière purement éventuelle qu'elles servent subséquemment à la nutrition: elles ne sont pas faites exprès. On en doit dire autant des substances qui, chez les végétaux, paraissent servir à la nutrition de l'individu dans lequel elles existent. Ces substances appartiennent à des organes dont les fonctions, nécessaires et actives à une certaine époque, ont été postérieurement abolies. Dès lors, les substances composantes de ces organes prennent, si leur nature le permet, l'utilité éventuelle de servir, par leur absorption et leur transport, à la nutrition des autres parties du végétal. Ainsi, pour revenir au solanum tuberosum, les tubercules, après l'époque de leur maturité, et lors de leur végétation, livrent leur substance composante, comme matériaux de nutrition, aux nouvelles tiges et aux nouvelles racines qu'ils produisent; alors ils ont cessé de vivre par eux-mêmes, et ils ne tardent pas à se flétrir, et à subir la décomposition putride qui détruit ceux de leurs élémens organiques que les nouvelles productions n'ont pu absorber. Ce que je viens de dire des tubercules du solanum tuberosum, peut et doit s'appliquer également aux substances nutritives qui accompagnent ordinairement les embryons végétaúx. 
ues eonsidérations me ramènent naturcllement à mon coint de départ : je veux dire aux fonetions de la moelle. Je ne eontesterai point à M. Dupetit-'Thouars jon opinion sur l'usage qu'il attribuc à cette partie ın véçétal, d’être le réservoir de la subsınee alinentaire des bourgeons, ou des embryons fixes, comme il les appelle; eela me paraîl même très-vrai. Hais je reeonnaîtrai, dans celte même partie, unne inction antérieure à eet usage, et cette fonetion doit être fort importante, à en jugrer par le volume considérable de la moelle dans les branehes nouvelcement sorties des enveloppes du bourgeon. Le sysième ceutral de ees jeunes branehes n’est véritablement que de la moelle enviromnée d'un étui médulaire. Or, si l'on considère que c'est à cette époque le formation première que la vie végćlale a le plus ll'aetivité, on sera porté à admeture qu'elle le doit aux organes qui entrent dans la eomposition de la mroduction nouvelle; ces organes sont les innombraoles corpuscules grlobuleux qui sont situés dans les parois des eellules de la moelle. Or', si chez les animaux le système nerveux est le siége spéeial de la vie, il pourra être permis de eonsidérer comme analoğues au système nerveux des animaux, les orgyanes ldes végétanx auxquels paraîl exclusivement due l'aeiivité du mouvement vital, activité qui se manifeste par la rapidité de l'aeeroissement. On voit, par ees considérations, que ee n'est pas tout à fait sans raison Ique je eonsidère les eorpuseules globuleux des végétaux eomme des organes nerveux; je n'entends par- 
lì que des organes spécialement dépositaires ou producteurs de la force particulière aux étres visuns, et que l'on nomme force vitale.

Ainsi, il est dénontré que la moelle étrangère à l'ascension de la sève lymphatique, admet dans ses cellules la sève élaborée qui lui fournit les matériaux nécessaires à la formation et au développement -des nombreux corpuscules que j'appelle nerveix $x$, et qui existent en nombre plus ou moins considérable dans les parois de ces mêmes cellules. Or, comme la moelle est fort abondante dans les jeunes branches récemment sorties des enveloppes du bourgeon, il me paraît fort probable que, selon l'opinion de M. DupetitThouars, la matière nutritive nécessaire à leur développement leur est fournie par la moelle de la branche mère, moelle qui, par l'abolition de ses fonctions vitales, n'a plus d'autre usage que d'être un reservoir de substance nutritive qu'elle livre à l'absorption des nouvelles branches avec lesquelles elle communique.

Il résulte des faits qui viennent d'être exposés, que la sève lymphatique et la sève élaborée ont chacune des organes spéciaux de transmission; ces deux sèves tendent à une diffusion générale chacune dans le systènı d'organes qui est spécialement affecté à sa transmission : si la sève lymphatique est la plupart du temps ascendante, cela provient de ce qu'elle tire principalement son origine de la partic terrestre du végétal; si la sève élaborée, au contraire, est la plupart du temps descendante, cela provient de ce qu'elle tire exclusivement son origine de la partie aérienne 
vćgétal; mais, dans plusicurs circonstances, ces ?mx sèves peuvent, comme nous venons de le voir, uervertir la direction la plus générale de leur marze. Dans ce mouvement de diffusion généralc, la we lymphatique affectc quelquefois une marche deszzndante, et la sève élaborée une marche ascendante. iinsi il n'y a point, à proprement parler, de circuution chez les végétaux; chez eux, il n'y a point de stour de la masse du fluide au point duquel il était arti, comme cela a lieu dans la circulation des aniנaux : tout se réduit dans les plantes à la diffusion :únérale de deux sortes de sèves, dont l'une tire gééralenicnt son origine des racines, et l'autre des ppendices de la tiğe, et qui doivent par conséquent Ifecter dans celte diffusion une marche inverse.

Dans cet exposé dc la marche générale de la sève, n'ai point fait mention de l'épanchement de ce huide cutre le bois et l'écorce, épanchement qui, dans ees arbres, a lieu deux fois l'année, au printemps et u mois d'août. L'origine de cet épanchcment séveux i'est pas cncore bien connue; on ignnore s’il est dû la sèvc lymphatique ou à la sève élaborée. Cornme 'cst spécialement au printcups que ce phénomène ce montre dans tout son développement, c'est-à-dire à "époque où la sève lymphatique monte avec abonlance dans la tigge, on serait, il senble, autorisć à yenser que cet épanchement serait produit par cette lernière sève. Cependant, une obscrvation qui m'est propıe, semble prouver que c'est la sève élaborée qui fournit la matièr: de cet épanchement. J'ai vu un 
gros tronc de noyer (juglans regia) abattu pendant l'hiver, chez lequel l'épauchentent de sève entre le bois et l'écorce ne laissa pas d'avoir lieu au printemps. Ici, cet épanchement ne pouvait être opéré qu'aux dépens de la sève conservée dans le tissu de l'arbre depuis l'année précédente; il est probable par conséquent que c'était de la sève élaborée dont l'usage, dans cette circonstance, est de servir à la nutrition et au développement des deux nouvelles couches de liber et d'anbier. Celte sève paraît sortir du tissu ligneux de l'arbre par nne marche horizontale, et je pense qu'elle est versée par le tissu cellulaire articulé horizontalement qui compose les rayons médullaires. Voici l'observation sur laquelle je me fonde à cet égrard; je la tiens des ouvriers qui travaillent à décortiquer les jeunes chênes pour les besoins de la tannerie, et j’ai étć plusieurs fois à mème d'en constater l'exactitude. Lorsque les chênes sont en pleine sève, et qu'ils se décortiquent par conséquent avec beancoup de facilité, s'il survient un vent froid du nord, l'épanchement de sc̀ve entre le bois et l'écorce disparaît presqu'entièrement, et il n'est plus possible d'enlever l'écorce. Cette disparution de la sève épanchée ne peut évidemment avoir lieu d'une manière aussi subite, que parce qu'elle rentre dans le tissu du végétal. L'observation que je viens de rapporter touchant ce tronc de noyer qui, quoiqu'abattu et privé de ses branches comme de ses racines, ne laissa pas de produire un épanchement séveux entre le bois et l'écorce, sert encore à prouver que c'est par une 
narche transversale ou par une diffusion horizontale nrculaire que s'opère eet épanchement de sève qui zntre par la nıème voie dans les tissus qni l'ont verée Jorsque la température vient tout à eoup à baisser. ïgnore eomment la température agit dans cette eirmnstance, je m'en tiens à l'exposé du fait, qui peut corter à penser que c'est par les rayons médullaires tue s'opère eette diffrnsion horizontale de la sève d'où ¿sulte son épanehement entre le bois et l'éeoree. An esste, il paraîtra bien probable que cet épanchement, u son abondance, est produit à la fois par la sève Itaborée et par la sève lymphatiqne qui se mèle avee Ille. Lopinion que j'émets iei sur les fonctions des ayons médullaires, que je eonsidère eomme les oranes qui épanehent la sève entre le bois et l'écoree, est rrtifiée par les eonsidérations snivantes. On sait que es rayons médullaires appartiennent exelusivement unx végétaux dieotylés, qui seuls aussi s'accroissent en iiamètre par la produetion de eorrehes successives et concentriques. La production de ees nouvelles conthes est le résultat de l'abondance de la sève nourriiière, qui se porte entre le bois et l'éeoree. Or, les véétaux dicotylés ayant seuls des rayons médullaires, t ayant seuls aussi eet épanehement de sève entre e bois et l'éeoree, il est presque démontré par eela teul, que les rayons médullaires sont les organes xclusif's de eet épanchement, lequel, à son tour, est sa cause immédiate de la formation ou du développenent des eouches sueccssives de liber et d'aubier, par 'abondanee des matériaux nutritifs qu'il apporte dans 
cet endroit. J'avais déjà entrevu l'importance de la coexistenee de ees faits, dans mes Recherches sur l'accroissement et la reproduction des végétaux (I). Voiei mes expressions: L'accroissement par couches concentriques est généralement lié avec l' existence des rayons médullaires; j'ignore quel est le rapport précis qui existe entre ces deux pliénomènes, mais leur coexistence étant générale, et les rayons médullaires existant avant l'accroissement par couches, l'existence des rayons médullaires peut étre considérée comme la condition sans laquelle ce noode d'accroissement ne peut avoir lieu. Alors je n'avais point encore fait de la marehe de la sève l'objet d'une étude spéciale, je n'avais pu par conséquent saisir la nature du rapport qui existait entre les deux phénomènes de l'accroissement par couches et de l'existence des rayons médullaires, phénomènes dont j'apercevais seulement la liaison intime et constante. A ujourd'hui je vois l'existence des rayons médullaires constamment liée au phénoniène de l'épanchement cntre le bois et l'éeorce de la sève, qui, par son abondance, provoque la formation des eouehes nouvelles chez les végétaux dicotylés. Je vois de plus, que ehez les végétaux monoeotylés, lesquels n'onı jamais de rayons médullaires, il n'y a jamais non plus d'épanehentent de sève sous l'éeorce; or, chez eux, il n'y a point de formation de couehes nouvelles. L'absenee de formation de ees dernières tient done évidemment à l'ab-

(1) Mémoires du Mruséum d'histoire naturelle, t. 7, p. 425. 
ssence de la sève épanchée, et l'absence de cet épancchement tient à l'absence des rayons inédullaires. Une aautre eonsidération fortifie encore eette assertion. La uransmission de la sève s'opère le plus naturellement cet le plus facilement par les organes creux disposés en lligne droite; c'est génćralentent, selon la dircction - desorganes linéaires, que s'opère eette transmission ffacile, e'est-à-dire selon la direetion des tubes lympphatiques, qui sont tous des tubes articulés, et selon Ha direetion du tissn eellnlaire artieulé, qui ne diffrère véritablement des tubes lymphatiques que par Ha petitesse de ses parties artieulées; enfin, selon la direetion des elostres. Or, les rayons médullaires sont ccomposés de tissu eellulaire articulé dans le sens transversal; en sorte que les organes linéaires qui les composent sont tous horizontanx. Ces séries reetiliynes de eellules sont donc émineminent propres à la transmission de la sève dans le sens horizontal, et calles doivent verser ce fluide par leur extrémité, qui aboutit à l'intervalle du bois et de l'éeoree. J'ai fait woir, dans mes Recherches sur l'accroisement et la reproduction des végétaux, que le système eortieal possède ses rayons médullaires partieulier's, qui viennent reneontrer ceux du système eentral sans se eontinuer avec eux. Ainsi la sève doit arriver, et du système central et du système cortieal, par les canaux de leurs rayons médullaires respeetifs, pour s'épancher dans l'intervalle de ces deux systèmes, et fournir ainsi les élémens nutritifs abondans, desquels résulte la formation des couches nouvelles de chaeun de ces deux 
systèmes. Une dernière considération vient encore à l'appui de mon opinion. Les feuilles sont composécs d'une énanation du système central et d'une émanatiou du systène cortical; leur pétiole a par conséquent son écorce particulière. Or, on ne voil jamais, même chez les végrétaux dicotylés, celle écorce se séparer des organes subjacens par l'épanchement de la sève. Cela provient évidemment de ce qu'il n'y a point de rayons inédullaires daus les pétioles, comme on peut facilement s'en convaincre par l'inspection des énormes pétioles que possèdent les feuilles de certaincs plantes herbacées, telles que le chou (brassica oleracea). Celte absence dans les pétioles d'organes de transmission circulaire de la sève dans un sens perpendiculaire à l'axe, fail qu'il n'y a jamais d'épanchement séveux entre leur système central et leur système cortical, qui sont toujours intimement unis. Aussi les pétioles des feuilles ne s’accroissent-ils jamais par couches concentriques; ils ne grossissent que par ce que j’ai nommé l'accroissement en largeur dans unes Recherches sur l'accroissement et la reproduction des végétaux (I). Ainsi nous voyons l'épanchement de la sève entre le système central et le système cortical constamment lié à l'accroissement en diamètre par couches successives; nous voyons, d'un antre côté, cet épanchement de sève constamment lié à l'existence des rayons médullaires; nous voyons entin que les rayons médullaires sont éminemment

(1) Rićmoires du Muséum, t. 7 , p. $39^{3}$ et 397 . 
rpropres, par leur organisation, à opérer la transmission ccirculaire de la sève et son épanchement entre les deux systènes. Nous sommes donc autorisés à consicdérer ces divers phénomènes comme étant sous une idépendance mutuelle et nécessiare, les uns comme causes, les antres comnie effets; car c'est par la conssidération de la constance de la narche des phénomènes qui se montrent tonjours associés, que nous ssommes conduits à regarder leur enchaînement comme ccelni de la cause à son effel.

La marche de la sève, chez les vérétaux monocottylés, n'a point encore été étudiée d'une manière spécciale : cette étude, d'ailleurs, présente de grandes (difficultés, à raison du défaut de distinction qui existe, lla plupart dı temps, entre leur système cortical et leur système central, défaut de distinction qui me paraît t tenir à l'absence des rayons médullaires. Nous avons -vu en effet plus haut que cette absence produit ce mêne défaut de distinction des deux systèmes dans les pétioles des fenilles, chez les végétaux dicotylés; nous ne pouvons donc établir la détermination de la marche de la sève, chez les végétaux monocotylés, que sur des analogies de structure anatomique. En effet, les organes élémentaires de ces végétaux sont les mêtmes que ceux des végétaux dicotylés; ils possèdent, comme eux, des tubes lymphatiques, des clostres, des trachées du tissu cellulaire médullaire, etc. Il y a grande apparence qu'ici les mêmes orgăanes servent aux mêmes usages. Les tubes lymphatiques doivent servir à l'ascension de la sève; les clostres 
doivent servir à son monvement descendant; les tracliées doivent conduire m liquide vivifiant puisé dans les fenilles; le tissu cellulaire médullaire, épars dans toutes les parties de la tige des monocotylés, et rempli de corpuscules nerveux, doit avoir le même usage que la moelle des dicotylés : aussi s̉e remplit-il de même quelquefois d'une substance éminemment nutritive, conme cela s'observe chez le palmier-sagoutier (sagus genuina Labillardière), substance qui sert à nourrir la fructification de l'arbre, et dont l'eusploi comme coniestible, sous le nom de sagou, est bien connu.

Une question qui se rattache de fort près à celle de la marche de la sève, est celle de l'orighine et de la formation des tubes qui servent de canaux à ce fluide. Tous les organes qui entrent dans la structure intime des véçétaux, dérivent Irès-évidemment de la cellule, dont ils sont des modifications; il n'y a d'exception, à cet égard, que pour les trachées, dont l'origine est tout à fait mystẹ́rieuse. Les tubes lymphatiques, et sous ce nom je comprends ceux que l'on a désignés sous les noms divers de tubes poreux, de fausses trachées ou tubes fendus, et de tubes en chapelet, sont bien évidemment composés de cellules placées les unes à la suite des autres : ce que je nomme le tissu cellulaire articulé, n'en diffère que par la petitesse des cellules. Dans l'origine, ces tubes sont divisés intérieurement par des diaphragines, ou par des cloisons articulaires formées par les parois juxtaposées des deux cellules contiguës : ainsi, les cavités de ces cellules ne conmuniquent point les unes avec les 
autres. I'ai noté ce lait dius mes liecherches sur Ia structure intime des mimaux et des végétaux: (page 25), et je l'ai observé sur les tubes lymphatiques dn bois de vigne jeune encore. Mais lorsque ce bois est âgé de quelques mois, l'organisation de ses - tubes lymphatiques se trouve modifiée : alors, les cloi:sons articulaires de ces tubes ont disparu presque entièrement; il n'en reste à chaque articulation qu'nu petit bourrelet circulaire, qui indique en dedans du tube la place qu'occupait auparavant la cloison dont il est le reste. Ainsi, les tubes lymphatiques ne forment un canal continn que par la disparition des cloisons articulaires qu'ils possédaient dans le principe. Ceci nous rend raison de la différence que présente le nnouvement de la sève de la vigne dans les jennes branches de récente formation, et dans le vieux bois. On sait qu'une incision faite an bois de la vigne, au commencement dı printenıps, donne lieu à une abondante émission de sève, qui est chassée an dehors par une force à tergo assez énergique. Or, à la nuème époque, les blessnres failes anx jemnes branclics rócemment sorties du bourgeon, ne versent point the sève: ce fait pronve que la force ì tergo, qui cliasse la sève hors dn vieux bois, éprouve un obstacle à son action dans les tigges récemment développées. Or, l'observation anatomique nous apprend que cet obstacle se trouve daus les cloisons articulaires que possèdent, dans les premiers temps, les tubes lymphatiques: alors, la sève ne peut pénétrer dans les articles successifs dont ces tubes sont formés, que par rine lente 
imbibition; tandis que, dans le vieux bois, l'absence des cloisons articulaires fait, des tubes lymphatiques, des canaux dont la cavité non interrompue n'oppose ancun obstacle au cours de la sève.

Les vaisseaux propres sont tous des tubes articulés; ils dérivent, par conséquent, aussi du tissu cellulaire. Les clostres ne sont évidemment que des cellules modifiées d'une manière particulière; il n'y a donc véritablement dans les végétaux que les trachées, dont les fils spiraux, réunis de manière à former des lames spirales, ne présentent aucune analogie avec le tissu cellulaire, et ne paraissent point, par conséquent, en irer leur origine. Ainsi, les plantes ne possèdent que deux élémens organiques réellement différens : la cellule et le fil trachéal. Je n'expose ici qu'en passant cette considération, qui me paraît importante, et je m'empresse de revenir à mon sujet. 


\section{CHAPITRE IL.}

De la prétendue circulalion du suc jaune daus la granto chéliıloinc.

Nous venons de voir qu'à proprentent parler, il n'existe point de circulation de la sève, et que ce fluide est seulement soumis à une diffusion générale, laquelle affecte denx directions opposées, l'une ascendainte et l'autre descendante. Ce mouvement de la sève est nécessairement fort lenı. Or, des observations dues à M. Schultz, médecin à Berlin, tendent à faire croire qu'il existerait, chez certains vércétaux, une circulation extrêmement rapide ( 1 ). C'est spécialement sur la grande chélidoine (chelidonium majus L.) que cet observateur a fait celte remarque : il affirme qu'en examinant au nucroscope, et avec le secours des rayons solaires, les nervures demi-transparentes des feuilles ou des pétales de la chélidoine, on aperçoit deux courans fort rapides, dirigés en sens inverse, l'un ascendant, l'antre descendant. M. Schuítz admet que c'est le suc jaune de la plante qui se ment ainsi, en présentant en ontre un mouvernent de tré-

(1) Le Mémoire de M. Schultz a ćtẻ traduit eu français, et inséré par MI. Jourdau aux tomes i6 et 17 du Journal complémentaire du Dictionnaire des scicnces médicales. 
pidation dans les globules dont il est composé. J'ai dû m'empresser de répéter ces observations; et pour suivre en tous points les indications de cet auteur, j'ai commencé par observer les feuilles de la chélidoine sur la plante enracinée. Je n'ai pas tardé à me convaincre de la vérité de l'assertion de M. Schulız, relativement à l'apparence d'un mouvcment de trépidation très-rapide dans les nervures transparentes des feuilles de celte plante, lorsqu'on les examine au microscope au moyen de la lumière du soleil, rélléchie par le miroir. La nervure de la feuille semble être un vaisseau mnique, dans lequel se meuvent en tremblotant des corps qui semblent être des golobules. M. Schulız prétend avoir observé que ces globules ont un mouvement de transport, et que le fluide qu'ils forment par lenr assemblage offre deux courans, l'un ascendant et l'autre descendant. Quelque attention que j'aie apportée dans mes observations, il m'a été impossible d'apercevoir rien qui pùt faire soupçonner l'cxistence de ces deux courans, rien même qui pût autoriser à penser qu'il y eût là un véritable mouvement de progression d'un fluide. On n'aperçoil, comme je viens de le dire, qu'un mouvement de trépidation fort rapide. Au reste, je me suis convaincu qu'il n'est point du tout nécessaire, pour voir ce mouvement, d'observer les feuilles de la plante pendant que celle-ci tient au sol; car une feuille cueillie offre ce mouvement tout aussi rapide que la feuille qui lient à la plante enracinée. Ce n'est mème, comme l'a observé M. Schultz, que lorsque la feuille est complètement fanée quion 
iesse de l'apercevoir. Je l'ai observé, tont anssi rapide ue dans le principe, dans une feuille à demi-flétrie ni était cueillie depuis deux jonrs. Ce phénomène te peut s'apercevoir qn'en illuminant la feuille avec eas rayons du soleil ; la lumière diffuse du jour ne fait percevoir aucun mouvement. On ponrrait croire que, lans cette dernière circonstance, la lumière ne posì̀de pas assez d'intensité pour percer au travers des tervures de la fenille, et apporter à l'œil l'image du monvement qui a lieu dans l'intérieur de ces nervnres. ¿ependant on peut rendre cette lumière diffuse extrêmement vive, et très-suffisante pour bien illuminer 'intérieur des nervures, sans qu'elle y fasse apercecoir le moindre mouvement. Il suffit, pour cela, de jlacer la feuille que l'on observe an microscope sur' mne lame de verre dépoli dont on éclaire la face inférieure avec les rayons dn soleil réfléchis par le niroir ioncave du microscope, qui fait converger sur ce point une grande quantité de rayons lumineus, lesfruels ne traversent le verre et la fenille qu'il suppoorte que sous l'état de lumière diffuse très-vive. Je lle répète, cette observation ne fait apercevoir aucun umouvement dans les nervures des feuilles de la chéliidoine. Ces faits me donnèrent des doutes sur la réalité de ce mouvement. D’ailleurs, il ne ne paraissait guère probable qu'un mouvement d'une rapidité aussi extraordinaire se maintînt dans une fenille séparée de lla plante, et cela pendant mn aussi long temps. D'un autre côté, la cessation de ce mouvenient, quand la ivie avait complètement abandonné la feuille, sem- 
blait attester qu'il y avait là une cause vitale. Pour éclaircir ces doutes, j’ai soumis les feuilles de la chélidoine à plusieurs expériences. J'ai commencé par faire l'anatomie des nervures de la feuille de cette plante. J'y ai trouvé des trachées en petit nombre, des tubes corpusculifères rayés en travers (fausses trachées de M. Mirbel), des tubes articulés contenant le suc propre et du tissu cellulaire. Lorsqu'on observe la feuille entière avec les rayons du soleil, on ne voit point du tout cette strucuure intéricure. J'ai examiné ensuitc le suc propre au microscope; je l'ai trouvé composé d'nne multitude de globules d'une excessive petitesse, et tout à fait inapercevables avec un grossissement médiocre, lequel suffit cependant pour voir les prétendus globules tremblotans dont je viens de parler. Ainsi, il me fut prouvé que ce ne sont point les globules du suc jaune que l'on voit ainsi se mouvoir en tremblotant. Mais on pouvait penser que ce inouvement résultait de la confraction et de la dilatation alternatives des tubes articulés, des ccllules et des autres organes creux fort petits qui existent dans les nervures des feuilles. Ce monvement de systole et de diastole, si son existence était démontrée, serait un fait d'une haute importance en physiologrie végétale; je ne négligeai donc rien pour éclaircir tous les doutes à cet égard. Je résolus d'examiner ces feuilles au microscope solaire. Si le mouvement intérieur de leurs nervures était une illusion d'optique, cette illusion devait disparaître ici, puisque, dans ce genre d'observations, la vue n'est point dirigrée sur l'objet lui-même, mais 
uIr son image considérablement grossie. Te sommis onc des feuilles de chélidoine au microscope solaire; zurs nervures fort transparentes n'opposaient aucun tbstacle à la transmission de la lumière du soleil ; on ess voyait se peindre en lignes luminenses qui conrastaient avec les intervalles obscurs occupés par le arenchyme vert de la feuille. Or, dans celte observaiion, tout me parut complètement inmobile; il n'y ivait dans les nervures aucune apparence de mouvement. Mais il se trouve ici un obstacle à l'observation. ¿objet observé se trouvant précisément alu foyer de a grande lentille du microscope, est bientôt brîlé par a concentration des rayons solaires. Pour obvier is cet tuconvénient, je collai une feuille de chélidoine sır une lame de verre avec un peu d'huile d'olive, et je pprésentai cette lame de verre au microscope solaire, een plaçant la feuille du côté opposé à celıi par lequel narrivaient les rayons du soleil. De cette manière, la uransparence des nervures était encore plus parfaite, tet la feuille resta en expérience sans se brûler. Or, je m’aperçus aucun mouvement de trépidation dans ses inervures. Cependant, avant l'expérience, j’avais vu ce imouvement, avec le microscope ordinaire, dans cette Imême feuille huilée, et j̣e le vis de même après l'exipérience. Dès lors il me parut prouvé que ce nouvement n'existait point daus la feuille, mais que son a apparence était ${ }_{3}^{8}$ le résulat d'une illusion d'optique produite par un certain jen de lnmière. Ve crus trouver la cause de cette illusion dans les réfiactions multipliées produites par les petits organes transparens 
qui contiennent les fluides, réfraetions sans cesse. variables par le mouvement inaperçu de l'œil de l'observateur. Ce fut dans celte idée que je publiai une note sur cet objet en 182.4 (I).

Depuis ce temps, jai fait de nouvelles observations qui m'ont prouvé que le phénomène déeouvert par M. Sehulı est très-réel, et que son apparenee n'est point le résultat d'une illusion d'optique, eomme je l'avais présumé. J'étais déjà revenu de mon opinion erronée à eet égard, lorsque M. Savi a fait paraître, dans le Nuovo giornale de' letterati (2), un travail qu'il a fait sur eette même natière. M. Savi admet que le nouvement de trépidation que l'on observe dans les nervures des feuilles de la grande ehélidoine, provient des ehangemens rapides qui surviennent dans les intersections des anneaux lumineux produits par les globules du fluide contenu dans les vaisseaux de eette plante, et il en conelut que ce fluide se meut par un mouvement eirculatoire. Il rejette la cause d'illusion d'optique que j'ai eru pouvoir admettre, et que, du reste, j'abandonne tout à fait. M. Savi ne fonde ses assertions sur aucune observation nouvelle; il n’a fait que répéter les observations de M. Sehultz, et constater eomme moi leur réalité.

L'une des raisons les plus fortes que l'on puisse produire pour prouver que le mouvement de trépida-

(1) Dans le Journal complémentairc du Dictionnaire des scienecs médiculcs, t. 1.), p. 289.

(a) Janvier el février 182.5 . 
כon offert par la grande chélidoine n'est point dû à ne illusion d'optique, est l'intermitten ee qui s'observe iéquemırent dans ee mouvenent. Cette intermiltenee iété notée par M. Sehulı, et je l'ai très-souvent obrrvée comıne lui. Ce phénomène est fort remarqua.ce. Au moment où le mouvenent de trépidation est ınne vîtesse extrême, on le voit tout à coup s'arrêrr, puis reeommeneer avec la même vîtesse l'instant a après. Cẹtte stase est générale dans tout le ehamp du iicroseope. Quelle est la nature de ce mouvement? st-il produic par une eirculation des fluides $d a$ la isante, on résulte-t-il simplement d'un mouvement noléeulaire qui aurait lieu dans ees fluides? On conjit en elfet que ees deux eauses sont également sussptibles de produire ee phénomène, qui indique inontestablement des déplaeemens rapides et multipliés ans les petits corps qui réfraetent la lumière transnise an travers des nervures de la feuille. M. Sehultz rétend que ce mouvement indique une circulation; 11. Savi est du même avis. Il nı'est impossible de artager leur opinion à cet égrard. Si ee phénomène rrovenait d'une eireulation, elle serait plus rapide sue ne l'est eelle des animaux, ee qui est ineompaible avec la strueture des organes des plantes. D'ailsurs, une pareille eirculation se maintiendrait-elle ans perdre de sa rapidité, pendant plısicurs jours, dans une feuille séparée de la plante, et même pres[ue totalement fanée, ainsi que je l'ai observé avee II. Sehultz? J'ai vu d'ailleurs qu'en eoupant transversalement la nervure d'une feuille dans deux en- 
droits peu distans l'un de l'autre, on continuait à observer le mouvement dans le fragment isolé; ce qui n'aurait pas lieu s'il y avait là une circulation. Il faut douc renoncer ì cette idée, et se porter vers celle d'un mouvement dars les molécules ou dans les globules dont le fluide jaune de la plante est composé. Or, quelle scrait la nature de ce mouvement moléculairc? Un premier soupçon m'est venu à cet ćugard. On sait que le calorique inprime une inpulsion rẹcciproque aux molécules des corps. Ne serait-il pas possible que les globules de suc jaunc, frappés par les rayons solaires, éprouvassent entre cux des changemens rapides dans leurs rapports mutnels, par le scul fait de la chaleur que leur communique ces rayons? Ce mouvement molćculaire suffirait en effet pour varicr à l'infuni et sans cesse les intersections des petits anncanx luminenx, et pour prodnire, par consćquent, l'apparence de trépidation que l'on observe; mais si telle était la cause du phénoùc̀ne, il n'offrirait point d'intermittence. D'ailleurs les observations suivantes prouveront d'une manière décisive que ce n'est point à de la chalcur actnellement comıuniquéc qu’est duc la production du phénomène dont il s'agiı; mais que ce phénomène est véritablement vital. L'influence dn froid suspend chez les plantes les phénomènes de la vie. Il était intéressant de savoir si le mouvement de trépidation de la chélidoine serait suspendu par cette cause. Lors donc des premières gelées qui se manifestèrent aux approches de l'hiver, je m'cmpressai d'observer leur effet sur le phénomène qui nous oc- 
spe. Un matin, le thernomètre était descendu à degré R.; je pris des feuilles de chélidoine qui uaient couvertes de gelće blanche, et je les soumis au nicroscope. Je trouvai dans la plupart des nervures the absence complète de mouvement de trépidation. In n'apercevait ce mouveninent que dans quelquesmes des uervures de moyenne grosseur, et il y était mterinittent. J'ai vu une de ecs nervures dont te miteu seul offrait ec nouvement; ses deux parties laténles étaient immobiles. Je vis une autre nervure comllètenıent privéc de mouvement, tandis que les deux ranches dans lesquelles elle se divisait oflraicnt une répidalion très-vive. Quclques jours après, par un roid de -2 degrés R., je ne tronvai aucune trace de irépidation dans les feuilles de la chélidoinc. En :ain elles furent réchauffécs par les rayons du soleil [ue rasscmblait le miroir concave du microscope, il ue se manifesta aucun monvement dans leurs nertures pendant plus d'une heure que je les observai. Toutes les chélidoines qui avaient été soumises à l'imoression du froid offraicnt la mème absence de monvement. Je tronvai cependant une trépidation faible intermittente dans les feuilles de l'une de ecs plantes qui avait étć garantie du froid par un abri.

Je répétai ces observations pendant deux hivers, afin d'ètre bien ccrtain de leurs résultats. Je vis toujours le mouvement de trépidation cesser dans les feuilles de la chélidoine, lorsque le thermomètre était descendu à I degrié au-dessous de zéro. Les feuilles soumises à ce degré de froid élaient quelque- 
foị susceptibles de reprendre leur trépidation, lorsqu'elles étaient réchanffées par les rayons du soleil que rassemblait sur elles le miroir concave du microscope; mais lorsque le froid avait été plus intense, les feuilles de la chélidoine qui y avaient été soumises n'étaient plus susceptibles de présenter le phénomène de la trépidation lorsqu'on les réchauffait. C'est en vain qu'alors je les mis séjourner dans un appartement dont la température constante était de $\nmid 7$ à 8 degrés R., elles ne reprirent point leur trépidation. Cependant ces feuilles, dont le pétiole trempait dans l'eau d'un vase, conservèrent pendant plus de quinze jours toute leur' fraîcheur. Ainsi l'action du froid avait anéanti complètement le mouvement de trépidation dans les nervures de ces feuilles, et le retour d'une température plus douce ne suffisait pas pour reproduire ce mouvement dans des feuilles séparées de leur tige, et conservées au moyen de l'immersion de leur pétiole dans l'eau.

Tant que dura le froid de l'hiver, il ne se manifesta aucun indice de trépidation dans les feuilles de la chélidoine. Lorsque le dégel survint, je m'empressai de les observer, mais je n'y aperçus aucune trépidation, quoique le thermomètre se maintînt pendant plusieurs jours à +3 et 4 degrés R., et même à des degrés plus élevés Les gelées qui avaient lieu souvent pendant la nuit, pouvaient être la cause de cette absence de la trépidation dans les feuilles de la chélidoine, malgré le retour d'une température qui, avant l'abolition de ce mouvement, était suffisante 
Ipour qu'il se maintînt. Cependant, lorsqư'il eut tout ¿à fait eessé de geler, je n'observai point encore de ttrépidation.

Le 20 janvier, je eueillis plusienrs feuilles de ehéllidoine, dans lesquelles on n'apercevait aucnn monivement, et je les eonservai en mettant tremper leurs Ipétioles dans des vases pleins d'eau. La température, dans l'appartement où se trouvaient ces feuilles, ne Ifut point au-dessous de +5 degrrés, et elle fut, la Imajeure partie du, temps, de +8 à - 10 degrés. $\left(\mathrm{Or}^{\mathrm{r}}\right.$, pendant plus d'un mois et demi que je conservai cces feuilles dans l'état de vie et de fraîeheur, je n'obsservai ancune trépidation dans leurs nervures. Ce ne ffut que le 8 mars que ;e commenẹai à apereevoir ceette trépidation. Alors la tenpérature de l'appartement était de +13 degrés. Cependan les ehélidoimes du dehors n'offraient encore aueun mouvement. Mais le 10 mars, la tompérature extérieure étant de + 15 degrés, japerẹus la trépidation dans la nerivure de quelques-unes des feuilles. Ce phénomène ne ffut généralement établi que plusieurs jours après.

Il résulie de ces observations, que la trépidation que l'on aperẹoit au microscope dans les nervures des feuilles de la ehélidoine, est un phénomène trèsrréel, et que son apparenee n'est point due à une illıssion d'optique, eomme je l'avais pensé d'abord. Si le mieroseope solaire ne fait point aperecvoir eette trépidation, cela provient probablement de ee qu'elle est abolie par la forte ehaleur que produit, sur la feuille en expérience, la eoncentration des rayons 
solaires opérée par la grande lentille du microscope. Cette trépidation, aux approches de l'hiver, subsiste dans toute sa force, malgré l'abaissement de la température jusqu’à zéro du thermomètre. Ce n'est qu'audessous de ce degré de température qu'elle disparaît tout à fait, pour ne plus se reproduire que lorsque la température environnante s'est maintenue, pendant un certain temps, jusqu'à +13 ou +15 degrés $R$. Ainsi, cette trépidation ne dépend point directement de l'influence actuelle de la chaleur, puisqu'un certain degré de température qui maintient très-bien ce phénomène est incapable de le reproduire. Il faut donc reconnaître que ce phénomène est vital. Toutes fois, nous ignorons en quoi il consiste essentiellement. Ce qu'il y a de certain, c'est qu'il n'indique point une circulation des fluides, comme une certaine apparence l'a fait croire à M. Schultz. Il me paraîl probable que ce phénomène est le résultat d'un certain mouvement moléculaire qui a lieu dans le suc janne de la plante; car une trépidation tout à fait semblable, dans ses apparences, se fait apercevoir dans le sang des animaux immédiatement après leur mort. Or, ce dernier phénomène résulte incontestablement d'un mouvement des molécules du fluide sangruin. Je ne puis me dispenser de jeter ici un coup-d'œil sur ce second phénomène, quoiqu'il paraisse bien éloigné du sujet qui m’occupe spécialement. C'est également à M. Schulız que l'on doit les premières observations sur cette matière ( 1 ).

(1) La traduction françaisc du Mémoire de MI. Schulte cst in- 
Si l'on examine au microscope éelairé par les mayons du soleil, les parties transparentes d'un ani:mal fraîehement tué, on voit, dans ses vaisseaux sanguins capillaires, un nouvement de trépidation texaetement semblable à celui que l'on observe dans Ha chélidoine. Les raisons qui m'avaient porté à con: sidérer le mouvement de trépidation de la chélidoine comme une illusion d'optique, m'avaient condnit à Iporter le même jugrement par rapport au mouvement ide trépidation qu'offre le sanğ des animanx; mais j’ai 'dû revenir de mon opinion à eet égrard. Si, eomme ll'indique M. Sehuitz, on observe au mieroscope, avee les rayons solaires, l'oreille d'une souris eoupée à l'a: nimal vivant ou réeemment tué, on voit, dans les vaisseaux sangruins, un mouvement de trépidation fort rapide, qui présente l'apparence d'une eireulation qui aurait son cours des trones dans les ramifieations des vaisseaux. Pour faire eommodément eette observation, il faut placer l'oreille de souris sur une lame de verre, et la mouiller pour prévenir la dessieation, qui serait prompte sous l'influence des rayons solaires. De eette manière, j’ai observé, pendant vinģtcinq minutes, ce mouvement de trépidation dans les vaisseaux sanguins de l'oreille de la souris. Ce monvement, en tout semblable à eelui de la ehélidoine, était, comme lui, sujet à des intermittenees : il cessait tout à fait pendant un quart de seconde, puis il re-

sérée au tume 19 du Journal complémentaire du Dictionnaire des stiences midicales. 
coınmençait tout à coup avec rapidité. Cela avait l'air, en quelque sorte, de spasmes interrompus par des monens de repos et de calıne. Lorsqu'il a été sur le point de cesser, il s'est graduellement ralenti, ne présentant plus, sur la fin, qu'une trépidation fort lente. J'ai soumis à la niême observation une portion détachée du mésentère de la souris : j’y ai vu le même phénomène de trépidation dans l'intérieur des vaisseaux sanguins, et ici il m'a été facile de voir qu'il n'y avait point de circulation, comme une apparence trompeuse l'a fait croire à M. Schultz. En effet, le vaisseau sanguin que j'observais ćtait assez court, et divisé transversalentent à ses deux extrémités. Or, quoiqu'il parût y avoir un courant dans son intérieur, il ne sortait rieu de ses extrémités ouvertes. Une heure après la mort de la souris, je fis l'extraction d'une nouvelle portion de mésentère. J'y observai encore le mouvement de trépidation dans les vaisscaux sanguins; mais il était devenu intermittent : il avait lieu par saccades irrégulières, et avait perdu beaucoup de sa rapidité. Après quelqucs minutes, il sc ranima, et reprit toute sa rapidité première, puis diminua de nouveau, et cessa tout à fait au bout de dix minutes. Le sang extrait des vaisseaux d'un animal vivant, offre le même mouvement de trépidation. Il suffit, pour faire cett'c observation, de mettre une couche mince de sang sur une lane de verre, et de l'éclairer àvec les rayons solaires, pour l'observer au microscope. Le mouvenent de trépidation est fort rapide, et il offre des intcrmittences, comme les offre 
sangr renfermé dans les vaisseanx, comne les ollice chélidoine : il diminue ğradụellement de rapidité, finit tout à fait lorsque le sang est coagulé. J'ai vu ue le sang artériel comparé au sang veineux, que le ungr des nammifères comparé à celui des reptiles, n'ofait aucune diflérence sous le point de vue de la rapité du mouventent de trépidation dont il paraît animé. On pourrail croire que le mouvement moléculaire, ui, daus le sangr $110 n$ circulant, produit cette appamce de trépidation, ne serait autre que celui qui and à amener la coasulation. Dans ce dernier phéomène, en effer, il y a mne tendance générale des obules sanguins à se rémir'; tandis qu'ils son consmnent dissociés daus le sang circulant. Par l'eflet ? cette tendance, les globules sanguins se nieuvent our se rapprocher les nns des autres, jusqu’à ce que urr réunion soit complète et intime. Or, ce mouvecent des globules sanguins snflirait ponr produire des nangemens rapides el unltipliés dans les intersecons des anneaux lumineux produits par ces gloules; cela suffirait, par conséquent, pour donner zu à l'apparence de trépidation que l'on observe cor's. Il serait donc possible de regarder la tendance u sang à la coagulation, comne la senle cause du hénomène dont il s'agit; mais cette explication ne sut point s'appliquer à la chélidoine, chez laquelle phénomène de trépidation est véritablement un bénonı̀ne vital, ainsi que nous l'avous vu plus haut. reci doit nous porter à. admetlre que, dans le sang, phénomène de la trépidation est égralement dù à la 
vie, et que s'il ccsse de se montrer quand la coagulation est complète, ccla provient de ce que lc sang possède une vic propre dont l'extinction est marquée par la coagrulation.

Le sangs, quoique privé de mouvement de transpor't dans les vaisseaux sanguins d'une partie fraîchement détachée d'un animal vivant, paraît cependant se mouvoir encorc des troncs vers les rameaux : en un mot, on croirait, si l'on se contentait d'une observation superficiclle, que le sang circule cncore, quoiquc privé de l'impulsion du cour. Nous avons acquis la certitude que cette apparence de circulation est une illusion d'optique, et ceci nous confirme dans l'opinion qu'il en est de mêne pour la chélidoine, chez laquelle il n'y a point de transport rapide d'un fluide, comme l'a pensé M. Schuliz. Mais il reste démontré que, dans la chćlidoine et dans plusieurs autres plantes à fluides laiteux, il existe, comme dans le sang des animaux nouvellement morts, un mouvement moléculaire d'une nature inconnue, mouvcment qui imprime, aux rayons de la lumière solairc, une grande variété de réfractions sans cessc changeantes, d'où résulte le changement rapidc et muliplié des intcrsections des anncaux lumineux, et par suitc l'apparencc de trépidation que l'on obscrve alors. Je ne me pernettrai aucune hypothèse sur la nature dc ce mouvemcnt molćculaire qui a lieu dans l’intérieur de certains fluides organiques; mais ce phénomène ıne paraît mériter de fixer l'attention des physiologristes. 


\section{CHAPI'TRE III.}

De la cause de la progression de la sive.

LA recherche des causes qui président à la jurorression de la sève a enfanté plusieurs systèmes, et $\therefore$ a conduit à aucun résultat positif. Malpighi pennait que la dilatation et la condensation alternatives Ice la sève, par l'effet de la température environnante, taient la cause de la progression de ce fluide; plus ard, Sarrabat admit que le mouvement de la sève Levait être attribué à la raréfaction et à la condensaiion alternatives de l'air, qu’il prétendait ĉlre contenı lans la moelle et dans les trachées; d'auties physi:iens ont considéré l'ascension de la sève comme le iimple résultat de la capillarité du tissu végétal : enrin, plusieurs physiologistes prétcndent que les vaisseaux des végétaux sont pourvus d'une forcc contracvile qui chasse de prochc en proche le fluide séveux. De ces quatre hypothèses, les deux dernières senles méritent de fixer notre attention. Nous allons voir cquel est lc degré de confrance que nous pouvons lcur accorder; et pour cela, nous devons étudier avec soin lles conditions appréciables dont l'existence cst nécessaire pour que la sc̀ve opère son ascension dans les | plantes.

Les expériences de Hales ont appris avec quelle 
force la sève monte dans la vigne : ce célèbre expérimentateur ayant adapté un tube de verre rempli de mercure à un cep de vigne tronqué, vil ce métal s'élever à 33 et à 38 pouces au-dessus de son niveau primitif, par l'effet de la force avec laquelle la sève tendait à sortir par l'extrémité tronquée du cep. Cette expérience, qui prouve bien évidemment que, dans cette circonstance, la sève monte en vertu d'une force impulsive à tergo, avait été mise en doute par Sennebier et par d'autres; mais ces résultats ont été rendus incontestables par MM. Mirbel et Chevreul, qui, ayant répété l'expérience de Hales, virent le mercure s'élever, dans cette expérience, à 29 pouces au-dessus de son niveau. Or, si l'on coupe une branche de vigne dans le moment où elle chasse la sève au dehơrs avec tânt de force par son extrémité tronquée, et qu'on la plonge dans l'eau par son extrémité inférieure, elle poinpera de l'eau par cette extrémité inférieure, mais elle ne versera plus de sève par son extrémité supérieure tronquée. La force inıpulsive à tergo a donc cessé d'exister dans cette dernière circonstance, puisqu'il n'y a plus d'expulsion de la sève par l'extrémité supérieure de la branche; et cependant la sève continue de monter dans celte dernière. Ceci nous fait voir que l'ascension de la sève, dans les plantes, est un phénomène complexe; elle est le résultat de deux causes qui, au premier abord, paraissent être différentes : la prennière de ces causes est une inıpulsion à tergo, dont nous ferons plus bas une élude spéciale; la seconde est le résultat de l'action des or- 
anes intérieurs de la plante sur le liquide qu’elle aborbe. Nous allons tenter de déterminer quelle est la ature de eetle action.

Tout le monde sait qu'en mettant un végétal coupé remper dans l'eau par sa partie inféricure tronquéc, e végétal absorbe l'eau, en opère l'aseension dans on intérieur, la transmel à toutes ses parties, ct par e moyen se maintient à l'état de vie el de fraîcheur rendant un temps quelquefois très - long. Or, l'aborption de l'eau dans eette eirconstance est soumise - eertaines lois qu'il est intportant de déterminer. En iénéral, la quantité d’eau absorbée est proportioncelle à la quantité de l'émanation aquense opérée par es feuilles de la plante soumise à cette expérienee. 1)r, la quantité de cette émanation aqueuse est en axison de la température de l'air ambiant, el en raionn de son état hygrométrique. Plus l'air est à la fois thaud et see, plus il y a d'émanation aquense opérée ar les feuilles, et plus en même temps il y a d'eau lbsorbée par la partie inférieure de la tige. Cepenrant, eomme on le pense bien, cet équilibre entre "'absorption et l'évaporation ne peut exister' qu'autant que celte dernière n'est point trop cousidérable; ear Lors l'absorption ne peut plus suffire à réparer les nertes eausées par l'évaporation, et la plante sc fléril ou se fane : plus il y a de fenilles sur lis tige, blus il y a d'évaporation, et plus il y a par conséquent l’absorption. Cela est si vrai, qu'on voil eette dernière diminuer subitement de quantité lorsqu'on reranche des feuilles à une tige de végétal dont on a 
auparavant évalué la force absorbante. Ciest d'après ces observations que Hales a été conduit à considérer les feuilles comme ayant pour usagre spécial d'élever la sève dans le végétal. On pourrait penser que cet usage des feuilles ne serait point le résultat d'une action spéciale qu’elles exerceraient; qu'elles n'opéreraient point directement l'ascension de la sève, mais que leur rôle se bornerait, dans cette circonstance, à favoriser l'évaporation de ce fluide par l'étendue de la surface qu'elles présentent à l'air, ce qui suffirait pour déterminer une absorption proportionnelle à l'évaporation. Les expériences qui vont être rapportées prouveront que cette manière de voir, plansible au premier coup-d'œil, est cependant fausse, et que les feuilles exercent une action vitale qui leur est propre pour attirer la sève et opérer son ascension.

La lumière influe d'une manière bien sensible sur les quantités respectives de l'absorption et de l'évaporation. On sait en effet, par les expériences de Hales, que les plantes transpirent beaucoup plus à la lumière que dans l'obscurité. On conçoit que la transpiration d'une plante soit augmentée par son exposition au soleil, dont les rayons directs, en échauffant les feuilles, augmentent l'évaporation des fluides qu'elles contiennent; mais on ne comprend pas de même comment l'exposition d'une plante ì la lumière diffuse qui ne produit point de chaleur, ausmente cependant son émanation aqueuse. Ce fait cependant est très-certain, et je m'en suis assuré par de nombreuses expériences sur des branches de végétaux 
Ifui absorbaient l'eau par leur extrémité inférieure tron[ruée. J'avais soin de peser le matin et le soir la plante et le vase rempli d'eau dans lequel elle était plongée par son extrémité inférieure, et j’ai vu qu'il y avait toujours excès de l'évaporation sur l'absorption penHant le jour, et au contraire excès de l'absorption sur l'éraporation pendant la nuit; en sorte que la plante Himinuait de poids dans le premier cas, el augmentait He poids dans le second. Ainsi, bien que l'absorption fùt en général proportionnelle à l'évaporation, cepenHanı la présence ou l'absence de la lumière diffuse, suffisaient pour changer la nature du rapport qui ezxistail entre ces deux qnantités : lorsque l'évaporaliion était forte on faible, l'absorption l'était aussi; mais en suivant ainsi l'éraporation dans sa marche, ll'absorption lui restait un peu inférieure pendant le ijour, et lui était un peu supérieure pendant la nuit. Deux résultats se déduisent de ces observations : le prenier est que la vacuité des vaisseaux des parties supérieures du végétal produite par l'évaporation des lliquides qui y sont contenus, est une des causes déterminantes de l'absorption opérée par les vaisseaux de la partie inférieure; les vaisscaux et les cellules des parties supérieures ayant perdu par l'évaporation Il'eau qui les remplissait, cette perte est réparée an fur eet à mesure par l"iutroduction des liquides qui remplissent les organes situés au-dessons, el cette conununication s'étend ainsi de proche en proche jusqu'aux orifices des vaisseaux qui pompent l'eau dans Haquelle la tige est plongée par son extrémité infé- 
rieure. Le second résultat est que la capillarité du végétal n'est pas la seule cause de l'absorption de l'eau et de son élévation dans la tige, car la cápillarité ne varie point, et l'on ne voit pas par conséquerit pourquoi pendant la nuit elle introduirait dans le végétal plus d'eau qu'il n'en est dissipé par l'évaporation, et pourquoi le contraire aurait lieu pendant le jour. Il y a donc évidemment ici une action vitale qui est modifiée par la présence ou par l'absence de la lumière. Nous allons voir celte conclusion confirmée par des expériences d'un autre genre.

Lorsque la tige d'une plante est coupée el abandonnée ainsi sur le sol, elle ne tarde point à se flétrir. Si la tigre est herbacée, elle devient flasque et pendante; les feuilles deviennent molles, et cessent d'avoir leurs pétioles redressés. Si cet état de dessication n'est pas porté trop loin, la tige dont il est ici question est susceptible de reprendre son état de vie et de fraîcheur, en la mettant tremper dans l'eau par la partie inférieure. Bientôt on voit la tige pendante se redresser; les feuilles perdent leurs plis et leur état de mollesse; leurs pétioles se relèvent; en un mot, il se manifeste dans toute la plante un état turgide opposé à l'état de flaccidité qui existait auparavant. Cet état turgide provient manifestement de la réplétion des vaisseaux et des cellules de la plante. Or, il était important de savoir quelles sont les conditions nécessaires pour que cet état turgide puisse se reproduire; quelles son celles qui s'opposent à leur retour. Dans cette vuc, j’ai fait plusieurs expériences que j’ai 
śpétées un grand nombre de fois, et sur plusieurs e.s¡èces de végétaux, afin de m'assurer de la constance t de la généralité des résultats auxquels je suis pareenu. Je ne eitcrai de ces expériences que celles qui ont faites avec une même espèce de plantes; et afin e les rendre comparatives autant que possible, je les faites tontes en même temps, ct j'ai eu soin d'emHoyer des plantes à peu près de la même taille, et riactement dı même poids. Je coupai une tigge de vercuriale (mercurialis annua), et je l'abandonnai anns cet état jusqu'à ce qu'elle cût perdu, par l'ćvaoration, les 0,15 de son poids. Ses feuilles et ses umeaux étaient dans un état complet de flaccidité. Llors je la mis tremper dans un flacon plein d'eau ue j'avais pesé avec soin auparavant. Au bout de uatre heures, par une température de + 12 degrés . la plante avait repris complètement son état tur(de; cependant elle n'avait pas repris tout à fait son ids primitif. Je trouvai que, pendant ces quatre preiières heures, la plante avait absorbé 20 grains et zmi d'cau par heure, et avait évaporé par ses feuilles :grains et demi d'eau également par heurc. Dans les natre heures suivantes, et pendant le jour également, température et l'état hygurométrique de l’air étant s mêmes dans l'appartement où se faisait cette ex"śrience, la plante absorba senlement g grrains et demi ean par heure, et elle en évapora par ses fenilles grains également par heurc. Pendant la nuit qui ivit, la plante absorba 7 grains et deui d'ean par "ure; elle en évapora 7 grains également par heure. 
Alor's elle avait repris son poids primitif. Le lendemain et les jours suivans, l'état de l'air ambiant ayant très-peu changé, la plante continua à absorber de 7 à 8 grains d'eau par hemre, et l'évaporation qu'elle opéra suivit à peu près la même proportion; seulement elle était plus forte que l'absorption pendant le jour, et plus faible que cette dernière pendant la nuit. En un mot, la plante continua à se comporter comme un végétal auquel rien ne manque pour exercer les fonctions de la vie dans toute leur plénitude. Il résulte de celte expérience, qu'une mercuriale qui a perdu les 0,15 de son poids par l'évaporation, et qui par-là est devenue flasque et fanée, peut reprendre complètement son état turgide et sa fraîcheur, quand on lui fournit de l'eau à absorber par l'extrémité inférieure de sa tige; il en résulte encore que la vacuité des vaisseaux eı des cellules, opérée par l'évaporation, et poussée seulement jusqu'au point qui vient d'être indiqué, augsmente considérablement la rapidité de l'absorption et de l'ascension de l'eau dans le vésétal. En effet, nons voyons dans cette expérience une plante qui absorbe 20 grains et demi d'eau par heure, lorsque ses vaisscaux et ses cellules sont dans un certain état de vacuité, et qui, rendue à peu près à son état turgide naturel, n'absorbe plus que 7 à 8 grains d'eau par heure, l'état de l'air ambiant n'ayant point changé. Cette expérience vient à l’appui de celles qui, plus. haut, nous ont conduit à penser que la vacuité des vaisseaux des parties supérieures de la plante était une des causes déterminantes de l'absorption opérée pas 
partie inférieure. Il y a évidemment ici d'autant us de facilité pour l'introduction et pour l'ascension : l'eau, qu'il y a plus d'organes vides qui attirent ce nide dans leurs cavités. On pourrait penser, d'après Ha, que le tissu végétal se eomporte comme une monge, qui est d'autant plus avide d'eau que ses cellles en sont moins remplies. Mais les expériences iivantes vont nous faire voir que celte eomparaison It inexacte.

Une mercuriale compée depuis vingt-quatre heures zait perdu les o,36 de son poids primitif. Alors je la iis tremper dans l'eau par son extrémité inférieure nonquée. Celte expérience se fit en même temps que précédente; en sorte que leurs résıltats ne peuvent canquer d'être eomparables. La plante était dans un aat de flaceidité eonsidérable; mais aucune de ses uilles n'était desséchée. L'absorption de l'eau fut. c̀s-peu considérable pendant le premier jour; car lle ne s'éleva qu'à deux graius un tiers par heure, noique la plante fùt à peu près de lia même taille, eût primitivement le même poids que celle qui a ié le sujet de l'expérience précédente, et qui, dans même temps et dans le même loeal, absorbait dans commencement jusqu'à 20 grrains et demi d'eau par sure. La plante qui fait le sujet de cette seconde exśrience ne regagna que i 2 grains de pesanteur penınt les vingt-quatre premières heures; e'était l'exss de l'absorption sur l'évaporation. Quelques-unes sos fenilles inférieures avaient repris leur fraîcheur leur état turgide. Le lendemain, la plante n'ab- 
sorba plus qu'un grain et demi d'eau par heure; ct comme ce qu'elle perdait alors par l'évaporation était plus considérable que ce qu'elle gragnait par l'absorption, elle commença à se dessécher, surtout aux extrémités des rameaux. Les jours suivans, cette dessication fut en augmentant, et bientôt il ne resta de vivant dans la plante que deux de ses petits rameaux les plus inférieurs. Nous voyons par cetle expérience, qu'une vacuité des vaisseaux et des cellules, poussée jusqu’à la perte des o,36 du poids primitif de la plante, diminue considérablement la faculté qu'avait le végétal d'absorber l'eau et de l'élever dans son intérieur, tandis que nous avons vu par la première expérience, que, par la perte des 0,15 de son poids seulement, la mercuriale avait augrmenté l'énergie et l'activité de sa faculté absorbante. Cela prouve bien évidemment que la vacuité des vaisseaux de la plante n’est pas la seule cause déterminante de l'ascension de l'eau dans son intérieur; cela prouve également que cette ascension n'est pas due à la seule capillarité; mais qu'elle est en majeure partie due à une action vitale qui se trouve abolie par une perte trop considérable des fluides qui remplissaient les vaisseaux et les cellules de la plante dans son état de fraîcheur.

En mème temps que ces expériences se faisaient, ¡’en faisais inarcher parallèlement une autre du même genre. Une mercuriale coupée depuis vingt-huit heures, avait perdu les 0,46 de son poids primitif; l'ayant mise tremper, par sa partie inférieure, dans un flacon rempli d'eau, je mis le tout sous un récipient de 
verre fermé avec de l'eau, qui couvrait entièrement la surface sur laquelle le récipient élait appuyé. De cctte manière, l'air renfermé sous le récipient avec la mercuriale fut bientôt saturé d'eau. La plante, dont la flaccidité était considérable, se trouvait ainsi dans la position la plus favorable pour récupérer promptement l'eau qu'elle avait perdue. Ses pertes ultérieures par l'évaporation devenaient à pen près nulles; il devenait ainsi bien plus facile d'étudier les progrès de l'absorption, et il n'y avait plus à craindre de voir la plante se dessécher, comme dans l'expérience précédente. Pendant les vinģt-quatre premières henres, la plante absorba seulement 50 grains d'eau : ce qui fait 2 grains $1 / 1_{2}$ par heure; le sccond jour, elle absorba à peu près I grain $1 / 2$ par heure. Celte lente absorption continua à peu près sur le même pied pendant quatre jours, au bout desfuels je trouvai que la plante avait récupéré, à peu de chose près, son poids primitif. Cette troisième expérience confirme pleinement le résultat de la deuxième, et fait voir, par l'extrênı lenteur de l'absorption dans ces deux circonstances, que la vacuité des vaisseaux de la plante, poussée jusqu’à un certain point, diminue considérablement sa faculté d'absorber l'eau dans laquelle elle cst plongée par l'extrémité inféricure de sa tige. Mais ici une réflexion se présente. L'imbibition préalable d'un tissu capillaire est nécessairc pour le disposer à absorber avec rapidité un liquide. Ainsi, une éponge parfaitement sèche pourra nager quelque temps à la surface de l'eau sans absorber ce liquide 
d'une manière sensible, tandis qu'une éponge préalablement imbibée d'eau, et ensuite fortement exprinlée, absorbera l'eau avec une rapidité prodigieuse. N'est-il pas possible que les plantes considérablement flétries qui font le sujet de ces deux dernières expériences, soient dans le cas d'une éponge sèche, comparativement à la plante qui fait le sujet de la première expérience, et qui serait dans le cas de l'éponge humide? De celte manière, les phénomènes qui viennent d'être observés rentreraient dans les phénomènes généraux de la capillarité. Pour apprécier cette objection, il faut se reporter à la cause qui fait que l'absorption est plus lente dans une éponge sèche qu'elle ne l'est dans une éponge hunide. Dans la première, l'air est adhérent aux parois des cellules : il faut qu'il en soit chassé peu à peu par l'eau qui s'introduit; dans la seconde, au contraire, c'est de l'eau qui est adhérente aux parois des cellules qu'elle mouille, et ici l'aturaction de ces parois pour l'eau est fortifiée par l'attraction que l'eau mouillante eserce sur l'eau qui s'introduit ensuite. On sait qu'en général un tissu capillaire absorbe de préférence le liquide qui mouille préalablement les parois de ses cavités capillaires; ainsi une éponge mouillée d'huile absorbera de l'huile de préférence à l'eau. Il en est de même de l'éponge mouillée d'air, si je puis m'exprimer ainsi; elle résistera à l'introduction de l'eau jusqu'à ce que tout l'air qui adhère à ses parties intimes soit remplacé par ce liquide, sur lequel alors elle exercera son action absorbante, sans aucun obs- 
tacle. Or, en nous reportant anx plantes fléuries qui n'opéraient qu'mue faible absorption de l'eau, nous voyons qu'elles n'étaient point dans cet état de siccité qui fait que c'est l'air et non:"plus l'eau qui mouille les parois des cavités ca pillaires. Ces plantes étaient molles; elles étaient fort éloignnées de cet état de desséchement qui est marqué ordinairement par une sorte de crépitation que font entendre les plantes sèches quand on ?es touche; elles contenaient évidemment encore beaccoup d'eau. Pour m'ell assurer d'une manière positive, je fis sécher complètement les plantes qui avaient servi aux densi derrières expériences, et je vis que, réduites à cet état de siccité, leur malière solide se trouvail peser dans l'une les 0,17 , et dans l'autre les 0,14 de ce que pesait la plante dans l'état frais. Par conséquent, Ja première contenait primilivement o,83 d'ean; elle en avait perdu o,36 lorsque je la mis en expérience : il lui restait par conséquent les 0,47 de la sève qu'elle possédait primitivement. La seconde plante, celle qui fut mise sous le récipient, possédait, dans le principe, o,86 d'cau sur son poids total: quand elle fut mise en expérience, elle avait perdu les o, 46 de son poids primilif : il lui restait par conséquent alors les o, fo de la sève qu'elle possédait dans l'état frais. On voit par-là que si les plantes très-flétries qui font le sujet de ces observations n'absorbaient l'ean qu'avec lenteur et difficulté, cela ne provenait point dır tout de la siccité des parois de leurs cavilés capillaires, puisquelles contenaient encore une quantité 
d'eau qui était presque la moitié de celle qu'elles possédaient dans l'élat frais. Par eonséquent, ce n'est ' point en vertu de sa seule capillarité, et à la manière d'une éponge, que le tissu végétal absorbe l'cau. Quelle est done la cause de eette absorption? Il est évident quil y a, dans cette eireonstance, une action vitale; l'observation peut nous apprendre en quoi elle eonsiste, et quel est son méeanisme. lei, pour mareher vers la eonnaissanee de la vérité, il faut observer et noter les changemens appréciables qui sont survenus dans le végétal, et qui accompagnent la perte ou la diminution de sa faeulté d'absorber et d'opérer. l'aseension de l'eau. Or, nous ne voyons eneore iei qu'un seul ehangement survenu dans ee végétal : c’est la diminution de l'eau, en quantité déterminće, dans le eavités capillaires de ses organes. Si e'est effeetivement à la présence d'une eertaine quantité de liquide dans les organes de la plante qu'est néeessairement attachée la faeulté d'opérer l'aseension de l'eau, il devra suffice de rendre à la plante ce liquide intérieur, pour lui rendre de suite, et dans toute sa plénitude, cette dernière faculté. C'est effectivement ce dont je me suis assuré par l'expéricnce suivante. Une mercuriale coupée et flétrie avait perdu les o,36 de son poids par l'évaporation. Je savais, par mes expériences antéeédentes, que dans eet état elle ne ponvait récupérer eomplèiement sa fraîeheur et son état turgide, en la mettant tremper dans l'eau seulement par la partie inféricure de sa tige, et en plein air. Je la couvris entièrement d'ean : au bout de donze 
hemes d'inmersion, lit plante avait repris complètement son état turgide et sa fiaìcheur. Alors je la tirai de l'eau, et ne l'y laissai plus tremper que par la partie inférieure de la tige. La plante absorba et éleva l'can dans son intérieur conme l'aurait fait nue plante fraîchement conpée; je la ugardai pendant quinze jours dans cet état de fraîchenr, qui me prouvait qu'clle n'avait point sonffert dn tout de l'expérience à laquelle elle avait été sounise, ct qu'il ne lni manquait rien de sa faculté d'opérer l'ascension de l'eau. L'immersion totale de la plante avait favorisć chez elle l'absorption de l'can par toute sa surface, et avait ainsi occasionné la prompte et complète resitution du liquide qui avait été enlevé à son tissu par l'évaporation. Sans cette restitution, la plante n'eût opéré qu'une très-faible absorption d'eau par la partie. inférieure de sa tige; avec cette restitution, la plante s'est trouvée pourvue de toute la plénitude de sa faculté d'opérer l'ascension de l'eau; c'est donc indubitablement à la présence d'une suffisante quantití de liquide dans les organes capillaires du végétal qu'est due, an moins en partic, la faculié yn'il a d'opérer l'ascension de l'ean. Ile poussai plus loin la dessication sur denx antres mercuriales : l'une perdit les 0,61 et l'antre les 0,72 de son poids prinitif par l'évaporation. La première avait encore la plupart de ses feuilles dans l'état de souplesse; les feuilles de la seconde, à demi-souples, offraient déjà cette crépitation que font entendre les feuilles sèches lorsqu'on les tonche; cependant elles avaient encore un certain degré de 
souplesse. Ces deux plantes ayant été ensuite entièrement plongées dans l'eau, la première, celle qui avait le moins perdu, reprit son état turgide et sa fraîcheur dans quelques-uns de ses rameaux; les autres, malgré leur immersion, demeurèrent dans leur état de flaccidité. Sortie de l'eau et mise seulement le pied dans ce liquide, cette plante absorba de l'eau pour maintenir la frâicheur de ceux de ses rameaux qui l'avaient récupérée; les autres rameâx se desséchèrent. Quant à la mercuriale qui avait perdu le plus par l'évaporation, son immersion dans l'eau ne rendit à aucune de ses parties leur fraîcheur et leur état turgide; cependant ses tetilles é:aienı imbibées d'eau, mais elles étaient flasques. Ta piante ayant été retirée de l'eau au bout de vingt-quatre heures, et mise seulement le pied dans ce liquide, conserva sa flaccidité, n’absorba aucunement l'enu dars laquelie irempait sa tige par son extrénité inférieure, et ne tarda pas à se dessécher complètement. Ici de nouveaux résultats se présentent à untre observation: nous voyous qu'une plante dont la dessication a été portée trop loin, perd tout à fait la faculté de redevenir turuside et d'opérer l'ascension de l'eau dans son intérieur. Cependant toutes ses parties ont récupéré l'eau qui leur avait été enlevée; cependant ses feuilles livrent l'eau qu'elles ont recupéré à l'évaporation. Or, par la vacuité progressive qui résulte dans leur issu de cette évaporation, il semblerait que l'eau devrait êire sollicitée à monter dans l'intérieur de la tige pour venir remplacer cette eau évaporée, comme cela a lieı dans un 
ıvégétal frais. Mais ce phénunène n’a point lieu. Lai ¿capillarité du végétal ne serait-elle plus la mème? -Mais il est évident que eette eapillarité n'il pu ètre :altérée par une évaporation Iente de l'eau contenne ¿dans les cavités eapillaires du tissı organique. Que Imanque-t-il done à la plante pour absorber et élever ll'eau? la simple observation des faits nous l'indique. Nous avons vu qn'en restituant l'eau ou tissu orgaınique d'une plante qui n’en avait pas eonsidérablement perdu, nous Ini avons restitné premièrement : son état tnrgide, et par suite sa faeulté d'opérer l'aseension de l'cau, faeulté qu'clle avait perdne en grande partie. Tous venons de voir 'que c'est en vain qu'on restitue ćc l'eau á une plante dont la dessieation a été poussée trop loin; clle s'imbibe d'can eomplètement, et altant que le veut sa eapillarité, nrais elle ne reprend point son étal turgide, ni par suite sa faculté d'opérer l'aseension de l'cau. Ainsi ectte dernière faeulté esi nécessairement liée à l'existenec de l'état turgice.e. Ur, la faculté de reprendre eet état turggide disparaît eomplètement lors de l'évaporation trèsavaneéc des Auides organiques qui remplissent les organes capillaires du tissu végétal. En effet, lors de la dessication d'une plante, on peut juger, par l'odeur qu'clle exhale, qu'elle ne perd pas seulement de l'eau pure par l'évaporation, mais que eelte dernière dissipe véritablement les lluides organiques qui remplissaient les organes de la plantc. Par cette perte d'une partie de leurs élémens constituans, les fluides organiques se tronvant altérés dans lenr eomposition, 
la substanee verte qui remplit les eellules végétales manifeste spéeialement, par son ehangement de eouleur qu'elle a subi, une altération profonde. La plante desséehée et ensuite imbibée d'eau, ne reprend point sa fraicheur, c'est - ì-dire la belle eouleur verte qu'elle avàit auparavant. Cette eouleur est remplacée par une teinte brune et terne. C'est donc à l'existence et à l'intégrité des fluides organiques qu'est due la faculté que possède le végétal d'être turgide: cette faeulté n'est point altérée par une dessication légère, qui n'opère l'évaporation que de la partie aquéuse surabondante de ces fluides organiques : cette légère vaeuité des organes qui n'altère point leur faeulté de devenir turgides, augmente, eomme nous l'avons vu, l'activité de l'absorption et de l'ascension de l'eau. Lorsque la dessication de Ja plante est portée jusqu’à un certain point, qui, quoique déjà eonsidérable, ne l'est cependant point assez pour altérer d'une manière notable la composition des fluides organiques, la faeulté de reprendre l'état turgide se tronve eonsidérablement diminuée, et, par suite, la faculté d'opérer l'aseension de l'eau; mais la plante peut récupérer la plénitude des ses facultés vitales par l'immersion complète dans l'eau; ee moyen suffit pour lui rendre toute l'eau qu'elle avait perdue, il ne lui manquait que ccla. Ses fluides organiques n’étaient point altérés dans leur eomposition, la substanee verte avait eonservé sa eouleur; mais lorsque la dessieation est portée jusqu’à porter atteinte à la composition des fluides organiques ot des autres substances contenues 
Hlans les eellules du végétal, alors disparaît ehez ee Hernier la faeulté de devenir turgide, eı, par snite, le pouvoir d'opérer l'aseension de l'eau; par eonséquent "état turģide est lié d'une manière nécessaire ì l'intégorité des liquides, et, en général, des substances eonteenues dans les cellulẹs du végétal. Ainsi, voilà denx conditions indispensables pour l'existenee de l'état turgide ehez les végétanx: $I^{\circ}$ la présenee dans leurs srganes d'une eeriaine quantité de liquide; $2^{\circ}$ l'intéurité de la eomposition des substances organiqnes eonitenues dans ces nêmes organes. Lorsque ecs deux conditions eessent d'exister, alors disparaît dans le tissu organique végétal le pouvoir d'opérer l'aseension de l'eau, pouvoir néeessairement lié ì l'existenee de l'état turgride. Ainsi, ce qui distingue essentiellentent Ile végétal mort du végétal vivant, eonsiste en eeei: que chez le premier, le tissu végétal étant aussi plein d'eau que le veut sa capillarité, il n'est eependant point turgide, il demeure flasque. Le second, an contraire, remplit d'ean ses cavités eapillaires d'une manière tellement surabondante, qu'il en résulte un tétat umrgide général. Cetse faeulté de devenir urrgicle dépend d'une foree locale inhérente aux organes mêmes qui sont le siége de eet étai tnrugide; car on lle voit subsister pendant un certain temps dans des parties détaehées d'un végétal, telles que des fenilles, et même dans de petites portions de feuilles. Cette faeulté d'être turgide est nurc faculté vilale du végétal. Par eetle expression frculté vitale, je ne désigne point une faeulté oeenlte, comme on pourrait le 
croirc; nous verrons plus bas en quoi ellc consiste. Il nous suffi ici d'avoir déterminé quclles sont les conditions de l'existence de l'état turgride chez les végétaux, et d'avoir prouvé que l'état turgide des petits organes creux du végétal est la condition nécessaire de l'ascension de la sève par adfluxion. C'est par cette expression nouvelle que je désigne la progression de la sève lorsqu'elle coule en s'approchant du lieu où sc trouve la cause qui la sollicitc, ou qui l'attire. Ainsi, dans une tige coupée et qui trempe dans l'eau par son extrémité inférieure, c'est seulcment par adfluxion que l'ean monte au travers de la lige vers les feuilles qui opèrent celte ascension du liquide; mais chez un végétal qui tient à la terre par ses raciues, il y a, outre le mouvement de la sève par adfuxion, une progressior ce ce fluide par impulsion, résultal évident d'une force à tergo. Ici, la sève se meut en s'éloignant du licu où se trouve la cause de sa progression. L'observation va nous éclairer sur la nature de ce second phéiomène.

Au printemps, lorsque la vigue versait abondanment de la sève lyinphatique par les blcssures faites à son bois, je choisis une tige dc ce végétal longue de deux mètres, et j’en tronquai l'extrémité, de laquelle la sèvc sorti aussitôi en gyouttes qui se succédaient assez rapidement. Je laissai conler ainsi la sève pendant une heure, pour voir si son écoulemeut était établi d'une manière régulière el pcrmancnte. Après m'être assuré que cet écoulement ne souffrait aucune interruption, el quc les gouttes se succédaient à des 
uervalles de temps tonjours les mièmes, je fis eou'xr d'un seul coup la tige auprès de la terre. J'obseriis, pendant ce tenıps, l'extrémité qui versait la ive, et je vis que le moment même de la section fut Hui de l'interruption de l'écoulement de la sève. Ce iit me prouva que la sève, abondamment contenue uns la tige ainsi séparée du sol, ne trouvait aueune nuse d'impulsion dans eette mème tige; il fallait one ehercher dans les raeines eetle eause inıpulsive. m effet, la portion de lige qui restait en communiation avee les racines, après l'ablation de la longue ortion que javais fait conper près de terre, contiuait seule à verser abondamment de la sève par toute surface de sa seetion. Je fis enlever la terre pui couaait les raeines, ei je eoupai transversalement ces ernières; je vis ia sève sortir seulement de leurs porcons inférieures qui étaient encore plantées dans le bl. Je poursuivis cette recherche dans le même sens, l en descendant toujours plus bas; je vis constamMent la sève sortir exelusivement de la partie la plus nférieure des raeines, et ; arrivai ainsi jusqu'au cheelu, où se trourait évidemment l'origine de eetle orce impulsive. Ce chevelil est composé d'une imnnense quantité de radicelles, toutes ternuinées par un ietit eône blanehàtre: cc petii eône, qui esı en quelue sorte le bourgeon terminal de la racine, a reçn le M. Deeandolle le non de spongiole, qui exprine íusage de eetle partic eonnue pour être le siége à peu orès exelusif de l'absorption opérée par les racines. le eonserverai ce nom, quoiqu'il puisse avoir l'incon- 
vénient d'entraîner l'idée que ce serait à. la manière d'une éponge morte (telle qu'elle est pour servir à nos usages domestiques) que la spongiole s'imbiberait de l'eau qui l'environne; idée qui serait fort éloignée de la vérité. Le hasard me fil rencontrer quelques radicelles qui, plus grosses que les autres, étaient aussi terminées par des spongioles plus grosses. $\mathbf{J}_{\mathrm{e}}$ pris une de ces radicelles, et je plongeai son extrémité seulement dans de l'eau. J'observais, pendant ce temps, à la loupe, la surface de la section de l'autre extrémité de cette radicelle; je ne tardai pas à voir cette surface se couvrir d'ean, qui sortit par le système central exclusivement: le système cortical n'en fournit point. Cette expérience nie prouva que la spongiole est véritablement l'organe auquel est due la force impulsive qui produit l'ascension de la sève : cette partie est en même temps l'organe spécial de l'absorption de l'eau, ainsi que l'a pronvé Bonnel (1). J'ai vu, comme lui, qu'en plongeant des racines pourvues de leur chevelu dans un liquide coloré, il n'y a que leur spongiolè qui s'imbibe de ce liquide, lequel passe immédiatement de là dans le système central de la radicelle. 11 n'y a donc point de doute que la spongiole ne soit en même temps l'organe de l'absorption et l'organe de l'impulsion de la sève lynphatique : la force considérable avec laquelle on voil cette sève sortir des extrémités tronquées des tiges de la vigne, est le résultat et la somme de toutes les petites actions partielles exer-

(1) Rerherches sur l'usnge des Jeuilles, 50 Mémoirc, $\ 9 \%$. 
:źes par chaque spongiole. La sève introdıite dans les ubes lymphatiques, dont le canal est libre et ouvert repuis l'extrémité des raeines jusqu'à eelle des tiges, y trouve soumise à une pression qui la détermine à ortir par toutes les ouvertures qui sont faites à ees ubes; aussi l'ai-je vue sortir par l'extrémité tronquée "ıne racine cétachée dn sol, el qui ne eommunimait qu'avec le tronc de la tige, ainsi que je l'ai déjà apporté plus haut. Iei, la sève lymphatique affectait un monvement rétrourade par l'effet de la pression géıérale à laquelle elle était soumise dans l'ensenıblc des eavités tubuleuses qui la eontenaient. Ainsi, ec cont les spongioles qui sont le siége et l'origine de celte force à tergo qui produit la pression de la sève, th par suite sa progression ascendante par impulsion. 11 nous reste aetuellement à déterminer en quoi eonنiste eette aetion inpulsive des spongioles : avant d'aJorder eette question, il est nécessaire de jeter un soup-d'œil sur la strueture de ees organes.

Les spongioles de la vigne, observées au printemps, Bont de eouleur blanchâtre; leur tissu, tendre ct délicat, est entièrement composé de tissı cellulaire corpuseulifềr. Une partie de ee tissı est artieulée en séries longitudinales; e'est eelle qui occupe le eentre; il fait suite an système eentral de la radieelle, et ses cellules articulécs sont les élémens des tubes également artienlés que j’ai nommés tubes corpusculifères on lymphatiques, et qui serven exclusivement à l'ascension de la sève. Lc tissn cellulaire qui est simé en dchors de eelui-ci, et qui fait suite an système 
cortical de la radicelle, est tout à fait irrégulier, trèstransparent, et couvert de corpuscules d'une excessive petitesse. Si on le couvre d'une goutte d'acide nitrique, il se forme dans l'intérieur de ses cellules de petits caillots opaques, résulıat de la coagulation d'un fluide qu'elles contenaient. Cette couche corticale, d'une grande délicatesse dans le principe, devient plus solide en vieillissant, et se couvre d'un épiderme brunâtre, pareil à celui qui revêt le reste de la radicelle. C'est ainsi qu'on trouve les spongioies de la vigne vers la fin de l'automne. Alors la sève a cessé de monter des racines cians la tirye, et cette suspension du mouvement ascend avec l'absence des sponģioles, cu plutôt avec le changement survenu dans leur organisation; elles ont perdu, en vieillissant, la délicatesse de tissu qui paraît leur être nécessaire pour remplir leurs fonctions. An printemps suivant, on voit une nouvelle spongiole, délicate et blanchàtre, sortir de la pointe de la spongiole endurcie qui forme le bout du filament de chevelu, ou de la radicelle produite l'année précédente. Alors la spongiole ancienne devient une partie du corps de la radicelle; en sorte qu'il n'y a là qu'une production successive de parties nouvelles, de la même manière que cela a lieu dans les tiges. La partie de la radicelle qui est nouvellement formée, participe un peu de la délicatesse d'organisation que possède la spongiole; elle a comme elle un système cortical épais, composé de tissu cellulaire. Au bout d'nn an environ, cette couche corticale meurt et se putréfie, en sorte que le 
iilament de chevelu se tronve alors rédnit à son axe Ligneux central presque dépouillé d'écorce; aussi est11 plus grêle que le chevelu de production nouvelle Ifui lui fait suite. Ces observations m'ont appris que les rérétaux ne perdent point tous les ans leur chevelu, your le remplacer par un autre, comme quelques auceurs le pensent, assimilant ainsi le chevelu des raines aux feuilles des tiges. La seule perte annuelle Iue fasșent les racines consiste dans la couche cortiçale cellulaire, qui revêtait dans leur jeunesse les raHicelles qui composent lo chevelu. C'est cette couche çellulaire délicate qui est spécialement l'organe de ll'absorption.

La spongiole, toujours distincte par la délicatesse He son tissu de la radicelle, à laquelle elle fait suite, me s'en distingue pas toujours par un plus grand vollume. Chez beaucoup de plantes, les radicelles représentent des filamens à peu près d'une égale grossseur dans toute leur longueur, et se terminent en |pointe. Le végétal chez lequel la spongiole offre les caractères les plus tranchés pour se distinguer de la radicelle, est peut-être la lentil!e aquatique (lenticula gibba). Cette petite plante possède une spongiole trèsvolumineuse, relativement aux dimensions de la racine. Celle-ci est grêle et blanche; la spongiole qui la ttermine est renflée et de couleur verte. C'est par l'observation de cette plante qu'on peut se convaincre facilement que la spongiole est bien véritablement un organe distinct du corps de la radicelle.

L'observation nous a démontré que c'est dans les 
spongioles qu'existe la cause première de la pression qu'épronve la sève lymphatique dans les canaux qui la contieunent, et par conséquent que c'est de ces organes que dérive la force à tergo qui communique l'impulsion à ce fluide. Cherchons à déterminer quelle est la nature de cette force.

Deux hypothèses peuvent servir à expliquer l'état de pression où se trouve la sève dans les tubes lymphatiques : $1^{n}$ quelques faits ayant prouvé que cette sève est souvent mêlée d'air, on peut croire qu'il y aurait dans le véçétal une production de gaz, lesquels tendant à l'expansion à mesure qu'ils se forment, imprimeraient ainsi une impulsion à la sève, dans les canaux de laquelle ces gaz sont contenus; $2^{\circ}$ on peut croire, avec plusieurs physiologistes, que les organes creux qui contiennent la sève se contractent sur ce fluide, comme le cœur se contracte sur le sang des animaux.

La présence de l'air mêlé avec la sève dans les tubes lymphatiques, est un fait certain. On connaît l'expérience de Conlon, que j’ai déjà rapportée plus hatut: mu trou horizontal pratiqué avec une tarière jusqu'au centre d'un peuplier, fait entendre, lorsque la sève monte, un bruissement continuel dû au dégagement de l'air qui sort, avec la sève, des orifices ouverts des tubes qui contiennent ce fluide. Hales, dans plusieurs de ses expériences, a vu de l'air sortir par la section transversale des tiges et des racines. La cause de ce phénomène n'est pas difficile à pénétrer. Lorsque dans ıne plante herbacée l'évaporation est beau- 
coup plus abondante que ne l'est l'afllux de la sève ascendante, cette plante se flétrit, toutes ses parties diminuent de volume par l'affaissenent des tubes et des cellules, dont les parois, à raison de leur mollesse, se prêtent avec facilité à cet affaissement. Ce même effet a lieu, mais d'une manière moins sensible, lorsque l'excès de l'évaporation sur l'afflux de la sève est peu considérable; alors la plante ne se flétrit point, elle doit seulement diminuer un peu de volume. Cette diminution de volume n'a point lieu dans le bois d'nn végétal ligneux : là les tubes conservent invariablement leur diamètre, en raison de l'impossibilité où sont leurs parois de se rapprocher par afIfaissement: les parois de ces tubes sont dures, comme lle tissu ligneux dont elles font partie; elles sont fortement adhérentes aux parties contiguës. Il résulte de llà que lorsque ces tubes cesseront d'ètre complètement remplis de sève, le vide qui résultera de l'absence de ce liquide devra nécessairement être rempli par de l'air, qui pénétrera à travers le tissu du végétal. C'est ainsi qu'on trouve de l'air atmosphérique pur dans la cavité centrale qu'olfre la tige des planthes fistuleuses. Cette présence de l'air dans les tubes lymphatiques, lorsque la sève cesse de les remplir, est un fait certain; on peut s'en convaincre en examinant le bois de la vigne en automme. Alors la sève n'abonde plus dans la tige de ce végútal, et l'on trouve la plupart de ses tubes lymphatiqnes entièrement vides de liquide: ils ne contiennent que de l'air. C'est d'après une obscrvation semblable quo 
Link a soutenu que les tubes de ce genre ne sont destinés qu’à condrire de l'air (I) : ce qui est une erreur. La présence alternative ou simulıanée de l'air et de la sève dans les tubes lymphatiques des végétaux ligneux, suffit pour rendre raison du dégragement d'air qui a lieu chez ces végétanx. Lorsque la sève monte, an printemps, dans ces tubes, clle en chasse l'air qu'elle y rencontre, et le force à sortir par les issues qui lui sont artificiellement livrées. De là ce bruissement entendu par Coulon, dans son expérience citée plus hant. La sève épronve, dans les végétaux, un balancement continuel dû aux alternatives du chaud et du froid de l'atmosphère, à l'augmentation et à la diminution de la transpiration. Il résulte de là que les vaisseaux des plantes doivent éprouver des variations continuelles dans le degré de leur réplétion. Si l'excès de l'évaporation sur l'absorption y produit un vide, l'air s'y précipite pour le remplir; si le contraire a lieu, et que la sève vienne à remplir les vaisseaux, elle chasse l'air précédemment introduit: ceci, comme je viens de le dire, ne peut avoir lieu que chez les végétaux à tissu solide, el spécialement chez les végétaux ligneux, dont les tubes sont endurcis. Jamais on ne trouve d'air dans les tubes des productions vérétales nouvelles et encore herbacées. Il résulte de ce balancement continuel de la sève, un balancenent également continuel dans l'air tour à

(1) Recherches sur. l'unatomic des plantes, dans les Amnalus du. Musémm d'histoire naturelle, 1. 19. 1. 330. 
our introduit dans le végétal, el chassé de son intérieur. De là vient que Coulon observa le bruissement dont il a été question plus hant, pendant toute la belle saison; de là vient également cet air que Hales a vu sortir de la seetion transversale des tiges et des racines des végétaux lignneux. J'ai observé, avee beaueoup de soin, l'effusion de la sève par les rameanx tronqués de la vigne; lorsque celte effusion commenẹait à s'opérer, j’ai vu des bulles d'air sortir avec la sève; mais lorsque eette effusion était devenue extrêmement abondante, il ne so:tait pas une seule bulle d'air avee elle. Cependant, è êt été alors qu'il cn aurait dû sortir le pluns, si l'exparsion de ee gaz en̂t été la cause de l'expulsion de la sève. Ceci ma pronvé que les bulles de gaz dont j’avais observé la sortie au commeneement, ne provenaient que de l'expulsion de l'air atmosphérique introduit dans les tubes lymphatiques depuis l'automne précédent, expulsion qui , était opérée par l'afflux de la sève au printemps. Nous avons vu d'ailleurs que c'est dans les spongioles qu'il faut chereher la eause de l'impulsion de la sève. Or, l'observation de ces organes ne $\mathrm{m}$ 'a pas fait apercevoir une seule bulle d'air dans leur intérieur; renon' ẹons done à trouver, dans la produetion contiunelle ret intérieure d'un gaz, la cause de l'impulsion de la sève, et voyons si nous devons reeourir, pour exipliquer ee phénomène, à la contraction des or'ganes.

L'irricabilité existe ehez les plantes, et sa nature 'y est la même que chez les animaux. J'ai démontré 
cette vérité dans un précédent ouvrage (I), et jy ai fait voir que cette faculté consiste dans la propriété que possède le tissu organiqne de pouvoir s'incurver, ou donner de la courbure à quelques-unes de ses parties. Ainsi, il n'est point impossible que les organes qui contienment la sève se contractent sur ce fluide. Il est un fait sur lequel on a spécialement insisté pour étayer cette opinion. Si l'on coripe en travers une plante laitense, telle qu'une euphorbe, ou une laitue par exemple, on voil le suc propre sortir des surfaces, divisées en quantité souvent assez considérable; il sor't également de la portion de tige qui est restée fixée au sol, el du fragment de tige qui a été enlevé; cette émission a également lieu chez ce dernier lorsqu'on le tient dans une position renversée, en sorte que le suc propre, pour sortir, surmonte la force de la pesanteur. Il est fort évident que ce suc est chassé hors des vaisseaux qui le contiennent, par une force intérieurc. Si l'on veut chercher une origgine éloignée à cette force d'impulsion, on ne lui en trouve aucune : elle n'a point, en effet, son origine dans les racines, puisqu'elle se manifeste dans un fragment détaché de la tige; elle n'a point non plus son origine dans les fenilles, pnisqu'en coupant une tige d'enphorbe au-dessous de l'insertion des feuilles, on observe, dans la portion de tige fixée au sol, une émis-

(1) Recherches sur la structure intime des amimaux el des "ígéfrux, ef sur leur motilite. 
sion de suc propre qui se manifeste à chacune des sections successires que l'on fait de haut en bas à cettc tigge. Ánsi, c’est dans le licu même où sc fait l'émission du liquide qu'cxiste la force qui l'cxpulse. Celte expulsion n'est point opéréc par l'cxpansion d'mu gaz, car le suc propre cst torjours exenipt de bulles d'air. Cependant, il cst bien évident que le suc propre est sommis à nue pression dans les váisscaux qui le contiennent, et que c'est par l'cflet de cettc pression qu’il s'échappe par tontes les onvertures que l'on fait à ces vaisscaux. Cette pression estelle produite par la contraction des vaisscaux? Il semble tout naturel de l’admettre; car on n’inagine pas qu'il puisse cxister ici unc autré cause d'cxpnlsion. Ainsi, la force de contraction des vaisseaux se trourcrait ici prouvćc non pas directentent, nais par l'cxclusion de toutes les autres canses conmucs qui seraient susceptibles de produire le même cffet. Une cxpéricnce de Van-Marmu semble venir à l'appui de ccttc hypothèse. Ce physicien ayant fait passex unc forte décharche électrique au travers de la tigge d'une cuphorbe, vit que cette plante ne versait plus de suc ipropre lorsqu'on la coupaiı en travers, bien qu'clle ı cn versât encore cn la. comprimant. Or , comme on. - sair yuc les fortes décharches électriques détruisent ll'imitabilité des animaux, il paraît tont natmel de Ipenser qu’ici la méme canse anrail démmit l'irritabilité des vaisscaus qui conticruent le suc propre, le'quel n’étant plus pressé par la contraction de ces 'aisseanx, ne fait plus aucnu nouvement pour cu 
sortir, à moins qu'on ne l'y furce par une eompression extérieure.

Une autre expérienee de Brugmans et Coulon semblait aussi venir à l'appui de l'hypothèse par laquelle on admet dans le lissu végétal une eontraction semblable à celle qui existe dans le tissu des organes des animaux. On sait que l'applieation de eertaines substanees astringentes arrête l'éeoulement du sang, par la eonstrietion que ees substanees produisent dans les vaisseaux eapillaires. Or, Brugmans et Coulon (1) ont eru observer que les astringens, et notamment l'alun et le sulfate de fer, arrêtaient l'éeoulement du sue laiteux des euphorbes; mais eette assertion a été eontredite par Van-Marum, par Linek et par Tréviranus. Je puis joindre, dans eette eirconstanee, mon témoignage à eelui des observateurs que je viens de eiter; ainsi, il n'y a point de doute que Bruymans et Coulon ne se soient trompés. Malgré eela, entraîné par la néeessité d'admettre une eause queleonque pour expliquer l'état de eompression où se trouvent les fluides dans les organes eapillaires des végélaux, je regardais eomme infinimeut probable l'opinion qui fait dériver la progression de la sève de la eoniraetion des organes dans lesquels elle est eontenue, lorsqu'une seule observation est venue me désabuser à eet égard. On sait que le figuier (ficus curica) eontient une grande quantité de suc laiteux non seulement dans le système eortieal, mais aussi dans le système eentral

(1) Sournal de physique, t. 51, p. $21 \%$ 
lie ses jeunes branches. Tant que le tissu ligneux de ess branches est à l'ćtat herbacé, on peut croire que es parois des tubes sont susceptibles de sc contracter mr le fluide laitcux qu'ils contiennent; mais lorsque ce tissu est endurci ct devenu bois, tonte idéc de conrraction de la part des vaisscaux doit nécessairement llisparaîtrc. Or, si l'on examine les jeunes branches Hu fignicr vers la fin d'octobre, on trouve le tissu lingneux de lcur système central endurci à tcl point, [[u“il se rompt avec éclat; on s'aperçoit éggalcment de a durcté, cn l'cntamant avec un instrument tranthant. L'agrégation des tubes et des clostres dont il st composé est tellcment solide, qu'il faut plus d'un fuart d'hcurc d'ébullition dins l'acide nitrique pour a détruire; cepcridant on voit lc suc laitcurs sortir ort abondammcnt des tubes de ce tissu ligneux, quand on le coupe ou quand on le rompt transversalenicnt. (ci, je le répc̀te, il est impossible d'adnicurc une conraction des vaisseaux; et ccttc obscrvation concluante rouve que c'csı à une antre causc, qucllc qu'cllc soir, [[u'il faut rapportcr l'ćtat de pression on sc trouvent ies Aluides contenus dans les cavités organiques des 'éécétaux. Dc̀s lors, nous voyons qu'il est possible que sc nc soit pas la contraction du tissu des spongioles fui imprime l'impulsion à la sève ascendantc, puisque 'obscrvation prouve l'cxistence d'unc causc inconnue le pression des fluides dans les tubcs végétaux, cause [ [ui n'est certainement pas la contraction de ces tubes. D'aillenrs, cn cxaminant à la loupe les spongioles sur les racines dévcloppées dans l'eau quc conticnt un 
vase de verre, on ue remarque pas le plus léger mouvement de dilatation et de contraction alternatives: on sent que cela devrait avoir lieu, si c'était la contraction du tissu des spongioles qui chassât dans les racines l'eau qu'elles pompent sans cesse. Tout ce que l'obsèrvation montre à cet égard, c'est que les spongioles sont dans un état turgíde continuel et trèsmarqué. La cause de cet état turgide est évidenıment la même que celle qui entretient l'état turgide de la tige el de ses appendices; cause qui, comme nous. l'avons vu, n’est point la simple capillarité. Cet état turgide provient de la réplétion avec excès des cavités organiques qui contiennent les fluides. C'est parce que les tubes qui contiennemt les sucs propres sont turgides, c'est-à-dire remplis avec excès, qu'ils se vident en partie quand on coupe la plante qui les contient. Il reste à déterminer quelle est la cause de cet état turguide ou de cette réplétion avec excès, puisqu'il est certain que cette cause n'est pas la simple capillarité. La recherche de cette cause inconnue n'eût offert aucune chance de succès; il faut une direction aux recherches, pour qu'elles soient fructueuses : or, ici l'on ignore quelle direction l'on doit suivre. Si donc jai fail, dans celte circonstance, quelques pas ver's la découverte d'une vérité nouvelle, je le dois au hasard, auteur de la plupart des découvertes. Il est vrai de dire, cependant, que j’ai fait nâ̂tre en ma faveur les chances du hasard, par la multitude des objets que j’ai soumis ì mon observation. 


\section{CHAPITRE IV.}

Observations et expéricnces sur la cause et sur- Ies effels de l'élat turgide.

Les observations ct les expériences qui vont être xposées, sont du nombre de celies pour lesquelles la oliysiologrie végétale est tout à fait inséparable de la blysiologrie animale. Ainsi, quoique ce soit la phybiologie des plantes qui nous occupe exclusiventent ci, cependant il nous faudra prendre les sujets de los observations, autant, et nlême plus, dans le rè„gne animal, que dans le règne végétal, détcrminé en cla par la plus grande opportunité des conditions farorables aux observations et aux expériences.

Un très-petit poisson dont j’avais coupé la quene, t que je conservais vivant dans un vase plein d'eau, offrit sur la surface de sa plaic la production d'une iorte de nuvisissure aquatique à filanens assez longs, tesquels étaient terninés chacun par un petit renflement très-facile à apercevoir à l'oeil nu. J'ens la curiosité d'observer an microscope cette plante qui végétait sur un animal vivant. Les filanens de la moisissurc "śtaient trunsparens; les renflenıens qui les terminaient, et qui ressenblaient aux capsules d'un végétal, élatent terninés en pointe, et complètonent spatues. Je conpai quelques-uns de ces filanens, et je les placai dans mo cristal de monte avec m pou 
d'cau, afin de les observer à loisir an mieroseope. Je ne tardai pas à voir quelques - unes des eapsules dont je viens de parler, expulser par une ouverture située à leur pointe, une multitude de globules; pendant ecte expulsion, la eavité de la capsule se vidait seulement à sa partie inférieure opposée à la pointe qui donnait issue anx globules; la masse de ees derniers, qui remplissait eneore la partie supérieure de la eavité eapsulaire, scmblait pressée, et fortement ehassée en hant par l'acenmulation de l'ean dans la partie inféricure de celle eavité eilpsulaire, qui ne diminuait aucunement de eapaeité, eu sorte qu'il me fut bien prouvé que l'expulsion des globules n'était point due à une eontraction de la eapsule. L'eau, par son introduetion dans la partie inféricure de la eavité eapsulaire, semblait faire iei l'office du piston d'une seringue, pour chasser eu hant, et cxpriser par la pointe de la capsule, l'anas de globules qui primitivement remplissait eette dernière en entier. Dans l'espaee de deux ou trois seeondes, tons les globules furent expulsés de leur eapsule, qui demeura pleine d'ean sans aroir rien perdu de ses dimensions primilives. Il semble inutile de dire que j’avais la eertitude que ee n’était pas de l'air qui s'étaic développé dans la eavité eapsulaire pour remplaeer la masse des globnles qu'il auraut expulsée, ear l'air eontenu dans les petits organes transparens plongés dans l'eau, est très-facile à voir et à distinguer an mieroseope. Je crus d'abord que les globules dont je venais d'observer l'expulsion étaicnt sortis de leur propre mouvement, et que e'é- 
ieul des animalcules. On sait que M. Bory de Saintincent a découvert, dans les plantes singulières qu’il nommé zoocarpées, une émission de globules anicés de mouvemens spontanés, ģlobules qu'il considère mme de véritables animalcules. Je me rappelai que reedham avait fait mention d'un semblable phénocène, et précisément chez une moisissure aquatique. t observateur avait vu Ies capsules terminales de tte moisissure donner naissance par émission à de ritables animalcules, assertion qui fut vivement mbattue par Spallanzani ( I). Je ne doutai point que Imoisissure aquatique que j'avais observée, ne fùt la iême que celle qui s'était présentée à l'observation : Needham, et je résolus de l'observer de nouveau tec beaucoup de soin, afin de voir si véritablement ; globules émis par les capsules étaient des animaliles. Il me fut facile de reproduire la plante dont (est ici question. Je prenais de l'eau du bocal où viiit le petit poisson, sur lequel cette moisissure s'était 'veloppée la première fois, et j'y plongeais des frayiens de substance animale, lesquels ne tardaient pas sse couvrir d'une épaisse production de cette plante. sci d'abord une pronva que celte production ne teait point du tont à la vie de l'animal sur lequel je avais d'abord observée. Il faut quelques jours pour ue cette plante acquière le degré de maturité nécesire, pour qu'elle puisse donner lieu à l'émission des obulcs contenus dans ses capsules. A cette éporque,

(1) Obsemations et enperiences sur les animalenles, chap. ה. 
je pris quelques-uns de ses filamens que j’isolai dans un cristal de montre rempli d'eau très-pure, et exempte d'animaleules. Par une observation assidue, je trouvai le moment où l'une des eapsules opérait l'émission. de ses globules. Ce phénomène se passa exaetement comme je l'ai décrit plus haut. Je vis les globules répandus dans l'eau enviromnante, s'agiter en divers sens pendant un instant, puis ils se préeipitèrent au fond de l'eau, où ils demeurèrent immobiles. Ainsi, il me fut prouvé que ees globules n'étaient point des animalcules; leur mouvement, en apparenee spontané dans l'eau, un instant après leur sortie de la eapsule, n'était évidemment que le résultaı du mouvement imprimé à ee fluide par le fait même de cettc émission : ce mouvemeut de l'eau étant apaisé, les globules eessaient aussi de se mouvoir. Je ne doute done point que ces globules ne soient les graines de la plante; aussi ne pouvais - je reproduire eette dernière qu'avec l'eau dans laquelle elle avait végété. A yant mis les deux moitiés d'un grain de blé, l'unc dans cette eau, et l'autre dans de l'eau très-pure, il n'y eut que la première qui se couvrit, sur la surface de sa seetion, de la moisissure dont il est ici question; la seconde moitić n'offrit aueune végétation. Les globules eontenus dans les eapsules de la noisissure n'é tant point des animalcules, on ne peut attribuer leut sortie de la eapsule à leur nouvement spontané : unc dernière considération confirme ee r'ésultat. Si c'étaient des animalcules qui sortissent par un mourement spontané de l'intérieur de la eapsule, on verrait 
?zider la première la partie de cette cavité la plus oisine de l'ouverture qui leur donne issue. Or, c'est ul eontraire la partie opposée qui manifeste sur le hamp un vide que remplit l'ean. Il n'y a donc point ce doute que cet amas de globules ne soit une masse herte, qu'une foree it tergo ehasse vers la pointe de t eapsule, et de là au-dehors, exaetement de la même uanière que l'eau est ehassée hors d'une seringue par : piston. L'ean introduite dans la partie inférieure ce la cavité eapsulaire, est évidemment l'instrument uéeanique de cette foree ì tergo qui produit l'imulsion de l'amas de globules qui remplit le reste de utte cavité. D'où vient celte eau? qnelle est la foree ui la pousse dans l'intérieur de la eapsule? J'avais ensé d'abord qu'elle élail poussée dans la eapsule ar les organes intérieurs du filanent, au sommet uquel cette capsule est située; mais j’a dû rejeter ette idée, en voyant des eapsules détachées de leurs lamens opérer de mème, et par le même mécanisme, ténission de leurs globules. Il me fallut done alors lacer ce phénomène au nombre de cenx dont la ause est tout à fait ineonnue. Cette observation étant nieroscopique, peut, par eela même, être frappée "une eerlaine défaveur anx yeux de eeux qui savent e eombien d'illusions d'optiques le mieroseope est souree. Je me contentai done de noter ce fait, et : n'y pensais plus, lorsqu'un fait du même genre, et ont l'observation n'avait plus besoin de l'emploi du nieroseope, vint s'offrir à moi. Ce fut le règne animal ui me la fournit. 
L'accouplement des limaces offre une particularité bien remarquable, et qui n’a point encore été notée. La verge de ces molusques est, avant l'accouplement, revêtue d'une gaîne épidermoïde imperforée, qui ne lni est point adhérente. L'accouplement étant effectué, le sperme, qui est pâteux chez ces animaux, sort par l'extrémité de la verge, et s'accumule dans la gaîne imperforée qui lui sert de fourrean, en sorte que la verge se trouve refoulée à mesure que le sperme s'accumule dans cette espèce de sac. Lorsque ce dernier est entièrement rempli, il se brise à la racine de la verge; l'accouplement cesse alors d'avoir lieu, et il reste ainsi dans l'organe femelle de la génération, un petit sac rempli par la pâte spermatique. Si l'on trouble l'accouplement lorsqu'il est sur le point de finir, on détermine sur-le-champ le détachement du sac dont il est ici question, et sa séparation de l'organe mâle. La limace, effrayée, se contracte avec force, et chasse hors de son organe femelle ce petit sac rempli de sperne. Ces animaux, comme on le sait, sont hermaphrodites, et ont besoin d'un accouplement réciproque; en sorte que, dans une seule observation de ce genre, je pus me procurer deux petits sacs remplis de pâte spermatique. Ces sacs ressemblent à de petites cornues; ils sont fortement courbés sur eux-mêmes, et phus gros à leur extrémité aveugle qu'ils ne le sont à leur entrée. Ils ont ordinairement environ quinze millimètres de longueur; leur diamètre est de trois millimètres à leur extrémité renflée, el d'un peu plus d'un millimètre à leur antre extrémité. Comme j’ai l'habitude 
d'observer les objets délicats en les couvrant d'ean, je plongeai inn de ces petits sacs dans un vase rempli ide ce fluide, et je l'y laissai sans y toucher. Ayant télé distrait de mon observation, que je repris nne demi-henre après, je fus singulièrenent surpris de trouver ma petite cornue vide de sperme, et remIplie d'eau dans sa partie la pliss grosse ou dans son ffond; la pàte spermatique avait été chassée par le col de la cornue, et s'était répandne dans l'eau, où ielle conservait sa consistance pâteuse. La moitié eniviron de la petite cornue était encore remplic de sperme, que je ne tardai pas à voir s'écouler hors de lla cornue. Cet écoulement, on plutôt cettc émission, cétait bien évidemment l'effet d'une impulsion opérée par une force à tergo; la pâte spermatique sortait avec ceffort par le col de la cornue, qu'elle remplissait exactement; en sorte que ce n'était bien ccrtainement point par ce col que s'introduisait l'ean qui remplacait le sperme dans le fond de la cornue à mesure qu'il en sortait. Cette eau, accumulée de plus en plus dans ce fond de cornue, était bien évidemment l'agent mécanique de la pression qui déterminait la pâte spermatique à sortir par l'ouverture du col de la corınue; anssi cette dernière était-elle tırgide; clle était distendue avec excès par l'eau; en un mot, il me fut facile de voir ici, à l'œil nu, et sans crainte d'aucune illısion d'optique, la répétition et la confirmation des observations que j’avais faites précédemment au micróscope sur la moisissure aquatique. Lorsque la cornue fut presque entièrement remplie d'eau, et qu'il 
n'y cut plus qu'une dernière portion de sperme à expulser, je fus témoin d'un phénomène qui ne me laissa aucun doute sur la cause de eette expulsion. Cette dernière portion de sperme sortit de la eornue avee un eourant d'ean que l'on distinguait à la répulsion qu'il exerçait sur les eorps légrers qui flottaient dans le liquide; ce courant devint de suite très-faible, et eessa au bout d'une minute d'être sensible. La petite eornue, ainsi vidée de la pâte spermatique qu'elle eontenait dans le principe, conserva exactement son diamètre primitif. Il ne se manifesta aucune contraetion dans ses parois, lesquelles n'éprouvèrent non plus aucun affaissement. J'avais conservé au sec la seeonde des deux petites eornues que je m'étais procurées. Elle était restée renuplie par la pâte spermatique. Je me hâtai de la mettre dans l'eau pour jouir encore une fois de l'observation de ce eurieux phénomène. Afin d'éviter tonte cause d'crreur, au lien de la plaeer horizontalement comme la première, j'eus soin de lui donner une position inelinée; en sorte que l'orifice du col de la petite eornue était tourné en haut, et se trouvait plus élevé que le fond. De cette manière, on ne pouvait attribuer l'émission de la pûte spermatique hors de la eornue à un simple éeoulement. Bien que la consistanee pâteuse du sperme s'opposât à ce qu'on pût adopter eette idée, j’étais eependant bien aise d'avoir une preuve de plus eontre son adoption. Or, dans cette seconde observation, les ehoses se passèrent exactement comme dans la première, excepté qu'il fallut un peu plus de temps pour que l'expulsion du sperme 
fùt eomplète; ce qui arriva au bout d'une heure et demie. Je vis, eomme dans la première observation, la pâte spermatique pressée, refoulée vers l'ouverture du eol de la eornue, sortir avec effort par eette ouverture. Peut-être pourrait-on penser que cette sortie étail le résultat d'un mouvement spontané, exécuté par des animaleules, ou plutôt par les globules mouvans, qui ordinairement abondent dans le sperme des animaux; mais il n'en est rien. J'ai examiné au imieroseope ce sperme de la limaee, et j’ai vu qu'il eonhenait une immense quantité de globulcs iner'tes et sans : aneun mourement. Ce sperme est tcllcment consistant, qque, nalgré son aceumuiation dans le sac qui le eonitient, et où toutes les parties de sa masse son t pressées les - unes contre les autres, il eonserve ecpendant sa disposiItion en un fil eontinu, disposition qui lni a élé donnée llors de son émission en passantpar l'ouverturc ćlroite de lla verģe du mâle. D’après ces observations et eelles qui iviennent d'être exposées, il est de la plus grande évidenee que la sortie de la pàte spermatique hors des petites cornues qui la eontiennent, n'est ni le résulltat d'un éeoulement ni eclui d'un mouvernent spontané cxéeuté par des animalcules. C'cst, comme je l'ai dit, une véritable expulsion produite par l'aecumnlation de l'eau ù tergo. Il est dione prouvé que l'eau, saccumulée de plus en plus dans l'intérieur des petites eavités organiques, y devient un agent mécinique d'impulsion, y devient une force ì tergo qui produit l'expulsion, lors de cees pelites carités, des suisiatances quo!les contenaient amparant. Iar vi cete 
eau sans cesse aftlnente s'introduit-elle? Il est évident que ce ne peut ètre qu’au travers des parois de la cavité dans laquelle elle s'accumule, puisqu'il n'existe dans ces parois aucune ouverture sensible. Ainsi nous voyons dans ces observations de petits organes creux qui sont doués de la faculté singulière d'introduire avec violence, dans lenr cavité et au travers de leurs parois, l'eau qui baigne leur surface extérieure, et cela de manière à chasser hor's de cette cavité les substances qui y étaient précédemment contenues. Lá cause de ce phénomène, ou de celte action physicoorganique nous échappe ici; mais nous devons noter une condition qui paraît nécessaire pour sa production.

Nous avons vu que l'introduction sans cesse aclive de l'eau dans les pelits sacs spermatiques de la limace, n'a eu lieu que tant qu'il a existé un reste de sperme dans ces petits sacs. Lol'sque toute celle substance pàteuse a été expulsée, nous avons vu qu’il sortait par l'ouverture de ces sacs un courant d'eau qui s'est promptement affaibli, et qui enfin a cessé de se montrer. Ainsi l'introduction forcée de l'eau au travers des parois de la cavilé organique a cessé, lorsque celte cavité, délivrée du corps consistant et dense qu'elle renfermait, n'a plus contenu que de l'ean pure. La présence d'un corps plus dense que l'eau dans les pelites cavités oryaniques, est donc une des conditions nécessaires pour y déterminer l'exercice de l'action physico-organique qui introduit avec violence l'ean dans leur intéricur.

'íels sont les denx faits sur l'ubservation desfuels 
'ítablis l'existence de la nouvelle action physicoorganique que je viens d'indiquer; action dont le Houble effet est de rendre turgides les petits organes reux, el de produire l'expulsion des substances que ces organes contiennent; double effet qui trouve sa cource dans une cause unique, dans l'accumulation continuelle et avec excès de l'eau dans l'intérieur de ces petits organes creux. Cette action physicorrganique, dont l'observation est neuve, a besoin "être désigntée par une expression nouvelle. Je désinerai donc celte action, en vertu de laquelle les wetits organes creux se remplissent d'un liquide qui. eomble être poussé et accumulé avec violence dans rur cavité, sous le nom d'endosmose (I). L'existence ce cette action physico-organique ou vilale étant rouvéc par l'observation des opérations spontanées ce la nature organique, nous allons chercher à lui onner des preuves confirmatives par le moyen de expérience.

L'observation de la turgescence acquise par les acs spermatiques de la limace, me fil penser que je ourrais obtenir un résultat analogue avec les intesins de petits animaux; intestins dans lesquels j'incoduirais, avant de les plonger dans l'eau, un liquide rganique plus dense que ce fluide ambiant. J'espéis, par ce moyen, obtenir une endosmose ou une troduction de l'eau dans la cavité intestinale, fernée de toutes parts; les intestins de ponlets âgés

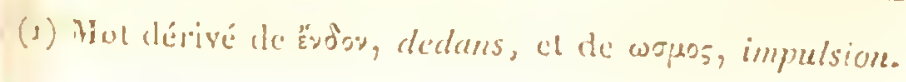


d'environ trois mois, me parurent propres à ce genre d'expériences. Je pris donc un cocum de poulet; et après l'avoir bien nelloyé intérieurement par des injections d'eau pure, je le remplis à moitié avec du lait, et je fermai son entrée avec une ligature. Je plongreai ensuite ce coecum dans un vase rempli d'ean de pluie : il pesait, avec le lait qu'il contenait, r 96 grains. Vingt-quatre heures après, je le tirai de l'eau; et l'ayant pesé, je trouvai son poids de 269 grains : il avait gagné $7^{3}$ grains par l'eau qu'il avait introduite. Je replaçai le cœcum dans de l'eau que j'avais toujours soin de renouveler soir et matin; douze heures après, je trouvai son poids de 3 r 3 grains. Ainsi, dans l'espace de trente-six heures, le cœcum avait introduit dans sa cavité I I 7 grains d'eau : il était devenu très-turgide. A partir de cet instant, le poids de cet intestin diminua continuellement: il perdit son état turgide; et sa vacuité, toujours croissante, occasionna l'affaissement de ses parois. Trente-six heures après le commencement de cette diminution de poids, le cœcum ne pesait plus que 259 grains : il avait perdu 54 grains de l'eau qu'il avait introduite. Prévoyant, par la continuité de cette déperdition, qu'elle ne ferail qu'aller en augmentant, je mis fin à l'expérience; j'ouvris le cocum, et le trouvai rempli de lait caillé et putride. La température, pendant la durée de l'expérience, s'était maintenue de 18 à 2 I degrrés $R$. Ayant nettoyé l'intérieur du cœcum par des injections d'eau pure, j'en remplis de nouveau la moitié de la cavité avec du lait fiais, eı je le plaçai, comme 
it l'ordinaire, dans de l'eau. Vingt-quatre heures après, ce trouvai son poids augmenté de 2 I grains. A partir He eet instant, le poids du exeum conmenẹa à diminuer progressivement. Je l'ouvris vingt-quatre heuces après, et trouvai eneore dans son intérieur du lait lléeomposé et putride. Le fait de l'endosmose se rerroduit manifestement à nous, dans cette expérience. Wous voyons, en effet, un organe crenx qui introduit Hans son intérieur, et au travers de ses parois, le fluide Hui l'environne, et eela de manière à devenir turogide. Ge phénomène, bien évidemment, ne dépend point He la capillarité; e'est une aetion physieo-organique thont la eause ne nous est pas eneore connue. La qualité du fluide organique eontenu dans le eøcum a खxercé une influenee bien marquée sur eette action whysico-organique, ear nous l'avons vue eesser lorsHue le lait eontenu dans cet intestin s'est altéré dans sa eomposition; et nous avons vu ensuite eette aetion se renouveler, mais eependant avee bien moins d'indensité, lorsque du lait frais a été substitué au lait eorrompu, pour eesser de nouveau et plus promptement que la prenière fois, lorsque le lait s'est altéré. Alors, le eœeum en macération dans l'eau depuis six jours, par une température élevée, avait pris lui-même une odeur putride; il était, par eonséquent, altéré dans sa eomposition, et par eela même moins propre qu'il ne l'était dans le prineipe à exereer l'aetion physicoorganique que je nomme endosmose, aetion vitale qu’il exerçait avec énergie dans l'état frais. Cette expérience, qui nous prouve la réalité de l'influzence 
qu'exerce sur la production de l'endosmose le fluide contenu dans la cavité organique, nous prouve en même temps que la dégénérescence putride de ce fluide anéantit complètement cette action physicoorganique. Alors, le liquide du dehors n'est plus sollicité à pénétrer dans l'intérieur de l'organe creux; c'est, au contraire, le liquide intérieur qui est sollicité à se porter au dehors.

Il faut qu'un oliquide organique plus dense que l'eau, et pourvu de ces /qualités "chimiques particulières qui constituent l'état sain, existe dans un organe creux, pour provoquer énergiquement chez lui l'exercice de l'endosmose : cela est vrai goénéralement; mais cette condition;, nécessaite pour l'énergie de l'endosmose, ne l'est point pour son existence. Les expériences suivantes m'ont prouvé cette vérité.

Je remplis à moitié d'eau de pluie un cœcum de poulet : il pesait, dans cet état, 64 grains, et je le plongeai dans l'eau. II introduisit de l'eau dans son intérieur, et augymenta ainsi de poids pendant trentesix heures. Au bout de ce temps, il se trouva peser 12 I grains : il avait introduit 57 grains. A partir de ce moment, le cocum diminua continuellement de poids jusqu'au troisième jour, dans le courant duquel je le vidai de l'eau putride qu'il contenait. Après l'avoir nettoyé, j’y introduisis de nouveau de l'eau pure, et je le replaçai dans l'eau : il pesait alors 63 grains. Pendant trente-six heures, le cœcum introduisit de notiveau"de l'eau dans son intérieur; mais cette quantité fut très-petite, car elle ne s'éleva qu'ì 
7 grains. Le eoeum eommenẹa alors à diminuer de poids. Je le vidai de nouveau de l'eau putride quil sc trouva eontenir ; et après l'avoir nettoyé, j'y introduisis du lait: dans eet état il pesait 66 grains; je le remis dans l'eau. Alors l'endosmose, qui étail devenue très-faible, aequit sur le ehamp une énergie nouvelle. L'introduetion de l'eau dans le eœeun s'éleva, au bout de trente - six heures, à 58 grains : à partir de eette époque, le poids du eoeemm eommenẹa à diminuer; je l'ouvris, et je trouvai le lait caillé. Le' eœeum lui-mıême, soumis à la macération dans l'eau, depuis sept jours et derri, par une température de + 20 à 24 deşrés R., avait éprouvé une altération putride. C'est eette altération qui, lors de la seeonde introduetion de l'eau, l'avait rendu bien moins propre à exercer l'endosmose qu'il ne l'avait été dans le prineipe; et eependant, nalggré eette altération, eneore augmentée par deux jours de maećration, l'introduetion du lait rendit au eceum son aetion endosmosiqne, avec, plus d'énergie qu'il n’en avait nontré dans le prineipe, lorsqu'il ne contenait que de l'eau pure. Cette expérienee prouve que s’il n’est pas néeessaire, pour l'existence de l'endosmose, que l'organe ereux qui l'cxeree possède, dans son intérieur, un liquide plus dense que le liquide ambiant, au moins est-il certain que eetue plus grande densıté dı liquide intérienr exerce une grande influcnce sur l'augmentation de l'énergie de l'endosmose. Nous voyons encore iei que les liquides putrides contentis dans les organes crenx abolissent eomplètenent l'en- 
dosmose de ces organes tant qu'ils y sont présens, et que l'ablation de ces liquides putrides suffit pour rendre aux organes creux qui ont cessé de les contenir, la portion d'énergie endosmısique qu'ils peuvent encore posséder, d'après l'état d'intégrité où se trouve lenr tissu, car l'état putride de ce tissu est une autre cause de l'abolition de l'endosmose.

L'existence de l'endosmose, lorsque l'organe creux ne contient que de l'eau, étant démontrée, il s'agyit actuellement de savoir si cette action existe de même quand l'organe creux est complètement vide. Pour éclaircir ce donte, je pris tune anse d'intestin de ponlet, bien nettoyée intérieurcment, soigneusement évacuée d'eau, et fermée par une ligature à chaque bout : elle pesait 27 grains; je la plongeai dans l'eau. Au bout de douze heures, je trouvai 13 grains d'eau dans son intérieur. Je ne poussai pas plus loin cette expérience, qui, par son résultat, me donnait par l'affirmative la solution de la question que je m'étais proposée. Ainsi, l'endosmose est une action qui appartient au tissu des parois de l'organe creux; elle n'a point besoin, pour s'exercer, d'être sollicitée par la préexistence d'aucune substance dans la cavité de l'organe, bien que cette préexistence ait, dauls certains cas, une influence sur le degré de l'énergie de cette action'physico-organique.

Je pensais que l'ordre de superposition des membranes intestinales pouvait être une des causes déterminantes de l'introduction de l'eau ou de l'endosmose. Pour m'en assurer, je pris deux anses d'in- 
cestin de poulet, que je retournai, mettant ainsi la nnembrane nuqueuse en dehors, el la membrane peiitonéale en dedans; dans l'une je mis un peu d'eau, laissai l'autre entièrement vide; et les ayant fernées toutes les deux à chaque bout, avec une ligaure, je les plongeai dans l'eau. Tontes les deux inrroduisirent de l'eau dans leur intérieur : l'anse ntestinale, qui contenait déjà de l'eau, en introduisit Hus, dans un temps égal, que celle qui était primititement vide. Je pris deux autres anses intestinales, tue je dépouillai de leur membrane muqueuse : j’en cetournai une, le péritoine en dedans; l'autre fut reHacée dans son état naturel, c'est-à-dire le péritoine n dehors. Un peu d'eau fut mise préalablement dans thacune de ces deux anses intestinales : toutes les deux introduisirent de l'eau dans leur intérieur, et à ou près en égale quantité. Je répétai cette dernière :xpérience, en ne mettant point préalablement d'eau lans les anses intestinales : il y eut également introluction d'eau. Ainsi, il me fut démontré que l'endosnose ne dépendait, dans celte circonstance, ui de iordre de superposition des membranes, considérées le deliors en dedans, ni dı nombre de ces membraues elles-nêmes. Je vis par-là qu’il suffisail que des arois organiques fussent disposées de manière à forner une cavité, pour que le liquide ambiant fùt poussé, Jar une force incomnue, dans l'intérieur de cette cavité qui n’était point capillaire, car les coecum que iemployais avaient généralement de ro à r 2 millinètres de diamètre; les anses intestinales, plus pe- 
tites, il est vıai, avaient cependant 5 millimètres de diamètre, et plus": ce qui ne constiuse point des cavités capillaires.

Convaincu, par les expériences précédentes, de l'influence exercée par la densité du liquide intérieur sur l'augmentation de l'énergrie de l'endosmose, je résolus d'employer un liquide organique plus dense que le lail, que j'avais employé jusqu'alors. L'albumen, ou blanc d'œuf, me parut propre pour cela; et il me parut avoir sur le lail cet avantage, qu'il était moins promplement altérable. Je résolus aussi d'employer de l'eau fortement chargée de gomme arabique, solution encore moins promptement altérable que l'albumen. Mais avant d'employer ces substances, je devais massurer de la manière dont elles se comportaient lorsqu'elles étaient en contact immédiat avec l'eau pure. En effel, il élait possible que ces liquides denses, quoique possédan $\mathrm{d}$ déjà beaucoup d'eau, eussent une tendance à s'en pénétrer davantage, et à se gronfler ainsi par de nouvelle eau introduite entre leurs molécules. On sen q que si celte tendance à se gonfler par l'absorption de l'eau cût existé dans les substances dont il est ici question, cette propriété eût alıéré l'exactitude des résultats que je me proposais d'observer. En effet, ces substances étaut introduites dans des organes creux clos de tontes parts, et environnés d'eau, ce liquide, par' la tendance qu'il aurait eue à gonfler les substances introduites, aurait opéré la distension de ces organes creux, el j’aurais pris pour un effet de l'endosmose l'introduction de 
l'ean, qui n'aurait été que le simple effet d'une altraction chimique. Ponr lever tous les doutes à cet égard, je pris un de ces larges tubes gradućs et fermés par un bout, qui servent à l'eudiométrie. Je le remplis à moilié avec de l'albumen, et je notai le degré du niveau occupé par celte substance. Alors, inclinant légèrement le tube, je fis couler de l'eau dedans, goutte à goutle, ei en glissant le longy des parois. De celte manière, l'eau superposée ne se mêla aucunement avec l'albumen, dont le niveau ne fut point dérangé. Le tabe étant rempli d'ean, on distinguait parfaitement la lišne de démarcation qui séparait les deux fluides. Je praçai le tube dans une position verticale; et l’ayant examiné vingt-quatre heures, et ensuite quarante-huit heures après, je trouvai que l'albumen avait conservé exactement son niveau primilif. Cela me pronra ou'il n'avait aucune tendance à absorber l'eau et à se gonfler par cette addition. Je fis la même expérience avec une solution épaisse de gomme arabique, solution que j’avais teinte très-légèrement en rouge avec du carmin, afin de nieux distinguer la ligne de séparation avec l'ean superposée. J'eus un résultat exactement semblable. Ainsi, il me fut pronvé que la cause d'erreur que je craignais n'existait pas, et je fis l'expérience dont je vais donner le détail circonstancié.

Je pris un cocum de ponlei qui, dans l'état de distension, avait 12 millimètres de largeur, et qui, courbé en arc, avait ıo centimètres de longueur. Je le remplis à peu près à moitié avec de l'albunnen. Dans 
cet état, il pesait 58 grains. Le cœcum fut plongé dans de l'eau de pluie. J'avais expérimenté que cette eau très-pure était plus propre à ce genre d'expérience que ne l'était l'eau très-chargée de carbonate de chaux fonrnie par les puits et par les fontaines du pays que j'habite. Huit heures et demie après le commencement de l'expérience, le cœcum pesait ı 3 o grains. Il avait gagné 72 grains, el était devenu très-turgide. Cet élat turgide se maintint pendant trois jours; et le cœcum, plein à peu près autant qu'il pouvait l'être, ne gagna que 3 grains de pesanteur durant cet espace de temps. Au commencenıent du quatrième jour, il commença à perdre de son poids; et au commencement du cinquième jour, il avait perdu 22 grains. Je l'évacuai d'un liquide putride et floconneux qu'il contenail; et après l'avoir lavé par des injections d'eau pure, j’y remis de l'albumen; il pesait, dans ce nouvel étal, 60 grains. Huit heures après, le cœcum pesait 92 grains; il avait gagné 32 grains de pesanteur. Douze heures plus tard, je trouvai qu'il avait perdu 5 grains de son poids. Celte perte continua à se manifester pendant deux jours, et la diminution totale du poids fut de 20 grains. Alors jouvris de nouveau le cœcum, que je trouvai rempli d'un fluide putride. Je le lavai, et j’y introduisis une solntion aqueuse, assez chargée de gomme arabique. Dans ce nouvel état, le cœcum pesait 48 grains. Cinq heures après son immersion dans l'eau, le cœcum pesait 124 grains; il avait ga gnné 76 grains, et était devenu très-turgide. Il se maintint dans cet état, sans aucune variation de 
poids, pendant deux jours entier's. Le troisième jour, iil eommença à diminuer un peu de poids; il perdit :seulement 4 grains. Le quatrième jour, il perdit 48 grains. Je l'ouvris alors, et je trouvai le fluide ' qu'il eontenait devenu très-putride. Le cœeum lui; même se putréfiait, et je eessai de l'employer. La temipérature, pendant la durée de ces expériences, s'ćtait : soutenue entre 18 et 2 I degrrés $R$. Ces résultats me Ifournirent la confirmation de eenx que j'avais obte: nus préeédemment. Il me fut pleinement démontré 'que l'introduetion de l'eau dans la eavité organique - dépendait entièrement de la nature du fluide, plus i dense que l'cau que eontenait eette eavité. Tant que ee fluide conservait son intégrrité de conprosition, l'endosmose avait lieu; mais dı moment qu'il était devenu putride, l'endusmose ecssait, et l'eau, au licu d'entrer dans la cavité eomme auparavant, en sortait, et mêne assez rapidement. J'ivais d'abord aturibné eetue sortie de l'cau à ec que l'endosmose ayant ecssé, les parois du coenu eomprinaient par leur pesanteur le fluide contenu dans sa eavité, et le forçaient ainsi ì fiturer au travers de ees nıèmes parois. Cependant, la rapidité de ecte sortie de l'eau ine donna lieu de soupçonner que eetle sortie était le résultal d'unc aetion partieulière opposée dans sa direction à l'endosmose, et due à la même eause. L'expérience suivante me prouva que ec soupecon était fondé. Je remplis aux trois quarts un eœeum de poulet avce de l'can 'qui tenait en solution $1 / 5$ o de son poids de gomme. arabique; et après l'avoir fermé par une ligature, je 
le plongeai dans l'eau pure. Il pesait 6 I grains. Au bout d'une henre d'immersion, je trouvai son poids augmenté de 6 grains. Alors je le retirai de l'eau pure, et je le plongeai dans de l'eau qui tenait en solution $1 / 1$ o de son poids de gomme arabique. L'ayant pesé de nouveau au bout d'une heure d'immersion, je trouvai quil avait perdu 30 grains de son poids. Celte perte augmenta si rapidement, qu'au bout de deux heures le cœcum étaii presque entièrenent vide. Cette expérience, que je répétai plusieurs fois, el toujours avec le même résultat, me prouva que, dans celte circonstance, l'eau est poussée au traver's des parois de la membrane organique, de manière à ce que son courant est toujours dirigé vers le plus dense des deux fluides qui baiggnent les deux parois opposés de cette membrane. Ainsi, lorsque c'est le plus dense des deux fluides qui est dans la cavité, l'eau y est introduite par l'action que j'ai nommée endosmose; lorsqu'au contraire c'est le plus dense des denx fluides qui est hors de la cavité, le fluide le moins dense, qui est au dedans, est poussé au dehors par une action inverse que je nommerai exosmose (1). C'était par un effet de cetle action expulsive que le liquide intérieur, lorsqu'il était devenu putride, était chassé hors des cavités organiques, dans les expériences qui ont été rapportées plus haut. Cependant ce fluide putride n'avait pas cessé d'ètre plus dense que l'eau environnante; il anrail dû, par conséquent, en celte

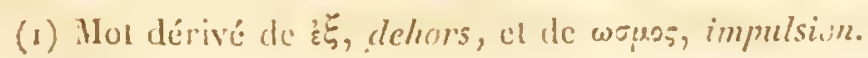


qualité de fluide płus dense, continuer à déterminer ll'endosmose; si, au contraire, il a déterniné l'cxosmose, cela prouve qu'il y a d'autres conditions que la Hifférence de densité, qui sont propres à déterminer le Houble phénomène de l'endosmose el de l'exosmose. wes conditions nouvelles me parturent devoir se trouver dans la nature chimique des fluides. Pour m'en ussurer, je fis de nombreuses expériences en in troduiiant dans des cœzum de poutet de l'eau faiblement uhargée d'un acide ou d'un alkali; je f'ermais ces oryganes avec une ligature, et je les plongeais dans l'eau oure. J'ai vu constamment dans ces expériences les hlkalis produire l'endosmose, et les acides produirc l'cxosmose. J'avais soin, comme je viens de le dire, He n'employer ces agens chiniques qu'à des doses asiez faibles, afin d'éviter tes altćrations qu'ils n'auraient pas manqué d'apporter dans la texture des membranes orsaniques. Il y avait une cause d'erreur à éviter Hans ces expériences. L'adjonction d'un acide ou d'un alkali à l'eau en change la densité, et il serait possioale que les effets d'endosmose ou d'exosmose qui se manifestent alors, fussent les résultats de cette modification de la densité du liquide introduit dans les savités organiques. Celte objection peut ètre fondéc, par exemple, lorsqu'on voit une cavité organique contenant de l'cau chargée d'un peu de potasse caustifue, produire l'endosmose, et devenir turgide par l'introduction de l'eau pure qui est an teliors. Alors le fluide intérieur est plus dense que le fluide extérieur, et cente différence de densité sulfit pour déter- 
miner l'endosmose. Rien ne prouve ici d'une manic̀rc certaine que cette endosmose soit due à la qualité alkaline du fluide intérieur. Cette objection disparaît complètement en employant l'ammoniaque, alkali moins dense que l'eau, et qui, par conséquent, doit diminuer la densité de ce fluide lorsqu'on l'y ajoute. Voici l'une des expériences que j'ai faites avec cet alkali : J'ai mis dans un cœcum de poulet de l'eau de pluie mêlée avec une très-petite quantité d'ammoniaque. Il pesait 52 grains. Je le fermai par une ligature, et je le plongeai dans l'eau pure. Après une heure d'immersion, je pesai le cœcum, et je trouvai son poids augmenté de 20 grains; il s'était considérablement gonflé. Ici le liquide intérieur était moins dense que le liquide extérieur, et cependant il y avait eu endosmose. Cela prouve bien évidemment que c'cst à la qualité alkaline du fluide intérieur que cet effet doit êtré exclusivenıent attribué. Ce premier point étant déterminé, je fis l'expérience en sens inverse. Je mis de l'eau dans un cœecum de poulet, et après l'avoir fermé par une ligature, je le plongeai dans de l'eau qui était mêlée avec une certaine quantité d'allmoniaque. Le cœcum, qui, au commencement de l'expérience, pesait 49 grains, perdit 6 grains en une heure; vingt-quatre heures après, il avait perdu 20 grains. Ainsi, dans cette circonstance, la qualité alkaline du fluide extérieur avait déterminé l'exosmose; et cependant, sa qualité de fluide moins dense que le fluide intérieur, aurait dù déterminer l'endosmose, ce qui pronve que ce n'était pas ici la difl'́rence 
de densité des deux fluides qui était la cause du mouvement de transport de l'un d'eux; mais que cette cause se trouvait dans la différence de leur constituttion chimique. En un mot, cette expéricnce prouve cque, lorsqu'un fluide alkalin est séparé de l'cau pure fpar une membrane organique, il s'établit un courant tqui porte l'eau an travers de la membrane vers le Ifluide alkalin; en sorte que, si ce fluide alkalin est dans l'intérieur d'une cavité organique environnée id'eall, il y a endosmose; si, au contraire, cette cavité organique remplié d'èan se trouve environnée ¿d'un fluide alkalin, il y a exosmose. Ces déductions rde l'expérience sont applicables à l'effet des acides, dont l'action est exactement inverse.

Ces résultats nous font déjà pressentir que l'impulssion qu'éprouvent les liquides dans ces expériences, idépend d'un courant électrique déterminé par le voisinage de deux fluides de dcnsité ou de nature chiImiquc différentes, fluides que sépare imparfaitement ume membrane perméable. Cette menibrane ne joue évidemment aucun rôle propre dans cette circonsitance; elle ne fait fonction que de moyen de séparation chtre les deux fluides auxquels elle est cepen(dant perméable : les liquides la traversent, soit dans un sens, soit dans l'autre, au gré de l'action réciproque des denx fluides qui baignent ses parois opposées. (Cependant nous avons vu, dans quelqucs-unes des expériences précédentes, quc la cavité organique étant vide et environnée d'eau, elle introduit cependant ce fluide dans son intérieur. Ceci tient à une cause qui 
trouvera son exposition plus bas, et n'infirme point les assertions que je viens d'émettre.

Lorsque les conditious de l'endosmose existent, les organes creux, qui en sont le siége, prennent un état turgide extrêmement prononcé : ils sont remplis avec excès; et si l'introduction du liquide extérieur paraît cesser lors de l'existence de cet état de réplétion, cela ne paraị̂t provenir que de l'obstacle qu'oppose à celte introduction nouvelle le fluide déjà introduit, et qui se trouve dans un état de pression dans l'intérieur de la cavité qui le contient, cavité dont les parois distendues rếagissent sur ce fluide par l'effet de lemr élasticité. Ces réflexions me conduisirent à penser que je pourrais faire monter l'ean dans un tube, en fixant autour de soḷ extrémité inférieure l'ouverture d'un organe creux qui serait dans les condilions de l'endosmose. Je pris donc un tube de verre ouvert par ses deux extrémités: son diamètre intérieur était de deux millimètres, et sa longueur de trente-deux centimètres. Au moyen d'vne ligature, je fixai autour de son extrémité inférieure l'ouverture d'un cœcum de poulet, que je remplis avec une solution d'une partie de gomne arabique danns cing parties d'cau. Je plongeai ce cœcum dans de l'eau de pluie, et je maintins le unbe élevé verticalement au-dessus. Bientôt le cœeum devint turgide, et le liquide qu'il contenait ne tarda pas à monter dans l'intérieur du tube. Cette ascension s'opéra avec une vîtesse de șept centimètres par heure; el quatre heures et demic après, le liquide, parvenu an sommet du uube, déborda par son ouverture, et 
s'écoula au dehors. Cct écoulcinent, apròs avoir duré pendant un jour ct demi, s'arrêta; ct bicntôt après, lc liquide commença à baisser dans lc tube. Vinğtquatre heures plus tard, l'abaissement du liquide était | parvenu à plus de la moitié du tube; alors jouvris lle cœcum, et jc le trouvai rentpli d'un fluide putride. La température, pendant cette expérience, s'était Imaintenue cutre 17 degrés el demi ct 19 degrés $R$. .Jc rccommençai cette curieusc expérience, en emIployant un tube de cinq millimètrcs dc diamètrc in"térieur et de six décimètrcs de longueur : le cocum, 'qui le terminait inférieurentent, fut rempli avec me solution d'une partic de gromme arabique dans cinc Iparties d'eau, et fut plongé dans de l'eau de pluie. IBientôt le cœcum devint turguide par l'cffet de l'endosmosc, et lc liquidc gommeux s'élcva dans l'intéricur du tubc avec une vîtesse d'environ vingt-cind Imillimètres par hcurc. Vingt-quatre heures après, lc lliquidc, sans ccsse ascendant, parvint au sommct du tube, et se répandit au dchors, mais cn très-pctitc quantité. Le jour suivant, cctte hautcur du liquide se maintint; ct cc ne fut qu'au commencencnt du troissième jour que l'abaissement du liquide dans le tube ccommença à se manifester, ct s'augrmenta progressiivcment. Le quatrième jour, j'ouvris lc cœeum, ct jc trouvai lc liquide qu'il contenait devenu putride. Il était important de savoir si des organes creux, autres quc des cœcum de poulct, ćtaient susccptibles d'offrì ce même phénomènc d'impulsion; je recommençai donc ces cxpériences avce des vessies natatoires de 
carpe, et jobtins les mêmes résultats : je vis qu'il n'était pas néeessaire, pour les produire, d'employer des solutions aussi ehargées de gomme arabique que celles dont j'avais fait usage. Un cinquantieme de gomme arabique dans l'ean m'a suffi, dans beaueoup d'expérienees, pour obtenir l'aseension de l'eau dans les tubes; mais je dois faire observer qu'alors l'ascension était moins rapide, et s'élevait moins haut que lorsque j’employais une solution plus dense. Il n'était pas moins important de savoir si des organes ereux végrétaux présenteraient le même phénomène : pour en faire l'expérienee, je me servis des gousses vésieulaires du colutea arborescens. J'ouvrais le bout de l'une de ses gousses; et remplissant sa eavité avee de l'eau chargée de yomme, je fixais par une liggature l'ouverture de la gousse autour de l'orifiee d'un tube de verre. L'endosmose ne tardait pas à se manifester dans ees gousses plongées dans l'eau; elles devenaient turgịdes, et bientôt le liquide montait dans le tube. J'ai obtenu eet cffet, même avee des gousses desséehées et devcnues presque transparentes après la maturité des fruits. Avant de les soumettre à l'expérienee, j’avais soin de les rendre souples par une immersion suffisamment prolongéc dans l'eau. Ainsi, l'état turgide par l'effet de l'endosmose, et l'impulsion des fluides par l'effet de eette mêmic endosmose, sont des phénomènes qui appartiennent également au règne animal et au règne végétal. Ces expérienees nous prouvent ainsi que l'endosmose, en rendant turgide ou en renplissant avec excès les organes ereux, devient par 
rcela mêne une cause d'impulsion pour les fluides que ccontiennent ces organes, lorsqu'un chemin leur est ovuvert pour en sortir. Ceci nous dévoile l'existence dd'une cause tout à fait ignorée d'impulsion pour les Muides des végétaux el des animaux. Il est temps, aavant d'aller plus loin, de rechercher quelle est la ccause qui fait passer l'eau an travers des menbranes corganiques, et qui, suivant les circonstances, produit oou l'endosmose ou l'exosmose.

Nous avons vu, par les expériences précédentes, que Ilorsque deux liquides d'inégale densité sont séparés par une membrane organique, il s'établit au travers ide cette menbrane un courant qui porte le liquide He unoins dense vers le liquide le plus dense. Ce résultat de la différence de densité de deux liquides ttrès-rapprochés, et presque en contact, doit d'abord lfaire penser qu'il est dù à une action électrique. Le rrapprochement ou le contact des corps de densité différente est, comme on sait, une cause de producttion d'électricité; et l'on sait aussi que le fluide électtrique accélère l'écoulement et la vaporisation des lliquides, ce qui prouve que ce fluide a prise sur les rmolécules des liquides pour leur commmiquer de ll'impulsion. Ce fait a été prouvé pour l'électricité ordinaire, par les expériences de Nollet. L'électricité galvanique possède d'une manière encore plus marquiée cette propriété de donncr de l'impulsion aux imolécules des liquides : ce fait est pronvé par une expérience très-curieuse de M. Porret, insérée aux Annales de physique et de chimie. Ce physi- 
cien ayant séparé un vase elı deux compartinens par un diaphragme de vessie, remplit d'eau l'un de ces compartimens, et n'en mit que quelques gouttes dans l'autre. Ayant alors placé le pôle positif de la pile dans le compartiment rempli d'eau, et le pôle négatif dans celui qui était à peu près vide, l'eau fut poussée au travers des parois de la vessie dans le compartiment vide, et elle s'y éleva à un niveau supérieur à celui auquel elle fut réduite dans le compartiment primitivement plein. Ce fait me parut tout à fait analogue à ceux dont l'observation vient d'être apportée. En effet, nous voyons dans l'expérience de M. Porret, l'eau poussée au travers d'une membrane organique par un courant dirigé du pôle positif (zinc, ou le inoins dense) vers le pôle négatif́ (cuivore, ou le plus dense). Or, dans les expériences précédentes, nous avons vu de même le liquide le moins dense être poussé au travers d'une membrane organique vers le liquide le plus dense. Cette similitude exacte dans les effets et dan's les conditions de leur existence, ne permet pas de douter qu'ils ne soient dus à une même cause, c'est-à-dire à un courant électrique. Nous allons voir cette assertion acquérir un complément de preuves, en produisant avec la seule électricité tous les phénomènes d'endosmose et d'exosmose qui ont été rapportés précédemment. Il ne s'agit pour cela que de varier un peu l'expérience de M. Porret, en faisant communiyuer le pôle négatif de la pile avec l'intérieur d'un organe creux fermé de tontes parts, vide, et plongé dans de l'eau, en communication avec le pôle positif: 
De cette manière, il devait y avoir une introduction rapidc de l'eau dans l'organe creux, oü clle serait pousscée par le couranı élecırique dirigé du pôle positif frers le pôle négratif. Je pris donc un cœcum de poulet uque je fixai par une ligature à l'ouverture d'un tube de verre. Un bouchon de lićgye qui fermait exactement ll'ouverture de ce tube, éripêchait la communicition de la cavité du coecını avec la cavité du tube. Ce thouchon était traversé par un fil de cuivre, lequel s'ćtendait un peu dans la cavité du cœcum, et traverssant de l'autre côtć toute la longueur du tube, était udestiné à établir la communication de l'intérieur du coecum avec le pòle négatif de la pilc. Le tube de verre dans lequel ce fil était renfermé avait pour office de l'isoler de l'eau dans lequel le cœcum fut plongé, eau qui était en communication avec le pôle positif de la pilc. Le cœcum formait ainsi un organe creux sans issue, et c'était au travers de ses parois Yu'était établie la communication cntre les deux pôles de la pile. Cettc pilc à ausges était composéc de 40 conples de 3 pouces et demi ( 9 centimètres $1 / 2$ ) en carré; Il'cau de la pile élait acidulée avec de l'acide sulfurique: cn 20 minutes, le coecum, qui élait entièrement vide au commencement de l'expérience, se trouva rempli de manière à ètre extrêmement turgide; les trois Iquarts de sa capacité étaient occupés par de l'eau introduite au travers de ses parois; l'autre quart était occupé par du gaz hydrogène dégagé par le pôle néyatif, et provenant de la décomposition de l'eau. Je répétai la même expérience, en faisant correspondice 
le pôle positif avec l'intérieur du cœcum; il n'y eut pas une seule gontte d'eau d'introduite. J'étais ainsi parvenu à produire une endosmose artificielle, au moyen de lat seule électricité; mais l'état turgide du cœcum ne dépendait pas seulement de l'eau introduite dans sa cavité, elle dépendait aussi en partie du gaz hydrogène développé.Pour obvier à cet inconvénient, j’ajoutai à mon appareil un tube de verre capillaire qui pénétrait dans l'intérieur du coecum, de la même manière que celui qui servait de conducteur au fil conjonclif: Ce tube, ouvert par ses deux bouts, s'éleyait verticalement à 15 centimètres au-dessus du niveau de l'eau. De cette manière, le cœcum ne formait plus une cavité sans issue, comme dans l'expérience précédente; mais cette cavité communiquait avec l'air extérieur par le moyen du tube capillaire, qui était disposé de manière à évacuer le gaz hydrogène à mesure qu'il serait produit. Le coecum mis en expérience vide et dans l'élat de flaccidité, ne tarda pas à se remplir d'eau introduite au travers de ses parois, et à devenir turgide. Bientôt après, je vis l'eau monter dans le tube, et parvenir rapidement à son ouverture supérieure : il ne me fallut qu'une demiheure pour obtenir ce résultal. L'eau s'écoula au-dehors par l'ouverture supérieure-du tube, et cet écoulement ne cessa que lorsque l'action de la pile se fut affaiblie. Nous voyons dans ces expériences l'endosmose et l'état turgide, et, par suite, l'ascension de l'eau dans le tube être le résultat de la seule action du courant électrique dirigé au travers de la ment- 
rrane organique du pôle positif (zince, ou le moints cense) au pôle négatif (cuivre, ou le plus dense). “on ne peut manquer d'être frappé de la similitude ce ces résultats avec ceux obtenus dans les expééences, où l'endosmose déterminée par la densité du tuide intérieur plus grande que la densité du fluide uxtéricur a produit d'abord l'état turgide de l'organe treux, et, par suite, l'ascension du liquide que coneznait cet organe dans le tube dont la cavité commuiiquait avec la sienne. Il est évident que, dans ces teux ordres d'expériences, c'est également à un couaant électrique dirigé d'un pôle positif, ou le moins llense, à un pôlenégatif, ou le plus dense, qu'est tue l'impulsion qui fait passer l'eau au travers de la nembrane organique, et qui l'accumule du côté du iôle négratif. Un autre rapprochement confirme encore ce résultat. On sait qu'en soumettant à l'action He la pile de l'eau chargée d'un sel à base alkaline, ce sel est décomposé, l'alkali se dépose au pôle négatif t'acide au pôle positif. Or, c'est vers le pôle négatif fue s'établit la direction du courant de l'eau, d'où résulte l'endosmose. Ainsi, si l'on fait cette expétience avec de l'eau chargée de sel, le courant de "'eau se trouvera dirigé vers le pôle où se dépose l'alkali. Or, nous avons noté précédemment un fait exactement semblable; l'endosmose résulte de la seule présence d'un alkali dans une cavité organique environnée d'eau, la présence d'un acide dans cette cavité produit l'exosmose. Nous voyons ici, comme dans l'action de la pile, le courant d'eau se diriger vers 
le côté de T'alkali, et fuir Je côté de l'acide. Tout concourt donc à prouver que, dans l'un comme dins l'autre cas, l'impulsion de l'eau est due ì un courant électrique diriggé du pôle positif (acide, ou le moins dense) vers le pôle négatif (alkali, on le plus dense). C'est de là que résulte, d'une part, l'état turgide de l'organe crenx, lorsque c'est dans sa cavité que se fait cette accumulation de l'eau; et d'une autre part, l'ascension de l'ean dans le tube qui communique avec la cavité de cet organe dans lequiel l'eau est saus cesse introduite an travers de ses parois.

A près avoir produit, avec la seule électricité, tons les effets de l'endosmose, on ne pouvait point douter qu'on ne produisît de même l'elfet d'exosmose. Je ne négligeai point cependaint de m'en assnrer par l'expérience. Ayant rempli d'eau nn cœcum de poulet, je le préparai comme dans les dernières expériences, ct je fis correspondre le fil qui pénétrait dans son intéricur avec le pôle positif de la pile, l'cau dans laquelle baignnait le cœenm correspondant avec le pôle négatif. Le courant électrique, dirigé du pôle positif au pôle nésatif, chassa l'eau de l'intérieur du cœcum au travers de ses parois; el en moins d'une demihomre le coecum se trouva presqu'entièrement vide. Jc répétai ces diveises expériences en employant, au lieu de cocum de poulet, des gousses de coluten arborescens; jobtins les mêmes résultats; et il me fut prouvé, de cette nunière, que toutes les menibranes organiques animales on végétales sont susceptibles de présenter le phénomène d’un courant d'cau 
tiriggé an travers de lcurs parois, courant dirigé du ổté positif vcrs le côté négatif, lorsque les deux côtés ze ces membrancs sont électrisés d'une manièrc diftércnte. Il n'y a pas de doute, dans ces dernièrcs excérienccs, que le courant de l'eau ne soit déterminé ara le courant électrique. Tcllc est parconséqucnt ussi la causc qui détcrminc le courant de l'eau au avers des membrancs organiques, lorsque leurs paois opposćcs sont en contact aveo des liquides dc censités différcntes. Áinsi, l'endosmose et l'exosmose épendent entièrement de l'électricité. Les organes rceux qui préscntent l'un ou l'autre de ces phénomèces sont, en quelque sorte, dcs bouteilles de Leyde parois perméables; leur intéricur est occupé par ne électricité opposée à cclle qui existc à lcur extéceur; et comme le courant du liquidc cst toujours iirigé vers le côté négatif', II en résultc que ces bou'zilles de Leyde offriront l'endosmose quand lcur utérieur sera négatif, lcur cxtćricur ćtant positif; et u'au contraire elles offriront l'exosmose lorsque leur utérieur sera positif, leur extérieur étant négatif. '́es considérations nous mettent à même d'expliuer pourquoi les organcs crcux, quoique ne conteant aucun fluidc, exercent cepcndant l'cndosmose, aiblement, il cst vrai, mais cependant d'unc manière ensible. Ceci, au premier coup-d'œil, scmblc ĉtre n contradiction avec l'assertion à laquclle nous a onduit l'expérience, que le courant élcctrique qui rroduit l'endosmose provient de la supériorité de la pensité du fluide intérieur sur la densité du fluide 
extérieur. Or, ici il n'y a point du tout de fluide intérieur', et cependant l'organe creux exerce l'endosmose. D'où provient donc ici le courant électrique qui fait entrer l'eau dans la cavité? Pour résoudre celle question, il faut se reporter à la composition des tissus organiques. J'ai démontré, dans un autre ouvrage (I), que le tissu végétal est essentiellement composé de vésicules agglomérées; il en est de même du tissu animal; nous reviendrons plus bas sur cette verité essentielle à établir. Chacune de ces vésicules est ordinairement, dans l'état naturel, remplie par une substance organique plus dense que l'eau; elle exerce par conséquent l'endosinose, par rapport à ce liquide, lorsqu'il baigne ses parois extérieures. Chacune de ces vésicules est donc une petite bouteille de Leyde, électrisée négativement en dedans et posilivement en dehors. Il résulte de là qu'un organe vésiculaire, comme un cœcum de poulet, fermé par une ligature, peut être considéré comme un organe creux dont les parois sont formées par l'agglomération d'une multitude de petites bouteilles de Leyde, tontes négatives à l'intérieur, et positives à l'extéricur. Or, d'après les lois connues de l'électricité, l'organe creux doit être lui-même une ggrande bouteille de Leyde, du même genre que les petites qui entrent dans sa composition. L'électricité positive qui occupe l'extérieur de chacune de ces petites bou-

(1) Recherches sur la structure intime des animaux et des végétaux, et sur leur motiliti. 
reilles de Leyde doit se porter tonte entière vers l'excérieur de l'organe erevix, par l'effet de la répulsion |fue l'éleetrieité négative exerce sur l'électrieité poiitive; par conséquent, l'intérieur de l'organe ereux cera à l'état négatif. Ainsi, si l'on pouvait construire une sphère ereuse en aggolomérant, pour faire ses parois, des petitcs bouteilles de Leyde en verre, les[uelles seraient toutes positives à l'extérieur, la sphère rense serait elle-même une bouteille de Leyde du même grenre. Ceei nous explique pourquoi un cœcum He poulet ne contenant aucun liquide, et fermé par une ligature, se trouve cependant, lorsqu'il est plongé llans l'eau, dans un état électrique positif en dehors négratif en dedans : d'où résulte l'introduction de l'eau, ou l'endosmose. Si les vésienles ou les petites Houteilles de Leyde eomposantes, au lieu d'ètre néuratives, étaient positives en dedans, elles exereeraient l'exosmose an lieu d'exereer l'endosnose, et l'organe ccreux en ferait autant. C'est aussi ce que l'expérienee prouve. J’ai démontré que lorsque les organes creux ceontienncnt un flnide putride, ils cxereent l'exosmose, quoique ee fluide putride soit cependant plus dense que l'ean enviromnantc. Dans eette eireonstance, e'est la constitution ehimique du fluide qui ll'emporte, eomme eondition de produetion d'électricité. Or, j’ai observé que lorsqu'un eœcum de poullet commence à se putréfier, et qu'on le plonge dans Il'eau étant vide, il n'introduit point d'eau dans son intérieur, il n'exeree plus l'endosmose; bien plus, I lorsqu'on le remplit d'cau pure, et qu'on le plonge 
dans l'eau, il tend à se vider, il exerce l'exosmose. Ceci provient évidemment de ce que chacune des vésicules, ou bouteilles de Leyde composantes, a interverti son état prinutif par la putréfaction des fluides qu'elles contiennnent. Lorsque ces fluides étaient dans l'ćlat sain, leur densité, plus grande que celle de l'eau, déterminait l'état négatif de l'intérieur des yésicules, et par conséquent l'état négatif de l'intérieur de l'organe crenx, et par suite l'endosmose. Lorsque ces mêmes fluides sont devenus putrides, ce nouvel état a déterminé l'état positil' de l'intéricur des vésicules, et par conséquent l'état positif de l'intérieur de l'organe creux, et par suite l'exosmose.

Les considérations précédentes doivent faire penser que les tissus organiques sont plus spécialement propres que les corps poreux inorganiques pour l'établissement du courant électrique, qui communique une impulsion à l'eau; c'est aussi ce dont je me suis assuré par les expéricnces suivantes. Jc pris un large tube de verre dont je fermai l'une des extrémités, en y fixant solidement, par une ligature, un morceau de vessie de cóchon. Je plongeai ce tube dans l'ean par sou extrémité ferméc, en sorte que son extrémité ouverte s'ćlevait un pen au-dessus de la surface de ce liquide, yni, par son poids, tendaịt ainsi à pénétrer dans l'intéricur du tube, en filtrant au travers de la membrane animale qui fermait son extrémité inférienre, et qui était pressée par une hauteur de 8 centimc̀tres d'eạu. Cet apparcil fut laissé en expérience pendant douze houres. An boul de ce temps, je trouvai 
"I* grains d'eau, que la filtration avait introduit dans te unbe. Alors, sans déranger la positiou de l'appareil, j'introduisis dans le tube, et jusqu'au contact de lia membrane obturatrice, le fil conjonctif communiyuant au pôle néşatif de la pile, tandis que le fil conironctif du pôle positif communiquait avec l'eau dı. rase, et ì peu de distance de la face extérieure de la membrane. Le courant électrique ne tarda pas à détcerminer l'introduction dans le tube d'une grande пuantité d'eau, qui filtra au travers de la membrane. Te répétai cette expérience en employant, an lieu de membrane de vessie, un morceau de peau de gant Hes plus minces. J'obtins à peu près le mêne résultat. Une inmuersion de donze heues n'introduisit que i6 grains d'eau dans le tube; l'application du courant voltaïque y fit entrer l'eaı avec rapidité. Il s’agissait actuellement de savoir si les corps poreux inorganiques se comporteraient de la même manière sous l'influence du courant électrịue.

Je lutai solidement une plaque de grès tendre de 44 millimètres d'épaissonr à l'une des extrémités d'un unbe de verre de 2 centimètres de dianètre, et je ploingreai cet appareil dans un vase plein d'eau, cn maintenant l'ouverture libre du tulse au-dessus de la surface de ce liquide. Au bout de huit henres, je trouvai 8 grains d'eau qui étaient entrés par filtration dans le ube, ce qui indiqne qu'il en entrait I grain par henre, sous une pression de 8 centimètres d'eau. Je mis 24 gyrains d'eau dans le tube, j’y introdnisis le fil conjonctif correspondant au pôle 
négatif de la pile, tandis que le fil conjonctif correspondant au pôle positif trempait dans l'ean dı vase, à pcu de distance de la plaque de grès. Le courant électrique fut entretenu, avec beaucoup d'énergie, pendant une heure. Au bout de ce temps, je pesai l'eau contenue dans le tube, et je n'en trouvai que 22 grains. Il aurait dû s'en trouver 25 grains, en supposant l'impulsion électrique nulle, et en ne tenant compte que de l'introduction en vertu de la porosité. Par conséquent, une partie de l'eau introduite avait disparu, ce que j'altribue à sa décomposition, par l'action électrique qui dégage de l'hydrogène au.pôle négatif. Il me paruı résulter de là que l'impulsion électrique était ici de nul effet. Je recommençai cette expérience, après m'êtrc assuré de nouvẹau que la plaque de grès n'introduisait par filtration qu'nn grain d'eau dans une heure. Cetle fois, je retrouvai dans le tube les 24 grains d'eau que j'y avais mis : résultat à peu près semblable au précédent, et qui, comme lni, me laissait dans le doute sur l'effet impulsif de l'électricité, tout en me prouvant que cet effet, s'il existait, devait être extrêmement faible : c'est ce qui me fut prouvé par l'expérience suivante. Je lutai à un tube de 35 millimètres de diamètre, une plaque de ce tuf très-poreux dont on se sert pour pierre à bâtiŕ; elle avait I centimètre d'épaisseur. Cel appareil fut plongé dans le même vase, et avec la même quantité d'eau que pour l'expérience précédente. Au bout d'une heure, je trouvai 5 I grains d'eau qui avaient été introduits dans ce tube par filtration. Je 
mis le tube vide dans l'ean dı vase, en faisant corrrespondre le fil conjonctif' dı pôle nérgatio avec la frace intéricure de la plaque do unf; l'can du vase correspondait avec le pôle positif. An bont d'uns theure, je trouvai 54 grains d'cau dans le unbe. Ainsi, ll'impulsion élcctrique s'ćtait manifestćc ici par l'introdnetion de 3 grains d'can de plus que ec que pouwait faire la senle porosité. $\mathrm{J}_{\mathrm{c}} \mathrm{m}$ 'assurai de nonvean He la qinantité d'cau que mon appareil pouvait introHuire dans l'espace d'unc heure sans le scconrs de l'électricité. Je tronvai cclte quantité un peu angmentée : l'cau introduite s'élevait à 53 gyrains. Alors ie recommenẹai l'expérienec avec le courant électriHue, et j'cus pour résultat l'introduction dans lc iube He 60 grains d'cau. Je m'assurai immédiatcment après He la quantité d'ean que mon apparcil introduisait, Hans l'espace d'unc heure, sans le sccours de l'éicctricjté, et je tronvai ccitc quantité de 55 grains. Jc ac sais à quoi attribner cette lésère augmentation orogressive de l'can introduite par simple filtration; coujours résultc-t-il de ces cxpériences, que j’ai répéGées plusicurs fois, et que j’ai variées, ponr ètre certain de lcurs résultats, que lc courant élcctrique, Adiriģé du pôle positif au pôle néggatif de la pile, ne produit qu'une impulsion très-faible sur l'eau po.xr la déterminer à passer au travers des eorps porcux inorganiques; ct que cet effet est nệe tout à fait insensible lorsque la porosité de ces corps est assez pen considérable pour ne permettre l'introduction que Ide quelques grains d'can par heure. Aussi, lorsque 
je laissais ces tubes se remplir jusqu'au niveau de l'ean du vase dans lequel ils plongeaient, et qu'alors je leur appliquais le courant électrique, comme dans les expériences précédentes, jamais il n’y avait de haussentent du niveau de l'ean daus le tube; j’ai seulement vu quavec le secours du courant électrique, l'eau qui pénétrait par filtration dans le tube arrivait un peu plus promptement au niveau de l'eau dı vase qu'elle ne le faisait par le seul effet de la pesanteur de l'eau. Ainsi, l'électricité voltaïque aggit ici comme l'électricité ordinaire, en accélérant l'écoulement de l'ean; mais il y a loin de cette impnlsion, qui est très-faible, à l’impulsion énergique que prodnit sur l'eau le courant électrique lorsqu’il la fait passer au traver's des nembranes organiques, dont la porosité seule, et sans le seconrs de l'électricité, laisse cependant assez difficilement passer l'eau. On ne peut donc se refuser à reconnaître que les membranes organiques, par certaines conditions qui leur appartiennent, sont, sinon exclusivement, au moins très-spécialement propres à transmettre l'ean par filtration, sous l'impulsion du courant électrique dirigé du pôle positif au pôle négatif. Ces conditions physiques, propres aux membranes organiques, ne sont point altérćes par la dessication, car jai expérinenté que des cœcım de ponlet, ou des vessics natatoires de carpe, qui avaient subi un dessćchenent complet et très-prolongé, étaient aussi propres que dans l'état frais pour tomes les expériences qui viemnent d'ètre rapportées; il suffisait de leur rendre leur souplesse 
par nue immersion suffisamment prolongée dans l'ean.

lievenons aetuellement à nos premières expérienees sur l'endosmose el sur l'cxosmose déterminćes par la dilférence de nature des deux liquides intérieur ct cextérieur.

Nous a vons déterminé les effets de la différence de idensité de ees deux liquides, et nous avons vu que le (courant se dirige toujours vers le liquide le plus (dense, à moins qu’il ne soit putride, on qu’il ne pos- ssède eertaines qualités ehimiques qui intervertiraient lla direction de ee eourant, que les faits observés jus'qu'ici nous ont porté à eonsidérer comme unique. $\mathrm{O}_{\mathrm{r}}$, (de nonveaux fiats vont nous apprendre que ce eourant n'est point unique, comme nous l'avons établi (d'après les apparences, mais quill y a effeetivement (deux courans opposés dans leur direction, et inégaux dans leur intensité : e'est ee dont je n'ai pas tardé à inı́apereevoir en étudiant les effets d'cndosmose produits par diverses substances ehimiques.

Pour étudier avee facilité les elfets d'endosmose ou d'exosmose que produisent les diverses substanees, j'emploie un moyen très-commode, dout l'idée m'a - été suggogérée par l'une de ines précédentes expériences. Je fixe une vessie natatoire de carpe, ou un coeum de poulet, à l'extrémité d'un tube de verre de irois millimètres de diansère intérieur; un rebord de eire à cacheter, mis à ce tube, sert à fixer la ligature qui attaehe la vessie. Au moyen d'une petite seringue de verre, jinjecte dans la vessie, par l'antre extrémité du tube, les liquides dont je venx étudier l'action. 
Lorsque la vessie est pleine, et que le liquide est même un peu élevé dans le tube, je plonge la vessie dans un vase rempli d'eau pure; le tube, suspendu verticalement au-dessus, correspond à une planchette graduée, destinée à indiquer l'élévation ou l'abaissement du liquide dans le tube. Il est évident que, šil y a endosmose dans la vessie, le liquide doit monter dans le tube; et que si, au contraire, il y a exosmose, le liquide descendra. Il y a une cause d'erreur à éviter dans ce genre d'expérience; il est possible que la nuembrane de la vessie se resserre ou se dilate sons l'influence des agens chiniques. On conçoit que, daus ce cas, l'élévation ou l'abaissement du liquide dans le tube ne dépendraient point de l'endosmose ou de l'exosmose. J'obviais à cette cause d'erreur, en pesant le tube garni de sa vessie et rempli du fluide en expérience, tant avant le commencement de cette expérience qu'après sa terminaison. .Te voyais par-là, d'une manièrc certaine, si les variations de hauteur du fluide dans le tube étaient le résultat d'un changement apporté dans la quantité du liquide que contenait la vessie. Avec cet appareil, qu'on pourrait appeler un endosmomètre, j’ai répété la plupart des expériences qui ont été exposées plus haut, et j’ai obtenu des résultats semblables. J'ai essayé, dan's le même sens, l'action de plusicurs sels en solution dans l'eau; et c'est alors qu'un phénomène dont je soupçonnais déjà l'existence, s'est plus complètement développé à mes regards. Si l'on net dans la vessie de l'endosmomètre une solution saline concentrée, une solution d'hydro- 
thlorate de soude, par exemple, on observe inne ascension assez rapide du liquide dans le tube : cette solution est, par conséquent, un agent producteur ll'endosmose. En même temps, l'eau du vase dans cequel trempe la vessie se charge de plus en plus de 3e même hydrochlorate de soude; ce qui prouve que a vessie perd par filtration une partie du liquide salé Iun'elle contieut. Cependant, le conrant établi dı denors an dedans de la vessie y introdnit sans cesse par iiltration l'eau qui baigne les parois extérieures; et il semblerait que celte vessie, qui acquiert sans cesse, me devrail rien perdre. L'observation prouve qu'il en est autrement. Quelques instans après que l'expérience est commencée, on peut, au moyen du nitrate H'argent, constater la présence de l'hydrochlorate de soude dans l'eau qui environne la vessie; et quelque temps après, la quantité de ce sel y est assez considérrable pour se manifester au goôtt. J'ai obtenu un résultat semblable avec tous les sels que j’ai employés à cee genre d'expérience, et même en général avec toutes les substances non chimiques qui, introduites dans la ivessie de l'endosmomètre, y produisaient l'endosmose; les liquides organiques sont, par conséquent, dans ce cas. La présence de ces liquides dans l'eau cenvironnante n'est pas, il est vrai, aussi facile à consutater que l'est celle des agens chimiques, dont les tréactifs décèlent avec facilité les moindres parties. I Cependant, en employant des liquides colorés, la transi mission de ces liquides au travers de la vessie de l'enidosmomètre, devient trèsfacile à constater. Ainsi, en- 
mettant dans la vessie de l'eau gommée colorée avec de l'indigo, on obticnt comme à l'ordinaire l'aseension du liquide dans le tube, et par eonséquent l'endosnose. Or, nıalggré cette iutroduction eontinuelle de l'eau, le liquide gommé eoloré filtre en sens inverse au travers de la vessie, et colore en bleu l'eau enviromnante. Celte filtration descendante du liquide intérieur n'est point produite par sa pesanteur; ear elle a lieu de même lorsque le liquide intérieur est plus léger que le liquide extérieur. Ainsi, lorsqu'on met dans la vessie de l'ammoniaque on de l'alkool, il y a produetion d'une vive endosmose, et eependaut on ne tarde pas à s'apereevoir de la présenee de ces liquides intérieurs dans l'ean environnante. Ainsi, il est démontré qu'outre le eourant dn dehors au dedans qui produit l'endosmose, il y a un seeond courant plus faible, dirigé du dedans au dehors, et qui produit ainsi l'exosmose. 11 résulte de là que l'endosmose et l'exosmose existent toujours simultanément, et que l'aecumulation dı liquide d'un côté on d'un autre de la membrane orşanique, n'est que le résultat de l'excès de foree el d'abondance d'un eouranı sur le eourant diantétralement opposé. Ainsi, quand nous dirons dorénavant qu'mue substanee queleonque est agent producteur d'endosmose, cela signifiera que celte substanee étant intérieure, et l'ean pure étant extérieure, il s'établit un fort eourant du dehors an dedans, et un eorrant plus faible dn dedans an dehors, d'où résulte l'auggnentation proggressive de la quantité dı liquide intérieur. L'existence 
Ide ees deux eourans, prouvée ici d'une manière directe, est, an reste, un résultat néeessaire de l'action éleetrique à laquelle ces eourans sont dus. On sait, en effet, que l'éleetrieité produite par eontaet de deux snbstances hétérogènes, donne tonjours naissanee à tlenx eourans électriques dirigés daus des sens diamétralement opposés. Tel est l'effet produit par la pile voltaique. Dans les expérienees précédentes, deux liquides hétérougènes en eontact produisent de même une électrieité qui manifeste ses effets par deux eourrans dirigrés dans des sens diamétralement opposés. Unn fait très-important qui résulte de ces expérienees, east la différence qui existe eonstamment dans la foree ddes deux eonrans éleetriques opposés. Je livre ee fait aux méditations des physiciens : il ne peut manquer He jeter quelque lumière sur la véritable théorie de l'éleetricité.

Nous avons vu plus hant qu'au moyen de l'électrricité voltaïque seule, on pouvait produire l'endosmose ou l'exosmose, selon qu'on faisail correspondre Uhe pôle négatif de la pile avec l'intérieur ou avee l'exnérieur de la vessie, le pôle positif oceupant alors la rposition opposée. Or, l'existenee connue des deux ccourans éleetriques de la pile nous indique qu'il existe dans eette expérience, comme dans eelles qui vieninent d'être rapportées, deux eourans d'eau opposés Hans leur direetion, et que l'endosmose et l'exosmose mxistent alors simultanément, mais avec une intensité idfiférente. Ainsi, l'aceumulation de l'eau dans la vessie n'est que le résultat de l'exeès de la force du eou- 
rant d'introduction sur la force du courant d'expulsion.

II serail curieux, il serait important d'étudier les effets d'endosmose qui seraient produits en mettunt les différens agens chimiquies en rapport les uns avec les autres, ou même en les étudiant isolément, sous ce point de vue, dans leur rapport avec l'eau pure. II serait également utile d'étudier ainsi les divers produits solubles que fournissent les végélaux et les animaux. Celte tâche est fort étendue, et je n'ai pu l'entreprendre. Je me suis borné à étudier l'action de quelques substances, et je me suis attaché spécialement à celles qui sont pour l'homme d'un usagge fréquent. Ainsi j’ai vu que tous les liquides qui peuvent nous servir d'aliment, comme le lait, le blanc d'œul', le jaune d'œuf, la solution de gélatine, l'ean gommée, l'eau sucrée, produisent l'endosmose. L'eau chargyée de divers iprincipes extractifs des véggétaux, nême d'opium, produil de même l'endosmose. L'alkool à 36 'degrés, introduit dans la vessie de l'endosmomètre, produit une ascension rapide daus le tube. Ceue substance est, par conséquent, un puissant aggent producleur d'endosmose. Or, par son infériorité de densité sur l'eau qui environne la vessie, il devrait prodnice l'exosmose, ou la diminution de la quantité dı liquide intérieur. Ici les qnalités chimiques de ce liquide intéricur sont tellement puissantes pou produire l'endosmose, qu'elles l'emportent sur la cause d'exosmose qui résulte de l'infériorité de densité. Cependant, lor'squon mel dar:s la icssic de l'endusmo- 
mètre de l'alkool lurt étendı d'eau, on obtient un effet inverse. Ainsi, ayant introduit dans la vessie un mélange de six parties d'eau (en volume) et d'une partie d'alkool à 36 degrés, le liquide s'ibaissa lentement dans le tube, et la vessie, plusieurs heures uprès, avait perdn une portion notable du fluide, Ini avait cessé de la remplir entièrenent. Il y avait ci production d'exosmose. Ainsi, l'alkool concentré oroduit un effet diamétralentent opposé à celui que oroduit l'alkool étendu d'eau. Il est facile de se rendre raison de ce phénomène. C'est en verul de son action chimique, et malggré son infériorité de llensité sur l'eau environnante, que l'alkool pur prolluit l'endosmose. Le mélange de l'alkool avec une orte proportion d'eau affaiblit tellement son action himique, qu'elle cesse d'être supérieure comme canse Il'endosmose à la cause d'exosmose, qui résulte de ce Ine le liquicle intérieur (alkool mêlé d'eau) est in“érieur en densité à l'eau pure, qui est le liquide $e x$ iérieur. La cause d'exosmose est ici la plus forte, et Hle évacue en partic la vessie. Un fait analogne, mais unverse dans ses résultats, se présente lorsqu'on met Ilans la vessie de l'endosmomètre un mélange d'eau tt d'acide sulfurique. Ici l'excès de la densité dn lluide intérieur sur la densité de l'cau enviromnante, Hevrait déterminer l'endosmose, el par conséquent 'ascension dn liquide dans le ube; mais au contraire e liquide s'abaisse graduellement, ot la vessie s'évane en partis; el cela, parce que les acides sont on énéral des agens productcurs l'cxosmose. Ici nous 
voyons eneore que l'action exercée par le liquide intérieur, en vertu de ses qualités ehimiques, l'emporte sur l'action opposée qu'il tend̀ à exercer en vertu de sa densité. Ces expériences prouvent combien il est important de distinguter les effets qui résultent de la lensité des effets qui résultent de l'action chimique. Pour bieu apprécier celle dernière, il sera nécessaire de n'employer comparativement que des solutions également denses. C'est ainsi que j'ai trouvé que l'eau fortement chargéce de sulfate de soude, et l'ean également ehargée d'hydrochlorate de soude, produisent l'ascension du liquide dans le tube de l'appareil décrit plus haut, avee des vîtesses qui sont entre elles comme 44 est à 3; ee qui indique que la sulfate de soude a, ponr produire l'endosmose, une énergrie bien plus considérable que eelle que possède l'hydrochlorate de soude, propriéré qưil doil à ses qualités chimicuries.

Il était inportant de savoir quel est le genre d'influenee qu'exeree la températıre sur les phénomènes eombinés de l'endosınose et de l'exosmose. J'ai fail dans ee but plusieurs expériences, qui toutes m'ont donné des résullats semblables. Voici l'une de ees expériences. J'ai mis dans la vessie de l'endosmomèu'e une solution d'une parlie de gomme arabique dans dix parties d'eau, el après avoir pesé eet appareil, j’ai plongé la vessie dans un vase qui contenait de l'cau à la température de +4 degrlés $R$. Pendant une heure et demie que je laissai l'endosmomètre en expérience, la température de l'eaı ne varia point, et j'eus 
your résultat une élévation de 33 milimnètres du liIfuide intérienr dans le tube, et une augmentation de ooids de 13 grains dans l'appareil. Alors je plongeai a vessie de l'endosmomètre dans un antre vase remsli d'eau, dont la température fut soignneusement encretenue à +25 ou 26 degrés $R$. Il est à remarquer Ifre les 13 grains d'eau introduits dans la vessie avaient Himinué un peu la densité du liquide gommeux intérienr, par conséquent l'endosmose devait être plus faible, en tant qu'on ne la considérait que comme un zeffet de cette seule densité. Or, un effet opposé se mamifesta. Le liquide intérieur monta dans le tube de 9.9 millimètres en mue heure et demie, et le poids de l'appareil se tronva augmenté de 23 grains. La quantité dont le liquide intérienr s'est élevé dans le tube, ii ces denx expériences, n'est point l'indice exact de Ha quantité comparative du liquide introdnit, parce que la dilatation du liquide intérieur par la chaleur est uci une canse accessoire de l'élévation de ce liquide dans de tube. C'est donc seulement le poids de l'appareil avant eat après l'expérience, qui indique d'nne manière certhaine la qnantité de l'eau introdnite dans la vessie par Il'endosmose. Or, nous avons vu que, par une température de +4 degrrés R., cette introdnction a été seulement de 13 grains dans une henre el demie, Itandis que dans le même temps, et par une température de +25 degrés, cette introduction a élé de 23 grains.

Ainsi, il est démontré que l'élévation de la temtpérature angmente l'intensité de l'endosmose. Coc fait 
est en harmonie avec les résulats des expériences par lequelles M. Becquerel a prouvé que l'élévation de la température des deux métaux dont le contact produit l'électricité, auggmente l'intensité du courant électrique. Cela prouve de plus en plus que l'endosmosé est due à l'électricité développée par le contact des deux liquides de densité différente.

Je citerai un dernier fait à l'appui de ceux qui viennent de nous démontrer que l'endosmose est due à une impulsion électrique. Lorsqu'un cœcum de poulet, presqu'entièrement rempli d'albumen d'œuf, est plongé dans l'eau, il introduit ce liquide dans son intérieur, et devient turgyide. Si l'on ouvre le cœcunı après quelques heures d'expérience, on trouve sa surface intérieure tapissée par une fausse nembrane formée d'albumen coagulé. Or, on sait que la coagulation de l'albumine est un des effets que produisent les courans de l'électricité voltaique.

Les expériences précédentes nous ont appris que les liquides différens de densité on de constitution chimique, lorsquils ne sont séparés que par une nembrane fine et perméable, donnent lieu à la production de courans électriques. Il serait fort important de déterminer en quoi consistent les conditions chimiques qui remplacent la densité du liquide pontr le rendre apte à prendre, dans celte circonstance, l'électricité négative. Nous avons vu que l'alkalinité est une de ces conditions chimiques. La combustibilité parâ̂t en être une autre. C'est pour cela que l'alkool se comporte comme un liquide très-dense. C'est 
unt-ĉtre anssi parce quïil contiont un radical trèsombustible, le soufie, que le suliate de soude prouit nome endosmose plus énergique qne l'hyd rochlorate ce soude à égale densité. Il est ici un rapprochement uricux à faire. On sait que le pouvoir réfringent des mabstances diaphanes est en raison de leur densité et e leur combustibilité, ce qui indique que ces deux nalités des corps produisent, dans ecrtains cas, des iffets semblables. Je me borne ì exposer ee rapprothement, qui indique l'existenee d'un mystère fort mportant à dévoiler.

Désirant savoir si les substanees porenses inorgaiiques sont propres à la production des phénonènes ¿éndosmose, j’ai fait les expériences snivantes. A onverture évasée de denx petits entonnoirs de verre, aai luté avec soin à l'un une plaque de tuf calcaire 'sudre, et propre à filtrer l'cau; à l'autre, une plaque cy gyrès tendre; la plaque de tuf avait 8 millimètreś, ' plaque de grès seulement 4 millimètres d'épaisseur. insuite, par le petit canal des entonnoirs, j'ai introuit dans leur cavité une forte solution de yonme cabiquze. Ces appareils ont de suite été plongés dans can de pluie et dans une simation renversée, en orte que les ouvertures des entomnoirs, munics de 'ur plaqne poreuse, étaient tournées en bas, tanis que les tubes opposés s'élevaient au-dessus de cenu. Ce liquide s'est introduit par filtration dans "cavité des entonnoirs, jusqu’à ce qưil ait aequis ans les tubes la hauteur du niveau de l'cau extć.eure, hantenr qui a été dépassée, par l'effet de l'autrac- 
tion capillaire des tubes, de 2 millimètres senlement; le liquide ne s'est point élevé plus haut. Ainsi, dans cetce circonstance, il n'y a point eu d'impulsion du liquide, il n'y a point en d'endosmose. Je ne sais si je dois attribuer l'absence de cet effet à ce que les substances inorganiques seraient incapables de le produire. Je suis plus porté à penser que cette absence d'impulsion électrique provient, dins ce cas, de l'épaisseur des plaqnes qui séparaient les denx liquides de densité différente. J'ai observé, en effet, que l'on obtient une endosmose beaucoup plus énergicune avec des oruganes membraneux, lorsqu'ils sont très-minces, que lorsque leurs parois ont une certaine épaisseur. Il me paraît donc que l'intensité de l'électricité produite en pareil cas, est en raison du rapprochement des deux liquides. Ceci expliquerait pourquoi il ne s'est point manifesté d'impulsion du liquide extérieur, lorsqu'il était séparé du liquide intérieur, par une plaque poreuse de 4 ou de 8 millimètres d'épaisseur. 


\section{CHAPITRE V.}

Application des observations précédentes à la statique vitale des végélaux.

Ex recherchant plus haut quelles sont les conditrions nécessaires pour que la progression de la sève ait lieu, nous sommes arrivés à la connaissance de (ce fait, qu’il existe dans le tissı végétal vivant, un iétat turgide résultant de ce que l'ean est accumulée idans les petits organes creux qui coniposent ce tissu ıvec excès, c'est-à-dire en plus ggrande quantité que ine l'opérerait la simple attraction des parois de ces organes capillaires. Nous avons vu que cet état turgide est la condition nécessaire de la progression de In sève. Or, la cause de cel état turgide est évidemment l'endosmose; elle seule, en effet, peut produire I'effet d'accumuler l'ean dans les organes creux avec excès, et de manière à les rendre turgides. D’aillcurs, les vésicules ou les cellules qui composent le tissu végétal, possédant ordinairement dans leur cavité des liquides organiques plus denses que l'eau, il en résulte nécessairement qu'elles doivent être le siége de l'endosmose, lorsque leurs parois extérieures sont en contact avec l'ean. Ici, ce n'est point une probabilité que j’expose, c'est mue nécessité physiqne. Ainsi, la nécessité de l'existence de l'endosmose chez les végétanx, est prouvée par l'existence nıême des conditions 
de cette aetion physieo-organique. Mais ee n'est pas tout : nous avons vu plus haut que l'existenee de ees conditions, e'est-à-dire la présence des liquides organiques denses dans les petits organes creux du tissu végétal, est une des conditions néeessaires ponr l'existence de l'état turgide de ees organes. Or, comme l'état turgille dérive de l'endosmose et ne peut dériver que d'elle, il en résulte que l'existence de l'endosmose chez les végétaux se trouve ainsi prouvée directement, d'une part, par l'existenee de sa eanse ordinaire et eonnue, et, d'autre part, par l'existenec de l'effet qu'il n'appartient qu'à elle seule de produire. Suivons ces preuves, nous allons les voir s'aecumuler. Nous avons vu, dans les expérienees préeédentes, que le fluide intérieur qui détermine l'endosmose cesse d'être propre à produire cet effet lorsque sa eonposition elimique s'est altérée, lorsque, par exemple, il est devenu putride. Or, nons avons vn plus hant que l'intégrité de composition des liquides organiqnes qui remplissent les petits organes creux du tissn végétal, est une des eonditions néeessaires pour l'existenee de l'état turgide de ee tissu. Ceci nous prouve eneore que cet état turgide dépend de l'endosmose, puisqu'il est, eomme elle, aboli par le seul fait de l'altération ehimique de la eomposition du liquide intérieur. La force exubérante, et sans eesse aetive qui produit l'endosmose dans les organes creux, chasse les liquides dans les tubes aseendans qui communiquent avec ces organes. Des expérienees directes nons ont démontré ce fait, qui nons dévoile la eause de l'ascension de lit 
sève lymphatique on de sa progression par imprlsion. IIei, nn effet eonnu déeoule si évidemment d'une cause iégalement connue, qu'il est impossible de méconmaître Jeur enchaînement. On ignorait complètement ceette eanse nouvelle, eette eause énerorique d'impulsion qui existe dans tout le règne organique; on ne ccomaissait guère que la contraetion des parois des corganes erenx pour imprimer un mouvement progresssif allx liquides que contiennent ees organes. Il élait ttout simple qu'on admît hypothétiquement eette seule canse eonnue d'impulsion, pour expliquer la progression de la sève aseendante qui est évidemment porss:sée par une force ì tergo. Or, j'ai proilvé que la eontraetion des parois des organes ereux sur les liquides Hüils eontiemnent, n'existe pas dans le tissu végétal; cceci est une preuve explétive en faveur de l'existence He l'endosmose, qui, seule avee la eontraction, est apte it produire l'impulsion des liquides ehez les êtres orz̧anisés. Les organes creux des tissus mous végétaux Histendus par l'accumulation du liquide dans leur incérieur, réagisseni, il est vrai, sur ee liquide en vertu 甘le leur élastieité; mais eeci n'est point un phénomène de contraction ou d'incurvation organique. 'Ainsi, ee fait n'infirme point ce que j’ai avaneé toushant l'absenee de la contraction proprement dite, :onsidérée eomme cause de progression des liquiHes ehez les végétaux : cette progression dépend de da seule endosmose. C'est elle qui produit en mênte emps la progression de la sève par impulsion. el sa progression par adfuxion. Nous allons expo- 
ser le mécanisme de ces denx modes de progression.

Les spongioles des racines sont, comme nous l'avons vu plus haut, les organes dans lesquels la sève ascendante reçoit l'impulsion qui la porte vers les parties supérieures du végétal. Ces organes, siéges exclusifs de l'absorption de l'eau, sont très-turgides, et ne le deviennent plus par le seul effet de leur capillarité, quand ayant subi une certaine dessication à l'air libre, elles sont ensuite replongées dans l'eau. Ceci prouve que leur état turgide dépend, cornme cclui des feuilles, de l'endosmose, et non de la simple capillarité. Environnées d'eau, les spongioles l'introduisent sans cesse dans l'intérieur des cellules qui composent spécialement leur tissu. Cette eau, sans cesse introduite par l'endosmose, et accumulée avec excès dans les organes qu'elle rend turgyides, ne tronve point, comme dans les feuilles, un moyen d'évacuation par l'évaporation. Dès lors il en doit résulter un mouvement d'impulsion qui chasse l'eau dans les tubes ascendans de la racine et de la tige. L'eau, sans cesse affluente dans les spongioles, par l'effet de l'endosmose, chasse vers les parties supérieures l'eau précédemnent introduite. Telle est la cause de cette pression considérable à laquelle est soumise la sève ascendante de la vigne dans ses canaux, pression supérieure à celle de l'atmosphère, ainsi que l'ont prouvé les expériences de Hales et celles de MM. Mirbel et Chevreul. Cet état de pression de la sève existe, quoique d'une manière moins marquée, dans tous les végétaux. Si l'on conpe en travers une plante herba- 
tée abondante en sève, mue eucurbitacée par exembe, on voit la sève sortir des denx portions de la igge divisée; et celle issue de la sève hors de ses casaux pronve qu'elłe y était dans un état de pression. itous avous vu plus hant que ce mênıe état de presion existe par rapport au sne laiteux dn figuier, et tu'il ne peut être rapporté à la contraction des vaisceallx.

Passons actucllement à la progression de la sève par udfuxion. Supposons une tige coupée et plouggée dans ceau par sa parlie inférieure. Les cellules et les vaiseaux situés à la surface des feuilles perdaut, par l'éaporation, une partic des fluides qu'ils contiennent, 'endosmose sans eesse active de ces organes remplit vide par l'introduction des fluides cmpruntés anx rganes voisins, et cette action, qui opère l'allfuxion ce la sève ver's les feuilles, s'étend de proche en prothe jusqu'à la base de la tigge qui trempe dans l'eau. ¿endosmose des feuilles, el en général des parties ıolles et herbacées du véģéıl, qui, comme les feuils, demeurent mrgides, teud sans cesse à introdure ans les petites cavités organiques les fluides fonrnis ar les tubes, dont les extrémités ouvertes trempent ans l'eau. Ainsi, e'est par une sorte de succion (si outefois il m'est permis de me servir de celte exrression inexacte) que l'eau du vase est déterminée monter dans les tubes de la tigre, qui penvent être, It qui souvent sont très-probablement inertes dans ette circonstance. Voici une expérience qui rend cette cernière assertion très-probable. Je mis une mercı- 
riale tremper par la partie inférieure de sa tige dans de l'eau, à laquelle j'ajoutai $1 / 48$ de son poids d'acide sulfurique concentré. Cẹ liquide délétère ne tarda pas à monter dans la tige, qui, par cette introduction, devint jaune, flasque et molle partout où l'acide avait pénétré. Au bout de quatre jours, l'acide avait monté dans la tige à la hanteur de 22 centimètres au-dessis du niveau du liquide que contenait le vase où se trouvait la plante. Celte tige, partout où l'acide avait pénétré, était jaune, molle, et de consistance presque pulpeuse. Sa mort était complète. Cependant clle ne cessair pas de pomper le fluide acide dans lequel plongeait son extrénité inférieure, ainsi que je m’en suis assuré en pesant chaque matin à la même heure le flacon qui contenait le fluide acide, flacon qui, par les précautions que j'avais prises, ne pouvait rien perdre directement par l'évaporation, je pesai de même la plante pour mesurer son évaporation. Le premier jour, la plante absorba 156 grains; elle évapora 154 srains; le second jour, absorption, go grains, évaporation, I 4 grains; le troisième jour, absorption, 42 grains, évaporation, 80 grains; le quatrième jour, absorption, 36 grains, évaporation, 64 grains : fin de l'expérience. La tige était morte dans toute son étendue, ainsi qu'une partie des rameaux; les feuilles scules étaient encore vivantes. On voit ici que l'ascension du liquide fut opérée dans une tigge complètement morte, et par conséquent inerte. Mais il est à remarquer que la quantité du liquide ascendant diminua chaque jour, à mesure qu'une plus grande étendue de 
Ila tige était frappée de mort, ce que l'on doit attritbuer à la destruction d'une partie de ses organes. Ceppendant l'évaporation n'éprouvait point de la diuninution dans la même proportion; et dans cette ccirconstance, l'absorption avait cessé d'être proporutionnelle à l'évaporation, comme cela a lieu dans l'éuat naturel. Les feuilles dans lesquelles le fluide. axcide n'avait pas encore pénétré, avaient conservé Heur friîcheur, c'est-à-dire leur couleur verte et leur é́tat turgide. Elles vivaient évidemment aux dépens the la sève contenue primitivement dans la tige, et fui, à mesure qu'elle élait transmise de proche en uproche aux fenillles, était remplacée par le fluide acide uscendant. L'ascension de ce fluide dans une tige éviHemment morte, mais qui conservait encore une pariie de ses canaux capillaires, dépendait évidemment the l'adfluxion sollicitée par l'endosmose des feuilles. [I] n'y avait point là, comme on pourrait le penser, uscension du liquide par le seul effet de la capillarité ldes tubes végétaux, ascension qui aurait été favorisée par le vide qu'avait opéré l'évaporation dans les feuilIdes, ascension qui aurait été ainsi le résuitat de la tendance du liquide à remplir le vide des tubes. Une autre expérience déjà rapportée plus haut, pronve sctte assertion. Une mercuriale qui avait été desséshée à l'air libre, au point de perdre les 0,72 de son poids, ayant été plongée entièrement dans l'ean penIdant un temps suffisant, s'imbiba complètement de ce fluide, nais sans redevenir turyide. Mise alors dans l'ean seulement par la partie inférieure de la tige, 
elle n'absorba aucunement ce liquide dont ainsi l'ascension ne fut point du tout déterminée par le vide que l'évaporation ne tarda pas à opérer dans les feuilles et dans les parties de la tige qui étaient dans l'atmosphère, et qui se desséchèrent promptement. Il se prouve donc de plus en plus que ce n'est point la capillarité qui produit l'ascension de l'eau dans la tige et dans les feuilles d'une plante coupée et immergée par sa partie inférieure, et que celte ascension dépend exclusivement de l'endosmose, qui produit l'élat turgide des fenilles, el qui leur donne par ce fait même le pouvoir d"imprimer à la sève un mouvement d'adfluxion dont elles sont le terne. Ces observations prouvent que l'ascension de la sève peut, dans certains cas, avoir lieu sans ancune action des tubes séveux, qui lui servent de conduits inertes jusqu'aux lieux où l'endosmose l'allire.

Chez les végétaux coupés et treınpés dans l'eau par la partie inférieure de leur tige, le phénomène de l'ascension de la sève par adfluxion existe dans toute sa simplicité, et exempt de toute complication. On conçoit que ce même phénomène doit avoir lieu lorsque le végétal est dans son état naturel, c'est-à-dire implanté par ses racines dans le sol imbibé d'eau. La sève ne peut manquer d'ètre sollicitée jusque dans les racines par la tendance d'adfluxion que prodnit l'endosmose des feuilles, puisque les tubes séveux offrent ordinairement une cavité non interrompue depuis l'extrémité des racines jusqu'à celle des tiges, ainsi que nous l'avons va plus haut. Je ne suis d'aillen's 
asssuré de ce fail par l'expérience suivante: J'ai pris wne mercuriale très-jeune et issue par rejeton d'une macine déjà ancienne. Cette mercuriale ne possédait tque quatre petites feuilles. Ses racines étaient assez considérables. Je plaçai ses racines, dépourrucs de tcerre el d'humidité extérieure, lans un vase que je renuplis de mercure, en tenant les racines plongées Hans ce métal par des nuoyens convenables; car leur liégrèreté spécifique les eût faiı surnager. La mercuriale, ainsi disposée, se conserva pendant trois jours nussi fraîche quı si elle en̂t été implantée dans le sol. Elle vivait alors aux dépens des liquides contenus dans "ßes racines, liquides qui étaiẻnt autirés dans les fenilìes rorr ad/luxion sculement; car il ne pouvait point exiswer ici d'impulsion de la part des racines, puisque, fplongées dans le mercure, elles n’introduisaient rien Hu dehors.

Les feuilles perdenı la faculté de devenir turgides, e par conséquent la faculté d'opérer l'ascension de lla sève par adfluxion, lorsqu'elles ont perdiu, par la Hessication, me certaine quanticé des liquides orgamiques qui remplissent leurs cellules et leurs tubes dans l'état naturel. Ces liquides organiques, plus idenses que l'eau, sont les agens producteurs de l'endosmose; leur absence on leur diminution notable enuraînent nécessairement l'absence ou la diminution de ll'endosmose el de ses effets; c'est-à-dire l'absence de ll'état tursyide et dn monvement d'adfluxion. L'altération chimique de ces liquides organiques produiı les mêmes reffes. Alors la plante meurt; l'état de vie du végé- 
tal n'est, dans'le fait, antre que l'étaf d'cndosmose, état de turyescence que les végétaux ne quittent effectivement jamais tant qu'ils vivent; car la mort de tonte partic végétale se manifeste par sa flaccidité, si cette partie est mollc. Ainsi, la progression de la sève par impulsion et sa progression par adfluxion dépendent également de l'endosmose. Tout organe, siégre de cette action physico-organique ou vitale, est nécessairement, et à la fois, origine d'impulsion et but d'adfuxxion pour les liquides. Voyons de quelle manière ces deux effets d'une même action se combinent aux deux extrémités opposées du végétal. Les racines environnées d'eau n'ont point le moyen de déplétion par l'évaporation que possèdent les feuilles; par conséquent, l'afflux de l'eau que l'endosmose accumule sans cesse dans les spongioles doit avoir pour effet principal el dominant l'impulsion de ce liquide vers les partics supérieures. Cependant, l'effet d'adfluxion ne doit pas être entièrement nul cher elles; elles aspirent fortement les liquides du dehors, et doivent aspire: faiblement les fluides contenus dans les parties supérieures de la plante. Les feuilles placées dans un milicu favorable à l'évaporation des liquides qui remplissènt lcur tissu, possédant ainsi une cause continuelle de déplétion, doivent au contraire aspirer avec énergie les liquides contemus dans la tige et dans les racines. C'est l'effet d'adfluxion qui, chez elles, est le résultat dominant de l'cndosmose; mais l'effet d'impulsion ne doit pas leur être étranger, il toil seulement être plus faible que celui 
Hes racines; car cet efret d'impulsion étunt le résultat He l'excès de plénitude des cellules dans lesquelles ce flnide séveux s"introdnit sans cesse, il est évident IIu'une cause exlérieure qui, comme l'évaporalion, lliminne sans cesse cet excès de plénitude, doil nézessairentent diminuer d'autant l'effet d'impulsion. Ze dernier effet existe cependant, et l'observation le prouve, car la sève descendante ne reconnaî́ pas inplement pour cause l'action de la pesanteur. J'ai expérimenté, en effet, que les bourrelets qui se déeloppent à la partic supéricure d'une décortication amnulaire, ne laissent pas de se développer de la anềne manière lorsqu'on reverse vers la terre l'extrémité supérieure de la branche sur laquelle cette dé:ortication annulaire esi pratiquée. Il est donc prouvé Hue la sève descendante obéit à une impulsion, et le siége de cette impulsion ne peut être que dans les enilles, siége de l'endosmose, qui produit à la fois "effet d'adfluxion el l'afiet "impulsion. En effet, la production du bourrelet, $k$. s de ia décortication annulaire, n'a point lieu lorsqu'on ôte toutes les fenilles He la branche sur laquelle elle est pratiquée, et qu'on Hes empêche de se reproduire. Ainsi, les feuilles et lites spongioles nous présentent, dans un rapport inwerse, la réunion des deux effets de l'endosmose. Les ffenilles sont le but d'une forte adfluxion, 'et l'origine d'une impulsion plus faible; les spongioles, au conuraire, sont l'origine d'une forte impulsion, et le but 'd'une p!us faible adfluxion de la part des fluides séiveux comtenus dans les parties supérieures. C’est de 
la eombinaison de ees divers effets de l'endosmose que résultent, d'une part, la progression aseendante de la sève, et, de l'autre part, la progression descendante de ee fluide.

L'aseension de la sève par impulsion n'a lieu, d'une manière bien marquée, que dans les unbes lymphatiques, qui, eomme nous l'avons vu plus haut, sont des tubes dont la eavité s'étend quelquefois depuis l'extrémité des raeines jusquà eelle des tiges; mais lorsque la contiruité de eette cavité des tubes se trouve interrompue par des eloisons on par l'interposition de masses de tissu eellulaire, alors l'impulsion direete opérée par les spongioles des raeines se trouve arrêtée. Nous avons vu plus hant que, daus la vigne, la sève ne monte par impulsion que daus le bois déjà d'un eertain âye, shez lequel seul les tubes lymphatiques offrent une cavité non interrompue, les diaphragnmes qui les eloisonnaient de distance en distanee ayant disparu; ces eloisons diaphragmatiques existent eneore dans les tubes lymphatiques des jennes branehes de la vignne; et la sève aseendante, en raison de cela, n'y parvient spéeialement que par adfluxion. Aussi ees jeunes branehes ne versent-elles point une sève abondante comme le vieux bois, lorsqu'on les eoupe an printemps. Il est des végétaux, eomne les graminées, ehez lesquels les tubes Jymphatiques sont interrompus, de distanee en distanec, par des nœuds eomposés de tissu eellulaire. On erolrait, au premier eoup-d'œil, que eela devrait être un obstaele à la progression de la sève; mais en y réllé- 
hissant, on voit quatr eontraire cette disposition est faite pour favoriser la progression de ce fluide. Les résicules agglomérées qui composent le tissu celluaire des næu :ulière, qui les rend but d'adfluxion et origine d'imoulsion. Il doit résulter de la réunion de leurs aeiions particulières, un effet gुénéral d'adfluxion opéré principalement du côté ou la sève arrive déjà par plus forte impulsion, e'est-à-dire du côté d'er bas, et .m effet général d’impulsion opéré principaienent dn côté où la sève est déjìt aspirée par plus forte adlluxion, c’est-à-dire du côté d'en haut. Ánsi, les moeuds sont véritablement des onganes moteurs plazés de distanee en distanee pour rivoriser la prorression de la sève, qui, sans ee secours, ne pourrait mrobablement arriver jusqu'au sommet des plantes, ordinairement grêles et for't allongées, dans lesquelles zette organisation existe.

Quoiqu'il soit certain que les fluides des véggétaux ظoient, en général, soumis à deux mouvemens génécaux opposés, l'un aseendant et l'autre deseendant, sependant il n'y a point ici de véritable cirenlation. Ainsi que nous l'avons déjà dit plus haut, la sève liymphatique ascendante et la sève élaborée deseenHante sont véritablement soumises à une diffusion şénérale, qui intervertit souvent la direction la plus zónérale de leur marche. Cette diflusion générale dépend de l'endosnose particulière de chacun des oryanes crenx qui eomposent le lissu végétal, et qui, 'en vertu de cette endosmose, exercent, les uns sur 
les autres, une succion dont les effets se font senlir de proehe en proehe. Aussi avons-nous vu plus haut que la sève ly mphatique, grénéralcment ascendante, est cependant quelquefois deseendante, et que la sève élaborée, grénéralcment deseendante, devient aseendante dans quclques eirconstanees. Toute partie cn développement reçoit nécessairement ees deux sortes de sève, et e'est son endosmose qui les attirc. Quclque forte que soit l'impulsion de la sc̀ve, elle ne fait point pénétrer ce liquide dans une partie morte. C'est parce quc les corolles meurent, e’est-ì-dirc ecssent de posséder l'endosmose, qu'clles sc flétrissent après la floraison; l'embryon, lans un fruit féeondé, est, en raison de son cndosmose, un but d'adfluxion de la sève. Si l'cmbryon meurt, le fruit se flétrit et tombe, parez cune la sève n'y est plus attirée. Lc déreloppement, co phénomènc propre aux scmls êtres vivans, esi cneore un résultat de l'endosmosc, ainsi que la nutriticr, cau; imnédiate de ec dévcloppement. Le mécauisme de cetıe fonetion cst faeilc à déduire des faits qua l'observation vient de nous apprendre. Chaque vésieulc possédaut dans sou intérieur un fluide plus dense que ne l'est la sc̀ve que eontiennent des organcs voisins, attire ce fluide nourricier en vertu de l'endosmose, et tend à l'introduirc dans sa eavité déjà remplie. Mais ee eourant introductcur n'existe pas seul, eomne nous l'avons vu plus haut. L'électrieiıć, eausc de ee phénomène, donne lieu à denx courans opposés daus leur direetion, et inćgaux en intensité. Ainsi, outre le conrant d'introduction, 
Il y a un courant d'expulsion, qui est plus faible, de corte que charque vésicule opère à la fois l'endosmose "t l'exosmose; mais comine la première est la plus corte, la vésicule est tonjout's lormplic avec excès, tt conserve son état turgide. Cette introduction vioeente du liquide extérieur dans la cavité de la vési"ule, doit nécessairement avoir pour effet de lui faire unbil une dilatation; de ì̀ résulte l'augmentation He grosseur des parties organiques toutes composées He vésicules agglomérécs : e’est une des causes du Héveloppement. Un autre effet, de celte introduction riolente du liquide extéries..: dans la cavité des vésisules, est l'expulsion des stiastauces précédemment mtroduites : ce second effet sceonde la faible exosmose qu'exerce chaque vésicule: c: favorise ainsi le cenouvellement continuel du liquide qu'elle contient. "est cn cela que consiste le mouvement continuel Ile composition et de décomposition qui constitue la uutrition. Les vésicules voisines échangent sans cesse ecurs liquides; celte échange ne s'opère point sans Ifue ees liquides ne snbissent des modifications dans ceur composition chinique; et cela parec que ee sont Hes eourans électriques qui déterminent ces échan,cs. On sait, en effet, qu'il n'y a point de courans bectriqùes dans des conducteurs liquides, sans altétation chimique de Ja composition de ees liquides, somme on sait, par les expériences de M. Becquerel, [u'il n'y a point d'action chimique sans production the courans électriques. Ainsi, les courans électriques fui transfèrent les liquides d'une vésicule à une au- 
tre, n'opèrent point ce déplacement sans modifier la composition chimique du liquide, lequel sort d'une vésicule par l'effet d'un courant d'exosmose, et entre dans la vésicule voisine par l'effet d'un courant d'endosmose. De cette manière, la composition chimique du liquide intérieur des vésicules ne reste jamais stationnaire; et c'est de là que provient la différente qualité des sucs que contiennent les fruits aux différentes époques de leur existence, depuis leur origine jusqu'à leur maturité. C'est de là que vient le changement de l'aubier en durremen; car le durcissement du bois, dans ce cas, ne provient que de l'état de solidité que prend, par l'effet de sa modification chimique, la substance contenue dans les clnstres. Alors cesse tout phénomène d'introduction nouvelle. Il n'y a plus d'endosmose, plus de nutrition. La vésicule remplie d'une substance totalement concrète n'appartient plus à la vie. Considérée sous ce point de vue, la nutrition consiste dans une véritable sécrétion. Chaque vésicule sécrète le liquide qu'elle contient; et ses parois sont ainsi de véritables filtres chimiques, qui ne laissent passer que des molécules d'une nature particulière, comme les filtres mécaniques ne laissent passer que des particules d'une certaine grosseur. Tel est, chez les végétaux, le mécanisme général de la sécrétion, qui fait une partie essentielle de la nutrition, mais qui ne la constitue pas toute entière. En effel, outre la production du liquide intérieur des vésicules, outre l'augmentation du volume de ces dernières, il y a encore, dans le 
thénoniène de la mutrition et dil développement, le nit de la multiplication de ces mèmes vésicules. Il aaraît qu'elles sont toutes formées dans les fluides rrganiques, et qu'il est dans la nature de ces fluides 'être composés de molécules vćsiculaires. Aussi, on ss examinant au microscope, les voit-on composés ce globules nagreant dans in fluide aqueux. C'est a ans les parois des cellules ou des vésicules que panissent d'abord et se développent ensuitc les nouezlles cellules; et cn effet, ces petites vésicules, que $\because$ considère conme des corpuscules nervenx, ne mnt que des rudimens de cellules développées dans iintéricur des parois des grandes cellules. Toute urtic nouvelle cst tonjonrs le résultat d'une prodlucon médiane; c'est-à-dire qu'elle est produitc envimnnée de toutes parts de parties organiques. Jamais :ue production n'est véritablement extérienre, uoiqu'elle paraisse l'êtrc souvent. J'ai démontré cette úrité dans un précédent onvrage (I), où j’ai émis nelques idécs surr la nutrition des végétaux (2); j’ai iancé qu'il n'y a point ches enx ce remplacement - molécules anciennes par des molécules nou'zlles, tel qu'on suppose qu'il existe chez les aniraux. Cette assertion ne pent être vraie que par rpport anx vésicules, ou plus généralement par rapbrt aux organes creux, qui sont les parties conte-

(1) Recherches sur l'accroissement al la reproduction des végéıx.-Mémoires du Mfuséum, t. 7 et 8.

(2) Idcm, t. 8 , p. 288 et suivantes. 
nantes; ces parties, effectivement une fois formées, restent invariablement en place; mais il n'en est pas de même des fluides, ou plus généralement des $p$ ties contenues; celles-ci sont assujetties à des transports continuels d'un organe crenx dans un autre; et. c'est dans cette partie seulement du phénomène de la nutrition qu'il s'opère un remplacement des substances anciennes par des substances nouvelles, chez les végétaux.

Chaque ce!lule ou vésicule qui entre dans la compo. sition du tissu végétal absorbe et exhale continuellement. Ces deux actions sont, comme nous venons de le voir, le résultat nécessaire des deux courans électriques qui déterminent le transport des liquides du dehors au-dedans et du dedans au-dehors. Ainsi, l'ahsorption et l'exhalation dépendent de l'endosmose et de l'exosmose : la capillarité est tout à fait étrangère à ces deux actions, du moins dans l'état naturel de la vie. En effet, l'attraction capillaire est une cause bornée dans ses effets. Il y a dans tous les corps un point de saturation pour l'introduction des liquides par l'effer de la capillarité. Un minéral poreux plougé dans l'eau, se sature de ce liquide, ou s'en imbibe jusqu’à la pléniunde de ses cavités capillaires; ce terme étant atteint, il n'y. a plus d'indroduction. Or, il n'en est pas de même chez les véggétaux vivans. Une plantc aquatique prend de l'accroissement, quoiqu'entièrenient plongée dans l'eau, ce qui prouve que, malğré son immersion prolongée, et par conséquent, malgré la saturation de son action capillaire, elle absorbe les 
substances du dehors. L'absorption est donc tout à Ifait indépendante de la capillarité, c'est l'endosmose ssenle qui l'opère. Ce qui est évident ici, par rapport ia une plante entièrement plongée dans l'ean, ne l'est pas moins par rapport à un végétal dont les racines ssont dans un sol humide, et dont la tige est dans l'air. Toutes les parties de ce végétal sont turgides, effet que ine peut produire la senle capillarité, ainsi que nous mous en sormmes assurés plus haur par l'expérience. Les organes creux végétaux, par cela neeme qu'ils ssont turgides, son donc au-delà du terme de la saturation de leur attraction capillaire. Ce n'est donc point ccette attraction qui produit l'absorption qui ne cesse ppoint de s'opérer, malygré la quantilé de liquide déjà iintrodnite asec excès, on ten plus grande quantité ¿que ne le pourrait faire cette attraction capillaire. II cest évident que c'est à la seule'endosmose qu'est due ll'absorption, et que la capillarité n'y contribue en Irien, tant que les organes végétaux sont dans l'état turgide, qui est pour eux l'état de vie. Mais il n'en eest pas de même lorsque, par un desséchement acciidentel, ils ont perdu cet état turgide jusqu’à un point inférieur à celui de la saturation de leur attraction ccapî̉laire. Alors celte attraction doil agir pour opérer M'introduction de l'eau, et cela jusqu'à la plénitude des cavités capillaires, mais sans les distendre avec excès, sans les rendre turgides; car il n'appartient qu'à l'endosmose de produire cet effet. Ainsi, l'introduction de l'eau par l'efiet de l'auraction capillaire, n'est rloz los végétaux, qro'un phóngmène acciden- 
tel qui ne méritc pas, à proprement parler, le nom d'absorplion : ce n'est point un phénomène vital. II n’en est pas de mêne de l'introduction de l'cau par l'effet de l'cndosmose; c'est lì le phénomène véritablement vital aıquel le nom d'absorption doit être exclusivement appliqué.

Les vésicules du tissu végétal ćtant toujours dans l'état turgide, et introdnisant néanmoins sans cesse de nouveaux fluides dans leurintérieur, l'exhalation qu'clles opèrent doit être en partie l'effet indirecı au subséquent de l'endosmose; car cette exhalation est anssi l'cffet de l'exosmose qui leur cst propre. Dans le courant de cet ouviage, j’ai constamment désigné par le simple nom d'évaporation, la perte de liquides que font les végétaux par l'cffet de l'action dissolvante de l'air atmosphérique. Mais plusicurs faits prouvent que cette perte de liquides ne sc fait pas entièrement counme se ferail, par exemple, cclle qu'éprouve une étoffe mouilléc qui se sèche : ici, c'est l'action dissolvante de l'air, qui seule agit sur l'étoffe pour lui enlever l'eau qui l'imbibc. Il n'en est pas tout à fait de même pour le végétal vivant; il exhale, et livre ainsi à l'action dissolvante de l'air, ses liquides surabondans. Il y a chez le végétal une véritable transpiration; aussi, la perte qu'il fail par l'évaporation n'estelle point soumisc anx scules Jois qui président à l'évaporation des liquides, comme cela a lieu pour les corps inorganiques imbibés d'ean. La présence de la lumière augrmente considérablement l'exhalation des plantes, exhalation qui n'est plus la même clans l'obs- 
curité, quoique l'ćtat thermométrique et hygrométrique de l'air n'ait point varié. Cela provient évidemment de ce que la lumière exerce une influence d'un genre inconnu sur l'énergie de l'endosmose et de l'exosmose végétales. Aussi, arrive-t-il quelquefois que le liquide qui est chassé au-dehors soulève l'épiderme en forme de petites vésicules, lorsqu'il ne peut sortir assez librement : c'est ce que l'on voit dans le mesembryanthemum crystallinum, dont les petites vésicules ou boursonflures de l'épiderme ne sont jamais plus remplies que lorsque la plante est exposée au soleil, parce que la lumière et la chaleur augrmentent alors l'action vitale. L'exhalation des végétaux a lieu de même, lorsqu'ils sont entièrement plongés dans l'eau. J'ai pu me convaincre de cette vérité par l'observation de la spongille fluviatile, que j’affirme être nu véritable végétal doné d'une composition chimique analogue à celle des animaux. Les innombrables cellules qui entrent dans la composition de cet être singulier, sont tapissées par une membrane mince et diaphane; leur intérieur contient une substance caséiforme; cette même membrane diaphane revêt toute la surface de la spongille. Souvent il se forme des boursouflures remplies d'eau sur celte membrare superficielle, et ordinairement ces boursouflures finissent par se percer à leur sommet; alors il s'établit par l'ouverture un courant d'eau continu du dedans an-dehors, et qui entraîne de temps en temps avec lui de petites portions de la substance caséiforme intérieure dont j'ai parlé tout à l'heure. Ce courant 
d'eau très-uniforme, et qui n'éprouve aucune interruption, nie paraît être le réstiltat de l'afflux vers l'onverture de tout le fluide intérieur quï tend à être chassé par exhalation; trouvant une issue libre par la perforation accidentelle de la membrane extérienre qu'il avait d'abord soulevée, le liquide, chassé vers le delurrs, et destiné à filtrer péniblement au travers de la membrane enveloppante, se précipite vers cette ouverture, qui lui livre un passage facile.

Ce n'est point par des orifices vasculaires, particulièrement destinés à cet usage, que se font l'absorption et l'exhalation chez les végétaux. Ce transport des liquides du dehors au-dedans et dı dedans andehors s'opère par filtration au travers des parois des meunbranes or'ganiques. Nous n’avons aucune idée de la disposition des pores qui transmettent les liquides dans cette circonstance, et l'on se tromperait beaucoup si l'on croyait pouvoir les apercevoir au microscopc. La ténuité des molécules de l'eau est immense, et hors de toute proportion avec des pores que le microscope nous ferait apercevoir. C'est par des voies capillaires, dont la forme est inconnue, que les liquides sont transmis; mais, comme cela a été pronvé plus hant, ce n'est point en veru de la capillurité, ou en vertu de l'attraction capillaire que se fait cette transmission, qui s'opère entièrement sous l'influence des comans électriques. Ainsi, c'est spécialement à l'agent. électrique qute sont dus les mouventens divers de la sève; les autres causes qui peuvent avoir de l'influcnce sur ces mouvemens sont purement acci- 
dentelles ou accessoires. 'Telles sont la capillarité, qui agyit dans toutes les circonstances que j’ai indiquées Iplus haut, la pesantertr, qui influe probablement un Ipeu sur la sève descendante, et enfin l'influence de ll'agitation par le vent. M. Knight a expérimenté 'qu'en rendant tout à fait immobile une partie de lla tige d'un jeune arbre, au moyen d'un étai solide, ccette partie immobile prenait moins d'accroissement ten grosseur que la partie libre de celte mênie tigge uqui pouvait être agitée par le vent. M. Knight a conccli de cette expérience, que l'agitation des végétanx ipar le vent est une des causes de la progression de la sève. Effectivement, on conçoil que les mouvemens the flexion des parties du végétal doivent occasionner ides compressions locales, lesquelles ne peuvent manquer d'accélérer la progyiession des liquides contenus dans ces parties. On sait combien les mouvemens de llocomotion des animaux ont d'influence sur la rarpidité de la progression de leurs liquides intérieurs; lees végétaux, qui ont peu de mouvemens spontanés, .rouvent un supplément à ce qui leur manque à cet syard, dans ¿l'agitation de leurs parties mobiles par le rent. C'est, en quelque sorte, leur manière de prenthe de l'exercice.

Un certain degré d'élévation dans la température 'st nécessaire pour que la proggression de la sève Lit lieu : c'est pour cela que la végétation s'arrête mendant l'hiver et reconmence au printemps. $\mathrm{Or}_{\mathrm{r}}$, mous trouvons une explication complète de ce phévomène dans les expériences rapportées plus haut, 
et qui nons ont pronvé que l'intensité de l'endosmose est augmentée par l'élévation de la température el diminuée par l'abaissement de cette même température. Comme e'est à l'endosmose qu'est due l'introduction et la proggression de la sève, on conçoit eomment le froid, en suspendant ou en diminuant considérablement eette aetion vitale, suspend le eours des fluides, et par conséquent la végétation. Au reste, il existe de grandes différences entre eertains végétaux, sous le point de vue de la température qui eonvient à chaeun d'eux; les uns ne se plaisent que dans les zônes glaeées, les autres ne peuvent vivre que dans les elimats brûlans. Toutes ees différenees, on n'en peut douter, tiennent à eertaines qualités physiques qui les rendent propres à produire des courans éleetriques sous une température déterminée, et don t le degré est différent selon la différence de ees mêmes qualités physiques, dont lá détermination ne doit pas être très-diffieile.

L'existence démontrée de eourans éleetriques dans les vésieules remplies d'une substanee plus dense que l'eau qui les environne, nous donne une explication très-satisfaisante des mouvemens spontanés qu'exécutent quelquefois ces vésicules que, jusqu'à ee jour, les observateurs n’ont point hésité à prendre pour des animaux. Ces vésieules, opérant à la fois l'introduction de l'eau environnante et l'expulsion du liquide qu'elles contienuent, doivent, par l'elfet de eette dernière aetion, agir sur l'eau dans laquelle elles nagent, comme une finsée aggit sur l'air, c'est-à-dire qu'elles 
doivent se mouvoir dans une direction opposée à celle idu courant d'ean qui sort de leur cavité. Cet. effet ne doit avoir lieu que lorsque le courant du liquide exIpulsé esı asscz énergíque pour opérer par réaction le idéplacement de la vésicule : dans le cas contraire, ıcette dernière doit rester immobile. Ceci nous donne lla solution d'un problème de physiologyie qui, dans ces derniers temps, a beaneoup occupé les naturalisItes : je veux parler des célèbres zoncarpées de M. Bory ide Saint-Vincent. J'ai eu occasion de les observer, ces :zoocarpées; j’ai vu leurs mouvemens spontanés toujours de peu de durée; j’ai vu même, dans certaines circonstances, les globules de la matière verte de Priestley se mouvoir spontanément, mais s'arrêtcr Ibientôt; je n'ai vn, dans tout cela, que des vésicules 'qui sont mues par des courans électriques : ce ne sont certainement point des animaux qui se meuvent volontairement. J'en dirai autant de tous ces prétendus animalcules infusoires qui sont simplement globuJeux on ellipsoides, et chez lesquels on n'aperçoit aucune de ces parties qui constituent incontestablement l'animal. 'Tels sont, par exemple, ces prétendus animalcules qui constituent, par leur aggglomération, ces pellicules qui se forment à la surface de l'eau dans laquelle des substances animales ou végétales sont en macération : ce sont des vésicnles tanlôt en mouvement, tantôt constamment immobiles, et cela selon le degrré de la température, ou selon d'antres circonstances qu'il n'est point dans mon plan d'exposer ici. Dernièrement, M. Edwards, en cxaminant 
au microscope des cellules détachées mécaniquentent d'une partic végétale et plongées dans l'eau, vit ces cellules se mouvoir spontanément, et crut pouvoir en conclure que, dans cette circonstance, des parties végétales prenaient de l'animalité. Ce phénomène, comme les précédens, dépend entièrement des courans électriques qui existaient dans ces vésicules. Ainsi disparaissent des merveilles imaginaires devant le flambeau de l'observation; la nature possède assez de merveilles réelles pour nous consoler de cette perte. Mais n'est-ce pas gagner que de perdre des erreurs?

Il résulte de tout ce qui vient d'être exposé, que la vie de nutrition des végétaux consiste toute entière dans l'endosmose et dan's l'exosmose; et comme ces actions physico-organiques reconnaissent pour cause l'agent électrique, il en résulte, en dernière analyse, que cet agent est le principe de la vie de nutrition des végétaux. Probablement est-il aussi le principe de leur vie de relation; mais ceci doit attendre la confirmation de l'expérience.

Je viens de parler de l'existence d'une vie de relation chez les végétaux; et cette assertion, qui a droit de surprendre, demande une explication que je ne puis me dispenser de donner ici, quoique cela soit étranger au sujet principal dont je m'occupe dans ce travail.

'Tous les physiologistes savent que Bichat a divisé les fonctions de la vie en denx classes : la première, sous le nom dr vic animale, comprend les sensations 
et les aetions volomaires : cette ie animale est tont centière sous la dépendance du cervean; la seeonde classe de fonetions, sous le $110 m$ de vie organique, comprend toutes les aetions spontanées par lesquelles Ila vie se soutient, par lesquelles la maehine vivante ss'entretient et se répare. On a donné anx fonetions ide la vie animale le nom de fonclions de relation, cet aux fonetions de la vie organique celui de foncitions de nutrition. D'après les idées adunises à cet iégard, les animaux seuls possèdent les fonctions de rrelation; les végétaux sont bornés aux fonetions de mutrition. Ces idées, quoique lumineuses, ne sont cependant pas tout à fait justes. En eflet, les véggétaux ont bien certainement des fonetions qui les mettent wn relation avee certaines choses qui lenr sont exteérieures. Par exemple: ils dirigent quelques - unes dde leurs parties vers la lumière, et cette direetion sspéeiale a lieu en vertu d'un mouvement spontané. La ssensitive a des relations assè nombreuses avee les choses du dehors, puisqu'elle se meut spontanément. à l'oeeasion des variations de la température, à l'oeccasion des ehoes, etc. Les végétanx ont donc des foncthions de relation distinetes de leurs fonctions de nutriıion. Ces fonctions de relation ne se rapportant point chez eux à un centre nerveux, ou à un sensorium rentral, ils n'ont ni sensations ni volonté. Leurs mou'vemells, quoique spontanés, e'est-à-dire dépendans immédiatement d'une eatrse intérieure et vitale, sont méeessairement exéentés à l'oecasion de l'influenee de la eause extérieure qui a le promoir de les provo- 
quer. En cela, lenrs mouvemens spontanés ressemblent tout à fait aux mouvemens ésalement spontanés qu'exécutent quelques-unes des parties intérieures des animaux, à l'occasion de leur relation avec certaines choses qui leur sont extérieures. Ainsi le cœur se contracte sur le sang, la vessie sur l'urine, les intestins sur les matières qu'ils contiennent, l'utérus sur le produit de la conception sans ancune participation de la volonté, sans aucune intervention du cerveau, centre et régulateur des fonctions de relation. Ces actions cependant sont fondées sur des relations de l'organisme avec des choses extérieures. Elles doivent donc aussi faire partie des fonctions de relation, et l'on en doit dire autant des actions spontanées des végétaux. Ceci nous indique la nécessité de faire une légère modification à la classification des fonctions adoptées par Bichat. Les fonctions de relation sont de deux ordres : $I^{\circ}$ celles qui reconnaissent le cerveau, ou le sensorium central comme centre et comme régulateur; $2^{\circ}$ celles qui sont indépendantes d'un sensorium central. Les animaux possèdent ces deux ordres de fonctions de relation; les végétaux ne possèdent que le second. Les fonctions de nutrition appartiennent également aux végétaux et aux animaux; les fonctions de génération sont également l'apanage de tous les êtres vivans. Le tableau suivant offre la classification des fonctions de tous les êtres vivans, d'après les principes que je viens d'exposer. 
Classifuation des fonctions duns les deux règnes négétal ct animal.

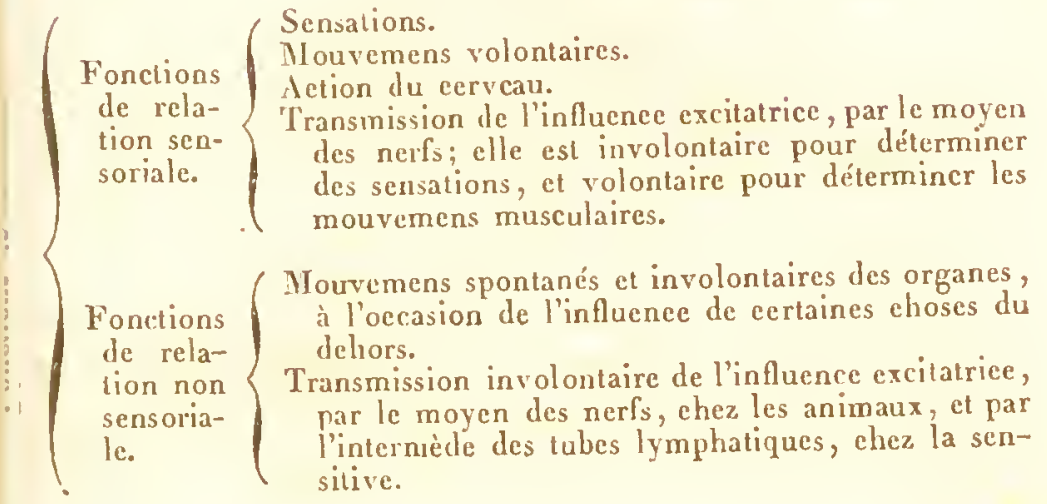

Absorption et exhalation.

Sécrétions.

Nutrition, ou composition et déeomposition des organes.

Progression des fluides $\left\{\begin{array}{l}\text { Cireulation ehez les animaux. } \\ \text { Aseension et descente de la séve ehez }\end{array}\right.$ dans leurs canaux. $\quad \begin{gathered}\text { Ascension } \\ \text { les végétaux. }\end{gathered}$

Elaboration des subs-
tances alimentaires. $\quad\left\{\begin{array}{l}\text { Digestion et ehilification ehez les ani- } \\ \text { maux. } \\ \begin{array}{c}\text { Production de la sève élaborée ehez } \\ \text { les végétaux. }\end{array}\end{array}\right.$

Vivifieation ou entretien du (Par l'oxigénation respiratoire mouvement vital, par les ehez les animaux.

deux causes les plus géné- Par l'insolation, ou par l'inrales de la production de la fluenee de la lumière chez chaleur. les végétaux.

Ponetions le \{ Génération sexuclle. Appartiennent toutes les deux génération. $\{$ Génération gernmipare. $\}$ aux animaux et aux plantes. 


\section{SECTION II.}

Application des observations précédentes à li slalique vitule des animaux.

Les faits qui nous ont dévoilé l'existence de l'endosmose et de l'exosmose, nous ont prouvé que ces actions physico-organiques appartiennentégalement aux végétaux el aux animaux. Nous avons vu que c'est par elles que s'opère la progression des liquides dans les cavités capillaires des végétaux; elles doivent, par" conséquent, présider aussi à la prougression des liquides dans les cavités capillaires des animaux. Mais chez ces derniers, la progression des fluides ne dépend pas d'une cause unique, comme cela a lieu dans le règne végétal. Chez les animaux à circulalion, les fluides ne marchent dans des cavités capillaires que pendant une partie du trajet qu'ils parcourent dans l'organisme. 'Tant que le sang est dans les artères, il se meut spécialement par l'impulsion que lui comnunique la contraction du cœur; mais arrivé dans les vaisseaux capillaires, le sang obéit à une force nouvelle, force à laquelle est spécialement due la circulation veineuse. Chez les animaux dépourvus de circulation, les fluides marchent exclusivement par des voies capillaires. C'est ainsi, par exemple, que s'opère la diffusion du fluide nourricier chez les 
useetes. Chez eux la marehe des fluides ne praraît mint avoir d'autre cause que celle à laquelle est due progression de la sève ehez les végétiux. Pour nous $n$ assurer, reeherehons si les conditions fondamenlles de l'endosinose existent ehez les animaux eomme lles existent chez les végétanx. Nous avons vil que us eonditions fondamentales sont, d'une part, la Iructure vésiculaire, ct, d'une autre part, l'existenee uns les vésicules d'un fluide organique dense. J'ai iit voir, dans $11 n$ précédent ouvrage (I), que les vé¿átax sont composés de vésieules agyglomérés. Ces śsicules ou eellules, tantôt arrondies, tantôt pothèdres, forment alors ce que l'on appelle le tissu allulaire. Lorsqu'elles sont plus ou moins alongées, surtout lorsque leurs eloisons terminales ayant disuru elles communiquent entre elles, elles forment ss tubes. Or, l'observation nieroseopique apprend ue tous les orgyanes des animaux sont aussi composés vésicules agogrelomérées; mais ces vésicules sont orinairement bien plus petites que ne le sont eelles ':s véçétaux, lesquelles sont quelquefois apereevaces à l'œil nu. C'est spécialement ehez les mollusucs que eette strueture vésienlaire est faeile à voir, urce que leurs vésieules, sont plus grandes que ne le nt eelles des animaux vertébrés. Avee un bon mi'oseope, on peut même voir, dans eertains organes 'ss mollusques, que les parois de leurs vésieules sont

(1) Recherches sur la structure intime des animaux et des véitant. 
formées par une aggrgégation de vésicules plus petites (1). Toutes ces vésicules sont remplies par des substances organiques. Ainsi, les conditions fondamentales de l'endosmose existent chez les animnux. Ici je dois m'arrêter un instant pour jeter un coupd'œil sur la distinction générale que l'on fait des parties constituantes des êtres vivans, en solides et en liquides : distinction qui me paraît peu philosophique. Revenons ici, pour un instant, aux végétaux. La meilleure distinction générale que l'on puisse faire de leurs parties constituantes, est celle des parties contenantes et des parties contenues. Les parties contenantes sont les organes vésiculaires ou tubulaires; les parties contenues sont les substances que contiennent ces organes creux, substances tantôt liquides, tantôt pâteuses, tantôt très-solides. Il est évident que ce ne sont point ces substances contenues qui, lorsqu'elles sont solides, forment les véritables solides organiques chez les végétaux. On ne doit donner ce nom qu'aux parois des vésicules ou des tubes, c'est-à -dire aux parties contenantes. Or,

(1) Si l'on cst curicux de s'assurer, d'une manièrc très-positive, dc l'existence de ces vésicules, il faut prendrc un fragment de cct organc, quil, semblable à un filet blanclıâtrc, cuvelopje l'estomac de l'helix pomatia, et qui est la glande salivaire de ce mollusque. On met ce fragment de glaude dans un cristal de montre, avcc de l'eau chargée d'une petite quantité d'lıydrate de potasse, et on le place sous le microscope. A incsure que l'alkali dissout les parois extrêmement minces des vésiculcs, on les roit crever, et s'évanouir commc des bulles de savon. 
il en est de même chez les animanx. Toutes leurs parties sont composées de vésicules aggolomérées, lesquelles contiennent des substances tantôt liquides, tantôt pâteuses, tantôt solides. $\Lambda$ insi, le tissu osseux, vu au microscope, paraît, comme tontes les antres parties, composé de globules agglomérées; ce sont des vésicules remplies de phosphate de chaux, comme lle cerveau est composé de vésicules remplies de subsltance nerveuse, comme les organes sécréteurs sont 'composés de vésicules remplies par le fluide sé‘crété, etc. Ainsi, ce que l'on appelle ordinairement ıun solide organique chez les animaux, cst un agré乡gat de vésicules remplies par des substances plus ou moins deuses. Mais ce ne sont pas ccs substances qui, llorsqu'elles sont solides, méritent le nom de solides corganiques : ce nom doit appartenir par excellence :aux parois des vésicules. Ces parois étant elles-mêmes (composées de vésicules plus petites, il en résulte qu'on iignore oì s'arrête cette texture vésiculaire, et où se trouve le solide essentiel et primitif, celui qui scrait composé de molécules solides aggglomérées, et non de ivésicules. Probablement que ce solide n'existc que (dans les molécules constituantes, et que le composé d'oxigène, d'hydrogène et de carbone, auquel s'adjjoint souvent l'azote, composé qui forme essentiellement les parties organiques, cst, par sa nature, essenıtiellement vésiculaire jusque dans ses molécules intégrantes.

Futre les vésicules qui composent le tissu organique des animaux, rampent les vaisseaux sanguins, chez 
les animaux à circulation; ces vésicules sont appliquées sur les parois des vaisseaux; et il est certain que la cavité des vésicules ne communique point immédiatement avec la cavité des vaisseaux, puisque le même fluide n'existe point dans leur's cavités. Ce fait est très-facile à vérifier, en examinant au microscope le tissu d'un organe sécréteur chez un mollusque gastéropode, cclui du foie par cxemple. On voit toutes les vésicules de cet organe remplies par la bile, que l'on distingue à sa couleur, tandis que les vaisseaux sanguins qui côtoient ces vésicules n'ont que la diaphanéitć que 'eur donne l'état incolore du sang qui les remplit. Ainsi, les vaisseaux sanguins n'existent que comme des moyens d'irrigation pour les vésicules qu'ils côtoient; et ce ne peut être que par filtration que le fluide sanguin pénètre, en se modifiant jusque dans ces vésicules élémentaires. Le système sanguin, considéré dans son cntier, forme une cavité sans issue dans laquclle rien ne peut entrer, et de laquelle rien ne peut sortir autrement que par filtration. Les extrémités artérielles et les radicelles veineuses sont des fables physiologiques. La continuité exacte des artères et des veines est très-visible au microscope, chcz les très-jeunes salamandres. En général, chez les reptilcs, la division en ramuscules dn système sanguin est moins considérable qu'elle ne l'est chez les mammifèrcs et chez les oiseaux; mais c'est à cela seul que se rédnit la différence qui existe à cet égard entre ces animaux : chez les uns comme chez les antres, le système sanguin forme une cavité sans is- 
ssue, mais plus ou moins divisée en ramifications. Ce ssont les ramifieations qui opèrent l'irrigation des orgganes, et qui portent ainsi à leurs vésienles élémenuaires les nouveaux matériaux organiques qu'elles leur transnictent par filtration, et cela en vertu de l'enudosmose, conme nous allons le prouver tout à l'heure. Nons venons de voir que les conditions fondamentales ide l'endosmose existent ehez les animaux; aetnelleiment mous allons voir que cette action pliysico-orgamique existe effectivement chez eux.

Tout le monde connaît l'état morbide que l'on déigne sous le nom d'inflammation. On sait que, dans cet état, les parties molles deviennent turgides, et Ifu'elles attirent avee abondanee les fluides dans leur imtérieur. Les liquides organiques qui environnent une partie enflammée sont soumis ì un mouvement U'adfluxion qui les porte vers eette partie, dans laII uelle se trouve la cause ineonnue qui les attire, on Ifui les appele, eomme l'ont dit quelques physioloyistes modernes dans leur langage improprement fi¡uré. Iei, il est impossible de méconnaître l’endosmose, qui se manileste à la fois par l'existenee de "état turgide el par le mouventent d'adfluxion, Houble elfet qu'il n'appartient qu'ì l'endosmose de roduire. Les artères qui se rendent à une partie enHammée augrmentent de ealibre pour y conduire le ang que l'adfluxion dirige en abondance de ee eôté; ces veines qui viennent de eette même partie enflamméc se dilatent sons l'impulsion du sang, que cette warlie lenr envoie en plus grande abondance el avec 
plus de force qu'à l'ordinaire. Ainsi, la partie enflammée est à la fois but d'adfluxion el origine d'impulsion pour le sang. Nous avons vu plus haut que tels sont exactement les effets de l'endosmose chez les végétaux. Il ne peut donc exister de doutes à cet égard; l'inflammation est un phéuomène d'endosmose : or, ce phénomène, dont les effets sont si visibles dans l'état morbide, existe de même dans l'état normal, mais avec un degré d'intensité moindre. Le sang des arlères est attiré dans leurs ramifications capillaires, et c'est à celte adfluxion qu'est due la vacuité des artères après la mort, lorsque l'impulsion du cœur a cessé. Le sang des veines reçoil dans les ramifications capillaires une impulsion dont, jusqu'à ce jour, les physiologistes ont vainement cherché la cause; cette cause est évidemment dans l'adfluxion des liquides qui chassent, en les remplaçant, les liqnides précédemment existans dans les cavités capillaires : c'est ainsi que s'opère la progression de la sève par impulsion, et c'est de neeme que s'opère la progréssion du sang veineux. Au reste, diverses causes accessoires peuvent favoriser et favorisent en effet le mouvement du sang dans les veines. Lorsqu'il n'y a point de ramifications capillaires entre une artère et une veine, l'impulsion du cœur suffit pour imprimer le mouvement au sang artériel et au sang veineux. C'est ainsi, par exemple, que cela a lieu chez les salamandres nouvellement écloses : on voit cn effet chez elles un seul vaissenu sanguin, qui, parvenu à l'extrémité de la queue, se réfléchit pour revenir vers le cœur, en 
sorte qu'il ne présente aucune distinetion en artère $\mathrm{et}$ en veine. On ne peut douter que l'impulsion du cœur ne se fasse sentir iei dans tout le trajet eireulatoire. Il paraît que e’est pour avoir spécialement étudié la circulation chez les reptiles, que Spallanzani a ćté conduit à émettre l'opinion que e'est l'impulsion du eœur qui est la eause du morrement du sang veineux : eela n'est vrai que pour les artères, qui, sans se ramifier beaneoup, se ehangent immédiatement en veines; mais lorsque les artères se divisent en d'innombrables ramifieations eapillaires avant de se changer en veines, l'impulsion dn eour devient de plus en plus insensible, et le sang marehe sous l'influenee d'une foree nouvelle. On admet généralentent que la contraetion des petits vaisseanx est une des causes de la marche du sang dans les eapillaires; mais il faudrait alors admettre dans ces vaisseaux capillaires une diastole et une systole semblables à eelles du cœur. Ceci est purement hypothétique. J'ai beaucoup observé la eirculation capillaire au mieroscope, et jamais je n'ai pu apereevoir le moindre mouvement de contraction ou de dilatation dans les parois des vaisseaux eapillaires. Il est certain, cependant, que les vaisseaux sanguins ont une aetion d'élasticité par laquelle ils réagissent sur le sang qui les distend; mais il n'est point eertain du tout que ee resserrement des vaisseaux soil dû à une contraction, e’est-à-dire à une incurvation de fibres. Dans ces derniers temps, M. Barry, médecin écossais, a cru pouvoir expliquer la progression du sang veineux par la pression de l'at- 
mosphère et par les mouvemens de la respiration; mais cette cause de progrression ne peut être qu'une cause accessoire propre seulement aux mammifères et aux oiseaux, qui seuls respirent en formant un vide dans leur poitrine. Ainsi, la circulation du sang est un phénomène complexe dû au concours de plusieurs causes très-différentes, mais à la tête desquelles se trouvent d'une part la contraction du cœur, et d'une autre part l'endosmose des capillaires, endosmose qui agit à la fois comme cause d'adfluxion et comme cause d'impulsion.

Ce que je viens de dire par rapport au mouvement du sangr veineux, s'applique également au mouvement des fluides que contiennent les vaisseaux chilifères et lyınphatiques; mais, ici, il y a quelque chose de plus. On sait que ces vaisseaux sont interrompus de distance en distance, dans leur cours, par des ganglions ou glandes lymphatiques, corps au travers du tissu desquels sont obligés de passer les fluides contenus dans les vaisseaux qui aboutissent à ces ganglions : ces corps semblent, au premier coup-d'œil, être des obstacles au cours du flnide; mais, en y réfléchissant un peu, on voit qu'ils sont placés là par la nature pour favoriser au contraire la marche du fluide lymphatique. En effet, les ganglions lymphatiqnes ont un tissu capillaire, dont l'endosmose doit opérer l'adfluxion du fluide contenu dans les vaisseaux afférens : cette mème endosnose opère l'expulsion des fluides précédemment introduits, et il en résulte une impulsion à tergo pour les fluides contenus dans les 
vaisseaux efférens. Ainsi, loin d'être des obstaeles au monvement du fluide, les ganglions lymphatiques sont, au eontraire, ponr ce fluide, des organes d'adfluxion el d'impulsion : placés de distance en distanee sur' le trajet des vaisseaux Iymphatiques, ils sont, pour les fluides que contiennent ces vaisseaux, des organes moteurs. Ces ganghlions remplissent iei le même offiee que les noeuds de tissu eellulaire qui existent de distanee en distance ehez les graminées, et sur lesquels nous avons jeté un eoup-d'œil plus haut; car tout tissu eapillaire vivant est nécessairement el à la fois, par l'effet de son endosmose, eause d'adfluxion et eause d'impulsion pour les fluides.

Ainsi, l'endosmose existe dans l'état sain ou normal chez les organes des animaux, par eonséquent l'inflammation se trouve être l'exagération de eetle action physieo-organique; c'est une hypérendosmose. On en doit dire autant de la turgyesecnee éreetile, hypérendosmose eompatible avee l'état sain de la partic dans laquelle ellc existc, et qui diffère essenriellement par-là de l'inflammation ou de l'hypérendosmose morbide, dont la eanse oceasionnelle est anssi bien différentc. L'hypérendosmose érectile ne̋ reeonnaît qu' une seule eause oeeasionnelle, e'est l'inflıence nerveuse; l'hypérendosmose morbide dépend de plusieurs eauses oeeasionnelles sur lesquelles il scra utile de jeter iei un eorp-d'oeil.

Une des causes les plus connues de linflammation, on de l'hypérendosmose morbide, est l'introduction dans les tissus orçinniques d'un corps étran- 
ger. Ceci nous reporte d'nne manière singulièrement frappante à l'une des causes déterminantes de l'endosmose, el qui existe en ceci, que la présence dans les cavités organiques d'une substance plus dense que le fuide qui les environne, est pour ces cavités une cause déterminante d'endosmose. Ceci nous explique pourquoi les corps étranger's, plus dense que le sang, produisent l'hypérendosmose, ou l'inflammation des parties dans l'intérieur desquelles ils sont introduits, et pourquoi cette hypérendosmose ne cesse qu'après la sorlie de ces substances étrangères. Je suppose ici que ces substances sont dépourvues de toute action chimique sur les organes, et n'agissent que par leur simple contact; car, dans le cas d'une action chimique de leur part, ce serait une complication de phénomènes. Toutes les fois donc que les substances introduites dans le tissu des organes n'ont point d'action chimique sur eux, et ont une densilé inférieure à celle du sang, elles ne produisent point d'inflammation; c'est ainsi qu'on peut impunément introduire dans l'organisme certains liquides et plusienrs gaz. Nous avons vin plus haut, que l'endosmose perd d'autant plus de son énergie, que le liquide intérieur est rendu moins dense par l'addition de l'eau pure. Or, on sait que l'introduction de l'eau dans l'organisme est un des moyens les plus efficaces pour faire cesser l'inflammation. C'est à ce moyen que se rapportent les cataplasmes aqueux dont on couvre les parties enflammées, les bains et les boissons aqueuses abondantes dont on fait usage 
cen pareil cas. L'introduction de l'eau en abondance aa, dans cette circonstance, le double effet de dinniinuer la densité des liquides productenrs de l'hyperendosmose, et de remplacer ces liquides qu'elle expulse Ipar sou introduction continuelle. Pour bien saisir ce rmécanisme, qui est en même tenps celui de la nutrition et celui de la guérison de l'hypérendosmose, iil est nécessaire de se reporter aux notions qui ont tété exposées plus haut tonchant la composition des solides oryaniques, et touchant les lois de l'endosmose, ret de l'exosmose. Les solides organiques mous sont 'des agrésgats de vésicules renıplies ordinairenıent par une substance pâteuse plus dense que ne l'est le sanğ qui baigne leur's parois extérieures, ou qui n'est séparé de ces vésicules que par les parois extrêmement minces des vaisseux capillaires. Il résulte de celle disposition, que le fluide sanguin doit tendre continuellement à s'introduire dans les vésicules, lesquelles deviennent alors siégres de deux courans électriques, l'un dominateur, qui produit l'endosmose, l'autre plus' faible, et qui prodnit l'exosmose. Par l'effet du premier, les parties constituantes du sang sont introduites avec violence dans les vésicules; par l’effet du second, ces mêmes vésicules expulsent, et versent dans le courant sanguin une partie dı liquide qu'elles contiennent. Cette expulsion qu'opère l'exosmose, est favorisée et augmentée par la pression que prodıit l'introduction violente du liquide extérieur dans les vésiculcs déjà pleines avec excès : c'esı ainsi que doit s'établir l'équilibre entre l'introduction et l'expulsion, 
malgyré la différence qui existe dans l'énergie des deux courans électriques auxquels sont dus l'endosmose et l'exosmose. L'introduetion de nouvelles substanees dans les vésicules élénıentaires, constitue le phénomène de composition; l'expulsion des substanees que contiennent ees vésieules ennstitue le phénomène de décomposition; l'ensemble de ces deux phénomènes, en y joignant eelui de la produetion des nouvelles vésieules, constiutue la fonction de nutrition, laquelle existe avec le même mécanisme chez les végétaux, ainsi que nous l'avons vu plus haut. Or, c'est à l'action sans cesse continnée de ee méeanisme qu'est due la guérison de l'miflammation on de l'hypérendosmose morbide. Cette affection ne peut se développer spontanément, que paree que les substances contenues dans les vésicules qui eomposent les solides organiques ont acquis accidentellement ou une densité trop considérable, ou des qualités elimiques inaccoutumées, $\mathrm{Cl}$ propres à déterminer une augrmentation de leur endosmose naturelle. Dès lors le sangg, qui est pour ces vésicules le fluide extéricur, se trouve déterminé à pénétrer dans leur intérieur en plus gुrande abondance que dans l'état naturel; il les gonfle, et augmente considérablement leur état turgide. Une violente tendance d'adfluxion se nunifeste par le fait nuême de la violence de l'endosnose; une forte impulsion pour le sang veineux résulte de la nê̂me cansc. Cependant les vésicules, siégres d'un double courant d'introduction et d'expulsion, tendent par cela même à renonveler entièrement leur substance 
intérieure. L'époque de ee renouvellement est eelle de la eessation eomplète de l'hypérendosmose morIbide, si eette affeetion est déterminée par une altération dans la eomposition ehimique de la substanee intéricure des vésienles : mais si eette affeetion reeonnaît simplement ponr eause une augmentation de la densité de la substance intérieure, elle eessera lorsqu'il se sera introduit dans ees vésieules une quantité de fluide aquenx suffisante pour diminuer eonvenablement eette densité de lenr substanee intéricure. Ainsi, la force médicatrice n'est auire ehose que le jeu naturel et eontinuel du double mouvement d'endosmose et d'exosmose qui constitne une partie essentielle da phénomène de la mutrition.

Il résulte de eette théorie, qui est une déduction rigoureuse des faits observés, que l'inflammation ou l'liypérendosmose morbide dépend essentiellement de l'altćration des substanees eontenues dans les vésieules élémentaires. Ces vésieules elles-mêmes peuvent, dans eet état morbide, avoir eonservé leur intégrité. Ainsi la vésienle ou la partie contencinte, (pui est le véritable solide organique, est néeessairement étrangyère à l'inflammation. Ce n'est poim eette vésicule qui est altérée, c'est la substance contenue dans son intérieur, substanee plus ou moins fluide. C'est done véritablement l'altératiou des fluides qui eonstitne les maladies par hypérendosmose; aussi est-ce par les fluides que les maladies eontagienses se eommmiquent. Un virus introduit dans l'économie fait épronver anx thides une altération semblable à celle qu'il possèrte 
lui-même, et cette altération se propağe de plus en plus, jusqu'à produire une altération générale des lluides.

Toutes les substances du dehors qui sont susceptibles de provoquer l'hypérendosmose agissent, comme les virus, par leur introdaction et par leur mélange, avec les liquides organiques que ce mélange alı̀re. II est impossible de concevoir qu'une substance puisse enflammer un tissu vivant sans y être introduite. Si inn agrent impondérable tel que le calorique produit l'inflammation, c'est par l'altération qu'il apporte dans la nature des substances contenues dans les vésicules des tissus organiques soumis à son action; enfin, s’il existe des inflamnıations spontanées, el sans cause extérieure connue, cela provient d'un changemeut survenu dans la nature des substances contenues, par l'effet d'une cause intérieure. Autant il pourra exister de changremens ou de modifications spéciflques dans la nature des substances contenues, autant il y aura de causes spéciales ou spécifiques d'hypérendosmose; de là ces inflammations si variées dans leur naturc; car ce n'est pas seulement par la différence des tissus qu'elles affectent que les inflammations different cntre elles. C'est ainsi que l'inflamımation des ganglions lymphatiques par le virus syphilitique n'est point semblable à l'inflammation de ces mêmes gaugglions par le vice scrophuleux; c'est ainsi que l'in. flammation d'un ulcère cancéreux ne ressemble point à l'inflanmation d'un autre ulcère dont le siége serait semblable. Il y a daus les parties affectées d'une inflammation spécifique, des fluides altérés d'une 
manière spéeifique; et il arrive souvent que ees fluides, ransmis à un individu sain, déterminent ehez lui une rnflammation de la même nature que celle de la pariie malade de laquelle ils sont origrinaires; et eela, parce jue le liquide morbifique introduit eommunique sa manière d'être aux liquides sains; ear, je le répète, coute inflammation dérive nécessairement de l'altéation des liquides.

Le mouvement d'adfluxion que produit l'hypérenlosmose morbide ne se fait pas sentir seulement sur ce sang eontenu dans les vaisseaux. Une partie dont "endosmose est augrmentée se gonfle en absorbant les luides eontenus dans les tissus voisins. Ceux-ei, déouillés d'une portion des fluides qui remplissaient deurs eavités, tendent, en vertu de leur endosmose, i se remplir de nouveau, et cela produit la déplẹtion des tissus organiques qui les avoisinent, du côté opposé à celui par lequel ils eorrespondent à la partie hypérendosmosée. Ainsi il s'établit de proche een proche une adfluxion qui porte les fluides vers la partie hypérendosmosée comme vers un eentre. Si la partie hypérendosmosée possède un moyen d'évaccuation au dehors pour les fluides afluens, l'adfluxion en devient plus énergique. Dans tous les cas, ce moyen d'évacuation existe toujours au dedans, par le moyen des vaisseaux sanguins. Aussi les veines de la partie thypérendosmosée prennent - elles très - promptement un volume bien plus eonsidérable que celui qu'elles Ipossédaient dans l'état normal. Elles servent alors de canal d'évacuation, non seulement pour le sang ap- 
porté directenent par les artères, mais aussi pour les fuides, qui sont transmis de proche en proche à la pariie hypérendosmosée, par les parties voisines. Plus l'hypérendosmose est intense, plus l'adfluxion se propage, et fait sentir au loin son effet. C'est sur l'observation de cet effet qu'est fondée la pratique de la dérivation, si fréquemment el si avantagreusement employée dans la médecine. Une partie essentielle à la vie est-elle devenue, par son hypérendosmose, un centre dangereux d'adfluxion, on suscite une hypérendosmose plus énergique, si cela est possible, dans une antre partie moins essentielle, et alors les routes de l'adflxion se tronvent changrées; les fluides cessent de converger uniquement vers leur prenter centre d'adfluxion; ils convergent vers le centre artificiel d'adfluxion que l'on a créé. C'est ainsi qu'agissent les vésicatoires; c'ost aussi de celte ntanière qu'agissent quelquefois les purgatifs. Les sangsues, les ventouses agissent aussi en déterminant une adfluxion dans la partie sur laquelle on les applique; et cela, parce que le vide qu'elles produisent tend sans cesse à être rentpli par l'endosmose des parties évacuées. Si un venin tel que celui de la vipère a été introduit dans une partie, et qu'il ait déjà tendu à se propager de proche en proche, en arrête ce mouvemient de propagation en appliquant une ventouse sur la partie par laquelle l'introduction du venin a été faite. Alors la direction du mouvement de propagation des fluides de proche en proche se trouve intervertic; l'adfluxion, diriggée vers l'extéricur par la ventouse, fait rétrogra- 
ter les fluides infeetés, et leur propagation se trouve iinsi arrètée. C'est de eette manière qu'il faut, à mon ovis, 'expliquer les bons effets que produit la ventouse lans cette circonstanee, bons effets dont on doit la léeonverte, eomme on sait, au doeteur Barry. Ainsi 'adfluxion peut être déterminée de deux manières : - par l'hypérendosmose, qui, par l'adfluxion qu'elle oeeasionne dans la partie où elle existe, détermine la narehe générale des fluides vers cette partie; $2^{\circ}$ par mide opéré dans le îssu organique au moyen d'une ueeion artifieielle. Alors le tissu organique, vidé en wartie par eette suceion extérienre, attire les fluides les parties voisines, en veru de son endosmose naurelle; et eet effet, propagé de proehe en proehe, oroduit une adfluxion dont l'elfet se fait sentir plus ou moins loin dans l'organisme.

Les moyens de eombattre l'inflammation ou l'hysérendosmose morbide, sont connus seulement d'une nanière empirique, et par eonséquent d'une manière ort imparfaite. Ce ne peut être que par la eonnaisanee physiologique de la nature de l'inflammation et le son méeanisme organique, que l'on peut arriver à ine théorie rationnelle, et par conséquent eertaine, les moyens de euration. Ce n'est ainsi véritablement que par la physiologie que l'on peut aequérir des eonaissanees positives en médeeine; car, dans l'art de guérir, l'expérienee est si souvent trompeuse, qu'on te peut que fort rarement en tirer des eonclusions iévères.

D'après la théorie que nous venons d'exposer, 
les moyens de combature et de guérir l'hypérendosmose morbide sont les suivans :

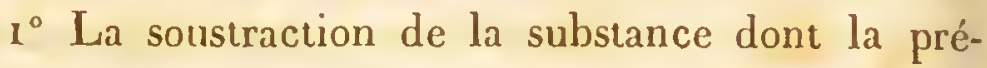
sence dans le tissu organique cause l'hypérendosmose. Ainsi, lorsqu'un corps étranger est la cause de l'inflammation, son extraction la fait cesser. Les sangsues, les ventouses scarifiées, appliquées immédiatement sur une partie hypérendosmosée, procurent l'évacuation des fluides altérés, dont la présence dans le tissu organique occasionnait son état morbide. Ce moyen de remédier à l'inflammation est un des plus puissans.

$2^{\circ}$ La saignée générale, ou l'évacuation des vaisseaux sanguins, remédie à l'hypérendosmose en diminuant l'afflux du sanğ artériel dans toutes les parties, et par conséquent dans la partie hypérendosmosée. Alors le vide opéré dans les gros vaisseaux se remplit au moyen de la déplétion générale des capillaires. $\mathrm{Par}$ conséquent, la partie hypérendosmosée perd alors une partie du sang qui abondait dans son tissu. Elle devient par conséquent moins turgide.

$3^{\circ}$. Les sangsues ou les ventouses scarifiées, appliquées dans le voisinage de la partie hypérendosmosée, et non sur cette partie immédiatement, ont deux modes d'action pour remédier à l'hypérendosmose :

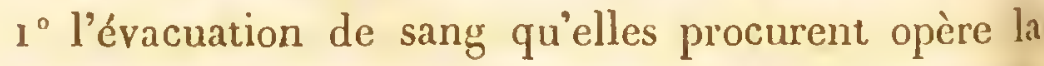
déplétion générale des vaisseaux; $2^{\circ}$ leur succion opère une dérivation ou un changement dans la direction de l'adfluxion. Les fluides qui se dirigeaient vers la partie hypérendosmosée tendent, par l'eflet de la suc- 
cion artificielle qui attire le sang au dehors, à se dirigger dans le sens de cette nouvelle adfluxion.

$4^{\circ}$ Une hypérendosmose énergique, suscitée dans une partie, tend à faire cesser ou à diminuer l'hypérendosmose qui existe dans une autre partie. Cet effet est le résultat au changement opéré alors dans la direction de l'adfluxion. Les fluides se dirigent vers la partie où existe l'hypérendosmose la plus énergique. C'est ainsi qu'agissent les vésicatoires. La dérivation opérée par ce moyen est d'autant plus efficace, que l'évacution qu'il produit est plus abondante; car l'évacuation continuée des fluides est, à elle seule, un excellent moyen d'opérer l'adfluxion, et par conséquent d'opérer la dérivation.

$5^{\circ}$ Nous avons vu plus haut que l'addition de l'eau, en diminuant la densité du fluide intérieur, ou en affaiblissant les effets de ses qualités chimiques, diminuait, par cela même, l'intensité de l'endosmose. Ceci nous explique pourquoi l'introduction de l'eau dans les tissus organiques est un si puissant moyen de combattre l'inflanmation, ou l'hipérendosmose morbide, puisque ce dernier liquide n'est point apte, par ses qualités physiques et chimiques, à provoquer l'endosmose.

$6^{\circ}$ Il existe des inflammations ou des hyperendosinoses morbides, pour la curation desquelles les moyens indiqués ci-dessus n'ont que peu ou point d'efficacité. La thénric indique ici l'emıploi d'uı sixième moyen de curation. Nous savons que les fluides introduits par l'endosmose chassent, en les rem- 
plaçant, les fluides précédemment existans dans les tissus organiques. Or, s'il existait dans ces tissus des fluides altérés, causes d'hypérendosmose, et dont aucun des moyens indiqués précédemment ne puisse opérer l'expulsion, on pourrait parvenir à ce but en introduisant, par l'absorption, dans les tissus organiques, des substances propres, par leurs qualités chimiques, à produire une hypérendosmose plus énergique que celle qui existerait déjà. Ces nouvelles substances, en augmentant l'activité de l'endosmose, procureraient l'expulsion de la cause antécédente d'hypérendosmose, cause qui pourrait être délétère par sa natıre même, et contre laquelle la force d'expulsion naturelle serait impuissante. De cette manière, on substituerail une cause d'hypérendosmose don la nature vivante pourrait se débarrasser facilement, à une antre canse d'hypérendosmose contre laquelle cette même nature vivante serait sans force. Or, ce que nous supposons ici théoriquement, a lieu tous les jours dans la praique médicale, mais d'une manière tout à fait empirique; car c'est ainsi, à mon avis, que le mercure guérit la syphilis. L'introduction de cette substance métallique dans l'économie y prodnit une hypérendosmose qui procure l'expulsion complète du virus syphilitique : cause antécédente d'liypérendosmose, contre laquelle la nature vivante était impuissante. Après cette expulsion, la nature se débarrasse toute seule de la cause d'hypérendosmose médicamentense; il arrive cependant quelquefois que lorsqu'on a tropin troduit de mercure dans l'économie, la 
mature devient impuissante pour s'en débarrasser, et il en résulte des hypérendosmoses merenrielics permanentes. C'est par un mécanisme semblable que se guérit sonvent l'hypérendosmose dissenteriquc, au moyen des purgatif's eonseillés dans eetle maladie par plusieurs médeeins célèbres. Les substances purgatives sont des agens produeteurs de l'endosmose. Leur aetion sur le eanal alimentaire ne peut avoir lieu sans leur absorption, sans leur introduetion dans le tissu organique de ee eanal. Or, dans le cas qui nous oeeupe, l'introduction de eette nouvelle cause d'hypérendosmose procure l'expulsion de la canse d'hypérendosmose dissenterique : eause delétère lont la nature vivante se serait peut-être diffieilement débarrassée toute seule. Il est inutile de faire observer que l'éeonomic se débarrasse ensuite faeilement, et à elle seule, de la cause d'endosmose introduite eomme moyen de euration. C'est eneore de eette manière qu'un vésicatoire appliqué sur un érysipèle, sur une dartre, en proeure la guérison. La substance irritante Hes eantharides, introduise, par l'absorption, dans le tissu hypérendosmosé, procure l'expulsion des fluides altérés qui oecasionnaient et entretenaient une hypérendosmose aiguë dangerense, ou une hypérenldosmose ehronique opiniâtre. Il y a gुrande apparence que l'on pourrait souvent, dans le traitement des maladies, obtenir ainsi de bons effets, en substituant une eause d'hypérendosmose saus danger, à une cause t'hypérendosmose dangereuse.

$7^{\circ}$ L'intensité de l'hypérendosmose pent être di- 
minuée par l'introduction daus l'économie de certaines causes d'exosmose. Nous avons vu plus haut que les acides sont, en général, des agens productenrs d'exosmose. C'est probablement cette qualité qui rend si utile, dans les inflammations, l'usage des acides légers en boisson, et qui les fail considérer comme rafraichissans. La théorie nous indique que l'ou doit, dans les inflanmmations, s'abstenir de toutes les substances propres à produire l'endosmose; rien, par conséquent, u'est moins convenable que ces boissons chargées de gomme, de substances extractives, ou de sucre, dont on abreuve ordinairement les malades, car toutes ces substances sont des agens producteurs d'endosmose, comme le sont les substances alimentaires, que l'instinct naturel repousse en pareil cas. Si les cataplasmes émolliens produisent un eflet salutaire, par leur application sur les parties hypérendosmosées, cela provient ou de l'eau qu'ils livrent à l'absorption, ou de l'effet d'exosmose qu'ils déterminent, en vertu de leur densité considérable. Nous avons vu, en effet, plus hạut, que l'exosmose a lieu lorsque le fluide extérieur est plus dense que le fluide intérieur. Or, un cataplasme de farine de graines de lin, par exemple, est véritablement un liquide d'une densité, très-supérieure à celle des liquides animaux contenus dans la partie sur laquelle ce cataplasme est appliqué. Il doit, par conséquent, déterminer, à la surface de cette partie, un courant électrique qui porte les fluides du dedans au dehor's, c'est-à-dirc yru'il prodnit un effect d'exosmose, d'où résulte la dé- 
blétion de la partie hypéreudosmosée. De là provient ce soulaggement très - marqué que procurent ces catablasmes dans les hypérendosntoses. Cette théorie peut Indiquer, dans certaines maladies, l'usage des bains Jaits avec des liquides plus denses que ne le sout nos iiquides organiques; alor's la peau doit exhaler abonHlamment au lieu d'absorber, comme elle le fait lors lle l'immersiou du corps dans l'eau pure, ou dans des iiquirles dont la densité est inférieure à celle de nos iiquides intérieurs. Ces réflexions nous conduisent maturellement à nous occuper ici de l'absorption et He l'exhalation.

Nous avons vu que chez les végétaux l'absorption n'est point du tout le résultat de l'atraction capilcaire, mais que cette introduction dés liquides du Hehors au dedans dépend entièrement de l'endosmose; il en est de même chez les animaux.

On a, jusqu'à ce jour, considéré l'absorption et 'exhalation comme les résultats de l'action des oriices béans de ccrtains vaisseaux absorbans et exha'ans. M. Magreudie a porté la première atteinte à cette théorie, relativement à l'absorption, en pronzant, par des expériences, que l'introduction des liquides daus l'organisme s'opère, par une véritable filtracion, an travers du tissn perméable des membranes. Iais ce physiologiste célèbre me paraît avoir poussé urop loin les dédıctions de ses expériences, lorsqu'il a cru pouvoir en conclure que l'absorption est le simple résultat de la capillarité. L'introduction des liquides 's'opère, il est vrai, par des voies capillaires, mais ce 
n'est pas l'attraction capillaire qui détcrmine cette introduction. J'ai prouvé cette vérité pour les vćgétaux, $\mathrm{ct}$ son application aux animaux cst dirccte. Les partics vivantcs sont sous l'influence continuelle dc l'ćlectricité, qui cntretient lcur état d'endosmose, ćtat de réplétion avec excès, qui fait que leurs cavités capillaires sont remplies par les liquidcs, au-delà du point de saturation de l'attraction capillairc. Ce n'est point, par conséquent, cctte attraction qui opc̀re une absorption subséquente. Conımcnt sc pourait-il, en effct, qu'un animal habitant de l'eau, qu'un poJype, par excmple, animal pulpeux cı sans vaisscaux, absorbât continuellemcnt, cn vertu de la seule attraction capillairc des petites cavités de son tissu? Cettc attraction cst bornée dans ses effets, ainsi que je l'ai déjà fait observcr plus haut. Lorsque les cavités capillaircs sont remplies, il n'y a plus d'introduction nouvclle, tout demeure cn repos. Il n'en est pas de même de l'introduction ou de l'absorption, résultat de l'cndoṣmose; elle est continuelle, parce qu'elle est accompagnéc par unc cxpulsion, ou par unc exhalation égalcment continuelle, ct qui est le résultat de l'exosmose. Cc sont donc véritablcment ces deux actions vitales qui scules opèrent l'absorption et l'exhalation, et cela par simple filtration au travers d'un tissu organique pcrmćable, et non, conme on l'avait supposé, par des orifices vasculaircs particulièrcment destinés à cct usagc. 'Toutes les parties organiqucs qui, par leur position naturelle ou accidentelle, se trouvent en rapport avec des liquides qui leur sont 
cextérieurs, absorbent ces liquides, lorsqu'ils sont dans lles conditions voulues pour cela, et les versent daus I'] système vasculaire, leur réceptacle naturel, chez lles aninaux pourvus de vaisseanx. L'observation Ipronve qu'il y a des absorptions électives. Ainsi, dans le canal alimentaire, le chile seul est absorbé; iil n'y a point d'absorption des matières fécales qui sont cependant mêlées avec le chile, dans l'intestin :grêle. Ce phénomène trouve son explication, de la Imanière la plus complète, dans l'expérience suivante, -combinée avec les expériences déjà rapportées plus lhaut. J'ai fait un endosmomètre avec un cœecun de poulet, dans lequel j’ai laissé les matières fécales qu'il contenait, et auxquelles j'ai ajouté de l'eau, pour les délayer d'une nanière suffisante. Ce liquide fécal s'élevait à une certaine hauteur dans le tube. Ayant plongé le cœcum dans l'eau, le liquide fécal n'a pas tardé à s'abaisser dans Je tube, et cet abaissement n'a point discontinué pendint tout le temps de l'expérience. Ce fait m'a démontré que la matière fécale est un agent producteur d'exosmose. Alors j’ai évacué le cœecun ; et l'ayant soighneusement lavé par des injections d'eau pure, j'y ai introduit du lait, fluide, comme on le sait, assez semblable au chile. Le cœcum étant plongé dans l'eau, le liquide intériemr a monté gradnellement dans le tube : il y a eu production d'endosmose. Les expériences rapportées plus haut nons avaient déjà prouvé ce dernier fait, que je ne reproduis ici que pour le metre en parallèle avec celui yui le précède. Il résulte de là que le chile et la na- 
tière fécale ont des qualités dianétralement opposées, sous le point de vue de la propriété de produire l'endosmose ou l'exosmose. Si done le ehile possède des qualités telles que le tissu orwanique soit déterminé à l'introduire ou à l'absorber', ee même tissu organique doit, par eela même, ne pas introduire, ne pas absorber la matière féeale. Ceei est un phénomène qui mérite toute l'attention des physiologistes et des médecins. Il y a des substances que l'absorption n'introduit point. Il suffit ' qu'une substanee appliquée au dehors de l'éeonomie soit plıs dense que les fluides intérieurs, ou qu'elle possède eertaines qualités e’hmiques qui équivalent, pour eet objet, ì l'exeès de densité, pour qu'elles ne soient point introduites, et e£la paree qu'elles sont des agens produeteur's d'exosmose, donés d'une suffisante énergie. Ceei expliquerait peut-être pourquoi des médieamens aetifs donnés à hartes doses dalis eertaines maladies, restent sans tffet; ils ıse son í point absorbés, ou plutôt ils le sont très-peu, et eela préeisément paree que leur dose est exagérée. Il est possible aussi que daus la maladie, l'éeonomie vivante soit plus voisine que dans la santé de eet état, qui est favorable à la manifestation de eette tolérance poitr les médicamens, toléranee qui ne me paraîl être que leur dériaut d'absorption.

Une belle expérience de M. Magendie a pronvé que, ehez les animaux, on augmente l'énergie et l'aetivité de l'absorption en désemplissant les vaisseaux sanguins. Mes expérienees sur les végétaux m'ont douné un résultat tout à fait semblable, et elles mont 
pronvé en même temps quil ne fallait pas généraliser celte assertion. En effet, nous avous vir que la rvacuité du lissn végélal, poussée jusquàà un certain point, augmente l'activité de son absorption; nuais mons avons appris ensnite que lorsque cette vacnité idu lissu végétal est portée au-delà de certaines limuites, elle diminue considérablement l'acrivité de Il'absorption, bien loin de l'angninenter. Il est fort probable qu'il en est de même chez les animaux. Mais con ne peut gnc̀re, je crois, s'en assurer par l'expéarience, à raison de ce que les animanx à circulation périssent tous par la seule perte dn sang qui remplit lleurs vaisseaux, sang qui n'est qu'une faible portion du li(quide dont est imprédıué le tissu de tous les organes. La séparation du chile de la matière fécale à laquelle il est mêlé dans l'intestin, est une véritable filtration chimique. La nuembrane muqueuse intestimale, véritable filtre chimique dans cette circonsitance, ne laisse passer que les substances pourvues de 'qualités chimiques déterminées. C'est donc une sorte 'de sécrétion qui s'opère dans ce cas; mais celte sécrétion n’est ici qu'une séparation de substances mêlées, scmblable, sous ce point de vue, à la sécrétion de 'l'urée par les reins; car on sait, par les expériences de Mill. Prevost el Dumas, que l'urée existe tonte formée dans le sang des animaux. Mais toutes les sécrétions ne sont pas de même de simples séparations de substances mêlées; la plıpart sont le résultat d'une séparation et d'une nouvelle association des élémens du fluide nourricier. Nous avons exposé plus hant, en 
traitant de la statique vitalc des végétaux, la thcoric générale de la sécrétion; cette théoric est applicable anx animanx. En cffet, les organes sécréteurs des aniniaux sont comme ccux des végćtaux, des organes creux, au travers des parois desquels est introduit le fluidc sécrété. Cette disposition cst manifeste chez les insectes; elle ne l'est pas moins chez les mollusques, dont les organes sécrétcur's sont composés par une aggolomćration de vćsicules très-visibles au microscope, et entrc lesquelles rampent les vaisseaux sanguins et les canaux excrétcurs. Les parołs de ces vésicules sont de véritables filtres chimiques, qui, sous l'influence d'un courant électrique, transmettent, en les modifiant, tels ou tcls élémens du fluide nourricier. Le fluidc sécrété est ensuitc expulsé des vésicules, tant par l'cffet dc leur exosmose quie par l'afflux continuel du Guide sécrété détcrminé par l'endosmose. Cette expulsion a lien, dans l'état naturel, vers lcs canaux excréteurs; mais si ces canaux sont obstrućs par accident, l'expulsion a licu vcrs les vaisscaux qui servent à la circulation, d'où résultent certains accidens morbides, dans la plupart des circonstances. Considéréc sous ce point de vue, lá sécrétion devient un phénomène général, auqucl la nutrition cllemême vient se rćunir, du moins sous le point de vue de la production de la substance qui remplit les vésicules élémncntaires des organes. Ainsi, la vésicule nerveuse sécrète la substancc nerveruse qui la remplit; la vésicule musculaire sécrète la substance à laquelle elle doil ses qualités vitales particulièrcs, etc. 
Chacune de ces résiculcs expulse en mème tcmps les substances précćdemment sćcrétées, et ces substances expulsées rctombent dans lcs canaux sanguins, sculs canaux excrétcurs de la sécrétion de nutrition.

Nons avons vu plus haut quc, chcz les végétaux, lcs substances contenucs dans les vésiculcs sont sculcs sounises au mouvcment continuel dc composition et de décomposition; et que les vésicules cllcs - mèmes, ou plus généralemcnt les organes creux qui contienneni les fluides, u'épronvent point de renouvellcment. Ce sont donc csscntiellcment les parties contenantes qui vieillissen, prisquc le mouvcment dc rcmplaccment de nutrition nc s'opèrc que sur les substances rontenues. Nons igrnorons s'il en est de mênc chcz lcs animanx; nuais l'analogie rend cela trc̀s-probable. Le défant de renouvellement des vésicules élémentaires, est trčs - probablement unc des causes de l'état sénile des aninaux. Nous voyons que, chez les végćtaux, les vésiculcs ne soni proprcs que dans lcur jeune agge à la production des liquides qu'clles sont destinécs à contenir. En vieillissant, elles perdent leur propriété première, cn sorte qu'clles demeurcnt vides par l'effet de la dessication, ou bien elles demcurcnt remplics de substances concrètes, cc qui met fin à tout mourcment de remplacement ultćrieur. L'analogie nous indique qu'il cn doit ĉtrc de même chcz les animaux, dont les organes perdent avec l'âtge unc partic de leurs facultés prentičes, ce. qui atteste l'élaboration moins parfaite des substances qui les constituent. On sait, d'aillems, que les organes 
dı renouvellement, eet agent eesse de produire la nervimotion; il y a, pour me servir d'une expression métaphorique, épuisement de la nervimotilité, e'està-dire qu'il n'y a plus de production d'électricité. L'absence de l'agent du dehors, ou le repos, rend à la nervimotilité toute son intensité première, paree que le renouvellement des substances contenues s'est opéré paisiblement dans l'absenee de eet agent nervimoteur, et qu'alor's clles peurent se présenter de nouveau à son aetion, pourvues de toutes les qualités nécessaires pour être modifiées par lui. Je suis loin de songer à présenter celte théorie eomme une déduction rigoureuse des faits; mais on ne pourra, je crois, se dispenser de juger avec noi qu'elle se rattache aux faits d'une manière très-plausible. Il en résulterait que l'agent nerveux serait véritablemnent une manière d'être de l'électricitó; ec qui étaii soupẹonné depuis long-temps par les physioiogisies, surtout depuis que les déeouvertes de Galvani nous ont appris que l'éleetrieité peut remplacer l'action nerveuse pour déterminer la eontraction nuusculaire. Des esprits sévères n'ont voulu voir dans eette aetion de l'électricité, que l'influenee d'un excitant d'une nature partieulière, excitant qni agyssait comme tous les autres, cn déterminant l'exercice de l'aetion nervense, et. non en la remplaẹant. Mais celte manière de voir cesse d'être admissible, depuis qu'il est prouvé que les substances excitantes n'agrissent elles-niêmes qu'au moyen de l'électricité, dont elles déterninent la production. Il est donc démontré, autant que peuvent le faire de 
simples pronves indirectes, que l'agent nerven:i est l'électricité, on l'une de ses modifications.

Nous avons vu plus hatii que, si la végétation est ssuspendue pendant l'hiver, eela provient de ce que ll'abaissement de la température diminne l'intensité de l'état élcetrique prodnetcur de l'endosmose. Les animaux ont aussi besoin d'uu degrré déterminé de chaleur pour cque leur mouventent s'établisse et se maintienne. Ainsi le poulet, dans l'œuf fécondé, n’absorbe les substanees nutritives qui l'environnent, et. ne se développe que lorsqu'il est environné de la tenıpérature assez élevér que lui communique l’incubation de la poule. Les conditions physiques de sa composition sont telles, qu'il faut ce degré de chaleur pour déterminer ehez lui l'état électrique, et par suite l'exercice de l'endosmose, souree de l'absorption et du développement. L'œuf des batraeiens n'a besoin, pour développer le tétard, que de la chaleur cxtérieure qui rèğge ordinairement à l'époque où il est pondu. Les œufs des insectes qui sont pondus avant l'hiver, passent cette saison dans un état statiomaire; et ce n’est qu’au retour de la donce température du printemps, qu'ils donnent le jour aux êtres qu'ils renferment. En ggénélal, tous les animaux ont besoin d'une température particulière, eı différente selon les espèces, ce qui indique que ehez eux l'état électrique a besoin, pour s'établir, d'un degré de température qui n'est pas le inême pour tons.

Les raisons qui m'out porté à penser que les prétendus animalcules infusoires ne sont point des ani- 
maux, mais bien des vésicules dans lesquelles existe un double courant électrique, m'ont persuadé qu'il en est de même des prétendus animalcules spermatiques. Déjà M. de Blainville a émis des doutes trèsfondés sur l'animalité de ces petits corps mouvans : ce qu'il y a de certain, c'est que leurs mouvemens spontanés, et qui simulent presque une natation à la manière des poissons, sont faits pour en imposer, et pour faire croire que ce sont effectivement des animaux. Mais ces petits corps ne sont pas mouvans chez tous les animaux, quoiqu'ils existent constamment dans leur sperme. J'ai examiné au microscope le sperme du cerf-volanı (lucanus cervus); je l'ai trouvé rempli d'une multitude innombrable de globules sans aucun mouvement : il en est de même du sperme des mollusques gastéropodes. Il paraîı donc que ce mouvement tient à des circonstances particulières, lesquelles sont plus ou moins favorables à l'établissement de l'état électrique, source très - probable du mouvement spontané de ces globules vésiculaires.

Les expériences qui ont servi de base à cette théorie nouvelle de la vie, pourront peut-être jeter du jour sur la nature des maladies et sur l'action des médicamens. Ici s'ouvre une vaste carrière que je n'entreprendrai point de parcourir; c'est assez pour moi de l'avoir ouverte. Je me bornerai donc ici à quelques aperçus généraux.

Les expériences qui ont été rapportées plus haut, touchant la propriété que possèdent diverses substances de produire l'endosmose ou l'exosmose, ne 
mous éclairru que très-imparfaiteruent sur le mode id'action qu'excrcent ces substances lorsqu'elles sont introduites dans l'économie aninule. Ces expériences inous apprennent, par exenple, que l'ammoniaque, Ile sulfate de soude et l'alkool sont des agens producthenrs d'endosmose, en vertu de leurs qualités chimimiques. Or, combien l'action de ces substances sur ll'économie animale n'ollie-t-elle pas de dillérences? 'Si, introduites dans l'économie, elles y produisent 'également l'endosmose, il faut convenir que les effets ide cette endosmose sont loin d'avoir de la similitude. L'expérience nous a appris qu'en général les acides :sont des agens producteurs d'exosmose : or, chacun : sait que ces substances, introduites dans l'économie, : sont susceptibles de provoquer l'inflammation ou l’hyIpérendosmose. Comment concilier ces deux qualités copposées? L'hypérendosmose produite par les acides un peu énergiques, proviendrait-elle de ce que ces agens chimiques coagnlent les lluides organiques, et par cónséquent augmentent leur densité? Ceci nous pronve qu'il y a beaucoup à faire pour parvenir à la connaissance de la manière d'agir des substances chimiques introduites dans l'écononic animalc. Il est unc chose qu'il serait extrêmement important de connaître; c'est le genre de modification que l'introduction des liquides chimiques fait épronver aux liquides organiques par leur mélange ou par leur combinaison avec eux. Il me paraît quo c'est là presque tout le secret de l'action des nrédicamens. Les liquides organiqunes, modifiés d'une manière particulière par l'ac- 
cession des liquides chimiques introduits, deviennent, en raison de celte modification, des agens producteurs d'endosmose ou d'exosmose. Enfin, l'action particulière des substances médicamenteuses sur le système nerveux, el la réaction de ce système sur les phénomènes divers de la vie de nutrition des animaux, sont encore des causes de complication pour les phénomènes qui résultent de l'action des médicamens : cela nous fait sentir combien serait importante pour la médecine la connaissance exacte de la statique nerveuse. Ce n'est, je le pense, que par l'étude approfondie des phénomènes électriques, que celte statique pourra être connue.

Nous avons vu, plus hant, que la force médicatrice n'est autre chose que la continuité de l'action qu'exerce la nature vivante dans l'état normal; mais cette action prend, dans la maladie, un surcroît d'activité dont la cause est facile à apercevoir. L'inflammation, ou l'hypérendosnose morbide, en augmentant l'état électrique de l'organe malade, augmente l'action nerveuse générale. Or, le cœur est, en quelque sorte, le nervomètre de l'économie; aussitôt que l'action nerveuse est augmentée par une cause quelconque, telle que l'action musculaire énergique ou une passion violente, le cœur précipite ses mouvemens: il les précipite de même, lorsque cette même action nerveuse est augmentée par l'existence d'une hypérendosmose un peu étendue ou suffisamment intense. Dès lors, la circulation éprouvant un surcroît de rapidité, porte une plus grande quantité de sang aux 
rganes dans un temps donné; d'où il résulte que le mouvement vital de toutes les parties doit devenir plus rapide : l'expulsion des substanees altérées, et leur remplacement, doivent ainsi s'opérer plus promptement dans la partie hypérendosmosée, laquelle rer çoit proportionnellement plus de sanģ que les autres farties, parce qu'elle est devenue un eentre d'adAluxion. C'est ordinairement dans l'espace d'environ vingt jours que s'opère la guérison des inflammations aiguës; ce qui nous indique qu'il faut à peu près ee temps pour le renouvellement des substances contenues dans les vésieules élémentaires des parties molles, et eela avec l'aecélération fébrile. Cette période be renouvellemcnt doit. nécessairement être plus lente dans l'état normal de la cir'eulation; et c'est peut-être a cette cause qu'est duo la durée de la période menstruelle des femmes, période qui est ordinairement d'un mois.

La eonnaissance que nous avons aequise du mécanisme du mouvement vital, nous met à même de déterminer à priori quelles sont les prineipales a]térations que peut éprouver ee mouvement. Dans l'état normal, le mouvement vital eonsiste dans une prédominance nécessaire de l'endosmose sur' l'exosmose, prédominance de laquelle résulte l'élat turgide normal des parties. Le degré de cette prédominance est fixe et déterniné, par la nature partieulière de l'être vivant. L'intensité de l'endosmose peut être augmentée; elle pent aussi être diminnée; enfin, l'exosmose augmentée peut devenil prédomi- 


\section{ERRATA.}

Pag. 24, lig. 14, les tubercules, lisez les tuhercules (n sola num tuberosum.

3 个, note, 1825 , lisez 1905 .

87,30 , trouvant, lisez trouveut.

$9^{3}, \quad 25$, il fait, lisez elle fait. 


\title{
GATALOGUR
}

\section{DES LIVRES DE MEDECINE}

\author{
QUI SE TROUVENT
}

\section{A jutits,}

\section{CHEZ J."B. BAILEIIET,}

LIBRAIRE DE L'ACADÉME ROYALE DE MUEDECNE,

ET DU COLLÉGE MOYA DES CHIRURGIENS DE LONDRES, Rue de l'École-de-Nédecine, $\mathrm{n}^{\circ} \mathrm{1} 3$ bis;

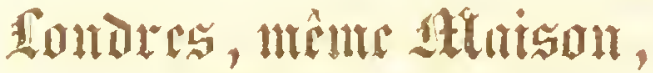

5 Bedford street, Bedford square.

\section{A sintutles,}

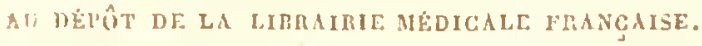

\section{Tooventie 1829 .}

P. S. J'adresscrai firanco le Caialoguc général de mes ivres de médecine français el ćtranygers aux personnes qui m'en feront la deminude par lettres affranchies. 


\section{ANATOMIE}

\section{P T THOLOGIOUE}

\section{DU CORPS HUMAIN,}

o v

\section{DESCRITTIONS AVEG FIGURES IITUGRARIIESS}

ET COLORIÉES DIES DIVERSES ALTÉlLATONS MORUIDI:S

DONT LE CORPS HUMA:N EST SUSCEPTIHLE;

\section{TAI! J. CRUVELLHER,}

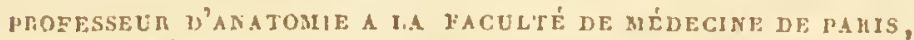
MÉMACIN DE LA MATSON TIUYALE DE SANTÉ,

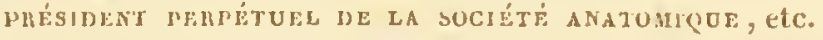

\section{Ies livraisons $1,2,3,4,5$ et 6 sont en vente.}

(Novembre 1820.)

\section{CONDITIONS DE IAA SOUSCRIPTION.}

Cel oumage sera pullié er fo livrnisens; rhacune contiendra six planclies dont plusieu:'s coloriées avee le plus grand soin, et an moins 6 fenilles de text in-fol. Grand-raista welin, carneiere neuf de 1 . Dielot. - Les livraisons se suivron résulièr'ment de six semaines en six semaines.

I'impossibilité de rendre en noir un stand nombre dialtérations nous forc d'avoir recours aux couleurs pour la pius sirande partic des planches qui eompro soront cet ouvrage;

Je prix de chaque livraison est de I I francs.

A la fin de l'onwrage on publiera! la liste des souseripteurs. - Tes dessins a la lithosiaphie sont confiés it MIM. A. Chasal ei J. G. Martin, exercés d. puis Longr-tersps a peindre l'anatomie, et qui, sous ce rapport, ont déjáz rendu tan le services $a$ la science.

Personne ne peut rivoquer ex dontel'utiliti des planclies d'anntomie pathologique. Ini l'ocension rst fug

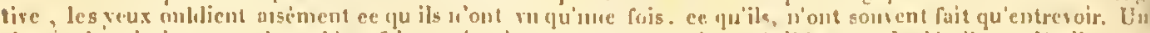

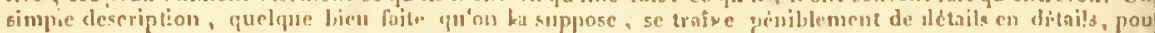

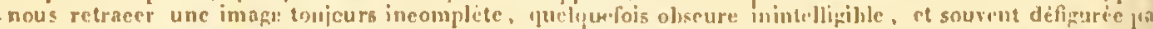

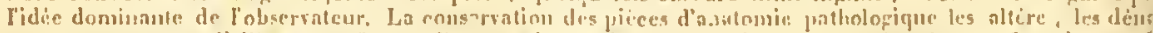

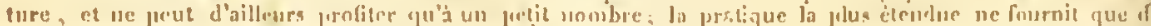

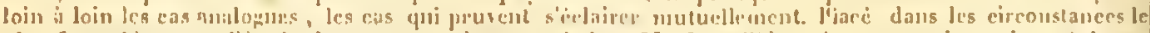

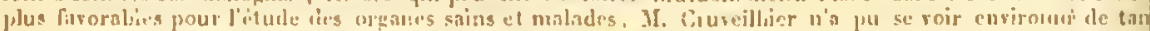

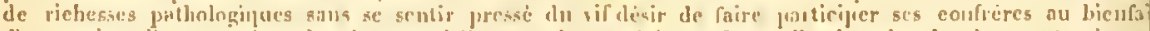

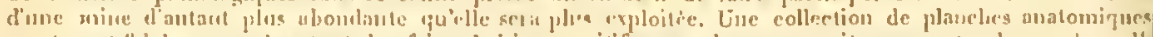

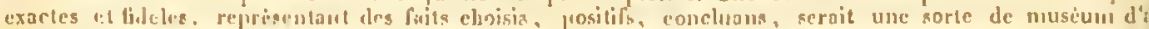

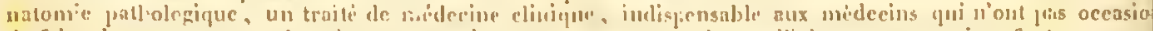

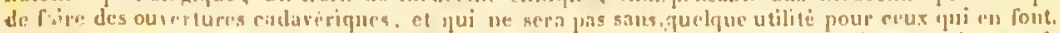

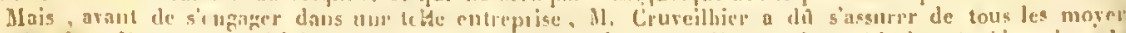

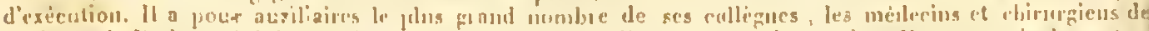

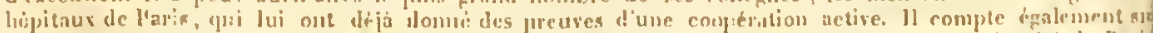

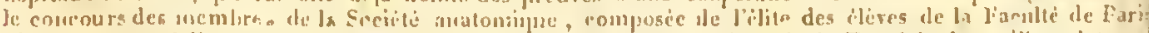

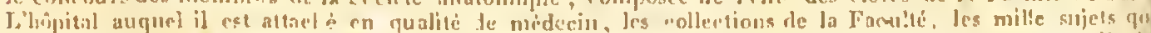

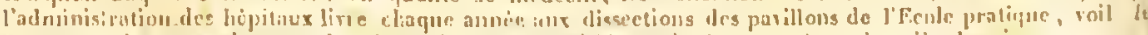

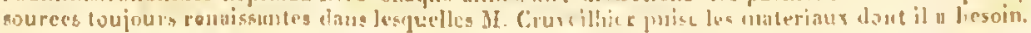




\section{DICTIONNAIRE}

D E

\section{MEDECINE ET DE CHIRURGIE}

\section{PRATIQUES;:}

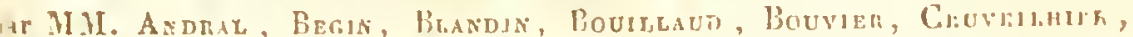

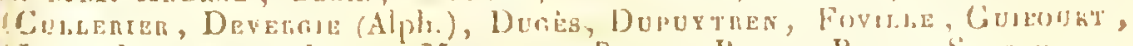
Jobly, Lallemo, Loxde, Magride, Ratien, Riyeli, Ruche, Saxun.

\section{5 volumes in-s".}

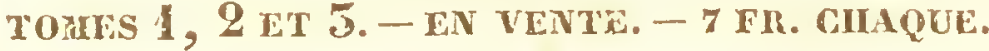

\section{CONDITIONS DE LA SOUSCRIPTION.}

Le Dictionnaire de Médecine et de Chirurgie pratiques sera romoséde 15 volumes, de 550 à (joo parges, caractiove petil rominin euf de H. Didol; 4 a lignes is lo pare. Les notes bibliographliques zront en petit-lexte. Par ce moyen, ce Dirlionnaire aura sur les -ulres l'avaizlage de contenir autanl de mationes, en moins de olumes, el d'offir. en plus les moles bibliographiques done la

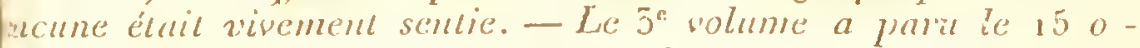

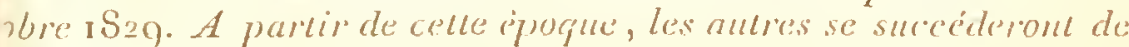
"ois mois en trois mois. - Le prix de rhaque volume sera de 7 fi., ¿ Sraile de port, par la poste, a fr.-Les édiceurs juennenl lenagement de liverer gratis, aux: souscripueurs, tous les volumes ui dépasseraiene le nombrequinze.

Afin de donner toutes les garauties desirables pour la pullication de cet ouvrage, il a ét:

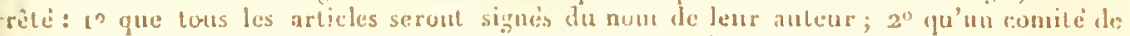

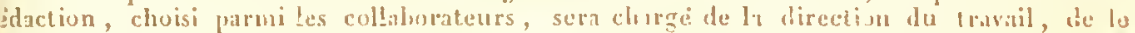
vision des articles, et de veiller a ce pu'il ne sy glisse ni ousission ui doulle emploi, ' enfin, qu'il ne serit aljomt aux collaloorateurs actu els nucnste ante personne sans uns

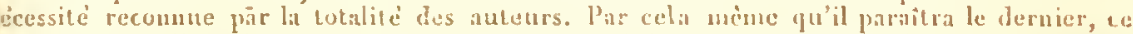
ictonnaire offira le precieux avantige de reproduire avec plaz de fielelifé que les juéceans l'etat présent de lis seicnce; mais toutes sts forlites scront emprembes de cot esprit

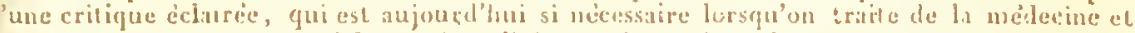
ta chirurgie pratiques. L'observation clinique, l. pratique des grands mattres, les expe-

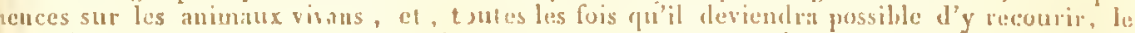

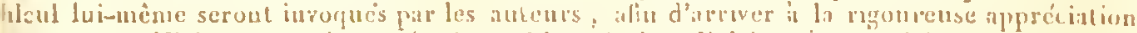

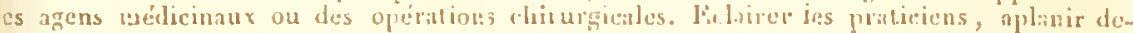

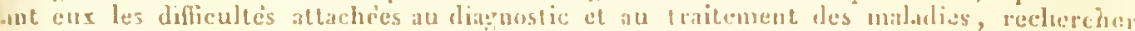
e qu'il y a de bou, de douteux, d'errone, ou de frutriare, dans les corseils qu'un ne

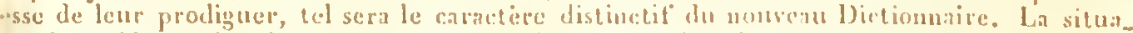

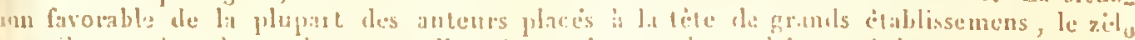

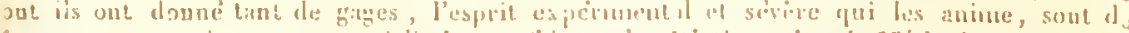

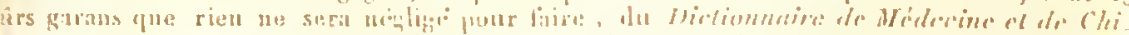

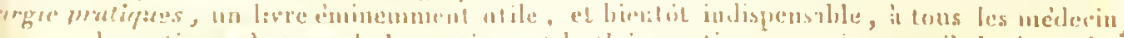

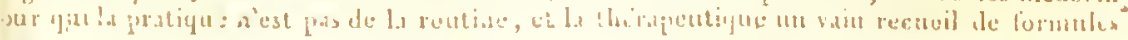




\title{
DICTIONNAIRE
}

\section{UNIVER SEL}

\section{DE MATIERE MEDICALE}

\author{
E T D E
}

\section{THÉRAPEUTIQUE GÉNÉRALE;}

CONTENATT I'INDICATION, LA DESCRTPT!ON F:T L'EMPLOI DE TOUS LAS MÉDJCAMENS CONNUS DATS LES DIVRRSE PAYTIES JUU GLOBE;

\section{PAT F. V. MERAT,}

Decleur en medechic de la Faculté de Paris, menubre de l'Acadén'e ropale de Médecine, cte., cte.

\section{ET A. J. DELENS,}

Docteur ell miderine de la Faculli de Paris. Inepectiur-qenéral des Etudes, Mlombre titulaire de l'scadenic royalo de Sledecinc, etc., etc.

6 VOLUMES IN-8n. - TOME Ier EN ITNTE. - PRIX: 7 FR.

\section{CONDITIONS DE LA SOUSCTHTION.}

Le Dictionnaire unisersel de Iratière médicale et de Thérapentique géntirale sera composi de six vol. int-So de 600 à joc pages, caractèr gaillarde neuf, 42 ' lignes à la page.-Le premier volume a paru le 20 Juillet 1820 . A partir de celle épogue, les autres se succéderont de 4 en 4 mois.

Le prix de chaque volume est de $7 \mathrm{fr}$, el firanc de port par la poste do $9 \mathrm{fi}$. Ala publication du $2^{\circ}$ uolume le prix de chaque volume sera. pour les non-sonscripleurs, de $\$ \mathrm{fi}$. et franco ro fr.

Les ciditeurs prement l'engragenent de livrer gratis anx sonscripteur: les volunes mit dépasseraient le nombre de six. Il sera public, arec le dernier volume. In liste des souscripteurs.

Pour donner une idce ciu radre immensa que Jes auteurs r.e ce Dictionnaire ont cumıasse fruit de dix aunces de recherclics, il wous suffic d'indig̨ter que, selou l'inportance du sujet l'histoire de charine meiticnment comprendra tous les articles du tableau ci-après :

o Noms Linuéen, official, commercial, rulgaire, ancian et noderne; défuition.

20 Décomerte; historique; eisement on lieu natal; extrartion nu récolte; ítal connercial ; especes, variótés, cortes, pululités.

30 Descriptinn phnrmacologique; clınix; preparaion pharmaceutique; alténtions, sophistications, substitutions.

$4^{\circ}$ Analyse chimique.

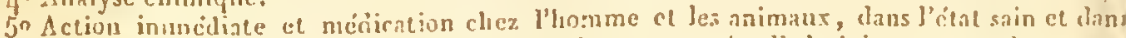

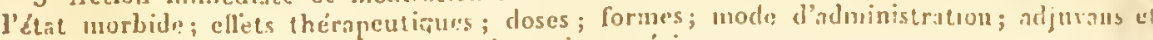
colrectifs, indications et rontre-indications; incouvéniens.

60 Opinions direrses des autenrs ; classifieation.

-o Combinaisons; mólanges; compnsés phoronaceutiģues.

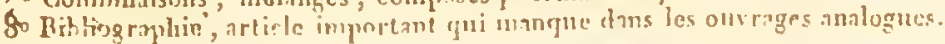




\section{TRAITE}

DES

\section{CHAMPIGNONS,}

OUVRAGE CONTENAMT

I'llistoire analytique el chronologique des découvertes et des travanx sur ces plantes, leur synonymie botanique et les tables nécessaires; la description détailléc, les qualités, les'effets, les différens usagres, non-seulement des Champipinons proprement dits, mais des Truffes, des Agarics, des Morilles, etc, avec une suite d'expériences sur les animaux, l'examen des principes pernicienx de certaines espèces, et les moyens de prévenir leurs effets ou d'y remédicr;

\section{par J.-J. PAUET, D. M. P.}

Correspondant de l'Institut royal de France, Médeciu du châtcou royal de Fontaucblea et de l'hópítal civil el nilitaire de là néme ville, membre d'm grand nombre de Socićtés savantes.

\section{HORTS VOLUMES IN-4', AVEC 30 EXVRAISONS DE PLANCHES,}

Composces ensemhle de 1 zo Planelies, gravées et colorices avce le plus gram? soin, et olfrant plus de 500 espéces de Champignons de gamdene et de couleur nitureities.

Quoique le Traitedes Champignons fit imprimé et pulylis depuis long-temps, devenait claque jour plus diflieile de se le proncurer. Nous eroyous done fair., re choss utile en annongant gue nous sommes ell possession du petit nombre exemplaires qui se sont irouves cliez, l'aulear npre's sil mort.

Le prix de eet onvrage, 2 vol. in $-4^{\circ}$, avec 30 livrasons contenant 1 go planelıes

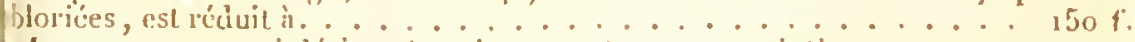
Les persomnes gai désireraient le recevoir en sonscription, pourrout refires. cux livraisons de planclies par mois, an prix de 5 fr. cliague. - I e premice

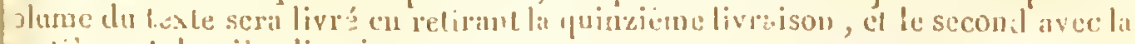
enticme et deruicue livinison.

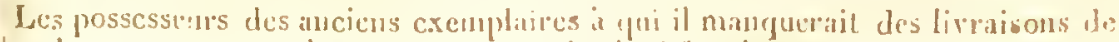

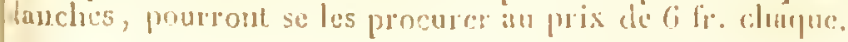


ANATOMIE PATHOLOGIQUE, DERNIER COURS DE Xav. BICHA' d'après un manuscrit autograplic de l'. A. BÉchand avec unc notice sur la ric, et les travaux de Brchat; par İ. G. Borsseav, D. M. P. membre des acadéunits royales de nédecine de Paris et ale Madrid, de la société nédicale d'ému. lition, etc. , ctc. Paris, 1825 , in-8., prortrait ct fac-simize......... $5 \mathrm{f}$.

Dans cet ouvrage les principales maladies He claruue tissu, de chaque organc, sont énn raéces ee

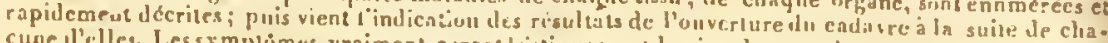
chac lelles. Les "y mplómes vraiment caraeteristiques, et le signalement des traces apries la mort, paraissent avoir dté be but que Bı̈

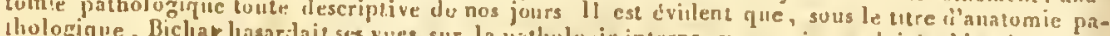
lhologique, Biclıa lıasardait ses vues sur la pathulogie interne, vues qui, exploités biontól par ses ct du sićce des manilics. du sicge des minailics.

- La notiec de N. Boisseau relrace en peu de nots les serviecs rendus h la seienre par Bichat, slle est eerile avec cet enthousiasme qu'on lui reconnat toules les fous qu'il s'agit de ce, grand.

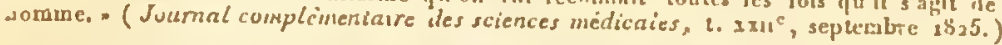

ANATOMIE DU CERVEAU , confenant. l'histoire de son développenent dans le foetus, avec une exposition comparative de sa structure dans les animanx. par Fr. Thedeans, prolesseur à. I'miversité de Heidelberg; traduite de l'al'lemand; avec un discours préliminairc sur l'étude de la physiologic eı générill, et sur celle de l'action du cervean en particulier; par A.-J.-L. Joundas. Paris, 1823, r vol. in-8. avec 4 plancines, br................ $\mathrm{f}$.

- M. Tiedemaun est, parmi les anatomistes de nos jours, un de ccux quj ont le mieux étal,li que l'anatomie comparée peut seule devciler le faitsi curicux de la maltipuicatien grauluelle des urganes, de leur déveluppemeut, de leur complieation sucessive, et du degre de leur importance relative pour l'entretien de la vie. Ces descriptieus nous out paru fort claires et les ficurce soignées en faeilitent encore l'intelligrnce; nous pensons que ce tuavail est un des plug renuarquables qai aicnt paru depuis long-tenps.o (Journal universel des sciences médicales, juio, 1823. )

\section{MICHERCHES EXPERIMENTALES, PHYSIULOGIQUES ET CHIMT-} QUES, SUR LA DIGESTION; considéréc dims les quatre classes d'amimaux vertébrés; par F. Theneman et L. Gaelix, professeurs à l'universilć de Hcidulberg; traduites de l'allemand pir A.-J.-L. Jounods, D.-M.-P'., Merubre de l'Académic royale de nédecine, ctc. Paris, $1827 ; 2$ vol. in-8., ivec siand 1.ombre de tableaux............................ is fi.

Cet oubrage, résıftat de plusieurs années d'espériences pénibles sar les diverses classes d'animaux, se recommante $\dot{n}$ tous les plyssiolopisto-s; c'est par des fails nombrenx et bien observes que les auteurs de cet ouvrage sont parvenus a faire mienx connaitse les phenoménes si importuns de la digestion; il ne s'apir pas ici de thenries fruits de l'imagrination. nunis d'expériences que tous les pliysiologistes qui aiment la vérité sont lorés de reconuaitre c:t je répéter.

CLESE (A.C.): TRAJTÉ DE LA MÉDECINE eu VII lives, landuction nonvelle par MMT. Fougunen, professenr de la fitculté de médecine de

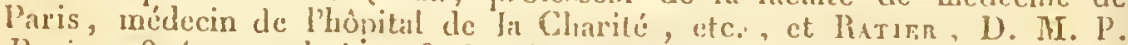
?'aris, i\$24, I vol. in -18 de 550 pages, imprimo sur papier tin, par E. Didot................................. 50 c.

(CESI ( A. C.) : DE RE MED[r:A LIP]RI OC'TO, edilio novit, curantibus '. Fouporen, ill saluberrima facultate parisiensi professore, el E.-S. Raten, 1). MI Parisies, $1 \$ 23,1$ vol. in-1S, iuprimé sur papier fin des Vosges, par

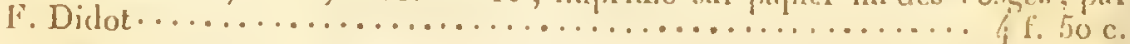

- Le même, pilpier vélia.......................... $\delta f$.

Il n'est pas de mederin qlii puisse se lispenset. llavoir dans se bibliullequne l'nuvrage de Celse, l'un des anteurs de l'antiquié chez lequel on Irouve le plus de comuaissonces posisives sur l'nrt de guérir, jnintes a un style sussi pur gu'slégant, qui l'a fait placer par les philoluanes au

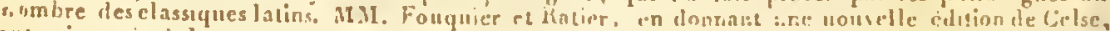

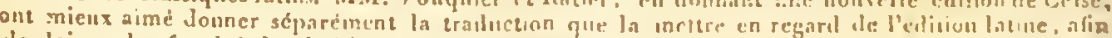
Ile haisser la faeulté de rhoisir : toutefois ees deus volunes, du urine format, juiguent au snérite d'unc correction parfaite colui diule exécution ty fogroplique trés-soiguéc.

CODEX MEDICAMENTAFIUS, sive l'harmacopar zallira, jussu regis op-

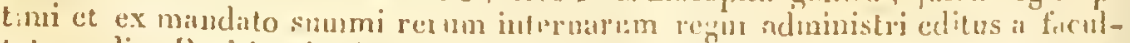

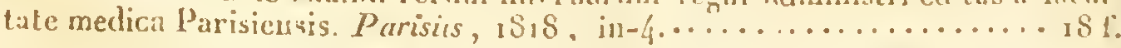

COUIS COMPLET DES MAIADIES ULS YEUX, suivi d'un prisis d'hy -

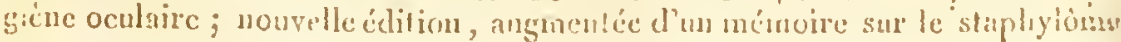


assez positires, asace supéricurce à celles du rutgaire pour qu'ils puisseut à ooup sar reconnaitre o distinguer de l'état normal cet etat irrégubor st extraordinaire?

Cos questlons sont traitées arec le dociblo oaractè je du talent et de la probite. Il y a dans le lirre de II. E. llegrault des eritiques qui frappeut justo et fort, des argumens dont les doctrines médicales iu peusent démoutrer la fausseté, et des conscils dout les méjeoing pourront faire leur profi. Toutes le personnes qni possitent les nuvrages de Georget doirent so procurer celui de M. E. Reguault, ayau examiné sous deux les nimanes questions arec des résultats diffírens.

- Séparément. Nouperaes Réfresions sur le degrẻ de compétence des médecine dans les questicus judiciaires, sur le suicide, et:. Paris, 1829 , in-8. 2 f. $50 \mathrm{c}$

DE LA SANTÉ TES GENS DE LETTRES, par Tissot; avec une notice sus ' la vicade l'isuteur, et des notes, par F.G. Bolsseau. Paris, 1826 , a volume

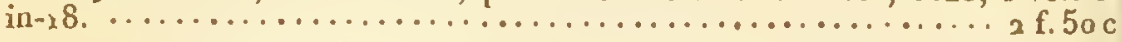

Ce petit onvraze manquait depnis long-tcreps dans le comorerce; nous pensons avoir fait un ehose utila en le réimprimaot. Les notes quo M. lo doeteur Psisseau y a ajoutde le renilent encorc plus intéressant : 'aussi nous nc doutons pas qu'il ne soit bien aceneilli des médecins ct de gens de letlecs, nuxquels il est spícialezoent, destine.

DE LA JARALYSIE, CONSIDÉRÉE CHEZ LES ALIÉNÉS, Recherche faites dans le service et sous les y cux do MM. Royer-Collard et Esquirol par L. F. Canmeil, D. M. P., médecin à la Maison royale des a'ienés de Charcnton. Paris, 1826 , in $-8 . \ldots \ldots \ldots \ldots \ldots \ldots \ldots \ldots \ldots \ldots$ fr. 50 c

- Résul tat de huít années l'oLservations faiies anx cliniques de la Sal pétriẹre et de la maison royal alc Charentca MI. Calmeil a fait une étule spéciale de ce genrc de malndie sur laquelle on n'avai que des idés confuses; son ouvrage, riche d'un gränd nombre d'observations pathologiques, doi fixer l'attention dans uu momens oil la pathologic du cerrcau est devenue l'objet d'une etode spé eiale.

DES CAUSES MORALES ET PHYSIQUES DES MALADIES MEN. TALFS, et de quelques autres affections nerycuses telles que l'hystéric, la nymphomanie et le satyriasis; par F. Vorsin, D. M. 1. , directeur de lí Miaison d'Aíićnés de Vanvies près laris, membre de plusicurs sociétés sa-

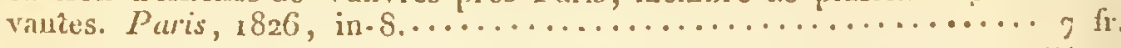

Denscet ouvrage M. Voısın exnınine quelle est l'infuence de l'éjucatisn, des institiztions politique religieuses, du fanatisme et de la superstition, des mreurs, les pis,lessivus, des áges et des sexes, d l'béré.lité, et généralemeut de teuces les passions qui peusea aliérer les facultés intellectuelle tant al6 woral qu'nu physinjue. Son livere est anssi bien esrit que bien pensé, il sera lu par I meilecin et le philosopbe, lc nagistrat et l'bomme du monde, tous y puisernat clés eonscils utiles.

DE LA LITHO'TRITIE, ou Lroienent de la pierre dans la vessic, par le doc. teur Civiale. Puris, 1827 , 1 vol. in-8, avec sept planches........ $7 \mathrm{f}$

LETTRES SUR LA LITHOTRITIE, ou Broicnent de la pierre dans la vessic, pour servir de suite et de complément ìl'ouvrage précédent, par le dorteur

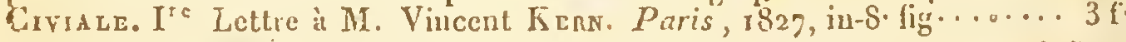

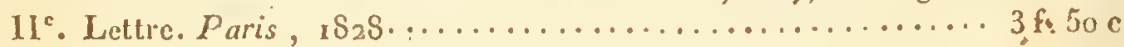
En $1836 \mathrm{ct}: 327, l^{\prime}$ astitut royal de France a récompensé $\mathrm{M}$. Cirule, pour le grand nombre l'u pérations qu'if a faites sur lr virant, et pour les beaux surcès qu'il a obteuus. C'est pont répondre a un suffragc aussi honorable que M. Civiatel a publié son premier ouvrage; et dan ses $L_{C}$ itresil indique les diverses modifications que see nombreuses obscrvations lui ont suggértes

DE LA I'ERCUSSION MEDIA'TE, et des signes obtenus '̇aide de ce nouveat moyen d'exploration, dans les maladies des organes thoraciques ct, abdominans par P.-A. Ṕomax, D. M. P. agrésé a la Faculté dic Médecine de Paris, mé deciu du Bıreau central des Hốpitaux, membre de l'A eadémie royale de Médes. cine, etc. Paris, 1828 , 1 vol. in-8, avec deux planches.......... 6 f

Linstitut royd de Franec vicnt d'accorder un prix i M. Piorry pour les arantares qui doiren résulter, pour le diagnostic des maladies de poitrinc, des nodifications qu'il a apportécs daus l'einplo de la percussion médiate.

DE L'ANATOUL PATHOLOGIQUE CONSIDEREE DANS SES VRAIS RAPPORTS, nvee li science des maladics; par. M. Rines, professeur de li

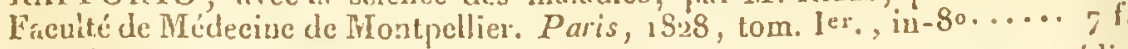

DE L'ÉTAT PlÉSENT DES IIOMMES, cossidérés sous le rapport médical ; par Luforit-Gouzr, méuecin du'Collégo royal de Toulouse, membred, p!usicurs Sociétés sivantes. Paris, $1 \$_{27}$, int $\$ . \cdots$

DES MALADIRS PROMUSS AUX. FEMMES, par M. Nachr, mériecin de 
la Société maternelle, et de l'Institution des Jeunes Aveugles, membre de piusieurs Sociétés savantes. Paris, 1829 , a rol. in-8, fig.... 10 f. 50 c.

Dana re nourel onrrage, M. Naurly a refondu, arce des additious, celui publió en 1916 sur les maladieg

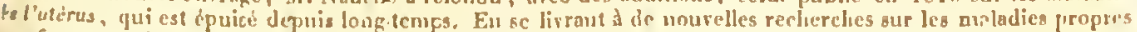
ux femmes, lauteur a réuni aux nonbreax faits que lui a feurri unc pratique tendue, ceux recurillis pur

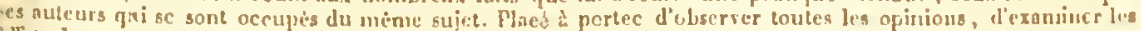
ISets de toutes les miblicdes euratises, il a alopte tout ce qui lui s paru vrai, conmure aussi il a rejete tout ¿qui ne lui a pas paru conforme á une expérience iehirie; parmi les nualadies 'ju il a díerites, il eu est peu lont il n'ait stovi les traitemens qu'il indique, il rapportc les circonstances daus lesquelles l'ant a cic utile ans erninure de citer cclles oủ il a èté inpuissiut.

OES DIVERSES MERHODES DEXPLORATIUN DE LA POI'TRINE E'T DE I.ECR AIPLICATION AU DIAGNOSTIC DE SES MALADIES, par V. Corlis , doeteur en médecine de la faeulté de Paris, interne des hópitaux eivils de la mêtme ville; deuxiène dition, eonsirlirablement augmencéc. Parls,

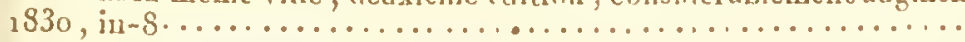

- Apres avoir examiné les monvemens respirntnires dans l'état sain et dnns l'étal morbide. l'au.

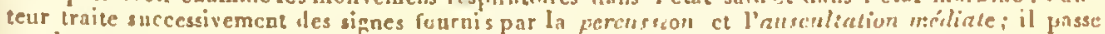
ensuite en revue tontes les malndies thoraciques, et cherche it les distinguer les une's cles autres. en assignant i elacune ses earacteres propres. Ce petit ouvrage pent itre coussderéc comme un

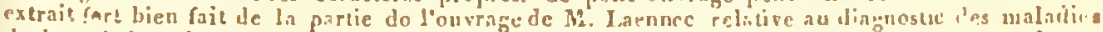
de la poitrine. Il scra tries-utile ans fleves qui suivent les conrs de elinique, at aus unedecins qui veulent se familiariser avec l'cmploi du stéthascupe et du plessinetre. "(Archives généralcs (ie médecine.)

DEE LINELAMMATION DES VAISSEAUX ABSORBATS, LYMPILATIQUES, DERMIOIDES ET SUUS-CUTANIS, maladic désinnée par les auteurs sous les différens noms d'éléphantiasis des Arnbes, d'oedème dur de hernie charnue, de maladic s!andulaire de Barbade, ete., avee quatic planches en taille-douee, représentant les diverses formes, ete.; par M. Aland, D. M. P. , membre de l'readémic royale de médecine, médecin de la maison royale de Saint-Denis, ete, deuxième éltition. Paris, 1824 , in-8..... G $\mathrm{f}^{\circ}$.

- Dans ect nuvrawe, M. Aroso strit l'inflammation des lymphatiques sous toutes les formes qu'ello pent revetir: il sumleve niec une rare saparite fes voiles eni la consrent daus ses divers dégui-

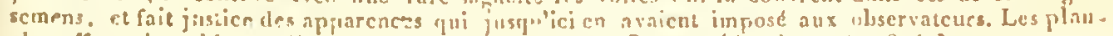

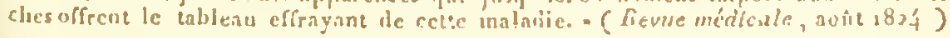

DU SIEGE ET' JEE LA NATYIRE DES MALADIES, OU Nowrules considérations touehant la véritable action du systéme alisnobant dans les plánomènes del'écononie animale; par M. A Asnn, memlire de l'Aeadémic royale de médecine, médecin de la maison rovale de Saint. Denis, te. Paris, 182:;

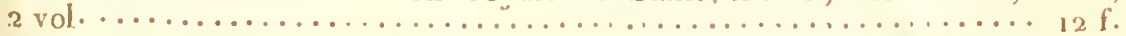

DU MAGNETISME ANTMAL EN FRANC W DES JUGEMENS QU'PN ONT PORTÉS LES SUCIÉTES SAVANTES, nvec le textedes divers rapports fait en 1784 pal les commissaires de l'Académie des Sciences de lit l'aeulté et de la buciété royale de Iriédecine, et me analyse des der -

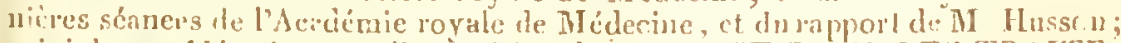
suivi de considérations sur l'appurition de l'EXTSE DANS LES TRAITEMLNS MLGNETIQUES, par Al, Bertraxis. docteur en médecine de la Feulté de Paris, anien éleve de l'Weole Polytechuirgue, ete. Paris, $18_{2} 6$,

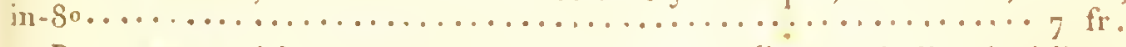

Dans un nenment oil Ie unagnétime nuimal est devesul l'olsict n'une grände diseussion ha l'Aca.

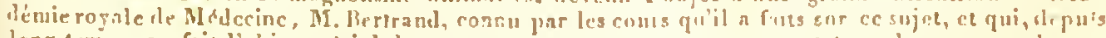

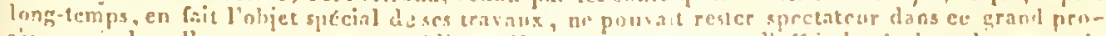

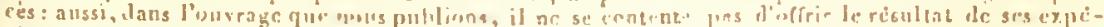

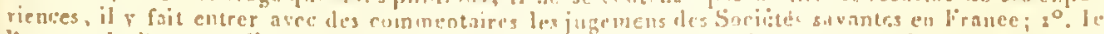

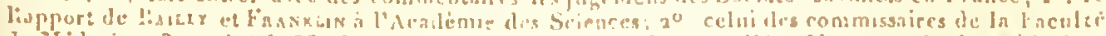

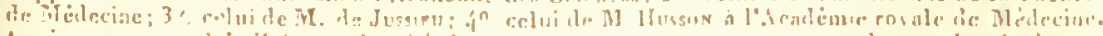

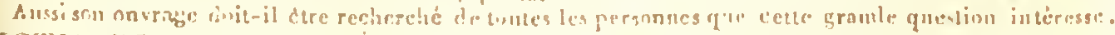

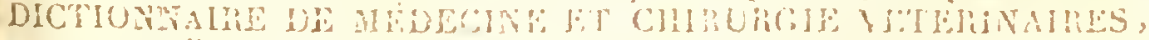

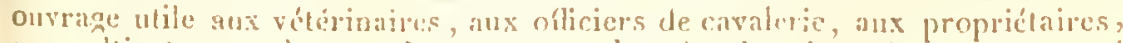

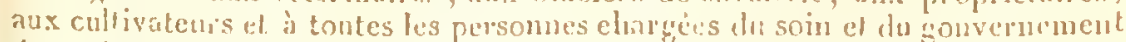

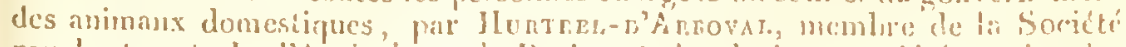

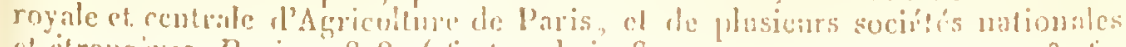

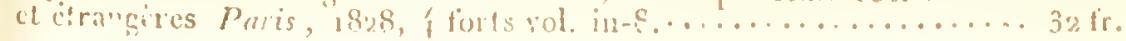


Depuis lung-ternps on iprnutvait genéralement le besoin thun liver daus lequel toutes les no-

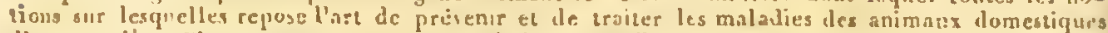

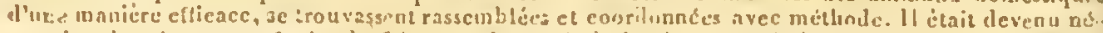

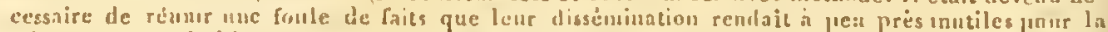

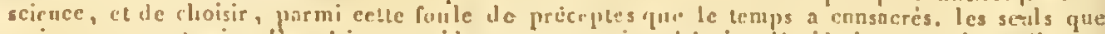

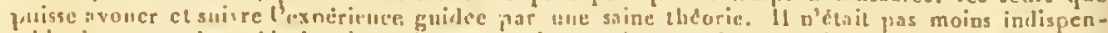

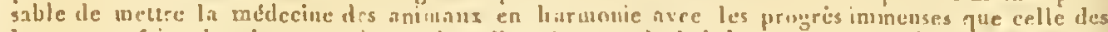
linmmes a frits depuis inı petit nombre d'anues, et de lui douner, cacame a eetle derniere.

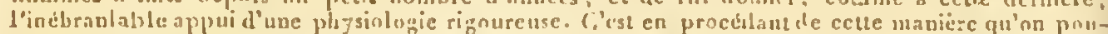

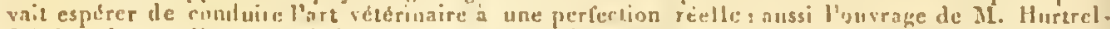
dArhoval peut-il être enosidéré comune un véribble traté de méllecinte el de ebururgite couparécs qui sera aussi asilc zux médecins qu'aux vétéribaires.

AYATOMIE CHIRURGICALE DES PRINCHAUX ANIMAUX DOMES-

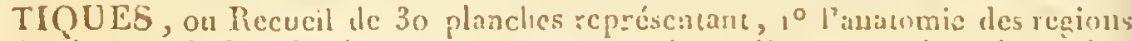
ducheral, du beuf, dit monon, ete. snr lesquelles on pratique les ciperin:ions les plus graves: $2^{0}$ les divers cetats iles deürs da elieval, du boent, da mouton, du chien, indiqnaut l’àge de res abimanx; 30 les insirnmens rhe chirurgie vétérinaire; $3^{\circ}$ nn tcxte cxplicatif; par U. Lencasc, mélccin vétciuaire, incien répétitenr à l'École roýlle vélérinajre d'Alfort; et A. Troessenc, docteur en medecine, agregé a la Faculte de Paris, professenr d'anatomie et de physiologic pathologígues comparécs. Atlas pour servir de suite et de compli:ment an Diclionnaire de né́lecine et de chirurgie vétérinaires ; par M. Hon-

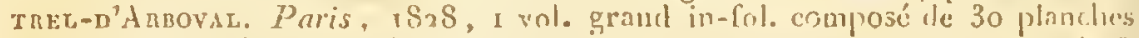

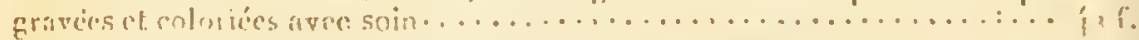

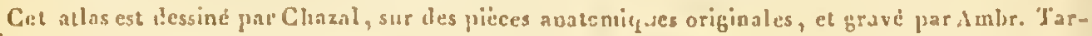
dicu.

DICTIONNAIR DES TERMES DE MEDECINE, CHIUURGIE, ART VÉTÉRINAIRE, PHARMACIE, HSTOIRE NATURELLY.BOTANIQUE, PHYSIQUE, CHIMLE, ele. , par MII. Bégis, Boisseau, Juen-

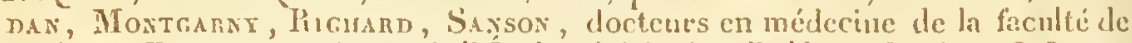
Paris, et Dorer, professeur à l'École vétérinaire d'Alfort. Paris, IS23. I v.

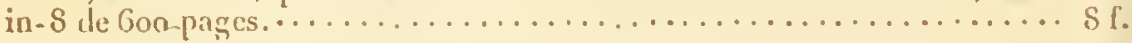

* Plus enmplet que celui ie Trsters, ce dictionnaire présente les mots avee leur étrmulogic r:

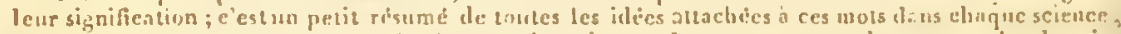
ct parce moyen un enurt résuns ile chacoune des scicuces dont on rapporte les terines; la dectrine physiningitue y trouve une bonne erplication de ces mots. 'Tontrs les comaissances chimique's s'y rencontrent, ics termes de l'art véteriwaire y sont au complet, entin l'auvrage est figalensent rn. richides termes de physique, d'histote naturelle ct de butauique. - (Annales de le méstecine ply)siologigue par Broussais, octobre, 1823.)

DISSERTATION SUR LES ANEVRYSMES DE L'AORTE, Pa G. NovFRE, docteur en midecine de la ficulté de l'aris. Paris, 1820 , in-\$... I f. 50 c.

ELENENS DE PIILOSOPIIE CHIMICUE, par II. D.rr, professcur de chimic a l'Instituition royale Backerienne, ututeur des Elcimens de Chimic agrienle ; trad. de l'anel. , aver: des additions, par Van-NIons, conespondant de

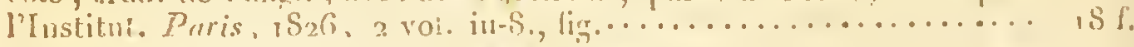

Le uom de Durr est connu depuis long-tenups; il necupe une place distinguée parmi les pre-

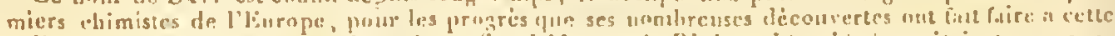

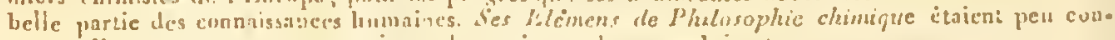
nus en liranec; nüs crogons avnir rendu service en les reprouluisaut.

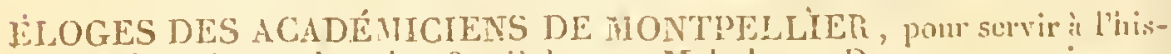
toire des scieuces dans le ise siecic, pur M. le biron Descenettias, insprecteur-génédel da service de sinité des armées, professem de la fitculté cle múde-

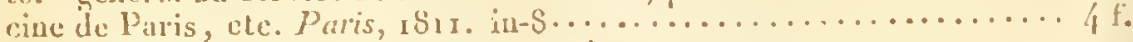

ESSAI SUR LA FIEVRE JAUNE D'AHẺliCUE, on Considérations sur les svmptòncs, la nature et le tritement (ie cette maladic; aves l'lisioire de l'épidémie de la Nonve!le-Orléans, en 1832 , et le rúsultiti de nonvelles lecener-

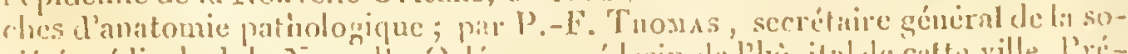

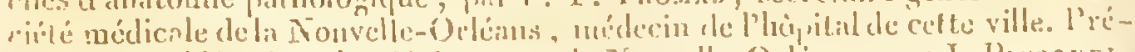

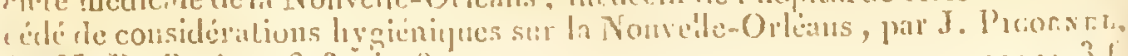
1). AI. '?. Paris, 1823 , iu-S. 


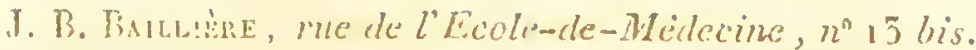

HESSAI DE CLOLOGIE, m MÉmoires pour servir is l'histoire nat melle du

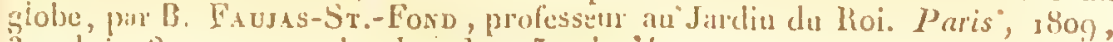
3 vol. in-8, arce 29 planches dont 5 colorićes................ 2i f.

PESAI PIYSIOLOGICO-PATHOLOGIOUE SUR LA NATURE DE LA FIEVRE, DE L'INELAMMATION ET DES PRINCIIALES NEVRO-

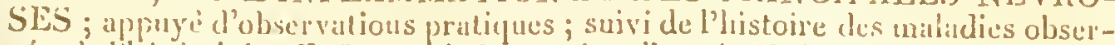
vées ì l'hôpital des Enfans rualeades peuclant l'umcée 1818 ; Mémoire conronné par lit lirculté de mídecine de Paris, le q novemalue 1821 ; par Ant. Dogís, 1). MI. P., professeur de la faculté de médecine de Montpellier, elc. Paris,

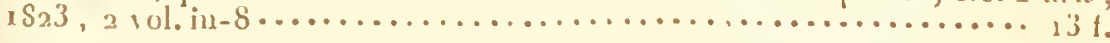

- Lonicur de ret ourrage semble nvuir eu pnur but de concilier les dnctrines les pla

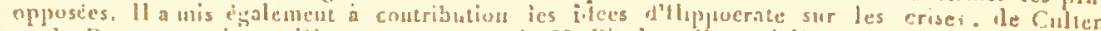

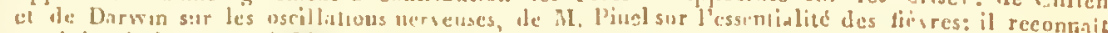
aussi dervir betacoup a M. Broussis et a l'ecole des contre-stimulistes. M1. Dugés es! done IIu

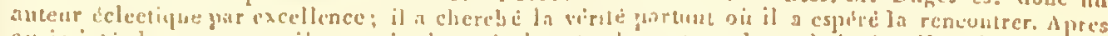

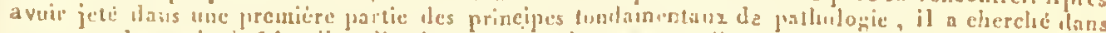

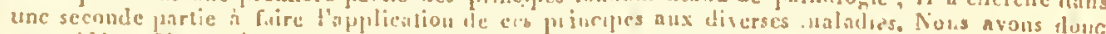

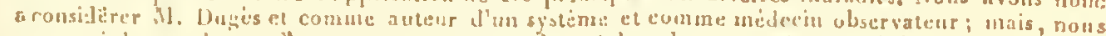

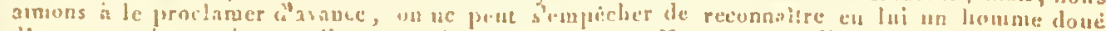

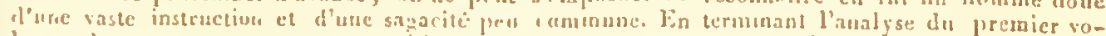

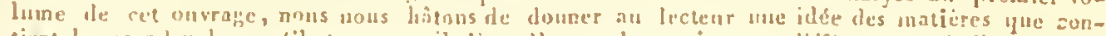

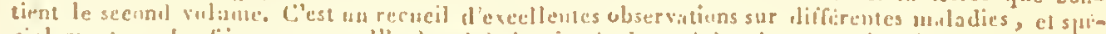

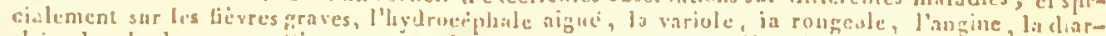

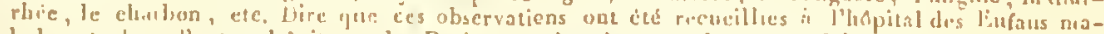

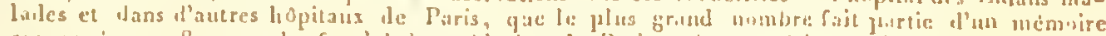

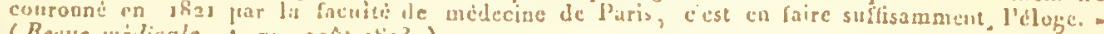

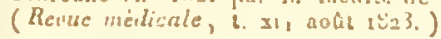

DE L'TNFIUENCE DESS SCIENCES MEDICALES FT ACCESSOIRES SUR LES !RUGRES DE lA CHIRURGIE MODERNE, PAR A.1.

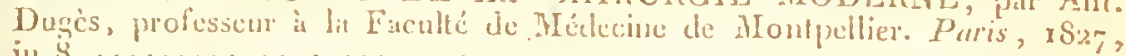

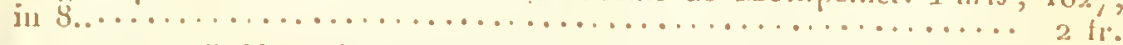

Dans re trarail, M. Duges a ruulu faire sentir la linison indiae qui existe ente les diverses

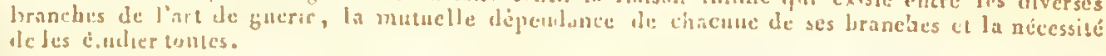

WLEN NE INTER ASCITLII ET IERITONITIDE: CHRGNICAM CERTA DISCRIMTNA QUIBUS DLAGNOSCI QULANT; auct. ANT.

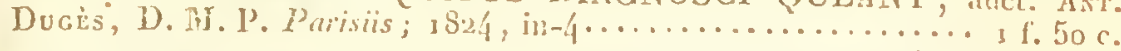

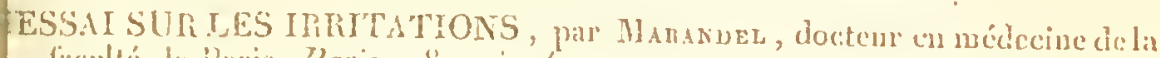

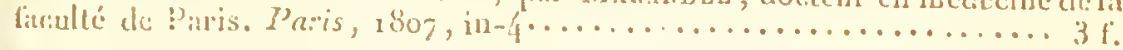

ESSAISUR LES MALADTES DE LOOHELLEINTERTE; par J.-A. S.MSSY,

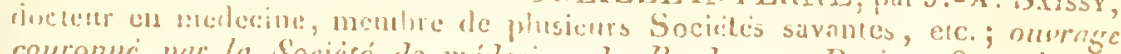
couronnci pur ln Société de médecine de Jordean.r. Partis, 1827, in-s, big................................. $50 . \ldots$ c.

ÉTUDES CLINIQUES SUR LES EMISSIONS SANGUINES ARTIEI-

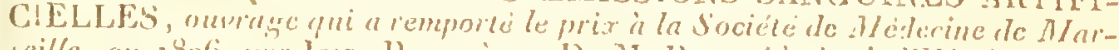

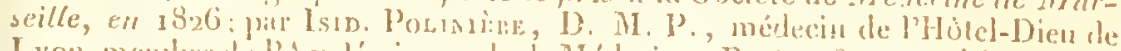

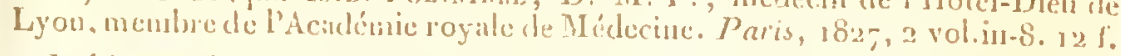

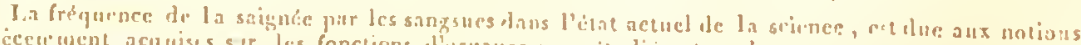

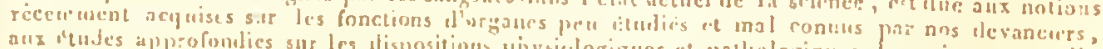

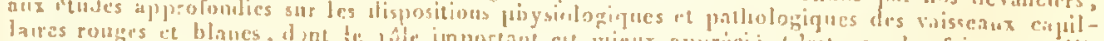

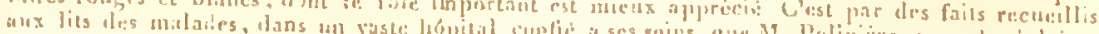

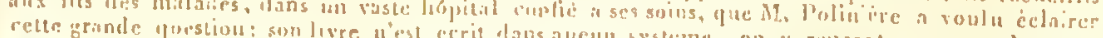

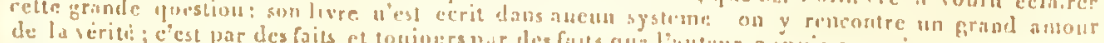

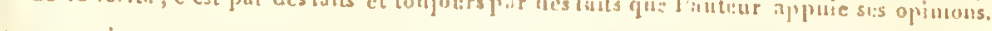

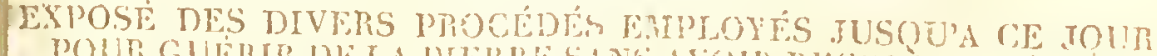

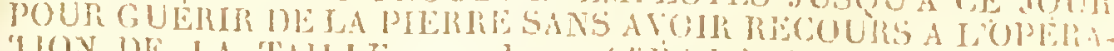

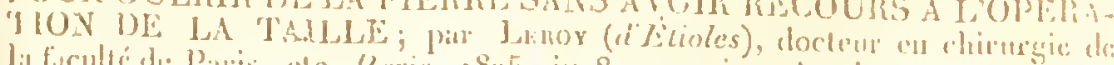

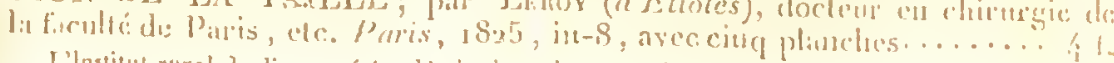

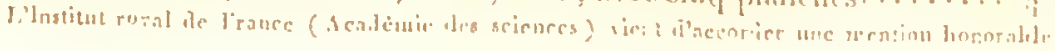


I6 J. B. Balluline, rue de l'Ecole-de-nKédecine, no 13 bis.

RECHERCHES SUR LES SYMIPATHIES ct sur d'autres phénomines qui sont orchiua:rement attribués comme cxclusiís du syzteme uervedx, par lo

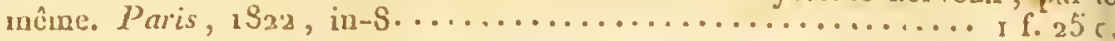

RECHERCHES EXPERIMENTALES SUR L'ABSORPTION ET L'EXHALATION, Alémoire conrumié par l'Iustitut royal de France; par le même. Paris, 1824 , in- 8 , avec une planclic coloriće............

DISCOURS SUR LA BIOLOGIE, on SCIENCE DE LA VIE, suivic d'us tableau des conuaissances naturelles, d'après leur nature et leur fliation; par le même. P'aris, 1826 ; ill-8.......................... 50 c.

MELANGES DE MEDECINE E'I DE CHIH URGIE, on Mémoınes snr les pansemens, les luxations, les nućrations chirurgicales, ius maladies syphil:tiques, Ia paralysie, etc., cle.; par M. Motıe, ancicn :lirurgien de l'Hốtel-Dicu d. Lyon, elc. Paris, $1812-1827$, a vol. in-8...................... 12 f.

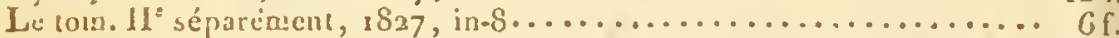

MEDECINE LÉGALE. Considérations sur l'infanticide; sur la manière de procéder à l'ouverture cles cadavres, spécialement daus le cas de visites indiciaires; sur les ćrosions et perforations de l'estomac, l'ecchynose, la sugiliation, la contusion, la meurtrissure; par MNI. Lecredx, Renamd, Laisné, Rieux, donteurs cu unédecine de la faculté de P’aris. ISr

- Cet ouvrafenous a ufferl beaucoun il'inlérél; on y trouve d'execlleus principes, exposés avee elarlé et ruétinde; partout nu y renionlre la ifoctrine, sonvent les expressins mèmes de il le polesenr Ciniessien. - (B.bliothénne médicale.) - Ces quatre dissertations nianqueot dans la biblio bique ale presque tous les gens che l'art; nous ne dunions pas qu'ils ne s'erapressent dese les jurncurer, et is loivent le faire puisqu'ils ue trouverout nulle part aillears les excelleus documens

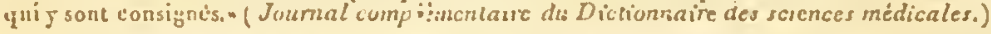

MÉDECINE LEGALE RELATIVE AUX ALIENES, AUX SOURDSMULTS, or les lois appliquées anx déscirdres de l'iutelligence; par Hosrbauen; Irad. de l'allemand par Chambeyron. D. MI. P., avec des noles,

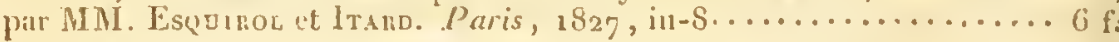

Lc besoin genóralemenl senti d'un traité de médecine légale appliquée aur dţerdres de l'inlelli. geoce, la juste réputatiou dont jonit crilui de M. Hofbaner, les noles numbrenses ci importątes qu'on ajouléres a ce travail MIII. Esiquirol, siur les aliends, et Itard sur les sonds-inuets, ira font un ouvrag. du premier ordre qui sc:a corisulté avec fruit par les mélneins, les gvocats, les juges, ele. Voiei lrs principales divisıns ile cet nuvrane. - Des nualadies mentales et de leurs suites légales. - Do l'eiresur ale sentment ct de's ualadies anilugues. - De la mane etiles maladies aualogues. - Du somnambu-

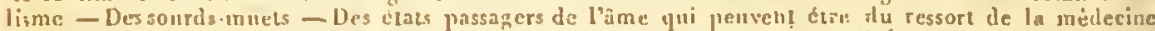
lenale - De l'ivresse. - De. l'èlat intermediaire de la veilte et du sonmeil.' - De l'ecraremert nioroentanó - De l'impulsion insslite. - De la noonomanie homicisle. - De l'ind́uesce qu'erercent sur Ja validitéd'ua temuin les maladies et les états indiqués ci.llessus. - Kègles feruerales pour reconnaître

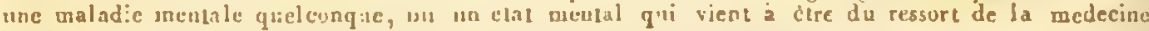
legale.

MÉMOITES DE LACADQUIIE ROYALE DE MÉDECINE.Paris, 182$\},$ tome les, 1 fort yol. ill-4., aree six planches................ $20 \mathrm{t}$

Ce premier solıme qุue nous annnnçous jent être roosidérét comme la suite el Je complément de Mémoirc: de la Sociélé ruya 'e de Mélecine et de l'Acraiemie ruyale de Chirargie. Les deus Sucietí célibes sont reprèsentèes alans la nourelle Acadénie par ce que la seienee a de plusil s-

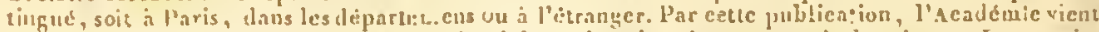
de ićponlre à l'attenle de tons les rgérlecins jalonx le suivre les prugres de la seienee. Le premies volumese eumpose des meinsires suitirans:

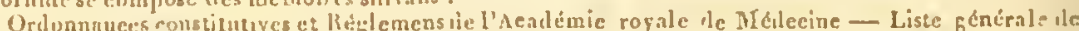

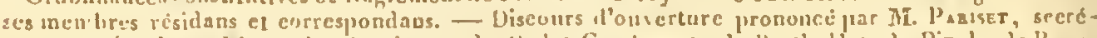
entre perpétnel - 1:10ues de Corvisart, de Cadet Conssicourt, de Berthollet, de Pizel, de Beau rliéce el de Bnurru, jar le máme. - - Kappurt de la Commissiun chargée de rediger un projet d" in struclion relativeme:t aus epidimies. par it Doubus. - Compte rendu des travaus de la Set lion de Mliderine par le même. - llisenurs sur l'histoire et lespro'gries des seienecs plarmacentiques

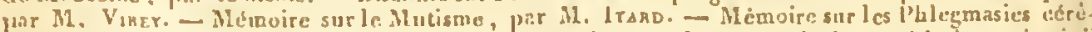
brales, par le méme. - lixiste-1.il de nos jours un plus gram! nnenbre de fous qu'ul n'en existaitil

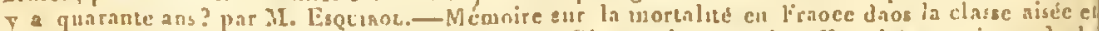

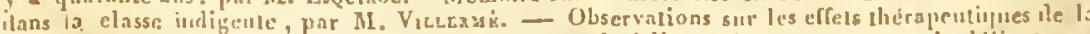
uncrphine ou narȩ́uc, par ML. liactr, - Mémoire sur la folie des ivrognes, nu sur le délire treu blaut, par M. Levest.i.. - Mémuire sur les plaies, pénélrantes de la poitrine, par Ml. le bator.

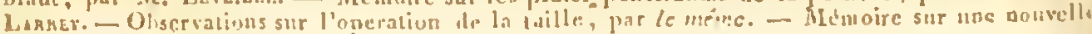




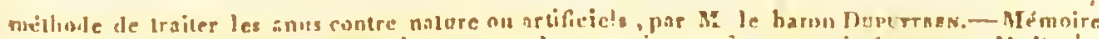

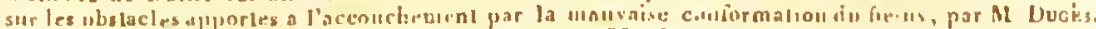

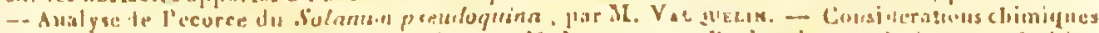

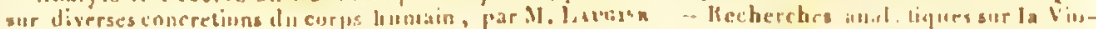

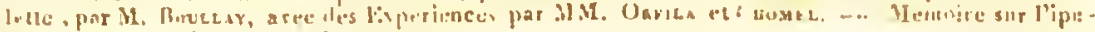

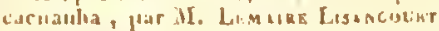

MEMOIRES DE LA SOCIETÉ MÉDICALE D'RHULATION DE PARIS,

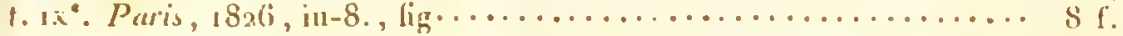

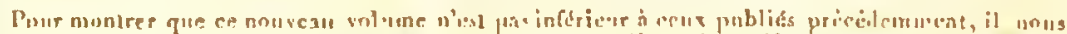

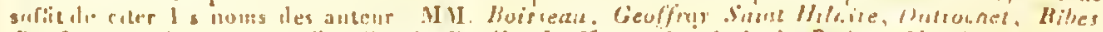

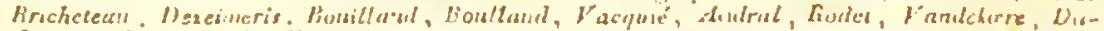
chisiesu, Chantot,relle, fourcaulte.

AA SOLITUDE, par J.-G. Znassamars, nonvelle traduction de l'allemand,

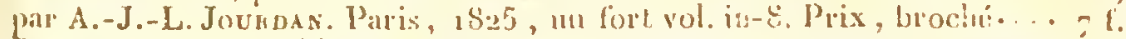

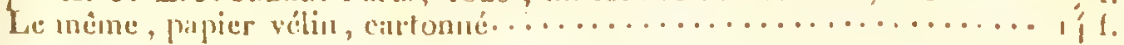

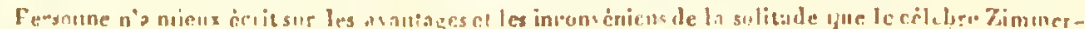

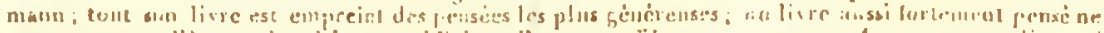

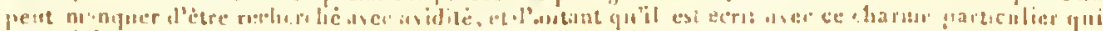

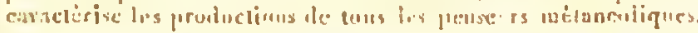

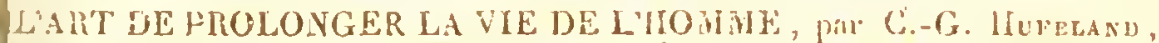

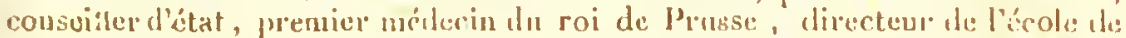
urédecinc de Berliu, ctc. traduit de l'allemand, pin A.-T.- L. Jounrax, D. M. I'.,

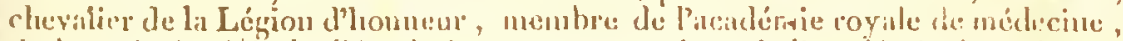

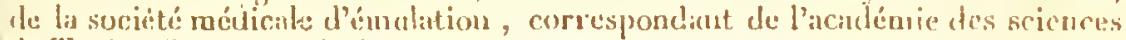

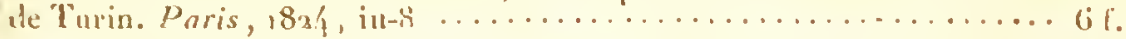

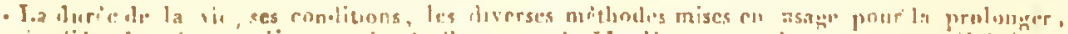

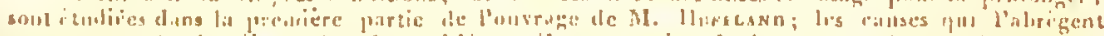

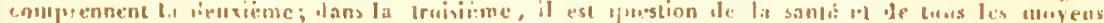

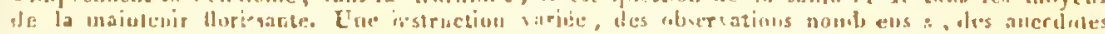

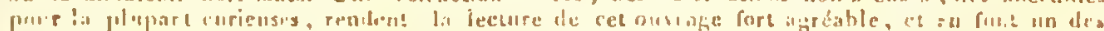

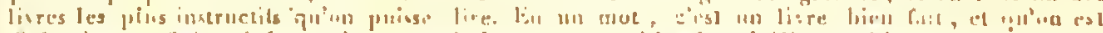

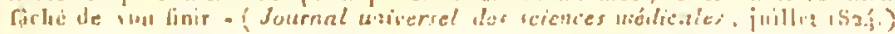

TRAITÉ DE LA MALADIE SC LOPHULEUSE, onvrage couromé pan l'aca-

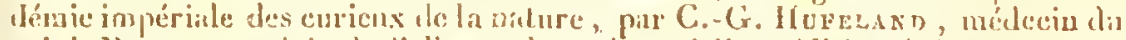

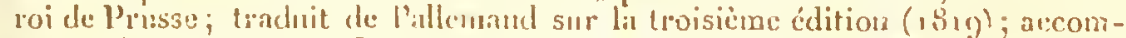

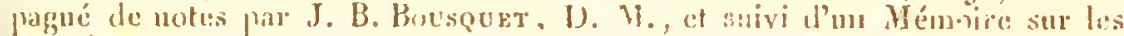

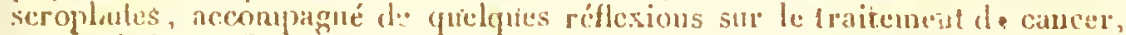
pal Ml. í biron Larkey. Paris, 1821 ; in-8. lign.............. $6 \mathrm{f}$.

1.ICCTURES RELATIVES A LA POTICE TiEDICALE, fuitus nn constil de

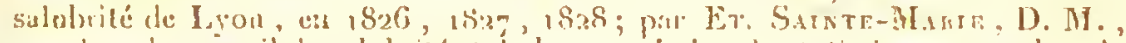

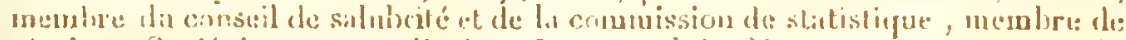

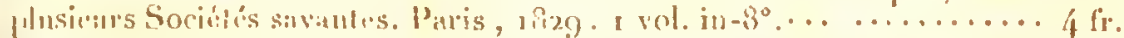

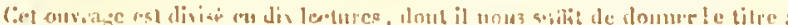

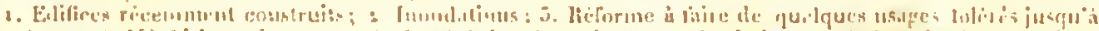

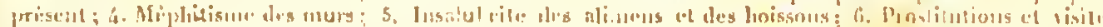

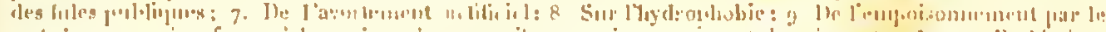

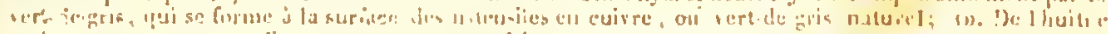

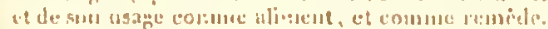

WhECiS ELEHENTAIRE DE PULICE MEDICALE; par le ménc. Paris,

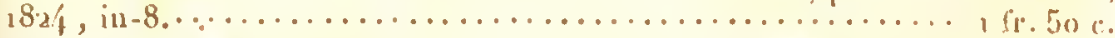

NOUVELLE METIODE POUR GUERIR LES MALADIJS VENL RIENNES INVETEREES qui ont résisté aux trintemens ordianires: pall

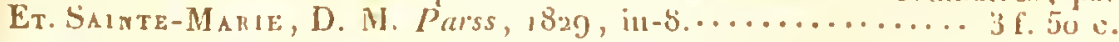

NOUVEAU FORMULAIRE MEDLCAL ET PHARMACEUTI()UF; piM le mime. Paris, 1820 , in-8........................ 5 f

DISSERTATION SUR LES MEDECINS POF'LES; ju te meme. Paris,

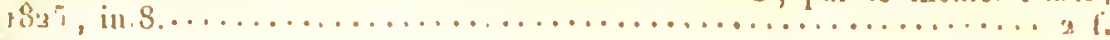


LE'TH'RE DE LOUIS-JACQUES BÉGIN A E.-Y.-J. BROUSSAIS, médeciu cu chef, turemicr professeur à l'hôpital militaire cl'instruction ch Valde-Grítce, oltevalizr de la Légion d'sonneur, unembre de l'ueadémic royale de múdecine, els. Paris, 1825 , in-\$................ I f. $20 \mathrm{c}$.

LETTRE A L'ACADEMIE DES SCIVNC;ES. Fxamen critique de l'ouvrage dı: M. le doetenr Civiale, intitulé: de la litholritic, ou broiensent de la pierre dans la vessie, et appréciation dis laits prosentés par ec médecin; par te harou Heortaloup, docteur en médecine. Paris, 1827 , in 8 , fig..... $3 \mathrm{f} .50 \mathrm{c}$.

MINUEL D'ANATOMIE GÉNÉRALE, DESCRIPTIVE ET PATHOLO-

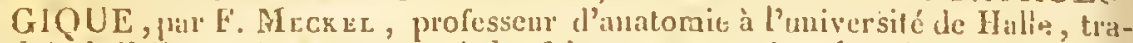
dnit de l'allemand, et angmenté des frits nonvcanx dent la scionce s'est enrichie jusqu'à ce jour ; par G. Bnescuex et A.-J.-L. Joenvali, L). Mi. P'. Paris, i 825,3 vol. in-S de Soo pages chricur, en ciractère petil-romain.. 28 f.

Depuis tong-temps on désirat un livre gui rénatt 10us les faitsimporlans te l'analomie: géné:a]e, ile l'analow'e descriptive, de l'analomie pathologique el de la physintogic. In pareil irarail exigeail iles connaissances anssi élenthes qu'apprufondus; il ve pouvail ćtre exćcuté que far l'un

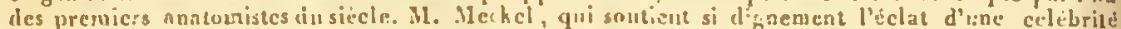
suédicale béréditaire dings sa familie, e'. a jui on doil plusieurs autres vuvages dn premict

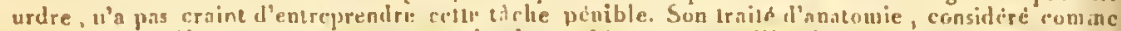

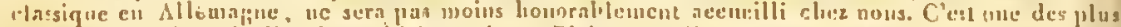
belles productions de l'école ale biebat, de ce Bicbat que l'turepe envic a la France, et aunuel

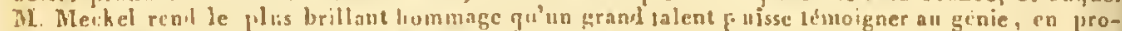
fessant pour lui unc almuration sans enthousiasunc. On n en simin, en faisant passer ce mauncl dlans untre lansuc, d'y ratucher lous les finis dout le domaiue de la scienee s'est onrichi depuis sa pu-

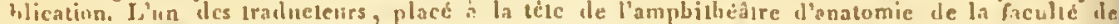
melecine sle Paris, est h mème. par sa pinsision, de s'assurer juurnelleneul de l'exactitude conase ic In veriles les descripliuus de Meckel : aussi ne crasgnons-nous pant de l'nffrir comme un ourrage cotierenent neuf, sous le duuble ropport llu plan et du mede deaceution.

MH:MULHE SUR L'EMTHOI UE L'IODE DANS LES MA LADIFS SCROPIIULLUSES; In à l'A cadémic royale des Sciences dans la siance du 22 juin

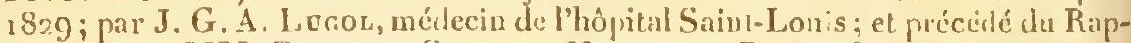
poit fait par MM. Dumbur, Senues et Magende. Paris, 1829, iu-8. 2 f. 50 c.

MUMOIRES SUR LE 'THAITEMENL' DES ANLS ARTIFICIELS, DES Pl.AIES DLS INTESTINS, E'I' DES PLAIES I'ENETKANTFS DE LA

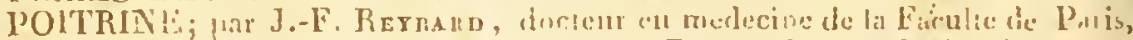

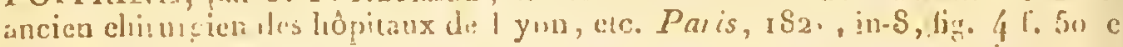

MENOIRE SUR LES HE IORRHAGIES INTERAES DE L'UTERUS, qMi a obteru le prix all concours ouvert par la Soricte de médecine de l'al is; pas inacime loovis, ex-survellante en chef de l'hospice de la Materuité, efe.

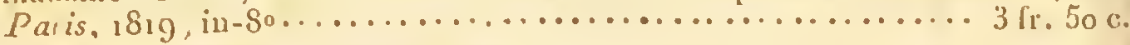

RECHERCHES SUR UNE DES CAUSES LES PLUS FREQQLENTES E'I' 1.A MOINS CONNUE DE L'AVORTEMENT, suivies d'un "émoire sur l'intro-pelvimetre, ou mensuralme interue du hassin; par madame reuve

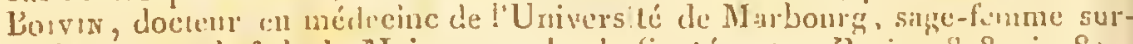
veillante en clirf de la MIaison royalo de Sunté, etr. Plivis, 1828 , in-8u.

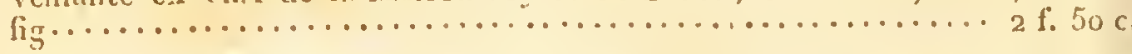

OHSERVATIONS EI REET.EXIUNS SUR I,ES CAS D'ABSORPTION 1)U PLACENTA, par le méme. Paris, 1829 , in-8......... if. 50 c,

IIONOGRAPHIE SUR LA RAGE, omrage couronne par te Cerele medical de l'aris, par A.-E.-C. De St,-Mantix, doeterw en médecine de la Ficulle de l'aris, cte., etc. Paris, 1826, in $8 \ldots \ldots \ldots \ldots \ldots \ldots \ldots \ldots \ldots \ldots \ldots \ldots$ of

NOUVEAUX ÉLËIENS DE PATHOLOFIE MIDICO.CHIRUHGICALE

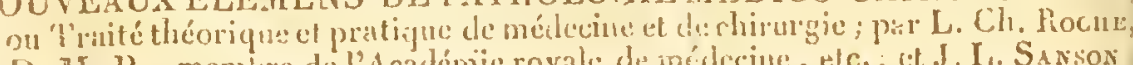
D. MI. L', mentire de l'A calćmie royale de médeciue, elc. et J. I. Saxion D. C. P., chirurgien en serund de l'llòtel-nien de Paris; seconte édition Par:is, 2828,5 vol. in-8., de $60 n$ pages charun .............. 351 
aecrolıre a à oe reparer: $z^{\circ}$ Je ees sutres fouctions par lisquelles sunı rejelés de l'd conomie le

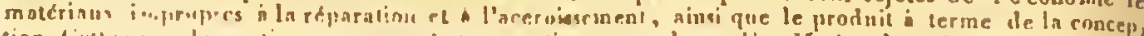

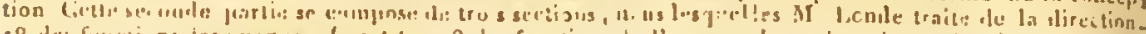

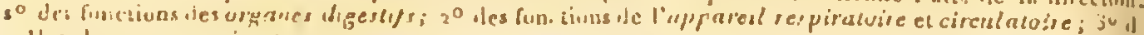
celles des arganes sécullevers

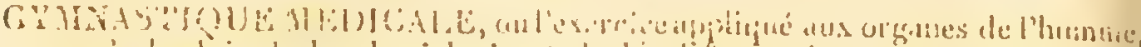

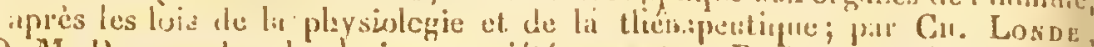

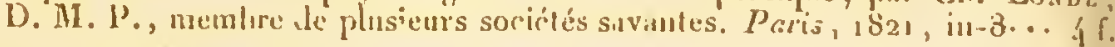

MOSOGRAPKiE ULGANIOUE, ou T'aité complet de mélecine pratique; par

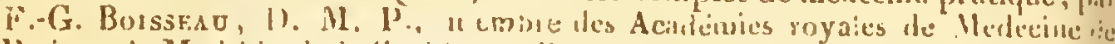

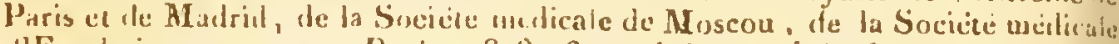
"f'Eumlation, etc., ctc. Paris, 1828-18ag, 4 forts vol. in-8........ 34 f.

lantroduction de la physiulogie, dano la pathologie le rappel a l'śl ide des organes, la décourerto

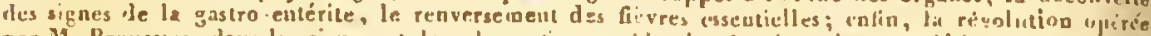

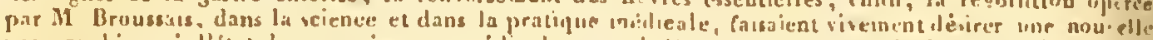

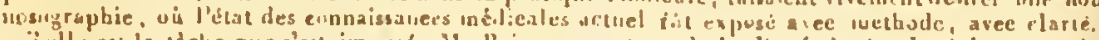

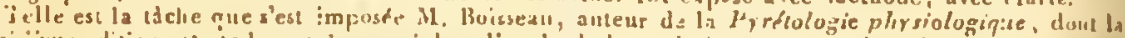

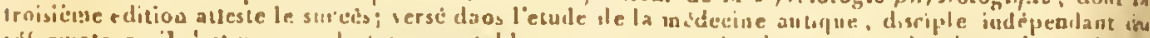

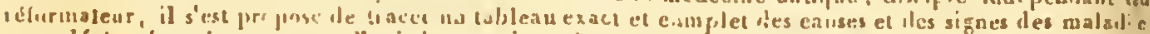

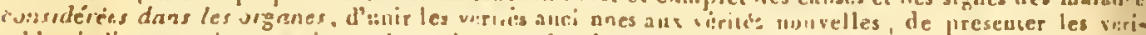

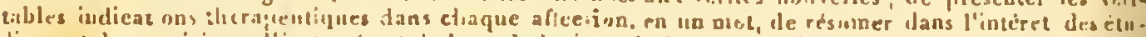

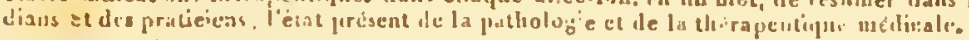

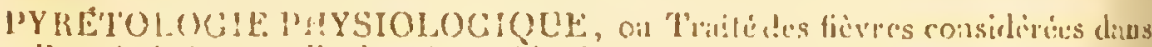

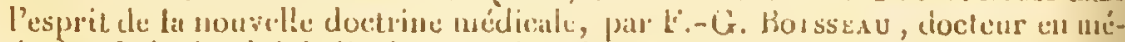
decine de la liaculté de l’aris, uerubre des acatómies royales de médeciue de

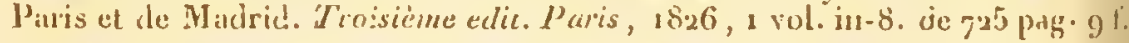

'Trois edilions en moins de Irois ans, voilà qui vaut mieux que tous les éloges qu'or puurrail doncer ś

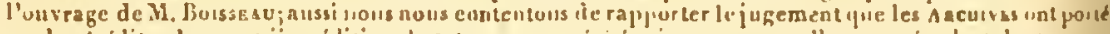

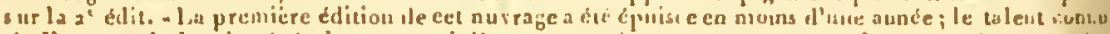
de l'anteur, le besoin généralepiest senti d'un onvage dece genre, propre a fare comaitre tunlesirs

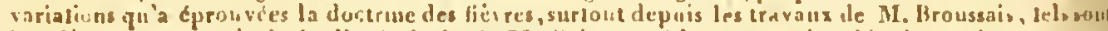

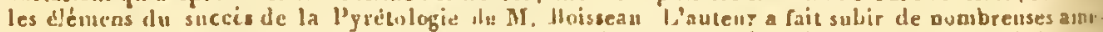
lioratious á snn travail; il a revu aves soin los furineipes de pliysiologie pathulngique yui furmurct

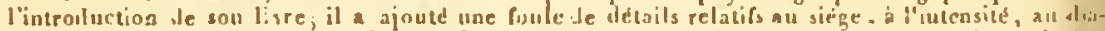

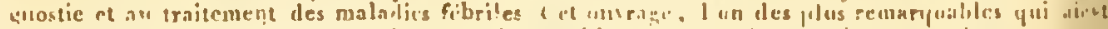

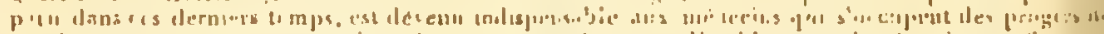

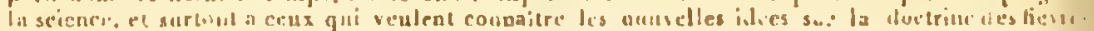

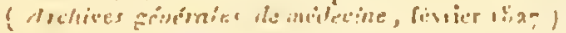

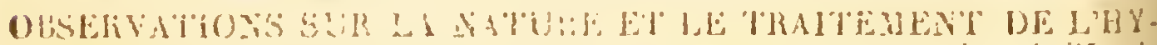

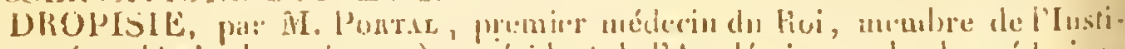
tut (atadénie des sciences), président de l'Acielemie royate de inćl: dius, Paris, $18: 4$, 2 vol. ilt. 8. $11 \mathrm{f}$.

- Cet ourrage, fruit ric la longue pratiqle d'un médecin dont le grand âge n'a point interrompri les irainux, se recommande aus prafiriens pas les uhervations clininues et les recherches quil enu' tient sur les livdropisies en géntrial et sur ehaque epier. dihydrupisie en partirulier. Il ebt isul le le ennsaeser de hungutes plirascs à sun éloge; lrom de M. Purtal est plus que surfisanI purur fiser

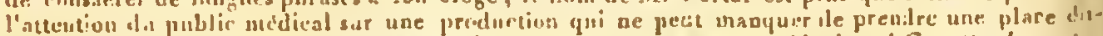

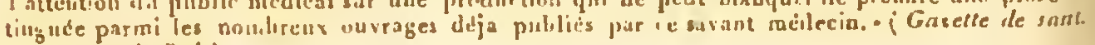
$\mu^{\circ} \mathbf{x i l l}_{1}, \mathrm{~min}$ is:i)

GBSERVATIONS SUR LA NATURE E'T LE 'TRATTEMENT DL L':JILEPSIE; par M. Pontal, premiet unédecín du Poi, membre de l'histitut, etc. Paris, 1827 , i vol. in-8.

\section{CEUVRES CHIRURGIC.ALES D'AS'LLEY COOPER E'T B. TRAVERS:} conteuant des menoires sur les luxalions, l'inflammation cle l'iris, la ligature de l'norte, li: phimosis te le piraphimosis, f'exustose, les ouvertmes contre nitture di: l'urcthre, les blessures et les ligat utes des veines, les fractures du col

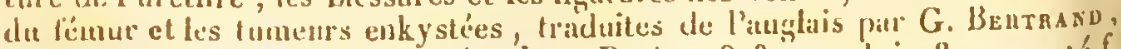
ducterar til médecituc, avec 21 planches. Paris, 1823 , 2 vol. in-8.... 14f.

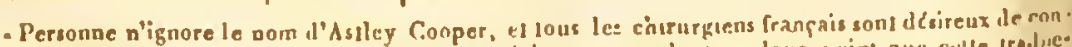

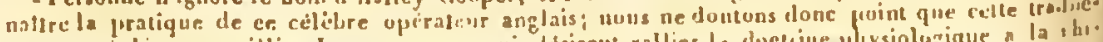

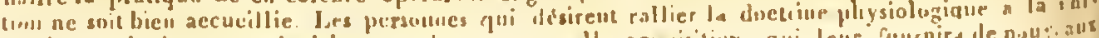

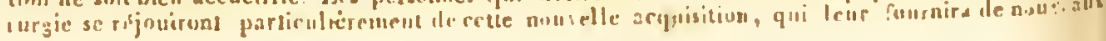




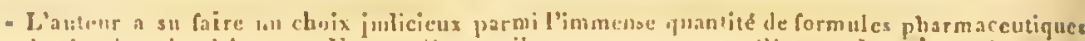

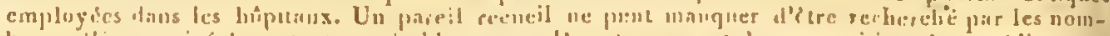

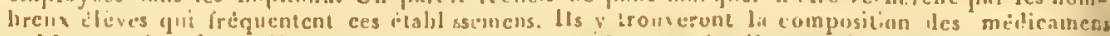

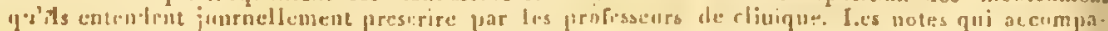

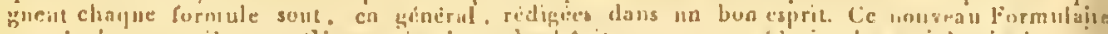

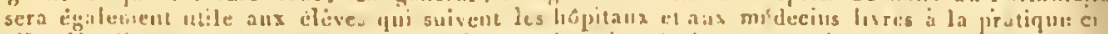

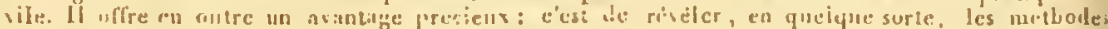

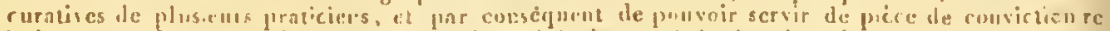

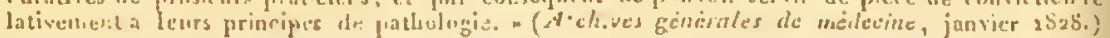

TRAITÉ ÉLEMENTAIRE DE MA TIERE MÉGICALE; par E.S. RATIFR, Docteur en méclecine de lin Farnlté de L'a, is, ntetubre de plusieurs Sociétés savantes. Paris, 1829,2 vol. in $8 . \ldots \ldots \ldots \ldots \ldots \ldots \ldots \ldots$ ro 8.50 .

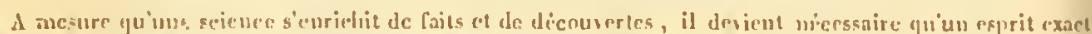

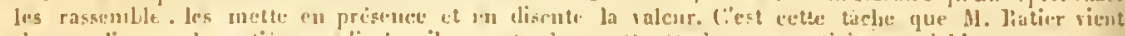
de renulir pour la matione medieale; il apporte daus eette itude un secpticisase qqui bien rarement y

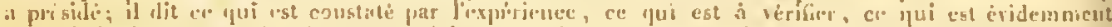

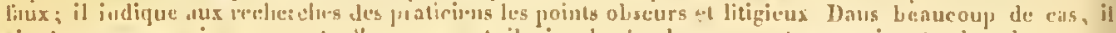

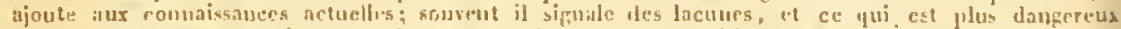

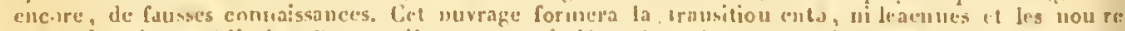

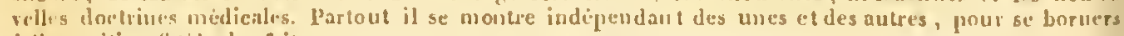
a : iexpositiou lintele des fuits.

PHYSIOLOGIE DES TEIIPERAMENS OU CONSTITUTIONS, nouvelle doctrinc applicalıle à la médecine-pratique, is l'bygriène, a l'histoire naturel!e et ì li philusophie, par MI. 'Jiromas (de Thoisvévres) D. M. I'. médeciu attaché à l'hôpital Beatrou. Paris, 1826 , in-S............ \& 1. 5o c.

'HILOSOHHLF ANATOMIOUE, par M. ET. Geoffnor-Saint-hilare,

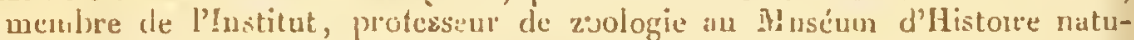

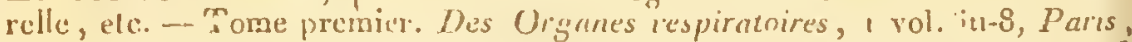

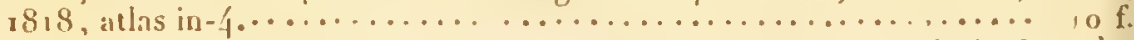
- Tome 11. Honstrunstes humaines, Paris, 1223, 1 vol. in-8, atlas

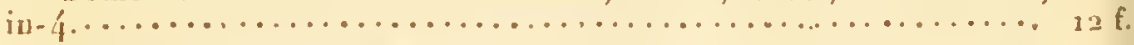

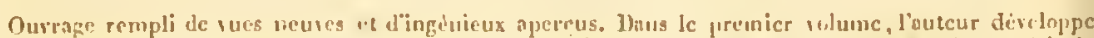

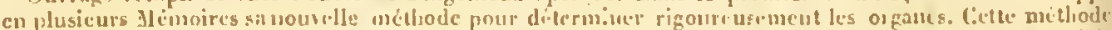

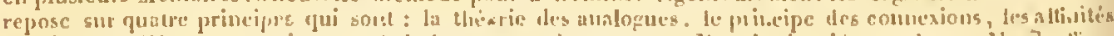

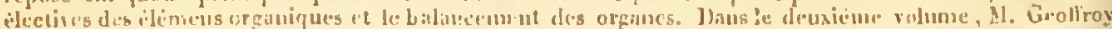

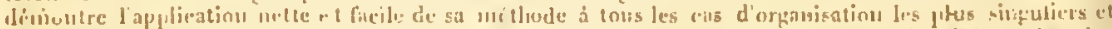

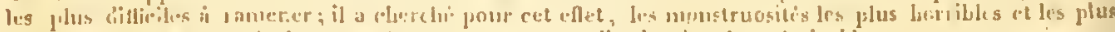

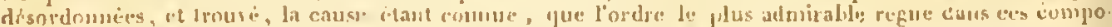

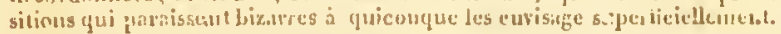

PRECIS DE BIRIIOGRAI'HLE MÉDICALE, coufenan l'in:ivation ct la

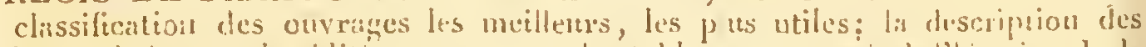

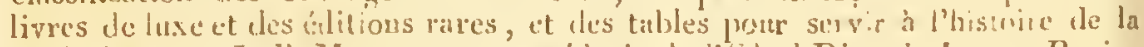

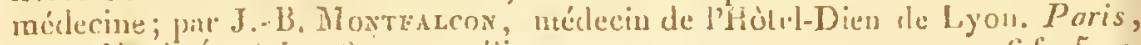

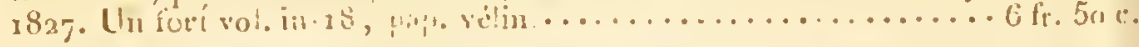

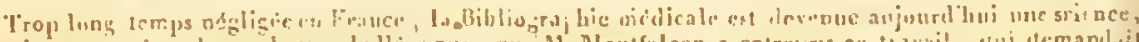

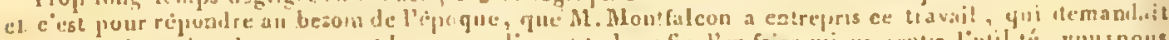

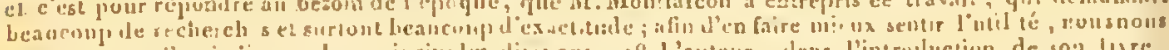

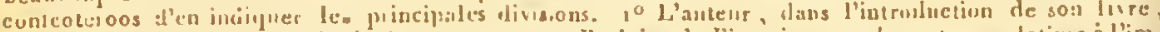

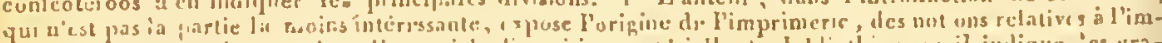

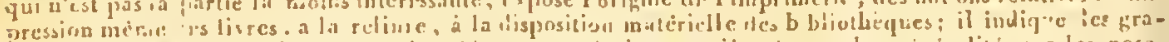

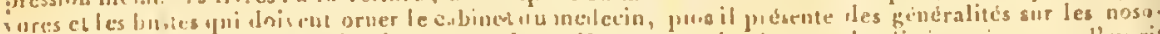

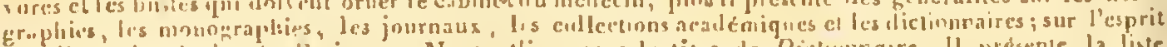

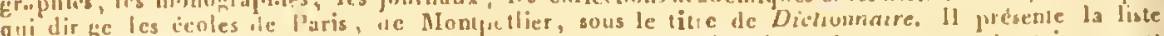

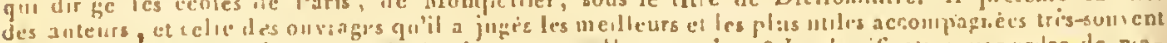

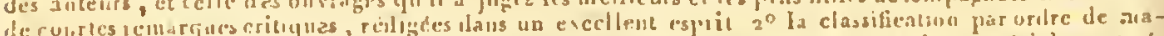
ace

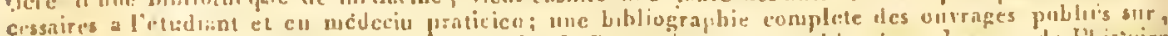

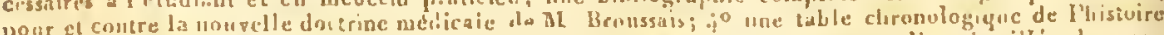

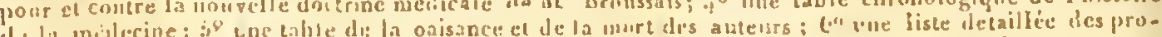
1. In mélecine; onterit sur la Hiblio orsiphic médicale

1RATQQUE DES ACCOUCILnIENS, on Mémoi es et obserations choisis

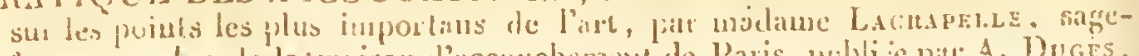

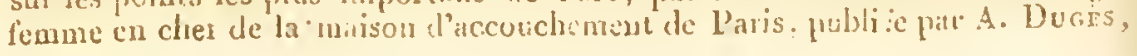


§son neveu, D. M. P', professeur d'accouchement à la faculté de médccise, I ontpellier, avec une notice sur la vie et les travaux de madame LACAPLLL,

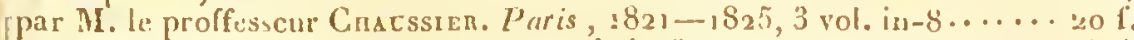
Les tomes II et III siparénent, 2 vo!. in $-8 \ldots \ldots \ldots \ldots \ldots \ldots \ldots \ldots$. 3 i.

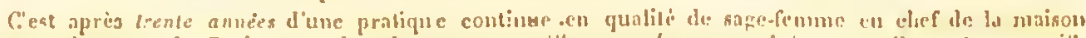

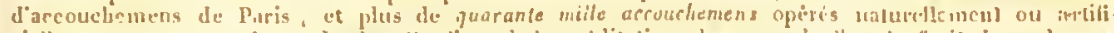

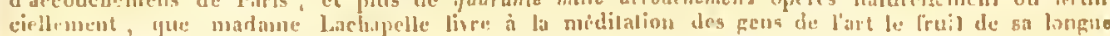
experinee. Son line ost un enurs de elivie, ue eomplet des accondienuens, et yui, pour nons strvir

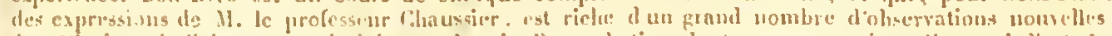

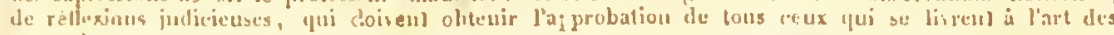
acculechemetus.

RECIS DESGRIPHE SUR LES INSTHUMENS DE CHIRURGIE ANEIENS ET MODERIES; contenant la description de claque iustrument, le nom de cexx gui y ont apporté des modificaticns, et ceux prélérés aujourd'lui par nos mejlenr's praticiens, et l'iudication des qualilés que l'on doif recherciner dins chaque instrument; par Hrsux, conteize, fabricant d'in-

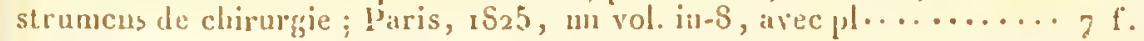

?RESSAGS TIRESS DU PUULS, d'après l'écult splyygmique, par J. LAvx,

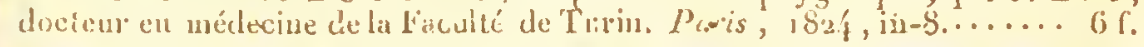

RINCIPES DE PHYSIOLOGIE MIEDICALE; Par Isid. BourdoN, D. M. I'. de l'Aca léarie royale de Médeciue, médeciu des Dispensaires, etc., 2 vol. in-S.................................... 1 2 .

Cet ouvragc, qniembrasse toute la science de l'homme sain ct malaje, est divisé eu sept livres, divisés en cent quatre-vingt-sept chapitres, dont bous cileross les suivans: Livae Ier. De la vie (dix-sept eluapilres). Caracteres de la vie. - Corps vivaus. - Organisalion de l'homme. - Caractère d: l'homme au physique et au moral. - Live II. Du systènenervenx (ving̨- cinq cliputres). Idée générale des nerfs. - Du cerveau et de ses lonctions. - Rôle des nerfs daus les maladies. - Liste des incilleurs autcurs quii ont - écrit sur ce sujet. - Livne III. Des seusatious (trente-trois chapilies). Sensibilité en fúluéral. - Exposé des cinq sens. - Histoire des avengles-nés de I'aris. - Seusationsiutéricures. - Lvre IV. De l'iutelligence (vingrt-cimy chapizres). Organisation nuisible on favorable à l'intelligence. - Siégc des passions. - Iufluence qu'out sur l'esprit, l'âgc, le sexe, les professions, l'lú:-ćditć, - Les dillormités, le climat, les gouvernemens, la médecine actuelle. - Systemu de Gill. - Du delire el de la folie. - Lavne V. Mouveuncus et fouctions de lis vie luuraine. - Civeulatiou du sang. - Ciuses des épidémies. - Comment recomnaitre les diverses maladies des ponmous, etc. - Livar. VI. Histoire du sommeil et des songes. - I Ive VII. Histoiredu sarg, de ses sources, de ses protuits. - Génératicu de l'homme. - Cet ouvrage est pricédé d'un discours sur l'ćtude générate de l'houme.

RECHERCHES SUR LE MÉCANISME DE LA RESPITATTION ct sur la circulation lu sang, essais sui ont obtedu une mention honomable au concous de l'Institut; par Isid. Bounnon. D. M. P. P'aris, 1820 , in-8... 2 f.

IDE J'TNELUENCE DE I.A I'ESANTEUR SUR QUELQUES PIITNOTIEYES DE LA VIE, par. Isicl. Bomodou, doctur en mélccine de la lit-

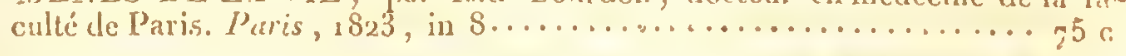

IRAPPORTS DU PHYSIQUE ET DU MORAL DE L'HOMME, par P.-J... ¿. CABaxis, de l'Institut, professeur de la faculté de médecine de l'alis, précédés d'une table analytique, par M. le conte Destutt DE Trarix, ct suivis d'uns: table alphabétique; nouvelle édition, Paris, 1824,3 vol. in-12 de 1,100

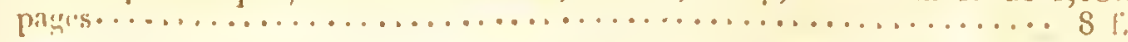

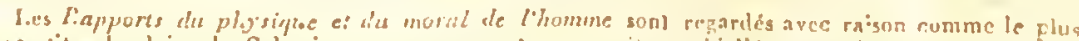

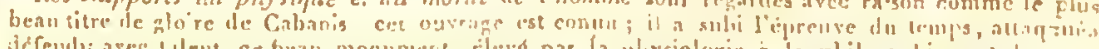

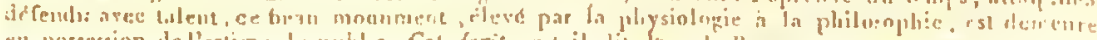

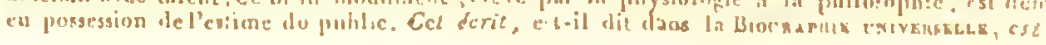




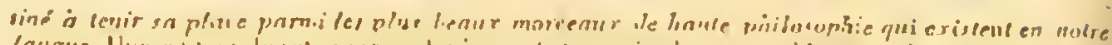

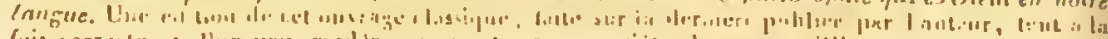

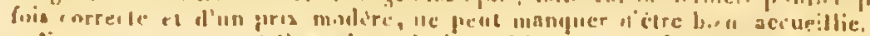

Nums avons eunserve l'eatrait aualgtique fait aec un si grant taleut par M. le conue Uesturt ale Craeg, et mons $Y$ avons jint une notice sur la vie de cobauis.

RAPPORT sus l'origine, les progrès, la propagation par voic de contagion, et la cessation de LA IHEVRE JAUNF qui a régné, en 1 \$al, a Barcelonc;

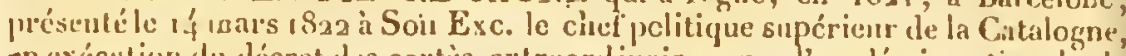
en cxéculion du décret des cortiss extraordinaire, par l'académie nationale de mirleciue de Barrelone, traduit de l'esplaguol par P. Raren, docteur 'n mé -

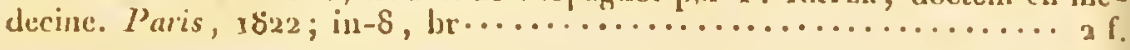

\section{RAPPORT IISTORIOUE SUIR LES PROGRES DES SCIENCES NATU.} RELLES deprois 17 Sy, et sur lear cetat actuel presente au gruvernement en

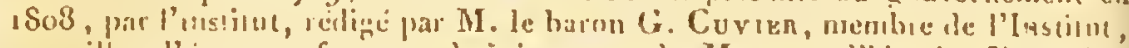

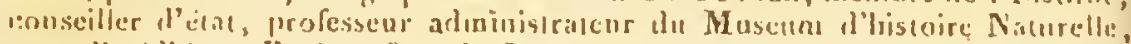

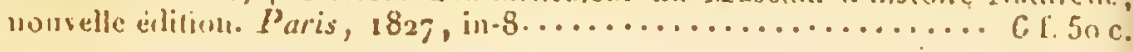

RECHERCHES ANATOMIQUES; PATHOLOGIQUES ET TIIER:PEUTIQUES sur lit milladie cunnue sous les nouns de gastuo - maténrre,

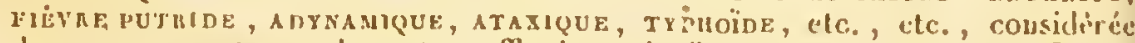
dins ses rapports arvec les tutres affections aiguës : par V. Lovis, D. II. P., uredecin de lhônital de lia Pit ié, membre de l'Aciulémie royale de Mídecine,

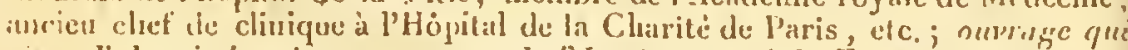
1'unt d'obtenir le prix au concours de l'lnstitul royal de Fiance. Paris, $1 \$ 28$,

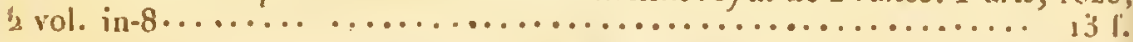

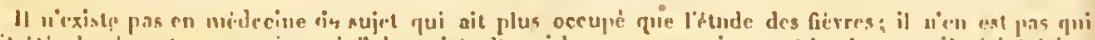

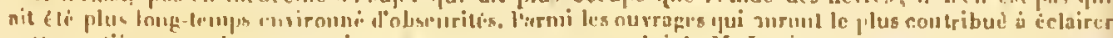

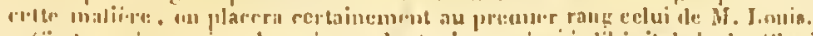

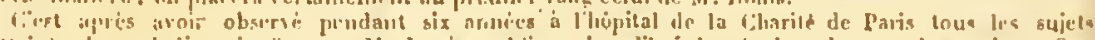

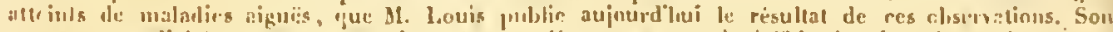

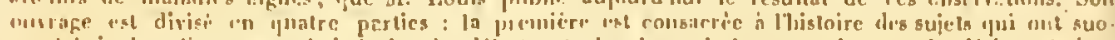

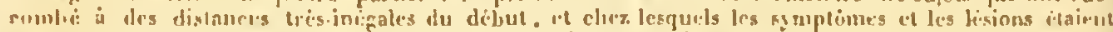

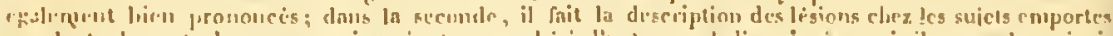

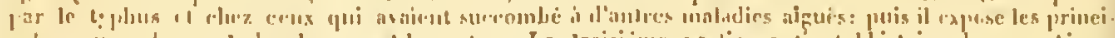

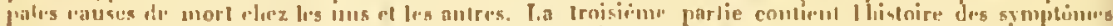

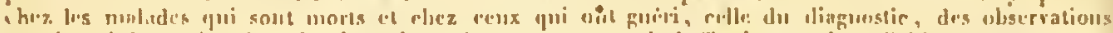

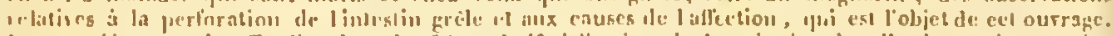

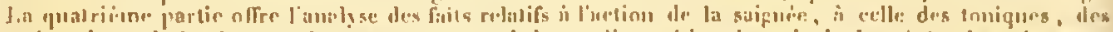

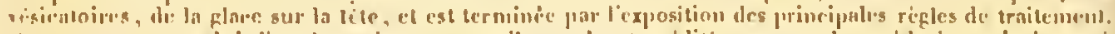
lit ourage est colui alun bon obseriatcur; il sera lu et miditi par touk les medecins qui aimenl a suivio: les jrogrés de lia seicnęe.

PLCIIERCHES SUR LA NATURE ET LE TRAITEMENI DU CANCER DE L'ESTOMAC; pis René l'nus, doctou en unedeciue de la liaculté de

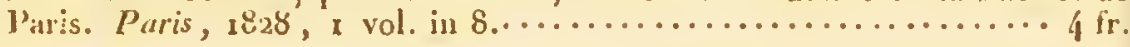

\section{RECIIERCHES ANATOMIQUES ET MHYSIOLOGIQUES SUR LA} STRUC̈IURE INTINE DES ANIMAUX E'T DES VEGETAUX E'T SLR L.EUR MO'TLITE, pill M. Dutnochet, D. MI. P., corresporilunt de l'Institut de France (acadéurie royalc des scieuces, ctc.). Pars, $1 \$ 24$, in-8,

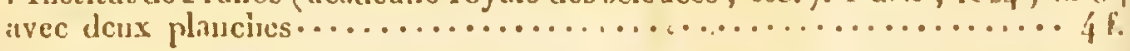

NOUVELLES RECHERCHES SUR I'ENDOSNOSE ET L'F.XOSMOSF, pat. H. Dutlocuet, D. M. '’, correspondiul de l'Institut. Paris, 1823 , in-8.

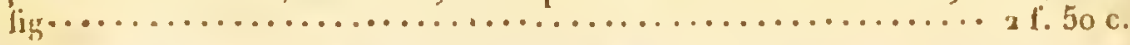

\section{¿'AGEN'T IMNÉDIAT DU MOUVEMEN'V VITAL DÉVOILE DANS} SA NATURE F'T DANS SON MODE. D'ACIION CHEZ LES ANIMAUX FT VEGliTAUX, ouraze faisant suitc au précédcne, par DUTHocnet. "Naris, 1826,1 vol. in.S................... $4 \mathrm{fr}$.

1.e celelure Ciuvier n'a pas cru pousoir mieur caraclériser le gene de mirile de M. Di:trochet

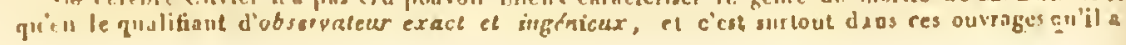




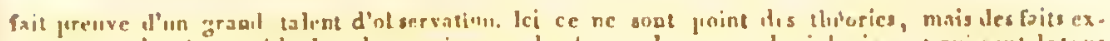

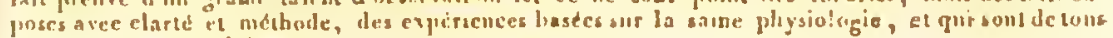
les piys, paree qu'elles suit exarics.

IECIIKCHES ANATOMIQUES ET PHYSIOLOGIQUES SUIR LES CAS

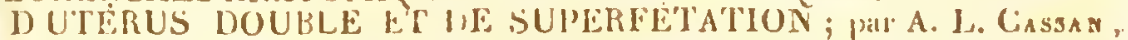

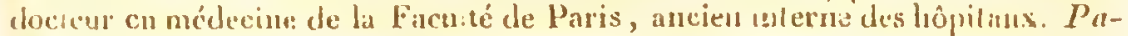

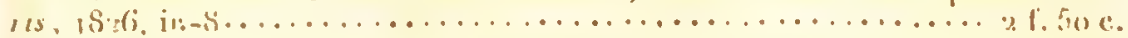

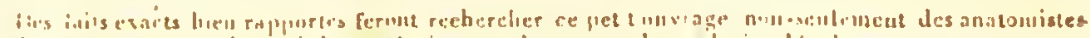

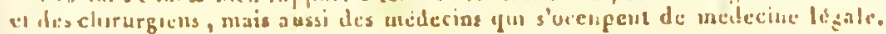

\section{HEFLFXIONS E'T OBSERVATIONS ANATOMICO-CHIRURGICALES} SUR L'ANEVRYSME SPUN'TANÉ EN GENERAL, et en pirticutie' sI" celni de l'artère fémorale; pirr J.-L.-L. Casamaxon, doeteur en médceiné de

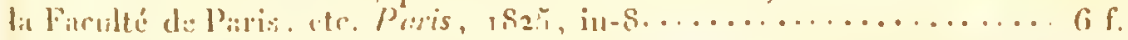

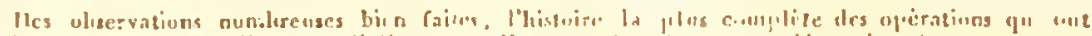

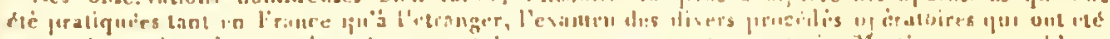

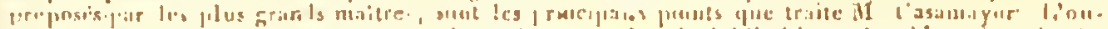

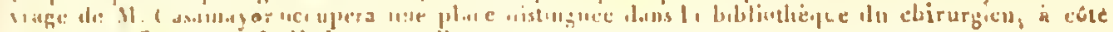
de censile Searpa el de llodgoun sur l'aucirysme.

IFYISION DES NOUVELLES DOCTRINES CHIMICO-PHYSIOLOGIOUES, suivic d'experiences rulatives à la respiration; par M. Coutanceav, 1). il. l', mrdecin et protesseur a l'hôpilai nilitaire d'unstrnction du Val.de:Gritec, nucubre de l'aenclémie royile di: médecine, de la légion d'lonnent",

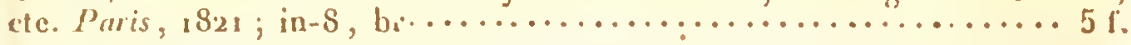

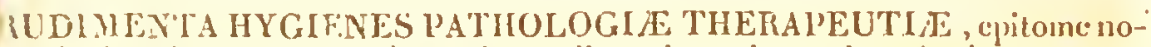
sologie ad instituendes chirurgia sludiosos in regio taurinensi at henwo; professobis II. Ganken:, chirurgi prinari in regioptocholophio. Turin, is21,

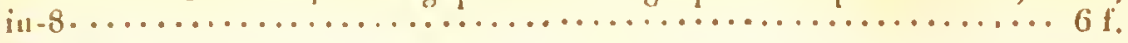

\section{BUR LES FONCTIONS DU CERYEAU ET SUR CELLES DE CHACUNE} DESES PAR'TIES, avec des observations sur lå possibilité de recounintre les instiucls, les penclians, les talens, ou les dispositions morales et intullectuciles des liommes et des animaux, par la configuration de leur cerveau et de lemr

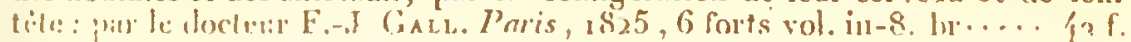

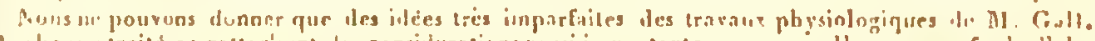

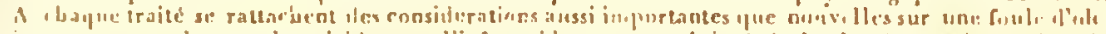

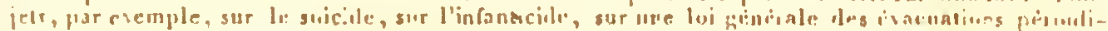

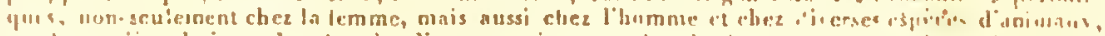

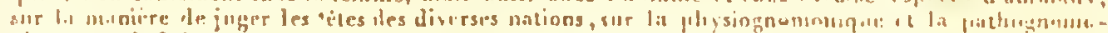

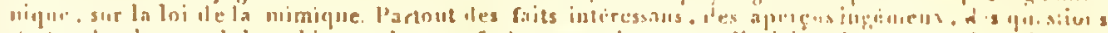

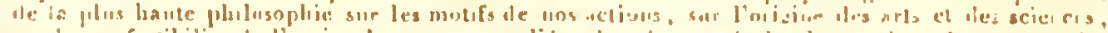

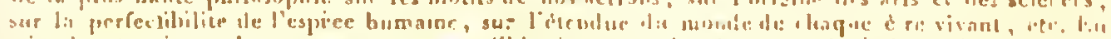

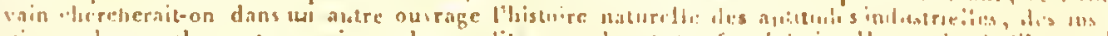

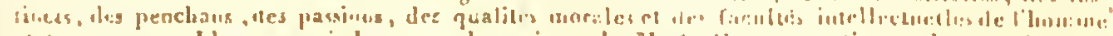

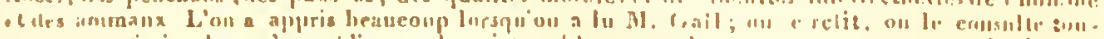

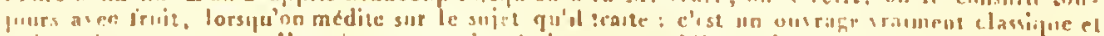

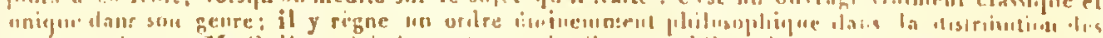

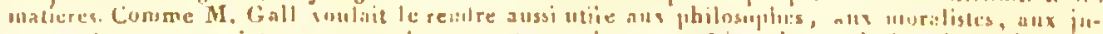

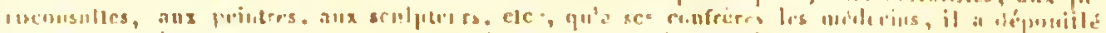

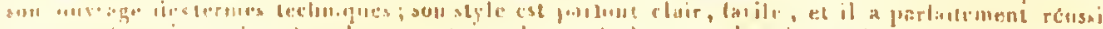

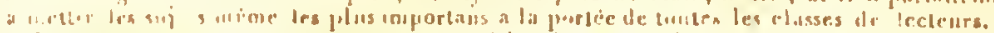

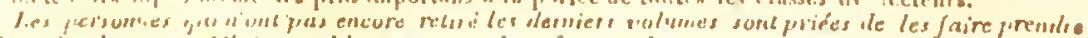
jans le plus courb deilsi pusstble, au prix de g francs chaque.

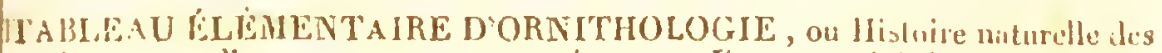

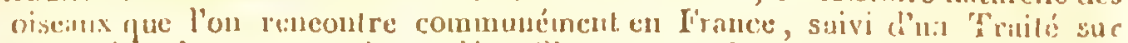
la misicie de conserver leurs dépouilles pour en former des ceillections, par

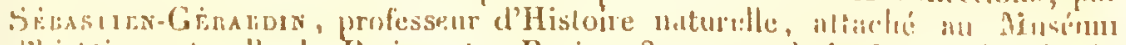

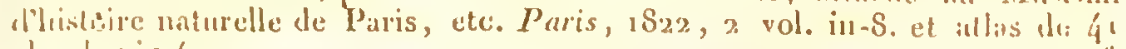

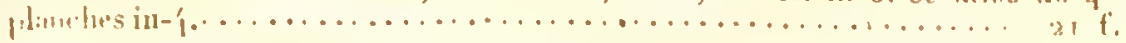

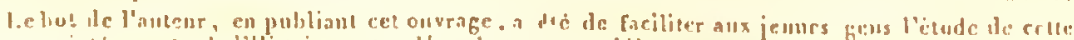

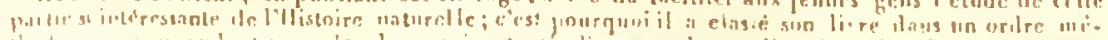

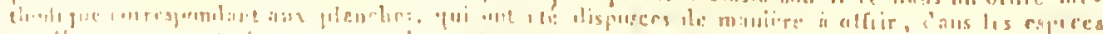

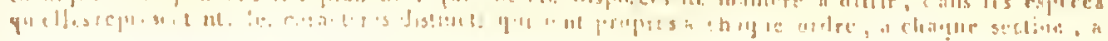


chaque famille, s chaque lribu ou a ebnque espece. l'husicurs planehes sont consacrés ponr zepré-

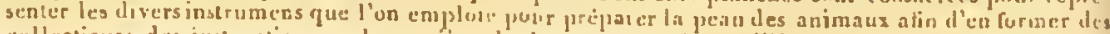
collections; des instructions jur la manére de disposer un caliuet d'Histnire naturelle, celle de se

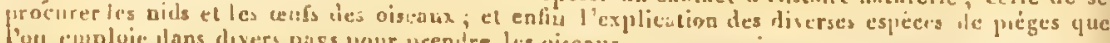
l'ou compluic ilans divers pays jour prendire les viscaus.

'THÉORIE NOUVELLE DE LA PITTHISIE PULHONAIRE, angmentée do la métlode préstryalive, pitt M. Lantnols, doctcur en médicinc, ete.

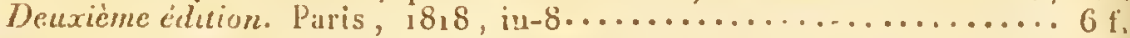

TORTI (F.) IHERAPEUTICE SPECIALIS AD FEIRES IERIUDICAS PERNILIOSAS; nova cditio edentibus el curastibus C. C. F. Tombeur et O. Brixlue, D. MI. Leodii et l'atisiis. $1 S_{3} 1,2$ vol. in-8. fig.......... $16 \mathrm{f}$.

TOPOCRAPHIE MÉDICALE DE PARIS, ou Ex amen général des causes qui penveut avoir une influence marquéc sur la santé des labitins de ectlo ville, le carractirede leurs maladies et le choix des précautions hy giénique's qui leor sont appiicables; dédióe à 11 . le comte Clabrol ds: Volvic, prélicl clu déprartemant di: lit Seine, par C. Lachasse, docterr en médecinc de la fircultẹ de l'aris, etc.

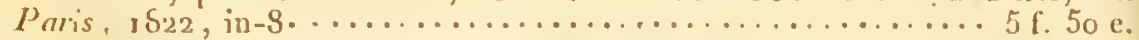

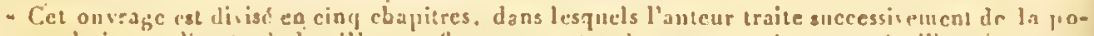
sition replative et directe de la ville. sa figure, son etepdue, sa teinpitrature, de l'histoire natu relle de Paris et de ses enviruns. Il passe en revite les causcs qü peuvent avirir une iuthuence sur la salıbrité de Paris. A cet.c uecasion, il Cait, a l'égard des inuze arrondiesemens municipanx yui composent la ville, des obstr.ations teis-importantes.ll recherche, dans 11 disposition des divers

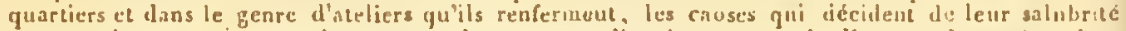
comparative, et propose, t'une part, des moyens d'assaiusscuent, de l'autre, des procicntions bygiéniques propres a soustraire les babitans a l'artion des causes insalubres. Ll exinmine l'hahitant de Paris tast an plystoque qu'au molal, el cermine par le tableau des constitutinis inćlicales. (Jourial général de m. decine, t. Lxxxt, uctobre 1822.)

TRAITÉ DE CHIMIE, par J. J. Lerzélius, traduit par A. J. L. JoundaA, sur les manuserit.s inédits de l'autcur, et sur la dernièré ćdition allenande. l’aris,

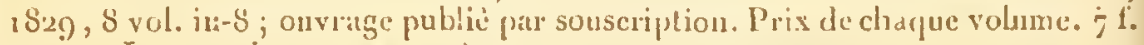
( Le inne lest en renle.)

Les nombreuses dienuredes ilont M. Berzilins a rnricli la scienec, surtout l'étallissenent de lat dere-

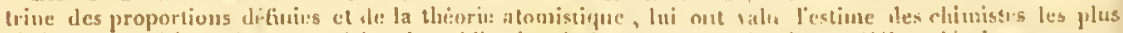

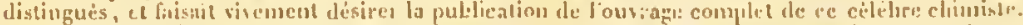

Les combreuses additions manuscrites que I autour a bien voula communiquer doisent faire re-

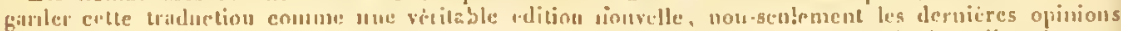

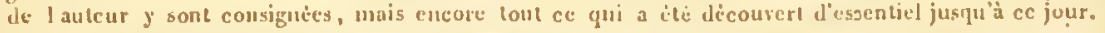

TIAITE DES MIA ADIES DU CCEUR ET DES CROS VAISSEAUX, par R.-J. Beirtax, professenr de la faculté de unćlecine de l'aris, méderin un chef

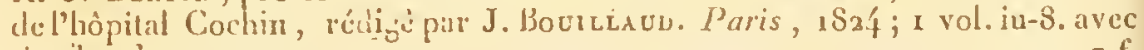

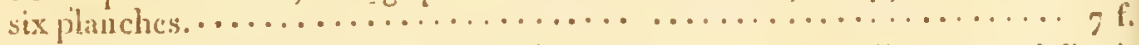

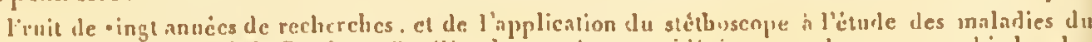
craur, l'ouvrage de MLM. Bertin et linwilland peut étre cousidere coinme la inynographice la plus

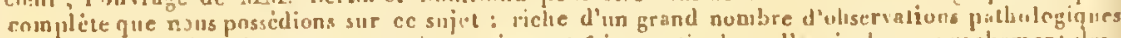

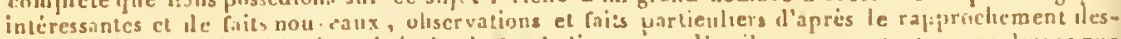
que!sa

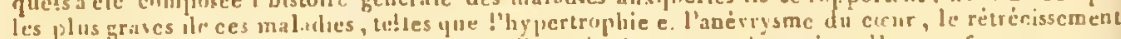

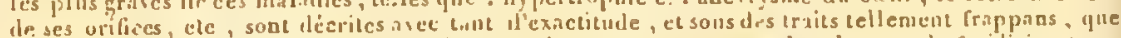

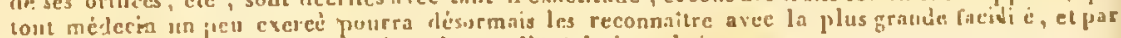
suite les traiter ave fll us de sueceis qu'on ve l'a Jait jusqu'ici.

TRAITÉ CLINIQUE ET PHYSIOLOGIQUE DE L'ENCÉPHALITE OU INELAMMATIOYY DU CERVEAU el de ses suites, telles que ie ramollissement, la suppuration, les tubercules, le syuirthe, le caneur, ctr.; par J. Bourluud, doeteur cn médecine de la ficulté de Paris, aucien interne des hôpitaux dé la mếme ville, metubre de plisicurs sociétés savantes. Paris,

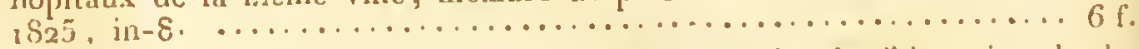

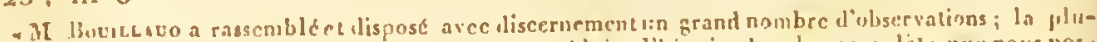

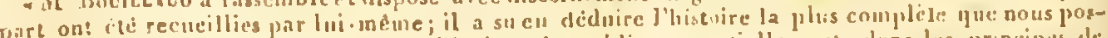
seffions sur l'cnecphalite. Le lirre quil vient te publier, essentiellemens dans les principu's de

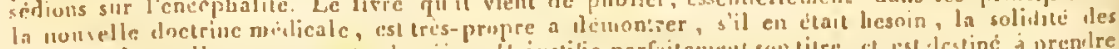

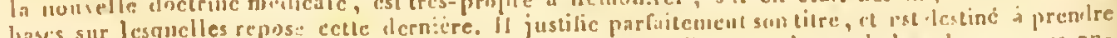

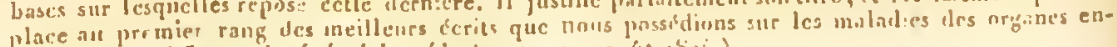

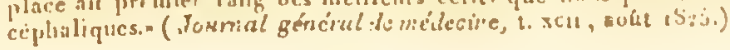


TRAITÉ CLINIQUE, ET EXPERIMENTAL DES FIEVRES DITES ESSENTIELLES ; par J. Bovllaud, docteur cnmédecine de la faculté de Paris, aucien interate des liópitaux de la méne ville, Membre de l'Aaradémic royale sle Médecine, ute. Paris, is 6 ; in-S..................... $7 \mathrm{fr}$.

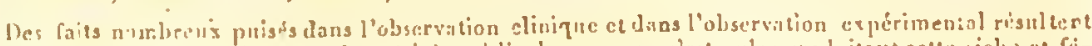

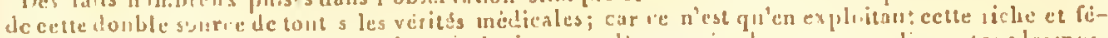

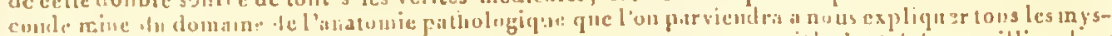

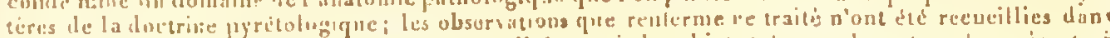

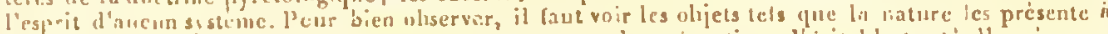
nus rearals, et pour les soir ainse, if faut ètre es emplt de prévention. Véritibl. tralté d'expérience

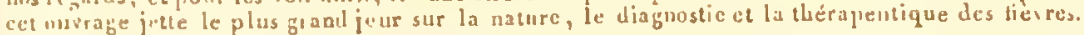

TRRAITE DES MILADIES DES ENFANS NOUVLAUX-NÉS E'I A LA MA.MELLE, fondé sur de nouvelles olsservations cliniques et d'anatomic p:ntholugique fitites à l'hópital d:'s Enfins. Trouvés de Paris, dans le service de

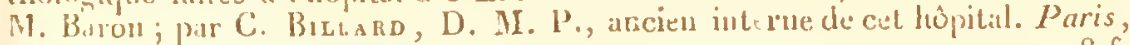

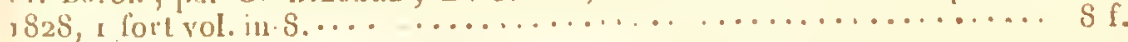

ATLAS D'ANATOMIE PATHOLOGIQUE, pour servir à l'histoire des malinlies les culans; par C. Bulland, D. M. P. Paris, 1828 , in-f, de dix planclies, ivec un texte explicat.f. ........................ to $\mathrm{f}$.

bes platches, exicutces sur les dessins de l'miteur, ont ite graves, impriaces en coulcur, et retoncbies au pinceau avec som par. II. Dumenil.

Dans ect ourrage. le but prim:ipal de l'autem est d'exposer les earactires des symplémes propres inx maladies des enfans, e't de les considerer dans le's's raptrorts avec les altereltious des orfanes. II a passé auceessiventent en revue tous les apporeils; il s'ent applipne à etudier lis sarietes de forme I t d'asuert de chaque organe considere dans l'itat sain, dous lietnt normal el dans l'état patlologique;

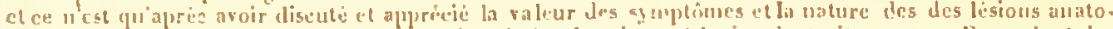

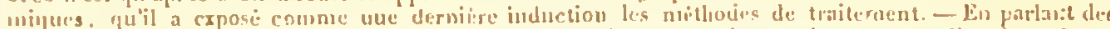
sices do conformation, il a particulierenent fait ressortir erux yui pouvaient donner lieu a quelques

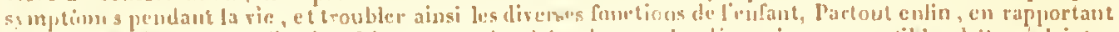

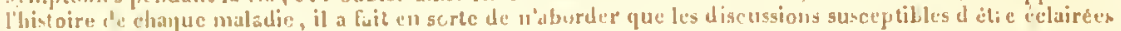
f.ur des litits.

TRAITE ELEMENTAIRE DE LART DE: ACCOUCHEMENS, ou principes de tocologie el d'embryologie; pitr A. A. L. M. Velpead, D. L. P.,

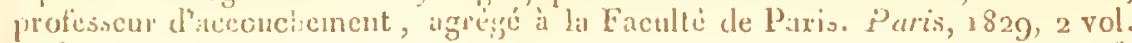

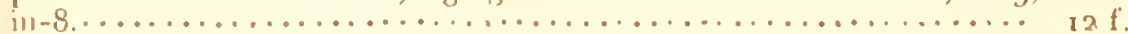

Cet ouwnge est le texte des lrcons publiques, futtes par lauleur ì un nombreuz anditoire depuispries

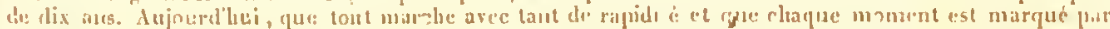

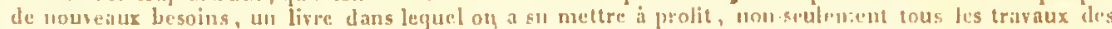

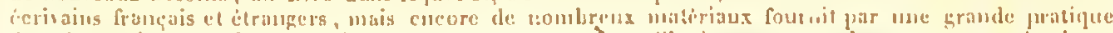

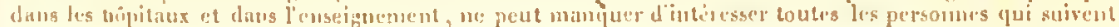
iys prozrios de la scieiece.

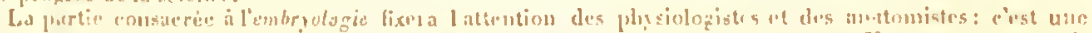

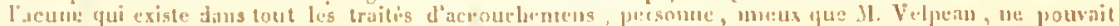

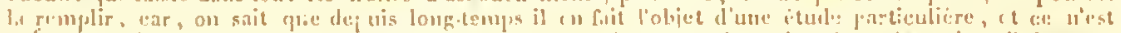

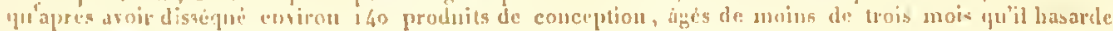
ses opini.me.

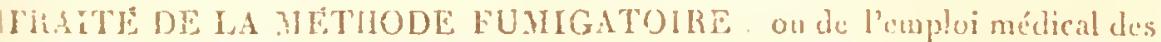
bains el douches de vapeurs, avec platuches pro: T. RAsoc , D. M. P., ancien

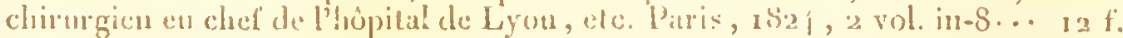

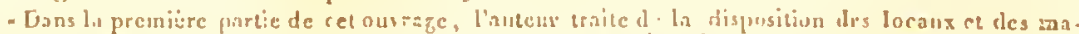

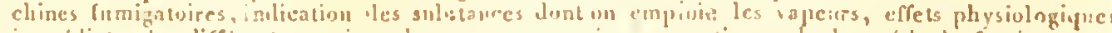

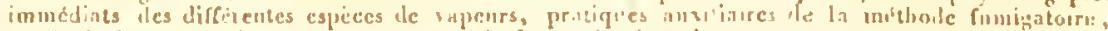

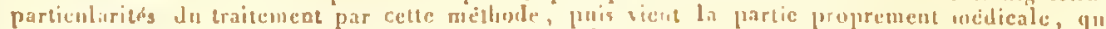

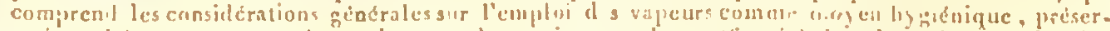

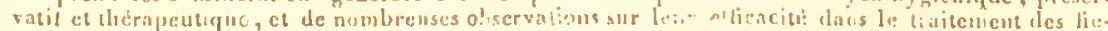

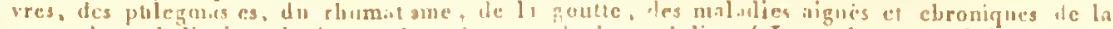

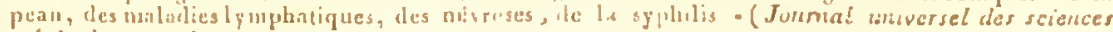
méaicales, airll traj. ?

IANNALES DF, LA METLODE FUMIGATOLRE, on Rccuril J'obsersi-

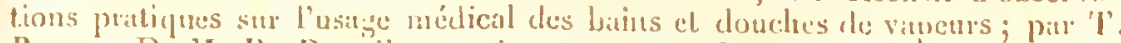
Rapov, D. M. P. Première partie reprisentanc les itonueanx appareils pror-

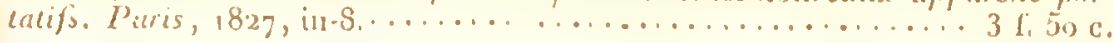


TRAITE DES MALADIES DES ARTISANS et de celles qui résultent des diverses professions, d'aprits Ramazzini ; ouvrange dais lequel on inilitue les prérantions que doivent prentle, sous le rapport de la sulubrité publique et particulicre, les administrutcurs, nanuticturiers, fabricans, chefs d'aleliers, ortistes, at toutes les personnes quii exereent des professions insilubres ; pir Pu. P'Atissier, docteur en médecine de la faculté de Paris, etc. Paris, is $2 \%$;

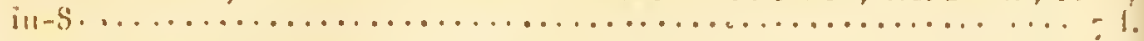

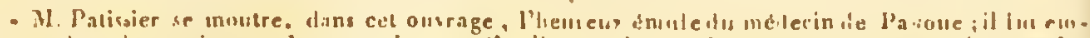

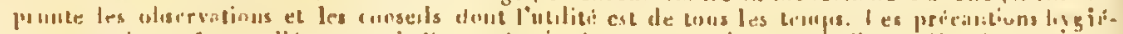

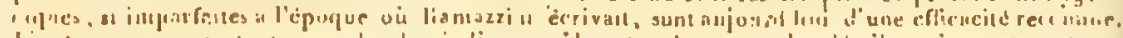

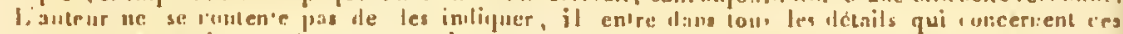

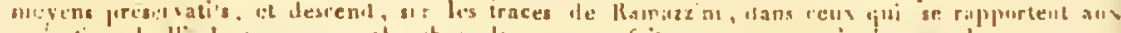

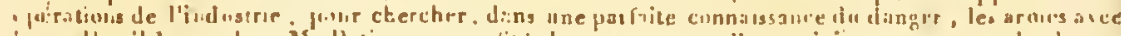

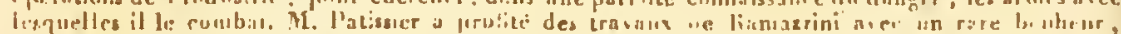

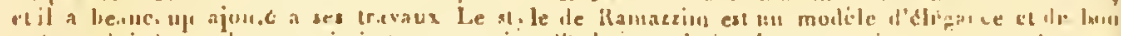

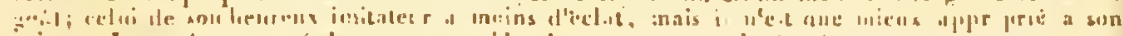

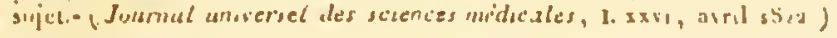

TRAITED DE THÉRAPEUTIQUE, rédigé suivant hes principes de lis nonve:l l: doclrine inúdicalle; par L. J. Bégıs, docteur en méd cine, m"mbrc de l'acadímic royiale de prédecinc, etc. Paris, , 1825, 2 vol. in-8............ 121.

Apres dix années cmployées ilisterter aur les enuses des maladics et sur la nature ules lésiuns "1.i leo coustituent, il est teups que les medecins observateurs sourcettent a une sévere atialyse t.a in unitire il'agir iles nunyens liggieniqu. mu médicomenteus dont ils prescrivect l'usage afiu le ro"

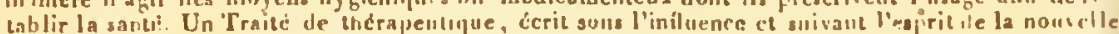

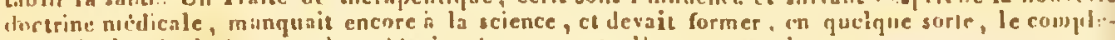
sicul de la révolisticn fue la néllccine éprouve actuellement parmi nous.

1.a thérapculipje a fait, Iepuis quelques anndes, f'imuenses, d'incuntestables progris: mais ils

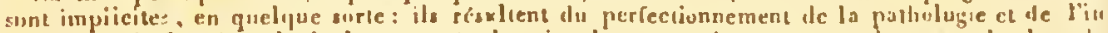

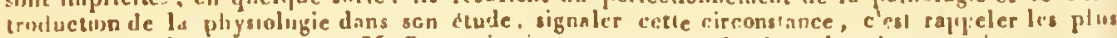

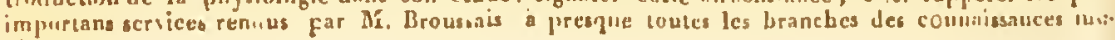
lličalęs.

TRAITÉ DE LA GRAVELLE, DU CALCUL VÉSICAL el des uUt'es miLadies qui se rattacient a un dérangement des fonctions des organ.`s uriuaires, pir Willian Provt, membre de lis société royale de Londres; Iratuit ale: l'an-

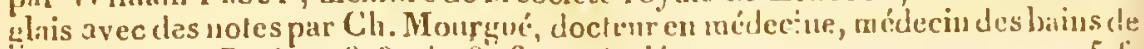

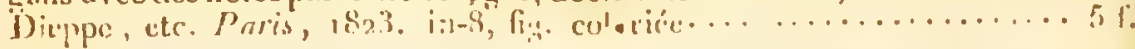

- L'ouvräe de MI Pand cal à cuup sfir un des uncilleurs trailés que nous agon sur les ur ies

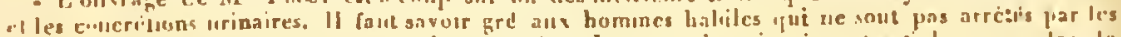

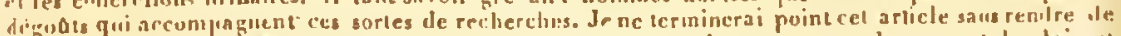

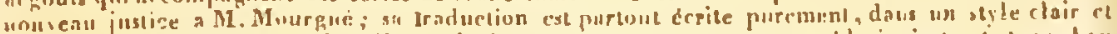

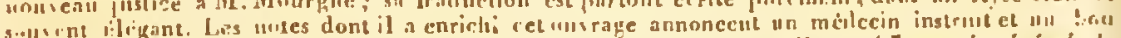

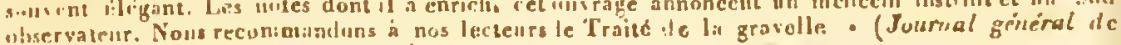

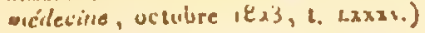

TP.SITE DE LAPOPLEXIE, on HÉnorthagic cérébrale ; considérations nonr:lles sur les hycurocéphales; description d'une hydropieic cérébrale partici-

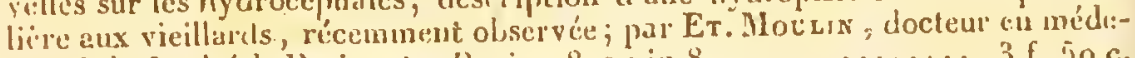

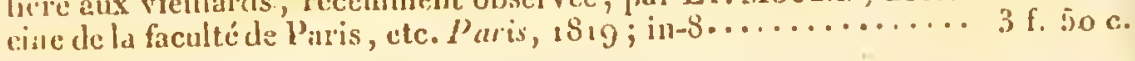

TRAITE THÉORIQUE IE' PRATIQUE DES MAIADIES DE I.A I'EAU, fondé sur de nowvelles rer.herehes l'anatornit et de plyysiologic pathologígues;

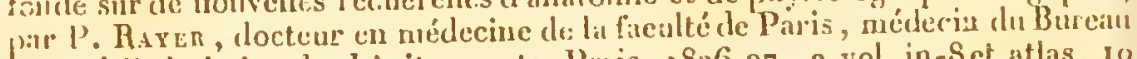
st: 1 trald'admission des hòpitaux, clc. Pill is, $183_{3} 6-27,2$ vol. in-Set atlas, I0 phanches coloricies, ollowil plus de 60 variéties de nuiladies cle peau. $27 \mathrm{f}$.

Rien un'ennonite par des mairs babiles, ccte partie si importnute de la pathologie dtait encorc

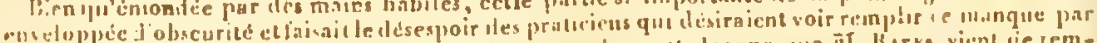

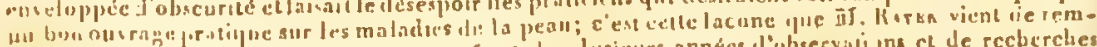

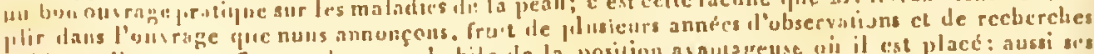
plir

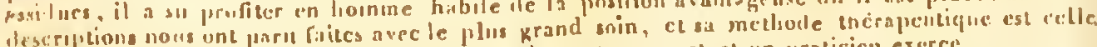
tien

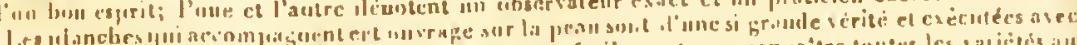

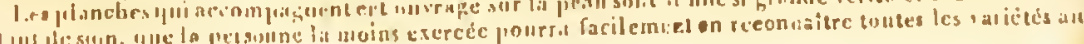
IIt 
"TRATÉ MÉDICO-CHIRURGICAL DE I:INFIAMMATION, JHI. J.

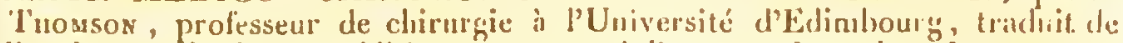
l'anglais sur la dernière édition et mugmenfé d'un grand nombre de nolés par. A. J. L. Journas et F. G. Boisseau, membres de l'Académic royate if: Médecine, etc. Paris 1827 , un fort vol. in $8 . \ldots \ldots \ldots \ldots \ldots \ldots .96 \mathrm{fr}$.

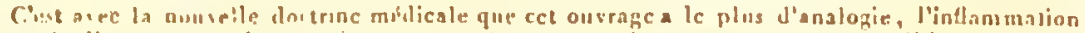

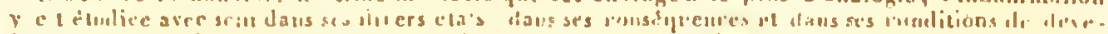

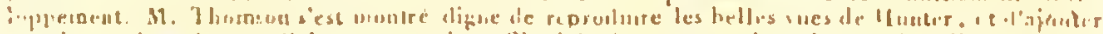

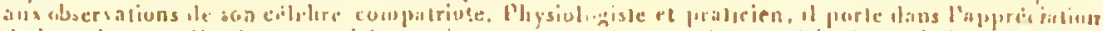

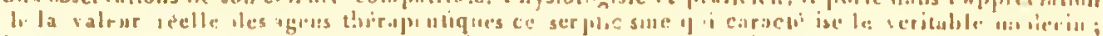

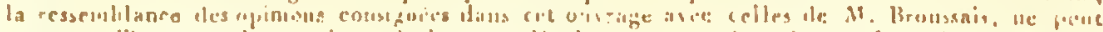

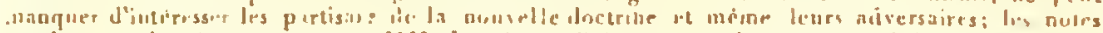

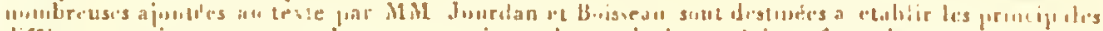

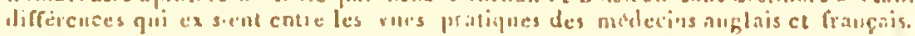

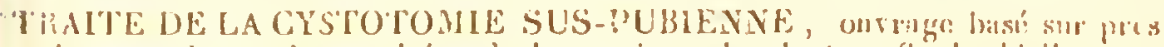
de ent olsservalions, tirées de lis pratique du docfeur Sonberbiclle, par

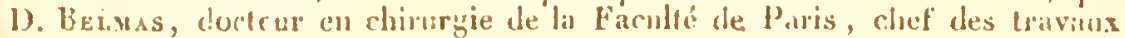

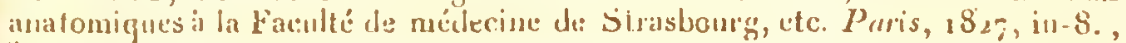

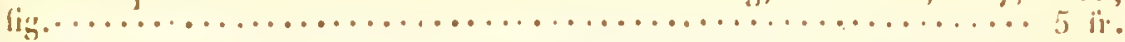

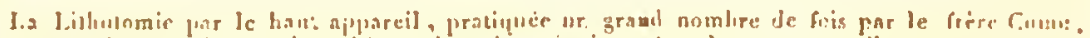

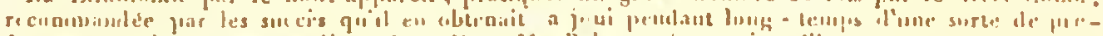

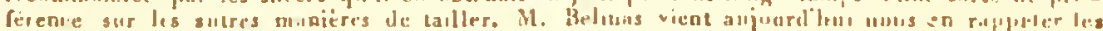

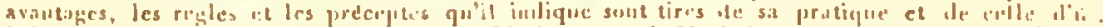

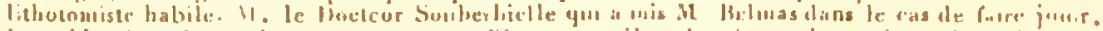

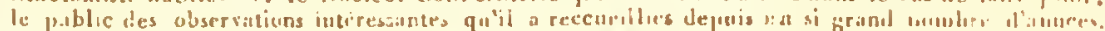

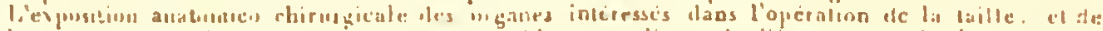

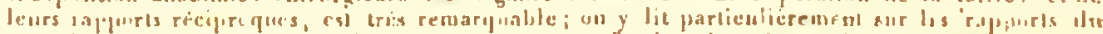

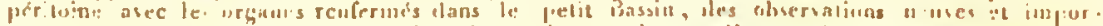

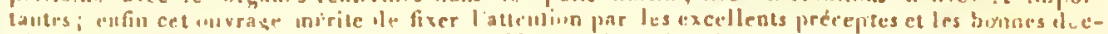

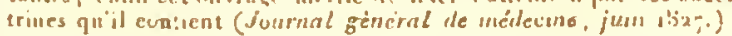

"TRAITE' THEORIQUE ET PRATIOUE DU CKOUP, d'Apris les principus de la doctrinte pliysiologique, précédé de réilexions sur l'orghanisation des éll -

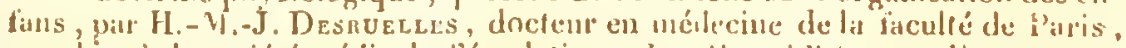
membie de lit suciété médicale d'émulation; deuxièmé édition, entiéremenf ré-

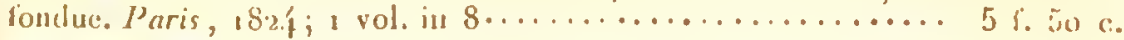

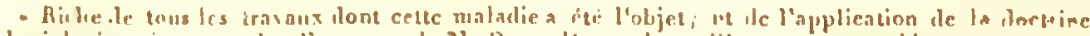

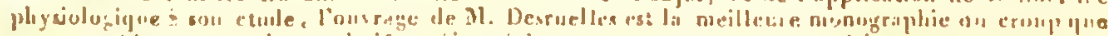

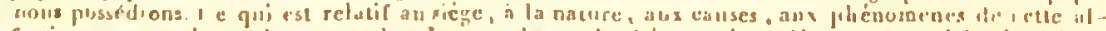

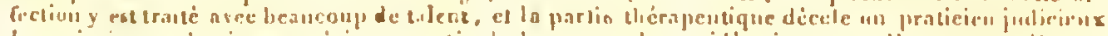

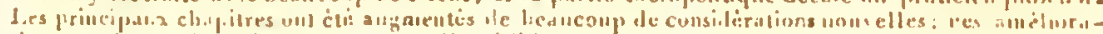

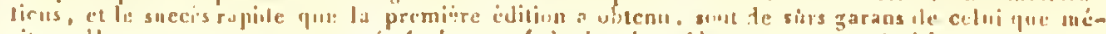

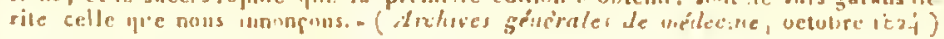

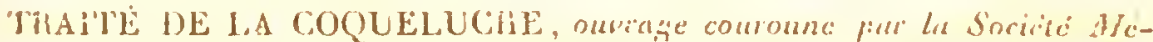
dico-pratigue de Paris; par H Desnues.tes, docteur en medecina de la lis-

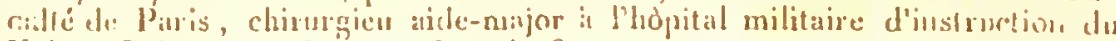

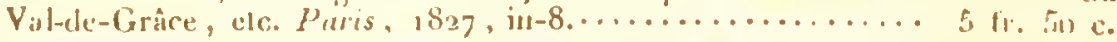

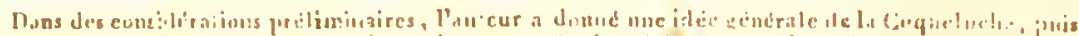

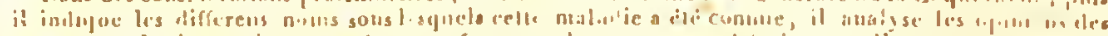

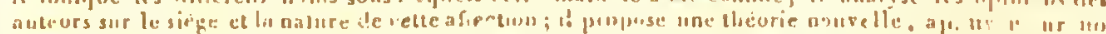

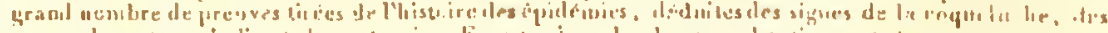

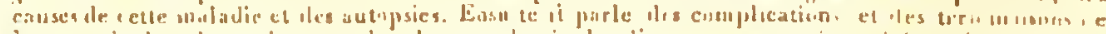

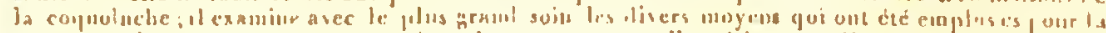

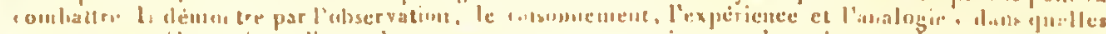

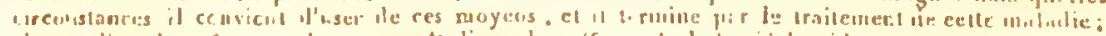

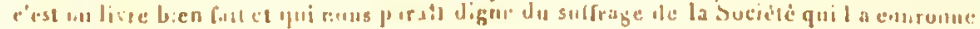

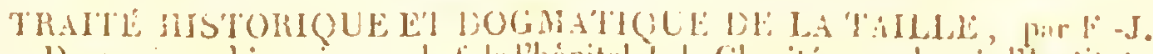

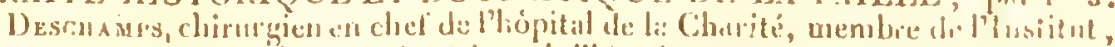

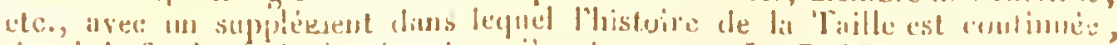

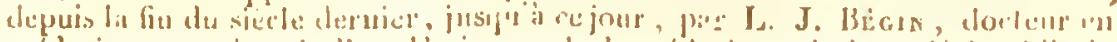

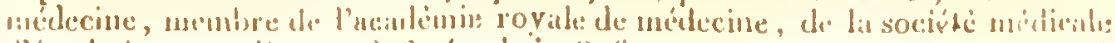

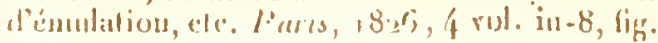

$201 \%$ 
TRAITE, OU OBSERVATIONS PRATIOUES ET PATIOLOGIOUES SUR LE TIAITEIIENT DES MIALADIES DE LA GLANDE PROS'TATE ; pa' Ercrard Hone, chirurgien en chef do l'hôpital Saint-Georges, cle; trad. de l'anglais, ive quatro planches, par Liou Manciant, D. M. Paris, i 820 ;

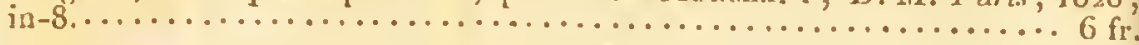

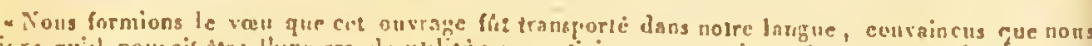

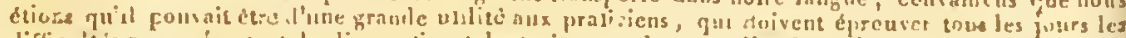
difliculitis que presentent le liacenstie el le iraitement des maladies des veiez urinaires: nous nous

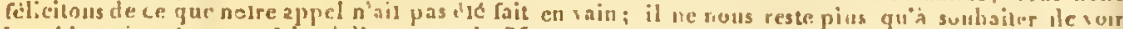

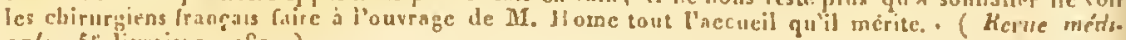
cale : 5 e livraisua, 1820.$)$

CONSIDERA'IONS GENERAITS SUR LES FIEVRES INTERMITTTENTES, CUNTINUES E' REMITTENTES; par MI. OLinet, doctcur en mélecine de lá faculté de Paris, etc. Paris, 1829 , in-8......... 2 i.

COUP.D'OEI, CRITIQUE SUR LA MEDECINE FRANGAISE AU XIXe SIECLE, et sur la nouvelle organisation cy'on projetle; suivi d'un aperçu sur les mesur'ss à prendre pour la tirer de l'état d'ivilissement où elle se trouve; par S Ermand, docteur cn médecine. Paris, 1829 , in-8. If $50 \mathrm{c}$.

COUP-D'OEIL SUR LES CLINIQUES MEDICALES DE LA FACULTE DE MEDECINE et des hôpitan civils de Paris; par F.S. Ratıen, doeLeur en mćdccine Paris, 1830 , in-S......................

DE LA FLUXION VULGAIREMENT APPELÉE PERIODIQUE, ou Recherches historiques, physiologiques et thérapeutiques sur cette maladic, anxyuelles on ajoule dres considéraitions sur le cornage, la pousse et la sectiou des uerfs pmeumogastriques; par. MI. Dupux, dirceteur et professeur de l'école

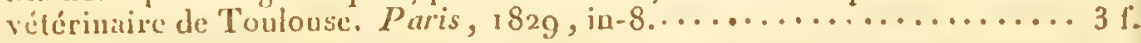

DE LINFLUENCE DES NOUVELLES DOCTRINES MEDICALES FRANÇAISES sur la comnaissance et le traitement des maladics aiguës ; par

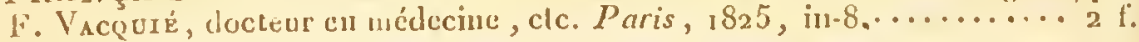

EXISTE-I'-IL TUUJOURS DES TRACES D'INFLAMMATION dans les viscìres aldominaux apres les lic̀res putrides et ataxiques? Cette inflammation est-cllc cause, effct on complication de la fièvre? par le même, Mémoire couronné par la sociéte de médecine pratique. Paris, ${ }_{1} \$_{2} 5$, in- 8 . I $f, 50 \mathrm{c}$.

FLORE ET FAUNE, de Virgile; on Hisioire naturelle des plantes et des aninaux les jus jutriressans à connintte, ct dont ce poc̀te a fait mention; par MI, J.J. Paulet, D. M. P., cortespoudant de l'I histitut. Paris, 1824 , in-s,

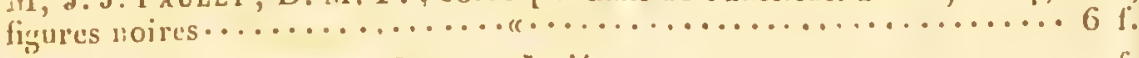

- Le mênc, papier véiin, figures colnoriées................. $12 f$.

HISTOIFE DE L'ANATUNIE ET DE I.A CHIPURGIE, contenant lorigine et les progrés de ces sciencen avec un tableau chronolngique des prinripalies decouvertes, etc.; par A. Poital, membre de l'Institut rogal de

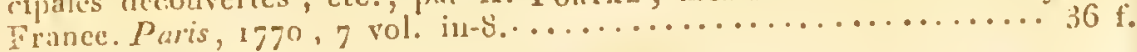

MANUEL PRATIQUE DE LA LITHOTRITIE, ou J.ettres à nn jeune médecin sur le broicment de ha pierre daus la vessic; par A. P. Baxcat, docteur en midecinc, suivi d'un rapporl fait à l'Instilut royal de France, par MlML. Percy, Chaussier, Deschamps, Pellelan ce Alagendic, en laveur de son nonvel instrument pour l'opération de la cataricte par extiaction et d'unc lettre descriplive de la maniere de proliquer au moyen de cet instrument. Paris, 1829 , 1 rol. in-8, avec cing plinches, le pormrait de MI. Dubois, et un finc simile de

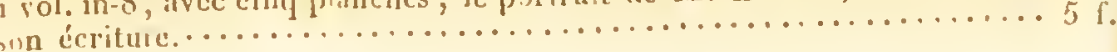


J. B. BAlLLtERE, me de l'Ecole-de-Midecine, no y bis. 3r

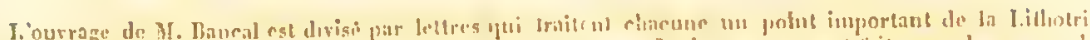

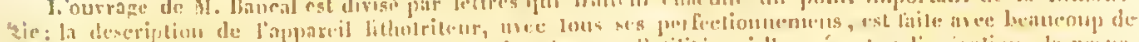

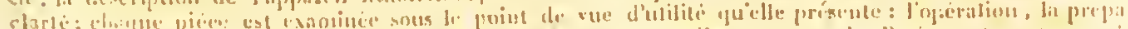

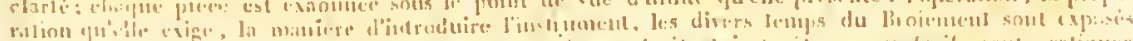

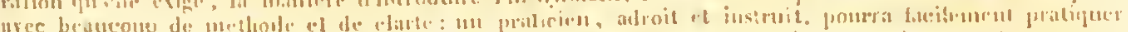

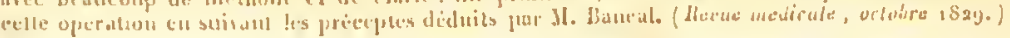

ME.MOIIE ET OBSERVATIONS CLINIQUES SUR LES MIALADIES DE POITRINE DLS ENFANS; par I . TEnReUx, docteur en medecine.

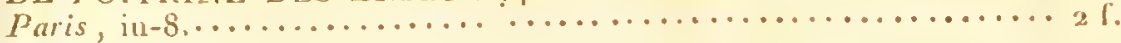

IIEMOIRFS POUR SERVIR A L'IISTOIRE GENERALE DES EAUX MINEliALES sulfurenses et des enux thermiles; par J. Arorada, professeur è la faculté de médeciue de Montpellier, etc. Pars, $18_{2} 8,2$ v. in-8. $12 \mathrm{f}$.

TOTE MEDICO-LEGALE SUR I,A MONOMANIE HUMICIDE; par M. le docteur lisqural. Paris, 1827 , in-8.............

DBSERVATIONS SUR LES MALALIES DQIS ENFANS; par le doctem.

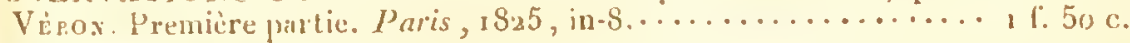

DSTEOGPAPHIE DE LA BALEINE échesuéc an port d'Ostende, précétéc d'une Notice sur ta elécouverle en la dissccion de ce cétacéc; par MI. Duman, cliful gien à Osıcule, membre de plusieus sociélés savantes. т 828,1 vol gr.

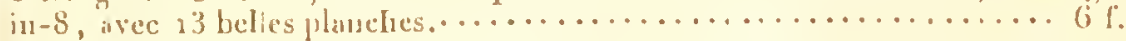

PRECIS D'ANATOHE PATHOLOGIQLF; par G. ANDAL, Jolessenr

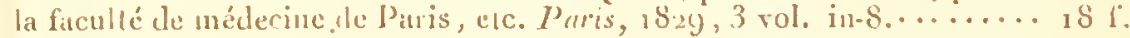

PRECIS D'UNE NOUVELLE DOCTRINE MEDICALE, fondce sur l'anatome quathologinge, et modifiant celles de Mil. Pinel, Bonss:as, Pomma-

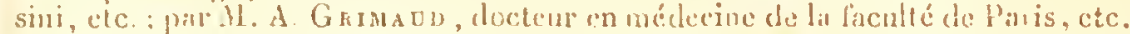

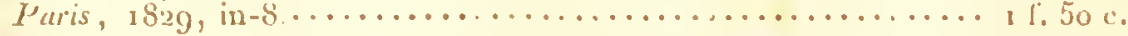

RICIERCHLS SUIK LE SIEGE ET LA NATURE IOES TEICNES; Pa

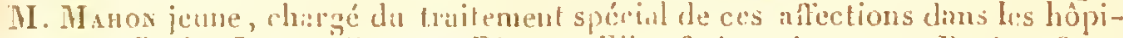

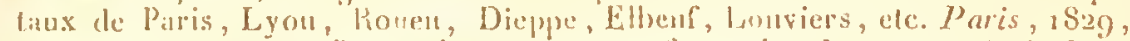
in-8, accompagné de 5 planelics colorices, offrant les diverses rarictés de ce

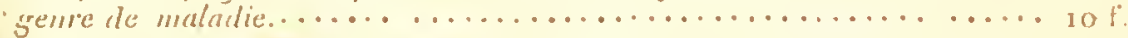

RECHERCHES D'ANATO UIE ET DE PIYSIOAOGL, PATHOLOGIOLES SUR PILCIEUPS HALADIES DES NOUVLAU-NLS; par P'. S. Devis, D. M. P., ancien interuc de l'hospice des entans. Commercy,

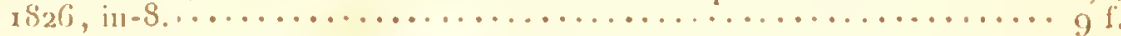

MILMOIRES SUR THOIS GENRES DE: CAS RARES duns l'orlic plyysio-

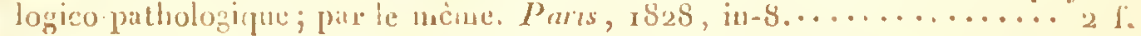

3ECHERCILS PAIOULS SUR LES PRINOIJALES DIFFORIITES

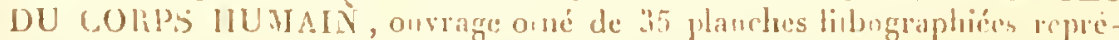

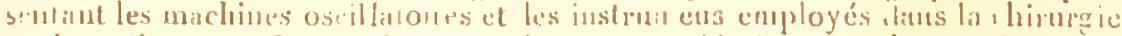

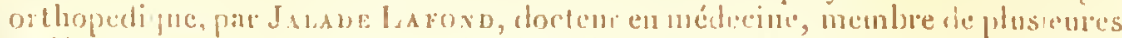

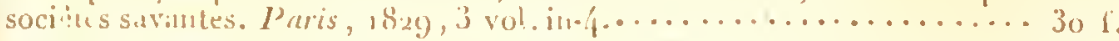

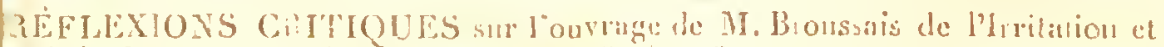

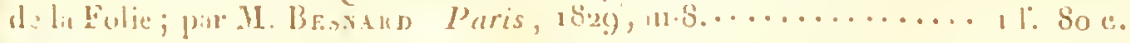

SERLS AUAOUFA, SONT EXPOSLS LES ENPANS (OUE I.EURS

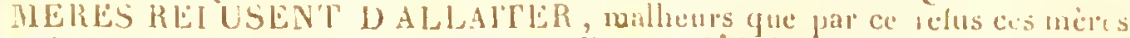

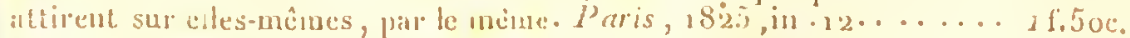

'ENTENDEMENT IIUMAIN MIS A DÉCOUVERT d'apris les prin-

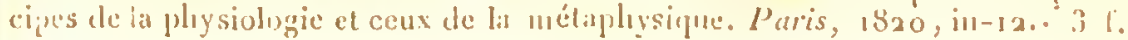


32 J.-B. BalLisine, rue de l'Ecole-de-Médecine, no 15 bis.

TABLE ALPHABÉTIQUE ET MÉTHODIQUE DES MATIÈRES contenues dans les 36 volunes de la denxième séric du JOURNAL GENERAL DE MEDECINE FRANCAISE, ou Recuinl périorlique des travaux de lit sociéte de médecine de Paris; par E. F. S. Gavltier de Cradray, docteur

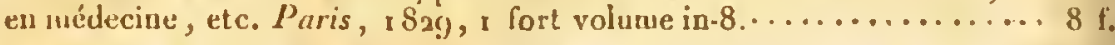

TRAITE DES POISONS tirés des rignnes minéial, végétal et animal, ou TOXICOLOGIE GENERALE considerce sous les rapports de lit pliysion. logie, de la pathologie ê de la médecine légale ; troisième édition ; par MI. ORFita, professeur à la Faculté de médeciuc. Paris, : 827,2 vol. in. $8 . \ldots$. 6 f, 

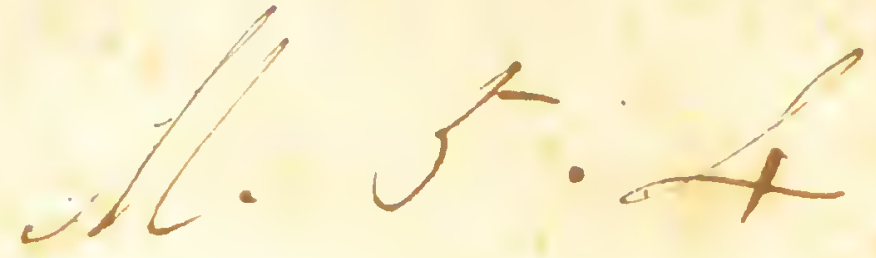

YOUVELLES RECHERCHES

SUR L'ENDOSMOSE

ET L'EXOSHOSE. 


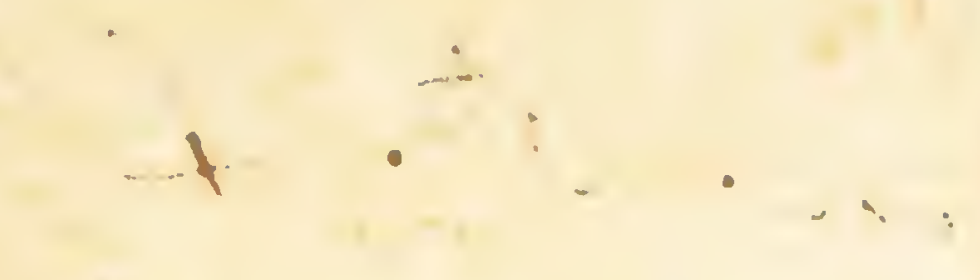

Ouvrages de M. Dutrochet qui se trouvent che: le même libraire.

Recherches anatomiques el physiologiques sur la structure inlime des animaux et des végélaux, el sur leur molililé. Paris, 1824 , in $-8^{\circ}$, fig.

L'Agenl immédial du mouvement vital dévoilé dans sa naure et dans son mode d'action chez les végétaux et les animaux. - Paris, 1826 , in- $8^{\circ}$. 


\title{
NOUVELLES RECHERCHES
}

\section{SUR L'ENDOSMOSE}

ET L'EXOSMOSE,

\author{
SUIVIZS
}

TDE L'APPLICATION EXPÉRIMENTALE DE CES ACTIONS PHYSIQUES

A LA SOLUTION DU PROBLEME

DE L'IRRITABILITÉ VÉGÉTAIE,

ET A LA DÉTERMINATION DE LA CAUSE

DE L'ASCENSION DES TIGES ET DE LA DESCENTE DES RACINES.

\section{PAR M. DU'TROCHE'T,}

'Correspondant de l'Institut dans l'A cadémie royale des Sciences, membre associé de l'Aca. démie royale de MIédecine, correspondant de la Socićté royale ct centrale d'Agriculture, re la Société horliculturale de Paris, des Sociélés horliculturale el inédico-botanique de Londres, de la Société d'agriculture d'Indre-el-Loire, etc., etc.

\section{A PARIS,}

\section{CHEL J.-B. BAILLIERE,}

LIBRAIRE DE L'ACADÉMIE ROYALE DE MÉDECINE,

RUE DE L'ÉCOLE-DE-MIÉDECINE, NO I 3 BIS;

LONDRES, MEME MAISON,

3, BEDEORT STREET, BEDFORT SQUARE;

BRUXELIES, AU DÉPốT DE LA LIBRAIBIE MÉDICALE. 1828. 


\section{ERRATUM.}

Pag. 45, lig. I. L'ouverture $d$, lisez l'ouverture $b$. 


\section{AVANT-PROPOS.}

$\mathrm{J}_{\mathrm{AI}}$ publié, en $\mathrm{I} 824$, ún ouvrage intitulé : Recherches anatomiques et physiologiques sur la structure intime des animaux et des végétaux, et sur leur motilité; en I 826 , j' ai publié un nouvel ourrage intitulé : L'Agent immédiat du mouvement vital dévoilé dans sa nature et dans son mode d'action chez les végétaux et chez iles animaux; depuis ce temps, j'ai fait ide nouvelles recherches qui ont confirímé, en leś modifiant, les résultats aux'quels j' étais parvenu dans ces deux ouvrages. Je réunis ici ces nouveaux travaux, dont quelques-uns ont déjà été Ipubliés dans les Annaies de physique et ide chimie. Par ces nouvelles recherches, Ile phénomène del' endosmose et del' exosimose, que j' ai découvert, se trouve déci'dément appartenir à un nouvel ordre de iphénomènes physiques; el soninterven- 
tion puissante dans les phénomènes vitaux, n'est plus à mettre en doute.

Les recherches de physiologie végétale que contient cette publication, ne sont qu'une partie détachée de travaux plus étendus que j’ai commencés sur cette matière. Mon projet était d'attendre, pour les publier, que la réunion de ces travaux eût fait une masse plus considérable. Je crains avec raison, en publiant prématurément mes moyens d'investigation, de mettre ceux qui courent la même carrière que moi, à même de m'enlever les découvertes commencées que j'ai en portefeuille, et que je ne suis point encore en mesure de publier; mais les circonstances dans lesquelles je me trouve m'ont déterminé à faire cette publịcation hâtive. 


\section{YOUVELLES RECHERCUES}

\section{SUR L'ENDOSMOSE}

\section{ET L'EXOSMOSE.}

Lorseve deux liquides de densité ou de nature ochimique différentes, sont séparés par une cloison membraneuse, il s'établit au travers de cette cloison deux courans dirigés en sens inverse, et inégraux en force. Il en résulte que la masse du liquide ¿accumule de plus en plus dans la partie vers laquelle est dirigé le courant le plus fort. Ces deux courans existent dans les organes creux qui composent les tissus organiques : c'est là que je les ai désignés sous les noms d'endosmose pour le courant d'introduction, $\because$ d'exosmose pour le courant d'expulsion. Un cédèbre mathématicien a cru pouvoir expliquer ces phénomènes par la simple attraction capillaire jointe à l'aflinité des deux líquides hétérogènes. Je vais ici retracer sommairement sa théoric (1).

(1) Note sur des effets qui peuvent étre produits par lu capilHarité et l'affinité des substances héttérogènes, par M. P'oisson. - Journal de physiologie expérimentale, tome 6, p. 36r, et Anuales de physique et de climie, tome 35 , p. $9^{8}$. 
quel jai lail ces expériences, et auquel je donnc lè nom d'endosmomiètre.

Cet apparcil consiste en un tube de verre de (fig. 1), muni inférieurement d'une partie évasée mobile, laquclle offre en bas une ouverture $a b$, qui est fermée avec un morceau de vessie fixé par une forte ligaturc dans la grorge circulaire $i i$. Celte partie évasée est ce que je nomme le réservoir de l'endosmomètre. C'est dans ce réscrvoir que je place lc liquide dont je veux éprouver la propriété d'endosmose. Ce réservoir se détache à volonté du tube, et l’on réunit ces deux pièces au moyen d'un bouchon de liége $c$, traversé par l'extrémitć inlérieure du tube; bouchon qui s'adapte au réservoir comme à une bouteille.

Après avoir rempli le réservoir avec le liquide que je veux éprouver, je le fixe au tube, lequel cst attaché sur une planchette graduée $p p$. Il ne reste plus alors qưà plonger lè réservơir de l'endosnomètre dans l'cau, au-dessus de laquelle le urbe s'élève verticalement. Lorsque le réservoir de l'endosmomètre esc fermé avec unc membrane organique, tel qu'un morceau de vessic, je fixc au-dessous de cette menbrane. une plaque métallique percée d'une multitude de trous. Cette plaque soutient la membrane, et l'cmpêche de se déprimer sous le poids du liquide contenu dans l'endosmomètre. On sent que si l'on ne prenai pas cctte précaution, la dépression de la membrane s'accroissant avec la hauteur du liquide contenu dans l'cndosmomètre, celle dépression loggerait une grande 
partie du liquide introduit par l'endosmose; en sorte que le monvement aseensionnel du liquide dans le uube n'indiquerait point du tout la quantité de l'endosmose.

Lorsqu'on met dans le réservoir de l'endosmomètre un liquide dense, tel qu'une solution de gomme, de suere, ou d'un sel queleonque, et que le réservoir de cet instrument. est plongé dans l'eau, il se manifeste de l'endosmose, et le liquide intérienr s'élève graduellement dans le tube vertical de l'endosmomètre, jusqu’à se déverser par son extrémité supérieure. On obtient le même effet en mettant dans le réservoir de l'endosmoinètre des liquides alkooliques, qui sont cerpendant moins denses que l'ean, mais qui se eomportent comme des liquides denses, en s'élevant peu, comme eux, dans les tubes capillaires. En même temps que l'effer d'endosmose a lieu, il se manifeste un effer d'exosmose. Le liquide contenu dans le réservoir de l'endosmomètre descend en filtrant an travers de la cloison, et se mêle à l'eau, qui est ordinairement le liquide extérieur. Ce mouvement de transport du liquide supérieur le plus dense vers le liquide inférieur le moins dense, pourrait être attribué à une simple filtration, qui serait l'effet de la pesanteur du liquide supérieur. Cette filtration a lieu effeetivement, mais le mouvement d'exosmose a lien d'une manière concomitante. II était essentiel de prouver l'existence isolée dı mouvement d'exosmose, ou plutôt du mouvement qui porte le liquide le plus dense vers to lig 
quide le moind ense. C'est ce que j'ai fait par l'expérience suivante. J'ai mis de l'eau distillée dans le réservoir d'un endosmomètre fermé avec un morceau de vessị. J'ai suspendu cet endosmomètre au-dessus d'un vase qui contenait de l'eau tenant en solution du sulfate de fer. La membrane de l'endosmomètre touchait la surface de la solution de sulfate de fer, sans s'enfoncer dedans. Ce dernier liquide étant plus dense que l'eau distillée contenue dans l'endosmomètre, il devait y avoir, au travers de la membrane, un courant fort qui portait l'eau en descendant vers la solution saline, et en même temps un courant plus faible qui portait en montant la solution saline vers l'eau. Ce dernier courant était ici contrarié par l'effet de l'écoulement, par l'action de la pesanteur; il ne laissa cependant pas d'avoir lieu; car au bout de deux heures ayant essayé l'eau de l'endosmomètre par le nitrate de baryte et par le prussiate de potasse, j'y constatai l'existence du sulfate de fer. Ainsi, l'existence des deux courans antagonistes et inégraux d'endosmose et d'exosmose, est démontrée d'une manière irréfragable: l'écoulement par l'effet de la pesanteur est un phénomène accessoire dont les résultats modifient plus ou moins ceux de ces deux courans antagonistes.

La membrane de l'endosmomètre, en opéranı l'endosmose, produir l'impulsion du liquide ascendant daus le tube de l'instrument; cette action d'impulsion sur le liquide supérieur attestc l'existence concomitante d'une action d'attraction ou d'adfluxion sur le 
Hiquide inférieur. Cette action d'adfluxion est mise ıen évidence par l'expérience suivante : Je prends un iendosmonètre $a b$ (fig. 2 ) fermé avec un morceau de wessic. Je fais correspondre son évasement à celui ' d'un autre endosmomètre renversé $c d$, privé de vessie. Je lute solidement ces deux instrumens l'un à l'autre dans cette position: de cette manière, les deux cavités des endosmomètres sont séparées l'une de l'autre par une seule cloison membraneuse. Je remplis le réservoir, et non le tube de l'endosmomètre ab, avec une solution aqueuse de sucre; je remplis entièrement le réservoir et le tube de l'endosmomètre $c d$ avec de l'eau pure, et je le renverse dans un vase $g$. rempli d'eau colorée. L'endosmose produit l'ascension du liquide sucré dans le tube $b$, et en même temps le liquide coloré du vase $g$ monte dans le tube $d$, et arrive dans la cavité $c$. Ainsi, il y a impulsion du liquide dans l'endosmomètre supérieur, et adfluxion du liquide dans l'endosmomètre inférieur.

Lorsqu'on met de l'eau dans le réservoir jusqu'au sommet du tube d'un endosmomètre, et qu'on plonge cet appareil tout entier dans un liquide dense, de manière à ce que l'extrémité supéricure du tube soil peu au-dessus dı niveau de ce liquide dense, l'eau intérieure sabaisse continuellement dans le tube au-dessous du nivean du liquide dense extéricur. Le mouvement de descente de l'eau au-dessous du niveau du liquide dense extéricur est dû à la même cause qui produit le mouvenent ascensionnel du liquide dense, 
lorsqu'il esi placé dans le réservoir de l'endosmomètre' et que l'eau est le liquide extérieur. Ces deux mouvemens d'ascension et de descente qui dépendent de la position inverse des deux liquides, sont soumis aux mêmes lois.

J'ai posé en principe que tous les liquides plus denses que l'eau produisent l'endosmose, lorsquils sont mis daus le réservoir d'un endosmomètre dont l'eau baigne la partie extérieure. L'acide sulfurique offre une exception remarquable à cet égard.

Si l'on met dans le réservoir de l'endosmomètre de l'eau chargée d'acide sulfurique, ce liquide, plus dense que l'eau, ne produit cependant point d'endosmose; au contraire, ce liquide s'abaisse graduellement dans le tube de l'endosmomètre, lorsque, par une addition de liquide, on l'a élevé au-dessus du niveau de l'eau dans laquelle plonge le réservoir de l'instrument. Dans mon ouvrage ( 1 ), j’ai attribué cet abaissement de l'acide sulfurique à ce que cet acide, au lieu de produire l'endosmose, aurait produit l'exosmose. Mais il n'en est rien; l'acide sulfurique s’écoule ici en filtrant au travers de la membrane, par le seul effet de sa pesanteur et de son élévation au-dessus du niveau de l'eau extérieure. On peut s'en assurer en faisant la contre-épreuve de l'expérience précédente. J'ai mis de l'eau pure dans le réservoir de l'endosmomètre, et j’ai plongé ce réservoir dans. de l'eau mêlée d'acide

(1) L’Agent immédiat, etc. 
inlfurique. L'eau s'est abaissée dans le tube de l'enkosmomètre, comne avait fait l'acide sulfurique dans “expérience précédente. Ceci pronve que cette descente du liquide est due, dans l'un comme dans "autre cas, à la filtration de ce liquide, par le seul fffet de sa pesanteur. Il n'y a aucun courant d'endosnose ni d'exosmose dirigé de l'eau vers l'acide sulfuirque, ni de l'acide sulfurique vers l'ean. Ainsi, je cois relever une erreur dans laquelle je suis tombé rrécédemment. L'observation de la manière dont se comporte l'acide sulfurique m'avait fait penser que les cides sont des agens producteurs d'exosmose; mais il 'en est rien. Le vinaigre, l'acide nitrique, l'acide ydrochlorique, placés dans le réservoir de l'endosnomètre, environné d'eau pure, produisent l'endosnose; l'acide hydrochlorique surtout produit une en'osmose très-énergique. Il se trouve que l'acide sulurique est incapable de produire celte action physique; is en rapport avec l'eau pure, il ne produit ni enosmose ni exosmose; bien plus, on trouve qu'il est innemi de celte double action, car il tend à l'anéanur lorsqu'elle existe. Ainsi, si l'on mêle une petite uantité d'acide sulfurique à une solution de gomme trabique que l'on introduit dans l'endosmomètre, ce (quide ne produit point d'endosmose, quoique la soution de gomme arabique, employée seule, produise nergiquement cet effet. Le liquide gommeux mêlé "acide sulfurique, s'abaisse graduellement dans le tube te l'endosmomètre. Si la quantité d'aciule sulfiurique 
cst evtrêmement petite, il reste encore un peu de force d'endosmose à la solution gommeuse; aussi voiton quelquefois cette solution acide, qui s'est abaissée d'abord dans le tube de l'endosmomètre, reprendre un peu de mouvement ascendant lorsque l'immersion prolongée de la vessie dans l'eau a dépouillé cette solution gommeuse d'une partie de l'acide qu'elle possédait primitivement. Ce fait, très-important, prouve qu'il y a des liquides inactifs, par rapport à la propriété de produire l'endosmose, et que ces liquides peuvent communiquer leur état inactif aux liquides qui ont, à cet égard, des qualités contraires, c'est-àdire qui sont des liquides actifs. Les liquides animaux putréfiés sont inactifs, comme l'est l'acide sulfurique. J'ai fait voir en effet que les liquides animaux qui, à l'état sain, produisaient énergiquement l'endosmose, cessaient de produire cet effet lorsqu'ils étaient putréfiés. Alors j'ai vu ces liquides, au lieu de produire l'endosmose on l'entrée de l'eau extérieure dans les organes creux qui les contenaient, produire au contraire un courant dirigé du dehors au dedans, courant qui évacuait en partie l'organe creux, et qui paraissait devoir ètre attribué à l'exosmose; mais il n'en est point ainsi. Cette filtration du dedans au dehors est un effel purement mécanique produit par la pesanteur du liquide que sa putrćfaction a rendu incactif, el qui, dans cet étal, ne produisant plus d'endosmose, n'obéit plus, dans sa filtration, à d'autres forces qu'à celles de la capillarité et de la pesanteur. 
Il est important de savoir quel est l'agent chimique auquel est due l'inactivité des fluides animaux putréfiés, c'est-à-dire l'inaptitude de ces liquides pour produire l'endosmose. La putréfaction développpe dans les liquides animaux une grande quantité de combinaisons nouvelles, et il était difficile de savoir auquel de ces nouveaux composés chimiques était due l'inactivité du liquide. Ce n'est donc que d'une manière indirecte que je suis parvenu à cette connaissance. En faisant mes expériences sur l'elfet d'endosmose produit par les différens liquides organiques, je ne négligeai pas id'essayer, dans cette vue, les liquides excrémentiels. Je trouvai que l'urine mise dans l'endosmomètre, environné d'eau, produisait l'endosmose. Je voulus essayer, dans la même vue, la matière liquide fécale. Je |pris dans les gros intestins d'une poule une matière fécale liquide, de couleur jaune, ayant fortement l'odeur propre aux excrémens; j'y ajoutai un égal volume d'eau, et je l'introduisis dans un endosmomètre fermé avec un morceau de vessie. Le liquide fécal : s'élevait à une certaine hauteur dans le tube. Ce liq quide ne tarda pas à s'abaisser dans le tube de l'endosmomètre, ce qui me prouva que le liquide fécal, malgré sa supériorité de densité sur l'eau dans laquelle le réservoir de l'endosmomètre était plongé, ne produisait point d'endosniose, et par conséquent était inactif. Pour constater ce fait d'une manière positive, il s'agissait de savoir si l'aljonction de ce liquide fécal inactif à un liquide actif, cnleverait à ce 
dernicr sa qualité d'activité. J'ajoutai au liquide fécal de la poule cinq fois son poids d'eau; et après l'avoir laissé reposer pour laisser précipiter toute la matière solide, je le décantai. J'obtins de cette manière un liquide légèrement jaunâtre, ayant fortement l'odeur d'hydrogène sulfuré propre aux matières fécales. Je mêłai ensemble parties égales de ce liquide et d'une solution aqueuse de gomme arabique, qui contenait 0,04 de son poids de gomme. Ia densité de ce mẹ́lange était $x, 005$, la densité de l'eau étant I. Ce liquide, mis dans l'endosmomètre, s'abaissa rapidement dans le tube, ce qui me prouva qu'il était inactif: cependant, une solution de gomme pure de pareille densité produit très-bien l'endosmose. Je mêlai ensemble parties égrales du même liquide fécal étendu d'eau et d'une solution aquense de gomme arabique qui contenait o, I de son poids de gomme. La densité de ce mélange était $\mathrm{I}, 017$; ce mélange, mis dans um endosmonètre, n'y produisit point d'endosmose : le liquide s'abaissa rapidement dans le tube. Je mêlai ensemble parties égales du liquide fécal étendu d'eau et d'une solution de gomme arabique, qui contenait 0,2 de son poids de gomme. La densité de ce mélange ćtait I,027; ce mélange étant introduit dans un endosmomètre, il y eut une endosmose très-faible durant une heure; au bout de ce temps, le liquide commença à s'abaisser lentement dans le tube, et cet abaissemenı ne discontinua point. Ces expériences prouvent que l'addition d'une petite quantité de lir 
¿quide fécal à dë l'eau chargée de gomme, suffit pour auéantir l'effet d'endosmose propre à cette substance en solution, c'est-à-dire pour la rendre inactive. On voit aussi par ces expériences, qu'en auggmentant la Hose de la gomme, on parvient à contrebalancer uni peu la tendance que manifeste le liquide fécal à lui communiquer son inactivité. A quoi tient cette inactivité bien démontrée du liquide fécal? Il me parut probable que cela dépendait de l'hydrogène sulfuré qu'il contient abondamment. Pour m'en assurer, je mis dans un endosmomètre de l'eau chargée de 0,025 ide 'gromme arabique, et j'y ajoutai 0,005 de son poids d'hydrosulfure d'ammoniaque sulfuré. Il n'y eut poin d'endosmose; le liquide s'abaissa graduellenient dans lle tube. Je recommençai la même expérience, en employant de l'eau chargée de 0,05 de son poids de yomme : il n'y eut point non plus d'endosmose, quoique ces solutions gommeuses fussent par elles-mèmes très-actives ou très-aptes à l'exercice de l'endosnose. IL'adjonction à ces solutions d'une très-petite quanItité de liquide hydrosulfuré suffisait pour leur enlever I toute leur activité, pour les rendre incapables d'opéırer l'endosmose. Si j’ajoutais à ces solutions gom। meuses une quantité plus considérable d'hydro-sulfurc d'ammoniaque, leur endosmose, loin d'être anéantie, semblait, au contraire, être augmentée d'énergie. C.e phénomène provient de ce que l'hydro-sulfure d'anımoniaque est, par lui-même, pourvu d'uctivité; il produit l'endosmose. Ce n'est que par l'hydrogène sul- 
furé libre que développe son addition à l'eau chargéc: d'une substance active, que l'activité de cette substance se trouve abolie. Or, il ne faut qu'une quantité extrêmement petite d'hydro-sulfure d'ammoniaque pour développer une très-grande quantité d'hydrogène sulfuré.

Ces expériences prouvent que c'est à l'hydrogène sulfuré qu'il contient, que le liquide stercoral doit son inactivité ou son inaptitude à produire l'endosmose; et l'on peut présumer de là que c'est à la même cause que l'on doit attribuer l'inactivité que l'on observe dans certains liquides animaux putréfiés, car toute putréfaction animale dégage de l'hydrogène sulfuré.

Il résulte de ces recherches, que nous ne connaissons encore que deux liquides inactifs; liquides non senlement incapables d'exercer ou de provoquer l'endosmose, mais véritablement ennemis de cette action physique. Ces deux liquides sont l'acide sulfurique et l'acide hydro-sulfurique ou hydrogène sulfuré, c'està-dire, d'une part, le soufre uni à l'oxiggène, et de l'autre part, le sonfie uni à l'hydrogène. Probablement l'expérience découvrira, parmi les nombreux agens chimiques, d'autres liquides inactifs.

J'ai voulu voir quel serait l'effet de l'addition de l'hydrogc̀ne sulfuré à l'eau dans laquelle est plongé le. réservoir de l'endosmomètre. Ayant donc introduit dans ce réservoir de l'eau chargée de 0,05 de son poids de gromme, sans addition d'hydro-sulfure d'am- 
I mioniaque, je mis dans l'eau environnante un millièmo de son poids de cet hydro-sulfure, ce qui suffit pour (charger cette eau d'hydrogène sulfuré. L'endosmose se manifesta, et continua pendant quatre heures : au lbout de ce temps, elle s'arrêta, et le liquide devint (descendant dans le tube. Ainsi, l'endosmose est égallement abolie par l'hydrogène sulfuré, lorsque cette ssubstance est mêlée au liquide intérieur, et lorsqu'clle ıst mêlée an liquide extérieur. J'ai fait, à cet égard, lla même observation par rapport à l'acide sulfurique. L'observation prouve que, dans ces deux circonstanices, l'endosmose n'est pas toujours abolie subitement. Dans la dernière expérience, nous avons vu, en ' effet, l'endosmose s'effectuer pendant quatre lieures: 'ce n'est qu'au bout de ce temps que cette action physique s'est trouvée abolie. Cela me fit penser que ce in'était point le simple contact du liquide hydro-snlfuré sur la vessie qui faisait cesser l'endosmose, mais qu'il fallait, pour produire cet effet, que le tissu capillaire de la vessie fût pénétré complètement par le liquide hydro-sulfuré. Pour juger de la validité de ce soupçon, je pris l'endosmomètre qui avait servi à la dernière expérience; je l'évacuai et le nettoyai soigneusement par des injections d'eau pure' ensuite, je remplis son réservoir avec de l'eau chargée de 0,05 de son poids de gomme arabique, et je le plongrai dans l'eau pure. Il ne se manifesta aucune endosmosc; le liquide s'abaissa graduellement dans le tube de l'endosinoniètre : ainsi la vessie, pénétrée d’hydro- 
gène sulfuré, ćtait devenue incapable d'endosmiose; elle était devenue inactive. J'évacuai l'endosmomètrc', je reinplis son réservoir d'eau, et je le laissai tremper pendant vingt-quatre heures dans l'eau pure; au bont de ce temps, je recommençai l'expérience. Alors, il se manifesta de l'endosmose; ce qui me prouva que le tissu de la vessie avait perdu, en totalité ou en grande partie, l'hydrogène sulfuré qu'il contenait. Ce résultat, que nous allons voir bientôt confirmé par une autre expérience, prouve que c'est dans les conduits capillaires de la membrane organique qu'existe la force qui produit l'endosmose. C'est lorsque ces conduits capillaires sont envahis par un liquide inactif, que l'endosmose se trouve abolie.

L'existence bien démontrée de liquides aclifs et de liquides inactifs, de liquides agens d'endosmose ct. de liquides ennemis de l'endosmose, devait faire présumer qu'il existait aussi des solides actifs et des solides inactifs, c'est-à-dire des solides capables d'exercer l'endosmose, et des solides privés d'aptitude par rapport à l'exercice de cette action physique. C'est effectivement ce que l'cxpérience m'a démontré. Tous les solides membraneux organiques sont actifs; tous, étant placés dans des conditions convenables, exercent l'endosmose; mais il n'en est pas de même des solides inorganiques perméables aux liquides, comme nous allons le voir.

Je n'avais d'abord employé que des membranes orgraniques pour fermer l'évasenent tcrminal du réser- 
voir de l'cndosmomètre : il s'agissait de savoil si des lames porøuses nimérales étant substituées, dans les expériences faites avec cet instrument, à la membrane organique, on verrait de même l'endosmose s'opérer. J'ai donc luté, à l'ouverture évaséc d'un réservoir d'endosmomètre, une lame de grès tendre, de six millimètres d'épaisseur; j'ai rempli son réservoir avec de l'eau chargée de 0,2 de son poids de gomme arabique, et je l'ai plongé dans l'eau pure, au-dessus de laquelle le tube vide de liquide s'élevait verticalement : il ne s'est manifesté aucune endosmose; le liquide gommeux intérieur ne s'est point élevé dans le tube au-dessus du niveau de l'cau cxtérieure. J'ai remplacé cette lame de grès par une autre lame de nlême substance, de quatre millimètres d'épaisseur; je n'ai encorc obtenu aucune endosmose : ces deux lames étaient faites avec du grès très-pur, c'est-à-dire exclusivement siliceux. J'ai employé à la même expéricnce une lame faite avec un grès dur et très-fersugincux; elle avait trois millimètres d'épaisseur : j’ai obtenu alors une endosmose très - faible, ou d'une lenteur telle que le liquide intérieur ne fut élevé que de trois millimètres dans l'espace dedeux jours, quoique le tube dans lequel s'opérait cette ascension du liquide gommeux n'eût que quatre millimètres de diamètre intéricur. J'adaptai à un endosmomètre une lame de carbonate calcairc poreux (picrre tendre à bàtir), de huit millimètres d'ćpaisseur : je n'obtins, par ce moyen, aucune endosmose. Pensant que l'ab- 
sence de cet effet d'endosmose ponvait provenir de lit trop grande épaisseur de cette lime, je la remplaçai par une lame de carbonace calcaire plus dır, mais cependant perméable à l'eau, et de trois millimètres d'épaisseur : je n'ai encore obtenu, par ce moyen, aucune endosmose. J'ai essayé, dans le même but, plusiemrs lames faites avec des variétés différentes de carbonate calcaire; je n'ai point eu plus de succès pour obtenir l"endosmose par leur moyen. Enfín, jai adipté à un endosmomètre une lanıe de marbre blanc, de deux millimètres d'épaisseur. Cette substance, quoique très-dense, n'est cependant pas imperméable ¿ l'eau; et j'espérais qu'à raison de son peu d'épaissenr, jobtiendrais ici de l'endosmose; mais mon attente fut trompée : il ne se manifesta aucune ascension du liquide gonımeux dans le uube de l'endosmomètre. Ainsi, il me fut démontré que le carbonate calcaire est un solide inactif, on dépourvu d'aptitude à exercer l'endosmose.

J'ai adapté à un endosmomètre une lame de plâtre (chaux sulfatée calcarifère), de quatre uilliniètres d'épaisseur : je n'ai obtenu, par ce moyen, aucune endosmose. J'ai employé pour la même expérience, et sans plus de succès, la chaux sulfatée cristallisće, qui, comme on sait, se divise en lames extrêmement minces. Mais ici le défant d'endosmose pouvait être auribué à ce que ces lames de substance cristallisée ne seraient pas perméables à l'ean : ainsi, je ne liens compte ici que de la première expérience, qui semble 
prouver que la chaux sulfatée est inactive, ou privée d'aptitude à prorluire l'endosmose.

Les solides siliceux et calcaires étant étudiés sous ce point de vue, il me restait à examiner l'eflet des solides alumineux. Je commençai par l'ardoise. Aı moyen d'une légère calcimation, on rend ce minéral facile à diviser en lames extrêmement miluces. J'obtins de celte manière une lame d'ardoise qui n'avait guère qu'un demi-millimètre d'épaisseur; je l'adaptai à un réservoir d'endosmomètre, que je remplis d'une solution fortement chargée de gomme : jobins un effet d'endosmose très-évident, quoique très-faible. Je pensais alors que l'effet d'endosmose produit par les cloisons perméables qui séparaient les liquides hétérogènes, pouvait dépendre de la très-petite ćpaisseur de ces cloisons, et cette dernière expérience semblait confirmer celle fansse manière de voir. Après avoir essayé dans l'ardoise l'effet d'endosmose produit par un solide alıminenx, il était naturel d'essayer, dins la mêne vuc, des lames d'argile cuite. J'adaptai donc à un endosmomètre une lane d'argile blanche cuite, d'un millimètre d'épaisseur : j'obtins une endosmose assez énergique, et peu différente de celle que j'aurais obtenue, dans le même cas, avec une nembrane organique : le réservoir de l'endosmomètre était rempli, comme à l'ordinaire, avec une solution de gromme arabique. Une lane de la mêne argile, de deux millimètres d'épaisseur, et une autre de cinq millimètres d'épaisseur, ayant été adaplées à des endosmomètres 
remplis ensuite de gomme arabique en solution, jobtins éggalement de l'endosmose. Enfin, des lames d'argile blanche, d'un centimètre et d'un centiniètre el demi d'épaisseur, adaptées à des endosmomètres, produisirent encore de l'endosmose : cependant, la plus épaisse de ces lames n'opéra qu'une endosmose très-lente; ce qui provenait de ce que sa grande épaisseur avait diminué sa perméabilité. Ces faits, qui me prouvaient que le peu d'épaisseur des cloisons perméables n'était point la condition nécessaire de l'effet d'endosmose, comme je l'avais d'abord pensé, me prouvaient en outre que les solides alumineux sont éminemment actifs, c'est-à-dire jouissent éminemment de l'aptitude à produire l'endosmose. J'ai voulu voir si l'addition d'un liquide inactif à la solution de gomme dont je remplissais les endosmomètres, dans ces dernières expériences, anéantirait l'effet d'endosmose, comme cela arrive lorsque l'endosmomètre est fermé avec une membrane organique. Je pris donc un endosmomètre fermé avec une lame d'argile blanche de deux millimètres d'épaisseur, et je mis dans son réservoir de l'ean tenant en solution 0,1 de son poids de gomme arabique, et je le plongeai dans l'eau : l'endosmose se manifesta. Ce premier essai était fait pour constater l'aptitude de mon appareil à exercer l'endosmose. Alors, jajoutai à la solution gommeuse une goutte d'hydrosulfure d'ammoniaque. Dans le premier moment, l'endosmose ent lieu; mais au bout d'un demi-quart d'heure, elle 
commença à s'arrêter, et bientôt il y eut suspension complète de l'endosmose : ainsi, l'hydrogène sulfuré agissait ici en sa qualité de liquide inactif, et paralysait l'action de la solution gommense, de la même manière que cela avait eu lieu lorsque l'endosmomètre était ferrné avec une membrane organique. J'évacuai l'endosinomètre; et après l'avoir soigneusement lavé intérieurement et extérieurement, je remplis son réservoir avec la même solution gommeuse que ci-dessus, mais pure, et je le plongeai dans l'eau. Pendant cinq heures que je le laissai en expérience, il ne se manifesta aucune endosmose. J'évacuai de nouveau l'endosmomètre; et après l'avoir bien lavé, je le remplis d'eau pure, et je le mis tremper dans l'eau pure pendant deux jours. Je renouvelai plusieurs fois l'ean pendant cet espace de temps : alors, je remplis de nouveau son réservoir avec une solution gommeuse pure, pareille à celle employée ci-dessus, et, l'ayant mis en expérience, j'obtins de l'endosmose, mais elle étail moins énergique que dans le principe. Ces expériences prouvent que les liquides inactifs n'exercent leur action pour abolir l'endosmose que lorsqu'ils ont pénétré dans les conduits capillaires de la cloison perméable qui sépare les deux liqnides hétérogènes, et que cette action reste abolie ou diminuée, tant qu'il reste dans ces conduits capillaires une certaine quantité de ces liquides inactifs. Nous avons observé plus haut le même phénomène avec les membranes organiques; ainsi, il est général. 
On pourrail penser que l'inaptiude des solides à produire l'endosmosc proviendrait de cc que, n'étant point assez capillaires, ils scraicnt trop facilcment perméables pour le liqnide contenu dans l'endosmomètre, liquide qui, en vertu de sa pesantenr, s'écoulerait ainsi par des canaux trop peu capillaires pour opposer un obstaclc à cet éconlement. C'est en effet ce qui a lieu quel fuelois. Ainsi, par exeruple, si l'on met cn expérience un endosinomètre fermé avec un morccau de parchemin très-nince, on obtiendra d'abord de l'endosmose, mais bientôt le lissu du parchemin, amolli et dilaté par l'eau qui le gonfle, devient trop facilement perméablc, et dìs lors il cesse d'opérer l'endosmose. Le liquide intérienr de l'endosmomètre s'écoule au traver's de la menbrane, en vertu de sa pcsanteur. Or, ce dernier elfel est pcut-être la cause qui produit l'absence de l'endosmosc, lorsqu'un endosmomètre est fermé avec une plaquc minérale très-facilement permćablc anx liquides. Ainsi, je n'alfirmcrai point ici positivcment que les solides siliceux soient inactifs, car je n'ai essayé que des lanies de gric̀s tendre très-facilement pcrinéables aux liquides. Quant à la lame de grès dur qui a prodıit un per d'endosmose, j'ignore si l'on doil aturibuer cel cf'el à sa capillarité plus considérablc, ou à sa nature particulière; car j’ai constaté par l'analyse chimique, que c'était un grrès très-ferrugineux. N'ayant essayé que des lames diversemcnt épaisses de la même chaux sulfatée assez perméable aux liquides, je n'ai poinc 
des domućes suffisantes pour aflirmer que cette substance soit inactive, quoique cela me paraisse fort probable.

Quant ì la chaux carbonatéc, ayant essayć des lames de celte substance pourvucs de tons les rlegrés possibles de capillarité, et avec tontes sortes d'épaisseurs, sans obtcnir le moindre cffet d'endosmose, je n'hésitc point à affirmer que cctuc substance est contplètement inactive. Il n'y a donc, parmi les solides minéraux, que les solides alumineux qui jonissent éminemment de la qualité que je nomme l'activité, ct qui consistc dans l'aptitude à produire l'cndosmosc. Je n'ai point expérimenté, à cet ćgard, la proprićté des solides magnésiens, ni celle des solides de baryte ou de strontianc. L'argile cuite pord complètcment la faculté d'općrer l'endosmose, lorsqu'elle cst imprégnnée d'hydrogène sulfuré; clle devient alors inactive, mais clle pcut reprendre son activité cn perdant l'hydrogène sulfuré qui la pénètrc. On peut en dire antant des mcmbranes organiques hydro-sulfurécs. La chaux carbonatéc est par elle-mênıc ce qu'cst l'arģile par l'adjonction de l'hydroğc̀ne sulfuré; clle est inactive ou incapable de l'endosmose, quoique pourvue de toutes les conditions de la simple capillarité, ce qui prouve bicu évidenıment que l'aturaction capilJaire n'est pas la cause de l'endosmose; car cette attraction existe dans toute son intégrité dans l'aryilo hydro-sulfuréc, dont la capillarité n'a point étć altéréc par l'bydrogène snlfurć. I es senls liquides dont l'inac. 
tivité soit démontrée, sont l'acide sulfurique et l'acide hydro-sulfurique ou hydrogène sulfuré. Ces deux liquides sont non seulement incapables d'exercer ou de provoquer l'endosmose, mais ils sont véritablement ennemis de cette action physique. Nous ignorons entièrement comment agissent, dans cette circonstance, ces deux liquides sédatifs de l'endosmose.

Quelquefois, lorsqu'on emploie des endosmomètres fermés avec des lámes d'argile cuite, l'endosmose s'arrête subitement, et le liquide intérieur s'abaisse dans le tube. Cela ne tient point, comme on pourrait le penser, à ce qu'il y aurait dans l'appareil quelque élément d'inactivité. Cette suspension de l'endosnıse tient à une autre cause qu'il importe de connaître. Lorsque la lame d'argile est mince et assez facilement perméable, il arrive que le liquide gommeux intérieur filtrant au travers de cette lame, se trouve entduire toute la surface inférieure de la lame qui baigne dans l'eaut. On s'en aperçoit à ce que cette surface, au lieu d'être rude au toucher, est glissante et onctueuse. Dès lors, tout accès est interdit à l'eau pour pénétrer dans les conduits capillaires de la lame d'argile, et par conséquent l'endosmose est suspendue; mais on la voit renaître sur le champ, en essuyant ou en lavant la face inférieure de cette lame d'arçile.

Il résulte de ces expériences que, par rapport à l'endosmose, il y a des solides actifs et des solides inactifs, et que les solides actifs peuvent posséder cette qualité d'activiéé à un degré plus ou moins émi- 
nent. Cesexpériences prouvent de même qu'il y a des liquides actifs et des liquides inactifs, et que les liquides actifs peuvent posséder la qualité d'activité à un degré plus ou moins éminent. Ainsi, l'endosmose résulte de l'influence réciproque des liquides actifs sur les solides actifs, et des solides actifs sur les liquides actifs. Il suffit qu'un seul de ces élémens d'action soir inactif, pour que l'endosmose n'ait point lieu. Ainsi, par exemple, tout étant convenablement disposé pour l'endosmose, cette action sera suspendue par l'addition d'un peu d'acide sulfurique ou d'acide hydro-sulfurique aux liquides, parce que ces deux acides sont inactifs. Ce sera de même en vain que denx liquides hétéroùènes seront actifs; si la cloison perméable qui les sépare est inactive, il n'y aura point d'endosmose. Ainsi, il demeure démontré que ce phénomène résulte de deux influences combinées : $1^{\circ}$ de l'influence des liquides sur le solide, influence qui détermine l'action de ce dernier; $2^{\circ}$ de l'influence du solide sur les liquides, influence de laquelle résulte l'impulsion que reçoivent ces derniers.

Les liquides que l'on peut désigner sous le nom de liquides organiques, opèrent l'endosmose sans discontinuité tant qu'ils ne subissent aucune altération dans leur composition chimique, tant qu'ils restent dans l'état sain. Ces liquides sont, par exemple, les solutions de gomme, de sucre, de gélatine, d'albumine, d'extractif, toutes les émulsions, etc. Il n'en est pas de même des liquides que je désigne sous le nom de 
chimiques, tels que les solutions salines et alkalines, les acides autres que l'acide sulfurique et l'acide hydro-sulfurique, l'alehıol, ete. Ces liquides opèrent tous l'endosnose, mais ce n'est pas sans discontinuite', comme ccla a lien pour les liquides organiques. Les liquides chimiques ont deux actions distinctes: l'une, qui est primitive et directe, par laquelle ils produisent l'endosmose; l'autre, qui est eonsécutive et indirecte, par laquelle ils diminuent ou abolissent cette action physique. Les expériences suivantes mettront eette vérité dans tout son jour.

Une solution de gomme arabique ou de suere étant mise dans un endosmomètre fermé avec un morceau de vessie, l'endosmose aura lieu pendant plusieurs jours, et ne s'arrêtera que lorsque ces liquides auront été altérés par la putréfaction eommenẹante de la membrane organique. Si l'on ajoute à ces solutions un agent chimique, leur aetion d'endosmose sera augmentée, mais elle ne durera pas très-long-teinps, surtout si la dose de l'agent excitateur chimique cst assez considérable; il y aura bientôt abolition de l'endosmose. Je pris une solution de sucre dans l'eau, dont la densité était 1,og5; je notai le nombre de deģrés que le mouventent aseensionnel de l'endosmose faisait pareourir à ce liquide pendant une heure dans le tube de l'endosmomètre fermé avec un moreeau de vessie. Alors j'ajontai an liquicle sucré une eertaine quantité d'hydroehlorate de soude, en sorte que, par cette addition, sa rensité fint portée à 1,2 I . Le mouvement 
ascensionnel du liquide dans le ubbe de l'endosmomère liut environ quatre fois plus rapide dans la premnière heure; mais dans les heuressuivantes, il diminua Graduellement de vîtessc; et enfin, au bout de cinq Ihremres, l'endosnose cessa complètenuent, et le liquide commenca à descendre dans le tube de l'endosmomètre. Cet abaissement du liquide intérieur continua jiusqu’à ce qu'il fût descendu au niveau de l'eau dans łaquelle était plongé le réservoir de l'endosmomètre. 'Alors je retirai le liquide sucré et salé du réservoir, t je trouvai sa densité réduite à 1,115 . Il s'agissait de avoir si l'abolition de l'endosmose était due à l'altéation de ce liquide intérieur, ou à l'altération de la membrane de l'endosmomètre. J'introduisis donc ce iiquide, extrait de l'endosnonètre ci-dessus, dans un mutre endosmomètre dont la nembrane de vessie étai n'aîche. Ce liquide opéra de l'endosmose pendant fluatre heures et demic; alors l'endosmose cessa enrore, et le liquide s'abaissa dans le tube. En même cemps, j’introduisis dans le premier endosmomètre Ifui avait cessé d'agir, une solution d'hydrochlorate de *oude; dont la densité était 1,08, c’est-à-dir'e qui concenait environ une partie de sel sur huit parties d'eau. Il n'y eut point d'endosmose. Je remplaçai cette soluion saline par une solution d'une partie de sucre dans roois parties d'eau, dont la clensité élaii r, 1 ro. L'enllosmose eut lieu, mais avec environ fuatre fois moins lle vîtesse que celle qu'clle avait manifestée an contucncement de l'expóricnco avec le licpuide sucré, dout 
la densité n'était que de r,og5. Ainsi, il est démontré que la membrane organique de l'endosmomètre avait subi, par l'action de l'hydrochlorate de soude, une altération particulière qui la rendait moins propre à opérer l'endosmose. C'est de là que provenait l'abolition de cette action dans les expériences qui viennent d'être exposées. Mais la membrane altérée possédait encore la faculté de produire l'endosmose, en mettant dans l'endosmomètre un liquide nouveau plus actif que celui dont l'action étail devenue impuissante. Quant au liquide sucré et salé qui avait servi à ces expériences, il conservait toujours sa propriété de produire l'endosnose, et cela en vertu de sa densité ou de ses qualités chimiques particulières. Si la solution d'hydrochlorate de soude, dont la densité était 1,08, n'a point produit d'endosmose avec un endosmomètre dont la membrane avait déjà été altérée par l'action de cette substance saline, cela ne provient point de ce que cette solution n'aurait point été apte par elle-même à opérer l'endosmose. Cette solution, en effet, contenait une partie de sel sur huit parties d'eau. Or, j'ai expérimenté qu'il suffit d'ajouter à l'eau deux millièmes de son poids d'hydrochlorate de soude pour la rendre apte à opérer l'endosmose avec un endosmomètre fermé par un norceau de vessie non altérée.

J'ai obtenu des résultats analoggues à ceux qui viennent d'être exposés, en associant l'eau sucrée au sulfate de sonde, à l'acide hydrochlorique, à la potasse caustique (hydrate de potasse), età l'alchool. Toujours 
iil y eut d'abord accroissement de l'endosmose, et cnsuite abolition de cette action au bout de quelques lheures. Cependant, cette abolition n'avait point lieu llorsque la quantité du liquide chimique associé au lliquide organique, était peu considérable; il n'y avait :alors que diminution de l'endosmose. J'ai obtenu des ırésultats analogues, en associant des liquides chimi(ques ì la gomme arabique. Ainsi, les liquides chimi'ques qui, par eux-mêmes, sont aptes à opérer l'cndosımose, augmentent à cet égard l'action des liquides corganiques, lorsqu'ils leur sont associés; mais ils exer(cent consécutivement une action d'abolition ou de dininution de l'endosmose, action qui dépend de ll'altération particulière qu'ils produisent dans la cloi:son membraneuse de l'endosmomètre. Il est bien reimarquable que cette action d'abolition consécutive :soil exercée par des liquides aussi différens entre eux (que le sont, par exemple, les acides et les alkalis, les solutions salines et l'alchool, ctc.

Il était important de rechercher si les liquides chiı miques exerceraient également une action consécutive d'abolition de l'endosmose sur une lanıe d'argile dont sserait fermé un endosmomètre. J'ai donc mis dans le réservoir d'un de ces endosmomètres unc solution :aqueuse de sucre, dont la densité était 1,226, et j’ai inoté la vîtesse de l'endosmose opérée par ce liquide; alors j'ai ajouté à ce dernier une quantité d'hydro' chlorate de soude, qui a porté sa densité à 1,271. La 'vîtesse de l'endosmose a été augmentéc dans la pro- 
portion de 12 à 13 , et cette action a continné sans éprouver beaucoup de diminution pendant vingtheures; alors j’ai augmenté la dose de sel, ce qui a porté la densité du liquide à r,339. La vîtesse de l'endosmose a été augmentée, et j’ai observé cette action pendant trois jours, sans en voir la fin. Ainsi, le liquide chimique introduit dans l'endosmomètre n'a produit, dans la lame d'argile qui le fermait, aucune altération capable d'abolir ou de diminuer l'endosmose. Cette action d'abolition consécutive n'a donc lien que par rapport aux membranes organiques. Or, il est trèsremarquable que l'abolition directe de l'endosmose par l'hydrogène sulfuré, a également lieu avec les membranes organiques et avec les lames d'argile. Ces deux phénomènes d'abolition de l'endosmose n'ont donc véritablement rien de semblable dans leur cause; l'une est une abolition directe, l'autre est une abolition indirecte.

Il résulte de ces expériences, que les liquides qui ont une action ou nne influence quelconque sur l'endosmose, peuvent être divisés en trois classes :

$I^{\circ}$ Les liquides qui ne possèdent d'ıne manière sensible que la senle action de production constante de l'endosmose. Ce sont ceux que je désigne sous le nom de liquides organiques;

$2^{\circ}$ Les liquides qui ne possèdent d'une manière sensible que la seule action d'abolition de l'endosmose. Je ne connais que deux liquides de ce genre, savoir: l'acide sulfurique et l'acide hydro-sulfurique 
wu hydrogènc sulfuré. Cie sont en quelque sorte des sédlatifs sle l'endosmose;

$3^{\circ}$ Les liquides qui possèdent à la fois les deux aclions de production ce d'abolition de l'endosmose. On peut désigner ces liquides par le nom d'excitans chimiques de l'endosmose. Leur action primitive ou directe est la production ou l'augmentation de l'endosmose; leur action consécutive ou indirectc est l'abolition on la diminution de cette action physiquc. Ces excitans chimiques n'aggissent qu'en détruisant on en diminuant dans le solide organique qu'ils traversent, les conditions en vertu desquelles lcur action iexiste.

Les expériences qui vicnnent d'être exposées prou'vent d'une manière incontestable quc la forcc impul- sive à laquelle est due l'endosmose, a son siége dans Iles conduits capillaires de la cloison perméable active 'qui sépare les dcux liquidcs hétérogènes; il s'agit actuellement de rcchercher quclle cst la nature de cette Iforce capillaire inconnue.

L'endosmose est le résultat immédiat de la difféırence de densité, ou plıs généralenıcnı de l'hétérogénéitć des deux liquides que sépare une çloison perméable active. Ce résulıat de la diffćrence de densité de deux liquides doil d'abord faire penser qu'il est dû à une action élcctrique; mais l'expérience physique prouve, ou du moins semble pronver qu'il ne rrésulte point d'électricité du contact des liquides de densité différente. M. Becqucrel a prouvé que le con- 
tact des liquides sur les solides produit de l'électricité; mais cet effet n'est prouvé que pour les liquides qui ont une action chimique sur les solides : or, le contact de l'eau et des liquides organiques sur les deux faces d'une membrane organique, ne produit aucune électricité appréciable au galvanomètre, ainsi que je m'en suis assuré par l'expérience. La cause de l'endosmose reste donc enveloppée de beaucoup d'obscurité. J'avais admis précédlemment que cette cause était l'électricité. Je penche encore à le croire, mais cela n'est point suffisamment-démontré; il n'existe, en fìveur de cette opinion, que des probabilités que je vais exposer. J'ai cité dans un précédent ouvrage (I), l'expérience de M. Porret, qui prouve que les courans électriques de la pile voltaïque impriment à l'eau une impulsion qui lui donne un mouvement ascensionnel, lorsque ces courans sont dirigés au travers d'une membrane organique que l'eau baigne des deux côtés. Ainsi, l'on peut, par ce moyen purement électrique, produire de l'endosmose sans hétérogénéité des li^ quides. Je mis de l'eau distillée dans le réservoir d'un endosmomètre, qui plongeait lui-même dans l'eau distillée. Je mis le fil conjonctif négatif d'une pile voltaïque en contact avec l'eau intérieure, en faisant plonger ce fil dans l'intérieur du tube. Je mis le fil conjonctif positif en contact avec l'eau extérieure. Bientôt je vis l'eau monter dans le tube, et parvenir

(r) L'Agent immédiat, etc. 
à son ouverture sypérieure. L'eau s'écoula aut-dehors, ct cet écoulentent ne cessa que lorsque l'action de la pile se fut affaiblie. Il résulte de ces expériences, qu'il existe deux causes d'endosmose : I I'hétérogénéité des liquides $; 2^{\circ}$ l'électricité de la pile voltaïque.

Nous avons vu plus hant que l'endosmose par hétérogénéité des liquides n'a lieu qu'avec des solides ac¿ifs. Il s'agit de savoir si cette même condition est nécessaire pour l'endosmose par électricité de la pile. Je pris un endosmoniètre fermé avec une lame de grès temdre. Je mis de l'eau distillée dans son réservoir, que je plongeai dans ce même liquide. Je mis le fil négatif de la pile en contacı avec l'eau intérieure, et le fil positif en contact avec l'ean extérieure. Je n'obtins aucune endosmose, et par conséquent aucune ascension de l'eau dans le tube de l'endosmomètre. Je substituai à la lame de grès tendre la lame de grès dur ferrugineux, avec laquelle j’avais obtenu un peu d'endosmose par hétérogénéité des liquides; je n'obtins avec cette lame de grès dur aucune endosmose sensible par l'électricité de la pile; l'eau s'abaissa au contraire dans le tube. Mais ici il y a une cause d'erreur qu'il faut signaler. Le fil conjonctif négatif, en contact avec l'eau intéricure de l'endosmomètre, décompose cette eau, et par conséquent diminue son volume, en sorte que ce liquide s'abaissera dans le tube de l'endosmomètre, si la quantité de l'ean introduite par l'endosmose est inférieure à la quantité de l'cau décomposée. C'est ce qui pouvait avoir lieu avec 
cette lame de grès dır, qui était difficilemeni perméable à l'cau : ainsi, cette expérience ne prouve rien. Cette même expérience, faite avec un endosmomètre fermé avec une lame de pierre à plâtre (chaux sulfatée calcarifère), ne donna aucun indice d'endosmose. Nous avons vu plus haut que cette même subslance ne produisait point non plus d'endosmose par le moyen de l'hétérogénéité des liquides. Mais ici il y. a une cause possible d'erreur qui existe également dans l'expérience faite avec une lame de grès tendre. Gette cause d'erreur consiste dans la possibilité qu'il y a que ces lames poreuses soient trop facilement perméables à l'eau. On sent, en effet, que l'ascension de l'eau dans le tube de l'endosmomètre ne peut s'opér'er lorsque la filıration descendante de l'eau intériemre, par l'effet de la pesanteur, est plus considérable que ne l'est son ascension ou son introduction par l'effet de l'endosmose. Ainsi, ces expériences sont sans résultats bien positifs. Il n'en est pas de même des expériences semblables que j'ai faites avec des endosmomètres fermés avec des lames de chanx carbonatée, pourvues de tous les degrés possibles de la capillarité, depuis la pierre tendre à bàtir jusqu'au maibre blanc. Je n'ai obtenu dans ces expériences aucun signe d'endosmose par le moyen de l'électricité de la pile. On se rappelle que je n’ai de même obtenı aucune cndosinose avec ces lames de carbonate calcaire, par le moyen de l'hétérogénéité des liquides : ainsi, cette subsıance est bien décidément inactive parr rap- 
port aux deux moyens que nous eonnaissons de pro duire l'endosmose. Cependant, j’ai expérimenté que l'impulsion électrique de la pile n'est pas tout à fait sans influence sur l'eau qui traverse les conduits eapillaires de cette substanee, quoique eette impulsion ne puisse élever, l'eau au-dessus de son niveau. Je lutai, à un tube de trente-cinq millimètres de diamètre, une lame de tuff ou pierre tendre à bâtir; elle avait un eentimètre d'épaisseur. Je plongeai vertiealement ce tube dans un vase plein d'eau, en maintenant l'ouverture libre du tube au-dessus de la surfaee de ce liquide : an bout d'une heure, je trouvai 51 grains d'eau qui avaient étć introduils dans ee tube par filtration au travers de la lame de chaux earbonatée, et sous une pression de huit eentimètres d'eau. Je vidai le tube, et je le replaẹai dans l'eau du vase, en faisant correspondre le fil eonjonctif négatif de la pile avec la face intérieure de la lame de ehaux carbonatée; l'eau du vase eorrespondait avec le fil conjonctif positif : au bout d'une heure, je trouvai 54 grains d'eau dans le tube. Ainsi, l'impulsion éleetrique s'était manifestée ici par l'introduction de 3 grains d'ean de plus que ee que pouvail faire la seulc porosité. Je m'assurai de nouveau de la quantité d'cau que mon appareil pouvait introduire, dans l'espace d'nne heure, sans le seeours de l'éleetricité : je trouvai cette quantité un peu angmentée; l'can introduite s'élevait à 53 grains. Alors, je recommençai l'expérience avec le courant élcetrique, et j'eus pour résultat l'introdue- 
tion dans le tube de 60 yrains d'eau: ainsi, le courant électrique dirigé du pôle positif au pôle négatif de la pile, exerce une légère impulsion sur l'eau, pour la déterminer à passer au travers du carbonate calcaire poreux; mais cette impulsion est trop faible pour déterminer l'ean négative intérieure à prendre un niveau supérieur à celui de l'eau positive extérieure. C'est cette faiblesse de l'impulsion électrique qui fait que, dans cette circonstance, il n'y a point d'ascension de l'eau. Ainsi, le carbonate calcaire n'est pas complètement inactif par rapport à l'endosmose au moyen de l'éloctricité de la pile; il est seulement trop peu actif pour produire l'ascension de l'eau. Il n'en est pas de même du grès. En effet, ayant répété l'expérience précédente avec un tube muni d'une lame de grès, je ne trouvai aucune différence dans la quantité de l'eau introduite par simple filtration, en vertu de la porosité, et la quantité de l'eau introduite sous l'influence ajoutée du courant électrique de la pile. Ceci prouve que ce courant électrique est ici d'une influence tout à fait nulle, et que par conséquent le solide siliceux est complètement inactif.

Il nous reste à examiner, dans ce genre d'expériences, l'effet des lames d'argile cuite, que nous savons être très - pourvues d'activité pour la production de l'endosmose par le moyen de l'hétérogénéité des liquides. J'ai donc pris un endosmomètre fermé avec une lame d'argile de deux millimètres d'épaissenr; le réservoir de cet cndosmomètre a été plongé infé- 
rieurement dans l'eau, etsa cavité a été remplie d'eau jusqu'au niveau de l'eau extérieure : alors, j'ai introduit le fil conjonctif négatif dans le tube, jusqu'au contact de l'eau intérieure, et j'ai mis le fil conjonctif' positif en contact avec l'eau extérieure. A l'instant, j’ai vu l'eau s'élever dans le tube de l'endosmomètre, et elle ne tarda pas à arriver au sommet et à s'écouler au-dehors. J'ai répété la mêrne expérience, et avec le même succès, avec une lame d'argile de cinq millimètres d'épaisseur, et avec une autre lame d'argile d'un centimètre d'épaisseur. Dans cette dernière expérience, l'ascension de l'eau dans le tube fut trèslente. Il résulte de ces expériences, que l'argile cuite est très-active pour la production de l'endosmose, par le moyen de l'électricité de la pile.

J'ai voulu, enfin, expérimenter siles liquides inactifs ou ennemis de l'endosmose, par le moyen de l'hétérogénéité, étaient également ennemis de l'endosmose, par le moyen de l'électricité de la pile. J'ai donc répété l'expérience précédente en mettant, au lieu d'eau pure, dans l'endosmomètre, de l'eau avec addition d'hydro-sulfure d'ammoniaque. Le courant électrique de la pile étant appliqué, comme à l'ordinaire, à l'endosmomètre pourvu de sa lame d'argile, l'endosmose a eu lieu sans diminution appréciable. Ainsi, les liquides ennemis de l'endosmose par hétérogénéité des liquides, ne sont point du tout ennemis de l'endosmose par électricité de la pile.

L'endosmose par hétérogénéité des liquides offre 
deux qualités qu'il est important d'étudier dans les variations qu'elles peuvent présenter. Ces deux qualités sont : $I^{\circ}$ sa vîtesse, $2^{\circ}$ sa force.

DE LA VÎTESSE DE L'ENDOSMOSE.

J'entends par vitesse de l'endosmose la quantité dont un liquide s'élève dans le tube d'un endosmomètre dans un temps donné. En général, plus le liquide que eontient l'endosmomètre est dense, plus il y a de vîtesse d'endosmose. Il était important de déterminer quel est le rapport qui existe entre la densité des liquides et la vîtesse de l'endosmose qu'ils sont suseeptibles de produire. Pour faire des expérienees eomparatives à eet égard, il faut d'abord qu'elles soient faites avec le même endosmomètre; il faut, en second lieu, ne comparer entre elles que des expériences qui se suivent immédiatement; car l'endosmomètre fermé avec une membrane organique, avec un morceau de vessie par exemple, offre des résultats très-variables; en sorte que deux expérienees faites l'une après l'antre, et avec lés mèmes liquides, n'offrent point toujour's exactement les mêmes résultats. Si ees deux expérienees sont faites long-temps l'une après l'autre, on obtient quelquefois des résultats qui diffèrent de la moitié. Ces variations proviennent des elıangemens apportés daus la densité, ou dans la perméabilité de la membrane par sa longne 
macération. Ainsi, lorsqu'on veut obtenir des résultats conmarables dans ce genre de recherches, il faut faire chacune des expériences dans le moins de temps possible, les faire immédiatement les unes après les autres, et recommencer plusieur's fois la même série d'expériences comparées, afun de ne point être induit. en erreur par des anomalies accidentelles. Il est tout à fait indispensable que la membrane de l'endosmomètre soil soutenue en dehors par la plaque métallique criblée de trous dont j'ai parlé plus haut. II faut, en outre, avoir soin que l'endosmomètre soit placé daus un local dont la température ne varie point; car, ainsi que je l'ai démontré, l'augmentation de la température accroît l'endosmose.

L'endosmomètre avec lequel j’ai fait les expériences suivantes, possède un réservoir de quatre centimètres ( I pouce $1 / 2$ ) de diamètre. Son tube a deux millimètres de diamètre intérieur. L'échelle graduée à laqquelle il esı fixé est divisée en dixièmes de ponce.

Première série d'expérience.

Je mis dans le réservoir de l'endosmomètre une solution d'une partie de sucre dans quatre parties d'ean. La densité de ce liquide était 1,083. Le réservoir, fermé avec un morceau de vessie, fuu plongé dans de l'eau de pluie. Au bout d'une heure et demie d'expérience, j’avais obtenu 19 degrés $1 / 2$ d'ascension. La densité du liquide sucré devait nécessairement 
avoir subi de la diminution par le fait de l'introduction de l'eau. Effectivement, je trouvai cette densité réduite à $\mathrm{I}, 078$; elle étail, au commencement de l'expérience, à 1,083 : cela donne une densité moyenne de $\mathrm{r}, 080$ pour cetle première expérience.

Immédiatement après, je mis dans le réservoir dn même endosmomètre une solution de deux parties de sucre dans quatre parties d'eau; sa densité était I, I 45. Après une heure et demie d'expérience faite comine ci-dessus, j’avais obtenu 34 degrés d'ascension. La densité finale se trouva être $\mathrm{I}, \mathrm{I} 38$, par conséquent la densité moyenne était r, 141 ponr cette seconde expérience, à laquelle je fis immédiatement succéder la suivante. Je mis dans le réservoir de l'endosmomètre une solution de quatre parties de sucre dans quatre parties d'eau; sa densité était 1,228. J'obtins en une heure et denie 53 desgrés d'ascension. La densité du liquide sucré était réduite à $\mathrm{r}, 216$, ce qui donna uno densité moyenne de 1,223 .

Les résultats de cette expérience prouvent que la vîtesse de l'endosmose n'est point dı tout proportionnelle aux quantités de sucre dissous dans l'eau. En effet, ces quantités sont $\mathrm{r}, 2$, 4: or, en prenant pour base d'une semblable progression le nombre de degrés de la première expłrience, qui est $19 \%$, on aurait pour les élévations ou pour les vîtesses proportionnelles des trois expériences, $19 \% 3,39,78$, tandis que l'observation donne $19 \% 2,34,53$. Ce résultat de l'expérience n'offre également aucun rapport avec les 
(densités respectives des trois liquides sucrés. Les den!sités moyennes de ces liquides sont I, 080, 1, I 4 I, I, 222 : เor, en établissant une progression semblable, dont le |premier terme serait $19 \frac{1}{2}$, on aurait $19 \% / 2,20,22$, i ce qui s’éloigne considérablement du résultat de lex|périence; mais ce qui s'en rapproche tout à fait, c'est ıne progression dont le premier terme serait de même $19 \frac{1}{2}$, el qui serait comme les nombres $0,080,0,54 \mathrm{I}$, 10,222, qui expriment la différence de la densité de ichacun des trois liquides sucrés avec la densité de ll'eau, qui est $r$. Cette nouvelle progression serait $191 / 2,34,54$ : or, l'observation donne $19 \% 2,34,53$. IIl n'y a évidemment entre ces deux résultats que la Ilégère différence qui ne peut manquer de résulter des iin exactitudes inévitables de l'expérience.

\section{Deuxième série d'expériences.}

Le même endosmomètre fermé avec un morceau 'de vessie, fut mis en expérience successivement avec lles trois liquides sucrés ci-après :

$1^{\circ}$ Eau sucrée, densité primitive, 1,045; densité finale, $\mathrm{r}, 043$; densité moyenne, 1,044 ; ascension du lliquide, 10 degrés $1 / 4$ en une heure et demie;

$2^{\circ}$ Eau sucrée, densité primitive, I, 075 ; densité Ifinale, $\mathrm{r}, 065$; densité moyenne, I, o7o; ascension $\mathrm{du}$ lliquide, 17 degrés en une heure et demie;

$3^{\circ}$ Eau sucrée, densité primitive, I, 145 ; densité finale, 1,133 ; densité moyenne, 1,139 ; ascension $\mathrm{du}$ liquide, 32 degrés $1 / 2$ en une heure et demie. 
Les ascensions on les vîtesses proportionnelles de l'eridosinose sont ici $10 \% / 4,17,32 \frac{1}{2}$. Les différences de la densité moyenne des trois liquides sucrés avec la densité dé l'ean, sont 0,044, 0,070, 0, I39: or, en établissant une progression semblable sur $10 \% \frac{1}{4}$, vîtesse de l'endosmose donnée par la première expérience, on aurait 10 $1 / 4,16 \frac{3}{10}, 32 \frac{3}{10}$. Ce résultat du calcul est, comme on le voit, presque entièrement semblable au résultat de l'expérience.

\section{Troisième série d'expériences.}

L'endosmomètre précédent fut fermé avec une lame d'argile très-compacte, épaisse de deux lignes et demie. J'y mis en expérience successivement les trois liqquides sucrés ci-après :

$I^{\circ}$ Eaı sucrée, densité primitive, I,049; densité finale, I,043; densité moyenne, 1,0/46; ascension du liquide, 9 degrrés en six heures d'expérience;

$2^{\circ}$ Eau sucrée, densité primitive, 1,082 ; densité finale, I, 076 ; densité moyenne, I, 079 ; ascension $\mathrm{du}$ liquide, 14 degrés $1 / 2$ en six heures d'expérience;

3. Eau sucrée, densité primitive, I, 145; densité finale, 1,136 ; densité moyenne, 1,140 ; ascension du liquide, 30 degrés en six henres d'expérience.

Les ascensions dans un temps égal, c'est-à-dire les vîtesses de l'endosmose, sont $9,141 / 2,30$. Les excès de la densité moyenue des liquides sucrés sur la densité de l'cau, sont o,o46, o, o79, 0, I 40 : or', en établissant une progression semblable, dont le premier 
rme est 9, on trouve 9, 15,6, 28. Ce résultat du illcul diffère assez peu du résultat de l'expérience, osur qu'on puisse admettre que leur différence tient à ess causes accidentelles d'erreur. Nous allons en actuérir la preuve tout à l'heure.

\section{Quatrième série d'expériences.}

Les trois expériences précédentes ont été faites avec me lame d'argile qui servait pour la première fois. ces expériences suivantes ont été faites avec la même urne d'argile qui servait sans interruption aux expécences depuis deux jours, et qui, par conséquent, zait plus complètement imbibée, et plus facilement srméable que dans le principe.

$1^{\circ}$ Eau sucrée, densité primitive, I, 047 ; densité nale, 1,043 ; densité moyenne, 1,045 ; ascension dı quide, 3 degrés $1 / 2$ en une heure et demie;

$2^{\circ}$ Eau sucrée, densité primitive, 1,258; densité male, 1,252; densité moyenne, 1,255 ; ascension du Fuide, 19 degrés $1 / 2$ en une heure el demie.

Les ascensions du liquide ou les vîtesses de l'enosmose sont $3 \frac{1}{2}, 19 \frac{1}{2}$. Les excès de la densité hoyenne des liquides sucrés sur la densité de l'eau, ont o,045, 0,255. Le calcul de l'ascension établi sur tte proportion donne $3 \frac{1}{2}, 20$, résultat évidemment mblable à celui que donne l'expérience. Ici nous couvons la cause de l'erreur que nous avons soupcon¿ée dans la troisième série d'expériences. Nous voyons ue, dans cette troisième série, l'eau sucrée, dont la 
densité moyenne est 1,046 , a produit une ascension de 9 degrés en six heures, tandis que, dans la quatrième série, l'eau sucrée, dont la densité moyenne est $\mathrm{I}, \mathrm{0} 45$, a produit trois degrés $1 / 2$ d'ascension en une heure et demie, ce qui donnerait 14 degrés en six heures. On voit par-là que la même lame d'argile peut, avec les mêmes liquides, donner des résultats d'endosmose très-différens. Lorsque cette lame est en expérience depuis un certain temps, et qu'elle est bien complètement imbibée, elle opère plus d'endosmose qu'elle n'en opérait dans le principe. C'est pour cela que la dernière expérience de la troisième série offre un résultat supérieur à celıi qui est donné par le calcul.

Il résulte définitivement de ces expériences, que les vîtesses de l'endosmose produites par des liquides intérieurs de diverses densités, sont proportionnelles aux excès de la densité de ces liquides intérieurs sur la densité de l'eau, qui est le liquide extérieur.

DE LA FORCE DE L'ENDOSMOSE.

Pour mesurer la force de l'endosmose, j'ai fail construire un appareil à peu près semblable à celui dont Hales, et, après lui, MM. Mirbel et Chevreul, se sont servis pour mesurer la force ascensionnelle de la sève de la vigne. Cet appareil est un endosmomètre (fig. 3) dont le tube, au lieu d'être droit, est courbé deux 
jis sur lui-même. Par l'ouverture supérieure $d$ de la rande branche ascendante, je verse du mercure, qui ombe dans la courbure inférieure $c$, où il se met de iiveau en $g$. Au sommet de la courbure supérieure st une ouverture $b$, par laquelle j'introduis le liquide rue je veux mettre en expérience dans le réservoir $a$. ce remplis du même liquide la partie $e b$, ainsi que a partie $b g$. La pression de la colonne $b g$ de liquide cfoule le mercure jusqu'en $f$, et le porte jusqu'en $i$ ians la branche ascendante $c d$; alors je ferme l'oucerture $d$ avec un bouchon très-solidement maintenu war un coin placé entre ce bouchon et un épaulement que porte la planche sur laquelle l'appareil est iixé. De cette manière, il n'y a point d'air dans la vartie ebf du tube; elle est remplie du même liquide rue contient le réservoir $a$. L'ouverture $o$ du résercoir est fermée avec trois morceaux de vessic superpo'és, lesquels sont fixés très-solidement, au moyen de igatures, dans les deux gorges circulaires dont le récervoir est muni. Je fortifie cet assemblage par dehors mar l'addition d'un morceau de fort canevas. L'ouverure $o$ du réservoir a cinq centimètres (un pouce dix iignes) de diamètre. Lorsqu'on veut faire marcher 'expérience, on plonge entièrement le réservoir $a$ lans un vase plein d'eau $h$, que l'on peut ôter et renettre à volonté sans déranger l'appareil. Dans l'état yù se trouve l'appareil par la description que je viens le donner, la membrane qui ferme l'ouverture $o$ de 'endosmomètre n'est pressée que par la colonne de 
liquide $e b$. La colonne $c i$ de mercure est érgale en pesantcur à la colonne $f c$ de mercure, plus la colonne $f b$ de liquide.

Cet appareil étant mis en expérience, l'endosmose introduit l'eau du vase $h$ dans le réservoir $a$. Le volume du liquide intéricur étant ainsi augrmenté, la surface $f$ du mercure est refoulée en bas, et la surface $i$ prend un molıvement ascensionnel. Le diamètre intérieur de la branche descendante $b c$ est beaucoup plus considérable que ne l'est le diamètre intérieur de la branche ascendante $c d$, en sorte qu'une faible dépression de la surface $f$ du mercure correspond à une ascension plus considérable de la surface du mercure en $i$. Sans cela, on ne pourrait observer en $i$ qu'une ascension égrale à $f c$, ce qui scrait trop peu considérable; d'ailleurs, la dépression du mercure en $f$ est diminuée par la dépression qu'éprouve la membrane oo, dépression qui est d'autant plus considérable, que la colonne de mercure est plus élevée en $i$, Cette dépression de la membrane oo est ici sans inconvénient, et la force de l'endosmose s'apprécie d'une manière exacte par la pesanteur de la coloune de mercure comprise entre les"deux niveaux $f, i$, en diminuant sur le poids de cette colonne le poids de ta colonne $f b$ du liquide, et en y ajoutant le poids de la colonne $e b$ du liquide intérieur, dont la pesantcur spécifique est connue. Ce calcul ne se fait qu’à la fiu de l'expérience, pendant le cours de laquelle il n’est besoin que de constater l'existence du mouvemen 
ascensiomel du mercure en $i$. Lorsque ce monvement ascensionnel s'arrête, l'expérience est terminée.

La gomme arabique et le sucre sont les seules subsllance en solution dont je me sois servi dans mes expériences sur la force de l'endosmose. J'ai fini par donner la préférence au sucre, qui a sur la gomme l'avantage très-considérable d'agir sur la membrane ide l'endosmomètre, comme substance conservatrice, ien retardant sa putréfaction, propriété tout à fait étrangère à la gomme. Lorsque le liquide intérieur acquiert me odeur putride, il cesse d'être propre à l'endosmose, et cela par l'effet de l'hydrogène sulfuré 'que développe toute putréfaction animale. Or, on prévient cet effel, en mettant dans le réservoir de l'endosmomètre une solution aqueuse de sucre suffisamment chargée; alors il 'n'y a plus que la partie extérieure de.la membrane dont la putréfaction commençante puisse imprégner d'hydrogène sulfuré l'eaı dans laquelle baigne le réservoir de l'endosmomètre. Lorsque cela arrive, l'endosmose s'arrête, mais elỉe recommence de suite, en mettant de nouvelle eau pure dans le vase où baigne le réservoir. D’après cette observation, j'avais soin de changer souvent cette eau extérieure. Une solution d'une partie de gomne dans trois parties d'eau, solntion dont la densité était r, 095, avait élevé le mercure à 75 centimètres ( 28 ponces). C'était la limite du tube de mon appareil, mais ce n'était pas celle de la force d"endosmose qui existait. dans cette circonstance. Je construisis donc un endos- 
momètre dont le tube avait plus d'étendue, êt je me servis exclusivement d'ean sucrée dans les expériences subséquentes. Ces expériences, que j’ai multipliées pendant plus de deux mois, exigrent de la patience. Ge n'est que par de nombreux tâtonnemens que je suis parvenu à des résultats tels que ront les offrir les expériences choisies que je vais exposer. Voici comment je procédais à ces expériences. Le réservoir de l'endosmomètre étant rempli du liquide sucré dont la densité m’était connue, et ce réservoir étant plongé dans l'eau, je versais du mercure dans la grande branche ascendante de l'endosmomètre par l'ouverture $d$, et cela jusqu'à une hauteur arbitraire, mais de beallcoup inférieure à la hauteur à laquelle la colonne de mercure devait être portée par la force de l'endosmose. Mes expériences antécédentes m'avaient fourni des données approximatives à cèt égard. J'attendais que le mercure eût monté dans le tube par l'impulsion de la force d'endosmose; alors j'ajoutais une certaine quantité de mercure à la colonne, en le versant par l'ouverture supérieure $d$ du tube. J'attendais encore que l'endosmose eût fait monter la colonne; alors j’ajoutais de nouveau mercure. Je cessais d'opérer cette addition à la hauteur de la colonne, lorsque je voyais, par l'extrême lenteur de son ascension, que la force de l'endosme approchait de sa limite; alors je laissais cette force opérer seule l'ascension du mercure, jusqu'au point où cette ascension s'arrêtait définitivement; alors je calculais, comme je l'ai dit plus haut, 
la pesanteur de la colonne de mercure soulevée par l'endosmose. J'évacuais ensuite le réservoir de l'endosmomètre par l'ouverture $b$, et je mesurais la densité ou la pesanteur spécifique du liquide sucré extrait de ce réservoir. Cette densité finale devait être seule prise en considération, puisque c'est sous son influence que s'ćtait terminée l'ascension de la colonne de mercurc. Ces explications donnécs, je vais exposer trois des expériences par lesquelles je suis parvenu à la comnaissance de la loi qui préside à la force de l'endosmose.

J'ai préparé trois solutions aqueuses de sucre, dont les densités étaient I,035, I,070, I, I 40. Cette dernière contenait un peu moins d'une partic de sucre sur deux partics d'eau. Les excès des densités de ces trois solutions sur la densité de l'cau étaient, comme. on voit, dans la progression I, 2,4 .

Je mis dans le réservoir de l'endosmomètre la solıtion sucrée $\mathrm{I}, 035$, et je le chargeai d'une colonne de mercure d'un pouce de liautenr. L'expérience fuı conduite comnıc il a été dit plus haut; et an bont de vingthuit heures, l'ascension de la colonne de mercure s'arrêta à̀ 286 inillimètres ( 10 pouces 7 lignnes). Je fais entrer dans cette estimation le poids de la colonne d'eau sucrée qui pesait immédiatement sur la meinbrane et l'endosinomètre. Le liquide sucré, pesé après l'expérience, se trouva réduit à la densitć de 1,025 , densité qui est ì peu près celle d'une solution qui conticnt une partic de sucre sur seize partics d'cau. 
Immédiatenient après cette première expérience, je mis dans le réservoir de l'endosmomètre la seconde solution sucrée $I, 070$, et je la chargeai d'abord d'une colonne de mercure de 27 centinètres ( 10 ponces) de hautciu. L'expérience dura rrente-six henres. An bout de ce temps, l'ascension de la colomne de inercure s'arrêta, et jévahuai sa hauteur à $6 \mathrm{I} 7$ millimètres ( 2.2 pouces ro lignnes). Le liquide sucré, pesé après l'expérience, était rédıit à la densité de 1,053 , densité qui esi à peu près celle d'une solution qui contient une partie de sucre sur sept partics d'eau.

Je mis ensnite en expérience le troisième liquide sucré 1,140 , et je le chargeai d'abord d'une colonne de mercure de 595 inilliniètres ( 22 ponces). L'expérience dura deux jonrs entiers. La colonne de mercure ayant terminé son ascension, je l'évaluai à I mètre 238 millimètres ( 45 pouces 9 lignes). Le liquide sucré, pesé après l’expérience, était réduit à la densité de I, I 10 , densité qui est exactement celle l'une solntion qui contient une partie de sıcre sur trois parties d'ean. Ces trois expérjences finent faites dans un local dont la température, qui ne variait uullement, filt constamment à $\rightarrow 16$ degrés $1 / 2 \mathrm{R}$.

On voit, par ces expériences, que la loi qui préside ì la for'ce de l'endosmose est la même que celle qui préside à sa vîtesse, résultat qui devait être prévu. Nous avons vin que la vîtesse de l'endosmose, produite par des liquides intérienr's de même nature et de densités diverses, l'ean étant toujours le liquide exté- 
rieur, que cette vîtesse, dis-je, est proportionnelle aux excès des densités des liquides intérieurs sur la densité de l'eau. Nous trouvons la même loi pour la force de l'endosmose. En effet, dans les trois expériences précédentes, nous avons des liquides intérieurs dont les densités finales sont 1,025, 1,053, r, 1 10. Les excès de densité de ces liquides sur la densité de l'eau, sont $0,025,0,053,0$, 1 0 . Or, élablissons une progression semblable, en prenant pour premier terme 286 millimètres ( 10 ponces 7 lignes ), hauteur de la colonne de mercure soulevée par l'endosmose dn premier liquide sucré, nous aurons $286^{\mathrm{nm}}, 606^{\mathrm{mm}}$, $\mathrm{r}, 258^{\mathrm{mm}}$, c'est-à-dire, 10 p. $71 ., 22 \mathrm{p} .51 ., 46 \mathrm{p} .61$. Or, l'observation donne $286^{\mathrm{mm}}, 6 \mathrm{I} 7^{\mathrm{mm}}, 1,238^{\mathrm{mm}}$, c'esta-dire, rop. $7^{l .,} 22$ p. $101 ., 45$ p. 9 l. Il n'y a évideminent ici, entre les résultats de l'expérience et ceux du calcul, que les différences légères qui sont inévitables dans les expériences de ce genre. Ainsi, il est démontré que la force de l'endosmose, produite par différentes densités d'un même liquiide in térieur, l'eau étant le liquide extérieur, et la tenıpérature étant constante, est proportionnelle aux quantités qui expriment, dans deux expériences coinparées, les excès de la densité des denx liquides intérieurs sur la densité de l'eau, qui est le liquide extérieur.

W'après cette loi, on peut calculer qu'avec l'endosmonètıc qui a servià ces expćriences, et par la mème température, le sirop de sucre, à la densité de 1,3 , 
produirait une endosmose capable de soulever unie colonne de 127 pouces de mercure; ou du poids de 4 atmosphères $1 / 2$.

Ceux qui tiennent encore à ne voir dans le phénomène de l'endosmose, qu'un simple effet d'attraction capillaire et d'attraction réciproque des liquides, croiront sans doute que si le liquide intéricur de l'endosmomètre, pressé par'une hante colonne de mercure, monte au lien de descendre, cela proviendrait, d'une part, de l'impossibilité où serait le liquide intérieur de filtrer, au travers de la membrane de l'endosmomètre, en raison de sa viscosité, et, d'une autre part, de la facilité avec laquelle l'eau pent traverser cette membrane; en sorte que l'attraction réciproque des deux liquides ayant lieu, et un seul d'entre eux pouvant traverser la cloison, il en résulterait que ce dernier marcherait seul au traver's de la membrane priar aller se réunir au liquide opposé, dont il augmen! sait ainsi le volume. Mais cette théorie, en apparence séduisante, est infirmée par l'expérience. J'ai rapporté plus haut qu'une solution d'une partie de gomme arabique dans trois parties d'eau, avait, par endosmose, élevé le mercure à 75 centimètres ( 28 ponces), et l'eùt élevé plus haut, si mon tube eùt cu plus de longueur. Je remplaçai l'eau dans laquelle baignait le réservoir de l'endosmomètre, par tule solution d'une partie de gomme arabique dans dix parties d'eau. Dès ce monent, le liquide gommeux intérieur s'abaissa dans le tube de l'endosmo- 
mètre: Cet abaissemeut extrêmement lent, étant arrivé à 72 centimètres, je replaẹai le réservoir do l'endosmomètre dans l'eau pure. Dès ee moment, le mercure reprit son mouvement ascensionnel eomme auparavant. Ainsi, le liquide gommeux intérieur avait la possibilité de filtrer au travers de la membrane, et cette filiration s'opérait sous la pression de la eolonne de mereure, lorsque le liquide extérieur. était augmentéde densité. Cependant, d'après les lois connues de l'hydrostatique, l'angmentation de densité de ee liquide extérieur, bien loin de favoriser l'éeoulement du liquide intérieur, aurait dù , au eontraire, le rendre plus diffieile. Il existe done, dans eette cireonstanee, une foree ineonnue qui met obstacle à l'écoulement du liquide intérieur, auquel la membrane livre eependant un passage suffisamment faeile par ses voies capillaires; e'est eette même force qui produit le mouvement aseensionnel de l'eau au travers de la membrane. Cette foree est ineontestablement une foree intra-eapillaire, mais ee n'est point l'atiraction capillaire connue jusqu'à ee jour; eette dernière est une foree d'ascension et de station qui ne porte jamais les liquides au-delà des voies capillaires; l'endosmose est le résultat d'une foree de perméation qui exigge le eoncours de deux liquides différens, et qui porte ees deux liquides en sens inverse an travers des voies capillaires, en les ehassant audehors. Tous les solides porenx et tous les liquides sont aptes à opérer l'ascension capillaire; certains 
solides et certains liquides seulement sont aptes à opérer la double perméation capillaire. L'augmentation de température diminue la force d'ascension capillaire; elle augmente la force de perméation capillaire. Ainsi, ces deux forces intra-capillaires paraissent être essentiellement différentes. 


\section{RECHERCHES}

SUR I, A CAUSE ET SUR LE MECA NISME

1)F:

\section{L'HRRITABILITÉ VÉGÉTAIE.}

Limpontance de la physiologrie comparée des vé¿gétaux el des animaux est aujourd'hui scmie par tous les bous esprits. La vie a des phénomènes généraux ı qui appartiennent au règne végétal comme au règne inimal. Il est donc nécessaire d'étudier comparative!nent ces phénomènes chez tous les êtres vivans sans rexception. C'est de cette étude que sortira la physioılogie générale, science qui est encore à créer, mais ipour laquelle il cxistc de nombrenx matériaux.

L'irritabilité est un de ces pliénomènes grénéranx "pui appartiennent aux végétaux comme anx animaux; unais chez ces denx classes d'êtres, ce phénomène Ipréscnte des nodifications très-remarcfuables, et telles "yuc ccrtains physiologistes ont pu douter si l"irritabiHité était véritablement m phénomène scmblable chez Iles végétanx el chez les animaux. Mais l'observation Iprouve que ces modifications ne sont dans le fait que des simplifications dı phónomène, en sorte que les 
végétaux présentent, dans le plus grand degré de simplicité, ce phénomène d'irritabilité que les animax ne présentent ordinairement qu'avec certaines complications. Ce sont donc les végétaux qui sont appelés à donner la solution de ce problême, l'un des plus importans de la physiologie, solution à laquelle l'étude des seuls animaux ne conduirait jamais.

J'ai annoncé, dars un précédent ouvrage (I), que l'irritabilité végétale consistait exclusivement dans la propriété que possèdent certaines partics des végétaux de prendre un ćtal de courbure élastique, et de s'y maintenir, tantôt d'une manière fixe et permanente, tantôt d'une manic̀re temporaire, en sorte que dans cc dernier cas l'incurvation alterne avec un état de redressement. Depuis que j’ai découvert qu'il existe chez les végétaux une irritabilité dont l'exercice ne se manifeste par aucune courbure, par aucume inflexion de parties, en sorte qu'elle consiste dans une véritable contractilité, j'ai étudié avec beaucoup d'attention ces deux ordres de phénomène d'irritabilité végétale, ct cette étude m’a conduit à la connaissance du mécanisme intime an moyen duquel il s'opère.

(1) Recherches anatomiques et phystologiques sur la structure intime des animaux et des végétaux, et sur leur molilité. 
OBSERVATIONS ET EXPERIENCES

SUR L'IRRITABILITÉ, DE LA BALSAMINE.

(Impatiens balsamina.)

On sait que les valves de l'ovaire de la balsamine, à l'époque de la maturité, se séparent les unes des autres, et que chacune d'elles se roule en spirale en deduns, c'est-à-dire que sa couvexité est en dehor's, ou du côté de l'épiderıue. Si on les redresse, elles retournent spontanément $\mathrm{cl}$ avec vivacité à leur état d'incurvation, lorsqu'on les abandonne à elles-mêmes. Si on les plonge dans l'eau, elles se courbent encore plus profondément; si on les laisse se dessécher à moitié, elles tombent dans l'état de flaccidité ou de relâchement, et perdent leur tendance élastique à l'incurvation. Ces premiers faits prouvent déjà que la présence de l'eau dans les organes qui composent le tissu de la valve, est une des conditions de l'existence de sa tendance à l'incurvation. Si l'on plongre dans l'eau la valve à moitié flétrie par l'évaporation de ses liquides intérieurs, elle absorbe ce liquide, reprend son état turgide vital, et son incurvation élastique, ou son irritabilité. Si on laisse dessécher presqu'entièrement la valve à l'air libre, elle ne reprend plus du tout son état turgide et son incursation lorsqu'on la plonge dans l'eati. Elle s'inmbibe entièrement, et jusquà complète saturation, mais elle u'abs orbe point 
l'ean avec excès comme elle le faisait anparavan; elle ne redevient point turgide; elle demenre constamment dans l'état de flaccidité; elle a conplètement perdu son irritabilité. Cette dernière expérience m'a conduit à penser que l'irritabilité tenait à l'existence du liquide organique qui remplissait les organes vésiculaires dont la vaive est composée, et que c'était, non par une simple imbibition, mais par endosmose que l'eau était introduite dans le tissu organique irritable. Les expériences qui vont être exposées confirmeront ce premier aperçu.

Le tissu organique qui compose la valve de l'ovaire de la balsamine, vu au microscope, se trouve composé par une agrégation d'utricules ou de vésicules. C'est, en totalité, ce que l'on nomme impropreneut un tissu cellulaire et qui sera mieux nomné tissu vésiculaire. Mais il y a une chose très-remarquable dans ce tissu vésiculaire, c’est quie les vésicules, grandes à la partie externe, vont tonjours en décroissant de grosseur, jusqu'à la partie interne, où elles sont le plus petites. Cette disposition dévoile complètement la cause de la tendance à l'incurvation. 'Toutes les vésicules étant pleines jusqu'à l'état turgide, l'incurvation de la valve en dedans en est le résnltat nécessaire. Les vésicules qui composent ce tissu sont, dans l'état naturel, remplies par un liquide organique plus ou moins dense. Lorsque ces vésicules épronvent extérieurement l'accession de l'ean, elles exercent l'endosmose, par cela seul qu'clles contiennent un liquide 
corganique plus dense que l'eau. Alors elles deviennent ttururides, et le tissu, distendn plus en dehors qu'en cdedans, prend un état d'incurvation en dedans (I). Lorsqu'une dessication prolongée a enlevé le liquide intérieur des vésicules, celles-ci s"imbibent dde l'eau dont elles éprouvent extérieurement l'accession, mais elle n'exercent plus d'endosmose; elles me deviennent plus turgides; le tissu demeure dans ll'état de flaccidité; l'irritabilité est abolie. Du moment qu'il me fut dénontré que l'accession extérieure de ll'eau était la cause de l'endosmose des vésicules qui contenaient un liquide organique dense, et que cette ezndosmose était la cause de l'état turgide du tissu; du moment qu'en outre il me fut démontré que l'incurration de ce tissu était le résultat de l'inégalité de ses vésicules, grandes en dehors, et petites en declans, Il me parut certain qu'en substituant à l'eau un liquide slus dense que celui que contenaient les vésicules, ce produirais, non plus de l'endosmose, mais de l'exosnose, et, par suite, une incurvation de la valve dans te sens opposé à celui de son incurvation naturelle. Je plongeai donc plusieurs de ces valves, qui étaient courbées ell dedans, dans du sirop de sucre. Elles ne

(1) Toutes les fois que je dirai, en parlant d'une parlic vécétale, qu'clle se courbe en dedrus on qu'elle se courbe en decors, cela signifiera, dans le prenuer cas, que la concavité de la our burc est tournće ver's l'intérienr ou le centre du végćtal, et, ans le sccond cas, que la concavitć de la combure est touruée rer's l'extéricur. 
tardèrent pas à perdre leur état d'incurvation, et. àt devenir droites. Bientôt après, elles se roulèrent en spirale en dehors. Ceı effel, que j’avais prévu, était un résultat nécessaire de l'exosmose, qui soutirait Je liquide organique moins dense que le sirop, liquide qui remplissait les vésicules du tissu de la valve. Ces vésicules étant désemplies, la valve se roulait en dehors, parce que, de ce côté, les vésicules, plus ugrandes, avaiemt plus perdu de liquide; il y avait, de ce côté, moins de matière solide qu'en dedans; dès lors, il devail y avoir incurvation de ce côté, lors de la soustraction d'une grande partie du liquide, qui, en gonflant ces vésicules, leur faisait occuper un espace considérable. Je transportai dans l'eau ces valves roulées en spirale en dehors; elles ne tardèrent pas à se dérouler, et, enfin, à reprendre leur état naturel d'incurvation en dedans; ici, leurs vésicules composantes exerçaient de nouveau l'endosmose, et l'incurvation en dedans en étail le résultat. Je transportai de nouveau mes valves dans le sirop. Elles se roulèrent en dehors; je les replaçai dans l'eau, elles se courhèrent en dedans. Je répétai ce double jeu d'incurvation neuf fois en cinq heures de tcmps. Alors, les valves cessèrent de se courber en dedans, lorsque je les plongeais dans l'eau; elles ne reprenaient plus assez pour cela leur état turgide, ce qui provenait de ce que l'action d'exosmose, provoquée par l'immersion dans le sirop, avait. soutiré en grande partie leur liquide dense julérieur; il ne leur en restait plus asscz ponr exercen 
ımne endosmose suffisante pour les replacer dans l'état tturgide; dès lors, il n'y avait plus d'incurvation en (dedans. Mais l'immersion dans le sirop prodnisait toujours le roulement en dehors, jusqu'au summum, pparce que cette incurvation était le résultat de l'exosimose, laquelle, loin d'éprouver de la diminution, alllait, au contraire, toujours en augmentant d'énergie, rpuisque le liquide intérieur des vésicules devenait de Imoins elı moins dense, l'eau ayant remplacé huit ou ınenf fois le liquide organique intéricur, soutiré par ll'exosmose qu'occasionnait l'immersion dans le sirop. .Te mis sous le microscope une lame mince de valve, fplongée dans du sirop de sucre. Je fus ainsi à même (de voil d'une manière immédiate le mécanisıne de sson incurvation. Je vis tontes les vésicules, et spécialement les plus grandes, qui occupaient son côté cextéricur convexe, perdre assez rapidement de leur (diamètre, par l'effet de leur déplétion, et l'incurvastion en dehor's de la lame de valve en fus l'effet.

Il résulte de ces expériences, que les valves de la Ibalsamine perdent lew irritabilité ou leur faculté d'incurvation élastique en dedans, lorsque le liquide organique dense qui remplit leurs vésicules esı souI tiré, soit par l'évaporation, soil par l'exosmose. C'est a donc à l'existence de ce liquide intérieur dense qu'est - due l'irritabilité. Si l'on pouvait rendre aux vésicules le liquide dense qu'elles ont perdu, on leur rendrait leur faculté de devenir turgides par endosmose, lors de l'accession extérieure de l'eau; on rendrait par 
conséquent aux valves leur faculté de prendre une incurvation en dedans, e'est-à-dire qu'on leur rendrait leur irritabilité perdue. C'est effectivement ce que j’ai fait par les deux expériences suivantes. J'ai fait desséeher à l'air libre des valves d'ovaire de balsamine, en ayant soin de les empêcher de se tortiller, et de les conserver dans la rectitude. Lorsque, eette dessieation me parut à peu près complète, j'achevai de la déterminer à l'aide de la chaleur douce du feu. Les valves ainsi desséchées étaient devenues cassantes et friables. J'en plongeai quelqnes-unes dans l'eau; elles s'imbibèrent jusqu’à saturation, et demeurèrent droites dans l'état de flacciditć. Je plongreai plusieurs autres de ces valves dans de l'cau très-sncrée; elles s'imbibèrent de ce liquide dense jusqu’à saturation, et demeurèrent de même dans l'élat de rectitude et de flaccidité. Lorsque je jugeai que les vésicules composantes de leur tissu avaient absorbé par imbibition du liquide sucré autant qu'elles pouvaient. le faire, en vertu de leur simple capillarité, je plongeai ees valves dans l'eau; eiles ne tardèrent pas à l'absorber par l'effet de l'endosmose, provoquée par la présen ee d'un liquide dense dans les vésicules; leur tissu vésiculaire devint turgide, et l'incurvation des valves en dedans eut lieu de la même manière que dans l'état naturel. Je transportai ees valves dans du sirop de sucre, elles sc roulèrent en dehors; je les replaęai dans l'eau, elles se eourbèrent de nouveau en dedans; en un mot, ces valves avaient repris leur ịritabilité par une véri- 
table résurrection; senlement lenr incurvation n'avait pas autant de force d'élasticité que dans l'élat naturel.

Je viens d'exposer comment l'exosmose produite par l'immersion alternative, sonvent répétée dans le sirop el dans l'ean, avait fini par sontirer la plus grande partie du liquide organique dense que contenaient originairement les vésicules, en le remplaçant par de l'eau. Il résultait de là l'impossibilité au tissı de la valve de reprendre dorénavant son étal turgide, et par conséquent son incurvation en dedans, or son irritabilité naturelle; urais, en abandonnant longtemps dans le sirop ces valves ainsi privées de lenr liquide dense naurel, ce liquide sucré tend à les pénétrer par imbibition. Les vésicules s'en remplissent, cu sortc qu'au bout de huit à dix jours, si l'on transiporte ces valves dans l'eau, elles quittent lent incurvation en dehors, et reprennent leur incurvation naturelle en dedans; clles ont récupéré leur irritabilité cen récupérant un liqnide dense dans l'intérieur de Heur's vésicules.

Il résulte de ces observations, que l'irritibilité de Ila balsamine consiste dans une faculté d'incurvation célastique qui résulte de l'état urgirde par endosmose I'ın tissı vésiculaire à vésicules larges et rares au (côté convexe, petites el serrées au côté concave. C'est Il'accession extérieure de l'eau sur ces vésicules remplies d'ın liquide organique dense, qui détermine Il'endosmose de ces vésicules, et par conséquent l'exerccice de l'irritabilité ou de l'incurvabilité, dont le mé- 
canisme se trouve ainsi dévoilé. Dans l'état naturel, c'est la sève lymphatique ascendante, qui n'est presque que de l'eau pure, qui remplit ici le rôle de liquide exiérieur, dont l'accession provoque l'endosmose des vésicules. On peut se convaincre de cette vérité, en laissant flétrir un rameau de balsamine détaché de la plante et chargé d'ovaires. En perdant une partie de l'eau qui les rend turgides, les valves de ces ovaires perdent une partie de leur irritabilité; elles lá récupèrent en plongeant l'extrémité du rameau dans l'eau. Ce liquide, pompé par la tige, arrive par les canaux lymphatiques jusqu'aux vésicules des valves, et son accession extérieure détermine leur endosmose, et par conséquent le retour de leur état turgide, ce qui ramène leur irritabilité.

Il était important d'apprécier l'action des différens agens chimiques sur l'irritabilité végétale. Je me suis assuré que les acides affaiblis augmentaient la force de la tendance à l'incurvation dans les valves de la balsamine. Ainsi, en plongeant une de ces valves dans l'eau pure, elle prenait un degré déterminé d'incurvation; si j'ajoutais à l'eau une petite quantité d'acide sulfurique, nitrique ou hydro-chlorique, l'incurvation de la valve devenait à l'instant plus profonde; mais l'incurvabilité de cette valve était altérée, en sorte qu'en la transportant dans du sirop de sucre, elle se redressait, mais sans se rouler en spirale en dehor's, comme cela a lieu ordinairement. Si l'action de cet acide affaibli était plus longue, la valve per- 
VËG ËTALE.

dait entièrement la faculté de se redresser dans le sirop; son irritabilité étail complètement détruite. Ce pliénomène étail le résultat de la coagulation du liquide intérieur des vésicules, congnlation opérée par l'action de l'acide. Alors les vésicules ne contenaient plus un liquide dense, mais simplement un coagulum; elles étaient par conséquent incapables d'exercer l'endosmose, dès lors l'incurvabilité était abolie. L'immersion suffisamment prolongée d'une valve d'ovaire de balsamine daus l'alchool, produit de même, et par la même raison, l'abolition de son incurvabilité. L'immersion suffisamment prolongée dans une solution de potasse canstique, anéantit également l'irritabilité de ces valves, et cela autant par l'altération chimique de lenr tissu, que par celle de leurs liquides intérieurs.

Je mis quelques valves de balsamine dans un verre d'eau, à laquelle j’avais ajouté trois gouttes d'hydrosulfure d'ammoniaque. Les valves se courbèrenı d'abord profondément en dedans; deux jours après, leur incurvation étail beaucoup diminuée. Je les transporItai dans l'eau pure; elles y demeurèrent immobiles. Je les transportai dans du sirop de sucre; elles se redressèrent jusqu'à la rectitude seulement, et ne se courbèrent point en dehors, comme cela a lieu ordiınairement: remises dans l'eau, elles affectèrent unc courbure très-légère en dedans. Ces valves étaicut wéritablement dans un état d'engourdissement on de stupéfaction, et cependant elles avaient conservé leur 
apparence de vie; elles n'avaient point perdu lecuir conleur verte, comme cela avait lieu lors de l'abolition de l'irritabilité de ces valves par des acides, par des alkalis ou par l'alchool. Ce fait coïncide avec les observations qui m'ont prouvé que l'hydrogène sulfuré est ennemi ou sédatif de l'endosmose.

\section{OBSERVATIONS ET EXPÉRIENCES}

SUR L'IRRITABILITÉ DU MOMORDICA ELATERIUM.

Le fruit du momordica elaterium, à l'époque de la maturité, se détache de son pédoncule. A l'instant de cette séparation, le liquide contenu dans la cavité centrale du fruit est expulsé avec violence, mêlé avec les graines, par l'ouverture qui provient de la séparation du pédoncule. A la seule inspection de ce phénomène d'irritabilité, on peut juger qu'il y a là une contraction des parois de l'organe creux sur le liquide contenu dans sa cavité. J'avais d'abord été porté à douter de ce fait; mais l'observation m'a ramené à le reconnaître. Il ne m’a fallu pour cela que mesurer d'une manière exacte les deux diamètres du fruit ellipsoïde, avant et après son évacuation. Ce fruit, après qu'il a expulsé son liquide central et ses graines par une violente expulsion, se trouve diminué environ d'un neuvième dans son petit diamètre, et environ d'un douzième dans son grand diamètre. J'ai pris ces mesures d'une manièrc extrêmement exacte, avec un 
compas de tourueur. Il n'y a donc point de doute; il y a ici une véritable contraction; l'organe creux s'est resserré sur lui-nềme dans tous les sens. Il s'agit actnellement de rechercher le mécanisme de cette contraction. Cette recherche est d'autant plus importante, qu'elle peut fournir par analogie des lumières sur la contractilité des animaux.

Avant sa maturité, le fruit du momordica elaterium ne manifeste aucune tendance à expulser le liquide, alors peu abondant, qui existe dans sa cavité centrale. Cependant, ce fruit vert donne des marques très-sensibles d'irritabilité. Si l'on en coupe une tranche longitudinale, comme on coupe une côte de melon, cette tranche se courbe profondément sous forme d'un croissant : cette illcurvation augmente encore en plongeant la tranche dans l'eau. Si l'on coupe le fruit par tranches circulaires transversales, et qu'on divise chacune de ces tranches circulaires en deux demi-cercles, chacun de ces demi-cercles se courbe profondément, jusqu'à former un petit cercle complet : cette incurvation augmente par l'immersion dans l'eau. Ainsi, il y a dans le fruit vert du momordica elaterium une tendance générale à l'incurvation: cette tendance, loin de comprimer le liquide central, tend au contraire à lui faire plus de place, puisque par elle le petit diamètre du fruit tend à s'agrandir. Ce n'est donc point cette tendance à l'incurvation qui comprime ce liquide, et qui l'expnlse à l'époque de la maturité. Effectivement, à cettc époquc 
et après l'expulsion dı liquide central, les tranches longitudinales du fruit ne tendent plıs à se courber en dedans sous forme de croissant. Elles conservent leur rectiunde, mème lorsqu'on les plonge dans l'eau. Ainsi, il y a eu un changement extrêmement notable dans le mode de l'irritabilité du fruit, comparé dans ses deux états de fruit vert et de fruit mûr. Nous allons déterminer, par l'expérience et par l'observation, quel est ce changement survenu.

Le tissu du fruit, examiné au microscope, se trouve spécialement composé de vésicules agglomérées. Ces vésicules vont en décroissant de grandeur de la circonférence au centre. C'est cettc grandeur décroissante des vésicules qui se retrouve ici comne dans les valves de l'ovaire de la balsamine, qui détermine de même la tendance à l'incurvation en dedans dans le fruit ver'; mais cette grandeur décroissante des vésicules existe aussi dans le fruit mûr. Pourquoi donc n'existe-t-il plus de tendance à l'incurvation en dedans chez ce dernier? c'est ce que l'observation va nous dévoiler.

Les vésicules qui composent par leur assemblage le fruit du momordica, contiennent un liquide organique dense. L'accession extérieure de l'eau ou de la sève lymphatique provoque l'endosmose dans ces vésicules, et par suite l'état turgide et l'incurvation en dedans. C'est pour cela que l'incurvation d'une tranche de ce fruit augmente en la plongeant dans l'eau. Si on la plonge dans du sirop de sucre, la densité de ce li- 
quide, plus ennsidérable que la densité du liquide intérienr des vésicules, provorfnera l'exosmose dans ces vésicules, et il en résultera que la tranehe perdra son ineurvation en dedans, et prendra une ineurvattion en dehors. Si l'on répète cc jeu d'incurvations alternatives dans l'eau el dans le sirop, il arrivera à la tranehe du fruit ee qui esi arrivé dans la même expérience à la valve de l'ovaire de la balsamine; elle perdra la faeulté de prendre de l'ineurvation en dedans, en eonservant eelle de se eourber en dehors. C'est le résultat de la sonstraetion dn liquide dense que eontenaient les vésicules, soristraetion qui a ćté opérée par l'effet eontinué de l'exosmose. Or, comme il arrive, lors de la maturité du fruit du momordiea, qu'il a perdu sa faculté de se eourber en dedans, et que eependant il eonserve ses vésicules décroissantes de dehors en dedans, il faıl nécessairement que ees vésicules aient perdu une grande partie du liquide dense intérienr qu’elles contenaient, lorsque le fruit. était vert. L'expérienee va nous dévoiler la eause de cette déperdition.

Le "centre dn fruit dı momordica elaterium eonlient une substance organique très-singulière, et qui ne ressemble à aueun autre tissu végétal. On le prendrait ponr un muens vert fort épais. $V_{4}$ an microscope, il paraît eomposé d'une immense quantité de globules fort petits, myoglomérés, tantôt confusément, lantôt de manière ì former des stries irrégulières. Cette substanee est pénétrée par un liquide blanehâtre, 
par une sorte d'émulsion, qui est d'autant plus dense, qu'on l'observe à une époque plus voisine de la maturité. Ce liquide aqueux s'épanche aussitôt qu'on ouvre le fruit vert. Au microscope, on voit des globules presque imperceptibles qui nagent dans ce liquide; l'époque de la maturité, ce liquide blanchâtre est beaucoup plus abondant, et en même temps beaucoup plus dense; les globules qu'il tient en suspension sont devenus beaucoup plus gros. Les graines détachées du fruit nagent dans ce liquide central, qui, par sa densité considérable, provoque l'exosmose des vésicules qui composent le tissu du fruiț; dès lors le liquide organique qui remplit ces vésicules tend, par l'effet de l'exosmose, à s'écouler vers le liquide central, dont la densité est supérieure à la sienne. Cette exosmose fait cesser la tendance à l'incurvation en dedans, qui existait dans toutes les parties du fruit; qui se trouve alors dans le même cas que s"il était en contact avec dư sirop de sucre; ses côtés tendent alors à la rectitude. La masse du liquide central est augmentée par l'addition du liquide qu'il soutire des vésicules. Les côtés du fruit sont courbés mécaniquement par cette accumulation de liquide dans sa cavité; et comme ces, côtés tendent avec force à la rectitude, ils pressent avec violence le liquide central, et ils le chassent rapidement dès qu'une issue lui est offerte. Cette expulsion n'est pas l'effet de la seule tendance à la rectitude des côtés du fruit; elle est aussi l'effet de la diminution de la capacité de sa cavité centrale, par 
șa eontraetion générale. Ces deux effets dépendent de la même eause, c'est-à-dire de l'exosmose des vésicules, produite par l'aceession extérieure du liquide central, plus dense que ne l'est le liquide qui remplit ces neêmes vésicules. La vérité de cette assertion est prouvée par l'expérience suivante. J'ai pris un nombre suffisant de fruits parvenus à leur maturité, et j’ai reeneilli dans un vase le liquide central qu'ils expulsaient, mêlé aux graines; alors j'ai pris un fruit vert, et je l'ai coupé par tranches longitudinales; ehacune de ces tranehes s'est courbée en croissant, en dedans, comme à l'ordinaire, et cette ineurvation s'est augmentée dans l'eau : c'était l'effet naturel de l'endosmose. Alors j’ai transporté ees tranehes dans le liquide que j'avais recueilli; elles n'ont pas tardé à diminuer de eourbure; ensuite elles se sont redressées eomplètement; enfin, elles se sont un peu eourbées en delırs. Il est prouvé par,cette expérienee, que le liquide central du fruit mûr agit comme eause d'exosmose sur les vésicules qui composent le tissu du fruit, ce qui prouve que ce liquide est plus deuse que ne l'est le liquide qui remplit ces vésicules. C'est done l'aceession ou le eontact de ce liquide central, devenu très-dense, qui fait eesser la tendance générale à l'incurvation en dedans, qui existait dans le fruit vert, par, l'effet ${ }_{\hookleftarrow}^{\complement}$ de l'endosmose.des vésicules, et qui lui substitue une tendance générale au redressement et à l'incurvation en dehors, par l'effet de l'exosmose de ces niêmes vésieules. 
Ainsi, il y a deux phases dans l'irritabilité du fruit du momordica claterium, savoir; tne tendance à l'incurvation en dedans par effet d'endosmose dans le fruit vert, et une tendance à l'incurvation en dehors par effet d'cxosmose dans le fruit mûr. Ce changement ne reconnaîl d'antre cause que l'angmentation survenue dans la densité du liquide qui occupe la cavité contrale du fruit.

Il résulte de ces observations, que l'irritabilité de l'ovaire de la balsamine el du fruit du momordica elaterium consiste daus une incurvabilité à laquelle sc joint une véritable contractilité. L'incurvabilité dépend de la grandeur décroissante des vésicules qui composent le tissu irritable; ce tissu offre, d'un côté, de la capacité en plus, ct de l'autre côtć, de la capacité en moins. Ces vésicules contiennent un liquide organique d'une densité toujours supérieure à celle de l'cau; lorsqu'elles subissent l'accession extérieure de l'eau ou de la sève lymphatiqne, qui diffère peu de l'cau pure, ces vésicules excrcent l'endosmose, et le tissu irritable se courbe, de manière que les plıs grandes vésicules occupent le côté convexe. Lorsque ces vésicules subissent l'accession d'un liquide plus dense que cclui qu'clles contionnent, elles excrcent l'cxosmose, et il en résulte deux effets; le premier est l'incurvation du tissu irritable, en sens inverse de celui qui avait licu par endosmose; alors ce sont les plus petites vésicules qui sont au côté convexe; le second effet est la contraction ou le raccourcissement du tissu 
VËG'XALE.

irritable : c'est le résultat nécessairc de l'évacuation partielle de toutes ses vésicules composantes. Par cette déplétion, le tissu devient moins volumineux, on, en d'autres termes, il se contracle.

\section{OBSERVATIONS}

SUR L'IRRITABILITÉ DE LA SENSITIVE.

\section{(Mimosa pudica.)}

Dans mes recherches sur la structure des orwanes irritables de la sensitive (1), j’ai fait voir quic ccs organes, auxquels j'ai donné le nom de bourrelets, sont composés d'un parenchime cellulaire. Ce parcnchime n'est autre chose que de la médulle corticale dans un grand ćtat de développement. Dans son centre cxiste un petit faisceau de tubes lymphatiques et de trachécs 'qui apparticnnent à l'étui médullairc du système ccnttral. Les vésicules articulées dont se compose le tissu du bourrelet, sont remplies par un liquide diaphane, (coagulable par la chalcur et par l'acide nitrique affailbli. Par ce moyen, on produil dans l'intéricur de (chacune de ces vésicules un petit coagulum globuleux (qui doit son apparence noire à son opacité. C'cst co qque j'ai représenté dans les figures s 6 et 17 de l'onwrage cité plus hant. On voit, dans les intervalles de cces corps globulcux, des lignes irrégulières qui indi-

(1) Recherches anatomiques et pliysiologiques sur la structure iintime des animaux et des végétaux, et sur leur motilité. 
quent les sections des parois contiguës des vésicules irrégulièrement divisćes par l'instrument tranchant. J'avais considéré les coagula globuleux dont il est ici qucstion, comme indiquant l'cxistcncc de vésicules globuleuses éparses dans une masse dc parcnchime cellulaire ordinaire; mais de nouvellcs observations m'ont ćclairé sur la véritable nature de ccs corps globuleux, qui n'existent point dans l'élat naturcl, et dont la formation purement artificielle est duc à ce quc l'acide nitrique froid et affaibli coagule subitement lc liquide organique contcnu dans chaque vésicule, et à ce que le coagulum se rcsscrre cn forme de boulc au centrc de la vésiculc. De nouvelles observations m'ont fait voir que le tissu du bourrelet ou dc l'organc irritable de la sensitive, est entièrement composé dc vésicules articulées remplies d'un liquide densc, ct décroissantes de grandcur de dehors en dedans. J'ai prouvé par des cxpériences, que c'est exclusivcment dans ce tissu vésiculaire quc réside l'irritabilité de la sensitive, et quc lc faisccau ccntral de tubes $\mathrm{ct}$ de trachécs est tout à fait étranger à cette proprićtć vitale; cnfin, j’ai détcrminé le mćcanisme des mouvcnens qu'exćcute la scnsitivc. Je rappellerai ici trc̀s-brièvement ces expćriences.

Le pétiole de la feuille de la scnsitive possèdc un double mouvement d'abaissement et de redrcssement, et c'est le bourrelct situé à sa basc qui est l'organe de ce double mouvcment. $\mathrm{Si}$, par une section longitudinale, on enlèvc la partie inférieure du bourrelet, 
lla partie simpérieure de cet organe, restée seule, se (courbe en arc, dont la concavité est dirigée vers la tterre, et, par ce moyen, le pétiole est maintenu consttamment dans l'état d'abaissement. Cet abaissement ın'est point le résultat d'un état d'affaissement des celllules du bourrelet, et n'est point un état de flaccidité; lle pétiole est maintenu dans cet état d'abaissement par la force d'élasticité de l'arc que forme le demilbourrelet supérieur. Si, par nne pareille section longitudinale, on enlève la partie supérieure du bourreIleı à une autre feuille, la partie inférieure de cet organe, restée seule, se courbe en arc, dont la concavité cest dirigée vers le cicl, et, par ce moyen, le pétiole cest maintenu constamment dans l'état de redressement. Ainsi, le bourrelet de la sensitive peut être conssidéré comme composé de deux ressorts courbés et hantagonistes : le ressort supérieur, en se courbant, aabaisse la feuille; le ressort inférieur, en se courbant à son tour, la relève. Lorsque chacun de ces deux resssorts existe seul, il maintient le pétiole dans une possition constante el invariable d'élévation ou d'abaissement. Le ressort inférieur, par exemple, existant sseul, le pétiole demeure invariablement redressé. Cependant, si l'on néglige d'arroser suffisamment la Iplante, on voit bientôt le pétiole s'abaisser. La plante ccependant n'est pas encore fanée ou flétrie par le manque d'eau, mais déjà il n'y a plus assez d'eau Hans le tissu du ressort pour entretenir son état d'éHasticité. Il tombe dans le relâchement par flaccidité, 
et la fenille entraine par son poids le pétiole dans l'état d'abaissement. Si on arrose la plante dans ce moment, on ne tarde pas à voir le pétiole se redresser par la force d'élasticité du ressort inférieur du bourrelet, qui reprend son état de courbure naturelle. Ces observations prouvent que l'élasticité des ressorts de l'organe irritable ou du bourrelet est produite par l'état turgide des cellules ou des vésicules qui le composent. Cet état turgide des organes vésicnlaires est le résultat de l'endosmose que ces organes exercent par l'accession extérieure de la sève lymphatique; ainsi, l'endosmose est la cause innmédiate de l'élasticité des ressorts de l'organe irritable de la sensitive, de la même manière que cela a lieu chez la balsamine et chez le momordica elaterium. La tendance à l'incurvation des parties du bourrelet de la sensitive est encore mise en évidence par les expériences suivantes. On enlève avec un instrument bien affilé des tranches minces du bourrelet; elles ne manifestent dans l'air aucun mouvernent; mais si on les plonge dans l'eau, à l'instant elles se courbent en arc, dont la concavité est tonjours tonrnée du côté qui regardait l'axe du pétiole. Si on les transporte dans du sirop de sucre, elles se redressent, et cnsuitc se courbent par exosmose en sens opposé ou en dehors; en les transportant de nouveau dans l'ean, elles reprennent par endosmose leur incurvation primitive en dedans. Ainsi, il n’y a point à douter que l'irritabilité de la sensitive ne soil due à l'endosmose d'un tissu vésiculaire dom 
ces vésicules sont décroissantes de dehors en dedans; 11 n'y a poiut de doute non plus que ce ne soit l'accession extérieure de l'eau ou de la sève lymphatiqne qui provoque l'endosmose de ces vésicules remplies sar un liquide organique très-dense. Ceci m'explique um phénomène dont je ne m'étais pas rendu compte cors de mes premières recherches, et que je m'étais xontenté d'exposer. Une excitation exercée sur une ceule des folioles de la sensitive se propage au loin IJans le végétal, et va détcrminer l'action de tous les rganes irritables ou de tons les bourrelets auxquels Ille parvient successivement. Des expériences posiiives mont prouvé que c'est par le moyen du liquide contenu dans les tubes Jyınphatiques que s'opère la ransmission de cette excitation, ou plutôt de cette wause excitatrice intérieure, si semblable en appaence à un influx nerveux. J'ai calculé la vîtesse de at marche de cette cause excitatrice intérieure chez a sensitive. Aujourd'hui, les nouveaux faits qui m'ont trouvé que l'action des organes irritables végétaux st toujours mise en jeu par l'accession d'un liquide a'indiquent ici que cette cause excilatrice, qui marthe dans les tubes Jymphatiques de la sensitive, "est autre chose que la sève Jymphatique elle-même, aquelle recoit, par l'action des excitans du dehors, in monvement d'impulsion qui se communique de roche en proche avec une vîtesse déterminée, et qui, ar' son accession, détermine l'action des organes irriubles. Mais il reste toujours à déterminer qu'elle est 
la force qui, dans cette circonstance, meut le liquide lymphatique dans ses canaux après l'influence d'une excitation du dehors. Il reste également à déterminer pourquoi l'accession de cette sève lymphatique fait prédominer l'incurvation du ressort supérieur du bourrelet, ce qui abaisse le pétiole. Il reste enfin à savoir pourquoi, après un peu de repos, le ressort inférieur du bourrelet reprend sa prédominence, ce qui relève le pétiole.

\section{OBSERVATIONS}

SUR L'IRRITABILITÉ DU SAINFOIN OSCILLANT.

\section{(Hedysarum girans.)}

La feuille dı sainfoin oscillanı a trois folioles comme la feuille du trèfle. La foliole du milieu, qui est la plus grande, est immobile, mais les deux folioles latérales, qui sont assez petites, sont dans un mouvement continuel d'élévation et d'abaissement alternatifs. Ces mouvemens s'exécutent au moyen de la flexion du pétiole très-grrêle de ces petites folioles; ainsi, c'est dans ce pétiole qu'existe l'organe des mouvemens des folioles qu'il supporte. L'extrême ténuité de ce pétiole rend son étude anatomique très-difficile. Il faut, avec un instrument tranchant, délicat et bien affilé, enlever une lame de tissu sur deux côtés opposés du pétiole. Alors, on soumet au microscope la partie moyenne extrêmement mince qui reste. On voit de 
cette manière, que le centre du pétiole est occupé par les tubes on vaisseaux qui se distribuent à la foliole. De chaque côté de ce faisceau central de tubes se trouve un parenchime composé de vésicules globulleuses d'une extrême petitesse, et dont la grosseur est idécroissante de dehors en dedans. Ces vésicules conttiennent un liquide incolore. C'est ce tissu vésiculaire 'qui est l'organe irritable.

Le sainfoin oscillant offre des phénomènes d'irriItabilité plus remarquables que ceux de la sensitive; (car le mouvement de ses folioles dépend d'une cause texcitatrice intérieure sans cesse agissante, et qui paıraîl complètement indépendante de toute excitation rextérieure. Les petites folioles de la feuille de celte Iplante s'élèvent et s'abaissent alternativement, et Itoujours par petites saccades; elles effectuent leur idescente en se fléchissant d'un côté, et elles opèrent lleur ascension en se fléchissant du côté opposé, en sorte que le sommet de la foliole décrit ure ellipse. (Cetle oscillation s'effectue dans l'espace d'une ou de (deux minutes. Elle a lieu même pendant la nuit, et s'arrête lorsque la plante est soumise à l'influence d'un soleil ardent. Alors les folioles cessent de se imouvoir, et leur pointe demeure fixement dirigée ivers le ciel; la grande foliole impaire prend la même (direction. C'est dans le pétiole des folioles qu'existe ll'organe irriable auquel est dû leur mouvement. Nous venons de voir que, semblable à tous les orgranes irritables végétaux, il est composé de vésicules 
dont la grosseur est décroissante; ainsi, il n'y a pas de doute que l'action de cet organe irritable ne dérive d'une tendance à l'incurvation : c'est effectivement ce que l'expérience démontre. J'ai divisé ce pétiole en deux moitiés longitudinales; à l'instant ces deux moitiés se sont courbées en arc dont l'épiderme occupait la convexité. Cette incurvation devint plus profonde en plongeant ces petits arcs dans l'eau. Ainsi, leur incurvation en dedans avait lieu par endosmose. Je transportai ces petits arcs dans le sirop de sucre; ils se redressèrent, et ensuite se courbèrent en dehors. Cette nouvelle incurvation avait lieu par exosmose. Ainsi, l'action de l'organe irritable du sainfoin oscillant est exactement semblable à celle de tous les autres organes irritables végétaux. Je divisai longitudinalement un pétiole en deux parties très-inégales; il n'y avaiı qu'une lame très-légère de tissu qui fût énlevée d'un côté. Le plus volumineux de ces fragmens de pétiole se courba en arc, dont la concavité était tournée du côté de la section. L'ayant plongé dans l'eau, il se redressa, et immédiatement ensuite il se courba de nouveau, s'agitant ainsi comme un vermisseau. La raison de ces deux mouvemens en sens opposé est facile à saisir. Le pétiole s'est d'abord courbé dans le sens voulu par la prédominance d'action d'incurvation du côté qui avait conservé son intégrité; ce côté ayant sa masse entière, l'emportait par cela même sur le côté affaibli par l'ablation d'une partie de sa masse; mais ce dernier, dont l'épiderme 
était enlevé, absorbait l'ean avec plıs de faeilité et de rapidité que ne le faisail son antagoniste; cette cause ayant fait prédominer sa force d'ineurvation, nnalggré son infériorité de masse, il opéra le redressement dı pétiole. Mais eet efiet ne ponvait être que momentané. L'eau ayant bientôt pénétré dans le tissu du côté intact, provoqua l'endosmose de ses vésieules, et lui rendit sa prédominance de foree d'incurvation. Après l'aecomplissement de ee dernier phénomène, le pétiole courbé en are conserve cette position, et reste immobile dans l'ean. J'ajontai une gontte d'acide nitrique à l'eau dans laquelle était plongé ee pétiole. A l'instant, le pétiole eourbé en are se redressa, pnis il se eourba de nouveau, et plus profondément qu'auparavant. Cette expérienee eoneourt avec eelles rapportées plus hant, pour prouver que l'aecession d'un acide provoque l'excreice de l'irritabilité on de l'incurvabilité végétale avec plus d'énergie, mais de la même manière que le fait l'accession de l'eau pure. Ce fail est très-remarquable, paree qu'il eoïncide avee ee fait eonnu, que les aeides provoquent l'exereiee de la contraetion chez les animanx.

Le pétiole de sainfoin oscillant, anquel on a conservé son intégrité, n'exéente aneun mouvement d'ineurvation quand on le plonge dans l'eau. Alors ee liquide pénètre également dans toutes les partics de son tissı; et de l'égralité d'endosmose qui en résulte, naît l'équilibre des forces antignonistes 
d'incmenation, yui existent dans l'organe irritable de ce pétiole.

II résulte de ces observations, yne le pétiole des petites folioles du sainfoin oscillant possède, connme le bonrrelet de la sensitive, lles ressorts antagonistes silués de chaque côté de l'axe commun, et qui tendent. tons à se courber en irc, dont l'épiderme occupe la convexité. Il existe autant de ces ressorts antagonistes qu'il y a de diamètres dans la compe transversale du pétiole; nais les denx ressorts supérieur et inférienr sont ceux don l'action est la plus énergique è la plus étendue. Cliez le sátinfoin oscillant, l'action successive de ces ressorts concentriques se manifeste dans le mode d'oscillation des folioles. J'ai dit plus hant que les folioles effectuent leur descente en se fléchissant d'uu côté, el qu'elles opèrent leur ascension en se Héchissant du côté opposé, en sorte que le somuıe de la foliole décrit une ellipse. Ainsi, il y a dans le pétiole une action d'incurvation qui est révolutive antour de l'axe du pétiole, mais cette action est prédomiriante dans les deıx sens supérieur et inférieur. En stupposant par la pensée un grand nombre de ressorts disposés autour de l’axe dı pétiole, et tendant tous à tourner vers lui la concavité de leur courbıre, nous verrions chacun de ces ressorts entrer successivement en action par l'effet d'une canse déterminante qui serail révolutive antour de l'axe du pétiole. Les ressoris supérieur etinférieur seraient ceux dont l'action anrait le plıss d'étendue. De là résulterait l'oscillation 
en ellipse, que présentent les folioles du sainfoin oscillant. Cette supposition est exactement ee qui existe, exeepté qu'il n'y a point dans le pétiole un grand nombre de ressorts, mais bien un seul ressort tubuleux dont toutes les parties ont une tendanee eoncentrique à l'incurvation, et agissent les unes après les autres, lorsqu'elles subissent l'aceession de la eause à marche révolutive, qui détermine l'endosmose de leurs vésicules, et par suite la prédominanee de leur foree d'ineurvation. Les deux ressorts supéricur et inférieur ont sur les ressorts latéraux une prédominanee d'action qu'ils doivent, à ee qu'il m'a paru, à la prédominanee de leur volume. L'aetion successive de ces ressorts dans le sens d'une révolution antour de l'axe dn pétiole, atteste qu'ils sont sueeessivement rendus turgides par l'aceession de la sève lymphatique, qui détermine l'endosmose de leurs vésieules eomposantes. Mais nous ignorons cntièrement quelle est cette eause impulsive de la sève lymphathique, qui, dans eette cireonstance, donne au liquide séveux une marche révolutive autour de l'axe du pétiole.

Il résulte de ees observations, que le mécanisme de l'irritabilité du sainfoin oseillant est exactement le même que eclui de l'irritabilité des antres végétaux irritables; il n'y a d'inconnu, ici eomme chez la sensitive, que la eause intérieure et vitale qui meut la sève lympathique pour opérer son aecession aux vésieules de l'organe incurvable. Cette motion de la sève, 
considérée comme cause excitatrice immédiate de l'in: curvation, paraît avoir lieu suivant une ligne droite chez la sensitive. Elle s'effectue en tournant autour de l'axe du pétiole, chez le sainfoin oscillant. 


\title{
DIRECTION DES TIGES
}

\author{
VERS LE CIEL,

\section{ET DES RACINES}

VERS LA TERRE.

$\mathrm{L}_{\mathrm{A}}$ tendance des tiges vers le ciel et la tendance inverse des racines vers le centre de la terre, est un des phénomènes les plus mystérieux de la végétation. J'ai démontré, dans un précédent ouvrage (I), que cette double tendance dérive d'une action organique et vitale exercée par le végétal, et qu'elle n'est point du tou le résultat d'actions immédiates extérieures, telles qu'une attraction qui attirerait les racines, ou bien une répulsion qui repousserait les tiges. Le mécanisme de cette action organique et vitale va être dévoilé par les observations et les expériences suivantes.

J'ai démontré, dans un précédent ouvrage (2), que

(1) Recherches anatomiques et plyssiologiques sur la structure intime des animaux et des végétaux, et sur leur motilité.

(2) Recherches sur l'accroissement des végétaux. 
le végétal est composé de deux systèmes concentriques, le système cortical et le système central, et que ces deux systèmes sont composés de parties senblables ou analognes, disposées en sens inverses. Dans le système central, la moelle ou médulle centrale occupe le centre; dans le système cortical, le parenchyme ou médulle corticale occupe la circonférence. Ce sont ces deux médulles et quelques vaisseaux et trachées qui composent toute l'organisation des tiges et des racines naissantes. Or, il est d'observation que, dans les tiges naissantes, la médulle centrale l'emporte en volume sur la médulle corticale. Au contraire, dans les racines naissantes, la médulle corticale l'emporte en volume sur la médulle centrale, dont l'existence est même difficilement appréciable dans la plupart des circonstances. Celte prédominence inverse des deıx médulles dans les tigges et dans les racines est un premier fait qu'il faut noter.

Les deux médulles, corticale et centrale, sont conposées de vésicules aggglomérées et remplies par 111 liquide dense. Or, une disposition organique très-importante de ces deux médulles, et qui n'a point encore été observée, est celle-ci : dans la médulle corticale, les vésicules, grandes en dehors, vont en décroissant de diamètre ver's le dedans, où elles sont le plus petites; au contraire, dans la méculle centrale, les vésicules petites en dehors vont en augnientant do diamètre vers le centre. Cette disposition est plus ou moins facile à voir chez tons les végétaux. La 
moelle offre tonjours de grandes vésicules dans son ccntre; ces vésicules vont en décroissant de grandeur jusqu'à l'éuni médullaire, dans le voisinage duquel elles sont le plus petices. On peut faire cette observation chez tous les végrétaux, unème chez ccux dont la tige est fistulense. Chez ces derniers, la moelle forme les parois diı canal central, et les vésicules composantes offrent comme à l'ordinaire une grandeur décroissante de dedans en dehors. Je citcrai ici le pissenlit (leotondon taraxacum) comme l'une des plantes herbacées chez lesquelles cette disposition est le plıs facile à observer. La tigge on hampe de ce végétal est fistuleuse; son caual médian occupe le centre de la médulle centrale, qui, blanche et diaphane, forme les parois immédiates de ce canal. En dehors existe le système cortical, dont l'épaisseur est moindre, qui est de couleur verte, et qui contient les vaisscaux du suc laitenx. Une tranche mince et longitndinale de cette tigge étant sounnise an microscope, on voit avec la plus grande facilité le décroissentent des vésicules de dedans en dehors; à l'intéricur, clles ont acquis iant de développement, que la surface interne du canal central s'est garnie d'une mulutude de plis l'ansversaux, résultal de l'augmentation disproporlionnelle de celle surface par le développement considérable des vésicules agglonérées qui composent Ir tissı médnllaire anquel elle appartient. Je système. cortical de la tige dn pissenlit est si mince, yu'il n'est. sulèe possible de voir l'ordre de décroissement des 
vésienles dont i! est eomposé; mais cela se voit sans diffieulıé dans le sysı̀̀me eortieal de la raeine de cette même plante. La raeine du pissenlit offre un système eortical très-volumineux et un système central trèsexigu. Une tranehe lonģitudinale du système eortieal étant soumise an microseope, on voit sans diffienlıé que les vésicules artieulées, dont elle paraît entièrement eomposée, sont déeroissantes de grandeur de dehors en dedans. Il résulte de eette organisation inverse du système eentral et du système eortieal, que ees deux systèmes érant isolés el divisés en lanières longritudinalcs, ees lanières, quand elles appartiennent au sysı̀ne eorlieal, doivent iendre à se courber en dedans; et quand elles appartiennent au système central, doivent tendre à se courber en dehors. C'est effeetivement ce que l'expérience démontre. Une lanière Jongitudinale d'éeoree, prise sur une plante herbaeée ou sur une branehe très-jeune d'un végétal liggneux, étant plongée dans l'eau, se courbe en dedans. Si on la plonge ensuite dans le sirop de sucre, elle se courbe en dehors. Pour que eette expérience réussisse bien, il faut, chez les vécrétaux ligneux, enlever l'épıderme qui sopposerait à la prompte et faeile absorption de l'eau par la partic quil reeouvre. Au eontraire, une lanière longitudinale du système eentral, prise sur une plante herbaeée ou sur une branche très-jeune de végétal liggneux, étant plongée dans l'eau, se eourbe en dehors; transportée dans le sirop de suere, elle se courbe en dedans. Les mêmes phénoniènes s'obṣer- 
vent sur le système cortical $\mathrm{ct}$ sur le systèmc central des racines. Ainsi, les tigges et les racines se ressenblcut cxactcment sous le point de vue de ce phénomènc physioloyiquc, et par conséquent sous le point de vuc de la dispositiou organique à laquellc ce phénomènc est dì. Il résulte de ccs obscrvations, que les mćdulles corticalc et ecnurale son de véritables oıgrancs irritables dont la tcndanee à l'incurvation a lieu dans des scns diamétralement opposćs. Or, comme iccs dcux systèmes sont cylindriques, et que lcs parties dianétralement opposćcs de chaquc cylindrc tendent à l'incurvation, toutes lcs dcux en dedans, ou itoutes les deux en dehors avcc unc même force, ii en r'ésulte que lc caudex vécócal conserve sa rcclitude; cllc cst lc résultat de l'ćquilibrc parfait de toutcs lcs ltendances concentriques à l'incurvation. Lcs expéniences qui vicmuent d’être rappor'tées prouvent que ceetle incurvation dépcund, comme eclle de tous les organcs irriables végétaux, $1^{\circ} \mathrm{dc}$ la grandeur dćcrois- sante de leurs vćsieules conposantcs, qui offrent d'un côtć de la caprcité cn plus, et de l'autre côté de la crupacité cn moins; $2^{\circ}$ de ce que ces vésicules contemant un liquide organiquc d'une dcnsité quelconque, c clles cxereent l'endosnosc lors de l'accession dc l'ean, cl l'exosmosc lors de l'accession extérieurc d'un liquidc plus rlcnsc quc cclui qu'elles contiennent. Ainsi, d'une part, capacité cn plus cu capacilé en moins des vésicules, ct d'une autre parl, densité en plus cı densilé en moins des denx liquides intérieur ct 
extérieur. Voilà les conditions fondanıentales de tontc incurvabilité végétale, et ce sont effectivemeut les causes des incurvations spontanćes qu'affectent les tiges et les racines. Ces caudex possèdent dans leurs nuédulles corticale et centrale des organes de nouvement en action d'incurvation permanente, et que l'équilibre parfait de leur antagonisme circulaire condamne au repos dans l'état naturel; mais qu'une cause quelconque vienne à rompre cet équilibre, cette égalité parfaite d'action d'incurvation, à l'instant les caudex vérgétaux se courberont dans le sens déterminé par l'action d'incurvation de celıi de leurs côtés dont la force sera prépondérante. Il ne s'agit donc que te déterminer les causes particulières qui, en détruisant l'équilibre auquel les caudex yérgétaux doivent lewr situation immobile, les détermine à se courber pour affecter des directions spéciales.

La prédominence de l'incurvation en un sens déterminé, dans une tige ou daus une racine, atteste nécessairement la rupture de l'équilibre qui primitivement maintenait chacum de ces caudex dans lit rectitude, par l'égalité des tendances concentriques ì l'incurvation. Le inoyen le plus simple de rompre cel équilibre est de fendre en deux, longitudinalement, chacun de ces caudex. Je fais cette opération, par exemple, sur une tige et sur une racine de haricot nouvellenent germé. Cousidérous séparément ici la ligge et la racine. La tige offre une prédominence du système central sur le système cortical; ces deux sys- 
tèmes tendent à sc courber en sens inverse : or, dans la nıoitié de lige il y aura une forte tendance du système central à se courber en dehors, et une tendance plus faible dı systènıe eortical à se eourber en dedans, en raison de la prédominence de masse du premier de ces systèmes. Si donc l'on plonge cette noitié de tige dans l'eau, elle se courbera en dehors par l'effet de l'endosmose, et avec une force qui scra égale à l'excès de la tendance à l'incurvation en dehors du système central sur la tendance à l'incurvation en dedans du système cortical. Si l'on transporte eette moitié de tige dans le sirop de sucre, elle perdra sa courbure en dehors et se courbera en dedans, par l'effet de l'exosmose.

La même expérience, faite sur la moitié de racine de haricot fendıe longitudinalement, donne des résultats inverses. La racine offre une prédominence du système cortical sur le système central; par conséquent la tendance du système cortical à se courber en dedans l'cmportera sur la tendance du sysic̀me central à se courber en dchors; ct la moitié de racine étant plongée dans l'cau, se courbera en dedans avec une force égrale à l'excès de la tendance du système cortical à se courber en dedans, sur la tendance du système central à se courber en dehors : eet effet sera dì à l'endosmose. Si l'on transporte cctue noilié de racine dans le sirop de sucre, elle perdra sa courbure cn dedans, et prendra nue courbure en dehors par l'effet de l'exosinose. 
Nulle rige ne manifeste avec plus d'énergie les tendances à l'incurvation dont il vient d'être question, que la tige ou hampe du pissenlit. Une lanière longitudinale de cette tige fistuleuse étant plongée dans l'eau, se roule en dehors sous forme d'une spirale très-serrée. Cette incurvation en dehors a lieu également sans plonger la lanière de tige dans l'eau; mais cette incurvation est bien moins profonde. $\mathrm{Si}$ l'on transporte cette lanière de l'eau dans le sirop de sucre, elle perd sa position roulée en dehors, se redresse, et se roule en spirale en dedans. Cette incurvation en dedans est le résultat de la déplétion générale des vésicules par l'effet de l'exosmose. Cela se voit de la manière la plus facile, en soumettant au nicroscope une petite lanière de tige de pissenlit plongée dans du sirop. On voit ses vésicules composantes, et spécialement les plus grandes, qui sont situées à la partie intérieure, se vider et devenir plus petites. Si on laisse une tige de pissenlit seflétrir un peuavant de la diviser en lanières longitudinales, ces lanières ne se courberont point en dehors dans l'air, comme cela a lieu pour ces mêmes lanières lorsqu'elles appartiennent à une plante fraîche, c'est-à-dire qui contient beaucoup de sève lymphatique. C'est donc l'accession de celte sève lymphatique sur les vésicules remplies d'un liquide dense, qui, dans l'état naturel, provoque l'endosmose de ces vésicules, et par suite l'incurvation du tissu qu'elles forment par' leur assemblage. Ces lanières à demi-flćtries sont dans l'état de flaccidité. Si 
on les plonge dans l'eau, elles reprennent promptement, par l'accession de ce liquide, leur tendance à l'incurvation en dehors. Ainsi, nous voyons que partout l'incurvabilité cxige, pour son exercice, l'accession d'un liquide extérieur sur les vésicules qui conposent le tissu incurvable, et que ce liquide extérieur est toujours la sève lyınphatique, lorsque l'incurvation a lieu par endosmose.

Nous venons de voir que l'incurvation inverse des moitiés longitudinales de tige et de racine cst le résultat du défaut d'équilibrc en sens opposé, qui existc rentre les tendances inverses à l'incurvation des sysItèmes cortical et central de chacune de ces moitiés de caudex végétal. Ceci va nous conduire à la connaissance de la cause qui détermine les tiges et les ra(cines à se courber dans leur entier ell sens opposé, :sous l'influence de la pesanteur.

J'ai couché horizontalement nne tige ou hampe de pissenlit, et je l'ai maintenue dans cette position au moyen d'un poids placé sur la moitié de sa longucur. Au bout de vingt-quatre heures, la tige couchée s'était redressée et dirigée vers le ciel, en se courtbant dansle voisinage de l'obstacle. Je détachai cette tige du sol, j'en retranchai les parties qui avaient cconservé leur rectitude. Je ne voulais étudier que la partie courbée. Je fendis longitudinalement cette partie courbée en deux, en suivant le sens de la sourbure ; jobtins de cette manière deux moitiés de iige courbées, l'une aa (furg. 4) dont l'épiderme oc- 
cupait la concavité dirigrée dans l'état natırel vers le ciel, l'autre $b b$ dont l'épiderme occupait la convexité dirigée dans l'état naturel vers la terre. Ainsi, la première, ou celle d'en haut, était courbée en dehors, et la seconde, ou celle d'en bas, était courbée en dedans. Or, il arriva que la première aa augmenta son incurvation en dehors, et que la seconde $b b$ perdit une partie de son incurvation en dedans, et tendit à se redresser. Ce phénomène devint encore plus seusible en retranchant deux lanières latérales à chacune de ces deux moitiés de tige fistuleuse, et en ne conservant ainsi qu'une seule lanière médiane pour clacune de ces moitiés. La lanière médiane de la portion supérieure aa se courba plus fortement en dehor's, la lanière médiane de la portion infériemre $b b$ se redressa complètement. Cette observation prouve que la moitié inférieure bb étail courbée en dedans malgré elle, on dans le sens opposé à celui de sa tendance naturelle à l'incurvation. Etant abandontée à elle-même par sa séparation de la moitié supérieurc $a a$, elle tendait au redressement et à l'incurvation en dehors, qui étail le sens naturel de sa tendance, mais cette tendance naturelle à l'incurvation en dehors était affaiblie, elle n'était pas à beaucoup près aussi énergique que celle de la portion supérieure a Ainsi, dans la plante vivante et sur pied, les deux moitiés longitudinales de tige $a a$ et $b b$ tendaient tontes les deux à l'incurvation en dehor's, comme c'est l'ordinaire. Mais cette tendance à l'incurvation en 
lelors's étant affublie dans la moitié longiundinale infinieure bb, et la moitié longritudinale supérieure au ayant conservé sa tendance à l'incurvation en dehors dans toute soll intégrrité, il est résulté de cette rupture d'équilibre, que la moitié de tigge supérieure aa, par sa prédominence d'action d'incurvation en dehors, a conrbé la tige toute entière dans le sens d'incurvation qui lui est propre. La moitié de tige infélieme $b b$ ayant une action d'incurvation en dehors I moindre, a ćté vaincue el entraînée malgré elle clans ınn étai de courbure contraire à celıi qui résulte de sa tendance naturelle. Ainsi, la courbure que prend łme tige couchée horizontalement, pour diriger son -sommet vers le cicl, dépend de la rupture de l'équillibre ou de l'égalité d'action d'incurvation en deHors dans ses deux moitiés longitudinales supérieme cet inférieure. Cette dernière, qui regarde la terre, iétant affaiblie, et son antagoniste, qui regarde le ciel, ayant conservé toute sa force, la tige toute entière est courbée dans le sens d'incurvation en dehors et en Hant, qui est propre au côté vainqueur, et le sommet He la tige se trouve ainsi dirigé vers le ciel. Passons inctuellement à la cause de la direction des racines vers la terre.

J'ai pris un haricot germé, dont la radicule, paraitcment droite, avait acquis une longrueur d'environ in ponce. Je domnai à cette radicule une position hoizontale, et bientôt elle se courba pour diriger sa nointe vers la tcree. Je détachai cette racine courbée, 
et je la fendis longitudinalement en deux, en sıiváné le sens de la courbure. J'obtins, de cette manière, denx moitiés de racine courbées, l'une aa (figr. 5 ), dont l'épiderme oecupait la convexité, dirigée, dans l'état naturel, vers le cicl; l'autre $b b$, dont l'épiderme occupait la concavité, dirigée, dans l'état naturel, vers la terre. Ainsi, la première, ou celle d'en liaut, était courbée en dedans, et la seconde, ou celle d'en bas, était courbée en dehors. Ayant plongé ces deux moitiés de racine dans l'eau, la moitié supérieure aa augmenta sa courbure; la moitié inférienre $b b$, au contraire, perdit la sienne et se redressa. Par conséquent, dans cette circonstance, la moitié inférieure bb était courbéc en dehors, malgré elle, ou dans le sens contraire à celui de sa tendance naturelle à l'incurvation, tendance qui, chez les racines, a lieu en. dedans, ainsi que nous l'avons vu plus haut. Cependant, cette moitié longitudinale de racine $b b$, plongée dans l'eau, ne fit que perdre sa position forcément courbée en dehors, clle atteignit la reetitude sans se courber en dedans, comme cela a lien ordinairement. Cette moitié longitudinale inféricure $b b$ a donc perd $ı$ une partie de sa tendance à l'incurvation en dedans: cctte tendance est affaiblie; or, comme cètte neême tendance naturelle à l'incurvation en dedans cxiste dans tonte son intégrité chez la moitié longritudinalc supérieure $a a$, il résulte de cette rupture d'équilibre, ou de cette inégalité de force d'incurvation en dedans, dans les deux côtés supéricur $a a$ et inférieur $b b$, quc 
re dérnier est vaincu par la prédominance de force d'incurvation, en dedans et en bas de son côté antagoniste $a a$; de cette manière, la pointe de la racine se trouve ramenée vers la terre.

Une conclusion importante se déduit de ces deux observations. Dans la tige courbée (fig. 4), comme dans 'la racine courbée (fig. 5), c'est toujours le côté supérieur $a a$ qui est vainqueur du côté inférieur $b b$, et qui lui iniprime de force le mode de courbure qui lui est propre. Cette prédominance d'action d'incurvation du côté supérieur aa provient, dans la tige comme dans la racine, de l'affaiblissement de l'action d'incurvation dans le côté inférieur $b 6$. Quelle est donc la cause qui, dans une tige ou dans une racine couchée horizontalement, affaiblit la tendance à l'incurvation qui est propre au côté de cette tige ou (de cette racine qui regarde la terre? C'est encore ll'expérience qui va nous résoudre ce dernier problême. Reportons-nous d'abord à nos connaissances précédemment acquises. Nous savons que la force H'incurvation est proportionnelle à la force de l'enHosmose des vésicules qui composent le tissu incurvable; par conséquent, l'affaiblissement de cette force l'incurvation provient de l'affaiblissement de l'endosmose. Il s'agit donc de déterminer quelle est, dans 'ette circonstance, la cause de l'affaiblissement de iendosmose. Cet affaiblissement peut avoir lieu de rois manières : $I^{0}$ par le défaut d'accession de la sève ymphatique en quantité suffisante; $2^{\circ}$ par la diminu- 
tion de densité du liquide intérieur des vésicules; 3॰ par l'augmentation de densité de la sève lymphatique, qui est ici le liquide extérieur aux vésicules. Il n'existe aucune raison pour qu'il y ait une diminution dans la quantité de sève lympathique que reçoit la partie latérale inférieure des caudex végétaux, couchés horizontalement; il n'existe, de même, aucune raison pour que le liquide intérieur des vésicules composantes de cette même partie latérale inférieure éprouve de la diminution dans sa densité par l'effet de la pesantenr. L'exclusion de ces deux premières manières dont peut avoir lieu l'affaiblissement de l'endosmose, nous met dans la nécessité d'adopter la troisième, et nous allons voir cette adoption confirmée et légitimée par l'expérience. Lorsque deux liquides, imparfaitement mêlés, sont ‘réunis dans un même vase, le plus dense se précipite vers la partie inférieure, et le moins dense occupe la partie supérieure. Or, la sève lymsphatique n'est point un liquide homogène et partont le même; lors de son introduction dans le végétal, ce n'est que de l'eau pure; cette eau acquiert peu à peı une densité plus considérable, par la dissolution qu'elle opère des liquides organiques. Ce fait est bien prouvé par les expériences de M. Knight. Lorsqu'un caudex végétal est couché horizontalement, la sève la plus dense doit se précipiter vers le côté qui regarde la terre; la sève la plus aqueuse, et par conséquent la plus légère, doit demeurer dans le côté qui regarde le ciel. 
Cette induction rationnelle est pleinement confirmiée par l'expérience. Je pris de jeunes tiges de bottrache dont j’avais sollicité le redressement vers le ciel, en les maintenant courbées vers la terre. Je retranchai les parties droites de ces tiges, et ne conservai que les portions courbées. Je fendis en deux ces tiges courbées par une section longitudiuale pratiquée dans le sens de la courbure, de la même manière que cela est représenté pour' la tige du pissenlit, dans la fig. 4. Je plongeai ces deux moitiés de tige dans l'eau : elles se précipitèrent au fond, parce que leur pesanteur spécifique était plus considérable que celle de l'eau. Je les transportai dans de l'eau sucrée, suffisamment dense pour que ces deux moitiés de tige surnageassent; alors j’ajoutai de l'eau peu à peu à la solution sucrée, et je diminuai ainsi sa densité d'une manière graduelle; bientôt je vis la moitié de tige inférieure, c'est-à-dire celle qui, dans l'état naturel, était située du côté de la terre, se précipiter au fond du liquide, tandis que la moitié de tige supérieure continuait de surnager. J'ai répété cette expérience un grand nombre de fois, et toujours avec le même résultat. Je dois faire observer ici que l'on ne doit faire cette expérience qu'avec des plantes dont la moelle est entièrement remplie de liquides, et ne contient point d'air du tout. Or, les jeunes tiges de bourache remplissent parfaitement à cet égrard les vues de l'expérimentateur; il faut avoir soin seulement qu'il ne reste point de bulles d'air adhérentes 
aux poils dont. l'écnrce de la plante est chargée. Ces expériences prouvent que la tige qui s'est courbée pour se redresser, offre une pesanteur spécifique plus grande dans sa moitié longitudinale inféricure que dans sa moitié longitudinale supérieure; ccllc-ci contient donc des liquides dont la densité est plus grande que ne l'est la densité des liquides contenus dans la moitié supérieure. Celte déduction est rigoureuse; car la matière solide du végétal, qui consiste toute entière dans les parois des vésicules ou des tubes, n'est pas susceptible d'augrmenter de pesanteur d'un instant à l'autre. La sève lymphatique, au contraire, peut devenir plus dense en très-peu de temps dans la partie latérale qui regarde la terre, chez une tige ou clicz une racine placée horizontalement, parce que la pcsanteur précipite nécessairement vers la partie inféricure la portion la plus dense ou la plus pesante de cette sève, dont la diffusion s'opère avec la plus grande facilité dans le tissu végétal. Les résultats de cette précipitation de la sève, la plus dense dans la partie latérale inférieure des caudex placés horizontalement, sonı faciles à déduire. Nous avons vu plus haut que l'accession extérieure de la sève lymphatique sur les vésicules composantes des tissus incurvables, est la cause de l'endosmose de ces vésicules, et par suitc la cause de l'incurvation des tissus qu'elles composent. Or, plus ce liquide extérieur est dense, moins il y a de force d'endosmose dans les vésicules, moins par conséquent il y a de force d'incurvation. La partic 
latérale des caudex horizontaux qui regarde la terre, contenant une sève lymphatique plus dense que ne l'est celle que contient la partie latérale opposée qui regarde le ciel, il en résulte une rupture de l'équilibre qui existait antérieurement entre les tendances concentriques à l'incurvation. Le côté inférieur se trouve affaibli, le côté supérieur a conservé toute la force de sa tendance à l'incurvation; dès lors ce dernier, doué d'une force prédominante, entraîne son antagoniste vaincu dans le sens d'incurvation qui lui est propre. Ce sens propre de l'incurvation est en dehors pour la tige et en dedans pour la racine, par. conséquént dans la tige horizontale, le côté qui regarde le ciel se courbant en dehors, dirige le sommet de cette tige vers le ciel; et dans la racine.horizontale, le côté qui regarde le ciel se courbant en dedans, dirige la pointe decette racine vers la terre. Ces deux caudex opèrent ensuite leur élongation, selon les directions opposées dans lesquelles ils sont constamment maintenus par la cause qui les y a placés. Voilà tout le mystère de ces deux directions spéciales opposées l'une à l'autre. Il n'y a point, à proprement parler, de tendance de la tige vers le ciel, ni de tendance de la racine vers la terre; il n'existe dans ces caudex végétaux que des tendances à l'incurvation dans des sens diamétralement opposés, et qui sont mises en jeı par laction de la pesantenr, ce qui fail que ces caudex régétaux affectent la direction verlicale.

Ce n'est pas sendement lorsque la racine et la tige 
sont horizontales, qu'elles se fléchissent pour se diriger, la première vers la terre, et la seconde vers le ciel. Le retournement de ces caudex végétaux a lieu également lorsqu'ils sont verticalement placés dans une position renversée, c'est-à-dire la racine en haut, et la tige en bas. Il semblerait que, dans cette circonstance, la théorie que je viens d'exposer ne serait point applicable, puisqu'il n'y aurait point de côté ou de partie latérale inférieure vers laquelle la sève la plus dense ait à se précipiter. Mais il ne faut pas perdre de vue que la, rectitude mathématique n'appartient poịt aux caudex végétaux; il en résulte qu'il est impossible de donner à ces caudex renversés une position verticale dans le sens rigoureux et mathématique. J'ai expérimenté que lorsqu'on dirige vers le ciel des radicules de graines en germination, l'inflexion de ces radicules, pour se retourner, a toujours lieu du côté où elles ont une inclinaison, même la plus légère. La même chose a lieu pour les tiges; mais il est nécessaire de faire observer que ces expériences doivent être faites dans une obscurité complète, car la lumière possède sur les tiges une grande puissance pour opérer leur direction. Ainsi, c'est toujours la partie latérale la plus basse ou la plus voisine de la terre, qui, dans les caudex végétaux, perd une partie de la force de sa tendance naturelle à l'incurvation. Il n'est pas nécessaire pour cela que cette partie latérale soit placée horizontalement; la plus légère déviation de la position verticale suffit pour produire 
cet effet. On sent que s'il était possible qu'une radicule fût pourvue d'une force d'incurvation mathématiquement égrale dans toutes ses parties latérales opposées, et qu'elle fût dirigée vers le ciel dans une position verticale mathématique, elle resterait dans cette position, n'y ayant aucune raison qui puisse la déterminer à opérer son inflexion d'un côté plutôt que d'un autre. Mais cette égalité mathématique dans les forces opposées qui animent les côtés opposés de la radicule n'existe point. Sa rectitude mathématique n'existe point non plus; par conséquent, sa position verticale mathématique est impossible; et quand bien même cette position serait possible, la radicule ne laisserait pas de trouver un moyen de commencement d'inflexion dans le défaut d'une égalité mathématique entre les forces d'incurvation de ses parties latérales opposées; et dès lors, l'action de la pesanteur agirait sur cette radicule fléchie, pour déterminer l'achèvement de son inflexion : le même raisonnement pent être fait par rapport à la tigé.

Au reste, ce n'est que dans leur jeunesse, et tant qu'ils conservent leur flexibilité, que les caudex végétaux peuvent opérer leur retournement, qui devient impossible lorsqu'ils ont acquis de la dureté; aussi les arbres, dont le bois est très-mou, conservent plus long-temps que les autres cette propriété de se fléchir spontanément.J'ai vu un peuplier (populus fastigiata) de la grosseur diu pnignet, qui, placé accidentellemeit dans une position inclinée, se courba pour ra- 
mener la partic supérieure de sa tige à la position verticale; mais il lui fallut toute une période annuelle de végétation pour opérer cette inflexion.

Lorsque des graines en germination sont fixées à la çirconférence d'une roue, soit verticale, soit horizontale, qui tourne avec une certaine rapidité, les tiges se dirigent vers le centre de la rotation, et les racines vers la circonférence. On doit la découverte de ce phénomène à M. Knight, et j’en ai confirmé la réalité par mes expériences. J'ai fait voir en même temps qne cette double direction des caudex végétaux n'a point lieu lorsque la rotation trop lente ne produit point de force centrifuge appréciable. La cause de cette double direction est facile à déterminer. Les deux caudex opposés d'une graine en germination $\mathbf{A}$ (fig. 6), sont disposés tangentiellement à la circonférence d'une roue qui tourne rapidement sur son axe; la force centrifuge projette la sève la plus dense vers le côté extérieur $b b$ de la tige et de la racine; de là résulte l'affaiblissement de la force d'incurvation de ce côté, et la prédominance de force du côté opposé $a a$; dès lors le côté $a$ de la tige, dont la force est prédominante, et qui tend à se courber en dehors, dirige le sommet de la tige vers le centre de la rotation; comme on le voit en $B$, le côté $a$ de la racine, dont la force est également prédominante, et qui tend à se courber en dedans, dirigre la pointe de la ra-. cine dans une direction opposée à celle de la tige.

Ces observations, comme on le voit, dévoilent 
complètement le phénomène jusqu’ici si mystérieux de l'ascension des tiges et de la descente des racines. Ce phénoniène est beaucoup plus simple qu'on ne paraissait le supposer. Certains esprits ont pu ètre tentés de croire qu'il existait là une sorte de polarité analogue à celle qui dirige les deux pôles opposés de l'aigguille aimantée vers les deux pôles de la terre, mais toutes les expériences portent à rejeter bien loin cette hypothèse. La double tendance qui résulte de la polarité appartient à toutes les parties dans lesquelles un aimant peut être divisé. Or, dans une tige séparée de sa racine, il n'existe plus de double tendance. C'est toujours sa partie demeurée libre et mobile qui se dirige vers le ciel. Ainsi, en supposant cette tigre suffisamment entretenue de sève lymphatique et placée dans une position horizontale, on verra sa partie inférieure se diriger vers le ciel lorsque sa partie supérieure sera fixée invariablement. Si celte lige horizontale est fixée par son milieu, ses deux moitiés se dresseront également vers le ciel; si cette tige horizontale et ployée en arc est fixée par ses deux extrémités, cel arc horizontal se dressera, et deviendra vertical; il est donc bien prouvé qu“il n'existe dans la tige aucune polarité, aucune tendance à diriger spécialentent son sonmuet vers le ciel; il n'y a point chez cette tige une disposition ou une organisation spéciale qui exige que son sommet soil en haut et que la base soit en bas. C'est simplement en sa qralité de partie libre et mobile, que le sommet de la tige est. dirigré vers le cicl. La base de cette tige peut ètre ar- 
tificiellement placée dans cette direction, sans qu'il en résulte aucun inconvénient pour le végétal. C'est ce qui arrive lorsqu'on plante des arbres la tête en bas. Ainsi, il n'y a point à douter que le phénomène de la direction spéciale quaffecte la tige et la racine n'ait sa cause toute entière dans le mode particulier d'incurvation qui est propre à cette tige et à cette racine.

Il y a des tiges qui dirigent leur sommet vers la terre comme des racines. Cela provient indubitablement de ce que, par anomalie, elles possèdent la même organisation que les racines. Je n'ai point encore assez étudié ce phénomène.

Il y a des parties des végétaux qui se dirigent vers la lumière, il y en a d'autres qui la fuient. Je possède déjà plusieurs faits pour l'établissement de la théorie de ces deux directions spéciales opposées, mais ce travail est encore trop incomplet pour pouvoir être publié. Je puis dire seulement ici que je regarde comme certain que tous les phénomènes de direction spéciale que présentent les végétaux, soit dans leur action de rechercher ou de fuir la lumière, soit dans leur sommeil ou dans leur nutation, dépendent des diverses manières dont l'équilibre ordinaire de leurs forces d'incurvation peut être altéré par la présence ou par l'absence de la lumière. Ici s'ouvre un champ très-vaste de recherches extrêmement curieuses.

FIN. 


\section{TABLE}

\section{DES MATIÉRES.}

Nouvelles recherches sur l'endosmose et l'exosmose. I Recherches sur la cause et sur le mécanisme de l'irritabilité végétale. ............ 55

De la direction des tiges vers le ciel, et des racines vers la terre..................... $8_{9}$ 



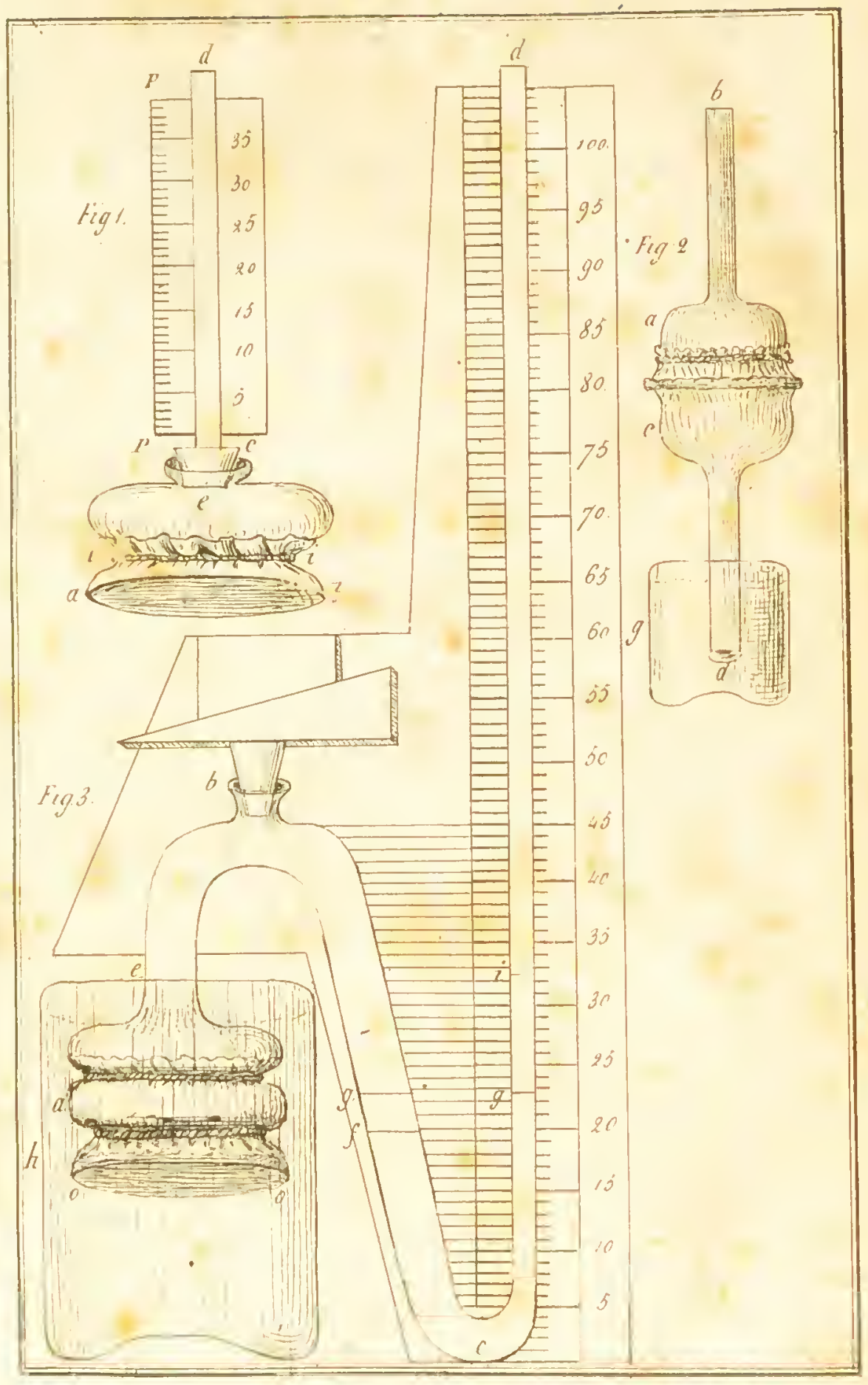




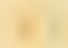

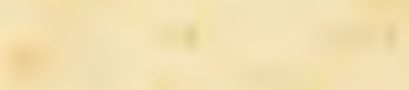

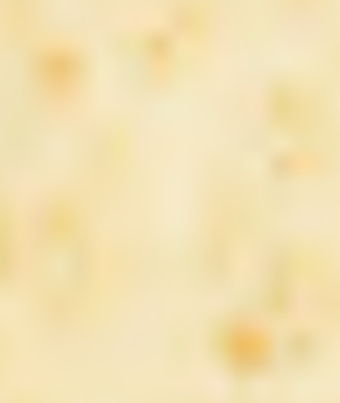

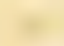

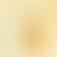

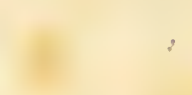

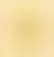

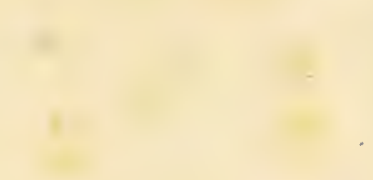




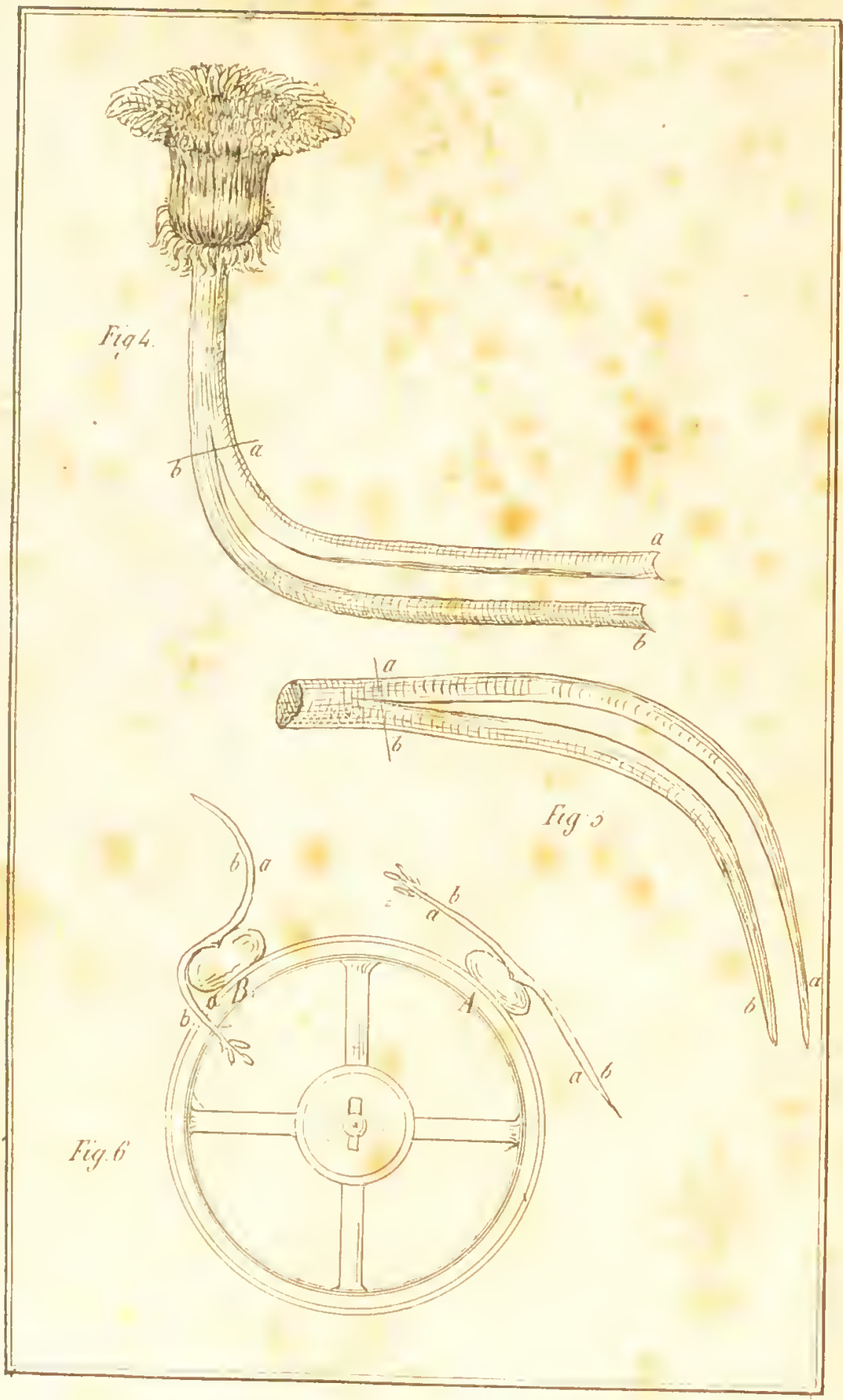




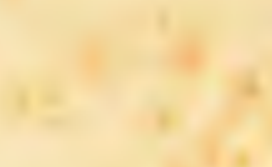

$$
\begin{aligned}
& \text { + }
\end{aligned}
$$

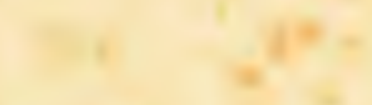

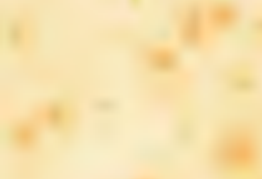

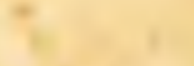

(2n

$\sqrt{2}$

$\sqrt{2}+x^{2}$

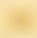

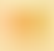
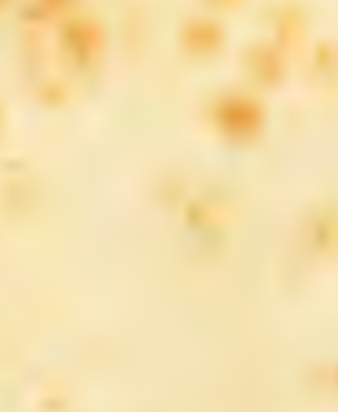

$+2$

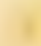

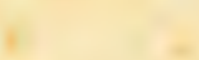

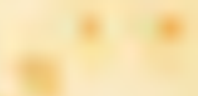

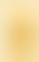

$\sqrt{2}$

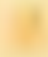

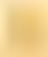





